Journal of

Clinical Medicine

\title{
Omega-3 Fatty Acids in Health and Disease
} Lindsay Brown, Bernhard Rauch and Hemant Poudyal Printed Edition of the Special Issue Published in Journal of Clinical Medicine 
Lindsay Brown, Bernhard Rauch and

Hemant Poudyal (Eds.)

\section{Omega-3 Fatty Acids in Health and Disease}


This book is a reprint of the Special Issue that appeared in the online, open access journal, Journal of Clinical Medicine (ISSN 2077-0383) from 2015-2016, available at:

http://www.mdpi.com/journal/jcm/special_issues/omega-3-fatty-acids

Guest Editors

Lindsay Brown

University of Southern Queensland

Australia

Bernhard Rauch

Institut für Herzinfarktforschung

Germany

Hemant Poudyal

Kyoto University

Japan

Editorial Office

MDPI AG

St. Alban-Anlage 66

Basel, Switzerland
Publisher

Shu-Kun Lin
Managing Editor

Allen Duan

\section{Edition 2016}

MDPI • Basel • Beijing $\bullet$ Wuhan $\bullet$ Barcelona $\bullet$ Belgrade

ISBN 978-3-03842-272-3 (Hbk)

ISBN 978-3-03842-273-0 (PDF)

Articles in this volume are Open Access and distributed under the Creative Commons Attribution license (CC BY), which allows users to download, copy and build upon published articles even for commercial purposes, as long as the author and publisher are properly credited, which ensures maximum dissemination and a wider impact of our publications. The book taken as a whole is ( 2016 MDPI, Basel, Switzerland, distributed under the terms and conditions of the Creative Commons by Attribution (CC BY-NC-ND) license (http://creativecommons.org/licenses/by-nc-nd/4.0/). 


\section{Table of Contents}

List of Contributors ......................................................................................................... VII

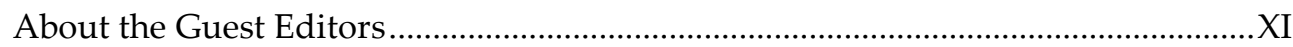

\section{Chapter 1: Omega-3 Fatty Acids and Cancer}

\section{Donatella D'Eliseo and Francesca Velotti}

Omega-3 Fatty Acids and Cancer Cell Cytotoxicity: Implications for Multi-

Targeted Cancer Therapy

Reprinted from: J. Clin. Med. 2016, 5(2), 15

http://www.mdpi.com/2077-0383/5/2/15.

Georgia Lenihan-Geels, Karen S. Bishop and Lynnette R. Ferguson

Cancer Risk and Eicosanoid Production: Interaction between the Protective Effect of Long Chain Omega-3 Polyunsaturated Fatty Acid Intake and Genotype Reprinted from: J. Clin. Med. 2016, 5(2), 25

http://www.mdpi.com/2077-0383/5/2/25.

Mandi M. Hopkins, Zhihong Zhang, Ze Liu and Kathryn E. Meier

Eicosopentaneoic Acid and Other Free Fatty Acid Receptor Agonists Inhibit Lysophosphatidic Acid- and Epidermal Growth Factor-Induced Proliferation of Human Breast Cancer Cells

Reprinted from: J. Clin. Med. 2016, 5(2), 16

http://www.mdpi.com/2077-0383/5/2/16. .59

\section{Homer S. Black and Lesley E. Rhodes}

Potential Benefits of Omega-3 Fatty Acids in Non-Melanoma Skin Cancer

Reprinted from: J. Clin. Med. 2016, 5(2), 23

http://www.mdpi.com/2077-0383/5/2/23 .79 


\section{Chapter 2: Omega-3 Fatty Acids and Psychiatric Disorders}

Paola Bozzatello, Elena Brignolo, Elisa De Grandi and Silvio Bellino

Supplementation with Omega-3 Fatty Acids in Psychiatric Disorders: A Review of Literature Data

Reprinted from: J. Clin. Med. 2016, 5(8), 67

http://www.mdpi.com/2077-0383/5/8/67. 95

\section{Gregor Berger}

Comments on Bozzatello et al. Supplementation with Omega-3 Fatty Acids in Psychiatric Disorders: A Review of Literature Data. J. Clin. Med. 2016, 5, 67

Reprinted from: J. Clin. Med. 2016, 5(8), 69

http://www.mdpi.com/2077-0383/5/8/69.

\section{Chapter 3: New Aspects in Nutrition}

\section{Stanislaw Klek}

Omega-3 Fatty Acids in Modern Parenteral Nutrition: A Review of the

Current Evidence

Reprinted from: J. Clin. Med. 2016, 5(3), 34

http://www.mdpi.com/2077-0383/5/3/34.

\section{Alicia I. Leikin-Frenkel}

Is there A Role for Alpha-Linolenic Acid in the Fetal Programming of Health?

Reprinted from: J. Clin. Med. 2016, 5(4), 40

http://www.mdpi.com/2077-0383/5/4/40.

Michael E. R. Dugan, Payam Vahmani, Tyler D. Turner, Cletos Mapiye, Manuel Juárez, Nuria Prieto, Angela D. Beaulieu, Ruurd T. Zijlstra, John F. Patience and Jennifer L. Aalhus

Pork as a Source of Omega-3 (n-3) Fatty Acids

Reprinted from: J. Clin. Med. 2015, 4(12), 1999-2011

http://www.mdpi.com/2077-0383/4/12/1956 
Agustina Creus, María R. Ferreira, María E. Oliva and Yolanda B. Lombardo Mechanisms Involved in the Improvement of Lipotoxicity and Impaired Lipid Metabolism by Dietary $\alpha$-Linolenic Acid Rich Salvia hispanica L (Salba) Seed in the Heart of Dyslipemic Insulin-Resistant Rats

Reprinted from: J. Clin. Med. 2016, 5(2), 18

http://www.mdpi.com/2077-0383/5/2/18.

\section{Chapter 4: Basic Research}

\section{Francesco Visioli}

Lipidomics to Assess Omega 3 Bioactivity

Reprinted from: J. Clin. Med. 2015, 4(9), 1753-1760

http://www.mdpi.com/2077-0383/4/9/1753

\section{Jennifer L. Watts}

Using Caenorhabditis elegans to Uncover Conserved Functions of Omega-3 and Omega-6 Fatty Acids

Reprinted from: J. Clin. Med. 2016, 5(2), 19

http://www.mdpi.com/2077-0383/5/2/19

Grace G. Abdukeyum, Alice J. Owen, Theresa A. Larkin and Peter L. McLennan Up-Regulation of Mitochondrial Antioxidant Superoxide Dismutase Underpins Persistent Cardiac Nutritional-Preconditioning by Long Chain $n-3$ Polyunsaturated Fatty Acids in the Rat Reprinted from: J. Clin. Med. 2016, 5(3), 32 http://www.mdpi.com/2077-0383/5/3/32

\section{Marijana Todorčević and Leanne Hodson}

The Effect of Marine Derived n-3 Fatty Acids on Adipose Tissue Metabolism and Function

Reprinted from: J. Clin. Med. 2016, 5(1), 3

http://www.mdpi.com/2077-0383/5/1/3. 



\section{List of Contributors}

Jennifer L. Aalhus Agriculture and Agri-Food Canada, Lacombe Research Centre, Lacombe T4L 1W1, AB, Canada.

Grace G. Abdukeyum Division of Medical and Exercise Science, School of Medicine, Faculty of Science Medicine and Health, University of Wollongong, Wollongong NSW 2522, Australia.

Angela D. Beaulieu Prairie Swine Centre, Inc., Saskatoon S7H 3J8, SK, Canada.

Silvio Bellino Centre for Personality Disorders, Department of Neuroscience, University of Turin, 10126 Turin, Italy.

Gregor Berger Department of Child and Adolescent Psychiatry and Psychotherapy, Outpatient Clinics and Specialized Care, Emergency Services, University Hospital of Psychiatry Zurich, Neumünsterallee 3, P.O. Box 1482, 8032 Zurich, Switzerland.

Karen S. Bishop Auckland Cancer Society Research Centre, University of Auckland; Private Bag 92019, Auckland 1142, New Zealand.

Homer S. Black Department of Dermatology, Baylor College of Medicine, Houston, TX 77030, USA.

Paola Bozzatello Centre for Personality Disorders, Department of Neuroscience, University of Turin, 10126 Turin, Italy.

Elena Brignolo Centre for Personality Disorders, Department of Neuroscience, University of Turin, 10126 Turin, Italy.

Agustina Creus Department of Biochemistry, School of Biochemistry, University of Litoral, Ciudad Universitaria, Paraje El Pozo, CC 242, (3000) Santa Fe, Argentina.

Donatella D'Eliseo Department of Ecological and Biological Sciences (DEB), La Tuscia University, Largo dell'Università, 01100 Viterbo, Italy; Department of Molecular Medicine, Istituto Pasteur-Fondazione Cenci Bolognetti, Sapienza University of Rome, 00161 Rome, Italy.

Elisa De Grandi Centre for Personality Disorders, Department of Neuroscience, University of Turin, 10126 Turin, Italy.

Michael E. R. Dugan Agriculture and Agri-Food Canada, Lacombe Research Centre, Lacombe T4L 1W1, AB, Canada.

Lynnette R. Ferguson Auckland Cancer Society Research Centre, University of Auckland; Private Bag 92019, Auckland 1142, New Zealand. 
María R. Ferreira Department of Biochemistry, School of Biochemistry, University of Litoral, Ciudad Universitaria, Paraje El Pozo, CC 242, (3000) Santa Fe, Argentina.

Leanne Hodson Oxford Centre for Diabetes, Endocrinology and Metabolism, University of Oxford, Churchill Hospital, OX3 7LE Oxford, UK.

Mandi M. Hopkins Department of Pharmaceutical Sciences, College of Pharmacy, Washington State University, Spokane, WA 99163, USA.

Manuel Juárez Agriculture and Agri-Food Canada, Lacombe Research Centre, Lacombe T4L 1W1, AB, Canada.

Stanislaw Klek Stanley Dudrick's Memorial Hospital, General Surgery Unit, Skawina 32-050, Poland.

Theresa A. Larkin Centre for Human and Applied Physiology, Graduate School of Medicine, School of Medicine, Faculty of Science Medicine and Health, University of Wollongong, Wollongong NSW 2522, Australia.

Alicia I. Leikin-Frenkel Bert Strassburger Lipid Center, Sheba, Tel Hashomer, Ramat Gan 52621, Israel; The Sackler Faculty of Medicine, Tel Aviv University, Tel Aviv 69978, Israel.

Georgia Lenihan-Geels Wageningen University and Research Centre, 6708 PB Wageningen, the Netherlands.

Ze Liu Department of Pharmaceutical Sciences, College of Pharmacy, Washington State University, Spokane, WA 99163, USA.

Yolanda B. Lombardo Department of Biochemistry, School of Biochemistry, University of Litoral, Ciudad Universitaria, Paraje El Pozo, CC 242, (3000) Santa Fe, Argentina.

Cletos Mapiye Department of Animal Sciences, Stellenbosch University, Stellenbosch 7602, South Africa.

Peter L. McLennan Centre for Human and Applied Physiology, Graduate School of Medicine, School of Medicine, Faculty of Science Medicine and Health, University of Wollongong, Wollongong NSW 2522, Australia.

Kathryn E. Meier Department of Pharmaceutical Sciences, College of Pharmacy, Washington State University, Spokane, WA 99163, USA.

María E. Oliva Department of Biochemistry, School of Biochemistry, University of Litoral, Ciudad Universitaria, Paraje El Pozo, CC 242, (3000) Santa Fe, Argentina. 
Alice J. Owen Centre of Cardiovascular Research \& Education in Therapeutics, School of Public Health \&amp; Preventive Medicine, Monash University, Melbourne VIC 3004, Australia.

John F. Patience Department of Animal Science, Iowa State University, Ames, IA 50011-3150, USA.

Nuria Prieto Agriculture and Agri-Food Canada, Lacombe Research Centre, Lacombe T4L 1W1, AB, Canada.

Lesley E. Rhodes Photobiology Unit, Dermatology Centre, University of Manchester, Salford Royal Hospital, Manchester M6 8HD, UK.

Marijana Todorčević Oxford Centre for Diabetes, Endocrinology and Metabolism, University of Oxford, Churchill Hospital, OX3 7LE Oxford, UK.

Tyler D. Turner Josera GmbH \& Co. KG, Kleinheubach 63924, Germany.

Payam Vahmani Agriculture and Agri-Food Canada, Lacombe Research Centre, Lacombe T4L 1W1, AB, Canada.

Francesca Velotti Department of Ecological and Biological Sciences (DEB), La Tuscia University, Largo dell'Università, 01100 Viterbo, Italy.

Francesco Visioli Department of Molecular Medicine, University of Padova, Via 8 Febbraio, 2-35122 Padova, Italy.

Jennifer L. Watts School of Molecular Biosciences and Center for Reproductive Biology, College of Veterinary Medicine, Washington State University, Pullman, WA 99164, USA.

Zhihong Zhang Department of Pharmaceutical Sciences, College of Pharmacy, Washington State University, Spokane, WA 99163, USA.

Ruurd T. Zijlstra Department of Agricultural, Food and Nutritional Sciences, University of Alberta, Edmonton T6G 2R3, AB, Canada. 



\section{About the Guest Editors}

Lindsay Brown is a pharmacologist now working at the University of Southern Queensland as Professor of Biomedical Sciences. His major research area is functional foods where his group determines whether foods, such as Queen Garnet plums, purple carrots, seaweeds, flavonoids and omega-3 unsaturated fatty acids, can reverse chronic inflammatory diseases such as obesity.

Hemant Poudyal was born in Sikkim, India and received his Masters of Biotechnology degree (2009) and his Ph.D. (2013) from the University of Queensland, Australia. Since 2014 he has been working at Hakubi Center of Advanced Research and the Graduate School of Medicine, Kyoto University as a program-specific Assistant Professor. His research interests include the effects of nutrients, including omega-3 fatty acids, and incretin hormones on the cardiovascular system. He has co-authored several peer-reviewed publications in these areas.

Bernhard Rauch (BR, born in Heidenheim/Brenz, Germany 1947) received his M.D. (1977), habilitation (1988), "venia legendi" (1989) and the degree of an extraordinary (apl) Professor (1995) from the University of Heidelberg, Germany. From 1976-1978, BR was a research fellow of the Max-Planck Society in Germany (membrane physiology and enzyme kinetics). BR completed his education at the University of Heidelberg (internal medicine 1987, cardiology 1989) and became senior physician (1987-1993). In between he had a stay at the University of Connecticut, U.S.A. as a research fellow of the American Heart Association Connecticut (1984-1985, research in membrane receptor biochemistry). From 1993-2001 BR was medical director of the rehabilitation center Waldkirch/Freiburg. Thereafter BR changed to the heart center Ludwigshafen, where he initiated a new cardiovascular rehabilitation center, of which he was medical director from 2009 until his retirement in 2013. Actually BR is senior scientific consultant of the "Institut für Herzinfarktforschung-IHF" (Institute for Heart Attack Research) concentrating on clinical sciences and registries. 



\section{Chapter 1:}

Omega-3 Fatty Acids and Cancer 



\title{
Omega-3 Fatty Acids and Cancer Cell Cytotoxicity: Implications for Multi-Targeted Cancer Therapy
}

\section{Donatella D'Eliseo and Francesca Velotti}

\begin{abstract}
Cancer is a major disease worldwide. Despite progress in cancer therapy, conventional cytotoxic therapies lead to unsatisfactory long-term survival, mainly related to development of drug resistance by tumor cells and toxicity towards normal cells. n-3 polyunsaturated fatty acids (PUFAs), eicosapentaenoic acid (EPA) and docosahexaenoic acid (DHA), can exert anti-neoplastic activity by inducing apoptotic cell death in human cancer cells either alone or in combination with conventional therapies. Indeed, n-3 PUFAs potentially increase the sensitivity of tumor cells to conventional therapies, possibly improving their efficacy especially against cancers resistant to treatment. Moreover, in contrast to traditional therapies, $n-3$ PUFAs appear to cause selective cytotoxicity towards cancer cells with little or no toxicity on normal cells. This review focuses on studies investigating the cytotoxic activity of n-3 PUFAs against cancer cells via apoptosis, analyzing the molecular mechanisms underlying this effective and selective activity. Here, we highlight the multiple molecules potentially targeted by n-3 PUFAs to trigger cancer cell apoptosis. This analysis can allow a better comprehension of the potential cytotoxic therapeutic role of n-3 PUFAs against cancer, providing specific information and support to design future pre-clinical and clinical studies for a better use of $n-3$ PUFAs in cancer therapy, mainly combinational therapy.
\end{abstract}

Reprinted from J. Clin. Med. Cite as: D'Eliseo, D.; Velotti, F. Omega-3 Fatty Acids and Cancer Cell Cytotoxicity: Implications for Multi-Targeted Cancer Therapy. J. Clin. Med. 2016, 5, 15.

\section{Introduction}

Cancer is a major burden of disease worldwide and, in certain countries, it ranks the second most common cause of death following cardiovascular diseases [1]. Furthermore, as elderly people are most susceptible to cancer and population aging continues, cancer is projected to become the leading cause of death worldwide in many countries. Despite progress made in recent years in cancer therapy, traditional cytotoxic therapies such as chemo- and radio-therapy have multiple limitations, leading to treatment failure, cancer relapse and unsatisfactory long-term clinical results [2]. These limitations are mainly related to two important issues: (1) conventional therapies lead to development of drug resistance by tumor cells 
and/or fail to destroy cancer stem cells (CSCs) or tumor-initiating cells (TICs), a population of self-renewing and drug resistant cancer cells [3,4]; (2) conventional therapies can cause normal cells to die in massive number, leading to local and systemic toxicity. Since cancer cell survival is driven by complex molecular interactions between growth and death signals [5], most oncologists think that targeting a single molecular component may not be sufficient to disrupt this process and combinational therapies, targeting multiple molecules, pathways, or networks are needed to eradicate the tumor and increase patients' survival [6].

Omega-3 ( $\omega-3$ or $n-3)$ fatty acids (FAs) are an important family of polyunsaturated fatty acids (PUFAs) and key nutrients, involved in normal growth and development of various human tissues [7-9]. Longer chain $n-3$ polyunsaturated fatty acids (PUFAs) are mainly composed of eicosapentaenoic acid (EPA) and docosahexaenoic acid (DHA). EPA has 20 carbon atoms and 5 double bonds (20:5n-3). DHA has a chain with 22 carbon atoms and 6 double bounds (22:6n-3), which makes it the longest chain and most unsaturated FA commonly found in biological systems. In the human body, DHA is either derived from $\beta$-oxidation of EPA or acquired from the diet. Cold-water oily fish are the main dietary source of essential $n-3$ PUFAs in humans, providing thus relatively large amount of EPA and DHA [10]. Beyond their role in physiological functions, $n$-3 PUFAs can affect some chronic diseases such as cancer $[8,9,11-13]$. Indeed, $n-3$ PUFAs or purified EPA and DHA can exert anti-neoplastic activity, playing a potential role either in cancer prevention or in cancer therapy [11-13].

Several decades ago, on the basis of human epidemiological studies, dietary oily fish and fish oil (FO) consumption have been associated with the protection against the development of some types of cancer, mainly colorectal, mammary and prostatic cancers $[14,15]$. Thereafter, most of the studies performed either in vitro or in vivo have demonstrated the protection by $n-3$ PUFAs against cancer risk. However, some reports question the effectiveness of these compounds in neoplastic prevention, and others argue that an increased $n-3$ PUFAs intake could induce some types of cancer [15-19]. Thus, the potential preventive role of $n-3$ PUFAs has become a subject of intense interest and debate. The biological effects of $n-3$ PUFAs on normal cells to prevent their transformation are not the topic of our dissertation, since exhaustive reviews have been written and have critically analyzed the data in the literature $[15,16,20,21]$.

During recent years, extensive studies have also considered the potential therapeutic activity of $n-3$ PUFAs against established solid and hematological tumors $[13,22]$. A number of biological effects that could contribute to this activity have been suggested, including induced alteration by $n-3$ PUFAs of cancer cell invasion and metastasization, as well as proliferation and apoptosis [21-25]. The induction of tumor cell apoptosis plays an important role in cancer therapy and 
represents a prominent target of many treatment strategies. Several studies have demonstrated that $n-3$ PUFAs, EPA and DHA have inhibitory effects on tumor growth by inducing cancer cell death via apoptosis, either alone [22-25] or in combination with conventional anticancer therapies [26-31]. Although all these studies have proposed molecular mechanisms that account for the pro-apoptotic activity of $n-3$ PUFAs in cancer cells, the mechanisms are still not completely understood, and a large number of molecular targets of $n-3$ PUFAs have been identified and multiple mechanisms appear to underlie the induction of apoptosis by these FAs. However, notably, the cytotoxic activity exerted by $n-3$ PUFAs is very peculiar for two main reasons. First, it has the potential to increase the sensitivity of tumor cells to conventional cytotoxic therapies, possibly improving the efficacy of these therapies against some types of tumors, especially those otherwise resistant to treatments [26-35]. Second, it appears to be selective, in that $n$-3 PUFAs cause cytotoxicity against cancer cells with little or no toxicity on normal cells [28,36-45]. This is a very important point, since in order for a therapeutic agent to be truly effective, it should be toxic to cancer cells without harming normal cells; conversely, conventional chemotherapeutics kill cancer cells but also strike the healthy cells, causing adverse effects and severe morbidity. All the above considerations greatly support investigations carried out to assess the role of $n-3$ PUFAs as adjuvant, to improve the efficacy and tolerability of traditional anticancer therapies.

This review focuses on studies investigating the cytotoxic activity via apoptosis of $n$-3 PUFAs against cancer cells and analyzes the cellular and molecular mechanisms underlying this activity. In particular, it will be highlighted the wide range of molecules potentially targeted by $n-3$ PUFAs to induce cancer cell apoptosis. Firstly, in Section 2, it will be examined the pro-apoptotic activity exerted by $n-3$ PUFAs in different cancer models in vitro and in vivo, as well as the apoptotic pathways triggered by these FAs. Concerning this point, it will be also considered the important potential capability of EPA and DHA of inducing cytotoxicity towards drug-resistant cancer cells such as CSCs or TICs. Next, in Section 3, it will be analyzed the molecular events upstream the triggering of apoptosis by $n-3$ PUFAs, highlighting the multiple potential molecular targets of these FAs. This review could allow a better comprehension of the potential cytotoxic therapeutic role of the principal long chain n-3 PUFAs EPA and DHA against cancer, providing specific information and support to design future pre-clinical and clinical studies, which lead to the development of a more proper and effective use of these FAs in human cancer therapy, mainly combinational therapy. 


\section{Induction of Cancer Cell Apoptosis by n-3 Polyunsaturated Fatty Acids (PUFAs) and Triggering of the Intrinsic and Extrinsic Apoptotic Pathways}

Apoptosis is a programmed cell death process, occurring in physiological and pathological conditions [46]. Caspases are central to apoptosis mechanism, as they are both the initiators and executioners of this process. There are three pathways by which caspases can be activated. The two commonly described initiation pathways are the intrinsic (or mitochondrial) and the extrinsic (or death receptor) apoptotic pathways. Both pathways eventually lead to a common pathway or the execution phase of apoptosis mediated by the executioner caspase-3, -6 and -7 . A third initiation pathway is the intrinsic endoplasmic reticulum (ER) pathway $[46,47]$. The intrinsic or mitochondrial pathway is activated by endogenous stress signals such as growth factor deprivation, DNA-damaging chemicals and reactive oxygen species (ROS), which increase mitochondrial membrane permeability by modifying the interplay between B cell lymphoma protein-2 (Bcl-2) family proteins, that interact with mitochondrial membrane voltage-dependent anion channels. Bcl-2 family proteins have either pro-apoptotic (e.g., Bak, Bax, or Bok) or anti-apoptotic (e.g., Bcl-2, $\mathrm{Bcl}-\mathrm{xL}$, or Mcl-1) roles; a Bcl-2 subfamily, the BH3-only protein family (e.g., Bad, Bid, Bim, Noxa or Puma) also modulate pro- and anti-apoptotic Bcl-2 protein interactions. Pro-apoptotic stimuli shift the balance towards apoptic proteins, promoting the mitochondrial outer membrane permeabilization (MOMP), the subsequent release of cytochrome $\mathrm{C}$ into the cytosol, followed by its complex formation with procaspase- 9 and apoptotic protease-activating factor 1 (APAF1), leading to the activation of the initiator caspase-9; then, caspase- 9 activates the executioner caspases. The extrinsic pathway of apoptosis is activated by signal originated by death receptors such as TNF $\alpha$-receptors, CD95 (Fas) and TNF-related apoptosis-inducing ligand (TRAIL)-receptors, following their interaction with their corresponding ligands, $\mathrm{TNF} \alpha$, FasL and TRAIL. Receptor activation leads to recruitment, to receptor associated lipid rafts, of adaptor molecules to form death-inducing signaling complexes (DISCs), which contains TNF receptor-associated death domain (TRADD), Fas-associated death domain (FADD), procaspase-8/FLICE and receptor-interacting protein kinase 1 (RIPK1). This complex induces the activation of caspase- 8 and -10 , which activate the executioner caspases. In addition, caspase- 8 can also truncate Bid (tBid), which can migrate to the mitochondria to associate with Bax, increasing membrane permeability and converging thus to the activation of the intrinsic apoptotic pathway. The intrinsic ER pathway of apoptosis is activated in response to diverse arrays of stress such as oxidative stress, calcium influx and ER stress. The ER has three main functions: (1) folding, glycosylation and sorting of proteins to their proper destination; (2) synthesizing cholesterol and other lipids; and (3) maintenance of $\mathrm{Ca}^{2+}$ homeostasis. Disruption of any of these processes causes ER stress and activates the unfolded protein response (UPR). However, following prolonged ER 
stress, imbalanced calcium storage will activate calpain, which can inactivate Bcl-Xl and also activate the executioner caspases, leading to apoptosis. Finally, the apoptotic cascade is regulated by regulatory proteins, such as FLICE-like inhibitory proteins (FLIPs), which inhibit the extrinsic apoptotic pathway by binding to FADD and causing dissociation of the FADD/caspase- 8 complex. Additionally, families of inhibitor of apoptosis protein (IAP) (e.g., XIAP, cIAP, and survivin) bind to caspase-3 and -9 , thereby inhibiting caspase activity. Moreover, XIAP associated factor 1 (XAF1) negatively regulates the antiapoptotic function of XIAP.

Evasion of apoptosis by tumor cells is a hallmark of cancer [5] and defects in cancer cell apoptosis have been described at any point along the apoptotic pathways, including impaired receptor signaling, disrupted balance of anti- and pro-apoptotic Bcl-2 family proteins, reduced expression of caspases and increased expression of regulatory proteins (e.g., IAPs).

\subsection{In Vitro and in Vivo Induction of Cancer Cell Apoptosis by n-3 PUFAs}

$n$-3 PUFAs, EPA and DHA can induce apoptosis in tumor cells in vitro and in vivo, in a dose- and time-dependent manner. They induce apoptosis in vitro, in tumor cell lines derived from a wide range of solid tumors including colorectal carcinoma [37,48-50], esophageal [51] and gastric cancers [52], hepatocellular carcinoma [53-55], pancreatic cancer [56-58], cholangiocarcinoma [59], breast [60,61], ovarian [62], prostate [63,64] and bladder [65] cancers, neuroblastoma [66] and glioma [67], lung cancer [68,69], squamous cell carcinoma (SCC) [42] and melanoma $[70,71]$. Apoptosis induced by $n-3$ PUFAs, EPA and DHA has been also described in cancer cell lines derived from hematological tumors such as myeloid and lymphoid leukemias and lymphomas [72-78], as well as multiple myeloma [44,79].

In addition, in these last years a great attention has been given to CSCs or TICs, a small population of cancer cells with self-renewal and drug resistance properties, involved in cancer initiation, maintenance, metastasis and recurrence $[2-4,80]$. Resistance of CSCs/TICs to standard anti-cancer therapies is responsible for ineffectiveness of these treatments, leading to tumor recurrence and metastasis [2-4]. Therefore, in order to establish efficient therapeutic strategies that can prevent tumor relapse and induce a long-lasting clinical response, it is important to develop drugs that can specifically target and eliminate CSCs/TICs. Remarkably, recent in vitro studies have indicated the capability of $n-3$ PUFAs to affect colorectal and breast CSCs [81-85]. Indeed, it was shown that both EPA and DHA $(10-70 \mu \mathrm{M})$, separately, induced apoptosis in cancer stem-like cells derived from the SW620 colon cancer cell line, and the effect was markedly increased when they acted simultaneously. Moreover, both compounds enhanced the efficacy of chemotherapeutics agents such as 5-fluorouracil (5-FU) and mitomycin $\mathrm{C}$ against the same target cells [82]. Accordingly, it was observed that EPA alone and (with 
increased efficacy) in combination with 5-FU + oxaliplatin (OX) (FuOX) induced apoptosis in FuOX-resistant HT-29 and HCT116 colorectal carcinoma cells, highly enriched in CSCs [83]. In addition, de Carlo et al. [84] found that $25 \mu \mathrm{M}$ EPA induced the differentiation of colon CSCs, by upregulating cytokeratin 20 and mucin 2 and downregulating CD133 expression; they hypothesized that the increased degree of colon CSC differentiation could be strictly related to the EPA-induced sensitization of $\mathrm{CD}_{133^{+}}$cells to 5-FU. More recently, in human triple negative breast cancers, it has been shown that DHA inhibited mammosphere formation of TICs [85]. The capability of $n$-3 PUFAs to eliminate CSCs/TICs and/or increase their sensitivity to conventional antineoplastic drugs have a very important therapeutic potential, further supporting the anticancer use of these FAs as adjuvants in cancer therapies.

Suppression of tumor cell growth by $n-3$ PUFAs has been confirmed in vivo, in pre-clinical studies using cancer animal models mainly rapresented by transgenic "fat- 1 " mice (bearing the Caenorhabditis elegans " $n-3$ desaturase" gene able to convert $n-6$ to $n-3$ PUFAs, resulting thus in elevated $n-3$ PUFAs tissue content) and xenograft nude mice implanted with different tumor cell types [13,22,24,41,86-89]. However, it should be noted that most studies have been performed in experimental settings evaluating the suppression of tumor development and only few investigations have been realized in therapeutic settings, evaluating the capability of PUFAs to eradicate established tumors [24,41,86-89].

Encouraging results concerning the in vivo anti-neoplastic activity of $n$-3 PUFAs have been also obtained from clinical studies, even though they were mainly set-up to investigate cancer prevention and support, rather than cancer therapy [25,30,31,90-101]. Indeed, the outcomes mainly investigated included $n-3$ PUFAs membrane incorporation, immune and inflammatory responses, oxidative status, as well as body weight and composition or quality of life [31,96,97,99-101]. Few studies addressed $n$-3 PUFAs supplementation and decrease of tumor size or extension of patient survival $[90-93,95,96,98,100]$ (Table 1).

Thus, although in these studies $n$-3 PUFAs supplementation was associated with improvement of clinical outcome and prognosis, the conclusion is limited because of the limited amount of data. 


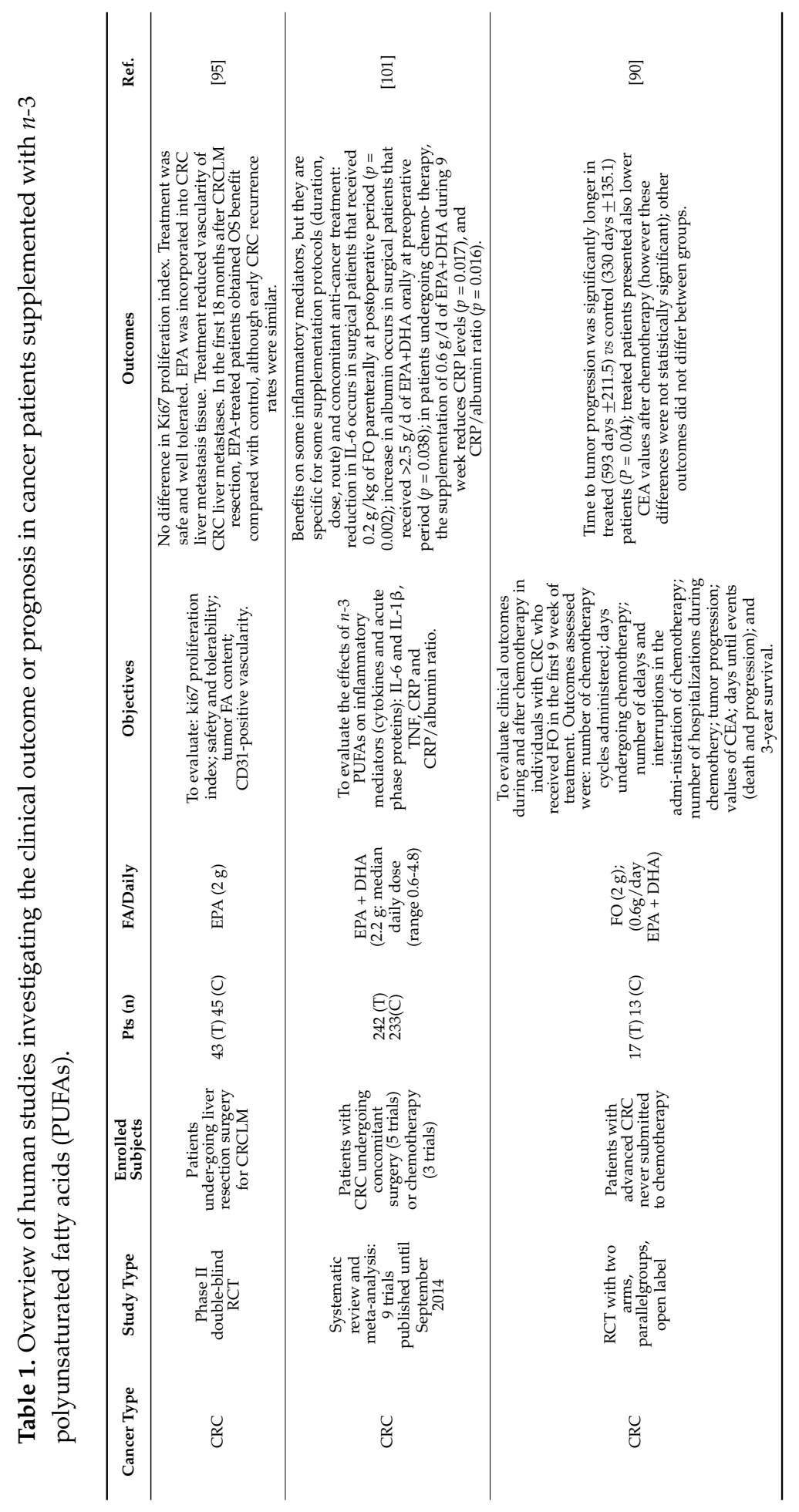




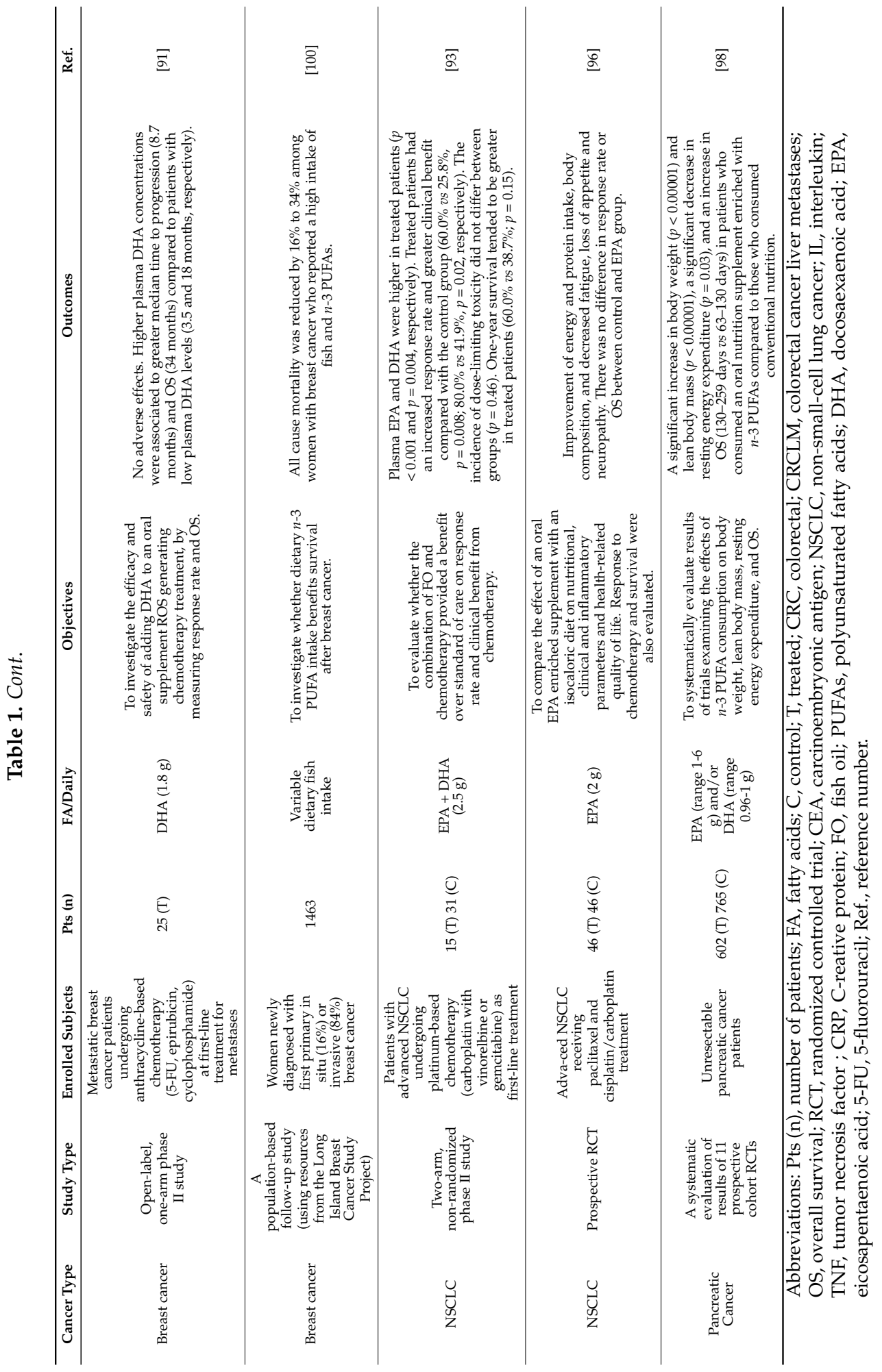




\subsection{Triggering of the Intrinsic and Extrinsic Apoptotic Pathways by n-3 PUFAs}

Many studies have reported that $n-3$ PUFAs induced apoptosis by triggering the intrinsic mitochondrial and ER pathways. In fact, EPA increased caspase- 3 and -9 , but not caspase-8, while inducing apoptosis in Ramos lymphoma cells [102]. Different studies in colon cancer (LS-174, HT-29, Caco-2 and COLO 201) cell lines showed that dietary FO [103] or DHA [104,105] modified the expression of Bcl-2 family proteins by increasing the levels of the pro-apoptotic proteins Bak and $\mathrm{Bcl}-\mathrm{xS}$ and decreasing those of the anti-apoptotic proteins Bcl-2 and Bcl-xL. Similarly, Sun et al. [54] observed that DHA induced apoptosis in human Bel-7402 hepatocellular carcinoma cells, by up-regulating caspase- 3 and Bax expression levels and downregulating the expression of Bcl-2 and Bim. Recently, Abdi et al. [44] demonstrated that EPA and DHA induced apoptosis in myeloma (L363, OPM-1, OPM-2 and U266) cells through mitochondrial perturbation and caspase- 3 activation, whereas both compounds did not affect the viability of normal human peripheral blood mononuclear cells. Moreover, the analysis of gene modulation by $n-3$ PUFAs in myeloma cells revealed the modulation of several signal pathways, including nuclear factor (NF)- $\kappa \mathrm{B}$, Notch, Hedgehog, oxidative stress and Wnt, indicating the possible involvement of multiple molecular signals in the initiation of apoptosis by the intrinsic pathway. Finally, the activation of the intrinsic ER stress pathway has been also proposed underlying DHA-induced apoptosis in colon cancer cells. Indeed, Jackobsen et al. [106] showed that DHA, while inducing cell death in the aggressive SW620 colon cancer cell line, also induced extensive changes in gene expression patterns (mRNA) of ER stress; they also found abundant presence of phosphorylated eIF $2 \alpha$, increase in cytosolic $\mathrm{Ca}^{2+}$ and disturbances in lipid metabolism, suggesting that cytotoxic effects of DHA are associated with signaling pathways involving lipid metabolism and ER stress.

On the other hand, other studies have indicated the activation of the extrinsic pathway in the induction of apoptosis by $n-3$ PUFAs. Indeed, increased expression of both caspase-9 and caspase-8 was reported in EPA- [107] and DHA- [108] induced apoptosis in human HL-60 leukemia and Caco-2 colon cancer cells, respectively. In the case of colon cancer cell apoptosis, tBid expression was also enhanced, indicating a contribution of caspase- 8 also to the activation of the mitochondrial pathway. Accordingly, in our laboratory we found the involvement of caspase- 8 in DHA-mediated apoptosis in pancreatic and bladder cancer cell lines [65].

Finally, both DHA and EPA could exert an important pro-apoptotic effect in different colorectal cancer (Caco-2, HT-29, HCT116, LoVo, SW480) cells by the downregulation of two key regulatory elements of the extrinsic and intrinsic pathways, FLIP and XIAP, respectively; interestingly, DHA and EPA did not affect the viability of normal human colon mucosal epithelium (NCM460) cells [39]. 


\section{Molecules, Signals and Networks Targeted by $n-3$ PUFAs: Upstream Events in the Triggering of the Apoptotic Pathways}

Cancer is often described as a disorder of the balance between cell growth and death [5]. On the one hand, defects in signaling pathways promoting cell growth and survival occur in cancer cells and high constitutive levels of MEK/ERK, PI3K/Akt, JAK/STAT or IKK/IкB/NF-kB pathways are frequently observed in human cancers $[5,109]$. On the other hand, as already mentioned in Section 2, defects along the apoptotic pathways also occur in cancer cells, leading to resistance to apoptosis $[5,110]$. Therefore, all the molecules, signals and networks involved in cancer cell survival and death are potential targets for apoptosis-based cancer therapies.

The mechanisms by which $n$-3 PUFAs induce apoptosis in tumor cells are not fully determined in molecular terms; however, the proposed main routes of action of n-3 PUFAs are: (1) incorporation into cell membranes, leading to changes in the distribution and function of key survival and death signals; (2) generation of lethal levels of intracellular oxidative stress; (3) modulation of eicosanoid metabolites; (4) binding to nuclear receptors, leading to changes in gene expression. These routes may underlie the pleiotropic and multifaceted effects of $n$-3 PUFAs, leading to the induction of apoptosis in cancer cells and/or to the sensibilization of tumor cells to traditional therapies. Therefore, in the context of these four routes of action of $n-3$ PUFAs, in this section we analyze studies investigating the mechanisms underlying the induction of apoptosis, highlighting the potential upstream molecular events targeted by $n-3$ PUFAs to trigger the apoptotic pathways in cancer cells.

\subsection{Cell Membrane Enrichment in n-3 PUFAs and Changes in the Distribution and Function of Key Survival and Death Signals in Cancer Cells}

Once ingested, n-3 PUFAs, EPA and DHA are uptaken and incorporated in tumor cell membranes by both passive or carrier-mediated transmembrane translocation [29]. The FA composition of membrane phospholipids can influence multiple cellular functions. It should be noted that DHA, for its high level of unsaturation and presence of several $\mathrm{CH}-\mathrm{CH}_{2}-\mathrm{CH}$ repeating units in its molecule, possesses an extremely flexible structure, more flexible than EPA, and it can rapidly isomerize through different conformational states [29]. Therefore, the enrichment of $n-3$ PUFAs in tumor cell membranes and the high molecular disorder originating from their (mainly DHA) incorporation into membrane phospholipids may affect physical-chemical properties of membranes, including their fluidity, permeability, deformability, as well as their lipid microdomain formation [29,111]. Plasma membrane is composed of microdomains of saturated lipids that segregate together to form "lipid rafts". Lipid rafts are enriched in glycosylphosphatidylinositol-linked proteins, contain several signaling proteins (e.g., epidermal growth factor receptor, 
EGFR) and play a key role in cell signal transduction, mainly by facilitating the association of signal molecules (e.g., those involved in cell survival). Cholesterol is a critical lipid component for lipid raft integrity and function, and DHA have poor affinity for cholesterol and influences lipid rafts, modifying their biochemical and biophysical features and changing their composition and/or the activity of raft-related signaling molecules. Therefore, concerning the regulation of apoptosis, $n-3$ PUFAs have the potential to modulate the function of death receptors, growth factor receptors, cytokines and hormones receptors, as well as oncogenes, tumor suppressor genes and signal transduction secondary messangers (e.g., adapter proteins, receptor-associated enzymes, protein kinases and phosphatases). As a consequence, $n-3$ PUFAs may alter the activation of transcription factors and expression of genes as well as the phenotype of tumor cells [111]. Thus, cell membrane enrichment in $n$-3 PUFAs can influence multiple cellular functions at multiple biological levels. Moreover, noteworthy, it has been reported that there are significant differences between tumor and normal cells in n-3 PUFAs uptake and membrane distribution, being tumor cells deficient in PUFAs (especially in arachidonic acid-ARA, EPA and DHA) as compared to normal cells, since they have decreased activity of $\Delta^{5}$ and $\Delta^{6}$ desaturases. Although the exact reason for the low activity of desaturases in cancer cells is not known, it has been proposed that it might be a defence mechanism adopted by tumor cells to protect themselves from toxic molecules such as free radicals derived from $n-3$ PUFAs peroxidation in cancer cells (see Section 3.2) [112,113]. Therefore, the specific enrichment of tumor cell membranes with n-3 PUFAs, EPA or DHA is one of the possible reasons underlying the capability of $n-3$ PUFAs to induce cytotoxicity in tumor cells, with no or little action on normal cells.

Discoveries over the last decade propose that $n$-3 PUFAs incorporation into cancer cell membranes is essential for apoptosis by n3-PUFAs in different cancer cell models [26,113-115]. However, currently, the precise mechanism of how a selective change in DHA and EPA content of membranes translates to a change in signaling events to induce apoptosis is not completely clear. Therefore, in the next sections (from Section 3.1.1 to 3.1.6), we analyze the studies investigating this issue. We take into consideration different possible actions by $n-3$ PUFAs, such as the displacement of lipid raft associated onco-proteins as well as the modulation of different survival signaling pathways in tumor cells, including Wnt/ $\beta$-catenin, MAPK/Erk, PI3K/Akt/mTOR, JAK-STAT and NF-kB pathways.

\subsubsection{Changes in Lipid Raft-Associated Onco-Proteins by $n-3$ PUFAs}

The involvement of the modulation of EGFR and HER-2 signals in $n-3$ PUFAs-induced apoptosis has been reported by different studies, performed in different types of cancer (mainly breast cancer) cells. In 2007, Schley and 
coworkers [116] showed that apoptosis induced by a combination of EPA and DHA in MDA-MB-231 breast cancer cells was due to changes in lipid raft composition, leading to a decrease of EGFR levels as well as an increase of EGFR and p38 mitogen-activated protein kinase (MAPK) phosphorylation. Accordingly, in oral SCC cells, it was found that DHA- and EPA-induced apoptosis was mediated by amplification of the EGFR/ERK/p90RS kinase (K) pathway (i.e., EGFR autophosphorylation, sustained phosphorylation of ERK1/2 and of its downstream target p90RSK); to note, the viability of normal keratinocytes was not affected [42]. In contrast with these results, in three different (A549 lung, WiDr colon and MDA-MB-231 breast) cancer cell models it was found that DHA-induced apoptosis was caused by the exclusion of EGFR from caveolin-rich lipid raft fractions, resulting in a decreased association of Ras with Sos1 and the subsequent downregulation of Erk signaling; these data were confirmed in vivo, using xenograft athymic mice implanted with A549 cells [117]. Similarly, a reduction of EGFR activation was observed in EPA- or DHA-induced apoptosis in breast cancer (MDA-MB-231 and MCF-7) cells, associated to a reduction of $\mathrm{Bcl} 2$ and caspase- 8 expression; moreover, DHA (probably related to its better capability to change lipid raft properties), but not EPA, also slightly reduced EGFR concentration [118]. More recently, in line with these results, it was found that DHA had the capability of decreasing cell surface levels of lipid rafts via their internalization and then fusion with lysosomes in MDA-MB-231 breast cancer cells. This implied that DHA displaced several raft-associated onco-proteins, including EGFR, Hsp90, Akt, and Src and also decreased total levels of those proteins via multiple pathways, including the proteasomal and lysosomal pathways, thereby decreasing their activities such as Hsp90 chaperone function [119]. Then, the therapeutic potential of DHA in the treatment of HER-2 positive breast cancers has been reported by two investigators. Ravacci [43], Mason [120] and coworkers showed that DHA induced apoptosis in transformed human mammary epithelial (HB4aC5.2) cells and in breast cancer (BT-474) cells, respectively, by the deplacement of HER-2 from lipid rafts and the decrease of Akt and ERK1/2 activation; no effects were observed in related untransformed (HB4a) cells.

New insight into the potential application of $n-3$ PUFAs in breast cancer treatment was also provided by a recent investigation in MCF-7 and T47D breast cancer cells, showing that DHA and EPA could shift the pro-survival estrogen signal to a pro-apoptotic effect by increasing the $G$ protein coupled estrogen receptor 1-cyclic adenosine monophosphate-protein kinase A (GPER1-cAMP-PKA) signaling response, blunting EGFR, Erk 1/2 and AKT activity [121].

It is of interest that it has been also demonstrated that pre-treatment of estrogen receptor negative MDA-MB-231 cells with DHA increased the anti-cancer effects of doxorubicin, by increasing the plasma membrane raft content of CD95 and FADD. [122]. 
Finally, in prostate cancer (PC3 and LNCaP) cells, growth suppression by DHA was due to changes in cell plasma membrane phospholipid content, leading to the alteration of phosphatidylinositol phosphates (PIPs) content, $\mathrm{PI}(3,4,5) \mathrm{P}_{3}\left(\mathrm{PIP}_{3}\right)$ and Akt localization, inhibition of Akt phosphorylation and thus of the AKT survival signaling pathway [123].

\subsubsection{Inhibition of the Wnt/ $\beta$-Catenin Pathway by $n-3$ PUFAs}

Wnt functions causing an accumulation of $\beta$-catenin in the cytoplasm and its eventual translocation into the nucleus, to act as a transcriptional coactivator of transcription factors that belong to the $\mathrm{T}$ cell factor/lymphoid enhancer factor (TCF/LEF) family. Dysregulation of Wnt signaling and $\beta$-catenin expression is believed to be central in the regulation of tumor cell apoptosis [109].

In 2007, Calviello et al. [124] proposed that DHA exerted pro-apoptotic effects in colon cancer cells through proteasomal-dependent degradation of $\beta$-catenin, leading to down-regulation of the expression of TCF- $\beta$-catenin target genes such as survivin (a IAP family member). Then, Lim [53,59], Song [125] and coworkers showed that DHA- and EPA-induced apoptosis in human cholangiocarcinoma, hepatocellular and pancreatic carcinoma cells was caused by the inhibition of the $\beta$-catenin signaling pathway through two systems of $\beta$-catenin degradation, such as the activation (by dephosphorylation) of glycogen synthase kinase-3 $\beta$ (GSK-3 $\beta$ ) and the induction of the formation of $\beta$-catenin/Axin/GSK-3 $\beta$ binding complex; similar results were obtained in vivo, in Fat-1 transgenic mice implanted with mouse pancreatic cancer (PANC02) cells [125]. More recently, the inhibition of the Wnt/ $\beta$-catenin pathway was also found to be involved in growth suppression of breast MCF-7 cancer cells in vitro and in therapy experiments in vivo, performed in Babl/c mice bearing 4T1 mouse breast cancer and fed with a 5\% FO diet [126].

\subsubsection{Modulation of the Mitogen-Activated Protein Kinase (MAPK)/ERK (or} Ras/Raf/MEK/ERK) Pathway by $n-3$ PUFAs

The MAPK/ERK pathway includes many MAPK proteins (originally called extracellular signal-regulated kinases, ERK), which function by adding phosphate groups to a neighboring protein, leading to change the expression of genes specific for molecules involved in cell cycle and apoptosis. This pathway represents a necessary step in the development and progression of many cancers. Although the activation of ERK is traditionally linked to cell survival and proliferation, recent studies have demonstrated that this is not always the case and ERK activation can also cause growth arrest or apoptosis [109]. Indeed, as reported below, both activation and inhibition of ERK have been associated with n-3 PUFAs-induced tumour cell apoptosis, suggesting that tissue- or cancer-specific mechanisms of $n-3$ PUFAs action might occur. 
In 2008, Serini et al. [68] showed that DHA-induced apoptosis was due to decreased levels of phosphorylated MAPKs, especially ERK1/2 and p38 in lung cancer cells. Accordingly, n-3 PUFAs induced apoptosis in breast cancer cells in vitro and in a Fat-1 mice breast cancer model, by inhibition of the MEK/ERK/Bad signaling pathway; the inhibition was induced through the increased expression of the integral membrane protein syndecan-1 (SDC-1) [127]. On the other hand, in gastric cancer cells, DHA-induced apoptosis was caused by the activation of ERK and c-Jun $N$-terminal kinase (JNK), leading to the activation of AP-1 transcription factor, which induced the expression of apoptotic genes [52].

\subsubsection{Inhibition of the PI3K/Akt/mTOR Pathway by $n-3$ PUFAs}

PI3K (phosphatidylinositol-3-kinase) is one of the intracellular pathways responsible for the transmission of anti-apoptotic signals by cell survival factors. Phosphatase and tensin homologue deleted on chromosome ten (PTEN) is a lipid phosphatase, which catalyzes the dephosphorylation of PIP3 and thus serves as a major negative regulator of PI3K/Akt signaling; when it is phosphorylated, it becomes inactive. Akt (or protein kinase $\mathrm{B}, \mathrm{PKB}$ ) is a serine/threonine kinase, activated in response to cytokines and growth factors through its translocation to the plasma membrane and its phosphorylation at two key residues (Thr308 and Ser473). Akt activation promotes directly cell survival and protect cells from apoptosis by inactivating components of the cell death machinery (e.g., caspase-9, Bad); in addition, Akt promotes indirectly cell survival and protect cells from apoptosis by activating transcription factors such as NF- $k \mathrm{~B}$, that induces the transcription of pro-survival and anti-apoptotic genes. Mammalian target of rapamycin (mTOR) is a protein kinase that integrates both intracellular and extracellular signals, and serves as a central regulator of cell metabolism, growth and survival. It regulates the activity of p70S6K and eukaryotic initiation factor (eIF)4E binding protein-1 (4E-BP1). The mTOR pathway is deregulated and activated in several types of cancer, significantly contributing to the enhancement of proliferation and the inhibition of autophagy; overexpression of downstream mTOR effectors 4E-BP1, S6K and eIF4E4 leads to poor cancer prognosis. Therefore, inhibition of mTOR activity disrupts the balance between pro- and anti-apoptotic proteins, enhancing tumor cell death [109].

It was shown that EPA and DHA induced apoptosis in MDA-MB-231 breast cancer cells in vitro [128,129] and in a xenograft animal model [129] by the inhibition of the survival Akt/NF- $\mathrm{kB}$ signaling pathway, due to the inactivation of PI3K, through increased PTEN expression by $n-3$ PUFAs. On the other hand, DHA-mediated apoptosis in colon cancer (Caco-2) cells, was due to the inactivation of PI3K induced by reduced PTEN phosphorylation by $n-3$ PUFAs. This inactivation promoted inhibition of Akt/PKB and thus of Bad and forkhead transcription factor (FKHR); to note, the viability of normal colon 
(NCM460) cells was not compromised [40,130]. Then, the suppression of the activity of (3'-phosphoinositide-dependent kinase 1)-PDK1/Akt/Bad signaling was demonstrated underlying $n$-3 PUFA-induced apoptosis in prostate cancer (PC3, LNCaP and DU145) cells in vitro and in vivo; moreover, the suppression was dependent on the upregulation of SDC-1, and 15-LOX-1-mediated metabolism of DHA was required for SDC-1 upregulation [64].

From recent studies, it has emerged that DHA can also simultaneously induce apoptosis and autophagy in cancer cells, and this process involves mTOR repression [131,132]. Indeed, DHA treatment in human cervical cancer cells led to autophagy via p53-mediated (AMP-activated protein kinase)-AMPK/mTOR signaling (i.e., mTOR inhibition and AMPK activation), and DHA-induced autophagy sensitized tumor cells to apoptosis [131]. Then, in non-small cell lung cancer cells, it was shown that DHA-induced apoptosis and autophagy were associated to mTOR suppression induced by both AMPK activation and PI3K/Akt inhibition; these data were confirmed in Fat-1 transgenic mice implanted with Lewis lung cancer cells [133].

\subsubsection{Inhibition of the JAK-STAT Pathway by $n-3$ PUFAs}

The JAK-STAT system consists of a receptor (activated by interferons, interleukins, growth factors, or other chemical messengers), the Janus kinase (JAK) and the signal transducer and activator of transcription (STAT) proteins. STAT proteins once activated translocate into the nucleus, where they bind to DNA, promoting the transcription of specific genes affecting basic cell functions such as cell growth and death. The activation of STAT3 pathway in tumor cells is mainly due to the effect of tumor released factors and plays a critical role in tumor cell-survival and chemo-resistance [109].

A very recent work by Rescigno et al. [134] demonstrated that DHA-induced apoptosis in aggressive SK-BR-3 breast cancer cells reduced both ERK1/2 and STAT3 phosphorylation; interestingly, DHA only arrested cell cycle progression of non-tumor MCF-10A breast cells, activating p21 Waf1/Cip1 and p53. Moreover, it was also shown that the elimination of aldehyde dehydrogenase positive cells and the inhibition of mammosphere formation of TICs in human triple negative breast cancer cells by DHA was due to the Src homology region 2 domain-containing protein tyrosine phosphatase-1 (SHP-1)-dependent suppression of STAT3 activation and of its downstream mediators c-Myc and cyclin D1 [85].

\subsubsection{Inhibition of the NF- $\kappa$ B Pathway by $n-3$ PUFAs}

NF-kB transcription factor plays a key role in many physiological processes, including inflammation, cell proliferation and death. The aberrant regulation of NF-kB and signaling pathways that control its activity are heavily implicated in 
promoting pro-survival signaling and may be critical for resistance to chronic oxidative stress (i.e., drug resistance) [2].

In prostate cancer (LNCaP, DU145, PC3) cells, it was shown that DHA synergistically enhanced the cytotoxic effect of docetaxel, through increased apoptosis by suppression of genes involved in the NF- $\mathrm{kB}$ pathway [135]. Then, always in prostate cancer (LNCaP and PacMetUT1) cells, it was reported that exposure of cells to DHA attenuated $\mathrm{H}_{2} \mathrm{O}_{2}$-induced NF- $\mathrm{kB}$ transcriptional activity and diminished the expression of the downstream anti-apoptotic target survivin; this activity was specific, since it was not observed in normal human prostate (PrEC) cells [136].

\subsection{Cell Membrane Enrichment in n-3 PUFAs and Increased Oxidative Stress in Tumor Cells}

One of the main characteristics of $n-3$ PUFAs is the fact that they are optimal substrates for oxidants inside the cell, undergoing thus nonenzymatic lipid peroxidation into cell membranes; moreover, nonenzymatic lipid peroxidation triggers a further increase of the formation of oxygen radicals and ROS [29]. Tumor cells contain higher levels of ROS compared to normal cells, principally due to their accelerated metabolism needed to maintain their high proliferation rate. Thus, ROS in tumor cells can react with intracellular $n$-3 PUFAs giving rise to nonenzymatic lipid peroxidation products that are highly toxic [38]. The methylene group, located between two double bonds $\left(-\mathrm{CH}=\mathrm{CH}-\mathrm{CH}_{2}-\mathrm{CH}=\mathrm{CH}-\right)$, is particularly vulnerable to radical attack by reactive species, thus entailing the abstraction of hydrogen [29]. Moreover, DHA, possessing an additional double bond with respect to EPA, is more susceptible to nonenzymatic lipid peroxidation, providing a variety of lipid hydroxiperoxides and aldehydic breakdown products such as malonaldehyde (MDA; a marker for lipid peroxidation) with toxic as well as prooxidant properties [13,24]. Indeed, the nonenzymatic lipid peroxidation triggers a further increase of the generation of intracellular radical species; the further increase of intracellular ROS levels and oxidative stress in tumor cells by $n-3$ PUFAs (EPA $>$ DHA) causes the disruption of the mitochondrial membrane potential, the release of cytochrome $\mathrm{C}$ and thus the triggering of the intrinsic apoptotic pathway (see Section 2). Moreover, DHA can be readly incorporated in mitochondrial membranes, altering their permeability and decreasing the mitochondrial membrane potential [29]. It has been also reported that DHA is mostly present in the mitochondrion in association with cardiolipins; cardiolipin-DHA molecules are under attack of radical species, with the consequent decrease of their binding affinity for cytochrome $C$, enhancement of its release and the release of other pro-apoptotic factors (e.g., Smac/Diablo) from mitochondria to cytosol, and the triggering of the intrinsic apoptotic pathway [29]. In addition, it is known that ROS can also oxidize and inhibit key signaling pathways involved in cell 
proliferation, survival and apoptosis, such as MAPK and NF- $\mathrm{B}$ p pathways $[55,137]$. Therefore, all these considerations indicate that lipid peroxidation and increased ROS levels play a key role in the induction of tumor cell apoptosis by $n-3$ PUFAs. Interestingly, it has been reported that there are significant differences in tumor vs normal cells not only in the uptake and distribution of $n-3$ PUFAs, but also in the ability to generate reactive species and oxidative stress from intracellular n-3 PUFAs. Indeed, as mentioned, tumor cells contain higher levels of oxygen radicals compared to normal cells and in presence of DHA they increase the production of cytotoxic lipid hydroperoxydes and other peroxides, undergoing apoptosis. In contrast to tumor cells, normal cells can use DHA to protect themselves from oxidative stress-induced apoptosis through a certain number of mechanisms, including the activation of the survival PI3K/Akt pathway as well as the increased production of cytoprotective molecules such as resolvins and protectins (see also Section 3.3) [138-140]. As reported in the next section, several investigators have shown that, as exogenous $n-3$ PUFAs are provided to cancer cells, these FAs can induce apoptosis by augmenting free radical generation and lipid peroxidation, whereas normal cells are not influenced [36,130,138].

Since ROS have been proposed as common mediators of apoptosis, the majority of cytotoxic anticancer agents (including ionizing radiations, most chemotherapeutic agents and some targeted therapies) work through ROS generation [29]. However, although they initially generate ROS production, most cancer cells following prolonged treatment with these drugs develop the capability to reduce ROS levels, resulting in drug-resistance. Evidence exists on the capability of $n$-3 PUFAs to increase both the efficacy of conventional anticancer therapies towards drug resistance and their tolerability towards normal cell damage. Indeed, $n$-3 PUFAs can increase the susceptibility of tumor cells to oxidative stress induced by conventional therapies, by maintaining high ROS levels in cancer cells, thereby precluding drug resistance $[27,29]$. Moreover, $n-3$ PUFAs can increase the tolerability to conventional therapies, by promoting both the selective induction of letal levels of oxidative stress in tumor cells and the selective production of protective lipid mediators in normal cells. Both activities have important therapeutic potential, further supporting the use of n-3 PUFAs as adjuvant in conventional cancer therapies.

Increased Oxidative Stress in Cancer Cells by $n-3$ PUFAs and Induction of Apoptosis

Early in vitro studies performed in breast [60] and pancreatic [56] cancer cells proposed the involvement of oxidative mechanisms in the induction of cancer cell apoptosis by EPA and $n$-3 PUFAs, respectively; interestingly, increased oxidative stress and apoptosis were not observed in human normal cells, such as fibroblasts [60]. Further studies have shown that oxidative stress in cancer cells was generated by 
n-3 PUFAs through both generating lethal ROS levels and decreasing anti-oxidant activities in tumor cells [38].

In our laboratory, we showed that DHA promoted apoptosis in the human PaCa-44 pancreatic cell line through the induction of an active extrusion process of intracellular reduced glutathione (GSH), depleting tumor cells of one of the endogenous antioxidant defences, and increasing thus tumor cell sensibility to lipid peroxidation and oxidative stress [57]. This data has important implications for cancer therapy, since elevated GSH levels in tumors have been associated with resistance to apoptosis and chemotherapy [29]. Similarly, Ding and co-workers [141] found downregulation of the antioxidant enzyme superoxide dismutase 1 (SOD1) expression in the DHL-4 lymphoid cell line undergoing apoptotsis by DHA. Then, in the same laboratory, it was shown that DHA-mediated cytotoxicity in human ovarian cancer cell lines was associated to a reduction of glutathione peroxidase (GPx)-4 protein expression and that DHA-mediated cytotoxicity was reversed by vitamin E, suggesting that GPx-4 downregulation was due to oxidative stress [142]. Moreover, it was reported that the in vitro and in vivo sensitization of MDA-MB-231 breast cancer cells to anthracyclines (doxorubicin) by DHA was caused by a decrease of cytosolic GPx-1 activity and a concomitant increase of ROS levels [143].

On the other hand, $n-3$ PUFAs can also promote apoptosis by increasing lipid peroxidation and intracellular oxidative stress. It has been shown that DHA enhanced arsenic-trioxide-induced apoptosis in arsenic-trioxide resistant HL-60 (myeloid leukemia), SH-1 (hairy cell-leukemia), and Daudi (Burkitt lymphoma) cell lines by an increase of lipid peroxidation and a reduction of the mitochondrial membrane potential; these effects were reversed by the addition of the antioxidant vitamin E [144]. Similarly, Lindskog et al. [66] showed that DHA-mediated neuroblastoma cell death was associated with production of ROS and depolarization of the mitochondrial membrane potential, whereas vitamin E inhibited both mitochondrial depolarization and cell death; of note, nontransformed fibroblasts were not substantially affected by DHA. Moreover, DHA also significantly enhanced the cytotoxicity of arsenic trioxide, nonsteroidal antiinflammatory drug (diclofenac) and conventional chemotherapeutic agents (cisplatin, doxorubicin and irinotecan) both in chemosensitive and in multidrug-resistant neuroblastoma cells. More recently, similar effects were found in human HT-29 colorectal adenocarcinoma cells treated with DHA-combined treatment with 5-FU, OX and irinotecan (IRI); the anticancer action of DHA, observed in presence of low doses of chemotherapeutic drugs (1 $\mu \mathrm{M}$ 5-FU, $1 \mu \mathrm{M}$ OX and $10 \mu \mathrm{M}$ IRI), was carried out by loss of mitochondrial membrane potential and caspase-9 activation [145]. Increased lipid peroxidation associated to the activation of the intrinsic apoptotic pathway was confirmed by other investigators as mechanism underlying DHA- or EPA-mediated apoptosis, in different human cancer colon (HT-29 and Caco-2) [146] and gastric (MGC and 
SGC) [147] cell lines. In addition, in DHA-induced apoptotic human papillomavirus (HPV)-infected cancer cells, it was reported that the overproduction of mitochondrial ROS by DHA promoted the activation of the cellular ubiquitin-proteasome system, which leads to the degradation of E6/E7 oncoproteins, essential in the maintenance of HPV-associated malignancies [148].

Furthermore, it has been found that oxidative stress could induce apoptosis by triggering not only the intrinsic pathway, but also by the extrinsic pathway. Indeed, Kang et al. [149] found that DHA promoted apoptosis in MCF-7 breast cancer cells in vitro and in vivo via both ROS formation and caspase- 8 activation, in that antioxidants or knockdown of caspase- 8 each effectively abrogated cytotoxicity by DHA. To explain caspase- 8 activation, the authors have hypothesized that ROS accumulation in plasma membrane lipid rafts might induce the assembly of DISC, triggering thus the extrinsic pathway. Then, the same investigators [58] also showed the induction of both ROS accumulation and caspase-8-dependent cell death by EPA and DHA, in human pancreatic cancer (MIA-PaCa-2 and Capan-2) cells in vitro and in xenografts athymic nude mice fed with $5 \%$ FO.

Recently, Jeong et al. [137] reported that the activation of MAPKs such as ERK/JNK/p38 was involved in DHA-induced apoptosis and that this activation was associated with mitochondrial ROS overproduction. Accordingly, Zhang et al. [55] showed that EPA caused apoptosis in HepG2 cells by evoking ROS formation, leading to both [Ca2+] accumulation and increased activation of JNK; both events promoted MOMP, the release of cytochrome $\mathrm{C}$ from mitochondria, and the activation of caspase-9 and caspase-3; to notice, EPA had no significant effect on the viability of normal liver (L-02) cells.

Finally, while it is well established that excessive ROS can instigate apoptosis, emerging data have also revealed a signaling role for ROS in the activation of autophagy. In PC3 and DU145 prostate cancer cells, with mutant p53 and exposed to DHA, it was found that ROS-mediated apoptosis and autophagy were caused by the inhibition of Akt-mTOR signaling [132]. According to these results, Zajdel et al. [150] showed that oxidative stress induced in human A549 lung cancer cells by EPA and DHA influenced apoptosis as well as tumor cell autophagy; the inhibition of the autophagic process suppressed cell death and decreased activation of caspase-3/7, indicating that EPA- and DHA-mediated autophagy could amplify cancer cell apoptosis.

\subsection{Cell Membrane Enrichment in n-3 PUFAs and Changes in the Level and Quality of Eicosanoid Metabolites}

Eicosanoids are generally considered as oxidized derivatives of 20-carbon FAs in the cell membrane, such as ARA (20:4n-6) and EPA. They include prostaglandins (PGs), thromboxanes (TXs), leukotrienes (LXs) and lipoxins (LXs). The major $n-6$ 
PUFA ARA, because of its prevalence in the phospholipids of cell membranes, is generally the major substrate for eicosanoid synthesis. Once released from membrane phospholipids, free ARA acts as a substrate for cyclooxygenases (COXs), lipoxygenases (LOXs) and cytochrome P450 enzymes; COX enzymes lead to 2-series PGs (e.g., PGE2) and TXs, and LOX enzymes to 4-series LTs and LXs, known as pro-inflammatory and pro-tumorigenic mediators. In fact, inflammation confers to tumor survival and drug resistance. On the other hand, EPA is also a substrate for COXs, LOXs and cytochrome P450 enzymes, giving rise to 3-series PGs (e.g., PGE3) and TXs and to 5-series LTs, known as anti-inflammatory and anti-tumorigenic mediators. These bioproducts bind specific receptors, usually $\mathrm{G}$ protein-coupled receptors, leading to the activation of signaling pathways involved in the regulation of cancer cell growth and death [9,13]. In addition, EPA and DHA give rise to anti-inflammatory and inflammation resolving metabolites, including resolvins produced from EPA (E-series) and DHA (D-series) and protectins and maresins produced from DHA [113,139,140,151,152]. Anti-inflammatory LXs, resolvins and protectins inhibit the expression of pro-inflammatory cytokines and adhesion molecules, thereby inhibiting tumor cell growth and invasion. Moreover, as mentioned in Section 3.2, it has been proposed that they behave as endogenous cytoprotective molecules for normal cells against lipid peroxidation-mediated damage by $n$-3 PUFAs. Indeed, enrichment of normal cell membranes in EPA and DHA, both in vitro and in vivo, may allow normal cells to produce enhanced amounts of resolvins and protectins, protecting themselves against toxic chemicals such as anti-cancer drugs [81]. Tumor cell membrane enrichment in n-3 PUFAs can induce changes in the level and quality of eicosanoid products by two main ways: (1) directly, by increasing specific metabolites derived from their metabolic conversion (e.g., PGE3); (2) indirectly, by inhibiting the conversion of ARA to pro-tumorigenic $n-6$ series eicosanoids (e.g., PGE-2). This second way might be pursued by displacing ARA from cell membranes (i.e., $n$-3 PUFAs membrane incorporation partially replace ARA, reducing its availability), by competing with ARA for enzymes (e.g., EPA can act as an alternative substrate for COX-2, leading to a reduction in PGE2 in favour of PGE3), or by inhibiting NF- $\mathrm{KB}$ activation, thus decreasing COX-2 enzyme expression [9,29]. Moreover, DHA can inhibit COX-2 activity by binding the substrate channel of COX-2 [9].

COX-2 is overexpressed in many types of cancer leading to the formation of excess of PGE2 [153], and the autocrine COX-2/PGE2 pathway can confer tumor cell resistance to apoptosis by different ways, including the up-regulation of the $\beta$-catenin and Ras/Raf/MEK/ERK signaling pathways [154]. 
Modulation of Eicosanoid Bioproducts by $n-3$ PUFAs and Induction of Cancer Cell Apoptosis

Several studies have indicated that the modulation of eicosanoid production by n-3 PUFAs (mainly EPA) may contribute to the induction of apoptosis in cancer cells.

Some investigations have demonstrated that $n$-3 PUFAs can inhibit the autocrine anti-apoptotic COX-2/PGE2 pathway in tumor cells, leading thus to cancer cell apoptosis. Early in vitro and in vivo studies reported that decreased PGE2 production was associated with decreased growth of prostate [155,156] and breast [157] cancer cells. Furthermore, $n$-3 PUFAs inhibited tumor cell growth in a xenograft prostate cancer model by decreasing PGE2 as well as COX-2 levels [158]. Later, Funahashi et al. [159] showed that EPA decreased the growth of COX-2-positive and COX-2-negative PaCa pancreatic cancer cells and the COX-2-dependent mechanism was mediated by the binding of PGE3 to EP2 and EP4 receptors. Accordingly, dietary intake of $n-3$ PUFAs decreased the pancreatic cancer cell growth in a xenograft model through increasing PGE3 and decreasing PGE2 in tumor tissues. The down-regulation of COX-2 by $n$-3 PUFAs might be a crucial mechanism underlying their apoptotic effect in other types of tumors, including colon cancer [160,161]. In colorectal cancer cells, it was shown that EPA not only decreased COX-2 expression and PGE2 formation, but also increased the COX-dependent formation of EPA-derived metabolites [153]. All these results suggest that EPA may act as a "natural COX inhibitor". Very recently, Zhang C. et al. [113] found that the tumoricidal action of $n$-3 PUFAs on LoVo and RKO colorectal cancer cells in vitro was associated not only with the decreased production of pro-inflammatory PGE2 and LTB4, COX-2, arachidonate 5-LOX and microsomal PGE synthase expression, but also with the increased formation of anti-inflammtory LXA4, supporting the hypothesis that LXs, resolvins and protectins have a direct growth inhibitory action on tumor cells; in contrast, 5-FU produced opposite effects on these indices. On the other hand, concerning DHA metabolites, Gleissman et al. [138] showed that the cytotoxic action exerted by DHA in neuroblastoma cells was related to its conversion by 15-LOX and, at much lower degree by autoxidation, to 17-hydroxydocosahexaenoic acid (17-HDHA), via 17-hydroxyperoxydocosahexaenoic acid (17-HPDHA), a compound with significant cytotoxicity potency compared to DHA. In normal nervous tissue, DHA was converted by 5-LOX to anti-inflammatory and cytoprotective resolvins and protectins. In contrast, although neuroblastoma cells contained both 15-LOX and 5-LOX enzymes, the complete conversion of DHA into resolvins and protectins did not take place in cancer cells; thus, 17-HPDHA accumulated and exerted high cytotoxicity. Moreover, DHA, similarly to EPA, inhibited the secretion of PGE2 and augmented the cytotoxic potency of the COX-2-inhibitor celecoxib, by competing with ARA metabolites and by binding to catalytic sites of elongases, desaturases, and COX-2. 


\subsection{Binding of Nuclear Receptors by n-3 PUFAs and Changes in Gene Expression}

Once released from the cell membrane, $n-3$ PUFAs can bind nuclear receptors such as peroxisome proliferator activating receptors (PPARs) in tumor cells [26], which, as ligand-activated transcription factors, regulate the expression of specific/target genes involved in several biological processes, including lipid metabolism and cell death. However, many of nuclear receptor-mediated effects of EPA and DHA are still unexplored.

It was shown that DHA-induced apoptosis in Reh and Ramos cells was mediated by PPAR $\gamma$, which in turn up-regulated the p53 protein, leading to the activation of caspase-9 and caspase-3 [77]. Moreover, in vitro treatment of breast [162] and prostate [163] cancer cells with DHA activated PPAR $\gamma$, which in turn up-regulated SDC-1 expression, inducing thus apoptosis. According to these results, O'Flaherty [164], Hu [165] and coworkers showed that 15-LOX metabolites of DHA, such as 17-HPDHA, 17-HDHA, 10,17-dihydroxy- and 7,17-dihydroxy-DHA, while exerting a more potent cytotoxicity on prostate PC3 cancer cells than DHA, like DHA induced apoptotic PC3 cells to activate a PPAR $\gamma$ reporter, which up-regulated SDC-1 expression; apoptosis was reduced by pharmacological inhibition or knockdown of PPAR $\gamma$ or SDC-1. In addition, 15-LOX-1-mediated metabolism of DHA was required to upregulate SDC-1 and to regulate the PDK/Akt signaling pathway that elicited prostate cancer cell apoptosis. 


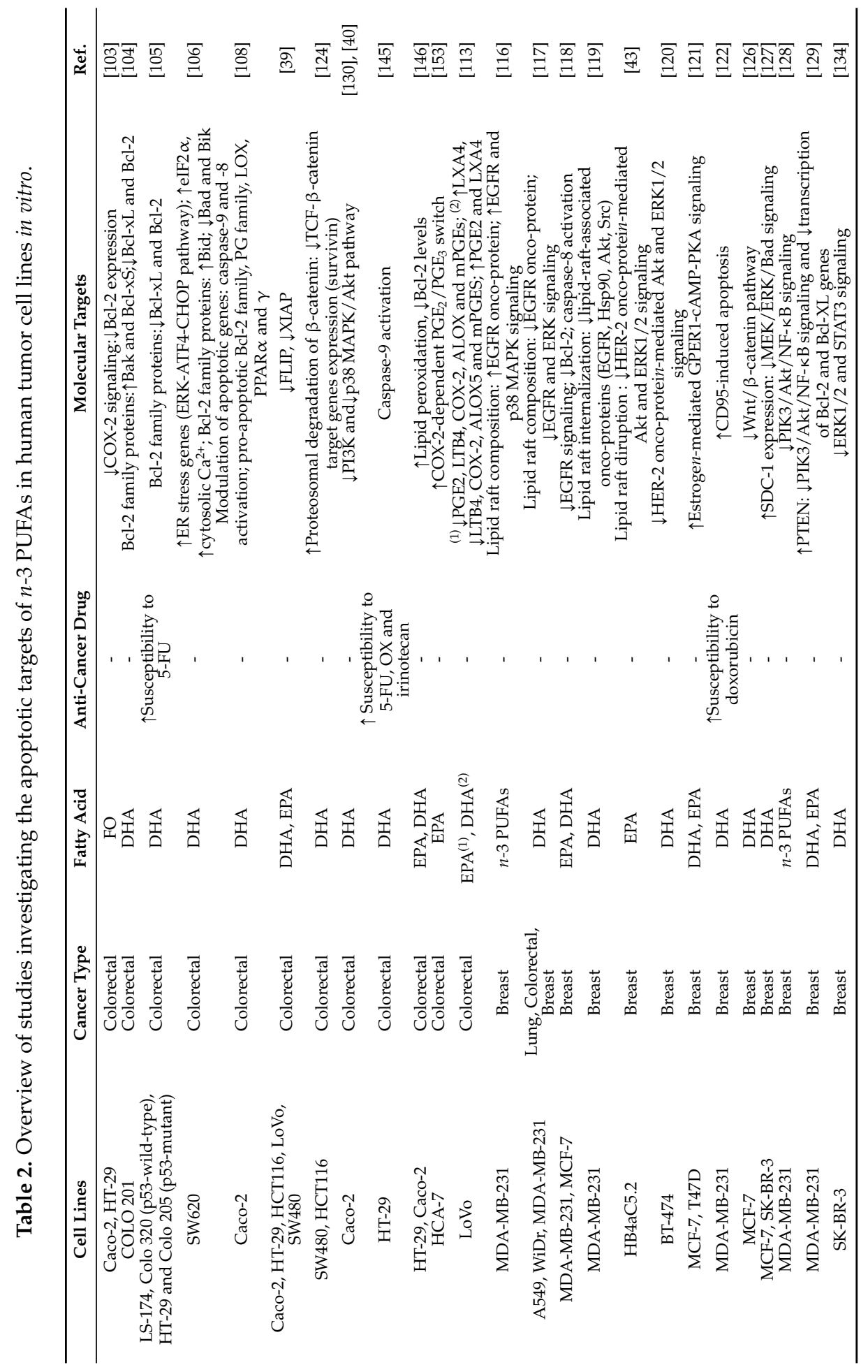




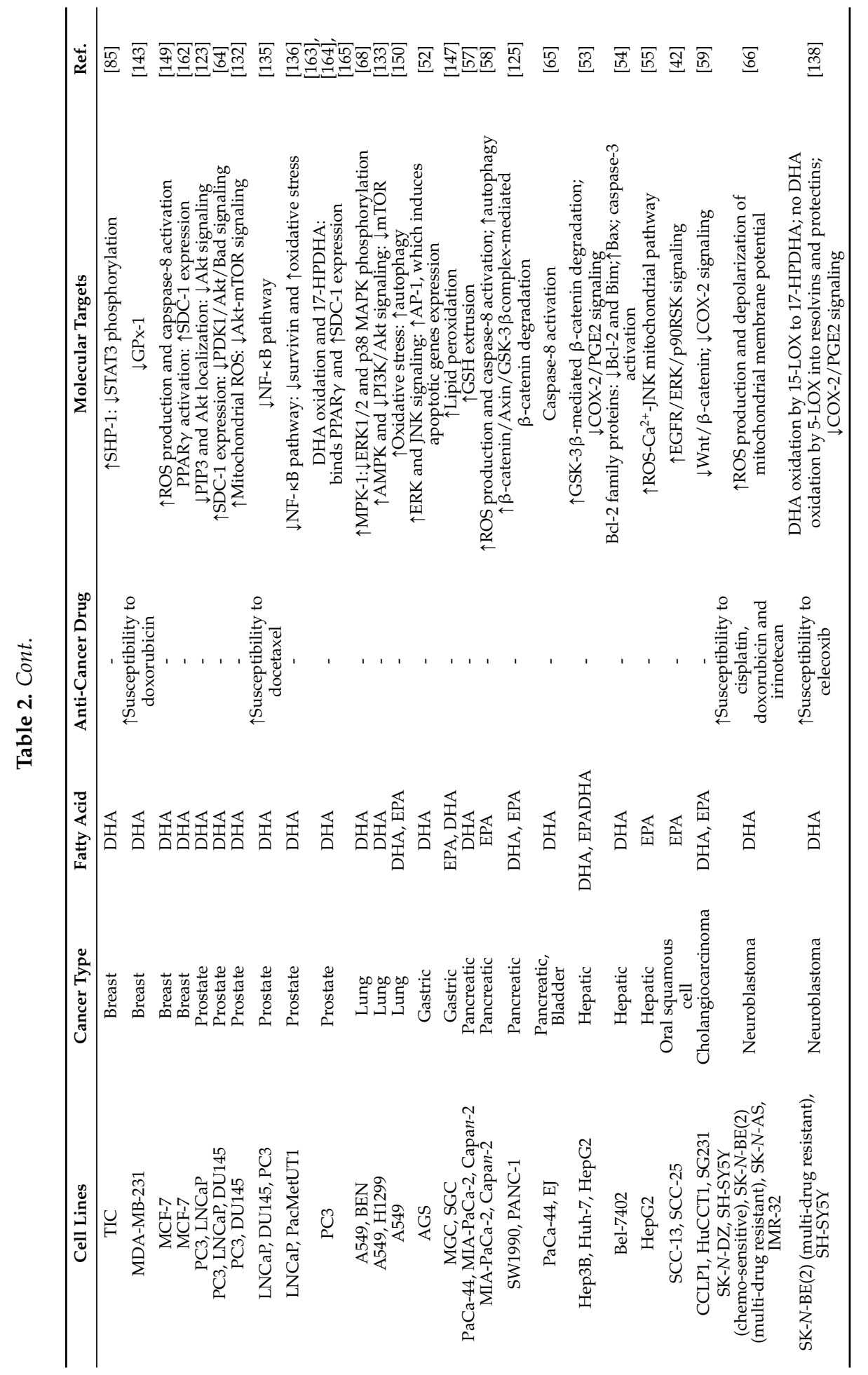




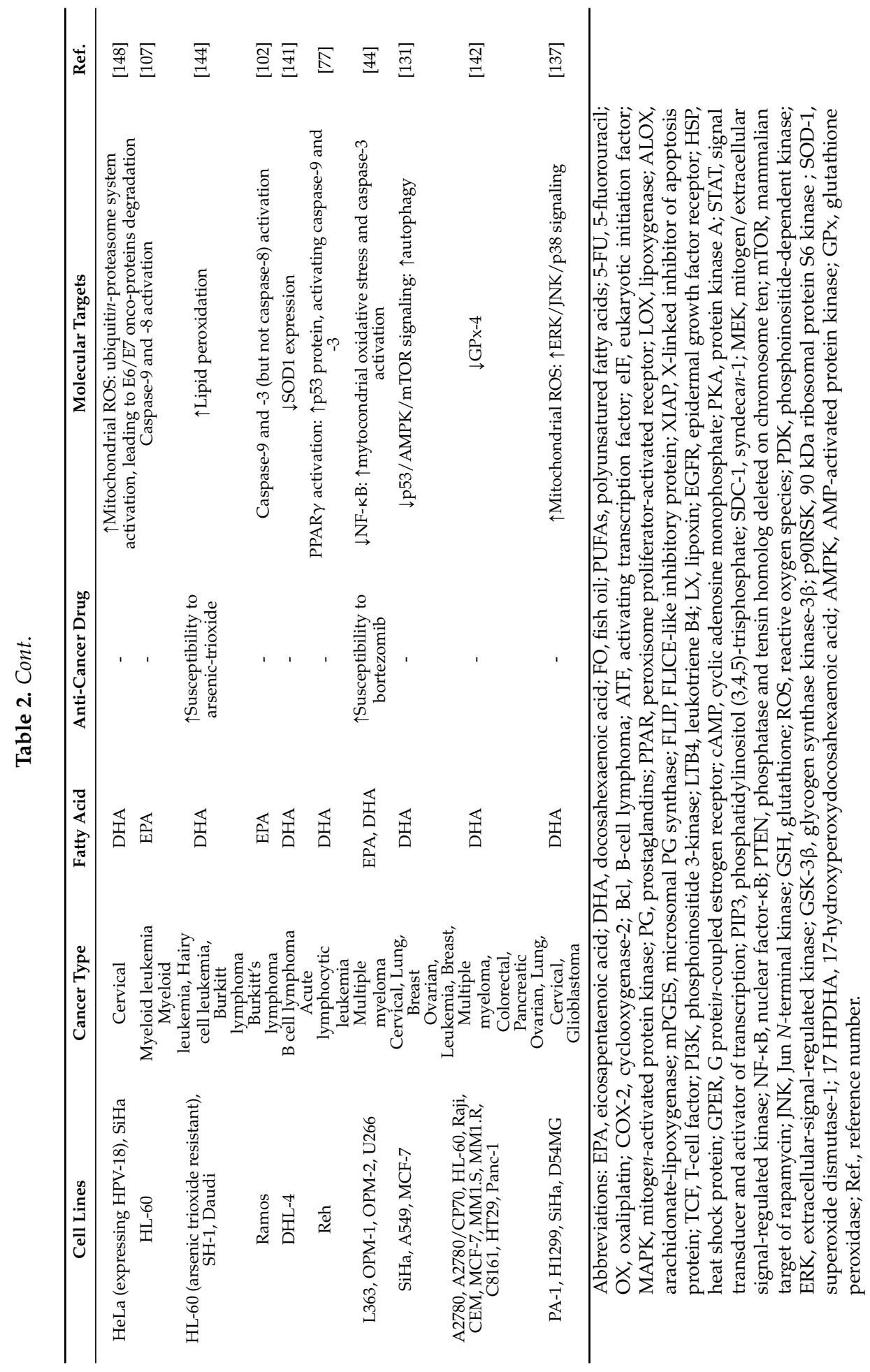




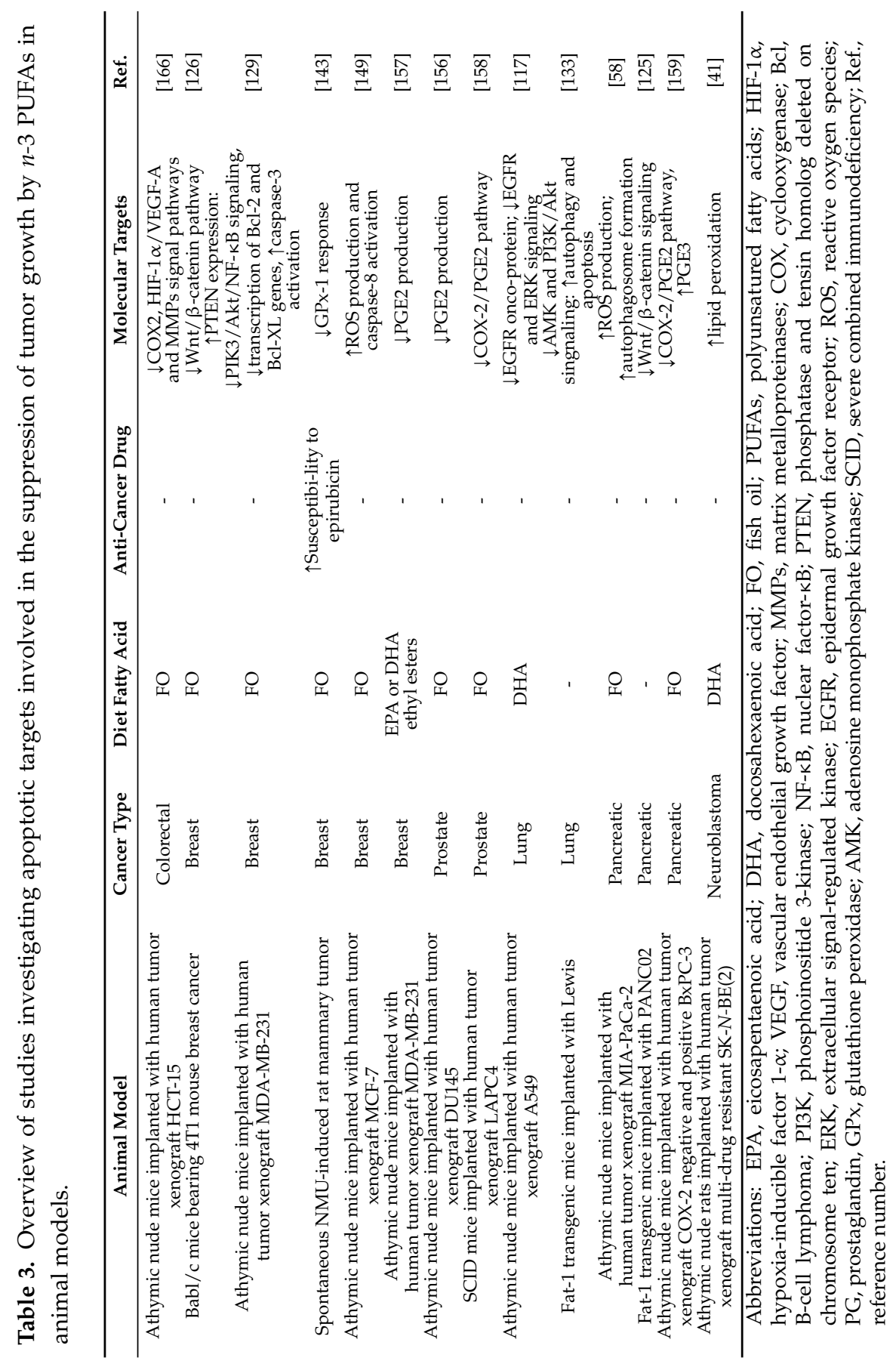




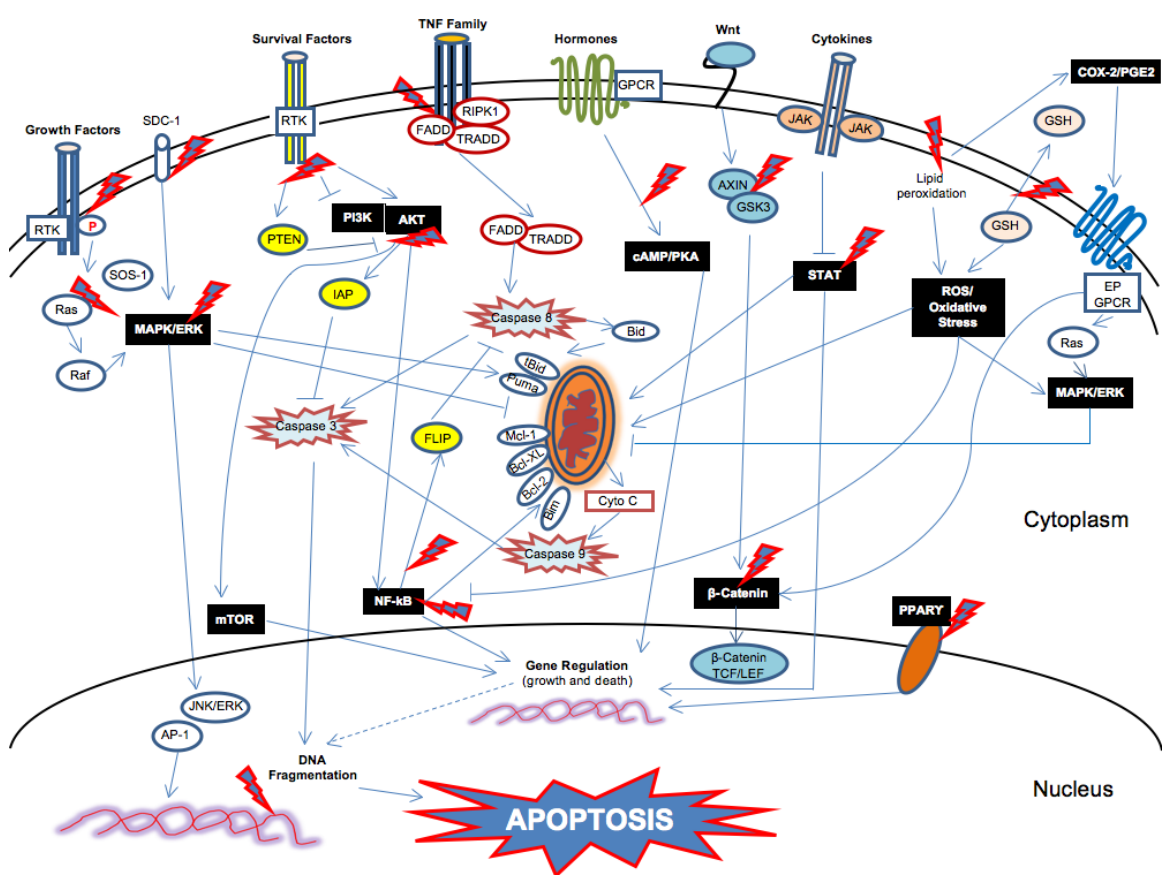

Figure 1. Multiple apoptotic molecular signals targeted by n-3 PUFAs in cancer cells. Abbreviations: RTK, protein tyrosine kinase; SOS-1, son of sevenless-1; Erk, extracellular-signal-regulated kinase; MAPK, mitogen-activated protein kinase; JNK, Jun N-terminal kinase; AP-1, activator protein-1; SDC-1, syndecan-1; PTEN, phosphatase and tensin homolog deleted on chromosome ten; PI3K, phosphatidylinositol-3-kinase; mTOR, mammalian target of rapamycin; IAP, inhibitor of apoptosis; NF- $\mathrm{B}$, nuclear factor-kB; FLIP, FLICE-like inhibitory protein; RIPK-1, receptor-like protein kinase-1; FADD, Fas-associated death domain; TRADD, TNF receptor-associated death domain; Bcl-2, B-cell lymphoma protein-2; Bim, Cyto C, cytochrome C; GPCR, G-protein coupled receptor; cAMP, cyclic adenosine monophosphate; PKA, protein kinase A; GSK, glycogen synthase kinase; TCF, T-cell factor; LEF, lymphoid enhancer-binding factor; JAK, Janus kinase; STAT, signal transducer and activator of transcription; ROS, reactive oxygen species; GSH, glutathione; COX-2, cyclooxygenase-2; PGE2, prostaglandin E2; PPAR- $\gamma$, peroxisome proliferator-activated receptor $\gamma$. arrows, activation; $\perp$, inhibition; dashed arrows, indirect action; red/blue flash, targeted by $n-3$ PUFAs.

\section{Conclusions}

The targeting of tumor cell apoptosis has important therapeutic potential. It is known that essentially all chemotherapeutic drugs and radiotherapy regimens that are in clinical use induce apoptosis of malignant cells when they work 
properly. However, the resistance to therapy, due to the modulation of the expression of multiple genes and gene products involved in cell death and survival, prompt oncologists to believe that, for a more effective apoptosis-based treatment, combinational therapies are needed to target multitude molecular signals involved in cancer cell death. Several studies have proposed the potential cability of $n-3$ PUFAs DHA and EPA to enhance the efficacy as well as the tolerability of conventional anticancer therapies. Taken together, the data presented in this review, showing the ability of $n$-3 PUFAs, DHA and EPA to induce cytotoxicity via apoptosis in different tumor cell types in vitro (Table 2) and in vivo (Table 3), indicate that these FAs potentially target multiple molecular signals involved in tumor cell death (Figure 1).

The use of multiple different pathways by $n-3$ PUFAs to trigger apoptosis in tumor cells may be partly related to the diverse activities possibly exerted in diverse cellular cancer models, but also to the different $n$-3 PUFAs used (EPA, DHA or FO), as well as to the different ways of administration, such as doses and kinetics. The context is complex and it might be even more complex if we consider that most of the molecular signals converge into the nucleus, altering gene expression. The pleiotropic nature of transcriptional changes induced by $n-3$ PUFAs have been recently illustrated by studies where global gene expression patterns were determined by microarray analysis in vitro and in vivo [44,166,167]. Several genes, potentially involved directly or indirectly in cancer cell apoptosis, appear to be regulated by $n-3$ PUFAs, underlining the complexity of the mechanisms involved in the induction of cancer cell apoptosis by these FAs. Therefore, further basic research is needed to show which pathways are crucial for the control of tumor cell apoptosis by $n-3$ PUFAs. Moreover, a clear need appears for further clinical studies, evaluating the potential role of DHA and EPA supplementation, mainly in combination with chemo- and radio-therapeutic anticancer regimens, in the improvement of patients' clinical outcome and survival.

Acknowledgments: This work has been funded by the" bando FILAS Regione Lazio Lr 13/2008" (Project "Innovazioni tecnologiche per migliorare i processi produttivi e le qualità nutraceutiche e salutistiche dei prodotti di specie vegetali del territorio laziale").

Conflicts of Interest: The authors declare no conflict of interest.

\section{References}

1. Ma, X.; Yu, H. Global burden of cancer. J. Biol. Med. 2006, 79, 85-94.

2. Basile, K.J.; Aplin, A.E. Resistance to chemotherapy: Short-term drug tolerance and stem cell-like subpopulations. Adv. Pharmacol. 2012, 65, 315-334.

3. Reya, T.; Morrison, S.J.; Clarke, M.F.; Weissman, I.L. Stem cells, cancer, and cancer stem cells. Nature 2001, 414, 105-111.

4. Maugeri-Saccà, M.; Vigneri, P.; de Maria, R. Cancer stem cells and chemosensitivity. Clin. Cancer Res. 2011, 17, 4942-4947. 
5. Hanahan, D.; Weinberg, R.A. Hallmarks of cancer: The next generation. Cell 2011, 144, 646-674.

6. Pritchard, J.R.; Bruno, P.M.; Gilbert, L.A.; Capron, K.L.; Lauffenburger, D.A.; Hemann, M.T. Defining principles of combination drug mechanisms of action. Proc. Natl. Acad. Sci. USA 2013, 110, E170-E179.

7. Burlingame, B.; Nishida, C.; Uauy, R.; Weisell, R. Fats and fatty acids in human nutrition: Introduction. Ann. Nutr. Metable 2009, 55, 5-7.

8. Riediger, N.D.; Othman, R.A.; Suh, M.; Moghadasian, M.H. A systemic review of the roles of $n-3$ fatty acids in health and disease. J. Am. Diet. Assoc. 2009, 109, 668-679.

9. Calder, P.C. Marine $\omega-3$ Fatty acids and inflammatory processes: Effects, mechanisms and clinical relevance. Biochim. Biophys. Acta 2015, 1851, 469-484.

10. Gil, A.; Gil, F. Fish, a Mediterranean source of n-3 PUFA: Benefits do not justify limiting consumption. Br. J. Nutr. 2015, 113, S58-S67.

11. Laviano, A.; Rianda, S.; Molfino, A.; Rossi Fanelli, F. w-3 Fatty acids in cancer. Curr. Opin. Clin. Nutr. Metab. Care 2013, 16, 156-161.

12. Bhagat, U.; Das, U.N. Potential role of dietary lipids in the prophylaxis of some clinical conditions. Arch. Med. Sci. 2015, 11, 807-818.

13. Murray, M.; Hraiki, A.; Bebawy, M.; Pazderka, C.; Rawling, T. Anti-tumor activities of lipids and lipid analogues and their development as potential anticancer drugs. Pharmacol. Ther. 2015, 150, 109-128.

14. Bang, H.O.; Dyerberg, J.; Nielsen, A.B. Plasma lipid and lipoprotein pattern in Greenlandic West-coast Eskimos. Lancet 1971, 1, 1143-1145.

15. Gu, Z.; Shan, K.; Chen, H.; Chen, Y.Q. n-3 Polyunsaturated fatty acids and their role in cancer chemoprevention. Curr. Pharmacol. Rep. 2015, 5, 283-294.

16. Serini, S.; Fasano, E.; Piccioni, E.; Cittadini, A.R.; Calviello, G. Dietary n-3 polyunsaturated fatty acids and the paradox of their health benefits and potential harmful effects. Chem. Res. Toxicol. 2011, 24, 2093-2105.

17. Chapkin, R.S.; DeClercq, V.; Kim, E.; Fuentes, N.R.; Fan, Y.Y. Mechanisms by which pleiotropic amphiphilic n-3 PUFA reduce colon cancer risk. Curr. Colorectal Cancer Rep. 2014, 10, 442-452.

18. Kiyabu, G.Y.; Inoue, M.; Saito, E.; Abe, S.K.; Sawada, N.; Ishihara, J.; Iwasaki, M.; Yamaji, T.; Shimazu, T.; et al. JPHC Study Group. Fish, $n-3$ polyunsaturated fatty acids and $n-6$ polyunsaturated fatty acids intake and breast cancer risk: The Japan Public Health Center-based prospective study. Int. J. Cancer. 2015, 137, 2915-2926.

19. Brasky, T.M.; Darke, A.K.; Song, X.; Tangen, C.M.; Goodman, P.J.; Thompson, I.M.; Meyskens, F.L., Jr.; Goodman, G.E.; Minasian, L.M.; et al. Plasma phospholipid fatty acids and prostate cancer risk in the SELECT trial. J. Natl. Cancer Inst. 2013, 105, 1132-1141.

20. Calder, P.C.; Deckelbaum, R.J. Dietary fatty acids in health and disease: Greater controversy, greater interest. Curr. Opin. Clin. Nutr. Metab. Care 2014, 17, 111-115.

21. Weylandt, K.H.; Serini, S.; Chen, Y.Q.; Su, H.M.; Lim, K.; Cittadini, A.; Calviello, G. $\omega-3$ Polyunsaturated fatty acids: The way forward in times of mixed evidence. Biomed. Res. Int. 2015, 2015, 143109. 
22. Berquin, I.M.; Edwards, I.J.; Chen, Y.Q. Multi-targeted therapy of cancer by $\omega-3$ Fatty acids. Cancer Lett. 2008, 269, 363-377.

23. Serini, S.; Piccioni, E.; Merendino, N.; Calviello, G. Dietary polyunsaturated fatty acids as inducers of apoptosis: Implications for cancer. Apoptosis 2009, 14, 132-152.

24. Gleissman, H.; Johnsen, J.I.; Kogner, P. $\omega-3$ Fatty acids in cancer, the protectors of good and the killers of evil? Exp. Cell Res. 2010, 316, 1365-1373.

25. Vaughan, V.C.; Hassing, M.R.; Lewandowski, P.A. Marine polyunsaturated fatty acids and cancer therapy. Br. J. Cancer 2013, 108, 486-492.

26. Biondo, P.D.; Brindley, D.N.; Sawyer, M.B.; Field, C.J. The potential for treatment with dietary long-chain polyunsaturated $n-3$ fatty acids during chemotherapy. J. Nutr. Biochem. 2008, 19, 787-796.

27. Siddiqui, R.A.; Harvey, K.A.; Xu, Z.; Bammerlin, E.M.; Walker, C.; Altenburg, J.D. Docosahexaenoic acid: A natural powerful adjuvant that improves efficacy for anticancer treatment with no adverse effects. Biofactors 2011, 37, 399-412.

28. Wang, J.; Luo, T.; Li, S.; Zhao, J. The powerful applications of polyunsaturated fatty acids in improving the therapeutic efficacy of anticancer drugs. Expert Opin. Drug Deliv. 2012, 9, 1-7.

29. Merendino, N.; Costantini, L.; Manzi, L.; Molinari, R.; D’Eliseo, D.; Velotti, F. Dietary $\omega-3$ polyunsaturated fatty acid DHA: A potential adjuvant in the treatment of cancer. Biomed. Res. Int. 2013, 2013, 310186.

30. Hajjaji, N.; Bougnoux, P. Selective sensitization of tumors to chemotherapy by marine-derived lipids: A review. Cancer Treat. Rev. 2013, 39, 473-488.

31. de Aguiar Pastore Silva, J.; Emilia de Souza Fabre, M.; Waitzberg, D.L. $\omega$-3 Supplements for patients in chemotherapy and/or radiotherapy: A systematic review. Clin. Nutr. 2015, 34, 359-366.

32. Das, U.N.; Madhavi, N.; Sravan Kumar, G.; Padma, M.; Sangeetha, P. Can tumour cell drug resistance be reversed by essential fatty acids and their metabolites? Prostaglandins Leukot. Essent. Fatty Acids 1998, 58, 39-54.

33. Slagsvold, J.E.; Pettersen, C.H.; Størvold, G.L.; Follestad, T.; Krokan, H.E.; Schønberg, S.A. DHA alters expression of target proteins of cancer therapy in chemotherapy resistant SW620 colon cancer cells. Nutr. Cancer 2010, 62, 611-621.

34. Kuan, C.Y.; Walker, T.H.; Luo, P.G.; Chen, C.F. Long-chain polyunsaturated fatty acids promote paclitaxel cytotoxicity via inhibition of the MDR1 gene in the human colon cancer Caco-2 cell line. J. Am. Coll. Nutr. 2011, 30, 265-273.

35. Gelsomino, G.; Corsetto, P.A.; Campia, I.; Montorfano, G.; Kopecka, J.; Castella, B.; Gazzano, E.; Ghigo, D.; Rizzo, A.M.; Riganti, C. $\omega 3$ Fatty acids chemosensitize multidrug resistant colon cancer cells by down-regulating cholesterol synthesis and altering detergent resistant membranes composition. Mol. Cancer 2013, 12, 137.

36. Das, U.N.; Begin, M.E.; Ells, G.; Huang, Y.S.; Horrobin, D.F. Polyunsaturated fatty acids augment free radical generation in tumor cells in vitro. Biochem. Biophys. Res. Commun. $1987,145,15-24$. 
37. Tsai, W.S.; Nagawa, H.; Kaizaki, S.; Tsuruo, T.; Muto, T. Inhibitory effects of n--3 polyunsaturated fatty acids on sigmoid colon cancer transformants. J. Gastroenterol. 1998, 33, 206-212.

38. Siddiqui, R.A.; Harvey, K.; Stillwell, W. Anticancer properties of oxidation products of docosahexaenoic acid. Chem. Phys. Lipids 2008, 153, 47-56.

39. Giros, A.; Grzybowski, M.; Sohn, V.R.; Pons, E.; Fernandez-Morales, J.; Xicola, R.M.; Sethi, P.; Grzybowski, J.; Goel, A.; et al. Regulation of colorectal cancer cell apoptosis by the $n-3$ polyunsaturated fatty acids Docosahexaenoic and Eicosapentaenoic. Cancer Prev. Res. 2009, 2, 732-742.

40. Toit-Kohn, J.L.; Louw, L.; Engelbrecht, A.M. Docosahexaenoic acid induces apoptosis in colorectal carcinoma cells by modulating the PI3 kinase and p38 MAPK pathways. J. Nutr. Biochem. 2009, 20, 106-114.

41. Gleissman, H.; Segerström, L.; Hamberg, M.; Ponthan, F.; Lindskog, M.; Johnsen, J.I.; Kogner, P. $\omega-3$ Fatty acid supplementation delays the progression of neuroblastoma in vivo. Int. J. Cancer 2011, 128, 1703-1711.

42. Nikolakopoulou, Z.; Nteliopoulos, G.; Michael-Titus, A.T.; Parkinson, E.K. $w-3$ Polyunsaturated fatty acids selectively inhibit growth in neoplastic oral keratinocytes by differentially activating ERK1/2. Carcinogenesis 2013, 34, 2716-2725.

43. Ravacci, G.R.; Brentani, M.M.; Tortelli, T.Jr.; Torrinhas, R.S.; Saldanha, T.; Torres, E.A.; Waitzberg, D.L. Lipid raft disruption by docosahexaenoic acid induces apoptosis in transformed human mammary luminal epithelial cells harboring HER-2 overexpression. J. Nutr. Biochem. 2013, 24, 505-515.

44. Abdi, J.; Garssen, J.; Faber, J.; Redegeld, F.A. $\omega-3$ Fatty acids, EPA and DHA induce apoptosis and enhance drug sensitivity in multiple myeloma cells but not in normal peripheral mononuclear cells. J. Nutr. Biochem. 2014, 25, 1254-1262.

45. Berstad, P.; Thiis-Evensen, E.; Vatn, M.H.; Almendingen, K. Fatty acids in habitual diet, plasma phospholipids, and tumour and normal colonic biopsies in young colorectal cancer patients. J. Oncol. 2012, 2012, 254801.

46. Thomas, G.C. Apoptosis and cancer: The genesis of a research field. Nature Rev. Cancer 2009, 9, 501-507.

47. Logue, S.E.; Gorman, A.M.; Cleary, P.; Keogh, N.; Samali, A. Current concepts in ER stress-induced apoptosis. J. Carcinogene Mutagene 2013.

48. Mengeaud, V.; Nano, J.L.; Fournel, S.; Rampal, P. Effects of eicosapentaenoic acid, $\gamma$-linolenic acid and prostaglandin E1 on three human colon carcinoma cell lines. Prostaglandins Leukot. Essent. Fatty Acids 1992, 47, 313-319.

49. Clarke, R.G.; Lund, E.K.; Latham, P.; Pinder, A.C.; Johnson, I.T. Effect of eicosapentaenoic acid on the proliferation and incidence of apoptosis in the colorectal cell line HT29. Lipids 1999, 34, 1287-1295.

50. Chen, Z.Y.; Istfan, N.W. Docosahexaenoic acid is a potent inducer of apoptosis in HT-29 colon cancer cells. Prostaglandins Leukot. Essent. Fatty Acids 2000, 63, 301-308. 
51. Kubota, H.; Matsumoto, H.; Higashida, M.; Murakami, H.; Nakashima, H.; Oka, Y.; Okumura, H.; Yamamura, M.; Nakamura, M.; Hirai, T. Eicosapentaenoic acid modifies cytokine activity and inhibits cell proliferation in an oesophageal cancer cell line. Anticancer Res. 2013, 33, 4319-4324.

52. Lee, S.E.; Lim, J.W.; Kim, H. Activator protein-1 mediates docosahexaenoic acid-induced apoptosis of human gastric cancer cells. Ann. N. Y. Acad. Sci. 2009, 1171, 163-169.

53. Lim, K.; Han, C.; Dai, Y.; Shenm, M.; Wu, T. $\omega-3$ Polyunsaturated fatty acids inhibit hepatocellular carcinoma cell growth through blocking $\beta$-catenin and cyclooxygenase- 2 . Mol. Cancer Ther. 2009, 8, 3046-3055.

54. Sun, S.N.; Jia, W.D.; Chen, H.; Ma, J.L.; Ge, Y.S.; Yu, J.H.; Li, J.S. Docosahexaenoic acid (DHA) induces apoptosis in human hepatocellular carcinoma cells. Int. J. Clin. Exp. Pathol. 2013, 6, 281-289.

55. Zhang, Y.; Han, L.; Qi, W.; Cheng, D.; Ma, X.; Hou, L.; Cao, X.; Wang, C. Eicosapentaenoic acid (EPA) induced apoptosis in HepG2 cells through ROS-Ca ${ }^{2+}-\mathrm{JNK}$ mitochondrial pathways. Biochem. Biophys. Res. Commun. 2015, 456, 926-932.

56. Hawkins, R.A.; Sangster, K.; Arends, M.J. Apoptotic death of pancreatic cancer cells induced by polyunsaturated fatty acids varies with double bond number and involves an oxidative mechanism. J. Pathol. 1998, 185, 61-70.

57. Merendino, N.; Loppi, B.; D’Aquino, M.; Molinari, R.; Pessina, G.; Romano, C.; Velotti, F. Docosahexaenoic acid induces apoptosis in the human PaCa-44 pancreatic cancer cell line by active reduced glutathione extrusion and lipid peroxidation. Nutr. Cancer 2005, 52, 225-233.

58. Fukui, M.; Kang, K.S.; Okada, K.; Zhu, B.T. EPA, an $\omega-3$ Fatty acid, induces apoptosis in human pancreatic cancer cells: Role of ROS accumulation, caspase-8 activation, and autophagy induction. J. Cell. Biochem. 2013, 114, 192-203.

59. Lim, K.; Han, C.; Xu, L.; Isse, K.; Demetris, A.J.; Wu, T. Cyclooxygenase-2-derived prostaglandin E2 activates $\beta$-catenin in human cholangiocarcinoma cells: Evidence for inhibition of these signaling pathways by $\omega 3$ polyunsaturated fatty acids. Cancer Res. 2008, 68, 553-560.

60. Rose, D.P.; Connolly, J.M. Effects of fatty acids and inhibitors of eicosanoid synthesis on the growth of a human breast cancer cell line in culture. Cancer Res. 1990, 50, 7139-7144.

61. Chamras, H.; Ardashian, A.; Heber, D.; Glaspy, J.A. Fatty acid modulation of MCF-7 human breast cancer cell proliferation, apoptosis and differentiation. J. Nutr. Biochem. 2002, 13, 711-716.

62. Sharma, A.; Belna, J.; Logan, J.; Espat, J.; Hurteau, J.A. The effects of $\omega-3$ fatty acids on growth regulation of epithelial ovarian cancer cell lines. Gynecol. Oncol. 2005, 99, 58-64.

63. Narayanan, N.K.; Narayanan, B.A.; Reddy, B.S. A combination of docosahexaenoic acid and celecoxib prevents prostate cancer cell growth in vitro and is associated with modulation of nuclear factor-kB, and steroid hormone receptors. Int. J. Oncol. 2005, 26, 785-792. 
64. Hu, Y.; Sun, H.; Owens, R.T.; Gu, Z.; Wu, J.; Chen, Y.Q.; O'Flaherty, J.T.; Edwards, I.J. Syndecan-1-dependent suppression of PDK1/Akt/bad signaling by docosahexaenoic acid induces apoptosis in prostate cancer. Neoplasia 2010, 12, 826-836.

65. Molinari, R.; D’Eliseo, D.; Manzi, L.; Zolla, L.; Velotti, F.; Merendino, N. The n3-polyunsaturated fatty acid docosahexaenoic acid induces immunogenic cell death in human cancer cell lines via pre-apoptotic calreticulin exposure. Cancer Immunol. Immunother. 2011, 60, 1503-1507.

66. Lindskog, M.; Gleissman, H.; Ponthan, F.; Castro, J.; Kogner, P.; Johnsen, J.I. Neuroblastoma cell death in response to docosahexaenoic acid: Sensitization to chemotherapy and arsenic induced oxidative stress. Int. J. Cancer 2006, 118, 2584-2593.

67. Faragó, N.; Fehér, L.Z.; Kitajka, K.; Das, U.N.; Puskás, L.G. MicroRNA profile of polyunsaturated fatty acid treated glioma cells reveal apoptosis-specific expression changes. Lipids Health Dis. 2011, 10, 173.

68. Serini, S.; Trombino, S.; Oliva, F.; Piccioni, E.; Monego, G.; Resci, F.; Boninsegna, A.; Picci, N.; Ranelletti, F.O.; Calviello, G. Docosahexaenoic acid induces apoptosis in lung cancer cells by increasing MKP-1 and down-regulating p-ERK1/2 and p-p38 expression. Apoptosis 2008, 13, 1172-1183.

69. Yao, Q.H.; Zhang, X.C.; Fu, T.; Gu, J.Z.; Wang, L.; Wang, Y.; Lai, Y.B.; Wang, Y.Q.; Guo, Y. $\omega-3$ polyunsaturated fatty acids inhibit the proliferation of the lung adenocarcinoma cell line A549 in vitro. Mol. Med. Rep. 2014, 9, 401-406.

70. Albino, A.P.; Juan, G.; Traganos, F.; Reinhart, L.; Connolly, J.; Rose, D.P.; Darzynkiewicz, Z. Cell cycle arrest and apoptosis of melanoma cells by docosahexaenoic acid: Association with decreased pRb phosphorylation. Cancer Res. 2000, 60, 4139-4145.

71. Denkins, Y.; Kempf, D.; Ferniz, M.; Nileshwar, S.; Marchetti, D. Role of $w-3$ polyunsaturated fatty acids on cyclooxygenase- 2 metabolism in brain-metastatic melanoma. J. Lipid Res. 2005, 46, 1278-1284.

72. Finstad, H.S.; Myhrstad, M.C.; Heimli, H.; Lømo, J.; Blomhoff, H.K.; Kolset, S.O.; Drevon, C.A. Multiplication and death-type of leukemia cell lines exposed to very long-chain polyunsaturated fatty acids. Leukemia 1998, 12, 921-929.

73. Finstad, H.S.; Drevon, C.A.; Kulseth, M.A.; Synstad, A.V.; Knudsen, E.; Kolset, S.O. Cell proliferation, apoptosis and accumulation of lipid droplets in U937-1 cells incubated with eicosapentaenoic acid. Biochem. J. 1998, 336, 451-459.

74. Chiu, L.C.M.; Wan, J.M.F. Induction of apoptosis in HL-60 cells by eicosapentaenoic acid (EPA) is associated with downregulation of BCL-2 expression. Cancer Letters 1999, 145, 17-27.

75. Chiu, L.C.; Wong, E.Y.; Ooi, V.E. Docosahexaenoic acid modulates different genes in cell cycle and apoptosis to control growth of human leukemia HL-60 cells. Int J Oncol. 2004, $25,737-744$.

76. Siddiqui, R.A.; Jenski, L.J.; Neff, K.; Harvey, K.; Kovacs, R.J.; Stillwell, W. Docosahexaenoic acid induces apoptosis in Jurkat cells by a protein phosphatase-mediated process. Biochim. Biophys. Acta 2001, 1499, 265-275. 
77. Zand, H.; Rhimipour, A.; Bakhshayesh, M.; Shafiee, M.; Nour Mohammadi, I.; Salimi, S. Involvement of PPAR- $\gamma$ and p53 in DHA-induced apoptosis in Reh cells. Mol. Cell. Biochem. 2007, 304, 71-77.

78. Yamagami, T.; Porada, C.D.; Pardini, R.S.; Zanjani, E.D.; Almeida-Porada, G. Docosahexaenoic acid induces dose dependent cell death in an early undifferentiated subtype of acute myeloid leukemia cell line. Cancer Biol. Ther. 2009, 8, 331-337.

79. Sravan Kumar, G.; Das, U.N. Cytotoxic action of $\alpha$-linolenic and eicosapentaenoic acids on myeloma cells in vitro. Prostaglandins Leukot. Essent. Fatty Acids 1997, 56, 285-293.

80. Ricci-Vitiani, L.; Lombardi, D.G.; Pilozzi, E.; Biffoni, M.; Todaro, M.; Peschle, C.; de Maria, R. Identification and expansion of human colon-cancer-initiating cells. Nature 2007, 445, 111-115.

81. Das, U.N. Essential fatty acids and their metabolites as modulators of stem cell biology with reference to inflammation, cancer, and metastasis. Cancer Metastasis Rev. 2011, 30, 311-324.

82. Yang, T.; Fang, S.; Zhang, H.X.; Xu, L.X.; Zhang, Z.Q.; Yuan, K.T.; Xue, C.L.; Yu, H.L.; Zhang, S.; Li, Y.F.; et al. n-3 PUFA shave antiproliferative and apoptotic effects on human colorectal cancer stemlike cells in vitro. J. Nutr. Biochem. 2013, 24, 744-753.

83. Vasudevan, A.; Yu, Y.; Banerjee, S.; Woods, J.; Farhana, L.; Rajendra, S.G.; Patel, A.; Dyson, G.; Levi, E.; Maddipati, K.R.; et al. $\omega-3$ Fatty acid is a potential preventive agent for recurrent colon cancer. Cancer Prev. Res. 2014, 7, 1138-1148.

84. De Carlo, F.; Witte, T.R.; Hardman, W.E.; Claudio, P.P. $\omega-3$ Eicosapentaenoic acid decreases CD133 colon cancer stem-like cell marker expression while increasing sensitivity to chemotherapy. PLoS ONE 2013, 8, e69760.

85. Xiong, A.; Yu, W.; Liu, Y.; Sanders, B.G.; Kline, K. Elimination of ALDH+ breast tumor initiating cells by docosahexanoic acid and/or $\gamma$ tocotrienol through SHP-1 inhibition of Stat3 signaling. Mol. Carcinog. 2015.

86. Rose, D.P.; Connolly, J.M.; Rayburn, J.; Coleman, M. Influence of diets containing eicosapentaenoic or docosahexaenoic acid on growth and metastasis of breast cancer cells in nude mice. J. Natl. Cancer Inst. 1995, 87, 587-592.

87. Yam, D.; Peled, A.; Huszar, M.; Shinitzky, M. Dietary fish oil suppresses tumor growth and metastasis of Lewis lung carcinoma in mice. J. Nutr. Biochem. 1997, 8, 619-622.

88. Boudreau, M.D.; Sohn, K.H.; Rhee, S.H.; Lee, S.W.; Hunt, J.D.; Hwang, D.H. Suppression of tumor cell growth both in nude mice and in culture by $n-3$ polyunsaturated fatty acids: Mediation through cyclooxygenase-independent pathways. Cancer Res. 2001, 61, 1386-1391.

89. Kato, T.; Hancock, R.L.; Mohammadpour, H.; McGregor, B.; Manalo, P.; Khaiboullina, S.; Hall, M.R.; Pardini, L.; Pardini, R.S. Influence of $\omega-3$ fatty acids on the growth of human colon carcinoma in nude mice. Cancer Lett. 2002, 187, 169-177.

90. Camargo, C.Q.; Mocellin, M.C.; Pastore Silva, J.A.; de Souza Fabre, M.E.; Nunes, E.A.; de Moraes Trinidade, E.B. Fish oil supplementation during chemotherapy increases posterior time to tumor progression in colorectal cancer. Nutr. Cancer, (in press). Available online: http://.doi.org/10.1080/01635581.2016.1115097 (accessed on 19 January 2016). 
91. Bougnoux, P.; Hajjaji, N.; Ferrasson, M.N.; Giraudeau, B.; Couet, C.; le Floch, O. Improving outcome of chemotherapy of metastatic breast cancer by docosahexaenoic acid: A phase II trial. Br. J. Cancer 2009, 1011978-1011985.

92. Cockbain, J.; Toogood, G.J.; Hull, M.A. $\omega-3$ Polyunsaturated fatty acids for the treatment and prevention of colorectal cancer. Gut 2012, 61, 135-149.

93. Murphy, R.A.; Mourtzakis, M.; Chu, Q.S.; Baracos, V.E.; Reiman, T.; Mazurak, V.C. Supplementation with fish oil increases first-line chemotherapy efficacy in patients with advanced non-small cell lung cancer. Cancer 2011, 117, 3774-3780.

94. Patterson, R.E.; Flatt, S.W.; Newman, V.A.; Natarajan, L.; Rock, C.L.; Thomson, C.A.; Caan, B.J.; Parker, B.A.; Pierce, J.P. Marine fatty acid intake is associated with breast cancer prognosis. J. Nutr. 2011, 141, 201-206.

95. Cockbain, A.J.; Volpato, M.; Race, A.D.; Munarini, A.; Fazio, C.; Belluzzi, A.; Loadman, P.M.; Toogood, G.J.; Hull, M.A. Anticolorectal cancer activity of the $\omega-3$ polyunsaturated fatty acid eicosapentaenoic acid. Gut 2014, 63, 1760-1768.

96. Sánchez-Lara, K.; Turcott, J.G.; Juárez-Hernández, E.; Nuñez-Valencia, C.; Villanueva, G.; Guevara, P.; de la Torre-Vallejo, M.; Mohar, A.; Arrieta, O. Effects of an oral nutritional supplement containing eicosapentaenoic acid on nutritional and clinical outcomes in patients with advanced non-small cell lung cancer: Randomised trial. Clin. Nutr. 2014, 33, 1017-1023.

97. Arshad, A.; Chung, W.Y.; Isherwood, J.; Mann, C.D.; Al-Leswas, D.; Steward, W.P.; Metcalfe, M.S.; Dennison, A.R. Cellular and plasma uptake of parenteral $\omega-3$ rich lipid emulsion fatty acids in patients with advanced pancreatic cancer. Clin. Nutr. 2014, 33, 895-899.

98. Ma, Y.J.; Yu, J.; Xiao, J.; Cao, B.W. The consumption of $\omega-3$ polyunsaturated fatty acids improves clinical outcomes and prognosis in pancreatic cancer patients: A systematic evaluation. Nutr. Cancer 2015, 67, 112-118.

99. Nabavi, S.F.; Bilottom, S.; Russom, G.L.; Orhan, I.E.; Habtemariam, S.; Daglia, M.; Devi, K.P.; Loizzo, M.R.; Tundis, R.; Nabavi, S.M. $\omega-3$ polyunsaturated fatty acids and cancer: Lessons learned from clinical trials. Cancer Metastasis Rev. 2015, 34, 359-380.

100. Khankari, N.K.; Bradshaw, P.T.; Steck, S.E.; He, K.; Olshan, A.F.; Shen, J.; Ahn, J.; Chen, Y.; Ahsan, H.; Terry, M.B.; et al. Dietary intake of fish, polyunsaturated fatty acids, and survival after breast cancer: A population-based follow-up study on Long Island, New York. Cancer 2015.

101. Mocellin, M.C.; Camargo, C.Q.; Nunes, E.A.; Fiates, G.M.; Trindade, E.B. A systematic review and meta-analysis of the $n-3$ polyunsaturated fatty acids effects on inflammatory markers in colorectal cancer. Clin. Nutr. 2015.

102. Heimli, H.; Giske, C.; Naderi, S.; Drevon, C.A.; Hollung, K. Eicosapentaenoic acid promotes apoptosis in Ramos cells via activation of caspase-3 and -9. Lipids 2002, 37, 797-802.

103. Llor, X.; Pons, E.; Roca, A.; Alvarez, M.; Mañé, J.; Fernández-Bañares, F.; Gassull, M.A. The effects of fish oil, olive oil, oleic acid and linoleic acid on colorectal neoplastic processes. Clin. Nutr. 2003, 22, 71-79. 
104. Danbara, N.; Yuri, T.; Tsujita-Kyutoku, M.; Sato, M.; Senzaki, H.; Takada, H.; Hada, T.; Miyazawa, T.; Okazaki, K.; Tsubura, A. Conjugated docosahexaenoic acid is a potent inducer of cell cycle arrest and apoptosis and inhibits growth of colo 201 human colon cancer cells. Nutr. Cancer 2004, 50, 71-79.

105. Calviello, G.; Di Nicuolo, F.; Serini, S.; Piccioni, E.; Boninsegna, A.; Maggiano, N.; Ranelletti, F.O.; Palozza, P. Docosahexaenoic acid enhances the susceptibility of human colorectal cancer cells to 5-fluorouracil. Cancer Chemother. Pharmacol. 2005, 55, 12-20.

106. Jakobsen, C.H.; Størvold, G.L.; Bremseth, H.; Follestad, T.; Sand, K.; Mack, M.; Olsen, K.S.; Lundemo, A.G.; Iversen, J.G.; Krokan, H.E.; et al. DHA induces ER stress and growth arrest in human colon cancer cells: Associations with cholesterol and calcium homeostasis. J. Lipid. Res. 2008, 49, 2089-2100.

107. Arita, K.; Kobuchi, H.; Utsumi, T.; Takehara, Y.; Akiyama, J.; Horton, A.A.; Utsumi, K. Mechanism of apoptosis in HL-60 cells induced by $n-3$ and $n-6$ polyunsaturated fatty acids. Biochem. Pharmacol. 2001, 62, 821-828.

108. Narayanan, B.A.; Narayanan, N.K.; Reddy, B.S. Docosahexaenoic acid regulated genes and transcription factors inducing apoptosis in human colon cancer cells. Int. J. Oncol. 2001, 19, 1255-1262.

109. Kolch, W.; Halasz, M.; Granovskaya, M.; Kholodenko, B.N. The dynamic control of signal transduction networks in cancer cells. Nat. Rev. Cancer 2015, 15, 515-527.

110. Huang, C.Y.; Yu, L.C. Pathophysiological mechanisms of death resistance in colorectal carcinoma. World J. Gastroenterol. 2015, 21, 11777-11792.

111. Glatz, J.F.; Luiken, J.J.; van Nieuwenhoven, F.A.; van der Vusse, G.J. Molecular mechanism of cellular uptake and intracellular translocation of fatty acids. Prostaglandins Leukot. Essent. Fatty Acids 1997, 57, 3-9.

112. Wassall, S.R.; Stillwell, W. Polyunsaturated fatty acid-cholesterol interactions: Domain formation in membranes. Biochim. Biophys. Acta 2009, 1788, 24-32.

113. Zhang, C.; Yu, H.; Ni, X.; Shen, S.; Das, U.N. Growth inhibitory effect of polyunsaturated fatty acids (PUFAs) on colon cancer cells via their growth inhibitory metabolites and fatty acid composition changes. PLoS ONE 2015, 10, e0123256.

114. Ibarguren, M.; López, D.J.; Escribá, P.V. The effect of natural and synthetic fatty acids on membrane structure, microdomain organization, cellular functions and human health. Biochim. Biophys. Acta 2014, 1838, 1518-1528.

115. Corsetto, P.A.; Cremona, A.; Montorfano, G.; Jovenitti, I.E.; Orsini, F.; Arosio, P.; Rizzo, A.M. Chemical-physical changes in cell membrane microdomains of breast cancer cells after $\omega$-3 PUFA incorporation. Cell Biochem. Biophys. 2012, 64, 45-59.

116. Schley, P.D.; Brindley, D.N.; Field, C.J. (n-3) PUFA alter raft lipid composition and decrease epidermal growth factor receptor levels in lipid rafts of human breast cancer cells. J. Nutr. 2007, 137, 548-553.

117. Rogers, K.R.; Kikawa, K.D.; Mouradian, M.; Hernandez, K.; McKinnon, K.M.; Ahwah, S.M.; Pardini, R.S. Docosahexaenoic acid alters epidermal growth factor receptor-related signaling by disrupting its lipid raft association. Carcinogenesis 2010, 31, 1523-1530. 
118. Corsetto, P.A.; Montorfano, G.; Zava, S.; Jovenitti, I.E.; Cremona, A.; Berra, B.; Rizzo, A.M. Effects of $n-3$ PUFAs on breast cancer cells through their incorporation in plasma membrane. Lipids Health Dis. 2011, 10, 73.

119. Lee, E.J.; Yun, U.J.; Koo, K.H.; Sung, J.Y.; Shim, J.; Ye, S.K.; Hong, K.M.; Kim, Y.N. Down-regulation of lipid raft-associated onco-proteins via cholesterol-dependent lipid raft internalization in docosahexaenoic acid-induced apoptosis. Biochim. Biophys. Acta 2014, 1841, 190-203.

120. Mason, J.K.; Klaire, S.; Kharotia, S.; Wiggins, A.K.; Thompson, L.U. $\alpha$-linolenic acid and docosahexaenoic acid, alone and combined with trastuzumab, reduce HER2-overexpressing breast cancer cell growth but differentially regulate HER2 signaling pathways. Lipids Health Dis. 2015, 14, 91.

121. Cao, W.; Ma, Z.; Rasenick, M.M.; Yeh, S.; Yu, J. n-3 poly-unsaturated fatty acids shift estrogen signaling to inhibit human breast cancer cell growth. PLoS ONE 2012, 7, e52838.

122. Ewaschuk, J.B.; Newell, M.; Field, C.J. Docosahexanoic acid improves chemotherapy efficacy by inducing CD95 translocation to lipid rafts in $\mathrm{ER}^{-}$breast cancer cells. Lipids 2012, 47, 1019-1030.

123. Gu, Z.; Wu, J.; Wang, S.; Suburu, J.; Chen, H.; Thomas, M.J.; Shi, L.; Edwards, I.J.; Berquin, I.M.; Chen, Y.Q. Polyunsaturated fatty acids affect the localization and signaling of PIP3/AKT in prostate cancer cells. Carcinogenesis 2013, 34, 1968-1975.

124. Calviello, G.; Resci, F.; Serini, S.; Piccioni, E.; Toesca, A.; Boninsegna, A.; Monego, G.; Ranelletti, F.O.; Palozza, P. Docosahexaenoic acid induces proteasome-dependent degradation of $\beta$-catenin, down-regulation of survivin and apoptosis in human colorectal cancer cells not expressing COX-2. Carcinogenesis 2007, 28, 1202-1209.

125. Song, K.S.; Jing, K.; Kim, J.S.; Yun, E.J.; Shin, S.; Seo, K.S.; Park, J.H.; Heo, J.Y.; Kang, J.X.; Suh, K.S.; et al. $\omega$-3-Polyunsaturated fatty acids suppress pancreatic cancer cell growth in vitro and in vivo via downregulation of Wnt/ $\beta$-catenin signaling. Pancreatology 2011, 11, 574-584.

126. Xue, M.; Wang, Q.; Zhao, J.; Dong, L.; Ge, Y.; Hou, L.; Liu, Y.; Zheng, Z. Docosahexaenoic acid inhibited the Wnt/ $\beta$-catenin pathway and suppressed breast cancer cells in vitro and in vivo. J. Nutr. Biochem. 2014, 25, 104-110.

127. Sun, H.; Hu, Y.; Gu, Z.; Owens, R.T.; Chen, Y.Q.; Edwards, I.J. w-3 Fatty acids induce apoptosis in human breast cancer cells and mouse mammary tissue through syndecan-1 inhibition of the MEK-Erk pathway. Carcinogenesis 2011, 32, 1518-1524.

128. Schley, P.D.; Jijon, H.B.; Robinson, L.E.; Field, C.J. Mechanisms of $\omega-3$ fatty acid-induced growth inhibition in MDAMB-231 human breast cancer cells. Breast Cancer Res. Treat. 2005, 92, 187-195.

129. Ghosh-Choudhury, T.; Mandal, C.C.; Woodruff, K.; St Clair, P.; Fernandes, G.; Choudhury, G.G.; Ghosh-Choudhury, N. Fish oil targets PTEN to regulate NFkB for downregulation of anti-apoptotic genes in breast tumor growth. Breast Cancer Res. Treat. 2009, 118, 213-228. 
130. Engelbrecht, A.M.; Toit-Kohn, J.L.; Ellis, B.; Thomas, M.; Nell, T.; Smith, R. Differential induction of apoptosis and inhibition of the PI3-kinase pathway by saturated, monounsaturated and polyunsaturated fatty acids in a colon cancer cell model. Apoptosis 2008, 13, 1368-1377.

131. Jing, K.; Song, K.S.; Shin, S.; Kim, N.; Jeong, S.; Oh, H.R.; Park, J.H.; Seo, K.S.; Heo, J.Y.; Han, J.; et al. Docosahexaenoic acid induces autophagy through p53/AMPK/mTOR signaling and promotes apoptosis in human cancer cells harboring wild-type p53. Autophagy 2011, 7, 1348-1358.

132. Shin, S.; Jing, K.; Jeong, S.; Kim, N.; Song, K.S.; Heo, J.Y.; Park, J.H.; Seo, K.S.; Han, J.; Park, J.I.; et al. The $\omega-3$ polyunsaturated fatty acid DHA induces simultaneous apoptosis and autophagy via mitochondrial ROS-mediated Akt-mTOR signaling in prostate cancer cells expressing mutant p53. Biomed. Res. Int. 2013, 2013, 568671.

133. Kim, N.; Jeong, S.; Jing, K.; Shin, S.; Kim, S.; Heo, J.Y.; Kweon, G.R.; Park, S.K.; Wu, T.; Park, J.I.; et al. Docosahexaenoic acid induces cell death in human non-small cell Lung cancer cells by repressing mTOR via AMPK activation and PI3K/Akt inhibition. Biomed. Res. Int. 2015, 2015, 239764.

134. Rescigno, T.; Capasso, A.; Tecce, M.F. Effect of Docosahexaenoic acid on cell cycle pathways in Breast cell lines with different transformation degree. J. Cell. Physiol. 2015.

135. Shaikh, I.A.; Brown, I.; Schofield, A.C.; Wahle, K.W.; Heys, S.D. Docosahexaenoic acid enhances the efficacy of docetaxel in prostate cancer cells by modulation of apoptosis: The role of genes associated with the NF-KB pathway. Prostate 2008, 68, 1635-1646.

136. Cavazos, D.A.; Price, R.S.; Apte, S.S.; de Graffenried, L.A. Docosahexaenoic acid selectively induces human prostate cancer cell sensitivity to oxidative stress through modulation of NF-kB. Prostate 2011, 71, 1420-1428.

137. Jeong, S.; Jing, K.; Kim, N.; Shin, S.; Kim, S.; Song, K.S.; Heo, J.Y.; Park, J.H.; Seo, K.S.; Han, J.; et al. Docosahexaenoic acid-induced apoptosis is mediated by activation of mitogen-activated protein kinases in human cancer cells. BMC Cancer 2014, 14, 481.

138. Gleissman, H.; Yang, R.; Martinod, K.; Lindskog, M.; Serhan, C.N.; Johnsen, J.I.; Kogner, P. Docosahexaenoic acid metabolome in neural tumors: Identification of cytotoxic intermediates. FASEB J. 2010, 24, 906-915.

139. Serhan, C.N.; Arita, M.; Hong, S.; Gotlinger, K. Resolvins, docosatrienes, and neuroprotectins, novel $\omega$-3-derived mediators, and their endogenous aspirin-triggered epimers. Lipids 2004, 39, 1125-1132.

140. Hong, S.; Lu, Y.; Yang, R.; Gotlinger, K.H.; Petasis, N.A.; Serhan, C.N. Resolvin D1, protectin D1, and related docosahexaenoic acid-derived products: Analysis via electrospray/low energy tandem mass spectrometry based on spectra and fragmentation mechanisms. J. Am. Soc. Mass Spectrom. 2007, 18, 128-144.

141. Ding, W.Q.; Vaught, J.L.; Yamauchi, H.; Lind, S.E. Differential sensitivity of cancer cells to docosahexaenoic acid-induced cytotoxicity: The potential importance of down-regulation of superoxide dismutase 1 expression. Mol. Cancer Ther. 2004, 3, 1109-1117. 
142. Ding, W.Q.; Lind, S.E. Phospholipid hydroperoxide glutathione peroxidase plays a role in protecting cancer cells from docosahexaenoic acid-induced cytotoxicity. Mol. Cancer Ther. 2007, 6, 1467-1474.

143. Vibet, S.; Goupille, C.; Bougnoux, P.; Steghens, J.P.; Goré, J.; Mahéo, K. Sensitization by docosahexaenoic acid (DHA) of breast cancer cells to anthracyclines through loss of glutathione peroxidase (GPx1) response. Free Radic. Biol. Med. 2008, 44, 1483-1491.

144. Sturlan, S.; Baumgartner, M.; Roth, E.; Bachleitner-Hofmann, T. Docosahexaenoic acid enhances arsenic trioxidemediated apoptosis in arsenic trioxide-resistant HL-60 cells. Blood 2003, 101, 4990-4997.

145. Granci, V.; Cai, F.; Lecumberri, E.; Clerc, A.; Dupertuis, Y.M.; Pichar, C. Colon cancer cell chemosensitisation by fish oil emulsion involves apoptotic mitochondria pathway. Br. J. Nutr. 2013, 109, 1188-1195.

146. Hossain, Z.; Hosokawa, M.; Takahashi, K. Growth inhibition and induction of apoptosis of colon cancer cell lines by applying marine phospholipid. Nutrition and Cancer 2009, 61, 123-130.

147. Dai, J.; Shen, J.; Pan, W.; Shen, S.; Das, U.N. Effects of polyunsaturated fatty acids on the growth of gastric cancer cells in vitro. Lipids Health Dis. 2013, 12, 71.

148. Jing, K.; Shin, S.; Jeong, S.; Kim, S.; Song, K.S.; Park, J.H.; Heo, J.Y.; Seo, K.S.; Park, S.K.; Kweon, G.R.; et al. Docosahexaenoic acid induces the degradation of HPV E6/E7 oncoproteins by activating the ubiquitin-proteasome system. Cell Death Dis. 2014, $5, \mathrm{e} 1524$.

149. Kang, K.S.; Wang, P.; Yamabe, N.; Fukui, M.; Jay, T.; Zhu, B.T. Docosahexaenoic acid induces apoptosis in MCF-7 cells in vitro and in vivo via reactive oxygen species formation and caspase 8 activation. PLoS ONE 2010, 5, e10296.

150. Zajdel, A.; Wilczok, A.; Tarkowski, M. Toxic effects of $n-3$ polyunsaturated fatty acids in human lung A549 cells. Toxicol. Vitro 2015.

151. Serhan, C.N.; Hong, S.; Gronert, K.; Colgan, S.P.; Devchand, P.R.; Mirick, G.; Moussignac, R.L. Resolvins: A family of bioactive products of $\omega-3$ fatty acid transformation circuits initiated by aspirin treatment that counter proinflammation signals. J. Exp. Med. 2002, 196, 1025-1037.

152. Wang, Q.; Hu, M.; Xu, H.; Yang, X. Anti-inflammatory and Pro-resolving effects of n-3 PUFA in Cancers: Structures and Mechanisms. Curr. Top Med. Chem. 2016, 16, 888-894.

153. Hawcroft, G.; Loadman, P.M.; Belluzzi, A.; Hull, M.A. Effect of eicosapentaenoic acid on E-type prostaglandin synthesis and EP4 receptor signaling in human colorectal cancer cells. Neoplasia 2010, 12, 618-627.

154. Poorani, R.; Bhatt, A.N.; Dwarakanath, B.S.; Das, U.N. COX-2, aspirin and metabolism of arachidonic, eicosapentaenoic and docosahexaenoic acids and their physiological and clinical significance. Eur. J. Pharmacol. 2015.

155. Karmali, R.A.; Reichel, P.; Cohen, L.A.; Terano, T.; Hirai, A.; Tamura, Y.; Yoshida, S. The effects of dietary $\omega-3$ fatty acids on the DU-145 transplantable human prostatic tumor. Anticancer Res. 1987, 17, 1173-1180. 
156. Rose, D.P.; Cohen, L.A. Effects of dietary menhaden oil and retinyl acetate on the growth of DU145 human prostatic adenocarcinoma cells transplanted into athymic nude mice. Carcinogenesis 1988, 9, 603-605.

157. Rose, D.P.; Connolly, J.M. Dietary fat and breast cancer metastasis by human tumor xenografts. Breast Cancer Res. Treat. 1997, 46, 225-237.

158. Kobayashi, N.; Barnard, R.J.; Henning, S.M.; Elashoff, D.; Reddy, S.T.; Cohen, P.; Leung, P.; Hong-Gonzalez, J.; Freedland, S.J.; Said, J.; et al. Effect of altering dietary $\omega-6 / \omega-3$ fatty acid ratios on prostate cancer membrane composition, cyclooxygenase-2, and prostaglandin $\mathrm{E}_{2}$. Clin. Cancer Res. 2006, 12, 4662-4670.

159. Funahashi, H.; Satake, M.; Hasan, S.; Sawai, H.; Newman, R.A.; Reber, H.A.; Hines, O.J.; Eibl, G. Opposing effects of $n-6$ and $n-3$ polyunsaturated fatty acids on pancreatic cancer growth. Pancreas 2008, 36, 353-362.

160. Narayanan, B.A.; Narayanan, N.K.; Desai, D.; Pittman, B.; Reddy, B.S. Effects of a combination of docosahexaenoic acid and 1,4-phenylene bis(methylene) selenocyanate on cyclooxygenase 2 , inducible nitric oxide synthase and $\beta$-catenin pathways in colon cancer cells. Carcinogenesis 2004, 25, 2443-2449.

161. Calviello, G.; Serini, S.; Piccioni, E. $n$-3 polyunsaturated fatty acids and the prevention of colorectal cancer: Molecular mechanisms involved. Curr. Med. Chem. 2007, 14, 3059-3069.

162. Sun, H.; Berquin, I.M.; Owens, R.T.; O'Flaherty, J.T.; Edwards, I.J. Peroxisome proliferator-activated receptor $\gamma$-mediated up-regulation of syndecan- 1 by $n-3$ fatty acids promotes apoptosis of human breast cancer cells. Cancer Res. 2008, 68, 2912-2919.

163. Edwards, I.J.; Sun, H.; Hu, Y.; Berquin, I.M.; O'Flaherty, J.T.; Cline, J.M.; Rudel, L.L.; Chen, Y.Q. In vivo and in vitro regulation of syndecan 1 in prostate cells by $n-3$ polyunsaturated fatty acids. J. Biol. Chem. 2008, 283, 18441-18449.

164. O'Flaherty, J.T.; Hu, Y.; Wooten, R.E.; Horita, D.A.; Samuel, M.P.; Thomas, M.J.; Sun, H.; Edwards, I.J. 15-lipoxygenase metabolites of docosahexaenoic acid inhibit prostate cancer cell proliferation and survival. PLoS ONE 2012, 7, e45480.

165. Hu, Y.; Sun, H.; O’Flaherty, J.T.; Edwards, I.J. 15-Lipoxygenase-1-mediated metabolism of docosahexaenoic acid is required for syndecan-1 signaling and apoptosis in prostate cancer cells. Carcinogenesis 2013, 34, 176-182.

166. Zou, S.; Meng, X.; Meng, Y.; Liu, J.; Liu, B.; Zhang, S.; Ding, W.; Wu, J.; Zhou, J. Microarray analysis of anti-cancer effects of docosahexaenoic acid on human colon cancer model in nude mice. Int. J. Clin. Exp. Med. 2015, 8, 5075-5084.

167. Sheng, H.; Li, P.; Chen, X.; Liu, B.; Zhu, Z.; Cao, W. w-3 PUFAs induce apoptosis of gastric cancer cells via ADORA1. Front. Biosci. 2014, 19, 854-861. 


\title{
Cancer Risk and Eicosanoid Production: Interaction between the Protective Effect of Long Chain Omega-3 Polyunsaturated Fatty Acid Intake and Genotype
}

\author{
Georgia Lenihan-Geels, Karen S. Bishop and Lynnette R. Ferguson
}

\begin{abstract}
Dietary inclusion of fish and fish supplements as a means to improve cancer prognosis and prevent tumour growth is largely controversial. Long chain omega-3 polyunsaturated fatty acids (LCn-3 PUFA), eicosapentaenoic acid and docosahexaenoic acid, may modulate the production of inflammatory eicosanoids, thereby influencing local inflammatory status, which is important in cancer development. Although in vitro studies have demonstrated inhibition of tumour cell growth and proliferation by LCn-3 PUFA, results from human studies have been mainly inconsistent. Genes involved in the desaturation of fatty acids, as well as the genes encoding enzymes responsible for eicosanoid production, are known to be implicated in tumour development. This review discusses the current evidence for an interaction between genetic polymorphisms and dietary LCn-3 PUFA in the risk for breast, prostate and colorectal cancers, in regards to inflammation and eicosanoid synthesis.
\end{abstract}

Reprinted from J. Clin. Med. Cite as: Lenihan-Geels, G.; Bishop, K.S.; Ferguson, L.R. Cancer Risk and Eicosanoid Production: Interaction between the Protective Effect of Long Chain Omega-3 Polyunsaturated Fatty Acid Intake and Genotype. J. Clin. Med. 2016, 5, 25.

\section{Introduction}

Cancer is a multifactorial, widely spread, variable and largely non-communicable disease, affecting populations in all parts of the world. Currently, some of the most significant cancers throughout the Western world include breast, colorectal and prostate cancers [1]. The role of nutrition in cancer risk and development is becoming increasingly recognised, particularly in regards to dietary intake of fresh fruit and vegetables, meat and meat products, and fish or fish oils, which may be related to their effects on inflammatory processes [2-6]. Intake of animal sources of fat, saturated and trans-unsaturated fatty acids are associated with all-cause mortality and death due to colorectal, breast and prostate cancers. On the other hand, plant based oils and fish oils are associated with a decrease in the risk and death due to the aforementioned cancers [7-9]. 
The predominant omega- $6(n-6)$ and omega-3 (n-3) fatty acids (FA) in the typical Western diet are linoleic acid (LA) (18:2n-6) and alpha-linolenic acid (ALA) (18:3n-3), respectively, known as the essential fatty acids. Through elongation and desaturation, these FA are converted to longer and more desaturated FAs via the $n-6$ and $n-3$ pathways (Figure 1). However, the conversion of LA and ALA to longer-chain FAs is limited by the enzymatic capacity of the desaturases, as well as dietary levels of LA and ALA, which compete for the same enzymes. For example, the conversion of ALA to eicosapentaenoic acid (EPA) (20:5n-3) ranges from between $0.2 \%$ and $21 \%$ [10].

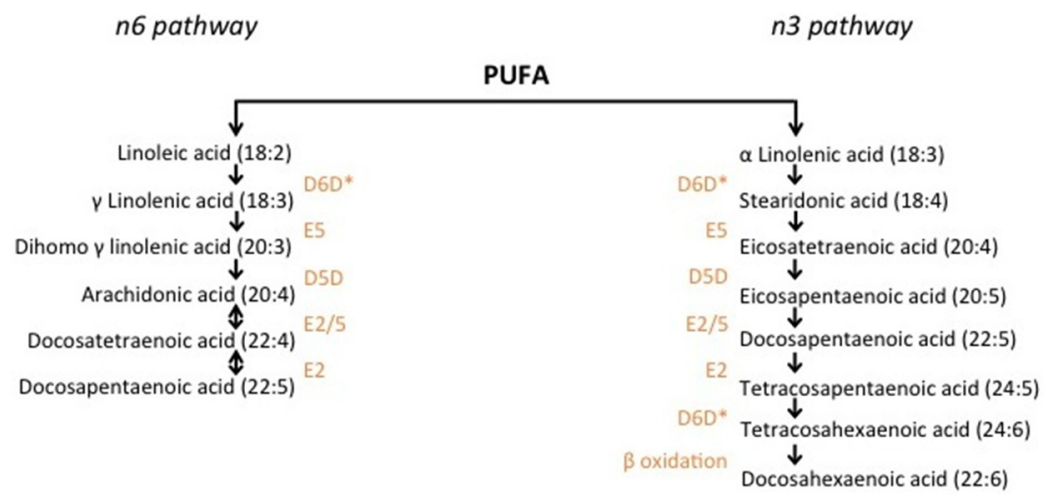

Figure 1. Synthesis of polyunsaturated fatty acids through the n-3 and n-6 pathways. Polyunsaturated fatty acid elongation from ALA and LA begins with desaturation by the D6D enzyme. Subsequent elongation and desaturation by the corresponding enzymes (orange) generates longer chain PUFA such as AA and EPA. n-3 and n-6 PUFA compete for the D6D, E5, D5D and E2 enzymes [11-15]. D5D: Delta 5 desaturase; D6D: Delta 6 desaturase; E: Elongase.*: Rate limiting step.

Long chain (LC) polyunsaturated fatty acids (PUFA) play a significant role in inflammatory processes, as they act as precursors for inflammatory mediators called eicosanoids. Eicosanoids are potent signaling molecules synthesized during inflammation and include leukotrienes, thromboxanes and prostaglandins [16]. A diverse set of enzymes are responsible for the synthesis of eicosanoids from PUFA, some of which are outlined in Figure 2. Cyclooxygenases catalyse the formation of series 2 and series 3 prostaglandins and thromboxanes, while lipoxygenases synthesise lipoxins and leukotrienes, which are further metabolized by glutathione transferases [16]. Additionally, dietary EPA and docosahexaenoic acid (DHA) are precursors for mainly anti-inflammatory eicosanoids, while arachidonic acid (AA) $(20: 4 n-6)$ is a precursor for mainly pro-inflammatory compounds and is in competition with EPA for eicosanoid production. Furthermore, the ratio of AA to EPA/DHA in cell membranes is thought to be informative in regards to 
inflammatory status. In fact, studies clearly show lower levels of circulating pro-inflammatory compounds such as cytokines and adhesion molecules with higher levels of membrane-bound and free EPA and DHA [17-20].

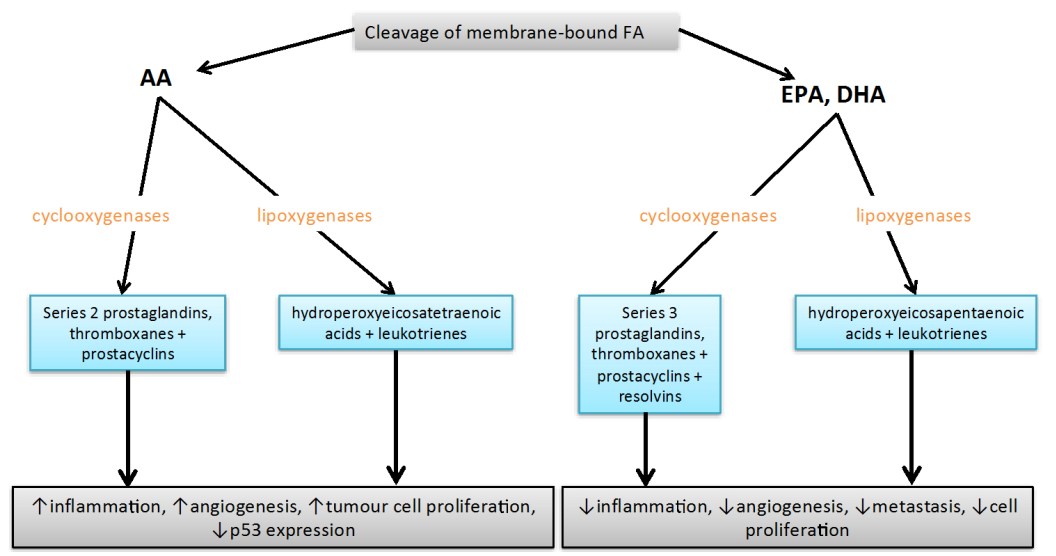

Figure 2. Effects of eicosanoids derived from AA and EPA/DHA. Cyclooxygenases and lipoxygenases act on AA, EPA and DHA to synthesise a range of different eicosanoids during an inflammatory response. AA-derived eicosanoids often generate pro-inflammatory compounds that enhance tumour growth, while EPA/DHA-derived eicosanoids often have anti-inflammatory properties and inhibit tumour growth [21,22]. FA: fatty acids; AA: arachidonic acid; EPA: eicosapentanoic acid; DHA: docosahexanoic acid.

Inflammation is a key event in the development of tumours and is known to promote tumour growth, angiogenesis and metastasis [23]. For example, the metabolism of AA to pro-inflammatory eicosanoids is characteristic of some colorectal and breast cancer cells [24-26]. Therefore, dietary LCn-3 PUFA intake is of great interest in the prevention and treatment of these cancers and as agents in reducing inflammation, although the topic remains largely controversial [9,27-29]. Discrepancies in both observational and experimental data may arise from multiple sources including: heterogeneity of cancers; confounding in epidemiological data; environmental contaminants, particularly from LCn-3 PUFA-rich marine sources; accuracy of dietary intake data; bioavailability; and/or genetic variation [9,12,30-33]. One challenging aspect in cancer epidemiology is that any factor reducing cancer risk will usually promote life-expectancy, which in itself is a risk for cancer [34].

It has come to light that efficiency of conversion of LA and ALA to LC PUFA is partially determined by the genotype of the fatty acid desaturase (FADS) family of genes, which code for the delta- 5 and delta- 6 desaturases that catalyse the rate limiting steps of the $n-3$ and $n-6$ pathways, which may therefore impact downstream eicosanoid production [9,14,35-38]. Interestingly, single nucleotide 
polymorphisms (SNPs) found in other genes, for example cytochrome $c$ oxidase (COX) and arachidonate lipoxygenase ( $A L O X)$, may also influence levels of eicosanoids produced from EPA and AA [26,39]. COX and ALOX genes code for the cyclooxygenase and lipoxygenase enzymes, respectively, and are responsible for generating a range of eicosanoid mediators [16] (Figure 2). Consequently, both levels of dietary fatty acids and variation at the FADS, COX and ALOX loci may impact inflammatory processes and carcinogenesis.

There is increasing evidence to support the view that LCn-3 PUFA, specifically EPA and DHA, inhibit the growth of colorectal, breast and prostate cancer cell lines [27,40-42] and inhibit tumour growth in animal models [43-45]. Current evidence in humans is less clear and epidemiological data is largely inconsistent $[9,27,28]$. As inflammation is a predominant hallmark in many cancers, the relationship between inflammation and dietary LC $n-3$ PUFA is of high interest. Furthermore, the impact of genetic polymorphisms on the production of eicosanoids is important to consider. In this review, we discuss the interaction between LCn-3 PUFA and genotype, related to eicosanoid production, which may have an impact on the development and progression of cancers. The genes or family of genes under consideration include the FADS genes involved in the desaturation of LCn-3 PUFA [46], the glutathione S-transferase (GST) family of genes involved in oxidative stress and inflammation [47], and the ALOX and COX genes that generate pro- and anti-inflammatory mediators [48].

\section{Methods}

Articles utilised in this review were selected using the PubMed and Google Scholar databases. Key words used in the searches included: eicosanoid/s; polymorphism/s; cancer; dietary; polyunsaturated fatty acid; omega-3. One of these words must have also been present: prostate OR breast OR colorectal OR colon OR rectal AND FADS/fatty acid desaturase OR COX/cyclooxygenase OR ALOX/lipoxygenase OR GST/glutathione transferase. Included articles focused on human studies only. Exclusion criteria were: review articles; articles in any language other than English; articles older than 1990; articles focused on another disease other than breast, prostate or colorectal cancer; and articles lacking data on diet specific to PUFA or fish. Following searches of combinations of the above keywords, a total number of 417 articles were found. The titles of these 417 articles were read and after applying the exclusion criteria, 56 articles were selected for the next stage. Adjusting to the same exclusion criteria left a total number of 10 studies, which are summarised in Table 1. 


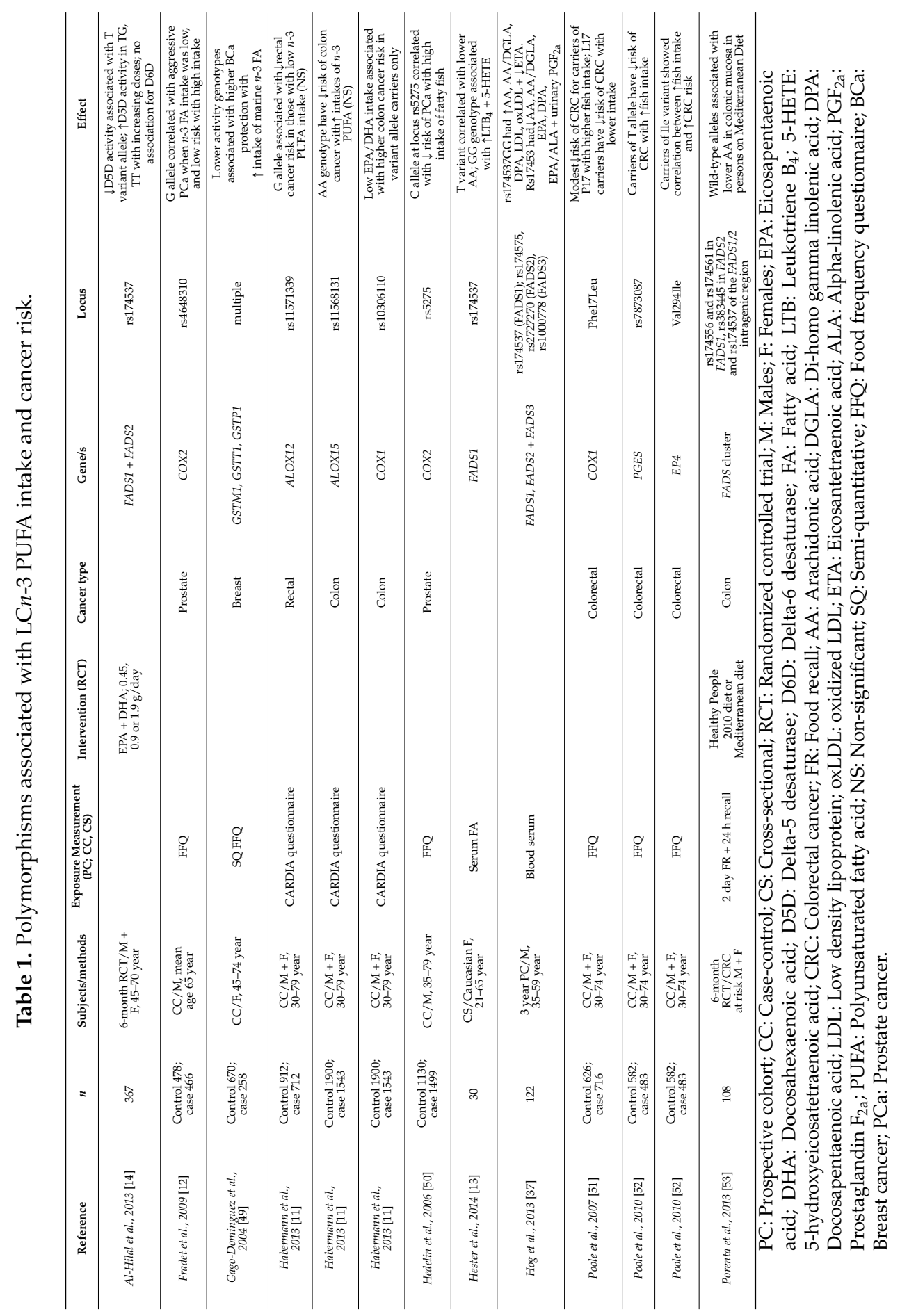




\section{The Role of Genetic Variation in Fatty Acid Desaturation}

The FADS gene cluster is located on a highly polymorphic region of chromosome 11 and includes FADS1 and FADS2, which encode delta 5 desaturase (D5D) and delta 6 desaturase (D6D), respectively [9]. These polymorphisms create a diverse set of haplotypes. The first demonstration of a relationship between FADS genotype and membrane-bound FAs was shown by Schaefer et al. (2006) [54] in serum phospholipids. Further evidence came from a study on infants, in which Danish infants carrying the FADS minor allele for locus rs1535 had a higher DHA level than those with the wild-type allele [55]. In contrast, those carrying the minor alleles of rs174448 (C) and rs174575 (G) had decreased DHA levels relative to wild-type [55]. Similarly, carriers of the T allele at rs174537 (in strong linkage disequilibrium with rs174546 and rs3834458) had lower levels of AA than the carriers of the G allele [37]. Additional examples have been presented by Al-Hilal et al. (2013) in which the minor allele of SNPs rs174537, rs174561 and rs3834458 correlate with higher amounts of ALA and lower levels of EPA, docosapentaenoic acid (DPA) and DHA, as well as lower activity of both D5D and D6D [14].

Of particular interest to the interplay with dietary LCn-3 PUFA, a 6-month intervention of an EPA/DHA supplement in individuals carrying a $\mathrm{T}$ allele at locus rs174537 showed rising activity of D5D with an increasing supplement dose [14]. Additionally, polymorphisms at FADS locus rs174546 correlated with serum triacylglycerides at baseline and 6 weeks following EPA/DHA supplementation [56]. At locus rs174537, the presence of a T-allele correlated with lower levels of AA, consistent with a similar study [37], and those carrying the GG genotype had higher levels of eicosanoids leukotriene $\mathrm{B}_{4}\left(\mathrm{LTB}_{4}\right)$ and 5-hydroxyeicosatetraenoic acid (5-HETE) [13]. LTB $_{4}$ and 5-HETE are pro-inflammatory compounds synthesized from AA by the leukotriene synthase and 5-LOX enzymes, respectively [39]. Therefore, it is possible that levels of circulating eicosanoids may be modulated by the interplay of diet and genotype. If these individuals are at particular risk for cancer, it would be advisable to increase the intake of LCn-3 PUFA.

Studies on colonic mucosal fatty acid compositions have revealed a diet-genotype effect. Lower concentrations of AA were observed in subjects carrying major alleles within the FADS gene cluster (rs174556 and rs174561 in FADS1, rs383445 in FADS2 and rs174537 of the FADS1/2 intragenic region) when consuming a Mediterranean diet compared to a Healthy Eating diet, due to increases in AA levels within the Healthy Eating group [53]. The Mediterranean diet has been extensively studied with regards to its effect on cancers. This diet is traditionally high in fat, but low in LCn-6 PUFA and trans fatty acids, and is typically high in olive oil, fresh fruit and vegetables [57,58]. The Mediterranean diet used in an intervention by Porenta et al. (2013) [53] was also high in fish and flaxseed. Additional studies are required to confirm these results, as diets were not strictly controlled and sample 
size was relatively small. A summary of the interaction between LCn-3 PUFA on prostate, breast and colorectal cancers as modified by FADS genotype, is provided in Table 1, alongside additionally discussed genotypes.

\section{Genetic Polymorphisms Modulate Leukotriene Synthesis in Cancer}

\subsection{Lipoxygenases}

Leukotrienes are eicosanoid inflammatory mediators produced by the oxidation of AA, and are implicated in inflammation and cancer [59]. Leukotriene synthesis begins with the formation of hydroperoxyeicosatetranoic acid (5-HPETE) and hydroperoxyeicosapentaenoic acid (5-HPEPE) from AA and EPA, respectively, by the lipoxygenases [16]. Although not statistically significant, a lower risk for colon cancer was demonstrated in wild-type homozygous individuals at locus rs11568131 of $A L O X 15$ when consuming high amounts of fish, an association that was absent in those carrying the variant allele [11]. Carriers of a $\mathrm{G}$ minor allele at locus rs11571339 of the $A L O X 12$ gene showed a lower risk for rectal cancer in those with low n-3 PUFA intake compared to higher intakes [11]. However, G allele carriers with high intakes showed no increased risk compared to the homozygous major allele reference group. Despite also demonstrating no statistical significance, this finding is particularly interesting, as a lower LCn-3 PUFA intake would generate fewer anti-inflammatory compounds than a higher intake. Furthermore, G allele carriers with lower intakes of LA and total PUFA showed a similar pattern. Studies investigating the differences in activity of 12-lipoxygenase due to this polymorphism could help to explain these findings.

A recent meta-analysis found that polymorphisms in the ALOX12 gene at the Gln261Arg locus may influence cancer risk in Asian populations but not in Caucasians [60]. Furthermore, carriers of the variant in homozygous or heterozygous form had an increased risk for breast cancer, also demonstrating differences across ethnic populations [61]. The same polymorphism also showed an association with risk of colorectal adenomas [62]. To our knowledge, the interplay of this polymorphism with EPA/DHA intake has not been previously explored and is worthy of further investigation.

\subsection{Glutathione S-Transferases}

The glutathione S-transferase (GST) enzymes implicated in various types of cancer are important for the detoxification of environmental pollutants and chemical carcinogens, and modulate signaling of pathways associated with cell proliferation, cell differentiation and apoptosis [47]. In addition, GSTs are involved in the synthesis of leukotrienes from 5-HPETE. Finally, GSTs are also important for the detoxification of reactive oxygen species [63] and may help protect against DNA damage [64]. 
Raised levels of anti-oxidants can help activate GST genes and this in turn may help to reduce the increased levels of DNA damage that are associated with prostate cancer $[36,64]$. GST phenotype (e.g., GSTT1 null genotype) is associated with risk of prostate cancer in Caucasians but this does not hold true for other races [47]. Unfortunately, no evidence appears to be available with respect to the modification of this effect by fatty acids in prostate cancer. However, van Hemelrijck et al. (2012) [65] identified an association between prostate cancer and the intake of heterocyclic aromatic amines (HCAs) that was modified by the genotype of HCA-metabolizing enzymes (e.g., MnSOD rs4880 and GPX4 rs713041). HCAs are mutagenic and are generated by cooking meat at high temperatures [66]. Meat is a common source of animal fat and the effect of some monounsaturated fatty acids (e.g., palmitic and stearic acids) as well as n-6 PUFA (e.g., AA) on prostate cancer may be confounded by the presence of HCAs. For this reason, we propose that while the genotype of HCA-metabolizing enzymes may appear to interact with type of fatty acid intake and prostate cancer risk, in fact it is the presence of HCAs that is interacting with genotype to influence disease risk.

In contrast, a clear association has been shown between the polymorphic GST genes, breast cancer and marine FA intake [49]. Women carrying variants resulting in higher activity of the GST enzymes show a correlation with marine $n$-3 PUFA intake and risk of breast cancer, in which lower intake demonstrates a higher risk compared to those with higher intakes of the same genotypes. These associations were found in Chinese and Singaporean women [49].

\section{Prostaglandin Synthesis}

Cyclooxygenase enzymes, also known as prostaglandin endoperoxide synthases, catalyse the rate-limited formation of inflammatory prostaglandins. Two isozymes (COX1 and COX2) exist, both of which are associated with injury and inflammation and demonstrate different tissue expression patterns [12]. Increased expression of COX2 leads to hyperproliferation of colon epithelial cells, a process which was decreased following the presence of EPA [67]. Furthermore, the inhibitory effects of non-steroidal anti-inflammatory drugs (NSAIDs) associated with colorectal cancer are thought to relate to their inhibitory activity at both COX1 and COX2 [68].

In vitro studies have shown inhibitory actions of LCn-3 PUFA on prostate cancer cell growth. In different prostate cancer cell models, namely LNCaP and PacMetUT1, DHA appeared to sensitise the cells by attenuating the NF- $\kappa B$ survival pathway that promotes cancer cell survival, resulting in decreased cancer cell survival [69]. On the other hand, NF-KB does not appear to be involved in the induction of COX2 expression in the prostate cancer cells, PC3, treated with DHA and EPA [70]. In regards to human studies, five of sixteen SNPs found within the COX2 region were tested in a Swedish population with and without prostate 
cancer, identifying a relationship between two SNPs and the presence of prostate cancer [50,71]. Subsequently, the same authors demonstrated that the presence of a C allele at locus rs5275 was significantly associated with a decreased risk of prostate cancer in men with a high intake of fatty fish [50]. Similarly, Fradet et al. (2009) [12] assessed diet alongside nine COX2 SNPs in men diagnosed with aggressive prostate cancer and found that LCn-3 PUFA intake was strongly associated with a decreased risk of aggressive prostate cancer. This effect was modified by the rs4648310 SNP, such that the increased risk of aggressive prostate cancer associated with a low intake of $n-3$ PUFA in those with the G allele (odds ratio $=5.49$ ) could be reversed by increasing $n$-3 PUFA intake [12]. Therefore, it is reasonable to say that carriers of a $\mathrm{G}$ allele at the rs4648310 locus could benefit from increasing LCn-3 PUFA intake.

COX1 SNPs at the rs10306110 locus may modulate colon cancer risk. Habermann et al. (2013) [11] demonstrated an association between low LCn-3 PUFA intake and the variant allele, with an odds ratio of 1.56 and 1.62 for EPA and DHA, respectively. Total and monounsaturated fatty acid intake was associated with the variant allele at rs10306122 of PTGS1, the gene encoding COX1, and increased rectal cancer risk, although marine LC PUFA showed no effect [11].

The P17L polymorphism, leading to sequence changes within the signal peptide of COX1, was associated with risk of colorectal adenomas, in which higher fish intake in those homozygous for phenylalanine at position 17 had a modestly lower risk of adenomas with increasing fish intake [51]. Interestingly, those carrying at least one leucine at position 17 had a decreased risk of adenomas when consuming less fish per week [51]. Importantly, these individuals demonstrated a higher risk for colorectal cancer with increasing fish intake. This is a highly interesting finding which highlights the occurrence of inconsistencies in studies of cancer and LCn-3 PUFA and the importance of designing and performing studies that will provide clarity in this regard.

The same authors [51] then analysed the risk of colorectal adenomas between those in an assumed low risk group (high fish intake + NSAID use) and an assumed high risk group (low fish intake + no NSAID use) and variable intermediate groups, to assess the dual implications of both NSAID use and fish intake in the relationship between P17L polymorphisms and adenoma risk. PP homozygotes benefited from including more than 2 servings of fish per week as well as regular use of NSAIDs. However, those with PL and LL genotypes showed no statistically significant associations [51]. These findings are unexpected and it is necessary to replicate these investigations in larger studies with more detail on type of fish in the diet, as well as other dietary information.

Polymorphisms within the gene for prostaglandin $E_{2}$ synthase-1 (PGES) also correlate with colorectal adenoma risk. PGES catalyses the formation of $\mathrm{PGE}_{2}$, a pro-inflammatory prostaglandin associated with increased cell proliferation [72,73]. 
Individuals carrying a T allele at rs7873087 had a lower risk for colorectal adenomas with increasing fish intake, whereas those homozygous for the A allele showed no significant association with fish intake [52]. Additional relationships were observed for polymorphisms within the 15-hydroxyprostaglandin dehydrogenase gene and the EP4 receptor gene, which code for proteins responsible for the breakdown of $\mathrm{PGE}_{2}$ and the corresponding $\mathrm{PGE}_{2}$ receptor, respectively. These studies highlight the importance of inter individual differences in genes involved in the prostaglandin synthesis pathways from AA and EPA, and their complex association with colorectal cancer and fish intake. This relationship warrants further investigation.

Limitations in these studies include recall bias in the FFQ and diet diaries, which are commonly used in large studies such as cohorts or case-control designs due to lack of affordable and better alternatives. Furthermore, ethnicity must be adjusted for in studies, as ethnicity may influence the relationship between dietary n-3 PUFA, cancer risk and genotype, as highlighted earlier [60,61]. Additional factors potentially influencing the outcome of the studies reviewed herein, include the stage of the disease, as LCn-3 PUFA may interact differently with genotypes as the physiology of the tumour changes, and fish contaminants. Dioxins may increase cancer risk, which could generate substantial confounding [33]. In addition, it is important to note that this review highlights the current knowledge of the interplay between genes involved in eicosanoid synthesis only, and that there are a range of other genes that are likely to contribute to the relationship between cancer risk and LCn-3 PUFA intake, such as polymorphisms in DNA repair- and apoptosis-related genes [74].

\section{Conclusions}

The effects of LCn-3 PUFA on prostate, breast and colorectal cancer modified by genotype are presented in Table 1 . It is clear that both dietary intake and polymorphisms of the FADS genes contribute to the concentrations of membrane-bound fatty acids such as EPA, DHA and AA. Although genetic variation within the FADS genes have not been directly associated with cancer, the effects on desaturase activity may influence the production of eicosanoids further downstream. Dietary LCn-3 PUFA (EPA, DPA and DHA) are inversely associated with aggressive prostate cancer [12] and prostate cancer risk. This protective effect can be modified by genotype including rs5275 [50] and rs4648310 [12] in COX2. On the other hand, the loss of expression of FADS2, in response to a mutation in FAD2, is associated with a more aggressive breast cancer tumour and reduced survival $[9,75]$. Breast cancer risk may also be modulated by dietary LCn-3 PUFA and activity of the GST enzymes [49]. Interestingly, an association between ALOX12 polymorphisms and breast cancer, which was modified by ethnicity $[60,61]$ could be further explored in regards to the relationship with LCn-3 PUFA intake. In regards to colon and rectal 
cancers, certain individuals may benefit largely from including LCn-3 PUFA in their diets while others do not, as demonstrated by polymorphisms in ALOX12, ALOX15 and PGES genes [11,52]. Furthermore, there exists a positive association between increased risk of colorectal cancers and increased fish intake in some genotypes of the COX1 gene, a relationship worthy of further investigation.

Compelling evidence from in vivo and in vitro studies has been presented on the inhibition of cancer progression. Here, evidence has been presented on the genotypic modification of response to LCn-3 PUFA and it is clear that we are on the brink of offering personalised nutritional advice with respect to these FAs. Such advice would ensure that people are correctly informed with respect to the types and amounts of LCn-3 PUFA they should consume in order to meet their specific requirements. In addition, further study could decipher the significance of the role of $n-3$ PUFA in cancer and inflammation, for example whether altered PUFA metabolism is a driver or a passenger in cancer [76].

Acknowledgments: The financial support of the Auckland Cancer Society Research Centre, Auckland, to KSB and LRF, is acknowledged.

Author Contributions: Georgia Lenihan-Geels and Karen S. Bishop conceived of the idea, conducted the literature search and wrote the article. Lynnette R. Ferguson edited the manuscript and provided guidance.

Conflicts of Interest: The authors declare no conflict of interest.

\section{References}

1. Ferlay, J.; Shin, H.-R.; Bray, F.; Forman, D.; Mathers, C.; Parkin, D.M. Estimates of worldwide burden of cancer in 2008: GLOBOCAN 2008. Int. J. Cancer 2010, 127, 2893-2917.

2. Yu, Y.; Zheng, S.; Zhang, S.; Jin, W.; Liu, H.; Jin, M.; Chen, Z.; Ding, Z.; Wang, L.; Chen, K. Polymorphisms of inflammation-related genes and colorectal cancer risk: A population-based case-control study in China. Int. J. Immunogenet. 2014, 41, 289-297.

3. De Marzo, A.M.; Platz, E.A.; Sutcliffe, S.; Xu, J.; Grönberg, H.; Drake, C.G.; Nakai, Y.; Isaacs, W.B.; Nelson, W.G. Inflammation in prostate carcinogenesis. Nat. Rev. Cancer 2007, 7, 256-269.

4. Baena, R.; Salinas, P. Diet and colorectal cancer. Maturitas 2015, 80, 258-264.

5. Shivappa, N.; Hébert, J.R.; Zucchetto, A.; Montella, M.; Serraino, D.; La Vecchia, C.; Rossi, M. Dietary inflammatory index and endometrial cancer risk in an Italian case-control study. Br. J. Nutr. 2015, 115, 138-146.

6. Dasilva, G.; Pazos, M.; García-Egido, E.; Gallardo, J.M.; Rodríguez, I.; Cela, R.; Medina, I. Healthy effect of different proportions of marine $\omega-3$ PUFAs EPA and DHA supplementation in Wistar rats: Lipidomic biomarkers of oxidative stress and inflammation. J. Nutr. Biochem. 2015, 26, 1385-1392. 
7. Pelser, C.; Mondul, A.M.; Hollenbeck, A.R.; Park, Y. Dietary fat, fatty acids, and risk of prostate cancer in the NIH-AARP diet and health study. Cancer Epidemiol. Biomark. Prev. 2013, 22, 697-707.

8. Richman, E.L.; Kenfield, S.A.; Chavarro, J.E.; Stampfer, M.J.; Giovannucci, E.L.; Willett, W.C.; Chan, J.M. Fat intake after diagnosis and risk of lethal prostate cancer and all-cause mortality. JAMA Intern. Med. 2013, 173, 1318-1326.

9. Azrad, M.; Turgeon, C.; Demark-Wahnefried, W. Current evidence linking polyunsaturated Fatty acids with cancer risk and progression. Front. Oncol. 2013, 3,224 .

10. Burdge, G.C.; Calder, P.C. Conversion of alpha-linolenic acid to longer-chain polyunsaturated fatty acids in human adults. Reprod. Nutr. Dev. 2005, 45, 581-597.

11. Habermann, N.; Ulrich, C.M.; Lundgreen, A.; Makar, K.W.; Poole, E.M.; Caan, B.; Kulmacz, R.; Whitton, J.; Galbraith, R.; Potter, J.D.; et al. PTGS1, PTGS2, ALOX5, ALOX12, ALOX15, and FLAP SNPs: Interaction with fatty acids in colon cancer and rectal cancer. Genes Nutr. 2013, 8, 115-126.

12. Fradet, V.; Cheng, I.; Casey, G.; Witte, J.S. Dietary omega-3 fatty acids, cyclooxygenase-2 genetic variation, and aggressive prostate cancer risk. Clin. Cancer Res. 2009, 15, 2559-2566.

13. Hester, A.G.; Murphy, R.C.; Uhlson, C.J.; Ivester, P.; Lee, T.C.; Sergeant, S.; Miller, L.R.; Howard, T.D.; Mathias, R.A.; Chilton, F.H. Relationship between a common variant in the fatty acid desaturase (FADS) cluster and eicosanoid generation in humans. J. Biol. Chem. 2014, 289, 22482-22489.

14. Al-Hilal, M.; Alsaleh, A.; Maniou, Z.; Lewis, F.J.; Hall, W.L.; Sanders, T.A.B.; O’Dell, S.D. Genetic variation at the FADS1-FADS2 gene locus influences delta- 5 desaturase activity and LC-PUFA proportions after fish oil supplement. J. Lipid Res. 2013, 54, 542-551.

15. Lenihan-Geels, G.; Bishop, K.S.; Ferguson, L.R. Alternative sources of omega-3 fats: Can we find a sustainable substitute for fish? Nutrients 2013, 5, 1301-1315.

16. Szefel, J.; Piotrowska, M.; Kruszewski, W.J.; Jankun, J.; Lysiak-Szydlowska, W.; Skrzypczak-Jankun, E. Eicosanoids in Prevention and Management of Diseases. Curr. Mol. Med. 2011, 11, 13-25.

17. Pischon, T.; Hankinson, S.E.; Hotamisligil, G.S.; Rifai, N.; Willett, W.C.; Rimm, E.B. Habitual dietary intake of $n-3$ and $n-6$ fatty acids in relation to inflammatory markers among US men and women. Circulation 2003, 108, 155-160.

18. Yli-Jama, P.; Seljeflot, I.; Meyer, H.E.; Hjerkinn, E.M.; Arnesen, H.; Pedersen, J.I. Serum non-esterified very long-chain PUFA are associated with markers of endothelial dysfunction. Atherosclerosis 2002, 164, 275-281.

19. Ferrucci, L.; Cherubini, A.; Bandinelli, S.; Bartali, B.; Corsi, A.; Lauretani, F.; Martin, A.; Andres-Lacueva, C.; Senin, U.; Guralnik, J.M. Relationship of Plasma Polyunsaturated Fatty Acids to Circulating Inflammatory Markers. J. Clin. Endocrinol. Metable. 2006, 91, 439-446. 
20. Calder, P.C.; Ahluwalia, N.; Brouns, F.; Buetler, T.; Clement, K.; Cunningham, K.; Esposito, K.; Jönsson, L.S.; Kolb, H.; Lansink, M.; et al. Dietary factors and low-grade inflammation in relation to overweight and obesity. Br. J. Nutr. 2011, 106, S5-S78.

21. Tuncer, S.; Banerjee, S. Eicosanoid pathway in colorectal cancer: Recent updates. World J. Gastroenterol. 2015, 21, 11748-11766.

22. Wang, W.; Zhu, J.; Lyu, F.; Panigrahy, D.; Ferrara, K.W.; Hammock, B.; Zhang, G. w-3 polyunsaturated fatty acids-derived lipid metabolites on angiogenesis, inflammation and cancer. Prostaglandins Other Lipid Mediat. 2014, 113, 13-20.

23. Hanahan, D.; Weinberg, R.A. Hallmarks of cancer: the next generation. Cell 2011, 144, 646-674.

24. Mazhar, D.; Ang, R.; Waxman, J. COX inhibitors and breast cancer. Br. J. Cancer 2006, 94, 346-350.

25. Eberhart, C.E.; Coffey, R.J.; Radhika, A.; Giardiello, F.M.; Ferrenbach, S.; DuBois, R.N. Up-regulation of cyclooxygenase 2 gene expression in human colorectal adenomas and adenocarcinomas. Gastroenterology 1994, 107, 1183-1188.

26. Kleinstein, S.E.; Heath, L.; Makar, K.W.; Poole, E.M.; Seufert, B.L.; Slattery, M.L.; Xiao, L.; Duggan, D.J.; Hsu, L.; Curtin, K.; et al. Genetic variation in the lipoxygenase pathway and risk of colorectal neoplasia. Genes. Chromosomes Cancer 2013, 52, 437-449.

27. Augustsson, K.; Michaud, D.S.; Rimm, E.B.; Leitzmann, M.F.; Stampfer, M.J.; Willett, W.C.; Giovannucci, E. A prospective study of intake of fish and marine fatty acids and prostate cancer. Cancer Epidemiol. Biomark. Prev. 2003, 12, 64-67.

28. Kantor, E.D.; Lampe, J.W.; Peters, U.; Vaughan, T.L.; White, E. Long-chain omega-3 polyunsaturated fatty acid intake and risk of colorectal cancer. Nutr. Cancer 2014, 66, 716-727.

29. Catsburg, C.; Joshi, A.D.; Corral, R.; Lewinger, J.P.; Koo, J.; John, E.M.; Ingles, S.A.; Stern, M.C. Polymorphisms in carcinogen metabolism enzymes, fish intake, and risk of prostate cancer. Carcinogenesis 2012, 33, 1352-1359.

30. Wallström, P.; Bjartell, A.; Gullberg, B.; Olsson, H.; Wirfält, E. A prospective study on dietary fat and incidence of prostate cancer (Malmö, Sweden). Cancer Causes Control 2007, $18,1107-1121$.

31. Corella, D.; Ordovás, J.M. Interactions between dietary $n-3$ fatty acids and genetic variants and risk of disease. Br. J. Nutr. 2012, 107, S271-S283.

32. Von Schacky, C. Omega-3 index and cardiovascular health. Nutrients 2014, 6, 799-814.

33. World Health Organization. Exposure to Dioxins and Dioxin-like Substances: A Major Public Health Concern. Available online: http:/ / www.who.int/ipcs/features/ dioxins.pdf (accessed on 14 January 2016).

34. White, M.C.; Holman, D.M.; Boehm, J.E.; Peipins, L.A.; Grossman, M.; Jane Henley, S. Age and Cancer Risk. Am. J. Prev. Med. 2014, 46, S7-S15.

35. Tintle, N.L.; Pottala, J.V.; Lacey, S.; Ramachandran, V.; Westra, J.; Rogers, A.; Clark, J.; Olthoff, B.; Larson, M.; Harris, W.; Shearer, G.C. A genome-wide association study of saturated, mono- and polyunsaturated red blood cell fatty acids in the Framingham Heart Offspring Study. Prostaglandins Leukot. Essent. Fatty Acids 2015, 94, 65-72. 
36. Bishop, K.S.; Erdrich, S.; Karunasinghe, N.; Han, D.Y.; Zhu, S.; Jesuthasan, A.; Ferguson, L.R. An investigation into the association between DNA damage and dietary fatty acid in men with prostate cancer. Nutrients 2015, 7, 405-422.

37. Hong, S.H.; Kwak, J.H.; Paik, J.K.; Chae, J.S.; Lee, J.H. Association of polymorphisms in FADS gene with age-related changes in serum phospholipid polyunsaturated fatty acids and oxidative stress markers in middle-aged nonobese men. Clin. Interv. Aging 2013, 8 , 585-596.

38. Chilton, F.H.; Murphy, R.C.; Wilson, B.A.; Sergeant, S.; Ainsworth, H.; Seeds, M.C.; Mathias, R.A. Diet-gene interactions and PUFA metabolism: A potential contributor to health disparities and human diseases. Nutrients 2014, 6, 1993-2022.

39. Simopoulos, A.P. Genetic variants in the metabolism of omega- 6 and omega- 3 fatty acids: their role in the determination of nutritional requirements and chronic disease risk. Exp. Biol. Med. (Maywood) 2010, 235, 785-795.

40. Zhang, C.; Yu, H.; Ni, X.; Shen, S.; Das, U.N. Growth inhibitory effect of polyunsaturated fatty acids (PUFAs) on colon cancer cells via their growth inhibitory metabolites and fatty acid composition changes. PLoS ONE 2015, 10, e0123256.

41. Mansara, P.P.; Deshpande, R.A.; Vaidya, M.M.; Kaul-Ghanekar, R. Differential Ratios of Omega Fatty Acids (AA/EPA + DHA) Modulate Growth, Lipid Peroxidation and Expression of Tumor Regulatory MARBPs in Breast Cancer Cell Lines MCF7 and MDA-MB-231. PLOS ONE 2015, 10, e0136542.

42. Rose, D.P.; Connolly, J.M. Effects of fatty acids and eicosanoid synthesis inhibitors on the growth of two human prostate cancer cell lines. Prostate 1991, 18, 243-254.

43. Akinsete, J.A.; Ion, G.; Witte, T.R.; Hardman, W.E. Consumption of high $\omega-3$ fatty acid diet suppressed prostate tumorigenesis in C3(1) Tag mice. Carcinogenesis 2012, 33, 140-148.

44. Van Beelen, V.A.; Spenkelink, B.; Mooibroek, H.; Sijtsma, L.; Bosch, D.; Rietjens, I.M.; Alink, G.M. An n-3 PUFA-rich microalgal oil diet protects to a similar extent as a fish oil-rich diet against AOM-induced colonic aberrant crypt foci in F344 rats. Food Chem. Toxicol. 2009, 47, 316-320.

45. Leslie, M.A.; Abdelmagid, S.A.; Perez, K.; Muller, W.J.; Ma, D.W. Mammary tumour development is dose-dependently inhibited by $n-3$ polyunsaturated fatty acids in the MMTV-neu(ndl)-YD5 transgenic mouse model. Lipids Health Dis. 2014, 13, 96.

46. Glaser, C.; Rzehak, P.; Demmelmair, H.; Klopp, N.; Heinrich, J.; Koletzko, B. Influence of FADS polymorphisms on tracking of serum glycerophospholipid fatty acid concentrations and percentage composition in children. PLoS ONE 2011, 6, e21933.

47. Zhou, T.-B.; Drummen, G.P.C.; Jiang, Z.-P.; Qin, Y.-H. GSTT1 polymorphism and the risk of developing prostate cancer. Am. J. Epidemiol. 2014, 180, 1-10.

48. Chen, C. COX-2's new role in inflammation. Nat. Chem. Biol. 2010, 6, 401-402.

49. Gago-Dominguez, M. Marine $n-3$ fatty acid intake, glutathione S-transferase polymorphisms and breast cancer risk in post-menopausal Chinese women in Singapore. Carcinogenesis 2004, 25, 2143-2147. 
50. Hedelin, M.; Chang, E.T.; Wiklund, F.; Bellocco, R.; Klint, A.; Adolfsson, J.; Shahedi, K.; Xu, J.; Adami, H.-O.; Grönberg, H.; Bälter, K.A. Association of frequent consumption of fatty fish with prostate cancer risk is modified by COX-2 polymorphism. Int. J. Cancer 2007, 120, 398-405.

51. Poole, E.M.; Bigler, J.; Whitton, J.; Sibert, J.G.; Kulmacz, R.J.; Potter, J.D.; Ulrich, C.M. Genetic variability in prostaglandin synthesis, fish intake and risk of colorectal polyps. Carcinogenesis 2007, 28, 1259-1263.

52. Poole, E.M.; Hsu, L.; Xiao, L.; Kulmacz, R.J.; Carlson, C.S.; Rabinovitch, P.S.; Makar, K.W.; Potter, J.D.; Ulrich, C.M. Genetic variation in prostaglandin E2 synthesis and signaling, prostaglandin dehydrogenase, and the risk of colorectal adenoma. Cancer Epidemiol. Biomark. Prev. 2010, 19, 547-557.

53. Porenta, S.R.; Ko, Y.-A.; Gruber, S.B.; Mukherjee, B.; Baylin, A.; Ren, J.; Djuric, Z. Interaction of fatty acid genotype and diet on changes in colonic fatty acids in a Mediterranean diet intervention study. Cancer Prev. Res. (Phila) 2013, 6, 1212-1221.

54. Schaeffer, L.; Gohlke, H.; Müller, M.; Heid, I.M.; Palmer, L.J.; Kompauer, I.; Demmelmair, H.; Illig, T.; Koletzko, B.; Heinrich, J. Common genetic variants of the FADS1 FADS2 gene cluster and their reconstructed haplotypes are associated with the fatty acid composition in phospholipids. Hum. Mol. Genet. 2006, 15, 1745-1756.

55. Harsløf, L.B.S.; Larsen, L.H.; Ritz, C.; Hellgren, L.I.; Michaelsen, K.F.; Vogel, U.; Lauritzen, L. FADS genotype and diet are important determinants of DHA status: A cross-sectional study in Danish infants. Am. J. Clin. Nutr. 2013, 97, 1403-1410.

56. Cormier, H.; Rudkowska, I.; Paradis, A.-M.; Thifault, E.; Garneau, V.; Lemieux, S.; Couture, P.; Vohl, M.-C. Association between polymorphisms in the fatty acid desaturase gene cluster and the plasma triacylglycerol response to an $n$-3 PUFA supplementation. Nutrients 2012, 4, 1026-1041.

57. Davis, C.; Bryan, J.; Hodgson, J.; Murphy, K. Definition of the Mediterranean Diet; A Literature Review. Nutrients 2015, 7, 9139-9153.

58. Trichopoulou, A.; Costacou, T.; Bamia, C.; Trichopoulos, D. Adherence to a Mediterranean diet and survival in a Greek population. N. Engl. J. Med. 2003, 348, 2599-2608.

59. Wang, D.; Dubois, R.N. Eicosanoids and cancer. Nat. Rev. Cancer 2010, 10, 181-193.

60. Shan, D.; Shen, K.; Zhu, J.; Feng, M.; Wu, Y.; Wan, C.; Shen, Y.; Xu, L. The polymorphism (Gln261Arg) of 12-lipoxygenase and cancer risk: A meta-analysis. Int. J. Clin. Exp. Med. 2015, 8, 488-495.

61. Prasad, V.V.; Kolli, P.; Moganti, D. Association of a functional polymorphism (Gln261Arg) in 12-lipoxygenase with breast cancer. Exp. Ther. Med. 2011, 2, 317-323.

62. Gong, Z.; Hebert, J.R.; Bostick, R.M.; Deng, Z.; Hurley, T.G.; Dixon, D.A.; Nitcheva, D.; $\mathrm{Xie}, \mathrm{D}$. Common polymorphisms in 5-lipoxygenase and 12-lipoxygenase genes and the risk of incident, sporadic colorectal adenoma. Cancer 2007, 109, 849-857.

63. Jin, Y.; Hao, Z. Polymorphisms of glutathione S-transferase M1 (GSTM1) and T1 (GSTT1) in ovarian cancer risk. Tumour Biol. 2014, 35, 5267-5272. 
64. Kanwal, R.; Pandey, M.; Bhaskaran, N.; Maclennan, G.T.; Fu, P.; Ponsky, L.E.; Gupta, S. Protection against oxidative DNA damage and stress in human prostate by glutathione S-transferase P1. Mol. Carcinog. 2014, 53, 8-18.

65. Van Hemelrijck, M.; Rohrmann, S.; Steinbrecher, A.; Kaaks, R.; Teucher, B.; Linseisen, J. Heterocyclic aromatic amine [HCA] intake and prostate cancer risk: effect modification by genetic variants. Nutr. Cancer 2012, 64, 704-713.

66. John, E.; Stern, M.; Sinha, R.; Koo, J. Meat Consumption, Cooking Practices, Meat Mutagens, and Risk of Prostate Cancer. Nutr. Cancer 2011, 63, 525-537.

67. Yu, W.; Murray, N.R.; Weems, C.; Chen, L.; Guo, H.; Ethridge, R.; Ceci, J.D.; Evers, B.M.; Thompson, E.A.; Fields, A.P. Role of cyclooxygenase 2 in protein kinase C beta II-mediated colon carcinogenesis. J. Biol. Chem. 2003, 278, 11167-11174.

68. Thun, M.J.; Henley, S.J.; Patrono, C. Nonsteroidal anti-inflammatory drugs as anticancer agents: Mechanistic, pharmacologic, and clinical issues. J. Natl. Cancer Inst. 2002, 94, 252-266.

69. Cavazos, D.A.; Price, R.S.; Apte, S.S.; deGraffenried, L.A. Docosahexaenoic acid selectively induces human prostate cancer cell sensitivity to oxidative stress through modulation of NF-kB. Prostate 2011, 71, 1420-1428.

70. Eser, P.O.; Vanden Heuvel, J.P.; Araujo, J.; Thompson, J.T. Marine- and plant-derived $\omega-3$ fatty acids differentially regulate prostate cancer cell proliferation. Mol. Clin. Oncol. 2013, 1, 444-452.

71. Shahedi, K.; Lindström, S.; Zheng, S.L.; Wiklund, F.; Adolfsson, J.; Sun, J.; Augustsson-Bälter, K.; Chang, B.-L.; Adami, H.-O.; Liu, W.; Grönberg, H.; Xu, J. Genetic variation in the COX-2 gene and the association with prostate cancer risk. Int. J. Cancer 2006, 119, 668-672.

72. Qiao, L.; Kozoni, V.; Tsioulias, G.J.; Koutsos, M.I.; Hanif, R.; Shiff, S.J.; Rigas, B. Selected eicosanoids increase the proliferation rate of human colon carcinoma cell lines and mouse colonocytes in vivo. Biochim. Biophys. Acta 1995, 1258, 215-223.

73. Sheng, H.; Shao, J.; Washington, M.K.; DuBois, R.N. Prostaglandin E2 increases growth and motility of colorectal carcinoma cells. J. Biol. Chem. 2001, 276, 18075-18081.

74. Stern, M.C.; Butler, L.M.; Corral, R.; Joshi, A.D.; Yuan, J.-M.; Koh, W.-P.; Yu, M.C. Polyunsaturated fatty acids, DNA repair single nucleotide polymorphisms and colorectal cancer in the Singapore Chinese Health Study. J. Nutrigenet. Nutrigenom. 2009, 2, 273-279.

75. Lane, J.; Mansel, R.E.; Jiang, W.G. Expression of human delta-6-desaturase is associated with aggressiveness of human breast cancer. Int. J. Mol. Med. 2003, 12, 253-257.

76. Azrad, M.; Zhang, K.; Vollmer, R.T.; Madden, J.; Polascik, T.J.; Snyder, D.C.; Ruffin, M.T.; Moul, J.W.; Brenner, D.; Hardy, R.W.; et al. Prostatic alpha-linolenic acid (ALA) is positively associated with aggressive prostate cancer: a relationship which may depend on genetic variation in ALA metabolism. PLOS ONE 2012, 7, e53104. 


\title{
Eicosopentaneoic Acid and Other Free Fatty Acid Receptor Agonists Inhibit Lysophosphatidic Acid- and Epidermal Growth Factor-Induced Proliferation of Human Breast Cancer Cells
}

\author{
Mandi M. Hopkins, Zhihong Zhang, Ze Liu and Kathryn E. Meier
}

\begin{abstract}
Many key actions of $\omega-3(n-3)$ fatty acids have recently been shown to be mediated by two G protein-coupled receptors (GPCRs) in the free fatty acid receptor (FFAR) family, FFA1 (GPR40) and FFA4 (GPR120). n-3 Fatty acids inhibit proliferation of human breast cancer cells in culture and in animals. In the current study, the roles of FFA1 and FFA4 were investigated. In addition, the role of cross-talk between GPCRs activated by lysophosphatidic acid (LPA), and the tyrosine kinase receptor activated by epidermal growth factor (EGF), was examined. In MCF-7 and MDA-MB-231 human breast cancer cell lines, both LPA and EGF stimulated proliferation, Erk activation, Akt activation, and CCN1 induction. LPA antagonists blocked effects of LPA and EGF on proliferation in MCF-7 and MDA-MB-231, and on cell migration in MCF-7. The $n-3$ fatty acid eicosopentaneoic acid inhibited LPA- and EGF-induced proliferation in both cell lines. Two synthetic FFAR agonists, GW9508 and TUG-891, likewise inhibited LPA- and EGF-induced proliferation. The data suggest a major role for FFA1, which was expressed by both cell lines. The results indicate that $n-3$ fatty acids inhibit breast cancer cell proliferation via FFARs, and suggest a mechanism involving negative cross-talk between FFARS, LPA receptors, and EGF receptor.
\end{abstract}

Reprinted from J. Clin. Med. Cite as: Hopkins, M.M.; Zhang, Z.; Liu, Z.; Meier, K.E. Eicosopentaneoic Acid and Other Free Fatty Acid Receptor Agonists Inhibit Lysophosphatidic Acid- and Epidermal Growth Factor-Induced Proliferation of Human Breast Cancer Cells. J. Clin. Med. 2016, 5, 16.

\section{Introduction}

Our group recently demonstrated that the inhibitory effects of $\omega-3$ fatty acids on prostate cancer cell proliferation are mediated by FFA4, a G protein-coupled receptor in the free fatty acid receptor (FFAR) family [1]. The purpose of the current study was to determine whether FFARs mediate similar inhibitory effects in human breast cancer cells. 
The dietary polyunsaturated $\omega-3$ fatty acids ( $n-3$ FAs) are alpha-linolenic acid (ALA), docosahexaenoic acid (DHA) and eicosapentaneoic acid (EPA). Although effects of $n$-3 FAs in prostate cancer have been debated [2], there is relatively strong evidence supporting a preventative effect of $n-3$ FA consumption on many human cancers [3], including breast cancer [4]. Multiple reports show that $n-3$ fatty acids inhibit growth of breast cancer cells, either in cell culture [5,6], or in xenograft tumors [7-10].

The prevailing mechanistic paradigm has been that $n-3$ FAs exert anti-inflammatory and potentially anti-cancer effects by competitively reducing production of eicosanoids, and/or more directly by generating metabolites with anti-inflammatory activity (e.g., "resolvins") [11,12]. However, the direct effects of n-3 metabolites on cancer cells, as compared to their anti-inflammatory effects, are under-studied [13]. In one report, resolvin D2, a DHA metabolite, unexpectedly increased proliferation of MCF-7 breast cancer cells [14].

Alternatively, it was shown in the last decade that $n$ - 3 FAs are agonist ligands for free fatty acid receptors (FFARs) that were formerly "orphan receptors" [15]. Two G-protein-coupled receptors (GPCRs), FFA1 and FFA4, bind long-chain polyunsaturated fatty acids that include $n-3$ FAs. The "de-orphanization" discovery has led to the ongoing characterization of the roles of the FFARs in cellular regulation, and to the rapid development of selective FFAR agonists with therapeutic potential [16-19].

Several studies have specifically explored the mechanism of the inhibitory action of $n-3$ FAs on breast cancer cells. The pathways implicated in the response include decreased Akt activation [5], increased neutral sphingomyelinase activity [20], increased BRCA levels [21], and increased PTEN levels [22]. GPCR-independent mechanisms have been reviewed [23]. To date, there is little information available concerning the roles of FFARs in breast cancer. It has however been shown that FFA1 is expressed in MCF-7 cells [24], and that MCF-7 and MDA-MB-231 cells express both FFA1 and FFA4 $[25,26]$.

One study investigated the role of FFA4 in breast cancer in a mouse model, focusing on the role of FFA4 in inhibiting inflammation [27]. In this study, $n-3$ FAs reduced tumor burden even when FFA4 was knocked out in the host mouse. The authors suggest that anti-inflammatory effects of $n-3$ FAs, mediated by FFA4, are not important for their anti-tumor effects. Using cultured cells derived from their mouse model, the investigators showed that DHA induced apoptosis in either wild-type or FFA4 knockdown cells when applied at high doses $(40-100 \mu \mathrm{M})$. The role of the alternative $n-3$ FA receptor, FFA1, has not been examined in breast cancer cell, to our knowledge.

In this study, we utilized two commonly used human breast cancer cell lines: MCF-7 and MDA-MB-231, as experimental models. MCF-7 is a luminal A estrogen 
receptor positive cell line, while MDA-MB-231 is a highly metastatic triple negative cell line. These two cell lines were used to explore the role of FFARs in the mechanism of action of $n-3$ FAs in breast cancer.

\section{Experimental Section}

\subsection{Materials}

EPA (prepared in ethanol) was from Cayman Chemical (Ann Arbor, MI, USA). The FFAR agonists TUG-891 (4-[(4-fluoro-4'-methyl[1,1'-biphenyl]2-yl)methoxy]-benzenepropanoic acid; prepared in dimethylsulfoxide (DMSO) and GW9508 (4-[[(3-phenoxyphenyl)methyl]amino]-benzenepropanoic acid; prepared in ethanol), were from Millipore (Billerica, MA, USA) and Cayman Chemical, respectively. AM966 (2-[4-[4-[4-[[(1R)-1-(2-chlorophenyl)ethoxy]carbonylamino]-3methyl-1,2-oxazol- 5-yl]phenyl] phenyl]acetic acid) was purchased pre-dissolved in DMSO from MedChem Express (Monmouth Junction, NJ, USA). Ki16425 (3-[[[4-[4-[[[1-(2-chlorophenyl)ethoxy]carbonyl]amino]-3-methyl-5- isoazoly]phenyl] methyl]thio]-propanoic acid; prepared in DMSO) was purchased from Cayman Chemical (Ann Arbor, MI, USA). Vehicle controls were included in all samples not receiving FFAR agonists or LPA receptor (LPAR) antagonists (final concentrations of $0.03 \%(v / v)$ ethanol or $0.01 \%$ DMSO). LPA (18:1; oleoyl) was obtained from Avanti Polar Lipids (Birmingham, UK), and was delivered to cells as a 1000X stock solution prepared in $4 \mathrm{mg} / \mathrm{mL}$ fatty acid-free bovine serum albumin (BSA). The vehicle control for LPA was a final concentration of $4 \mu \mathrm{g} / \mathrm{mL}$ BSA. EGF was from Sigma (St. Louis, MO, USA). Antibody recognizing CCN1 (lot \# F0509; 1:1000 dilution) was obtained from Santa Cruz Biotechnology (Santa Cruz, CA, USA). Anti-actin, obtained from BD Transduction Laboratories (Lexington, KY, USA) (lot \# 51711), was used at a 1:5000 dilution. Goat anti-rabbit secondary antibody (lot \#083M4752) was purchased from Sigma (St Louis, MO, USA) and used at 1:20,000 dilution, while goat anti-mouse secondary antibody (lot \#1124907A) was purchased from Invitrogen/Life Technologies (Grand Island, NE, USA) and used at a 1:5000 dilution.

\subsection{Cell Culture}

MCF-7 and MDA-MB-231 cells were obtained from the American Type Culture Collection (Manassas, VA, USA). The cells were grown in RPMI medium supplemented with 10\% FBS (Hyclone/Thermo-Fisher Scientific, Waltham, MA, USA). Both cell lines were grown in an incubator at $37{ }^{\circ} \mathrm{C}$ in $5 \% \mathrm{CO}_{2}$ on standard tissue culture plastic. 


\subsection{Cell Proliferation Assays}

Cells were seeded in 6-well plates at $3 \times 10^{5}$ cells/well in serum-containing medium. After 1 day, the medium was changed to RPMI 1640 without serum. On the next day, the medium was changed to RPMI 1640 with $10 \%$ FBS, $10 \mu \mathrm{M} \mathrm{LPA}$, or $10 \mathrm{nM}$ EGF, in the absence or presence of $100 \mathrm{nM}$ AM966, $10 \mu \mathrm{M}$ Ki16425, $20 \mu \mathrm{M}$ EPA or $1 \mu \mathrm{M}$ TUG-891. Control cells were incubated with the appropriate vehicle $(0.03 \%$ ethanol, $v / v ; 0.01 \%$ DMSO, $v / v$; and /or $4 \mu \mathrm{g} / \mathrm{mL} \mathrm{BSA})$. Duplicate wells were prepared for each experimental condition. Cell numbers were evaluated after 24, 48 , and $72 \mathrm{~h}$ by removing medium, incubating cells with trypsin/EDTA for $5 \mathrm{~min}$, adding trypan blue, and counting the suspended live cells (excluding trypan blue) using a hemacytometer.

\subsection{Cell Migration Assays}

MCF-7 cell migration was assessed using a modified Boyden chamber method, as previously described [28]. Cells were serum starved for $24 \mathrm{~h}$ and then seeded in serum-free medium at $2.5 \times 10^{4}$ cells per insert in the upper chambers of $8-\mu \mathrm{m}$ transwell inserts (BD Biosciences, San Jose, CA, USA). Cells were then treated with 10\% FBS, 100 nM AM966, $20 \mu \mathrm{M}$ EPA, $1 \mu$ M TUG-891, $10 \mu \mathrm{M}$ LPA, or 10 nM EGF, either alone or in combination, with appropriate vehicle controls as described above. Serum-free medium was added to the lower wells. Following a 6-h migration, the insert membranes were fixed and stained using methanol and crystal violet. Cells that invaded the lower chambers were counted by microscopy.

\subsection{Cell Incubations for Signal Transduction Assays}

Cells were grown in DME medium supplemented with 10\% FBS until $80 \%$ confluent. Cells were serum-starved for $24 \mathrm{~h}$ in RPMI 1640 medium, then incubated with $10 \mu \mathrm{M}$ LPA, $10 \mathrm{nM}$ EGF, and/or $100 \mathrm{nM}$ AM966 or $10 \mu \mathrm{M}$ Ki16425 for $10 \mathrm{~min}$. Cells were rinsed twice with ice-cold phosphate-buffered saline (PBS), harvested by scraping into $1 \mathrm{~mL}$ ice-cold PBS, collected by centrifugation at $10,000 \times \mathrm{g}$ for $10 \mathrm{~min}$ at $4{ }^{\circ} \mathrm{C}$, and resuspended in ice-cold lysis buffer $(20 \mathrm{mM}$ HEPES $(\mathrm{pH}=7.4)), 1 \%$ Triton X-100, $50 \mathrm{mM} \mathrm{NaCl}, 2 \mathrm{mM}$ EGTA, $5 \mathrm{mM} \beta$-glycerophosphate, $30 \mathrm{mM}$ sodium pyrophosphate, $100 \mathrm{mM}$ sodium orthovanadate, $1 \mathrm{mM}$ phenylmethylsulfonyl fluoride, $10 \mu \mathrm{g} / \mathrm{mL}$ aprotinin, $10 \mu \mathrm{g} / \mathrm{mL}$ leupeptin). Insoluble debris was removed after centrifugation.

\subsection{Reverse Transcription Polymerase Chain Reacton (RT-PCR)}

For analysis of FFAR expression, total RNA was isolated using an RNeasy Mini kit (Qiagen, Valencia, Spain). First-strand complementary DNA (cDNA) was synthesized with SuperScript II Reverse Transcriptase (Invitrogen) following 
the manufacturer's instructions using $20 \mu \mathrm{L}$ of reaction mixture containing $2 \mu \mathrm{g}$ RNA. PCR was carried out using Platinum Pfx DNA Polymerase (Invitrogen) and Integrated DNA Technology (San Diego, CA, USA) primers: FFA4 (F: 5'-CCTGGA GAGATCTCGTGGGA-3'; and R: 5'-AGGAGGTGTTCCGAGGTCTG-3'); FFA1 (F: 5'-CTCCTTCGGCCTCTATGTGG-3'; and R: 5'-AGACCAGGCTAGGGGTGA GA-3'); RPLP0 (F: 5'-CGCTATCCGCGGTTTCTGAT-3'; and R: 5'-AGACGA TGTCACTTCCACGA-3'). For each reaction, $5 \mu \mathrm{g}$ cDNA template was used. Products were separated by ethidium bromide agarose gel electrophoresis, and were then imaged using a ChemiDoc with Image Lab software (Bio-Rad, Hercules, CA, USA). For analysis of LPA receptor expression, total RNA was extracted from harvested cells using TRIzol solution (Invitrogen, Carlsbad, CA, USA) according to the manufacturer's protocol. Reverse transcription was performed using iScriptTMcDNA synthesis kit (Bio-Rad, Hercules, CA) in a reaction volume of $20 \mu \mathrm{L}$ under the conditions recommended by the manufacture. Total RNA ( $1 \mu \mathrm{g})$ was used as a template for cDNA synthesis. PCR was performed in a $50-\mu \mathrm{L}$ reaction volume with a buffer consisting of $10 \times$ iTaq buffer, $50 \mathrm{mM} \mathrm{MgCl}$, $10 \mathrm{mM}$ dNTP mix, iTaq DNA polymerase; and $0.25 \mu \mathrm{mol} / \mathrm{L}$ each primer. The primers were: LPA1/Edg-2 (F: 5'-TGTCATGGCTGCCATCTC-3'; and R: 5'-CATCT CAGTTTCCGTTCTAA-3'); LPA2/Edg-4 (F: 5'-CCCAACCAACAGGACTGACT-3'; and R: 5'-GAGCCCTTATCTCTCCCCAC-3'); LPA3/Edg-7 (F: 5'-GGACACC CATGAAGCTAATG-3'; and R: 5'-TCTGGGTTCTCCTGAGAGAA-3'); $\beta$-actin (F: 5'-TGACGGGGTCACCCACACTGTGCCCATCTA-3'; and R: $5^{\prime}$-CTAGA AGCATTTGCGG TGGACGATGGAGGG-3'). RT-PCR products were separated on a $2 \%$ agarose gel by electrophoresis and visualized and imaged under UV illumination.

\subsection{Immunoblotting}

Whole-cell extracts containing equal amounts of protein $(30 \mu \mathrm{g})$ were separated by SDS-PAGE on 10\% Laemmli gels, transferred to nitrocellulose, and incubated with primary (overnight at $4^{\circ} \mathrm{C}$ ) and then secondary (one to two hours at room temperature) antibodies. Blots were developed using enhanced chemiluminescence (GE Healthcare, Pittsburgh, PA, USA), and imaged using a Gel Doc system (BioRad, Hercules, CA, USA). Protein expression was quantified by densitometry using Quantity One software (Bio-Rad). Results were normalized to the actin loading control, and then to the value obtained for untreated control cells.

\subsection{Statistical Analysis}

Data were analyzed by two-way ANOVA followed by Tukey's multiple comparisons test. The only exceptions were assays in which there was only one time point (e.g., migration assays); these data were analyzed by one-way ANOVA 
followed by Tukey's mutliple comparisons test. All analyses were done using Prism software (Graphpad, San Diego, CA, USA).

\section{Results and Discussion}

3.1. Effects of Lysophosphatidic Acid (LPA) and Epidermal Growth Factor (EGF) on Breast Cancer Cell Proliferation

Before testing for effects of FFAR agonists on breast cancer cells, we first established conditions for using growth factors to stimulate proliferation. Cells were serum-starved before treatments in order to remove confounding effects of LPA contained in serum, and to provide a baseline for testing effects of growth factors. The effects of serum, LPA, and EGF on proliferation of serum-starved MCF-7 and MDA-MB-231 cells are shown in Figure 1. All growth factors significantly increased cell number as compared to control. Serum was significantly more effective in inducing proliferation than LPA or EGF at all time points tested, in both cell lines; this result was expected since serum contains multiple mitogens including LPA. There was no significant difference between responses to LPA versus EGF at any time point.

\subsection{Signal Transduction Responses to LPA and EGF in Breast Cancer Cells}

To further characterize responses to LPA and EGF in the breast cancer cell lines, we performed immunoblotting experiments to test for Erk and Akt activation (Figure 2). Both LPA and EGF increase activating phosphorylation of Erk and Akt in both MCF-7 and MDA-MB-231 cells (Figure 2A). These results are consistent with previous reports concerning the mitogenic activity of LPA and EGF in breast cancer cells $[29,30]$.

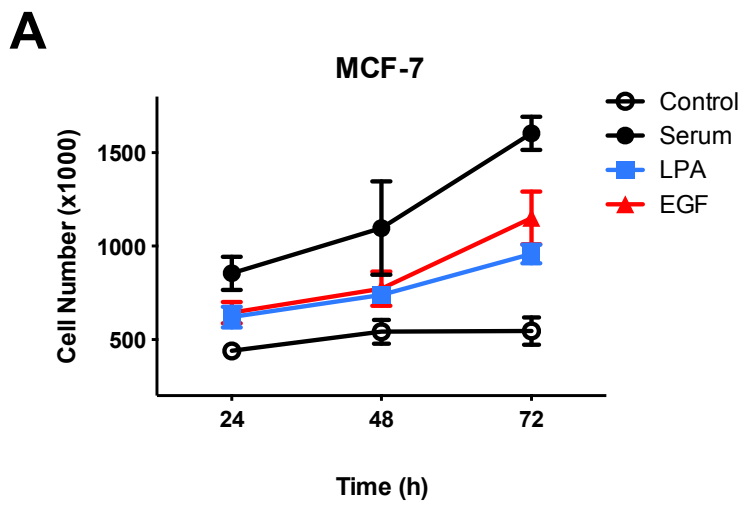

Figure 1. Cont. 


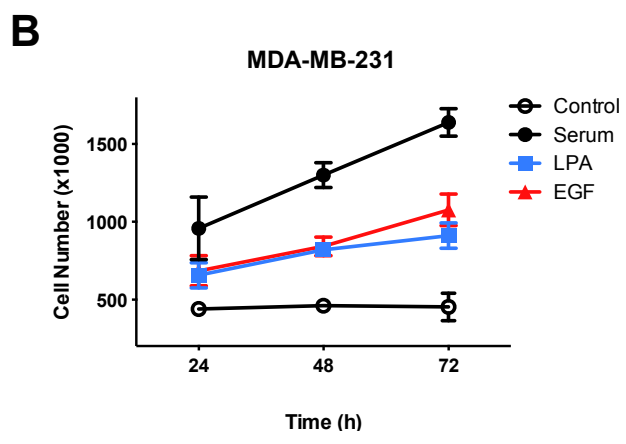

Figure 1. Effects of growth factors on proliferation of human breast cancer cells. Proliferation assays were conducted using serum-starved MCF-7 (A) or MDA-MB-231 (B) cells. Cells were incubated with or without 10\% FBS (serum), $10 \mu \mathrm{M}$ LPA, or $10 \mathrm{nM}$ EGF for the indicated times (growth factors were added at time " 0 "). Each data point represents the mean \pm SEM $(n=4)$ of values (number of live cells per well) from two separate experiments, each done in with two separate replicate wells of cells for each condition. Data analysis was performed using two-way ANOVA, followed by Tukey's multiple comparisons test. All growth factor values were significantly $(p<0.05)$ different from the control value at all time points shown, except for LPA at $24 \mathrm{~h}$ in MCF-7. Serum values were significantly different from lysophosphatidic acid (LPA) or epidermal growth factor (EGF) at all time points tested.

We also examined a more novel response to growth factors, CCN1 induction, in MCF-7 cells. CCN1 is an inducible matricellular protein whose expression is positively correlated with breast cancer progression [31,32]. As shown in Figure 2B, both LPA and EGF stimulate expression of CCN1 in MCF-7 cells. An increase in $\mathrm{CCN} 1$ protein levels was seen after only $30 \mathrm{~min}$ of treatment with either growth factor. Taken together, the results presented in Figure 2A,B confirm that both LPA and EGF activate pro-mitogenic signaling pathways in human breast cancer cell lines.

\subsection{Effects of LPA Antagonists on Breast Cancer Cell Proliferation}

Previous studies have shown that LPA receptors are expressed in breast cancer cell lines. One group performed a comprehensive analysis of LPA receptor expression in commonly-used breast cancer cell lines [33]. Their data show that MDA-MB-231 and MCF-7 cells, among other cell lines, express $\mathrm{LPA}_{1}, \mathrm{LPA}_{2}$, and $\mathrm{LPA}_{3} . \mathrm{LPA}_{1}$ has been determined to mediate many of the actions of LPA in breast cancer cells [34-37]. RT-PCR experiments conducted in our lab confirmed that $\mathrm{LPA}_{1}$ was expressed in both MCF-7 and MDA-MB-231 cells under the conditions used herein (Figure 3A). 


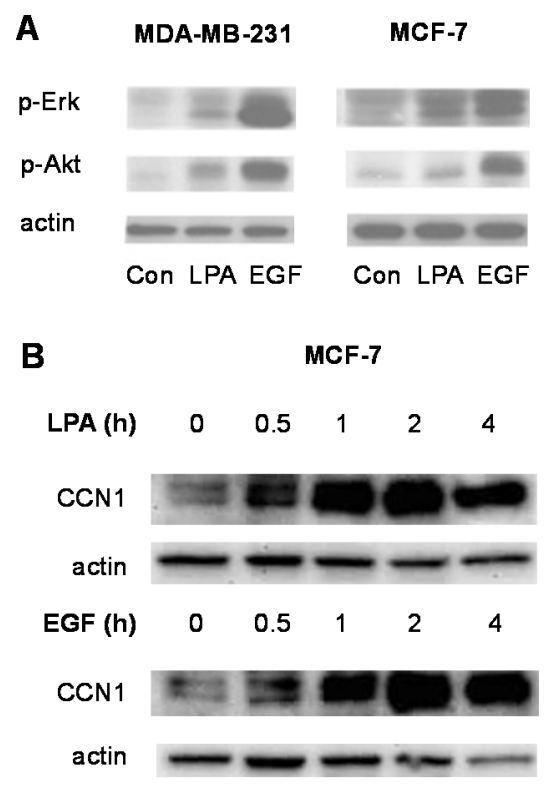

Figure 2. Effects of LPA and EGF on signal transduction events in human breast cancer cells. (A) Serum-starved MCF-7 and MDA-MB-231 cells were incubated with $10 \mu \mathrm{M}$ LPA or $10 \mathrm{nM}$ EGF for $5 \mathrm{~min}$. Whole-cell extracts were immunoblotted using antibodies recognizing phosphorylated active Erk and Akt. An immunoblot for total actin was performed as a loading control; (B) Serum-starved MCF-7 cells were incubated for the indicated times with $10 \mu \mathrm{M}$ LPA or $10 \mathrm{nM}$ EGF. Whole-cell extracts were immunoblotted using antibody recognizing total CCN1. An immunoblot for total actin was performed as a loading control. Each experiment is representative of at least three separate experiments.

Two LPA receptor pharmacologic antagonists were used to further study the role of $\mathrm{LPA}_{1}$ in breast cancer cells. Ki16425 is a selective inhibitor of $\mathrm{LPA}_{1}$ and $\mathrm{LPA}_{3}$ [38], while AM966 is an $\mathrm{LPA}_{1}$-selective antagonist [39]. We used a dose-response study (Figure $3 \mathrm{~B}$ ) to test whether $\mathrm{LPA}_{1}$ inhibitors inhibit LPA-induced proliferation in MDA-MB-231 breast cancer cells. As expected, based on their relative receptor affinities, the $\mathrm{LPA}_{1}$-selective antagonist AM966 was more potent $\left(\mathrm{IC}_{50}=32 \mathrm{nM}\right)$ in inhibiting LPA-induced proliferation than was the pan-LPA inhibitor Ki16425 $\left(\mathrm{IC}_{50}=904 \mathrm{nM}\right)$. Interestingly, the amount of AM966 needed to completely inhibit MDA-MB-231 cell proliferation was 100-fold higher than in our previously published work using DU145 prostate cancer cells [1], although the amount of Ki16425 required was similar in both MDA-MB-231 and DU145. The dose-response curve for AM966 was very shallow, suggesting the involvement of multiple receptors. These 
results suggest that $\mathrm{LPA}_{1}$ is not the only LPA receptor that mediates LPA-induced proliferation in these cells.

A

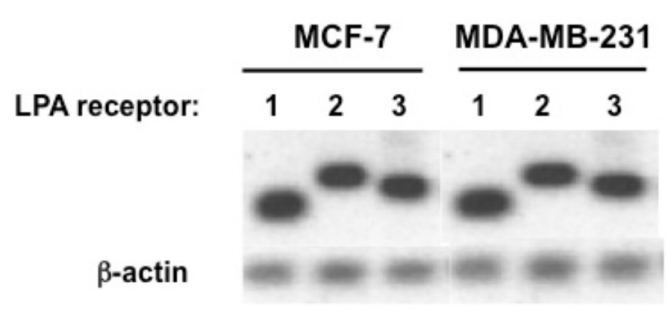

B

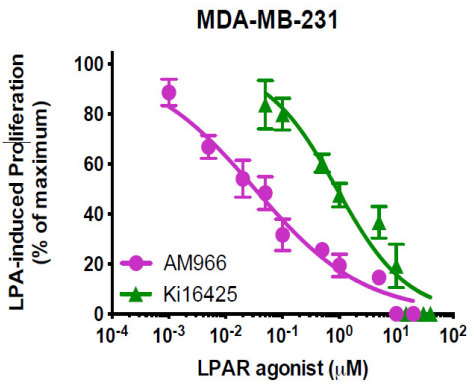

Figure 3. LPAR expression in breast cancer cell lines, and dose-response for the effects of $\mathrm{LPA}_{1}$ antagonists on MDA-MB-231 cell proliferation. (A) Total RNA was extracted from MCF-7 and MDA-MB-231 breast cancer cells. RT-PCR was performed (separate gels for each cell line) using the cDNA primers described in the Methods. $\beta$-actin was amplified as loading control; (B) Serum-starved MDA-MB-231 cells were incubated for $48 \mathrm{~h}$ with and without $10 \mu \mathrm{M}$ LPA in the absence and presence of the indicated concentrations of Ki16425 or AM966. The number of cells achieved in response to LPA alone was defined as 100\% response; the number of cells in the absence of LPA was defined as $0 \%$ response. Each point represents mean \pm SEM $(n \geqslant 4)$ for values obtained from at least two experiments, each performed with two separate replicate wells of cells for each condition.

In Figure 4, we further tested the effects of LPA antagonists. We asked 1) whether the inhibitory effects of LPA antagonists extend to EGF-induced proliferation, and 2) whether LPA antagonists have similar effects in MCF-7 and MDA-MB-231 cells. The results of this series of experiments, which are presented in Figure 4, clearly demonstrate that the LPA receptor antagonists block proliferation in response to both LPA and EGF. This response was observed in both MCF-7 and MDA-MB-231 cells.

Taken together, these results are consistent with a crucial role for LPARs in EGF response, as has been noted in other cell types [1,40]. 
A

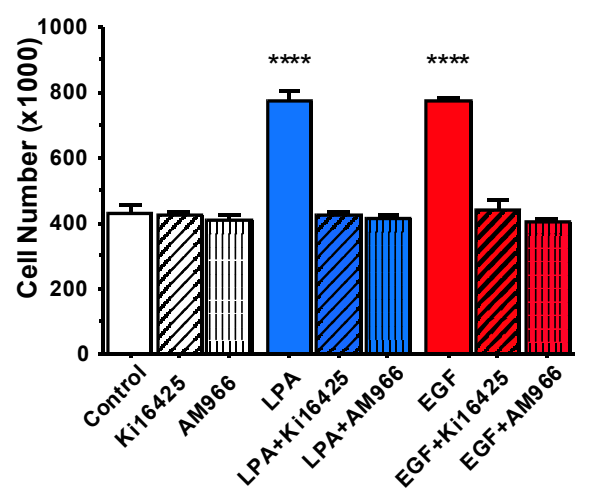

B

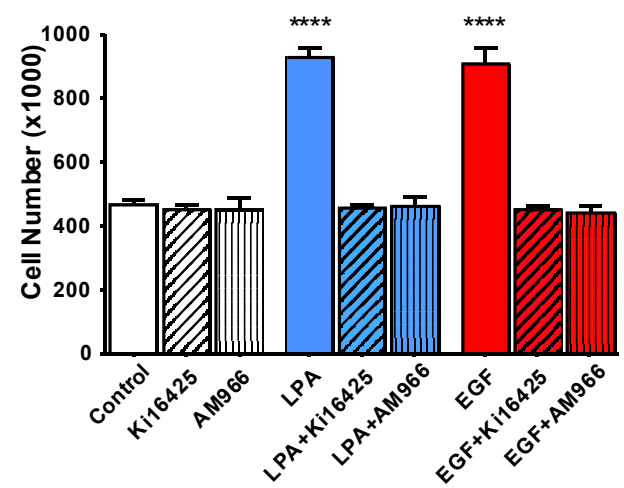

Figure 4. Effects of LPAR antagonists on breast cancer cell proliferation. Serum-starved MCF-7 (A) or MDA-MB-231 (B) cells were incubated with or without $10 \mu \mathrm{M}$ LPA or $10 \mathrm{nM}$ EGF in the absence and presence of $10 \mu \mathrm{M}$ AM966 or $10 \mu \mathrm{M}$ Ki16425. Each data point represents the mean \pm SEM $(n=4)$ of values (number of live cells per well) from two separate experiments, each performed with two separate replicate wells of cells for each experimental condition (**** $p<0.0001$ compared to control). The 48-h time is point shown; similar results were obtained at 24 and $72 \mathrm{~h}$. Data analysis was performed using two-way ANOVA, followed by Tukey's multiple comparisons test.

\subsection{Expression of Free Fatty Acid Receptors (FFARs) in Breast Cancer Cells}

We next turned to the effects of $n-3$ FAs. We examined whether FFARs are expressed in MCF-7 and MDA-MB-231 cells. Both receptors have previously been reported to be present in MCF-7 cells, based on flow cytometry [25]. Using RT-PCR, we assessed mRNA levels for FFA4 and FFA1, the two receptors for long chain free fatty acid (Figure 5A). Both PCR products were detected, although the PCR product for FFA4 was present at such low levels that it was difficult to visualize. To confirm that FFA4 was expressed, we performed immunoblotting using an antibody previously validated in our laboratory [1], and did detect FFA4 protein (Figure 5B). We were unable to validate an appropriate antibody for FFA1, but our PCR results suggest that FFA1 is likely expressed at higher levels than FFA4 in the two breast cancer cell lines. These results indicate that the roles of both FFA1 and FFA4 need to be considered in breast cancer cells. 
A

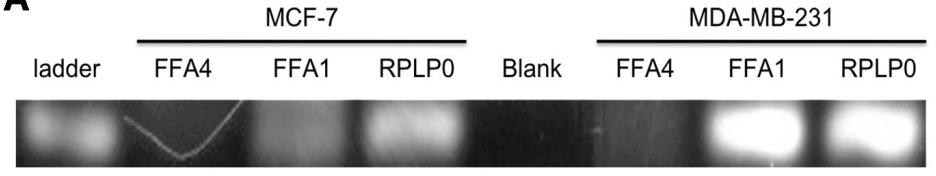

B

MCF-7 MDA

FFA4

actin

Figure 5. Expression of FFA1 and FFA4 in breast cancer cell lines. (A) RT-PCR reactions were carried out for human FFA4, FFA1, or RPLP0 (loading control) as described in Methods. Products were separated and visualized under UV light. Results shown are representative of two separate experiments, each done in triplicate. Faint signals for FFA4 were confirmed in additional experiments; (B) Whole-cell extracts, from MCF-7 and MDA-MB-231 cells growing in serum, were separated by SDS-PAGE and then immunoblotted for FFA4 and actin (loading control).

\subsection{Effects of FFAR Agonists on Breast Cancer Cell Proliferation}

We next performed a dose-response study (Figure 6) to test whether FFAR agonists inhibit proliferation of MDA-MB-231 cells. EPA, TUG-891, and GW9503 all inhibited proliferation of these cells. EPA was the least potent compound $\left(\mathrm{IC}_{50}\right.$ $403 \mathrm{nM}$ ), which was expected based on results with other cell lines [1]. The $\mathrm{IC}_{50}$ for the FFA4-selective agonist, TUG-891, was 24 nM. However, a 100-fold higher dose of TUG-891 was required to completely inhibit LPA-induced proliferation in the breast cancer cell line (Figure 6) as compared to our previously published results with Du145 prostate cancer cells [1]. In addition, the dose-response curve for TUG-891 was very shallow, suggesting the involvement of more than one receptor in the inhibitory response. While TUG-891 is selective for FFA4 over FFA1, neither TUG-891 nor GW9508 is specific for a single receptor. The FFA1-specific agonist GW9508 was 100 -fold more potent $\left(\mathrm{IC}_{50}=16 \mathrm{~nm}\right)$ in inhibiting LPA-induced proliferation in MDA-MB-231 (Figure 6) as compared to previous results in DU145 cells where FFA4 response predominates [1]. The published $\mathrm{EC}_{50}$ for $\mathrm{GW} 9508$ at FFA1 is $~ 50 \mathrm{nM}$. Thus, our results are consistent with a role for FFA1, and possibly also FFA4, in inhibiting proliferation of MDA-MB-231 cells.

We next tested the effects of $\mathrm{LPA}_{1}$ inhibitors on both LPA- and EGF-induced breast cancer cell proliferation (Figure 7). Neither the LPA antagonists AM966 and Ki16425, nor the FFAR agonists EPA, GW9508, and TUG891, had any significant effects on cell numbers on their own. However, all agents completely inhibited LPA- and EGF-induced proliferation in MCF-7 and MDA-MB-231 cells, when added individually, consistent with the results in Figures 4 and 6. Although EPA appeared to decrease cell numbers slightly below control values in the presence of LPA or EGF, the effect was not significant. There was no additional effect (e.g., cytotoxicity) on cell numbers when LPA antagonists were combined with FFAR agonists GW9508 
or TUG891 (Figure 7). Thus, both LPA antagonists and FFAR agonists can block the effects of LPA and EGF on breast cancer cell proliferation.

MDA-MB-231

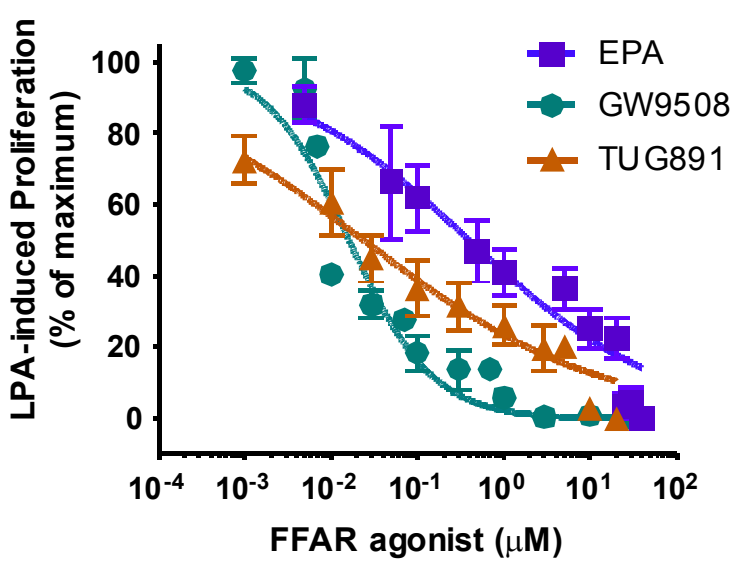

Figure 6. Dose-response for effects of FFAR agonists on breast cancer cell proliferation. Serum-starved MDA-MB-231 cells were incubated for $48 \mathrm{~h}$ with and without $10 \mu \mathrm{M}$ LPA in the absence and presence of the indicated concentrations of EPA, GW9508, and TUG-891. The number of cells achieved in response to LPA alone was defined as $100 \%$ response; the number of cells present in the absence of LPA was defined as $0 \%$ response. Each point represents mean \pm SEM $(n \geqslant 4$ for values obtained from at least two experiments, each performed with two separate replicate wells of cells for each experimental condition.

\subsection{Effects of FFAR Agonists on Breast Cancer Cell Migration}

Finally, we asked whether FFAR agonists inhibit migration of breast cancer cells. As shown in Figure 8, EPA and GW9508 both block LPA- and EGF-induced migration of MCF-7 cells. The LPAR antagonist Ki16425 has a similar effect. The combination of GW9508 and Ki16425 also yields complete inhibition; i.e., there is no additional effect of the FFAR agonist in the presence of an LPA antagonist. We conclude that either activation of FFARs, or antagonism of LPARs, can inhibit migration in response to either LPA or EGF. 

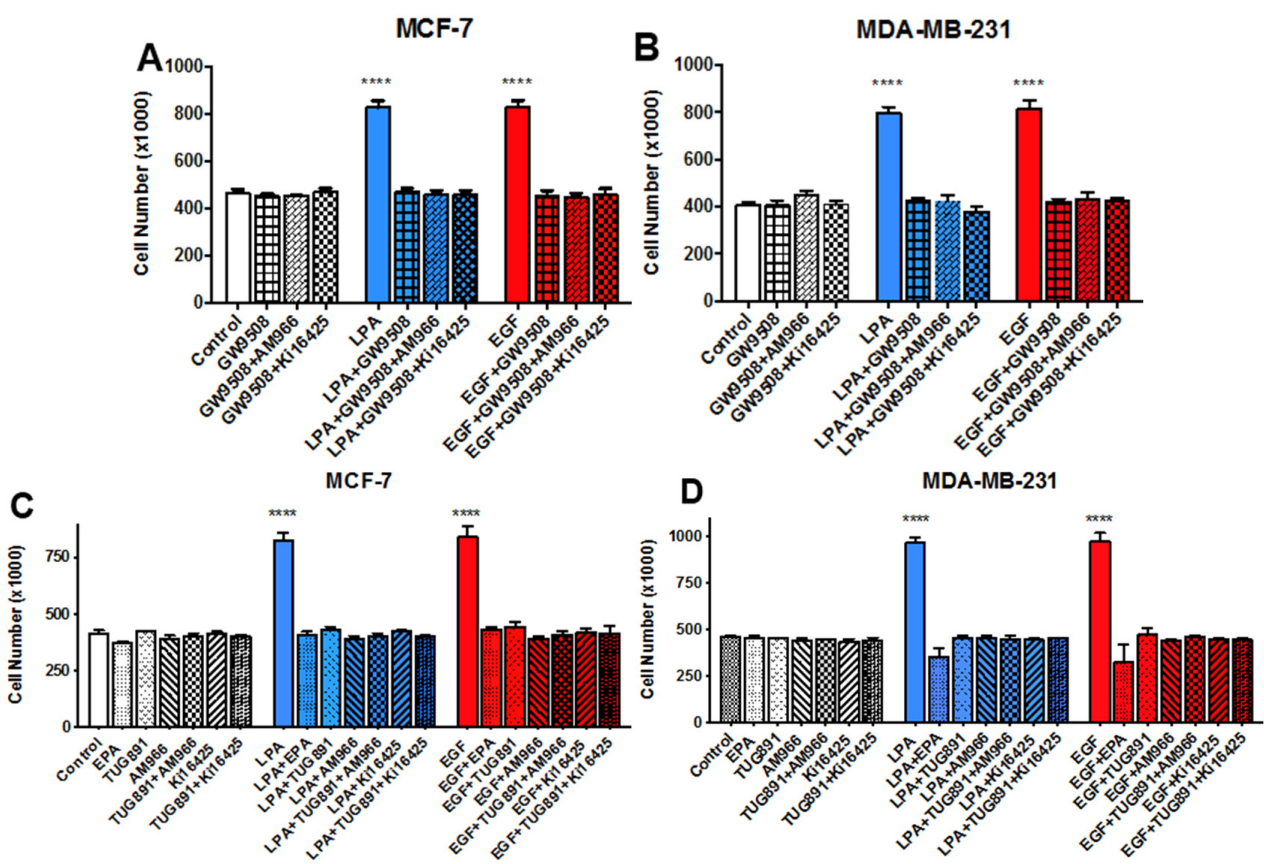

Figure 7. Effects of FFAR agonists on breast cancer cell proliferation. Serum-starved MCF-7 or MDA-MB-231 cells were incubated with or without $10 \mu \mathrm{M}$ LPA or $10 \mathrm{nM}$ EGF for $48 \mathrm{~h}$ in the absence and presence of $1 \mu \mathrm{M} \mathrm{GW} 9508,10 \mu \mathrm{M} \mathrm{Ki16425}$, and/or $10 \mu$ M AM966 (Panels A and B), or (Panels C and D), $10 \mu \mathrm{M}$ LPA or $10 \mathrm{nM}$ EGF in the absence and presence of $10 \mu \mathrm{M}$ TUG891, $10 \mu \mathrm{M}$ Ki16425, and/or $10 \mu \mathrm{M}$ AM966. In addition, Panels $C$ and D show the effects of TUG-891 as compared to that of $25 \mu \mathrm{M}$ EPA, and controls were included for the LPA antagonists alone. Some of the data points from Panels $\mathbf{C}$ and $\mathbf{D}$ were presented earlier in Figure 4, which was derived from the same series of experiments; panels (A) and (B) are from separate sets of experiments. Although only the 48-h time point is shown, similar inhibitory effects were observed at 24 and $72 \mathrm{~h}$. Each value represents the mean $\pm \operatorname{SEM}(n=4)$ of values (number of live cells per well) obtained from two separate experiments, each of which used two separate replicate wells of cells for each experimental condition $\left({ }^{* * * *} p<0.0001\right.$ compared to control). Data were analyzed done using two-way ANOVA, followed by Tukey's multiple comparisons test. 


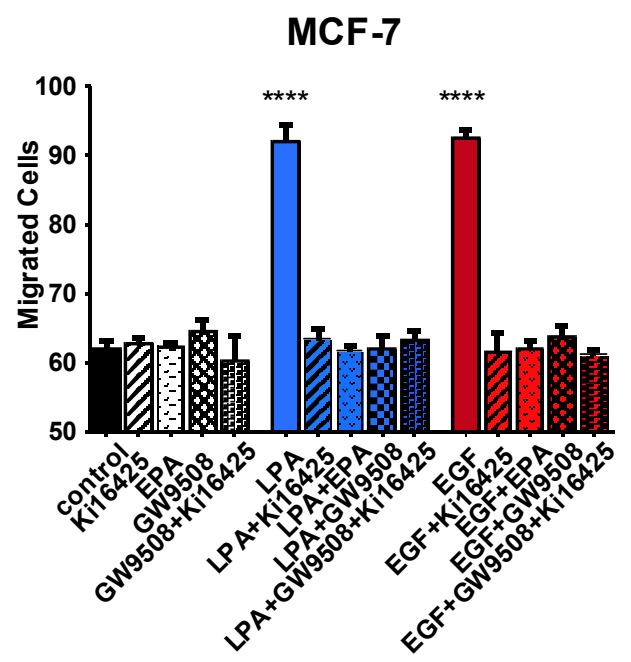

Figure 8. Effects of LPAR agonists on MCF-7 cell migration. Serum-starved MCF-7 cells were treated with $10 \mu \mathrm{M}$ Ki16425, $20 \mu \mathrm{M}$ EPA, $1 \mu \mathrm{M}$ GW9508, $10 \mu \mathrm{M}$ LPA, or $10 \mathrm{nM}$ EGF, either alone or in combination. After a 6-h migration period, cells were analyzed as described in Methods. Each bar represents the mean \pm SEM $(n=4)$ of values (total number of migrated cells/well) obtained from two separate experiments, each of which used two separate replicate wells of cells for each experimental condition ( ${ }^{* * *} p<0.0001$ vs. control). Data were analyzed using one-way ANOVA, followed by Tukey's multiple comparisons test.

\section{Conclusions}

In extending our ongoing studies of FFAR activation to breast cancer cells, the intention was to expand our knowledge of the roles of FFARs in cancer. While others have examined the effects of $n-3$ FAs on breast cancer, to our knowledge no other studies have tested the roles of FFARs in human breast cancer cells.

The current study used LPA as one of the growth factors, based on our previous work implicating LPARs in the mechanism of FFAR-mediated inhibition of cancer cell proliferation. LPA has multiple actions that support breast cancer growth, migration, invasion, and survival [37,41-44]. An LPA antagonist was shown to induce regression of breast tumors in a mouse model [45]. Others reported that either an LPA $_{1}$ antagonist or LPA 1 knockdown decreases MDA-MB-231 cell metastasis, but not primary tumor size, in mice [46]. Together, these data have established a potential role for the LPA axis as a therapeutic target in breast cancer cells, as reviewed by Panupinthu and colleagues [29]. However, the overall roles of LPA and its receptors in breast cancer cell proliferation have not been fully delineated.

FFA1/GPR40 was first reported to be present in MCF-7 cells in 2004 [24]. This was later confirmed by another group using flow cytometry [25] and also in the 
current study by PCR (Figure 5). Soto-Guzman and colleagues also detected FFA4 expression in MCF-7 cells [25]. Our data demonstrate expression of FFA1 and FFA4 mRNA, and FFAR protein, in both MCF-7 and MDA-MB-231 cells. Interestingly, the results of dose-response studies using two selective FFAR agonists were consistent with a major role for FFA1 in MDA-MB-231 cells (Figure 6). While these results do not exclude a role for FFA4, this is the first demonstration in our lab that FFA1 and FFA4 may similarly mediate inhibition of cancer cell proliferation.

Figures 4 and 7 demonstrate that LPAR inhibition not only impedes LPA-induced proliferation, but also EGF-induced proliferation. This result suggests that, in MCF-7 and MDA-MB-231 cells, EGFR is reliant on one or more LPARs. This result is similar to those obtained with prostate cancer cells [1,40]. LPARs have been described as "masters" of EGFR signaling [47], as confirmed by studies in various cell types $[1,48-53]$. Further studies will be required to determine whether FFA1 or FFA4 act directly on both LPARs and EGFR to result in inhibition, or indirectly inhibit EGFR via effects on LPARs.

FFAR activation through EPA, GW9508, or TUG-891 abolishes LPA- and EGF-induced proliferation and migration in MCF-7 and MDA-MB-231 cells (Figures 6-8). GW9508 yields a classical dose-response curve with an $\mathrm{IC}_{50}$ consistent with action at FFA1. At the higher dose of TUG-891 used to achieve complete inhibition in breast cancer cells $(10 \mu \mathrm{M})$, it is plausible that it is activating FFA1 in addition to, FFA4. Our results are consistent with other reports of inhibitory effects of $n-3$ FAs in breast cancer cells. In one study, low doses of $n-3$ FAs were shown to inhibit proliferation of MCF-7 cells, while high doses induced apoptosis [20]. This is consistent with our dose-response results for MDA-MB-231 cells, where inhibition of proliferation was achieved without cytotoxicity (Figure 6). The EPA concentration $(25 \mu \mathrm{M})$ used in subsequent experiments was chosen as the dose that maximally inhibited proliferation but did not decrease viability, since our focus was on inhibition of growth factor response rather than toxic responses that may occur at high doses.

Although our results suggest that both FFAR agonists and LPAR antagonists inhibit proliferation in MCF-7 and MDA-MB-231 cells, more work is needed to fully elucidate the mechanism of action. It remains to be definitively determined which FFAR is responsible for the inhibition in breast cancer cells. It is also unclear at this point whether FFAR activation directly influences EGF-mediated signaling, or whether FFARs act indirectly via interference with LPAR activity as appears to be the case in prostate cancer cells [40]. Since the synthetic FFAR agonists used in the current study are not metabolized to resolvins, and are designed to act selectively at GPCRs, utilization of these agonist drugs is helpful in distinguishing receptor-mediated effects from effects of $n-3$ FAs on lipid metabolism. In addition, the emerging small-molecule FFAR agonists have therapeutic potential for the prevention and/or treatment of breast cancer. In summary, the results presented herein demonstrate 
for the first time that FFAR activation results in inhibition of breast cancer cell proliferation and migration. The inhibitory response mediated by FFARs needs to be taken into account when considering effects of $n-3$ fatty acids on breast cancer cells.

Acknowledgments: This work was supported by the College of Pharmacy at Washington State University. Mandi Hopkins, a member of the Graduate Program in Pharmaceutical Sciences in the College of Pharmacy, also received support from the NIH Protein Biotechnology Training Program at WSU [T32 GM008336]. Zhihong Zhang received support from NIH 1K05AA017149 (G. Meadows, principal investigator). The authors thank Renae Hamilton and Colin Kennedy for assistance in early phases of this project.

Author Contributions: Mandi Hopkins: wrote manuscript, designed and performed experiments; Zhihong Zhang: designed and performed experiments; Ze Liu: performed experiments; Kathryn Meier: edited manuscript, supervised the project.

Conflicts of Interest: The authors declare no conflict of interest.

\section{References}

1. Liu, Z.; Hopkins, M.M.; Zhang, Z.; Quisenberry, C.B.; Fix, L.C.; Galvan, B.M.; Meier, K.E. $\omega$-3 Fatty acids and other FFA4 agonists inhibit growth factor signaling in human prostate cancer cells. J. Pharm. Exp. Ther. 2015, 352, 1-15.

2. Hopkins, M.M.; Meier, K.E. $\omega-3$ Fatty acids and their impact on prostate cancer risk. Curr. Nutr. Rep. 2015. in press.

3. Hardman, W.E. $\omega-3$ Fatty acids to augment cancer therapy. J. Nutr. 2002, 132, 3509S-3512S.

4. Zheng, J.S.; Hu, X.J.; Zao, Y.M.; Yang, J.; Li, D. Intake of fish and marine n-3 polyunsaturated fatty acids and risk of breast cancer: Meta-analysis of data from 21 Independent Prospective Cohort Studies. Br. Med. J. 2013, 346, f3706.

5. Schley, P.D.; Jijon, H.B.; Robinson, L.E.; Field, C.J. Mechanisms of $\omega-3$ fatty acid-induced growth inhibition in MDA-MB-231 human breast cancer cells. Breast Cancer Res. Treat. 2005, 92, 187-195.

6. Pogash, T.J.; el-Bayoumy, K.; Amin, S.; Gowda, K.; de Cicco, R.L.; Barton, M.; Su, Y.; Russo, I.H.; Himmelberger, J.A.; Slifker, M.; et al. Oxidized derivative of docosahexaenoic acid preferentially inhibit cell proliferation in triple negative over luminal breas cancer cells. Vitro Cell Dev. Biol. Anim. 2015, 52, 121-127.

7. Karmali, R.A.; Marsh, J.; Ruchs, C. Effect of $\omega-3$ fatty acids on growth of a rat mammary tumor. J. Natl. Cancer Inst. 1984, 73, 457-461.

8. Rose, D.P.; Connolly, J.M. Effects of dietary $\omega-3$ fatty acids on human breast cancer growth and metastases in nude mice. J. Natl. Caner Inst. 1993, 85, 1743-1747.

9. Sauer, L.A.; Dauchy, R.T.; Blask, D.E.; Krause, J.A.; Davidson, L.K.; Dauchy, E.M. Eicosapenaenoic acid suppresses cell proliferation in MCF-7 human breast cancer xenografts in nude rats via a pertussis toxin-sensitive signal transduction pathway. J. Nutr. 2005, 135, 2124-2129. 
10. Jiang, W.; Zhu, Z.; McGinley, J.N.; el Bayouny, K.; Manni, A.; Thompson, J.H. Identification of a molecular signature underlying inhibition of mammary carcinoma growth by dietary $n-3$ fatty acids. Cancer Res. 2012, 72, 3795-3806.

11. Dwivedi, S.; Patel, S.; Jain, K. The what, where, and how of resolvins. AsPac J. Mol. Biol. Biotechnol. 2010, 20, 45-54.

12. Zhang, M.J.; Spite, M. Resolvins: Anti-inflammatory and pro-resolving mediators derived from $\omega-3$ polyunsaturated fatty acids. Annu. Rev. Nutr. 2012, 32, 203-227.

13. Janakiram, N.B.; Mohammed, A.; Rao, C.V. Role of lipoxins, resolvins, and other bioactive lipids in colon and pancreatic cancer. Cancer Metastasis Rev. 2011, 30, 507-523.

14. Al-Zaubai, N.; Johnstone, C.N.; Leong, M.M.; Li, J.; Rizzacasa, M.; Stewart, A.G. Resolvin D2 supports MCF-7 cell proliferation via activation of estrogen receptor. J. Pharmacol. Exp. Ther. 2014, 351, 172-180.

15. Oh, D.Y.; Talukdar, S.; Bae, E.J.; Imamura, T.; Morinaga, H.; Fan, W.Q.; Li, P.; Wendell, J.L.; Watkins, S.M.; Olefsky, J.M. GRP120 is an $\omega-3$ fatty acid receptor mediating potent anti-inflammatory and insulin-sensitizing effects. Cell 2012, 143, 687-698.

16. Hara, T.; Hirasawa, A.; Ichimura, A.; Kimura, I.; Tsujimoto, G. Free fatty acid receptors FFAR1 and GPR120 as novel therapeutic targets for metabolic disorders. J. Pharm. Sci. 2011, 100, 3594-3601.

17. Holliday, N.D.; Watson, S.J.; Brown, A.J.H. Drug discovery opportunities and challenges at $G$ protein coupled receptors for long chain free fatty acids. Front. Endocrinol. 2011, 2, $1-12$.

18. Dranse, H.J.; Kelly, M.E.; Hudson, B.D. Drugs or diet? Developing novel therapeutic strategies targeting the free fatty acid family of GPCRs. Br. J. Pharmacol. 2013, 170, 695-711.

19. Hudson, B.D.; Shimpukade, B.; Mackenzie, A.E.; Butcher, A.J.; Pediani, J.D.; Christiansen, E.; Heathcote, H.; Tobin, A.B.; Ulven, T.; Milligan, G. The pharmacology of TUG-891, a potent and selective agonist of the free fatty acid receptor 4 (FFA4/GPR120), demonstrates both potential opportunity and possible challenges to therapeutic agonism. Mol. Pharmacol. 2013, 84, 710-725.

20. Wu, M.; Harvey, K.A.; Ruzmetov, N.; Welch, Z.R.; Sech, L.; Jackson, K.; Stillwell, W.; Zaloga, G.P. Siddiqui RA (2005) $\omega-3$ polyunsaturated fatty acids attenuate breast cancer growth through activation of a neutral sphingomyelinase-mediated pathway. Int. J. Cancer 2015, 117, 340-348.

21. Bernard-Gallon, D.J.; Vissac-Sabatier, C.; Antoine-Vincent, D.; Rio, P.G.; Maruizis, J.C.; Fustier, P.; Bignon, Y.J. Differential effects of $n-3$ and $n-6$ polyunsaturated fatty acids on BRCA1 and BRCA2 gene expression in breast cell lines. Br. J. Nutr. 2002, 87, 281-289.

22. Ghosh-Choudhury, T.; Mandall, C.C.; Woodruff, K.; St. Clair, P.; Fernandes, G.; Chouhury, G.; Ghosh-Choudhury, N. Fish oil targets PTEN to regulate NFkB for downregulation of anti-apoptotic genes in breast tumor growth. Breast Cancer Res. Treat. 2009, 118, 213-228. 
23. Monk, J.M.; Turk, H.F.; Liddle, D.M.; de Boer, A.A.; Power, K.A.; Ma, D.W.; Robinson, L.E. $n-3$ Polyunsaturated fatty acids and mechanisms to mitigate inflammatory paracrine signaling in obesity-Associated breast cancer. Nutrients 2014, 5, 4760-4793.

24. Yonezawa, T.; Katoh, K.; Obara, Y. Existence of GPR40 functioning in a human breast cancer cell line, MCF-7. Biochem. Biophys. Res. Commun. 2004, 314, 805-809.

25. Soto-Guzman, A.; Tobledo, T.; Lopez-Perez, M.; Salazar, E.P. Oleic acid induces ERK1/2 activation and AP-1 DNA binding activity through a mechanism involving Src kinase and EGFR transactivation in breast cancer cells. Mol. Cell. Endocrinol. 2008, 294, 81-91.

26. Navarro-Tito, N.; Robledo, T.; Salazar, E.P. Arachidonic acid promotes FAK activation and migration in MDA-MB-231 breast cancer cells. Exp. Cell. Res. 2008, 314, 3340-3355.

27. Chung, H.; Lee, Y.S.; Mayoral, R.; Oh, D.Y.; Webster, N.J.; Sears, D.D.; Olefsky, J.M.; Ellies, L.G. $\omega-3$ Fatty acids reduce obesity-induced tumor progression independent of GPR120 in a mouse model of postmenopausal breast cancer. Oncogene 2014, 34, 3504-3513.

28. Zhang, Z.; Knoepp, S.M.; Sansbury, H.M.; Han, S.; Ku, H.; Xie, Y.; Hallman, M.; Meier, K.E. Differential expression of FAK and Pyk2 in phorbol ester-sensitive and -resistant EL4 thymoma cells. Clin. Expt. Metastasis 2011, 28, 551-565.

29. Papupinthu, N.; Lee, H.Y.; Mills, G.B. Lysophosphatidic acid production and action: Critical new players in breast cancer initiation and progression. Br. J. Cancer 2010, 102, 941-946.

30. Masuda, H.; Zhang, D.; Bartholomeusz, C.; Doihara, H.; Hortobagyi, G.N.; Ueno, N.T. Role of epidermal growth factor receptor in breast cancer. Breast Cancer Res. Treat. 2012, 136, 331-345.

31. O'Kelly, J.; Chung, A.; Lemp, N.; Chumakova, K.; Yin, D.; Wang, H.J.; Said, J.; Gui, D.; Miller, C.W.; Karlan, B.Y.; et al. Functional domains of CCN1 (Cyr61) regulate breast cancer progression. Int. J. Oncol. 2008, 33, 59-67.

32. Jun, J.I.; Lau, L.F. Taking aim at the extracellular matrix: CCN proteins as emerging therapeutic targets. Nature Rev. Drug Discov. 2011, 10, 945-963.

33. Boucharaba, A.; Serre, C.M.; Gres, S.; Saulnier-Blache, J.S.; Bordet, J.C.; Guglielmi, J.; Clezardin, P.; Peyruchaud, O. Platelet-derived lysophosphatidic acid supports the progression of osteolytic bone metastases in breast cancer. J. Clin. Invest. 2004, 114, 1714-1725.

34. Chen, M.; Towers, L.N.; O'Connor, K.L. LPA2 (EDG4) mediates Rho-dependent chemotaxis with lower efficacy than LPA1 (EDG2) in breast carcinoma cells. Am. J. Physiol. Cell. Physiol. 2007, 292, C1947-C1933.

35. Li, T.T.; Alemayehu, M.; Aziziyeh, A.; Pape, C.; Pampilio, M.; Postovit, L.M.; Mills, G.B.; Babway, A.V.; Bhattacharya, M. Beta-arrestin/Ral signaling regulates lysophosphatidic acid-mediated migration and invasion of human breast tumor cells. Mol. Cancer Res. 2009, 7, 1064-1077. 
36. Swaney, J.S.; Chapman, C.; Correa, L.D.; Stebbins, K.J.; Bundey, R.A.; Prodanovich, P.C.; Fagan, P.; Baccei, C.S.; Santini, A.M.; Hutchinson, J.H.; et al. A novel, orally active LPA1 receptor antagonist inhibits lung fibrosis in the mouse bleomycin model. Br. J. Pharmacol. 2010, 160, 1699-1713.

37. Alemayehu, M.; Dragan, M.; Pape, C.; Siddiqui, I.; Sacks, D.B.; di Guglielmo, G.M.; Babway, A.V.; Bhattacharya, M. $\beta$-Arrestin2 regulates lysophosphatidic acid-induced human breast tumor cell migration and invasion via Rap1 and IQGAP1. PLoS ONE 2013, 8, e56174.

38. Sun, K.; Cai, H.; Duan, X.; Yang, Y.; Li, M.; Wu, J.; Zhang, X.; Wang, J. Aberrant expression and potential therapeutic target of lysophosphatidic acid receptor 3 in triple-negative breast cancers. Clin. Exp. Med. 2014.

39. Ohta, H.; Sata, K.; Murata, N.; Damirin, A.; Malchinkhuu, E.; Kon, J.; Kimura, T.; Tobo, M.; Yamazaki, Y.; Watanabe, T.; et al. Ki16425, a subtype-selective antagonist for EDG-family lysophosphatidic acid receptors. Mol. Pharmacol. 2003, 64, 994-1005.

40. Hopkins, M.M.; Liu, Z.; Meier, K.E. Cross-talk between lysophosphatidic acid receptor a, free fatty acid receptor 4 , and epidermal growth factor receptor in human prostate cancer cells. 2015, Submitted.

41. Boucharaba, A.; Guillet, B.; Menaa, F.; Hneino, M.; van Wijnen, A.J.; Clezardin, P.; Peyruchaud, O. Bioactive lipids lysophosphatidic acid and sphingosine 1-phosphate mediate breast cancer cell biological functions through distinct mechanisms. Oncol. Res. 2009, 18, 173-184.

42. Du, S.; Sun, C.; Hu, Z.; Yang, Y.; Zhu, Y.; Zheng, D.; Gu, L.; Lu, X. Lysophosphatidic acid induces MDA-MB-231 breast cancer cells migration through activation of PI3K/PAK1/ERK signaling. PLoS ONE 2010, 5, e15940.

43. Swamydas, M.; Nguyen, D.; Allen, L.D.; Eddy, J.; Dreau, D. Progranulin stimulated by LPA promotes the migration of aggressive breast cancer cells. Cell. Commun. Adhes. 2010, 18, 119-130.

44. Sun, K.; Duan, X.; Cai, H.; Liu, X.; Yang, Y.; Li, M.; Zhang, X.; Wang, J. Curcumin inhibits LPA-induced invasion by attenuating RhoA/ROCK/MMPs pathway in MCF7 breast cancer cells. Clin. Exp. Med. 2015, 1-11.

45. Zhang, H.; Xu, X.; Gajewiak, J.; Tsukahara, R.; Fujiwara, Y.; Liu, J.; Fells, J.I.; Perygin, D.; Parrill, A.L.; Tigyi, G.; et al. Dual activity lysophosphatidic acid receptor pan-antagonist/autotaxin inhibitor reduces breast cancer cell migration in vitro and causes tumor regression. Cancer Res. 2009, 69, 5441-5449.

46. Liu, S.; Umezu-Goto, M.; Murph, M.; Lu, Y.; Liu, W.; Zhang, F.; Yu, S.; Stephens, L.C.; Cui, X.; Murrow, G.; et al. Expression of autotaxin and lysophosphatidic acid receptors increases mammary tumorigenesis, invasion, and metastases. Cancer Cell. 2009, 15, 539-550.

47. Daaka, Y. Mitogenic action of LPA in prostate. Biochim. Biophys. Acta. 2002, 1582, 265-269. 
48. Marshall, J.C.A.; Collins, J.W.; Nakayama, J.; Horak, C.E.; Liewehr, D.J.; Steinberg, S.M.; Albaugh, M.; Vidal-Vanaclocha, F.; Palmieri, D.; Marbier, M.; et al. Effects of inhibition of the lysophosphatidic acid receptor 1 on metastasis and metastatic dormancy in breast cancer. J. Natl. Cancer Inst. 2012, 104, 1306-1319.

49. Kue, P.F.; Taub, J.S.; Harrington, L.B.; Polakiewicz, R.D.; Ullrich, A.; Daaka, Y. Lysophosphatidic acid-regulated mitogenic ERK signaling in androgen-insensitive prostate cancer PC-3 cells. Int. J. Cancer 2002, 102, 572-579.

50. Bektas, M.; Payne, S.G.; Liu, H.; Goparaju, S.; Milstien, S.; Spiegel, S. A novel acylglycerol kinase that produces lysophophatidic modulates cross talk with EGFR in prostate cancer cells. J. Cell. Biol. 2005, 169, 801-811.

51. Snider, A.J.; Zhang, Z.; Xie, Y.; Meier, K.E. Epidermal growth factor increases lysophosphatidic acid production in human ovarian cancer cells: Roles for phospholipase D2 and receptor transactivation. Am. J. Physiol. Cell. Physiol. 2009, 298, C163-C170.

52. Mausbacher, N.; Schreiber, T.B.; Daub, H. Glycoprotein capture and quantitative phosphoproteomics indicate coordinated regulation of cell migration upon lysophosphatidic acid stimulation. Mol. Cell. Proteom. 2010, 9, 2337-2353.

53. Colin-Santana, C.C.; Avendano-Vazquez, S.E.; Alcantara-Hernandez, R.; Garcia-Sainz, J.A. EGF and angiotensin II modulate lysophosphatidic acid $\mathrm{LPA}_{1}$ receptor function and phosphorylation state. Biochim. Biophys. Acta 2011, 1810, 1170-1177. 


\title{
Potential Benefits of Omega-3 Fatty Acids in Non-Melanoma Skin Cancer
}

\author{
Homer S. Black and Lesley E. Rhodes
}

\begin{abstract}
Considerable circumstantial evidence has accrued from both experimental animal and human clinical studies that support a role for omega-3 fatty acids (FA) in the prevention of non-melanoma skin cancer (NMSC). Direct evidence from animal studies has shown that omega-3 FA inhibit ultraviolet radiation (UVR) induced carcinogenic expression. In contrast, increasing levels of dietary omega-6 FA increase UVR carcinogenic expression, with respect to a shorter tumor latent period and increased tumor multiplicity. Both omega- 6 and omega-3 FA are essential FA, necessary for normal growth and maintenance of health and although these two classes of FA exhibit only minor structural differences, these differences cause them to act significantly differently in the body. Omega- 6 and omega-3 FA, metabolized through the lipoxygenase (LOX) and cyclooxygenase (COX) pathways, lead to differential metabolites that are influential in inflammatory and immune responses involved in carcinogenesis. Clinical studies have shown that omega-3 FA ingestion protects against UVR-induced genotoxicity, raises the UVR-mediated erythema threshold, reduces the level of pro-inflammatory and immunosuppressive prostaglandin E2 $\left(\mathrm{PGE}_{2}\right)$ in UVR-irradiated human skin, and appears to protect human skin from UVR-induced immune-suppression. Thus, there is considerable evidence that omega-3 FA supplementation might be beneficial in reducing the occurrence of NMSC, especially in those individuals who are at highest risk.
\end{abstract}

Reprinted from J. Clin. Med. Cite as: Black, H.S.; Rhodes, L.E. Potential Benefits of Omega-3 Fatty Acids in Non-Melanoma Skin Cancer. J. Clin. Med. 2016, 5, 23.

\section{Introduction}

Considerable interest has been focused on the potential health benefits of omega-3 fatty acids (FA) on a range of human diseases. This interest arose from a series of reports in which high dietary intake of these unsaturated FA among Greenlandic West Coast Eskimos was specifically associated with low incidence of ischemic heart disease, and inflammatory symptoms, in general [1-3]. Whereas the major focus has been on cardiovascular disease [4-6], studies have been extended to type II diabetes and the metabolic syndrome, inflammatory bowel disease, rheumatoid arthritis, renal disease, systemic lupus erythematosus, and osteoporosis [7,8].

Dietary lipids have also been implicated in the development of several kinds of cancer, e.g., breast, lung, bowel, bladder, pancreatic, and prostate [9-12]. Whereas, 
omega-3 FA have generally shown positive effects on cardiovascular disease, studies are equivocal for human cancers $[13,14]$. Among 43 risk ratios calculated across 19 cohorts for 11 different types of cancer and 5 different ways to assess omega- 3 FA consumption, only four were significant, and it was concluded that omega-3 FA do not reduce overall cancer risk [14]. A systematic review involving 20 cohorts and using up to 6 different ways to categorize omega-3 FA consumption similarly arrived at the conclusion that, overall, there was not a significant association between omega-3 FA and cancer incidence and that dietary supplementation was unlikely to prevent cancer [15].

Although women with high intake ratios of marine omega-3 FA, relative to omega- 6 FA have been found to have a reduced risk of breast cancer, not all case-control and cohort studies are in agreement [16]. In the first meta-analysis previously referenced [14], five estimates of risk for breast cancer did not show a significant association, and no effects were found for cancers of the aero-digestive tract, bladder, colorectum, ovary, pancreas or stomach, or for lymphoma. While a recent study observed that high levels of serum phospholipid omega-3 FA (a biomarker) were associated with a large increase in the risk of high-grade prostate cancer [17], subsequent systematic review and meta-analysis, including 12 studies of self-reported dietary intake of omega-3 FA and 9 biomarker studies, failed to find an association between omega-3 FA and prostate cancer [18]. These ambiguities require clarification and undoubtedly will require randomized, double-blinded intervention trials.

Inflammatory processes are involved in initiation, promotion, and progression stages of cancer and herein rests the rationale upon which omega-3 FA might be expected to reduce cancer risk [19]. In this regard, there has accrued a considerable body of evidence, albeit circumstantial at this point, that omega-3 FA could reduce cancer risk for the most common of cancers, i.e., skin cancer. The American Cancer Society [20] estimates that over 3.5 million cases of skin cancer will occur this year in the United States alone. The evidence to support a beneficial outcome for omega-3 FA supplementation on non-melanoma skin cancer (NMSC) is presented herein.

\section{Essential Fatty Acids}

Linoleic acid (LA) and $\alpha$-linolenic acid (ALA) cannot be synthesized by humans and are, thus, considered essential and must be supplied in the diet. These essential fatty acids (EFA) are the precursors of the omega- 6 and omega- 3 series of FA, respectively. These FA are often abbreviated by their chemical designation, e.g., LA is $18: 2 n-6$ where 18 indicated the length of the carbon chain, the 2 represents the number of double bonds and the $n-6$ indicates that the first of the double bonds begins at the sixth carbon atom from the methyl end of the carbon chain. ALA is abbreviated as $18: 3 n-3$, the $n-3$ signifies that the first double bond is at the third 
carbon from the methyl end of the chain. Longer chain polyunsaturated FA (PUFA) can be synthesized in humans from their respective precursor EFA (LA or ALA) through a series of elongation (addition of two carbon atoms) and desaturation (addition of a double bond) enzymatic reactions. The two series of EFA cannot be inter-converted in humans and thus compete for these enzymes. Because Western diets may contain 15-20 times more LA than ALA, greater levels of long- chain omega-6 FA (Arachidonic acid, 20:4n-6) result. Consequently under certain dietary conditions supplementation with EPA (Eicosapentaenoic acid, 20:5n-3) and DHA (Docosahexaenoic acid, 22:6n-3) may be essential for maintenance of good health.

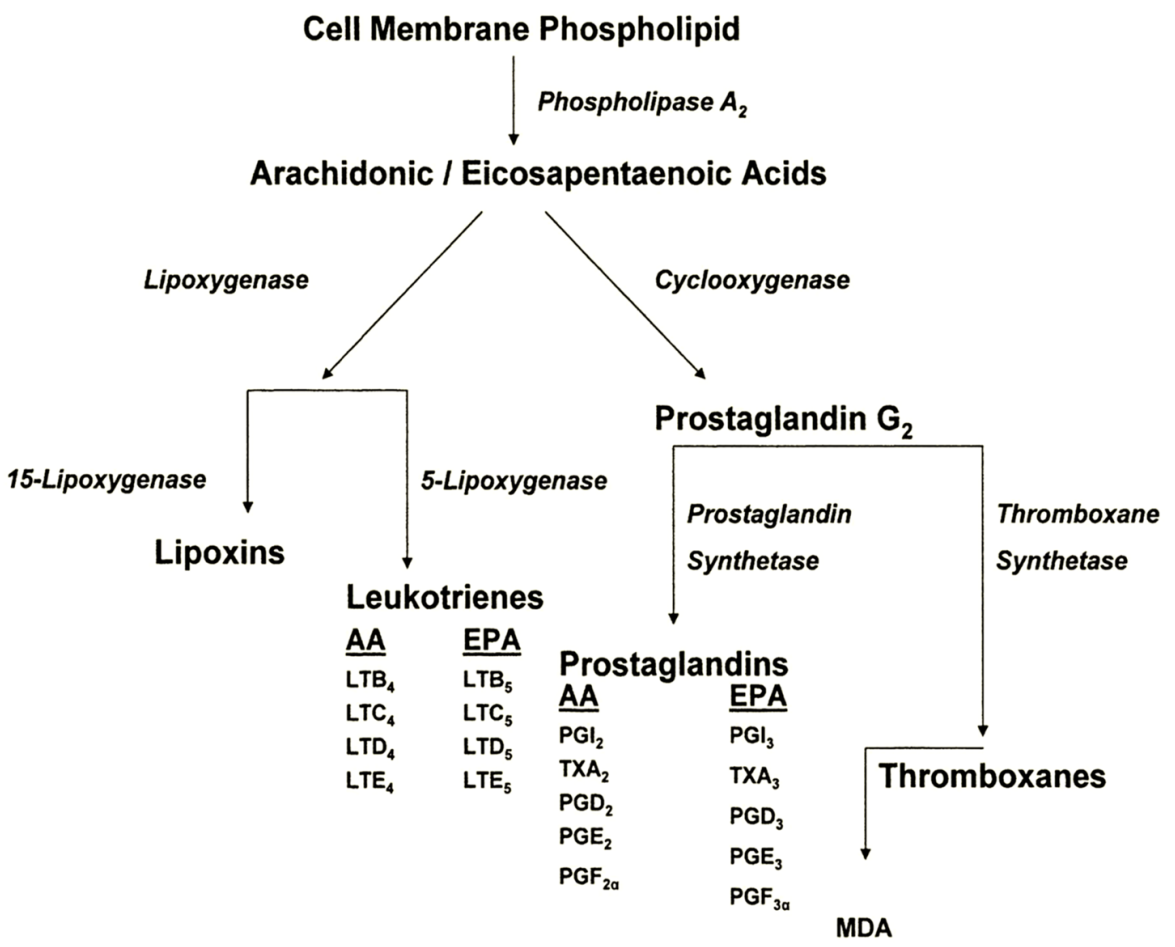

Figure 1. Differential eicosanoid metabolism from omega-6 and omega-3 FA sources. Arachidonic acid, 20:4n-6 (AA), is metabolized via lipoxygenase and cyclooxygenase pathways. Eicosapentaenoic acid, 20:5n-3 (EPA) acts as a competitive inhibitor to the cyclooxygenase enzyme complex with AA and produces different leukotriene and prostaglandin oxidation products. Malondialdehyde (MDA) is a product of prostaglandin and thromboxane metabolism and is commonly used as a measure of lipid peroxidation.

Not only do these two series of EFA compete for elongase and desaturase enzymes, but they also compete with the cyclooxygenase (COX) and lipoxygenase 
(LOX) enzymes and differentially influence the flux of metabolites through these pathways. These oxidative metabolites differ in hormonal potency. The omega- 6 FA derived products are more active than their omega-3 FA counterparts. Some of these metabolites are known to influence tumor biology. $\mathrm{PGE}_{2}$, derived from omega-6 FA oxidation via the COX pathway, acts as a tumor promoter and has been associated with aggressive tumor growth patterns in both basal cell carcinoma (BCC) and squamous cell carcinoma (SCC) in humans [21]. On the other hand, omega-3 FA compete with omega-6 FA for binding sites on COX and inhibit the production of $\mathrm{PGE}_{2}$, resulting in higher levels of the less potent PGE3. Omega-3 FA may also shunt potential PG precursors through the LOX pathway, resulting in products that inhibit tumor growth and in products that are involved in immune surveillance [21-23]. A simplified schema of eicosanoid metabolism is illustrated in Figure 1. Thus, the COX and LOX pathways, with their bioactive intermediates, provide a strong rational foundation for the cancer preventive potential of omega-3 FA [24].

\section{Evidence for Participation of Dietary PUFA in UVR-Induced Skin Cancer}

\subsection{Animal Studies}

The first report that dietary fat could potentiate UVR-carcinogenesis came in 1939 [25]. With the advent of World War II, this avenue of research lay fallow

for nearly 45 years until this lead was followed-up in a series of studies that demonstrated that an approximate linear relationship occurred between PUFA (dietary corn oil that contained roughly 50\% omega-6 FA) and UVR-carcinogenic expression [26,27]. Increasing dietary levels of omega-6 FA shortened the tumor latent period and increased tumor multiplicity. Partial hydrogenation of the PUFA resulted in a marked inhibition of carcinogenesis [26]. Reeve et al. [28] found that feeding a totally hydrogenated PUFA completely abolished UVR-carcinogenic expression while those animals fed the normal PUFA exhibited $100 \%$ tumor incidence. Furthermore, when the diet of animals fed the hydrogenated fat was reconstituted with a normal mixed fat, large numbers of tumors rapidly appeared. The authors suggested that UVR initiation of tumors had not been prevented by lack of PUFA, but that the EFA deficiency held the appearance of tumors in abeyance, probably at the promotion stage of carcinogenesis. Confirmation that omega-6 FA exerted their influence principally at the post-initiation stage of carcinogenesis came from cross-over feeding studies [29]. Animals were placed on a defined, isocaloric diet containing high $(12 \% w / w)$ and low $(0.75 \%, w / w)$ levels of corn oil. At completion of a regimen of UVR, and before tumors appeared, some diets were crossed to the contravening diet, e.g., high to low fat and low to high fat. Incidence curves and tumor multiplicity analysis provided confirmation that diets containing high levels of omega-6 FA enhanced UVR-carcinogenic expression and that enhancement occurred during the 
post-initiation, or promotion/progression, stages of carcinogenesis. Importantly, crossing from a high fat to a low fat diet after a cancer causing dose of UVR had already been administered, negated the exacerbating influence of high fat diets. This finding provided the rationale upon which a low-fat dietary intervention might act to ameliorate cancer expression.

Contrary to the tumor promoting effects of omega-6 FA, animals fed a diet containing menhaden oil as lipid source exhibited a marked inhibition of UVR-carcinogenic expression [30]. Menhaden oil is rich in omega-3 FA. Unlike omega-6 FA, cross-over feeding studies indicated that omega-3 FA exert their principal anti-cancer effects during the initiation stage of carcinogenesis. Animals fed with the omega-3 FA diet throughout the study exhibited an increased tumor latent period and decreased tumor multiplicity compared to animals receiving an equivalent level of corn oil (rich in omega-6 FA).

As noted previously, omega-3 FA compete with omega-6 FA for active sites on COX, a major enzyme in the eicosanoid cascade [24,31]. As such, the level of pro-inflammatory and immune modulating omega- 6 FA metabolites is reduced. As dietary omega-6 FA increase, the plasma $\mathrm{PGE}_{2}$ level increases. Omega-3 FA intake reduces $\mathrm{PGE}_{2}$ levels approximately 7 -fold in comparison to an equivalent level of omega-6 FA [32]. These data support the thesis that omega- 6 and omega-3 PUFA differentially influence not only $\mathrm{PGE}_{2}$ levels, but other pro-inflammatory and immune-modulating intermediates of the COX and LOX pathways.

Supporting evidence for a role of omega-3 FA in carcinogenesis has recently been acquired from studies with a transgenic mouse model designated fat-1 [33]. The fat- 1 transgenic mice are capable of producing omega-3 FA from omega-6 FA, i.e., the transgenic has received a gene encoding an omega-3 FA desaturase that converts omega-6 FA to omega-3 FA. This results in abundant omega-3 FA and reduced omega-6 FA in the animals' tissues without the need for omega-3 FA supplementation and eliminates many of the confounding variables encountered in dietary studies. With regard to skin tumorigenesis, $X i a$ et al. [34] showed that there was a dramatic reduction of melanoma formation and growth when fat-1 mice were injected with B16 melanoma cells, compared to their non-transgenic littermates. The levels of omega-3 FA and metabolite $\mathrm{PGE}_{3}$ were much higher in the transgenic animals and the omega-6/omega-3 FA ratio much lower. This transgenic model should be invaluable in future studies to elucidate the role and mechanism(s) of effects of omega-3 FA in NMSC.

As alluded to earlier, previous studies had indicated that carcinogenesis might be modulated immunologically and that this influence might occur at the promotion stage [32]. Notably, the systemic alteration induced by UVR that suppresses an animal's ability to reject highly antigenic UVR-induced allergens occurs during the chemical promotion stage of carcinogenesis [35]. Reeve et al. [28] had already shown 
that feeding mice an EFA (omega-6 FA) deficient diet inhibited the appearance of UVR-induced tumors and suggested that this inhibition might be due to the lack of eicosanoid precursors that, in turn, might prevent UVR induction of the immune state. In the case of omega- 6 FA, this would be deficient levels of $\mathrm{PGE}_{2}$, the gate-keeper for other eicosanoids. This would account for protection observed from UVR-initiated tumor outgrowth. Chung et al. [36] had shown that T-cell function was $\mathrm{PGE}_{2}$ dependent and that UVR-induced suppression of contact hypersensitivity (CHS) was abrogated by treatment with an inhibitor of PG synthesis.

It was subsequently shown that plasma $\mathrm{PGE}_{2}$ levels were directly related to omega-6 FA dietary intake, i.e., the highest level of $\mathrm{PGE}_{2}$ occurring with the highest level of omega-6 FA intake [32]. This, in turn, induced the greatest exacerbation of UVR carcinogenic expression. Importantly, omega-3 FA provided striking protection against UVR-induced immunosuppression. This observation was subsequently confirmed [37]. Delayed type hypersensitivity (DTH) and CHS are both regulated by T-cell function and share common pathways with immunological tumor rejection. DTH in UVR-irradiated animals is dramatically suppressed in animals fed high levels of omega-6 FA when compared to those receiving low levels of the FA or those receiving omega-3 FA [32,38].The ability of an animal to reject a transplanted tumor was related to the level of omega-6 FA intake. Moreover, the tumor rejection time for animals fed high levels of omega-6 FA was three times longer than animals fed low levels of the omega- 6 FA and occurred at a time when high omega-6 FA had been shown to exacerbate primary tumor expression [38].These studies suggest that one potential mechanism of omega-3 FA inhibition of carcinogenic expression is via immune modulation [31].

In summary, the following sequence of observations support the thesis that omega-6, -3 PUFA metabolism, through the LOX and COX pathways, leads to differential metabolites that influence inflammatory and immune responses involved in UVR-carcinogenesis:

1. Increasing levels of dietary omega-6 FA exacerbate UVR carcinogenic expression, with respect to both shortened tumor latent period and increased tumor multiplicity.

2. Dietary omega-3 FA inhibit UVR carcinogenic expression.

3. Omega-6 FA exert their principal effect upon the post-initiation, or promotion/progression stages of UVR carcinogenesis.

4. Omega-3 FA appear to exert their principal effects during the initiation stage of the carcinogenic continuum.

5. Pro-inflammatory and immunosuppressive $\mathrm{PGE}_{2}$ levels are increased linearly as dietary omega-6 FA levels increase. 
6. Pro-inflammatory and immunosuppressive $\mathrm{PGE}_{2}$ levels are dramatically reduced by dietary omega-3 FA intake.

7. Dietary omega-6 FA suppress the immunologic responses involved in tumor transplant rejection and the immunologic pathways involved in DTH and CHS.

8. Dietary omega-3 FA inhibit UVR-induced suppression of DTH and CHS.

\subsection{Clinical Studies}

The experimental studies employing a high-fat diet to low-fat diet cross-over, even after a cancer causing dose of UVR had been administered, negated the exacerbating influence of the high-fat diet and provided a rationale for undertaking a clinical intervention trial. This trial, involving 133 skin cancer patients, of whom 115 completed the two year study, clearly demonstrated that a low-fat intervention reduced the occurrence of NMSC [39,40]. The cumulative rate of occurrence of NMSC (cumulative NMSC/patient/time period) was 0.21 and 0.19 during the first 8-month period of the study and 0.26 and $0.02(p \leqslant 0.02)$ during the last 8-month period for control and intervention arms, respectively. The dietary parameters involved only a reduction in the calories consumed as fat, while maintaining total calorie intake and body weight. Efforts were made to maintain the P/S ratio (polyunsaturated/saturated fat ratio) of patients' diets going into the trial and there were no increases in omega-3 FA intake. Thus, the influence of fat on MNSC occurrence was primarily that resulting from lowering fat intake, primarily omega- 6 FA. Furthermore, the influence of this low-fat intervention was observed early in the study as a significant difference in the number of actinic keratoses (pre-malignant lesions) between control and low-fat diets occurred [41]. Patients in the control arm of the study (no dietary modifications introduced) were found to be at 4.7 times greater risk of having one or more actinic keratoses during the two-year study period than patients in the low-fat intervention arm.

Whereas lower intake of omega-6 FA reduces the risk of NMSC occurrence in skin cancer patients, a population based case-control study showed a consistent tendency toward a lower risk of SCC with higher intakes of omega-3 FA [42]. Their data also suggested a tendency for a lower risk of SCC with diets containing high omega-3/omega-6 FA ratios. Although this study was suggestive that omega-3 FA could influence NMSC risk, a number of human studies have provided a physiological rationale to support such a hypothesis. Encouraged by the experimental animal results, a short term supplementation study of mixed omega-3 FA was conducted in humans [43]. The patients received oral capsules of either $4 \mathrm{~g} /$ day of mixed omega-3 FA (2.8 g EPA $+1.2 \mathrm{~g}$ DHA) or a gelatin placebo. After four weeks there was a statistically significant increase in the minimal erythema dose (MED) to UVB in the active group. Serum triglyceride levels decreased by $40 \mathrm{mg} / \mathrm{dL}$. A second study examined the effects of omega-3 FA supplementation 
on UVB-induced erythema and lipid peroxidation [44]. This study employed a supplement of $3 \mathrm{~g} /$ day of mixed omega-3 FA (1.8 g EPA + 1.2 g DHA) administered over a 3-6 month period. The MED rose progressively with increasing time of omega-3 FA supplementation, and had more than doubled at six months. This increase in MED was accompanied by an increase in epidermal omega-3 FA composition and increased susceptibility to lipid peroxidation. The MED had returned to baseline two and a half months after omega-3 FA supplementation was halted.

As noted earlier, a number of cytokines and PG have been shown to be modulated by omega-3 FA. When human keratinocytes were cultured in the presence of omega- 3 FA, TNF- $\alpha$ and IL- $1 \alpha$ secretion was induced and PGE 2 and IL-6 level reduced [45]. Subsequently, further in vitro keratinocyte studies showed that EPA and DHA each inhibited basal and UVR-induced IL-8, a chemokine pivotal to UVR- induced skin inflammation and which exhibits pro-carcinogenic activity [46]. However, a double- blind, randomized trial of 28 patients supplemented with $4 \mathrm{~g} /$ day of $95 \%$ of ethyl esters of EPA or oleic acid for three months found no evidence that the MED response evoked by omega- 3 FA was mediated by the pro-inflammatory cytokines IL-8, TNF- $\alpha$, IL- 6 or IL- $1 \beta$. In contrast, there was a notable and significant reduction in cutaneous $\mathrm{PGE}_{2}$, the pro-inflammatory and immune-suppressor mediator [47]. Further, lipidomic analysis was performed in a human intervention trial of EPA-rich omega-3 FA, quantifying impact of supplement on eicosanoid levels in skin blister fluid [48]. This showed a significant reduction in the ratio of $\mathrm{PGE}_{2}$ : $\mathrm{PGE}_{3}$ in UVR-exposed skin, accompanied by a reduction in the ratio of the pro-inflammatory and tumor promoting 12-LOX product 12-hydroxyeicosatetraenoic acid (12-HETE): 12-hydroxyeicosapentaenoic acid (12-HEPE), EPA-derived homologue of 12-HETE [48].

A double-blind, randomized intervention examined the impact of oral omega-3 FA on UVR suppression of cell-mediated immunity, assessed through the nickel CHS response [49]. Seventy-nine nickel-sensitive adult females consumed encapsulated omega-3 FA (3.5 g EPA + 1.5 g DHA) or control lipid daily for 3 months, with compliance and skin bioavailability of omega-3 FA assessed by blood [49] and skin [48] assay, respectively. This indicated apparent abrogation of the photo-immunosuppression induced by low level solar simulated ultraviolet radiation (SSR; 95\% UVA, 5\% UVB) exposure. Following SSR exposure equivalent to $15 \mathrm{~min}$ of midday summer sunlight in Manchester, UK (latitude 53.5 $\mathrm{N}$ ), on 3 consecutive days, the UVR-suppression of the CHS response was $50 \%$ lower in the subjects taking omega-3 FA compared to those taking control.

Previous discussion provides clear evidence that omega-3 FA protect against the clinical sunburn response. Yet, there was no evidence of an EPA effect on direct UVR-induced DNA damage to DNA, i.e., cyclobutane pyrimidine dimer formation 
in skin [50]. There was, however, protection against UVR induction of cutaneous p53, considered to be a biomarker of DNA damage and which acts as a tumor suppressor gene. In addition, in ex vivo UVR-treated peripheral blood lymphocytes, omega-3 FA protected against DNA single-strand breaks [50].

Thus, human studies (and cell culture studies employing human cells) have shown:

1. Omega-3 FA supplementation significantly increases the erythema threshold to UVR.

2. Omega-3 FA modulate a number of cytokines (in human cells in vitro only) and eicosanoids that mediate inflammatory and immune responses.

3. Omega-3 FA inhibit certain genotoxic markers of UVR-induced DNA damage, e.g., UVR- induced cutaneous p53.

4. Omega-3 FA abrogate UVR-induced immunosuppression of cell mediated immunity assessed as nickel CHS

\section{Conclusions}

In Toto, the results of experimental studies, and the influence of omega-3 FA on UVR-induced erythema, early genotoxic markers and immune-suppression in human trials, suggest that supplementation of these photoprotective nutrients [51] could result, in the longer term, in a reduction in NMSC in humans. Based upon age-adjusted cancer incidence/UVR exposure plots, an omega-3 FA enhanced sun protection factor (SPF), even of the low reported magnitude could reduce incidence of NMSC by as much as 30\% [50-52]. Neither have observational studies, case-control or prospective cohort studies, provided clear evidence that dietary omega- 6 FA or omega-3 FA reduces the risk of NMSC. A recent meta-analysis has been suggestive, but lacked adequate data due to scarcity of trials in this area, to support the hypothesis that omega-3 FA protect against NMSC [53]. For the most part, both case-control and prospective cohort studies have failed to find a relationship between skin cancer incidence with dietary fat intake. Indeed these types of studies are fraught with methodological difficulties resulting from: (1) the complexity of the human diet in a free living population; (2) the difficulties in measuring food intake and analyzing dietary information; (3) the epidemiologist requires assess of dietary patterns that are stable over long periods, i.e., usually years if cancer induction is under study [54].

The authors have previously proposed that the most direct evidence for the preventive potential of omega-3 FA would be achieved through intervention trials in populations with high, and known, risk for NMSC - much in the manner that reduction in the percentage of calories consumed as fat was shown to influence NMSC occurrence in skin cancer patients [24,39-41,55]. Caveats to the design of 
such a study will include consideration of baseline omega-3 FA nutrition at study inclusion [56] and careful monitoring of the diets of study patients to assure that any potential benefits of omega-3 FA supplementation is not diminished by increasing omega-6 FA intake. The relative omega-6/omega-3 FA ratios will determine response and could be monitored by an easily determined parameter such as red blood cell membrane omega-6/omega-3 FA ratios [48,56]. This parameter could also be used to determine adherence to the supplement protocol. It is also important that an adequate level of omega-3 FA supplementation be employed. Omega-3 FA have a high safety profile and a daily intake of circa $4 \mathrm{~g} /$ day as employed in previous photoprotection studies is envisaged to be adequate. Because of the promising evidence from animal and clinical studies, it is imperative that the potential of omega-3 FA as a preventive agent for NMSC be fully explored.

Acknowledgments: LER acknowledges the financial support of the Association for International Cancer Research and the European Commission.

Conflicts of Interest: The authors declare no conflict of interest.

\section{References}

1. Bang, H.O.; Dyerberg, J. Plasma lipid and lipoprotein pattern in Greenlandic West Coast Eskimos. Lancet 1971, 1, 1143-1145.

2. Bang, H.O.; Dyerberg, J.; Hjorne, N. The composition of food consumed by Greenland Eskimos. Acta Med. Scand. 1976, 200, 69-73.

3. Bang, H.O.; Dyerberg, J.; Sinclair, H.M. The composition of the Eskimo food in North Western Greenland. Am. J. Clin. Nutr. 1980, 33, 2657-2661.

4. Jordan, H.; Matthan, N.; Chung, M.; Balk, E.; Chew, P.; Kupelnick, B.; Lawrence, A.; Lichtenstein, A.; Lau, J. Effects of Omega-3 Fatty Acids on Arrhythmogenic Mechanisms in Animal and Isolated Organ/Cell Culture Studies; Evidence Report/Technology Assessment No. 92; Publication No. 04-EO11-2. Agency for Healthcare Research and Quality: Rockville, MD, USA, 2004.

5. Balk, E.; Chung, M.; Lichtenstein, A.; Chew, P.; Kupelnick, B.; DeVine, D.; Lawrence, A.; Lau, J. Effects of Omega-3 Fatty Acids on Cardiovascular Risk Factors and Intermediate Markers of Cardiovascular Disease; Evidence Report/Technology Assessment No. 93; Publication No. 04-EO10-2. Agency for Healthcare Research and Quality: Rockville, MD, USA, 2004.

6. Wang, C.; Chung, M.; Lichtenstein, A.; Balk, E.; Kupelnick, B.; DeVine, D.; Lawrence, A.; Lau, J. Effects of Omega-3 Fatty Acids on Cardiovascular Disease; Evidence Report/Technology Assessment No. 94; Publication No. 04-EO09-2. Agency for Healthcare Research and Quality: Rockville, MD, USA, 2004.

7. Schacter, H.; Reisman, J.; Tran, K.; Dales, B.; Kourad, K.; Barnes, D.; Sampson, M.; Morrison, A.; Gaboury, I.; Blackman, J. Health Effects of Omega-3 Fatty Acids on Asthma; Evidence Report/Technology Assessment No. 94; Publication No. 04-EO13-2. Agency for Healthcare Research and Quality: Rockville, MD, USA, 2004. 
8. MacLean, C.H.; Mojica, W.A.; Morton, S.C.; Pencharz, J.; Hasenfeld Garland, R.; Tu, W.; Newberry, S.J.; Jungvig, L.K.; Khanna, P.; Rhodes, S.; et al. Effects of Omega-3 Fatty Acids on Lipids and Glycemic Control in Type II Diabetes and the Metabolic Syndrome and on Inflammatory Bowel Disease, Rheumatoid Arthritis, Renal Disease, Systemic Lupus Erythematosus, and Osteoporosis; Evidence Report/Technology Assessment No. 89; Publication No. 04-EO12-2. Agency for Healthcare Research and Quality: Rockville, MD, USA, 2004.

9. Nixon, D.W.; Rodgers, K. Breast Cancer. In Nutritional Oncology, 2nd ed.; Heber, D., Blackburn, G.L., Go, V.L.W., Eds.; Academic Press: San Diego, CA, USA, 1999; pp. 447-452.

10. Aronson, W.; Yip, I.; Dekernion, J. Prostate Cancer. In Nutritional Oncology, 2nd ed.; Heber, D., Blackburn, G.L., Go, V.L.W., Eds.; Academic Press: San Diego, CA, USA, 1999; pp. 453-461.

11. Clinton, S.K.; Michaud, D.; Giovannucci, E. Nutrition and Bladder Cancer. In Nutritional Oncology, 2nd ed.; Heber, D., Blackburn, G.L., Go, V.L.W., Eds.; Academic Press: San Diego, CA, USA, 1999; pp. 463-475.

12. Harris, D.M.; Kang, S.Y.; Go, V.L.W. Nutrient-Gene Interactions and Prevention of Colorectal, Liver, and Pancreatic Cancer. In Nutritional Oncology, 2nd ed.; Heber, D., Blackburn, G.L., Go, V.L.W., Eds.; Academic Press: San Diego, CA, USA, 1999; pp. 469-500.

13. Caygill, C.P.J.; Charlett, A.; Hill, M.J. Fat, fish, fish oil and cancer. Br. J. Cancer 1996, 74, 159-164.

14. MacLean, C.H.; Newberry, S.J.; Mojica, W.A.; Issa, A.; Khanna, P.; Lim, Y.S.W.; Morton, S.C.; Suttorp, M.; Tu, W.; Hilton, L.G.; et al. Effects of Omega-3 Fatty Acids on Cancer; Evidence Report/Technology Assessment No. 113; Publication No. 05-EO10-1. Agency for Healthcare Research and Quality: Rockville, MD, USA, 2006.

15. MacLean, C.H.; Newberry, S.J.; Mojica, W.A.; Khanna, P.; Issa, A.M.; Suttorp, M.J.; Lim, Y.W.; Traina, S.B.; Hilton, L.; Garland, R.; et al. Effects of omega-3 fatty acids on cancer risk: a systematic review. JAMA 2006, 295, 403-415.

16. Fabian, C.J.; Kimler, B.F.; Hursting, S.D. Omega-3 fatty acids for breast cancer prevention and survivorship. Breast Cancer Res. 2015, 17, 62-77.

17. Brasky, T.M.; Darke, A.K.; Song, X.; Tangen, C.M.; Goodman, P.J.; Thompson, I.M.; Mayskens, F.L., Jr.; Goodman, G.E.; Minasian, L.M.; Parnes, H.L.; et al. Plasma phospholipid fatty acids and prostate cancer risk in the SELECT trial. J. Natl. Cancer Inst. 2013, 105, 1132-1141.

18. Alexander, D.D.; Bassett, J.K.; Weed, D.L.; Barrett, E.C.; Watson, H.; Harris, W. Meta-analysis of long-chain omega-3 polyunsaturated fatty acids (LC $\omega-3$ PUFA) and prostate cancer. Nutr. Cancer 2015, 67, 543-554.

19. Lu, H.; Ouyang, W.; Huang, C. Inflammation, a key event in cancer development. Mol. Cancer Res. 2006, 4, 221-233.

20. American Cancer Society. Cancer Facts and Figures, Atlanta, GA. 2014. Available online: http:/ / www. cancer.org (accessed on 8 November 2015). 
21. Vanderveen, E.; Grekin, R.; Swanson, N.; Kragballe, K. Arachidonic acid metabolites in cutaneous carcinomas. Evidence suggesting that elevated levels of prostaglandins in basal cell carcinomas are associated with aggressive growth pattern. Arch Dermatol. 1986, 122, 407-412.

22. Malmsten, C. Leukotrienes: Mediators of inflammation and immediate hypersensitivity reactions. Crit. Rev. Immunol. 1984, 4, 307-334.

23. Werner, E.; Walenga, R.; Dubowy, R.; Boone, S.; Stuart, M. Inhibition of human malignant neuroblastoma cell DNA synthesis by lipoxygenase metabolites of arachidonic acid. Cancer Res. 1985, 45, 561-563.

24. Black, H.S.; Rhodes, L.E. The potential of omega-3 fatty acids in the prevention of nonmelanoma skin cancer. Cancer Det. Prev. 2006, 30, 224-232.

25. Baumann, C.; Rusch, H. Effect of diet on tumors induced by ultraviolet light. Am. J. Cancer 1939, 35, 213-221.

26. Black, H.S.; Lenger, W.; Phelps, A.W.; Thornby, J.I. Influence of dietary lipid upon ultraviolet-light carcinogenesis. Nutr. Cancer 1983, 5, 59-68.

27. Black, H.S.; Lenger, W.A.; Gerguis, J.; Thornby, J.I. Relation of antioxidants and level of dietary lipid to epidermal lipid peroxidation and ultraviolet carcinogenesis. Cancer Res. 1985, 45, 6254-6259.

28. Reeve, V.; Bosnic, M.; Boehm-Wilcox, C. Dependence of photocarcinogenesis and photoimmunosuppression in the hairless mouse on dietary polyunsaturated fat. Cancer Lett. 1966, 108, 271-279.

29. Black, H.S.; Thornby, J.I.; Gerguis, J.; Lenger, W. Influence of dietary omega-6, -3 fatty acid sources on the initiation and promotion stages of photocarcinogenesis. Photochem. Photobiol. 1992, 56, 195-199.

30. Orengo, I.F.; Black, H.S.; Kettler, A.H.; Wolf, J.E., Jr. Influence of dietary menhaden oil upon carcinogenesis and various cutaneous responses to ultraviolet radiation. Photochem. Photobiol. 1989, 49, 71-77.

31. Black, H.S. Omega-3 fatty acids and non-melanoma skin cancer. In Handbook of Diet, Nutrition and the Skin; Preedy, V.R., Ed.; Wageningen Academic Publishers: Wageningen, The Netherlands, 2012; pp. 367-378.

32. Fischer, M.A.; Black, H.S. Modification of membrane composition, eicosanoid metabolism, and immunoresponsiveness by dietary omega-3 and omega- 6 fatty acid sources, modulators of ultraviolet-carcinogenesis. Photochem. Photobiol. 1991, 54, 381-387.

33. Kang, J.X. Fat-1 transgenic mice: A new model for omega-3 research. Prostaglandins Leukot Essent Fatty Acids 2007, 77, 263-267.

34. Xia, S.; Lu, Y.; Wang, J.; He, C.; Hong, S.; Serhan, C.N.; Kang, J.X. Melanoma growth is reduced in fat- 1 transgenic mice: Impact of omega-6/omega-3 essential fatty acids. Proc. Natl. Acad. Sci. USA 2006, 103, 12499-12504.

35. Strickland, P.; Creasia, D.; Kripke, M. Enhancement of two-stage skin carcinogenesis by exposure of distant skin to UV-radiation. J. Natl. Cancer Inst. 1985, 74, 1129-1134. 
36. Chung, H.; Burnham, D.; Robertson, B.; Roberts, I.; Daynes, R. Involvement of prostaglandins in the immune alterations caused by exposure of mice to ultraviolet radiation. J. Immunol. 1986, 137, 2478-2884.

37. Moison, R.; Beijersbergen Van Henegouwen, G. Dietary eicosapentaenoic acid prevents systemic immunosuppression in mice induced by UVB radiation. Radiat. Res 2001, 156, 36-44.

38. Black, H.S.; Okotie-Eboh, G.; Gerguis, J.; Urban, J.I.; Thornby, J.I. Dietary fat modulates immunoresponsiveness in UV-irradiated mice. Photochem. Photobiol. 1995, 62, 964-969.

39. Black, H.S.; Thornby, J.I.; Wolf, J.E.; Goldberg, L.H.; Herd, J.A.; Rosen, T.; Bruce, S.; Tschen, J.A.; Scott, L.W.; Jaax, S.; et al. Evidence that a low-fat diet reduces the occurrence of non-melanoma skin cancer. Int. J. Cancer 1995, 62, 165-169.

40. Jaax, S.; Scott, L.W.; Wolf, J.E.; Thornby, J.I.; Black, H.S. General guidelines for a low-fat diet effective in the management and prevention of nonmelanoma skin cancer. Nutr. Cancer 1997, 27, 150-156.

41. Black, H.S.; Herd, J.A.; Goldberg, L.H.; Wolf, J.E.; Thornby, J.I.; Rosen, T.; Bruce, S.; Tschen, J.A.; Foreyt, J.P.; Scott, L.W.; et al. Effect of a low-fat diet on the incidence of actinic keratosis. N. Engl. J. Med. 1994, 330, 1272-1275.

42. Hakim, I.A.; Harris, R.B.; Ritenbaugh, C. Fat intake and risk of squamous cell carcinomas of the skin. Nutr. Cancer 2000, 36, 155-162.

43. Orengo, I.F.; Black, H.S.; Wolf, J.E. Influence of fish oil supplementation on the minimal erythema dose in humans. Arch. Dermatol. 1992, 284, 219-221.

44. Rhodes, L.E.; O'Farrell, S.; Jackson, M.J.; Friedmann, P.S. Dietary fish-oil supplementation in humans reduces UVB-erythemal sensitivity but increases epidermal lipid peroxidation. J. Investig. Dermatol. 1994, 103, 151-154.

45. Pupe, A.; Moison, R.; De Haes, P.; Beijersbergen van Henegouwen, G.; Rhodes, L.E.; Degreef, H.; Garmyn, M. Eicosapentaenoic acid, a n-3 polyunsaturated fatty acid differentially modulates TNF- $\alpha$, IL- $1 \alpha$, IL- 6 and PGE 2 expression in UVB-irradiated human keratinocytes. J. Investig. Dermatol. 2002, 118, 692-698.

46. Storey, A.; McArdle, F.; Friedmann, P.S.; Jackson, M.J.; Rhodes, L.E. Eicosapentaenoic acid and docosahexaenoic acid reduce UVB- and TNF-alpha-induced IL-8 secretion in keratinocytes and UVB-induced IL-8 in fibroblasts. J. Investig. Dermatol. 2005, 124, 248-255.

47. Shahbakhti, H.; Watson, R.; Azurdia, R.; Ferreira, R.; Garmyn, M.; Rhodes, L.E. Influence of eicosapentaenoic acid, an omega-3 fatty acid, on ultraviolet $\mathrm{B}$ generation of prostaglandin E2 and proinflammatory cytokines interleukin-1 beta, tumor necrosis factor-alpha, interleukin-6 and interleukin-8 in human skin in vivo. Photochem. Photobiol. 2004, 80, 231-235.

48. Pilkington, S.M.; Rhodes, L.E.; Al-Aasswad, N.M.I.; Massey, K.A.; Nicolaou, A. Impact of EPA ingestion on COX- and LOX-mediated eicosanoid synthesis in skin with and without a pro- inflammatory UVR challenge. Report of a randomised controlled study in humans. Mol. Nutr. Food Res. 2014, 58, 580-590. 
49. Pilkington, S.M.; Massey, K.A.; Bennett, S.P.; Al-Aasswad, N.M.; Roshdy, K.; Gibbs, N.K.; Friedmann, P.S.; Nicolaou, A.; Rhodes, L.E. Randomized controlled trial of oral omega-3 PUFA in solar-simulated radiation-induced suppression of human cutaneous immune responses. Am. J. Clin. Nutr. 2013, 97, 646-652.

50. Rhodes, L.E.; Shahbakhti, H.; Azurdia, R.; Moison, R.; Steenwinkel, M.-J.S.T.; Homburg, M.; Dean, M.; McArdle, F.; Beijersbergen van Henegouwen, G.; Epe, B.; et al. Effect of eicosapentaenoic acid, an omega-3 polyunsaturated fatty acid, on UVR-related cancer risk in humans. An assessment of early genotoxic markers. Carcinogenesis 2003, 24, 919-925.

51. Pilkington, S.M.; Watson, R.E.B.; Nicolaou, A.; Rhodes, L.E. Omega-3 Polyunsaturated Fatty Acids: Photoprotective Macronutrients. Exp. Dermatol. 2011, 20, 537-543.

52. Rhodes, L.E. Preventive oncology. Lancet 2004, 363, 1736-1737.

53. Noel, S.E.; Stoneham, A.C.S.; Olsen, C.M.; Rhodes, L.E.; Green, A.C. Consumption of omega-3 fatty acids and the risk of skin cancers: A systemic review and meta-analysis. Int. J. Cancer 2014, 135, 149-156.

54. Lyon, J.Y.; Gardner, J.W.; West, D.W.; Mahoney, A.M. Methodological issues in epidemiological studies of the diet and cancer. Cancer Res. 1992, 52, 2040-2048.

55. Black, H.S. Skin Cancer. In Nutritional Oncology, 2nd ed.; Heber, D., Blackburn, G.L., Go, V.L.W., Eds.; Academic Press: San Diego, CA, USA, 1999; pp. 405-422.

56. Wallingford, S.C.; Pilkington, S.M.; Massey, K.A.; Al-Aasswad, N.M.I.; Ibiebele, T.I.; Hughes, M.C.; Bennett, S.; Nicolaou, A.; Rhodes, L.E.; Green, A.C. Three-way assessment of long chain omega-3 polyunsaturated fatty acid nutrition: By questionnaire and matched blood and skin samples. Br. J. Nutr. 2013, 109, 701-708. 


\section{Chapter 2: \\ Omega-3 Fatty Acids and Psychiatric Disorders}





\title{
Supplementation with Omega-3 Fatty Acids in Psychiatric Disorders: A Review of Literature Data
}

\author{
Paola Bozzatello, Elena Brignolo, Elisa De Grandi and Silvio Bellino
}

\begin{abstract}
A new application for omega-3 fatty acids has recently emerged, concerning the treatment of several mental disorders. This indication is supported by data of neurobiological research, as highly unsaturated fatty acids (HUFAs) are highly concentrated in neural phospholipids and are important components of the neuronal cell membrane. They modulate the mechanisms of brain cell signaling, including the dopaminergic and serotonergic pathways. The aim of this review is to provide a complete and updated account of the empirical evidence of the efficacy and safety that are currently available for omega-3 fatty acids in the treatment of psychiatric disorders. The main evidence for the effectiveness of eicosapentaenoic acid (EPA) and docosahexaenoic acid (DHA) has been obtained in mood disorders, in particular in the treatment of depressive symptoms in unipolar and bipolar depression. There is some evidence to support the use of omega- 3 fatty acids in the treatment of conditions characterized by a high level of impulsivity and aggression and borderline personality disorders. In patients with attention deficit hyperactivity disorder, small-to-modest effects of omega-3 HUFAs have been found. The most promising results have been reported by studies using high doses of EPA or the association of omega- 3 and omega- 6 fatty acids. In schizophrenia, current data are not conclusive and do not allow us either to refuse or support the indication of omega-3 fatty acids. For the remaining psychiatric disturbances, including autism spectrum disorders, anxiety disorders, obsessive-compulsive disorder, eating disorders and substance use disorder, the data are too scarce to draw any conclusion. Concerning tolerability, several studies concluded that omega-3 can be considered safe and well tolerated at doses up to $5 \mathrm{~g} /$ day.
\end{abstract}

Reprinted from J. Clin. Med. Cite as: Bozzatello, P.; Brignolo, E.; De Grandi, E.; Bellino, S. Supplementation with Omega-3 Fatty Acids in Psychiatric Disorders: A Review of Literature Data. J. Clin. Med. 2016, 5, 67.

\section{Introduction}

The role of omega-3 highly unsaturated fatty acids (HUFAs) in human mental health has been widely studied in the last two decades.

Omega-3 fatty acids eicosapentaenoic acid (EPA) and docosahexaenoic acid (DHA) are derived from alpha-linolenic acid (ALA) and are dietary essential fatty 
acids. They cannot be synthetized de novo by mammals and are provided by supplementation, such as fish oil.

EPA and DHA act as competitive inhibitors of omega- 6 fatty acids causing a reduction in the synthesis of pro-inflammatory mediators [1]. In fact, the omega- 6 family of fatty acids is converted to arachidonic acid and then into prostaglandins and leukotrienes, which are responsible of the pro-inflammatory effects. Therefore, a diet rich in fish oil has been shown to decrease the incidence of inflammatory diseases.

In addition, HUFAs slow coronary atherosclerosis by optimizing cholesterol concentrations and lowering plasma triglyceride levels. HUFAs also have antithrombotic, antiarrhythmic and vasodilatory properties, providing the protection of the cardiovascular system and significantly diminishing cardiovascular mortality. These effects of omega- 3 fatty acids have supported indications in the secondary prevention of hypertension, coronary heart disease, type 2 diabetes and in some cases of rheumatoid arthritis, Crohn's disease, ulcerative colitis, chronic obstructive pulmonary disease and renal disease [2,3].

EPA and DHA are important for fetal development, including neuronal, retinal and immune functions [4].

In recent years, the interest of omega-3 fatty acids has grown in psychiatry, and their role in the treatment of several mental diseases has been investigated. HUFAs are important components of phospholipids and cholesterol esters of the neuronal cell membrane, especially of dendritic and synaptic membranes. The rationale for the use of these new agents in psychiatric disorders stemmed from their primary action in producing modification of the synaptic membrane [5]. Indeed, they modulate and are involved in brain cell signaling, including monoamine regulation [6-10], modification of receptor properties or the activation of signal transduction by receptors.

Among psychiatric diseases, long-chain omega-3 fatty acids have been tested in the treatment of schizophrenia, unipolar and bipolar mood disorders, anxiety disorders, obsessive-compulsive disorder, attention deficit hyperactivity disorder (ADHD), autism, aggression, hostility and impulsivity, borderline personality disorder, substance abuse and anorexia nervosa. The aim of this review is to provide an updated account of the empirical evidence of the efficacy and safety that are currently available for omega-3 fatty acids in the treatment of mental disorders. In particular, we focused on the data of efficacy and the adverse effects of different doses of HUFAs in the treatment of mental disorders, to determine which dose levels of single or combined fatty acids are related to a good clinical response of specific mental disorders with a low risk of significant adverse effects. 


\section{Methods}

This review presents and critically evaluates data from clinical trials, systematic reviews and meta-analyses published from 1980 to September 2015, after a systematic research on the Medline database provided by the U.S. National Library of Medicine (http://www.ncbi.nlm.nih.gov/pubmed?otool=iitutolib). The search terms were: omega-3 fatty acids; HUFAs; eicosapentaenoic acid; docosahexaenoic acid; ethyl-eicosapentaenoic acid; $\alpha$-lipoic acid; $\alpha$-linoleic acid; psychiatric disorders; psychotic disorders; schizophrenia; mood disorders; anxiety disorders; obsessive-compulsive disorder; attention deficit hyperactivity disorder; autism spectrum disorders; aggression, hostility, impulsivity; personality disorders; borderline personality disorder; substance dependence; anorexia nervosa; adverse effects; dose.

\subsection{Schizophrenia}

Studies of post mortem samples have shown that patients with schizophrenia have often low levels of EPA and DHA in their brain cells. The level of fatty acids is considered important to maintain the correct nerve cell-membrane metabolism [11]. The evidence is strong enough to have led to a 'membrane phospholipid hypothesis' of schizophrenia $[12,13]$. Based on findings that in individuals with schizophrenia or related psychotic disorders, certain omega-3 and omega-6 HUFA levels are reduced compared to healthy control samples, the idea that restoration of HUFA resources could be used as a treatment option in psychotic disorders has been widely discussed [14].

To date, there are 13 RCTs available concerning the role of omega- 3 fatty acids supplementation in schizophrenia or related disorders. For ethical and clinical reasons, most trials used the add-on strategy, including patients who already received neuroleptics or atypical antipsychotics. Two exceptions are the studies published by Amminger [15] and Markulev [16], which considered patients at high risk for developing psychosis. Peet et al. [17] and Emsley et al. [18] designed trials with omega-3 HUFAs as a monotherapy, but in both cases, almost all patients needed to be treated also with an antipsychotic drug during the course of the trial. Only six RCTs [15,17,19-22] reported that HUFAs had a benefit on positive or negative symptoms. The sample size of all of the reviewed studies was small, and the population investigated suffered from a considerable degree of heterogeneity. In fact, not only patients with a diagnosis of schizophrenia were included, but also subjects with schizoaffective disorder [23], first episode of psychosis [21,24] and resistant schizophrenia [19]. The omega-3 supplementation has been administered in a dosage ranging from 1-4 g/day. The duration of the trials has been limited to 8-16 weeks in the majority of the studies. The main issue is that no conclusions could be drawn 
regarding the medium- to long-term effects of HUFAs in schizophrenia, according to the findings of the four meta-analysis available on this topic [25-28] (Table 1).

Table 1. Double-blind controlled trials of highly unsaturated fatty acids (HUFAs) as an add-on strategy in the treatment of schizophrenia. PANSS, Positive and Negative Syndrome Scale; E-EPA, ethyl-EPA.

\begin{tabular}{|c|c|c|c|c|}
\hline Study & Drug and Dose & Sample & $\begin{array}{l}\text { Treatment } \\
\text { Duration }\end{array}$ & Results \\
\hline $\begin{array}{l}\text { Peet et al., } \\
2001[17]\end{array}$ & $\begin{array}{l}\text { EPA or DHA } 2 \\
\text { g/day }\end{array}$ & 45 patients & 12 weeks & $\begin{array}{l}\downarrow \text { psychotic symptoms measured with } \\
\text { PANSS in the group treated with EPA }\end{array}$ \\
\hline $\begin{array}{l}\text { Peet et al., } \\
2001[17]\end{array}$ & EPA 2 g/day & 30 patients & 12 weeks & $\downarrow$ positive symptoms measured with PANSS \\
\hline $\begin{array}{l}\text { Peet and } \\
\text { Horrobin, } \\
2002[19]\end{array}$ & E-EPA $1-4$ g/day & 115 patients & 12 weeks & $\begin{array}{l}\downarrow \text { positive symptoms measured with PANSS, } \\
\downarrow \text { depressive symptoms }\end{array}$ \\
\hline $\begin{array}{l}\text { Jamilian et al., } \\
2014[20]\end{array}$ & $1 \mathrm{~g} /$ day & 60 patients & 8 weeks & $\begin{array}{l}\downarrow \text { psychotic symptoms measured } \\
\text { with PANSS }\end{array}$ \\
\hline $\begin{array}{l}\text { Fenton et al., } \\
2001[23]\end{array}$ & ethyl-EPA 3 g/day & 87 patients & 16 weeks & $\begin{array}{l}\text { no significant differences in positive, } \\
\text { negative symptoms, mood or cognition }\end{array}$ \\
\hline $\begin{array}{l}\text { Berger et al., } \\
2007[24]\end{array}$ & ethyl-EPA 2 g/day & 69 patients & 12 weeks & no efficacy on specific psychotic symptoms \\
\hline $\begin{array}{l}\text { Amminger et al., } \\
\quad 2010 \text { [15] }\end{array}$ & $\begin{array}{l}\text { EPA } 700 \mathrm{mg} / \text { day }+ \\
\text { DHA } 480 \mathrm{mg} / \text { day }\end{array}$ & $\begin{array}{l}76 \\
\text { individuals } \\
\text { "UHR" }\end{array}$ & 12 weeks & $\begin{array}{l}\downarrow \text { progression in psychosis in young } \\
\text { UHR patients }\end{array}$ \\
\hline $\begin{array}{l}\text { Pawelzcyk et al., } \\
\quad 2016[21]\end{array}$ & $\begin{array}{l}\mathrm{EPA}+\mathrm{DHA} \\
2.2 \mathrm{~g} / \text { day }\end{array}$ & $\begin{array}{l}71 \text { patients } \\
\text { with FEP }\end{array}$ & 26 weeks & $\begin{array}{l}\downarrow \text { psychotic symptoms measured with } \\
\text { PANSS } \downarrow \text { depressive symptoms } \uparrow \\
\text { level of functioning }\end{array}$ \\
\hline $\begin{array}{l}\text { Bentsen et al., } \\
2013 \text { [22] }\end{array}$ & ethyl-EPA 2 g/day & 99 patients & 16 weeks & $\downarrow$ impairment of the course of psychosis \\
\hline $\begin{array}{l}\text { Emsley et al., } \\
2014 \text { [18] }\end{array}$ & $\begin{array}{c}\text { EPA } 2 \mathrm{~g} / \text { day }+ \\
\text { DHA } 1 \mathrm{~g} / \text { day }+ \\
\alpha-\mathrm{LA} 300 \mathrm{mg} / \text { day }\end{array}$ & 33 patients & 2 years & relapse prevention of psychotic symptoms \\
\hline $\begin{array}{l}\text { Emsley et al., } \\
2002[29]\end{array}$ & ethyl-EPA 3 g/day & 40 patients & 12 weeks & $\begin{array}{l}\downarrow \text { positive symptoms and negative symptoms } \\
\text { measured with PANSS }\end{array}$ \\
\hline
\end{tabular}

Abbreviations: $\mathrm{EPA}=$ eicosapentaenoic acid; DHA $=$ docosahexaenoic acid; ethyl-EPA $=$ ethyl-eicosapentaenoic acid; $\alpha$-LA $=\alpha$-lipoic acid; UHR $=$ ultra-high-risk; FEP $=$ first episode of psychosis; $\downarrow=$ decrease of; $\uparrow=$ increase of.

Peet and colleagues [17] performed a 12-week placebo-controlled trial in 30 males and 15 females on stable antipsychotic medication who were still symptomatic. Only 35 patients completed the trial. The authors found that EPA was more effective at the reduction of symptoms as assessed with the Positive and Negative Syndrome Scale (PANSS) in comparison to DHA and placebo. In the same year, Peet and colleagues [17] conducted a second RCT to test EPA (dose: $2 \mathrm{~g} /$ day) as a monotherapy for schizophrenia. Thirty patients who never assumed an antipsychotic agent with a recent diagnosis of schizophrenia were recruited. For ethical reasons, antipsychotic medications were permitted, so at the end of the 
study, only two patients remained without an antipsychotic treatment. The results indicated that patients treated with EPA, but also antipsychotics, improved more than placebo-treated subjects in psychopathology measured with the PANSS. In 2002, Peet and Horrobin [19] administered ethyl-EPA to patients with resistant schizophrenia for 12 weeks. One hundred and fifteen subjects, who were already in treatment with clozapine, olanzapine, risperidone, quetiapine or a neuroleptic, were randomly assigned to placebo or 1, 2 or $4 \mathrm{~g}$ /day of ethyl-EPA arm in addition to antipsychotic drugs. Authors reported a significant improvement of the PANSS total score and subscales scores in the $2 \mathrm{~g}$ /day EPA-treated group, but there was also a large effect of placebo in patients receiving only typical or new generation antipsychotics. In contrast, in patients on clozapine, there was little placebo response, but a significant effect of augmentation with ethyl-EPA on all rating scales, PANSS, PANSS subscales and the Montgomery-Asberg Depression Rating Scale (MADRS).

More recently, Jamilian and colleagues [20] compared $1 \mathrm{~g} /$ day of non-specified omega-3 with placebo in 60 patients with schizophrenia who already assumed a standard antipsychotic medication. Omega-3 outperformed placebo significantly, based on the PANSS score.

Less encouraging findings were presented by Fenton and colleagues [23] who designed a 16-week study to evaluate the efficacy of ethyl-EPA ( $3 \mathrm{~g} /$ day) vs. placebo in 87 patients with schizophrenia or schizoaffective disorder with residual symptoms in treatment with conventional antipsychotics. The results indicated no significant differences in positive or negative symptoms, mood and cognition. Similar results were reported by Berger and colleagues [24], who performed an RCT including 69 patients with first-episode psychosis who were treated for 12 weeks with a flexible dose of antipsychotic medication (risperidone, olanzapine or quetiapine) plus ethyl-EPA (E-EPA) ( $2 \mathrm{~g} /$ day) or placebo. The authors suggested that E-EPA may accelerate treatment response and ameliorate the tolerability of antipsychotics, but they remained skeptical about the specific efficacy of EPA in early psychosis.

Early treatment of the prodromal period of psychotic disorders has been linked to more favorable outcomes. The term 'ultra-high-risk' (UHR) identifies individuals who are at increased risk of developing full blown psychotic symptoms. Treatment with omega-3 HUFAs may be an interesting treatment option for UHR subjects due to the low incidence of adverse effects.

Amminger and colleagues [15] performed an RCT on 76 individuals with high risk for developing psychosis comparing omega-3 HUFAs (1.2 g/day) to placebo during a period of 12 weeks. This phase was followed by a further 40 -week monitoring period. This study showed that fatty acids may prevent the progression in psychosis in young UHR patients. Recently, in a multicenter double-blind randomized study, 304 participants meeting the 'at-risk' criteria were allocated to treatment with either omega-3 plus cognitive-behavioral case management (CBCM) 
or placebo plus CBCM. The total length of treatment was six months. The data collected are still under evaluation [16].

Another recent RCT was designed by Pawelczyk et al. [21] to compare the efficacy of $2.2 \mathrm{~g} /$ day of HUFAs or olive oil placebo added on to an antipsychotic medication with regard to symptom severity in patients suffering from first-episode schizophrenia. An improvement in psychopathology and the level of functioning was observed.

In the last few years, the first placebo-controlled trial on the association of omega-3 fatty acid and redox regulators for treating schizophrenia was conducted for 16 weeks by Bentsen and colleagues [22]. The participants were 99 patients with a diagnosis of schizophrenia or related psychoses who were divided in four groups (double placebo, active vitamins, active EPA or double active). The psychotic symptoms were assessed with PANSS. The results showed that adding E-EPA $2 \mathrm{~g} /$ day or vitamin E $364 \mathrm{mg}$. day/1 plus vitamin C $1000 \mathrm{mg} /$ day to antipsychotic drugs prevented the course of psychosis, but only among patients with low levels of erythrocyte HUFAs. On the contrary, combining EPA and the vitamins neutralized this detrimental effect. Emsley and colleagues [18] tested a combination of omega-3 polyunsaturated fatty acids (EPA $2 \mathrm{~g} /$ day and DHA $1 \mathrm{~g} /$ day) and a metabolic antioxidant, alpha-lipoic acid ( $\alpha$-LA $300 \mathrm{mg} /$ day), as prevention treatment of relapse after antipsychotic discontinuation in subjects who were successfully treated for 2-3 years after a first-episode of schizophrenia or schizoaffective disorder. Unfortunately, this study was affected by a small sample size and by premature termination of recruitment due to the high relapse rates and the severity of the relapse episodes. Finally, the results failed to support evidence that HUFAs $+\alpha$-LA could be a worthwhile tool to maintenance antipsychotic treatment in relapse prevention.

Emsley et al. [29,30] also investigated the efficacy of long-chain fatty acids in reducing side effects due to conventional antipsychotic treatment in two randomized controlled trials. In particular, they verified the effects of omega-3 fatty acids supplementation on extrapyramidal symptoms. The first study [29] found some beneficial effects of E-EPA ( $3 \mathrm{~g}$ /day) as add-on treatment in 40 patients with chronic schizophrenia, who had received a stable antipsychotic medication for at least six months on extrapyramidal symptoms assessed with the Extrapyramidal Symptoms Rating Scale. Moreover, the authors in this study also found a significant improvement of positive and negative symptoms measured with the PANSS. Unfortunately, the second study [30], disconfirmed these results in a larger sample of 77 patients with schizophrenia or schizoaffective disorders and tardive dyskinesia. In this trial, patients received E-EPA ( $2 \mathrm{~g} /$ day) or placebo for 12 weeks. The E-EPA arm had an initial improvement in dyskinesia, but this result was not stable beyond six weeks. 
Four meta-analyses [25-28] of RCTs were conducted in order to establish if the available data support the use of omega-3 fatty acids in patients affected by schizophrenia or other psychotic disorders. The authors concluded that the evidence in favor of omega-3s as psychotropic agents in schizophrenia is preliminary, and the findings remain yet inconclusive.

\subsection{Major Depressive Disorder}

In 1998, Hibbeln [31] discovered a direct and powerful inverse association between fish consumption and the prevalence of major depression in a study aimed to test the hypothesis that a high consumption of fish could be correlated with a lower annual prevalence of major depression. One year later, it was established that depression often co-occurs with cardiovascular disease, which is associated with elevated cholesterol and lower fatty acids plasma levels [32]. After this discovery, abnormal fatty acid compositions in the peripheral tissues (e.g., plasma, serum and red blood cells) of patients with depression have been reported extensively $[33,34]$.

Twenty randomized controlled trials were conducted in order to evaluate the effectiveness of omega-3, EPA and DHA, in the treatment of mild or moderate depressive disorder. Fatty acids were administered both as a monotherapy and as supplementation to ongoing pharmacotherapy or psychotherapy. Doses ranged from 0.4-4.4 g/day of EPA and from 0.2-2.4 g/day of DHA. Ten RCTs investigated the efficacy of omega-3 fatty acids as an individual treatment strategy, but only seven of them reported that EPA and/or DHA had a positive effect on core depressive symptoms. Seven RCTs aimed to determine whether administering omega-3 fatty acids provides any additional benefit to conventional patient treatment (e.g., fluoxetine, citalopram or sertraline) for major depression. Five of these studies obtained a significant improvement of depressive symptoms [35-39]. However, the validity of these findings is limited by the heterogeneity of methods among different studies, including the use of unstandardized assessment instruments of depressive symptoms, as well as considerable differences in doses and ratios of omega-3 fatty acids, the duration of the trials and the demographic and clinical characteristics of the samples. Further studies are needed to better understand the mechanisms of the antidepressant effects of HUFAs and to explain the reason for the discordant results published until now.

Peet and Horrobin [35] conducted a 12-week study that showed improvement in patients treated with $1 \mathrm{~g} /$ day of ethyl-EPA (E-EPA) and who were refractory to selective serotonin uptake inhibitor monotherapy for major depression. Findings showed that only $1 \mathrm{~g} /$ day of omega-3, but not 2 or $4 \mathrm{~g} /$ day, was effective in reducing depressive symptoms measured with the Hamilton Depression Rating Scale (HDRS), the MADRS and the Back Depression Inventory (BDI) (effect size: 0.92) in adults with ongoing depression. Nemets et al. [40] designed a four-week placebo-controlled 
study on 20 subjects already undergoing antidepressant therapy and found better outcome results in the treatment group with E-EPA at the dosage of $2 \mathrm{~g} /$ day. The effect of omega-3 was significant from the second week of treatment, similarly to the time of response to antidepressants and resulting in an improvement of core depressive symptoms on the HDRS. The same authors [41] suggested that omega-3 may have a therapeutic benefit in 6-12-year-old children with major depression. Of the 20 patients who entered data analysis, 10 received placebo and 10 received omega-3 ( $0.4 \mathrm{~g}$ /day of EPA plus $0.2 \mathrm{~g}$ /day of DHA) for 16 weeks. Depression symptoms were assessed with the Childhood Depression Rating Scale, the Childhood Depression Inventory and the Clinical Global Impression (CGI). Su and colleagues [36] conducted an eight-week RCT comparing EPA (at the dose of $4.4 \mathrm{~g}$ /day) plus DHA (at the dose of $2.2 \mathrm{~g} /$ day) with placebo in augmentation to antidepressant in 22 patients with major depression. They found encouraging results: participants who were treated with omega- 3 fatty acids had a significantly lower score of the HDRS. An interesting study on 36 pregnant women with major depressive disorder [42] compared omega-3 HUFAs monotherapy (2.2 g/day of EPA plus $1.2 \mathrm{~g} /$ day of DHA) with placebo. Twenty-four patients who finished the trial showed significantly lower depressive symptoms' ratings on the HDRS, the Edinburgh Postnatal Depression Scale (EPDS), and the BDI. The findings of this study are remarkable because omega-3s are preferentially transported to the growing fetus during pregnancy, which can deplete fatty acid levels in mothers.

The beneficial effect of omega-3 fatty acids supplementation on depressive symptoms was also confirmed in elderly women (aged 66-95 years) residents in a nursing home $[43,44]$. Within a context of an eight-week double-blind RCT, 46 women who received a diagnosis of depression were randomly assigned to the intervention group (1.67 g/day of EPA plus $0.83 \mathrm{~g} /$ day of DHA) or the placebo group. Depressive symptoms, assessed with the Geriatric Depression Scale, quality of life assessed with the Short-Form 36-Item Health Survey and phospholipids fatty acids' profile improved. Nevertheless, the same authors in another study [45] measured some immunological parameters and cytokines in the same sample, but they did not find a significant amelioration of the immunological function in the intervention group.

Currently, there is not a general consensus concerning the efficacy of omega-3s in the treatment of depression. Rees and colleagues [46] found that omega-3 fatty acids were not superior over placebo in treating perinatal depressive symptoms. They administered $0.4 \mathrm{~g}$ /day of EPA plus $1.6 \mathrm{~g}$ /day of DHA as a monotherapy to 26 pregnant women for six weeks. Similar results were collected by Freeman and colleagues [47], who did not detect significant differences between omega-3 HUFAs (EPA $1.1 \mathrm{~g} /$ day plus DHA at the dose of $0.8 \mathrm{~g} /$ day) and placebo on perinatal depressive symptoms measured with the HDRS and the EPDS. All 59 women enrolled in this trial received also a supportive psychotherapy for eight weeks. 
In a similar way, omega-3s were not found superior to placebo in treating depressive symptoms in three other studies. Marangell et al. [48], Silvers et al. [49] and Grenyer et al. [50] obtained no significant improvement in the symptoms of depression in patients who were treated with similar doses of EPA and/or DHA (ranging 2-3 g/day) as sole therapy or in addition to antidepressive drug. These studies were different for sample size, duration and instruments to assess depressive symptoms, but they drew the same conclusions.

Lespérance et al. (2011) [37] conducted an inclusive, double-blind, randomized controlled trial on 432 patients with a major depressive episode. They administered EPA (1.050 g/day) and DHA (150 mg/day) for eight weeks to a sample with a heterogeneous therapy $(40.3 \%$ of the patients already received an antidepressant at the baseline). Omega-3 supplementation resulted in being superior over placebo only for the patients without comorbid anxiety disorders.

Two studies investigated the effectiveness of omega-3s on depression and on cognitive functions. Rogers et al. [51] in a 12-week trial tested the efficacy of EPA plus DHA ( $0.6 \mathrm{~g} /$ day $+0.85 \mathrm{~g} /$ day) on mood and cognition in 218 patients with mild to moderate depression. More recently, Antypa and colleagues [52] treated 71 depressed patients with omega-3 fatty acids or placebo for four weeks. They evaluated cognitive reactivity with the Leiden Index of Depression Sensitivity-Revised (LEIDS-R) and the Profile of Mood States (POMS), and depressive symptoms were measured with the BDI. Both studies have not obtained favorable results on mood and cognition using omega-3s.

Two trials compared the effectiveness of different doses of EPA or DHA in the treatment of depressive disorders. Mischoulon and colleagues in 2008 [53] enrolled 35 depressed outpatients that were randomized into one of three double-blind dosing arms for 12 weeks: $1 \mathrm{~g}$ /day DHA; 2 g/day DHA; 4 g/day DHA. In this study, subjects who received $2 \mathrm{~g}$ /day or more DHA had a lower response rate (on the HDRS) compared to those who received $1 \mathrm{~g} /$ day. It is interesting to note that these findings are similar to Peet and Horrobin's results [35]: E-EPA, as well as DHA appear more effective at lower doses. In the same year (2008), Jazayeri and colleagues [38] compared EPA at the low dosage of $1 \mathrm{~g} /$ day with fluoxetine ( $20 \mathrm{mg} /$ day) in 60 patients with major depressive disorder for eight weeks. The results showed that a low dose of EPA may have similar therapeutic effects as fluoxetine.

A recent and larger eight-week RCT [54] compared the potential therapeutic effects of three differing doses, (i) EPA ( $1 \mathrm{~g} /$ day), (ii) DHA (1 g/day) or (iii) placebo (containing $980 \mathrm{mg}$ of soybean oil per cap; four capsules/day), in 196 depressed patients. The authors reported a significant improvement in depressive symptoms in all three groups; neither EPA nor DHA were superior to placebo.

Interesting results were obtained from one study that explored the association of omega-3 fatty acids and antidepressants in treating depressed patients [39]. 
The authors investigated the efficacy of combination therapy with citalopram plus omega-3 fatty acids $(1.8 \mathrm{~g} /$ day of EPA $+0.4 \mathrm{~g} /$ day of DHA $+0.2 \mathrm{~g} /$ day of other omega-3s) vs. citalopram plus placebo in 42 patients with initial depression. Combined therapy showed grater improvements in depression symptoms assessed with HDRS, but did not enhance the speed of antidepressant response.

The effects of fish oil supplementation were also investigated in patients with major depression associated with coronary heart disease [55]. Carney and colleagues (2009) obtained negative results testing omega-3 in cardiopathic patients. They performed a 10-week RCT to evaluate the effects of ethyl-EPA ( $0.93 \mathrm{~g} /$ day) plus DHA $(0.75 \mathrm{~g} /$ day $)$ in addition to sertraline on symptoms of depression in 122 patients with major depressive disorder and coronary heart disease. The authors did not find any superiority of treatment over placebo.

In conclusion, supplementation with HUFA in patients with major depression seems useful in improving depressive symptoms, but the findings are not univocal. Systematic reviews and meta-analyses also provided controversial conclusions. Appleton and colleagues [56] reviewed 35 RCTs that included 329 adults and concluded that trials investigating the effects of HUFAs on depression are increasing, but it is difficult to evaluate their results because of data heterogeneity. Bloch and Hannestad [57] outlined the methodological limits of available studies that affect the validity of results. On the contrary, other meta-analyses [58-60] indicated a more encouraging prospective in this field and concluded that omega- 3 fatty acids produce a significant antidepressant effect. In particular, EPA seems to be more efficacious than DHA in treating depression. A more recent comprehensive meta-analysis [61] of RCTs confirmed that the use of omega- $3 s$ as therapeutic agents was effective in patients with the diagnosis of major depression or depressive symptoms and suggested that patients with greater depressive severity and those meeting full criteria for a diagnosis of depressive disorder demonstrated greater treatment gains (Table 2). 
Table 2. Double-blind controlled trials of HUFAs in the treatment of depressive disorders. HDRS, Hamilton Depression Rating Scale; MADRS, Montgomery-Åsberg Depression Rating Scale.

\begin{tabular}{|c|c|c|c|c|}
\hline Study & Dose and Method & Sample & $\begin{array}{l}\text { Treatment } \\
\text { Duration }\end{array}$ & Results \\
\hline $\begin{array}{l}\text { Peet and } \\
\text { Horrobin, } \\
2002[35]\end{array}$ & $\begin{array}{l}\text { ethyl-EPA } 1,2 \text { or } 4 \mathrm{~g} \text { / day } \\
\text { add-on standard } \\
\text { antidepressant treatment }\end{array}$ & $\begin{array}{l}70 \text { patients resistant to } \\
\text { antidepressant } \\
\text { treatment }\end{array}$ & 12 weeks & $\begin{array}{l}\downarrow \text { depressive symptoms measured } \\
\text { with HDRS, MADRS and BDI in } \\
\text { the group treated with } \\
1 \mathrm{~g} / \text { day of HUFAs }\end{array}$ \\
\hline $\begin{array}{l}\text { Nemets et al., } \\
2002[40]\end{array}$ & ethyl-EPA 2 g/day & 20 patients & 4 weeks & $\begin{array}{l}\downarrow \text { depressive symptoms measured } \\
\text { with HDRS from the second } \\
\text { week of treatment }\end{array}$ \\
\hline $\begin{array}{l}\text { Nemets et al., } \\
2006[41]\end{array}$ & $\begin{array}{l}\text { ethyl-EPA } 0.4 \mathrm{~g} / \text { day }+ \\
\text { DHA } 0.2 \mathrm{~g} / \text { day }\end{array}$ & $\begin{array}{l}20 \text { patients } 6-12 \\
\text { years-old }\end{array}$ & 16 weeks & $\begin{array}{l}\downarrow \text { depressive symptoms measured } \\
\text { with CDRS, CDI and CGI }\end{array}$ \\
\hline $\begin{array}{l}\text { Su et al., } \\
2003[36]\end{array}$ & $\begin{array}{l}\text { ethyl-EPA } 4.4 \mathrm{~g} / \text { day }+ \text { DHA } \\
2.2 \mathrm{~g} / \text { day add-on existing } \\
\text { antidepressant treatment }\end{array}$ & 22 patients & 8 weeks & $\begin{array}{l}\downarrow \text { depressive symptoms } \\
\text { measured with HDRS }\end{array}$ \\
\hline $\begin{array}{l}\text { Su et al., } \\
2008[42]\end{array}$ & $\begin{array}{l}\text { ethyl-EPA } 2.2 \mathrm{~g} / \text { day }+ \\
\text { DHA } 1.2 \mathrm{~g} / \text { day }\end{array}$ & 36 pregnant patients & 8 weeks & $\begin{array}{l}\downarrow \text { depressive symptoms measured } \\
\text { with HDRS, EPDS and BDI }\end{array}$ \\
\hline $\begin{array}{l}\text { Rondanelli et al., } \\
2010,2011[43,44]\end{array}$ & $\begin{array}{l}\text { EPA } 1.67 \mathrm{~g} / \text { day + DHA } \\
0.83 \mathrm{~g} / \text { day add-on existing } \\
\text { antidepressant treatment }\end{array}$ & $\begin{array}{l}46 \text { elderly female } \\
\text { residents in a nursing } \\
\text { home }\end{array}$ & 8 weeks & $\begin{array}{l}\downarrow \text { depressive symptoms assessed } \\
\text { with GDS, improvement of } \\
\text { phospholipids fatty acids' profile }\end{array}$ \\
\hline $\begin{array}{l}\text { Rees et al., } \\
2008 \text { [46] }\end{array}$ & $\begin{array}{l}\text { ethyl-EPA } 0.4 \mathrm{~g} / \text { day }+ \\
\text { DHA } 1.6 \mathrm{~g} / \text { day }\end{array}$ & 26 pregnant patients & 6 weeks & $\begin{array}{l}\text { no benefits on } \\
\text { depressive symptoms }\end{array}$ \\
\hline $\begin{array}{l}\text { Freeman et al., } \\
2008 \text { [47] }\end{array}$ & $\begin{array}{l}\text { EPA } 1.1 \mathrm{~g} / \text { day }+ \\
\text { DHA } 0.8 \mathrm{~g} / \text { day }\end{array}$ & 59 women & 8 weeks & $\begin{array}{l}\text { no benefit on perinatal } \\
\text { depressive symptoms }\end{array}$ \\
\hline $\begin{array}{l}\text { Lespérance et al., } \\
2011 \text { [37] }\end{array}$ & $\begin{array}{l}\text { EPA } 1.050 \mathrm{~g} / \text { day + } \\
\text { DHA } 150 \mathrm{mg} / \text { day }\end{array}$ & $\begin{array}{l}432 \text { patients with a } \\
\text { major depressive } \\
\text { episode }\end{array}$ & 8 weeks & $\begin{array}{l}\downarrow \text { depressive symptoms only for } \\
\text { the patients without comorbid } \\
\text { anxiety disorders }\end{array}$ \\
\hline $\begin{array}{l}\text { Mischoulon et al., } \\
2008 \text { [53] }\end{array}$ & DHA 1,2 , or $4 \mathrm{~g} /$ day & 35 patients & 12 weeks & $\begin{array}{l}\text { measured in the group in } \\
\text { treatment with } 1 \mathrm{~g} / \text { day of HUFAs }\end{array}$ \\
\hline $\begin{array}{c}\text { Jazayeri et al., } \\
2008[38]\end{array}$ & $\begin{array}{l}\text { EPA } 1 \mathrm{~g} / \text { day vs. fluoxetine } \\
20 \mathrm{mg} / \text { day }\end{array}$ & 60 patients & 8 weeks & $\begin{array}{l}\downarrow \text { depressive symptoms } \\
\text { in both groups }\end{array}$ \\
\hline $\begin{array}{l}\text { Mischoulon et al., } \\
2015 \text { [54] }\end{array}$ & $\begin{array}{l}\text { EPA } 1 \mathrm{~g} / \text { day or DHA } \\
1 \mathrm{~g} / \text { day }\end{array}$ & 196 patients & 8 weeks & $\begin{array}{l}\text { EPA and DHA were not superior } \\
\text { to placebo }\end{array}$ \\
\hline $\begin{array}{l}\text { Gertsik et al., } \\
2012 \text { [39] }\end{array}$ & $\begin{array}{l}\text { EPA } 1.8 \mathrm{~g} / \text { day }+ \text { DHA } \\
0.4 \mathrm{~g} / \text { day }+ \text { other omega- } 3 \\
\text { fatty acids } 0.2 \mathrm{~g} / \text { day }+ \\
\text { citalopram vs. } \\
\text { placebo + citalopram }\end{array}$ & 42 patients & 9 weeks & $\begin{array}{l}\downarrow \text { depressive symptoms } \\
\text { measured with HDRS }\end{array}$ \\
\hline $\begin{array}{l}\text { Carney et al., } \\
2009 \text { [55] }\end{array}$ & $\begin{array}{l}\text { E-EPA ( } 0.93 \mathrm{~g} / \text { day }) \text { plus } \\
\text { DHA ( } 0.75 \mathrm{~g} / \text { day }) \text { on } \\
\text { depression in addition } \\
\text { to sertraline }\end{array}$ & $\begin{array}{l}122 \text { patients with } \\
\text { major depression } \\
\text { associated with } \\
\text { coronary heart disease }\end{array}$ & 10 weeks & $\begin{array}{l}\text { EPA and DHA were not } \\
\text { superior to placebo }\end{array}$ \\
\hline
\end{tabular}

Abbreviations: $\mathrm{EPA}=$ eicosapentaenoic acid; DHA $=$ docosahexaenoic acid; ethyl-EPA = ethyl-eicosapentaenoic acid; CDRS = Childhood Depression Rating Scale; $\mathrm{CDI}=$ Childhood Depression Inventory; EPDS = Edinburgh Postnatal Depression Scale; GDS $=$ Geriatric Depression Scale; $\downarrow=$ decrease of; $\uparrow=$ increase of.

\subsection{Bipolar Disorders}

In patients with a diagnosis of bipolar disorder, lower erythrocyte membrane levels of omega-3 fatty acids have been observed [62]. Based on this consideration, 
several investigators performed clinical trials to evaluate whether the implementation of these agents may impact the clinical features of this disorder. To date, only seven RCTs on omega-3 treatment for bipolar disorders have been published. Except for the first pioneering trial, they were all augmentation studies. Intervention length ranged from 6-16 weeks. Three of these [63-65] used EPA in addition to stable psychotropic medication. Two studies $[66,67]$ explored the combination of EPA and DHA in addition to mood stabilizers or other usual treatments. In one trial [68], alpha-linolenic acid was administered in association with stable psychotropic medications. Finally, in one add-on RCT [69], omega-3 was associated with cytidine or not as augmentation to a stable medication. Doses ranged from 0.3-6.2 g/day of EPA and from 0.2-3.4 g/day of DHA. The dose of alpha-linolenic acid was $6.6 \mathrm{~g} /$ day.

In the first trial, Stoll and colleagues [66] administered a combination of EPA (6.2 g/day) and DHA (3.4 g/day) as a monotherapy to 14 patients, while 16 patients received placebo for 16 weeks. This study was affected by a large number (13 subjects) of drop-outs because of depression, mania, hypomania and mixed state. Outcomes revealed the efficacy of omega- 3 in terms of the reduction and remission of depressive symptoms (HDRS), whereas no significant effects were registered on mania (Young Mania Rating Scale (YMRS)). These findings were replicated by Frangou and colleagues [63] in a 12-week controlled study including 75 patients treated with ethyl-EPA ( 1 or $2 \mathrm{~g} /$ day) as adjunctive treatment to stable psychotropic medications. The same authors [64] reported increased brain levels of $N$-acetyl-aspartate (NAA), a presumed marker of neuronal integrity, with $2 \mathrm{~g}$ /day of ethyl-EPA in 14 female patients with type I bipolar disorder that were treated with a stable dose of lithium. The study was controlled with placebo. The rise in NAA level provided the first evidence for a neurotrophic role of E-EPA treatment in bipolar disorder.

Concerning the manic phase, less encouraging findings were presented by Chiu et al. [67], who designed a four-week study to evaluate the efficacy of $4.4 \mathrm{~g} /$ day of EPA plus $2.4 \mathrm{~g} /$ day of DHA vs. placebo in addition to a fixed dose of valproate $(20 \mathrm{mg} / \mathrm{kg} /$ day) in 15 patients with acute mania. The authors found a reduction in both groups of the YMRS scores from baseline, with no significant differences between omega-3 and placebo. Similar results were obtained in a larger RCT [65], which involved 121 patients with bipolar depression or rapid cycling bipolar disorder. The adjunction of $6 \mathrm{~g} /$ day of EPA to at least one mood stabilizer did not produce meaningful differences in reducing the severity of depressive or manic symptoms, assessed respectively with the Inventory for Depressive Symptomology total and the YMRS.

Gracious and colleagues [68] conducted a double-blind RCT of alpha-linoleic acid (ALA) as flax seed oil in children and adolescents with bipolar I or II disorder, assuming a stable psychotropic medication. In this study, there was a high rate of 
noncompliance with taking supplements because of the large number of capsules required (up to 12 per day). Those who were treated with an adequate amount of ALA, demonstrated a significant improvement of overall symptom severity in comparison with the placebo group. Nevertheless, depression and mania measures did not show significant differences between treatment and placebo groups. More recently, therapeutic properties of omega-3 fatty acids in bipolar disorder were further denied by Murphy et al. [69] in a four-month RCT. Forty-five patients with a diagnosis of type I bipolar disorder, who were already treated with a mood stabilizer, were randomly assigned to three different groups consisting of omega-3 fatty acids plus cytidine, omega- 3 fatty acid plus placebo or only placebo. In this study, supplementation with omega-3 did not produce a significant improvement in affective symptoms over an extended period of treatment.

Overall, a moderate antidepressant effect was observed for adjunctive omega-3 agents compared to conventional therapy alone in the treatment of bipolar depression [66,67]. The small number of studies, the heterogeneity of HUFA doses and ratios and the small sample sizes were important limitations to obtain reliable data on this topic.

In summary, some beneficial effects of omega-3 HUFAs in bipolar disorders were observed. The conclusions of systematic reviews and meta-analyses [70-73] provided initial evidence that bipolar depressive symptoms, but not manic symptoms, may be improved by adjunctive administration of omega-3 fatty acids (Table 3 ).

\subsection{Anxiety Disorders}

The hypothesis that omega-3s may have anxiolytic properties was formulated in the light of the frequent comorbidity between mood disorders and anxiety disorders and the effectiveness of some conventional pharmacotherapy on both disorders. Furthermore, low omega-3 erythrocyte membrane levels have been observed in patients with anxiety disorders [74-76]. Unfortunately, to our knowledge, there are no RCTs that have systematically investigated the effect of omega-3 PUFA in anxiety disorders. Only a small trial [77] showed a reduction in the levels of anxiety and tension in 24 substance abusers. Participants received $2.2 \mathrm{~g}$ of EPA/day plus $0.5 \mathrm{~g}$ of DHA/day vs. placebo (vegetable oil) for three months. Anxiety continued to be significantly decreased in the active treatment group after three months' discontinuation. In each case, the evidence supporting the use of omega-3 PUFAs as an anxiolytic is currently insufficient [78]. 
Table 3. Double-blind controlled trials of HUFAs in the treatment of bipolar disorders (depressive and maniac phases).

\begin{tabular}{|c|c|c|c|c|}
\hline Study & Dose and Method & Sample & $\begin{array}{l}\text { Treatment } \\
\text { Duration }\end{array}$ & Results \\
\hline $\begin{array}{l}\text { Stoll et al., } \\
1999[66]\end{array}$ & $\begin{array}{l}\text { EPA } 6.2 \mathrm{~g} / \text { day }+ \\
\text { DHA } 3.4 \mathrm{~g} / \text { day }\end{array}$ & 30 patients & 16 weeks & $\begin{array}{l}\downarrow \text { depressive symptoms } \\
\text { measured with HDRS }\end{array}$ \\
\hline $\begin{array}{l}\text { Frangou et al., } \\
2006[63]\end{array}$ & $\begin{array}{l}\text { ethyl-EPA } 1 \text { or } 2 \mathrm{~g} / \text { day } \\
\text { add-on to stable psychotropic } \\
\text { medications }\end{array}$ & 75 patients & 12 weeks & $\begin{array}{l}\downarrow \text { depressive symptoms } \\
\text { measured with HDRS }\end{array}$ \\
\hline $\begin{array}{l}\text { Chiu et al., } \\
2003 \text { [67] }\end{array}$ & $\begin{array}{l}\text { EPA } 4.4 \mathrm{~g} / \text { day + DHA } 2.4 \\
\text { g/day vs. placebo in addition } \\
\text { to valproate } 20 \mathrm{mg} / \mathrm{kg} / \text { day }\end{array}$ & $\begin{array}{l}15 \text { patients with } \\
\text { acute mania }\end{array}$ & 4 weeks & $\begin{array}{l}\text { no significant differences } \\
\text { between omega- } 3 \text { fatty acids } \\
\text { and placebo }\end{array}$ \\
\hline $\begin{array}{l}\text { Keck et al., } \\
2006[65]\end{array}$ & $\begin{array}{l}\text { EPA } 6 \mathrm{~g} / \text { day in addition to at } \\
\text { least one mood stabilizer }\end{array}$ & $\begin{array}{l}121 \text { patients with } \\
\text { bipolar depression or } \\
\text { rapid cycling } \\
\text { bipolar disorder }\end{array}$ & 4 months & no significant differences \\
\hline $\begin{array}{l}\text { Gracious et al., } \\
\quad 2010[68]\end{array}$ & $\begin{array}{l}\text { ALA in addition to } \\
\text { psychotropic medication }\end{array}$ & $\begin{array}{l}\text { children and } \\
\text { adolescent with } \\
\text { bipolar I or II disorder }\end{array}$ & 16 weeks & $\begin{array}{l}\text { significant improvement of } \\
\text { overall symptom severity in } \\
\text { comparison with } \\
\text { placebo group }\end{array}$ \\
\hline $\begin{array}{l}\text { Murphy et al., } \\
2012 \text { [69] }\end{array}$ & $\begin{array}{l}\text { omega- } 3 \text { fatty acids plus } \\
\text { cytidine, omega- } 3 \text { fatty acid } \\
\text { plus placebo or only placebo in } \\
\text { addition to a mood stabilizer }\end{array}$ & $\begin{array}{l}45 \text { patients with type I } \\
\text { bipolar disorder }\end{array}$ & 4 months & $\begin{array}{l}\text { no benefits of omega- } 3 \text { fatty } \\
\text { acids on affective symptoms }\end{array}$ \\
\hline
\end{tabular}

Abbreviations: EPA $=$ eicosapentaenoic acid; DHA $=$ docosahexaenoic acid; ethyl-EPA $=$ ethyl-eicosapentaenoic acid; $\mathrm{ALA}=\alpha$-linoleic acid; $\downarrow=$ decrease of; $\uparrow=$ increase of.

\subsection{Obsessive-Compulsive Disorder}

Only one RCT about obsessive-compulsive disorder was published by Fux and colleagues [79], who administered $2 \mathrm{~g}$ /day of EPA or placebo in augmentation of a stable dose of SSRIs to 11 patients (four of these received $40 \mathrm{mg}$; two received $30 \mathrm{mg}$; and two received $60 \mathrm{mg}$ of paroxetine daily; one patient was on $250 \mathrm{mg}$ of fluvoxamine daily; and two were on $40 \mathrm{mg}$ of fluoxetine daily) for six weeks. The augmentation with HUFAs was not associated with significant improvement of anxious, obsessive-compulsive and depressive symptoms compared to placebo.

\subsection{Attention Deficit Hyperactivity Disorder}

Increasing attention has been given to the role of HUFAs in childhood developmental disorders. Since the 1980s, reduced HUFA levels have been reported in blood analysis of hyperactive children compared to healthy controls [80-83]. Recently, several double-blind placebo controlled trials have been conducted to assess the efficacy of omega-3 fatty acid in the treatment of children with ADHD [84-95], but results are still discordant and disputable. Due to the considerable heterogeneity of published investigations, additional large-cohort studies and well-designed clinical trials of ADHD patients are required. In particular, studies should be conducted with 
strict criteria concerning methodological issues, such as an accurate DSM-5 diagnosis of ADHD, a double-blind controlled design, the choice of reliable assessment instruments of symptoms and functioning, a clear definition of the doses and ratios of omega- 3 fatty acids and of the possible association with conventional medications.

If we examine the available data, seven [84,85,87-90] of thirteen RCTs investigating the efficacy of omega-3 fatty acids had a positive effect on ADHD-related symptoms. The remaining studies did not obtain significant improvement in ADHD symptoms [81,91-95]. Only two of these thirteen RCTs aimed to determine whether taking omega-3 fatty acids confers any additional benefit to conventional patient treatment (stimulant and non-stimulant medications): one found negative results [91] and one positive [90].

In particular, not very encouraging findings have been presented by six RCTs comparing the association of EPA and DHA to placebo. In a double-blind study performed by Stevens and colleagues [81], fifty patients with ADHD-like symptoms received either placebo (olive oil) or HUFA supplementation (480 $\mathrm{mg}$ of DHA, $80 \mathrm{mg}$ of EPA, $40 \mathrm{mg}$ of arachidonic acid and $96 \mathrm{mg}$ of gamma-linolenic acid/day) for four months. At baseline and at the end of the intervention period, both parents and teachers completed the Conners' Abbreviated Symptom Questionnaires (ASQ) and the Disruptive Behavior Disorders (DBD) Rating Scale. Other additional neuropsychological tests were administered: the Conners' Continuous Performance Test (CPT) and eight tests of cognitive ability of the Woodcock-Johnson Psycho-Educational Battery-Revised (WJ-R). No significant difference between active group and placebo was observed for any rating scale comparing patients who completed the trial. HUFAs supplementation led to a significant behavioral improvement over placebo on only two of the 16 outcome measures that were used (DBD-Conduct for Parents and the DBD-Attention for Teachers) and by intention-to-treat analysis only.

In 2004, Hirayama and colleagues [92] also reported no evidence of efficacy of omega-3 fatty acids compared to placebo in treating ADHD symptoms. In this double-blind placebo controlled trial, the majority of the 40 children did not receive ADHD medications (only six of them had been under conventional medications). They administered both DHA and EPA through fish oil-enriched food (the daily dose was approximately $100 \mathrm{mg}$ of EPA and $500 \mathrm{mg}$ of DHA) to twenty children with ADHD for two months. The control group $(n=20)$ took indistinguishable food without fish oil. The authors measured ADHD related symptoms according to the DSM-IV criteria, aggression, impatience and some cognitive features, but they did not find any significant changes in outcome measures.

In another study (Johnson and colleagues) [93], seventy-five children and adolescents 8-18 years old with ADHD were included and treated with $558 \mathrm{mg}$ EPA, $174 \mathrm{mg}$ DHA and $60 \mathrm{mg}$ gamma linoleic acid daily, compared to placebo. 
Only one of the patients had been previously treated with a conventional drug for ADHD (methylphenidate). Investigators found that only a subgroup of patients characterized by the inattentive subtype of ADHD and associated neurodevelopmental disorders showed a meaningful clinical response to omega-3 and omega- 6 treatment. They concluded the study results were essentially negative and did not support the superiority of HUFAs over placebo.

Milte [94] performed a double-blind RCT, including 90 children 7-12 years old with ADHD treated with EPA-rich oil (providing $1109 \mathrm{mg}$ of EPA and $108 \mathrm{mg}$ of DHA), DHA-rich oil (providing $264 \mathrm{mg}$ of EPA and $1032 \mathrm{mg}$ of DHA) vs. an omega-6 HUFA oil during a period of four months. Children were taking no other medication. Despite that this study demonstrated no statistically-significant differences between the two groups, the authors found that increased levels of erythrocyte DHA seemed associated with improved word reading and lower parent ratings of oppositional behavior. Interestingly, a subgroup of 17 patients with learning difficulties exhibited superior benefits from the supplementation with omega-3 fatty acids.

A more recent randomized, double-blind controlled trial (Widenhorn-Müller et al.) [95] was conducted in 95 ADHD patients aged between six and 12 years who received omega- 3 fatty acids or placebo for 16 weeks, not treated with medications for ADHD. The authors found less negative results than those from previous studies, but not yet satisfactory. Supplementation with EPA and DHA (600 mg of EPA and $120 \mathrm{mg}$ of DHA daily) improved working memory function, but had no effect on other cognitive measures or behavioral symptoms in the study population.

Only one study concerning the use of DHA has been reported: using a randomized, double-blind design, Voigt and colleagues [91] tested the effect of $345 \mathrm{mg}$ /day of DHA for four months upon 63 children (6-12 years old) with ADHD, all receiving maintenance therapy with stimulant medication. Despite blood phospholipid DHA content being increased in the active treatment group, there was no statistically-significant improvement in any ADHD symptoms compared to placebo.

On the other hand, some investigations have provided more promising findings. In particular, interesting results have been obtained by the seven RCTs in which EPA and DHA were administered to ADHD patients and compared to placebo.

Richardson and colleagues [84] published a pilot double-blind RCT investigating the efficacy of the combination of omega-3 and omega- 6 fatty acids (daily dose of $186 \mathrm{mg}$ of EPA, $480 \mathrm{mg}$ of DHA, $864 \mathrm{mg}$ of linolenic acid and $42 \mathrm{mg}$ of arachidonic acid) vs. placebo in 41 children with ADHD-related symptoms and specific learning disabilities. ADHD-type symptoms were assessed using the Conners's Teacher Rating Scale. After 12 weeks of treatment, mean scores for cognitive problems and general behavior improved more in the group treated with HUFAs than placebo. 
More recently, these findings were replicated by the same authors [85]. They conducted a larger double-blind placebo controlled RCT, including 117 children diagnosed with Developmental Coordination Disorder (DCD), which is characterized by deficit of motor functions and shows substantial overlap with ADHD in terms of difficulties with organizational skills and attention. The active treatment provided a high ratio of omega- 3 and omega- 6 fatty acids for three months. Despite no effects being reported on motor skills, HUFAs dietary supplementation led to improvement of reading, spelling and behavior in the treatment group compared to placebo. Subsequently, a one-way, uncontrolled crossover to active supplement followed for a further three months: similar changes were observed in the placebo-active group, while the original treatment group's scores continued to improve.

Sinn and Bryan (2008) on a large sample included 132 children aged 7-12 years with a diagnosis of ADHD [86]. Participants were enrolled in a 15-week double-blind controlled trial and were treated with $93 \mathrm{mg}$ of EPA, $29 \mathrm{mg}$ of DHA and $10 \mathrm{mg}$ of gamma-linolenic acid daily, with or without a micronutrient supplement, and were compared to placebo. Children were not treated with ADHD medications. The results outlined that both groups treated with HUFAs improved more than placebo subjects in some core ADHD symptoms, such as inattention, hyperactivity and impulsivity. These results were confirmed by the same authors (Sinn and Bryan) [87] in a 15-week crossover study.

Bélanger et al. (2009), in a Canadian double-blind, one-way, crossover randomized trial [88], measured the impact on ADHD of omega-3 HUFAs, using equivalent quantities of omega-6 HUFAs (sunflower oil) as the control condition. Thirty seven subjects were enrolled, but only 26 children succeeded in completing the study. Participants did not receive any other medication. They were divided into two groups (A and B) and participated in a 16-week, double-blind, one-way, crossover randomized study. In the first phase, Group A received the $n-3$ HUFA supplement, and Group B received placebo. During the second phase, Group B received the active $n$-3 HUFA supplement that was continued in Group A. In the first phase of the study (Weeks 0-15), a meaningful improvement in inattention and global ADHD symptoms emerged in patients who received omega-3 treatment $(20 \mathrm{mg} / \mathrm{kg} /$ day-25 $\mathrm{mg} / \mathrm{kg} /$ day of EPA and $8.5 \mathrm{mg} / \mathrm{kg} /$ day $-10.5 \mathrm{mg} / \mathrm{kg} /$ day of DHA). These positive results were maintained during the second phase (the treatment crossover, Weeks 16-30) but did not reach in this phase statistical significance.

Kirby et al. [89] have assessed the effects of the administration of $0.8 \mathrm{~g} /$ day fish oil (including $400 \mathrm{mg}$ of DHA and $56 \mathrm{mg}$ of EPA/day) on 450 healthy school-children. After a period of 16 weeks, the plasma ratio omega-6/omega-3 was measured, and patients were submitted to a series of cognitive tests to assess IQ, reading, language and writing skills, attention, working memory, impulsivity and hyperactivity. This RCT showed a significant improvement in impulsivity, evaluated 
with the Matching Familiar Figures Task (MFFT), handwriting and attentional capacity, evaluated with the Computerized Penmanship Evaluation Tool (COCOM) and a possible protective effect of omega-3s on behavioral dysregulation, compared to placebo.

In accordance with these findings, a six-month randomized double-blind controlled trial by Perera [90] indicated the efficacy of supplementation with a combined omega-3 and omega-6 preparation vs. placebo in 98 children with ADHD, refractory to methylphenidate treatment (all participants continued taking immediate-release methylphenidate during the study). In particular, omega-3 (600 mg/day) combined with omega-6 (360 mg/day) improved behavior and learning in restlessness, aggressiveness, completing work and academic performance, but not in inattention, impulsiveness and cooperation with parents and teachers.

A comprehensive meta-analysis conducted by Bloch et al. [96] of omega-3 fatty acids supplementation in children with ADHD found a small to modest effect size for EPA at high doses. Another recent meta-analysis conducted by Sonuga-Barke et al. [97] concluded that free fatty acids (omega-3 supplements, omega- 6 supplements and both omega- 3 and omega- 6 supplements) produce small, but significant reductions in ADHD symptoms. Besides, it provides evidence to justify the use of omega-3 fatty acids for ADHD as a supplement to other empirically-supported therapies. Gillies et al. [98] suggested that the efficacy of omega-3 fatty acids is supported only in combination with omega- 6 fatty acids. A recent review [99] found that randomized clinical trials with omega-3 HUFAs have reported small-to-modest effects in reducing symptoms of ADHD in children and that available findings need to be replicated in future investigations of nonpharmacological interventions in clinical practice (Table 4).

\subsection{Autism Spectrum Disorders}

The efficacy of HUFAs as a treatment consideration has also been taken into account in other developmental disorders, such as autism spectrum disorder [100,101]. There is some evidence to suggest that autism may involve a cellular functional deficiency or imbalance of omega-3 [100-102]. Studies focused on the deficit in the concentration of HUFAs complexed to membrane phospholipids in children with autism showed controversial results. Vancassel and colleagues [102] examined the concentration of fatty acids in plasma in a population of children with autism and in another group of children with learning disabilities. This study reported a $23 \%$ reduction of DHA levels in the group with autism compared to the control group, a reduction in the erythrocytes membrane concentration of omega-3 and a concomitant increase in the levels of saturated fatty acids [100,101]. 
Table 4. Double-blind controlled trials of HUFAs in the treatment of ADHD.

\begin{tabular}{|c|c|c|c|c|}
\hline Study & Dose and Method & Sample & $\begin{array}{l}\text { Treatment } \\
\text { Duration }\end{array}$ & Results \\
\hline $\begin{array}{l}\text { Voigt et al., } \\
2001 \text { [91] }\end{array}$ & $\begin{array}{l}\text { DHA } 345 \text { mg/day vs. } \\
\text { placebo; with ADHD } \\
\text { medication }\end{array}$ & $\begin{array}{l}63 \text { children } \\
\text { (6-12 years old) } \\
\text { with ADHD } \\
\end{array}$ & 4 months & $\begin{array}{l}\text { no statistically-significant } \\
\text { improvement in any ADHD } \\
\text { symptoms compared to placebo }\end{array}$ \\
\hline $\begin{array}{c}\text { Richardson et al., } \\
2002[84]\end{array}$ & $\begin{array}{l}\text { EPA } 186 \mathrm{mg} \cdot \mathrm{g} / \text { day }+ \\
\text { DHA } 480 \mathrm{mg} / \text { die }+ \\
\text { linolenic acid } 864 \mathrm{mg} / \text { die } \\
+ \text { arachidonic acid } 42 \\
\text { mg/die vs. placebo }\end{array}$ & $\begin{array}{l}41 \text { children with } \\
\text { ADHD-like } \\
\text { symptoms }\end{array}$ & 12 weeks & $\begin{array}{l}\text { mean scores for cognitive } \\
\text { problems and general behavior } \\
\text { improved more in the group } \\
\text { treated with HUFAs than placebo }\end{array}$ \\
\hline $\begin{array}{l}\text { Stevens et al., } \\
2003 \text { [81] }\end{array}$ & $\begin{array}{l}\text { DHA } 480 \mathrm{mg} / \text { day + EPA } \\
80 \mathrm{mg} / \text { day + arachidonic } \\
\text { acid } 40 \mathrm{mg} / \text { day + } \\
\text { gamma-linolenic acid } 96 \\
\text { mg/day vs. placebo; no } \\
\text { ADHD medications }\end{array}$ & $\begin{array}{l}50 \text { children with } \\
\text { ADHD-like } \\
\text { symptoms }\end{array}$ & 4 months & $\begin{array}{l}\text { no significant difference between } \\
\text { active group and placebo was } \\
\text { observed for any rating scale } \\
\text { comparing patients who } \\
\text { completed the trial }\end{array}$ \\
\hline $\begin{array}{c}\text { Hirayama et al., } \\
2004 \text { [92] }\end{array}$ & $\begin{array}{l}\text { EPA } 100 \mathrm{mg} / \text { die + DHA } \\
500 \mathrm{mg} / \text { die vs. placebo; } \\
\text { mostly without ADHD } \\
\text { medications (only six } \\
\text { subjects had been under } \\
\text { medications) }\end{array}$ & $\begin{array}{l}40 \text { children with } \\
\text { ADHD }\end{array}$ & 2 months & $\begin{array}{l}\text { no evidence of the efficacy of } \\
\text { omega- } 3 \text { fatty acids compared to } \\
\text { placebo }\end{array}$ \\
\hline $\begin{array}{c}\text { Johnson et al., } \\
2009 \text { [93] }\end{array}$ & $\begin{array}{l}\text { EPA } 558 \mathrm{mg} / \text { die + DHA } \\
174 \mathrm{mg} / \text { die + gamma } \\
\text { linoleic acid } 60 \mathrm{mg} / \text { die vs. } \\
\text { placebo; only one patient } \\
\text { with ADHD medication }\end{array}$ & $\begin{array}{l}75 \text { children and } \\
\text { adolescents } 8-18 \\
\text { year old with } \\
\text { ADHD }\end{array}$ & 3 months & $\begin{array}{l}\text { no evidence of the efficacy of } \\
\text { omega- } 3 \text { fatty acids compared to } \\
\text { placebo }\end{array}$ \\
\hline $\begin{array}{c}\text { Bélanger et al., } \\
2009 \text { [88] }\end{array}$ & $\begin{array}{l}\text { EPA } 20-25 \mathrm{mg} / \mathrm{kg} / \mathrm{die}+ \\
\text { DHA } 8.5-10.5 \mathrm{mg} / \mathrm{kg} / \text { day } \\
\text { vs. placebo; no ADHD } \\
\text { medications }\end{array}$ & 26 children & 16-week & $\begin{array}{l}\text { improvement in inattention and } \\
\text { global ADHD symptoms only in } \\
\text { the first phase of the study (Weeks } \\
0-15 \text { ) }\end{array}$ \\
\hline $\begin{array}{l}\text { Milte et al., } \\
2012 \text { [94] }\end{array}$ & $\begin{array}{l}\text { EPA-rich oil (providing } \\
\text { EPA } 1109 \text { mg and DHA } \\
108 \text { mg), DHA-rich oil } \\
\text { (providing EPA } 264 \mathrm{mg} \\
\text { and DHA } 1032 \mathrm{mg} \text { ) vs. an } \\
\text { omega-6 HUFAs oil; no } \\
\text { ADHD medications }\end{array}$ & $\begin{array}{l}90 \text { children } \\
\text { (7-12 year old) } \\
\text { with ADHD }\end{array}$ & 4 months & $\begin{array}{l}\text { no statistically-significant } \\
\text { differences between the two } \\
\text { groups }\end{array}$ \\
\hline $\begin{array}{l}\text { Widenhorn-Müller } \\
\text { et al., } 2014 \text { [95] }\end{array}$ & $\begin{array}{l}\mathrm{EPA} 600 \mathrm{mg} / \mathrm{die}+\mathrm{DHA} \\
120 \mathrm{mg} / \mathrm{die} ; \text { no ADHD } \\
\text { medications }\end{array}$ & $\begin{array}{l}95 \text { children } \\
(6-12 \text { years }) \\
\text { with ADHD }\end{array}$ & 16 weeks & $\begin{array}{l}\text { improved working memory } \\
\text { function, but no effect on other } \\
\text { cognitive measures or behavioral } \\
\text { symptoms in the study } \\
\text { population }\end{array}$ \\
\hline $\begin{array}{c}\text { Sinn and Bryan, } \\
2008 \text { [87] }\end{array}$ & $\begin{array}{l}\text { EPA } 93 \text { mg/day + DHA } 29 \\
\text { mg/day + } \\
\text { gamma-linolenic acid } 10 \\
\text { mg/day vs. placebo; no } \\
\text { ADHD medications }\end{array}$ & $\begin{array}{l}132 \text { children } \\
(7-12 \text { years }) \\
\text { with ADHD }\end{array}$ & & $\begin{array}{l}\text { improved in inattention, } \\
\text { hyperactivity and impulsivity in } \\
\text { most ADHD scales in parents' } \\
\text { reports; no improvement in } \\
\text { teacher reports; limits: no ADHD } \\
\text { diagnosis (reported ADHD } \\
\text { symptoms) }\end{array}$ \\
\hline
\end{tabular}


Table 4. Cont.

\begin{tabular}{|c|c|c|c|c|}
\hline Study & Dose and Method & Sample & $\begin{array}{l}\text { Treatment } \\
\text { Duration }\end{array}$ & Results \\
\hline $\begin{array}{l}\text { Perera et al., } \\
2012 \text { [90] }\end{array}$ & $\begin{array}{l}\text { omega- } 3+\text { omega- } 6 \text { vs. } \\
\text { placebo; with ADHD } \\
\text { medications }\end{array}$ & $\begin{array}{c}98 \text { children } \\
\text { (6-12 years) with } \\
\text { ADHD diagnosis }\end{array}$ & 6 months & $\begin{array}{l}\text { improved behavior and learning } \\
\text { in restlessness, aggressiveness, } \\
\text { completing work and academic } \\
\text { performance, but not in } \\
\text { inattention, impulsiveness and } \\
\text { cooperation with parents } \\
\text { and teachers }\end{array}$ \\
\hline Kirby et al. [89] & $\begin{array}{l}\text { DHA } 400 \text { mg/day + EPA } \\
56 \text { mg/day; no ADHD } \\
\text { medications }\end{array}$ & $\begin{array}{l}450 \text { healthy } \\
\text { school-children }\end{array}$ & 16 weeks & $\begin{array}{l}\text { significant improvement in } \\
\text { impulsivity, handwriting and } \\
\text { attentional capacity and a possible } \\
\text { protective effect of omega-3 on } \\
\text { behavioral dysregulation, } \\
\text { compared to placebo }\end{array}$ \\
\hline
\end{tabular}

Abbreviations: EPA = eicosapentaenoic acid; DHA = docosahexaenoic acid.

In addition, some evidence showed that omega-3 may improve the course of chronic inflammatory diseases [103], frequently associated with autism and potentially related to its pathophysiology [104].

Considering that data collected from uncontrolled studies are affected by severe limitations and that only three RCTs are available, evidence is insufficient at the moment to determine whether HUFAs are effective for autism spectrum disorder. Actually, only one study [105] supported HUFAs supplementation to treat autism-related symptoms, while the remaining did not find any positive effects [106,107].

The first RCT is a double-blind, randomized, placebo-controlled, pilot study, performed by Amminger in 13 children (aged 5-17 years) with autistic disorder [105]. After six weeks, the group treated with EPA $(840 \mathrm{mg} /$ day) and DHA (700 mg/day) showed an improvement of hyperactivity and stereotyped behaviors.

The second double-blind, placebo-controlled trial was conducted by Voigt [106] and presented different conclusions. In particular, the authors did not show any improvement in core symptoms of autism after a dietary DHA supplementation of $200 \mathrm{mg} /$ day for six months in a group of 48 children with autism.

The third randomized, double-blind, placebo-controlled trial was conducted by Mankad et al. [107]. They designed a six-month trial of omega-3 fatty acid supplementation (1.5 g of EPA and DHA/day) in comparison with placebo in 38 children ( $2-5$ years old) with autism. This study did not support the hypothesis that high dose supplementation of HUFAs in children with autism provides any efficacy in terms of improvement of core symptom domains or adaptive function.

Considering the scarcity of data in this field, we also reported the results from one case-report and one open-label study performed in patients with autism. The case report study [108] showed an improvement of symptoms in a child suffering from 
autism following treatment with EPA in the first time administered at a dose of $1 \mathrm{~g} /$ day, then at a dose of $3 \mathrm{~g} /$ day, for a period of four weeks. During the eight-month follow-up phase, the improvements were maintained. The open-label study [109] carried out on 20 autistic children reported a significant improvement of the disease after three months of treatment with omega-3, omega- 6 and omega-9 at a dose of $1 \mathrm{~g} /$ day (1 g/day).

To date, one systematic review [110] about the efficacy of HUFAs in the treatment of autism spectrum disorders is available. The conclusions showed that the omega-6/omega-3 fatty acid ratio's alteration during early life can affect major processes in brain development and induces aberrant behavior. Thus, changes in dietary omega-6/omega-3 supplies may contribute to reducing the incidence of symptoms related to autism. So far, the studies are still few and provided limited results; therefore, further investigations on a larger population are required to draw conclusions in this field.

\subsection{Aggression, Hostility and Impulsivity}

In the last two decades, growing attention has been given to the potential role of omega-3 in clinical conditions characterized by high impulsivity, hostility and aggressive behaviors [5]. The discovery of low levels of EPA and DHA in the central nervous system of patients with impulsive-aggressive, self-harm and parasuicidal behaviors [111,112] has encouraged the investigators to perform trials on omega-3 implementation in this clinical population characterized by a high level of impulsivity and aggression with favorable results in terms of mood symptoms and control of impulsivity [113,114].

Two randomized, double-blind, placebo-controlled trials have been performed to analyze the effects of HUFA supplementation in populations without psychiatric diagnosis on aggression and impulsivity. Both showed positive results, but available data are limited by the heterogeneity of the study criteria. The first RCT [113] was conducted by Hamazaki in a group of 41 healthy controls (21-30 years old) monitoring the episodes of aggressive behaviors and cognitive functions during three months. Participants daily received 1.5-1.8 g of DHA or placebo. Aggression toward others significantly increased in the control group, while it decreased in the DHA supplement group. No significant differences were observed on cognitive functions. Another trial was conducted by Itomura et al. (2005) in 166 healthy school children 9-12 years old in order to investigate whether fatty acid nutrition may affect physical aggression [114]. Eighty-three children received a fish oil-fortified diet, providing a daily intake of $3.6 \mathrm{~g}$ of DHA and $0.84 \mathrm{of}$ EPA for three months. Aggression against others and impulsivity were significantly decreased in the fish oil group, especially in girls. 
In a six-week RCT, Bradbury and colleagues (2004) [115] investigated the possible role of DHA in improving adaptation to perceived stress in a group of 47 healthy controls (18-60 years). They found a significant reduction in stress for both the fish oil (1.5 g/day of DHA) and the placebo groups, but the stress reduction for the fish oil group was significantly superior to that in the no-treatment controls. The fish oil group obtained a more substantial stress reduction than the olive oil group, but the differences between treatments did not reach statistical significance.

\subsection{Borderline Personality Disorder}

The effects of omega-3 fatty acids supplementation has been studied in patients with personality disorders, who often show high levels of impulsive-behavioral dyscontrol and aggressiveness. Only two RCTs with a double-blind, randomized, placebo-controlled design have been conducted [116,117]. Both indicated the efficacy of HUFAs on borderline personality disorder (BPD) core symptoms, although they were different in diagnostic criteria, contemporary administration of conventional medications and the doses and ratios of omega- 3 fatty acids.

Zanarini and Frankenburg [116] performed the first RCT in a group of 30 female patients with a diagnosis of BPD who were treated for eight weeks with $1 \mathrm{~g} /$ day of E-EPA or placebo and without any other psychotropic medication. The results showed a significant effect of E-EPA on aggressive behaviors measured with the Modified Overt Aggression Scale (MOAS) and on depressive symptoms assessed with the Montgomery-Åsberg Depression Rating Scale (MADRS) compared to placebo. In another study, published by Hallahan and colleagues [117], 49 patients with self-defeating behaviors (39 patients had received a diagnosis of BPD) were enrolled. Twenty-seven patients were randomly assigned to placebo, and 22 were treated with EPA at the dose of $1.2 \mathrm{~g} /$ day and DHA at the dose of $0.9 \mathrm{~g} /$ day for 12 weeks. Omega-3s were added to the standard psychiatric therapies. This RCT showed a significant improvement of affect (measured with the Beck Depression Inventory and the Hamilton for Depression Rating Scale (HDRS)), parasuicidal behaviors and stress reactivity in the treatment group. Aggressiveness, measured with the MOAS score, and impulsive behaviors, assessed with the Memory Delay Task, did not obtain a significant difference in the two groups.

On the basis of this background, our research group performed a RCT in order to assess the efficacy and tolerability of omega- 3 fatty acids in combination with valproic acid in a group of 43 BPD patients [118] who were randomly assigned to two treatments for twelve weeks: (1) valproic acid (800-1300 mg/day) (plasma range: 50-100 $\mu \mathrm{g} / \mathrm{mL})$; (2) EPA (1.2 g/day) and DHA (0.6 g/day) in combination with the same dose of valproic acid. Results indicated that the association of omega-3 fatty acids and valproate was effective in reducing the severity of characteristic BPD symptoms, measured with the Borderline Personality Disorder Severity Index 
(BPDSI), impulsive behavioral dyscontrol, assessed with the Barratt Impulsiveness Scale (BIS-11), outbursts of anger, evaluated with the item "outburst of anger" of the BPDSI, and self-mutilating conduct, measured with the Self-Harm Inventory (SHI) (Table 5).

Table 5. Double-blind controlled trials of HUFAs in the treatment of impulsivity and borderline personality disorder.

\begin{tabular}{|c|c|c|c|c|}
\hline Study & Drug and Dose & Sample & $\begin{array}{l}\text { Treatment } \\
\text { Duration }\end{array}$ & Results \\
\hline $\begin{array}{l}\text { Hamazaki et al., } \\
1996 \text { [113] }\end{array}$ & DHA $1.5-1.8 \mathrm{~g} /$ day & $\begin{array}{l}41 \text { healthy controls } \\
\text { (21-30 years) }\end{array}$ & 3 months & $\downarrow$ aggression \\
\hline $\begin{array}{l}\text { Bradbury et al. } \\
\text { (2004) [115] }\end{array}$ & DHA $1.5 \mathrm{~g} /$ day & $\begin{array}{l}47 \text { healthy controls } \\
\text { (18-60 years) }\end{array}$ & 6 weeks & $\downarrow$ level of stress \\
\hline $\begin{array}{l}\text { Itomura et al. } \\
(2005)[114]\end{array}$ & DHA 3.6 g/day + EPA 0.84 g/day & $\begin{array}{l}166 \text { healthy controls } \\
\text { (9-12 years) }\end{array}$ & 3 months & $\begin{array}{l}\downarrow \text { aggression, } \\
\downarrow \text { impulsivity }\end{array}$ \\
\hline $\begin{array}{l}\text { Zanarini and } \\
\text { Frankenburg, } \\
2003[116]\end{array}$ & $\begin{array}{l}\text { EPA } 1 \mathrm{~g} / \text { day (with no standard } \\
\text { psychiatric therapies) }\end{array}$ & 30 BPD females & 8 weeks & $\begin{array}{l}\downarrow \text { aggression, } \\
\downarrow \text { depression }\end{array}$ \\
\hline $\begin{array}{l}\text { Hallahan et al., } \\
2007 \text { [117] }\end{array}$ & $\begin{array}{l}\text { EPA } 1.2 \mathrm{~g} / \text { day }+ \text { DHA } 0.9 \mathrm{~g} / \text { day } \\
\text { (added to the standard } \\
\text { psychiatric therapies) }\end{array}$ & $\begin{array}{l}49 \text { patients with } \\
\text { self-defeating } \\
\text { behaviors } \\
\text { (39 BPD patients) }\end{array}$ & 12 weeks & $\begin{array}{l}\downarrow \text { depression, } \\
\downarrow \text { parasuicidal behaviors, } \\
\downarrow \text { stress reactivity }\end{array}$ \\
\hline $\begin{array}{l}\text { Bellino et al., } \\
2014[118]\end{array}$ & $\begin{array}{l}\text { EPA ( } 1.2 \mathrm{~g} / \text { day })+ \text { DHA } \\
(0.6 \mathrm{~g} / \text { day }) \text { in combination with } \\
\text { valproic acid }(800-1300 \mathrm{mg} / \text { day }) \\
\text { vs. valproic acid } \\
(800-1300 \mathrm{mg} / \text { day })(\text { plasma } \\
\text { range: } 50-100 \mu \mathrm{g} / \mathrm{mL})\end{array}$ & 43 BPD patients & 12 weeks & $\begin{array}{l}\downarrow \text { severity of BPDSI, } \\
\downarrow \text { impulsive behavioral } \\
\text { dyscontrol, } \\
\downarrow \text { anger, } \\
\downarrow \text { self-mutilating conduct }\end{array}$ \\
\hline
\end{tabular}

Abbreviations: $\mathrm{EPA}=$ eicosapentaenoic acid; DHA $=$ docosahexaenoic acid; ethyl-EPA $=$ ethyl-eicosapentaenoic acid; BPDSI $=$ Borderline Personality Disorder Severity Index; $\downarrow=$ decrease of; $\uparrow=$ increase of.

\subsection{Substance Dependence}

According to research, proinflammatory cytokines are responsible for the physical and psychological symptoms concomitant to craving, and EPA can neutralize these molecules' toxic effects in the brain [75,119]. The neuroprotective effect of omega-3s on the production of serotonin and its action on the prefrontal cortex may also help with maintaining executive ability, both compromised during withdrawal and craving. Only two studies have been conducted to assess the efficacy of omega-3 fatty acid in the treatment of substance dependence [75,120]. The first study [75] evaluated the efficacy of EPA + DHA (3 g/day) for a period of three months on anxiety symptoms in addicted patients. The group treated with omega-3s showed a significant reduction in anxiety when compared to placebo. These results were replicated and confirmed by the following study performed by the same authors [120]. 


\subsection{Anorexia Nervosa}

Two studies, focused on the deficit in the concentration of HUFAs complexed to membrane phospholipids in patients with anorexia nervosa, showed similar results. Holman and colleagues [121] examined the concentration of fatty acids in plasma in a population of eight hospitalized anorexia nervosa fasting females compared to 19 healthy female adults $<25$ years old. Subjects with anorexia nervosa had deficiencies of essential fatty acids and showed decreased concentrations of total omega- 6 and omega- 3 acids, compared to the control group; this indicates compensatory changes in non-essential fatty acids and can lead to a consequent problem in terms of membrane structure and fluidity. Furthermore, Langan and Farrell [122] reported a concomitant significantly reduction of omega-3 and omega-6 in plasma phospholipids' concentration in a group of 17 patients (16 females, one male) hospitalized for anorexia nervosa compared to 11 normal females.

Only two trials have been conducted to assess the efficacy of omega- 3 fatty acid in the treatment of anorexia nervosa. In the first pilot open label study, conducted by Ayton [123], seven patients between 13 and 22 years old with anorexia nervosa, restrictive subtype, received $1 \mathrm{~g}$ /day EPA in addition to standard treatment for three months. This small study showed a general improvement in sleep, mood, dry skin and constipation (measured with Weight 4 Height software, the Eating Disorder Inventory (EDI-2), the Morgan-Russell Average Outcome Scale (MRAOS), BDI-2, the Children's Global Assessment Scale (CGA-S) and CGI-S.

Negative results in this clinical population were reported by Barbarich and collaborators [124]. Twenty six subjects with anorexia nervosa (10 subjects were restricting-type; six subjects were restricting and purging only-type; and 10 subjects were binge eating/purging-type) participated in a six-month trial of fluoxetine (20-60 mg/die). Using a randomized, double-blind design, subjects were assigned to either nutritional supplements (600 mg of DHA and $180 \mathrm{mg}$ of arachidonic acid daily, tryptophan, vitamins, minerals) or placebo. Patients were evaluated with: the Frost Multidimensional Perfectionism Scale (FMPS), the State-Trait Anxiety Inventory (STAI-Y) and Yale-Brown Obsessive Compulsive Scale (Y-BOCS). They were also weighed at weekly intervals for the first eight weeks, at two-week intervals for the following six weeks and at 4-week intervals for a further 12 weeks. There were no significant differences in weight gain per week between subjects treated with fluoxetine plus nutritional supplements vs. fluoxetine plus placebo. Moreover, there were no significant differences between groups in mean changes of anxiety or obsessive and compulsive symptoms.

\section{Adverse Effects}

Omega-3 fatty acids did not induce serious adverse effects and were generally well tolerated: most common side effects reported in clinical trials were nausea and 
a fishy aftertaste, but they were mild and rarely induced discontinuation [125]. The Panel of The European Food Safety Authority (EFSA) concluded that the available data are insufficient to establish a tolerable daily intake (UL) of DHA, EPA and DPA individually or in combination, but the supplementation with EPA and DHA up to $5 \mathrm{~g} /$ day is not dangerous for the general population [126]. In particular, EPA and DHA are generally recognized as safe and well tolerated at dose up to $5 \mathrm{~g} /$ day in terms of bleeding risk, as pointed out by Yokoyama et al. [127] and Tanaka et al. [128]. In addition, doses up to $5 \mathrm{~g} /$ day, consumed for a maximum period of 12-16 weeks, do not significantly affect glucose regulation in both healthy and diabetic subjects $[119,129-131]$ and do not increase infection risk by the activation of inappropriate inflammatory responses [132]. The intake of EPA and DHA at the same dose and up to 16 weeks does not induce alteration of lipid peroxidation and does not increase cardiovascular risk [133]. Combined intake of EPA and DHA at the dose of 2-6 g/day and intake of DHA at the dose of 2-4 g/die are responsible for an LDL concentration increase (3\%), but do not affect cardiovascular risk. At last, an intake of EPA at the maximum dose of $4 \mathrm{~g} /$ day does not induce significant changes in LDL plasma levels [134].

\section{Conclusions}

In the last decade, the role of long-chain HUFAs in the treatment of several psychiatric diseases has gradually increased, as confirmed by the growing number of randomized controlled trials testing the efficacy of essential fatty acid, especially omega-3 HUFA, supplementation. Nevertheless, an overall consensus about their efficacy is still lacking, and the findings of most of trials are controversial and inconclusive. Differences in methods, including sample size, selection criteria, choice and dosage of fatty acids (i.e., EPA, or DHA, or a combination of the two, or the addition of omega-6 HUFAs) and the duration of supplementation often make results not comparable.

The main evidence for the efficacy of EPA and DHA has been obtained in mood disorders. In particular, omega- 3 fatty acids seem to be useful in preventing and improving depressive symptoms at a low dose of $1 \mathrm{~g} /$ day; EPA seems to be more efficacious than DHA; patients with more severe depression showed greater treatment gains. However, due to the considerable heterogeneity of the investigations, additional large cohort studies and well-designed clinical trials are warranted. Concerning bipolar disorder, the results of systematic reviews and meta-analyses suggested a potential beneficial role of omega- 3 fatty acids in addition to stable medications in treating depressive symptom at the approximate dose of $1-2 \mathrm{~g} /$ day, but did not support their use in attenuating mania. Furthermore, studies using a combination of EPA and DHA reported a statistically-significant improvement in symptoms of bipolar depression, whereas trials using a single compound did not. In 
schizophrenia, little evidence of a meaningful clinical effect was reported, and current data do not allow us either to refuse or support the use of omega-3 fatty acids in psychotic patients. However, adverse effects of antipsychotics, in particular metabolic abnormalities and extrapyramidal symptoms, may benefit from the addition of EPA or a combination of EPA and DHA. In ADHD disorder, several RCTs have been performed, but the main findings have reported small-to-modest effects of omega-3 HUFAs in reducing ADHD symptoms in children. Most promising results in this field have been reached by studies using EPA at high doses or the association of omega-3 and omega- 6 fatty acids. Anyway, the relative efficacy of these agents was modest compared to the currently available pharmacotherapies. To date, a small number of clinical trials have explored the impact of HUFAs on impulsive and aggressive behaviors and a growing number of studies has been conducted in order to test the efficacy of these agents in borderline personality disorder. Results are encouraging, although this area of psychopathology needs to be explored in depth by future investigations. In autism spectrum disorders, only two RCTs with opposite results are available, whereas there is a substantial lack of data about the use of omega- 3 fatty acids in anxiety disorders and obsessive-compulsive disorder. The majority of trials assessing patients with mood disorders did not investigate changes in anxiety symptoms, although anxiety is frequently associated with depression or mania. HUFAs were not found efficacious in treating eating disorders and substance use disorders. Concerning tolerability, RCTs considered in this review share a common finding: omega- 3 fatty acids did not induce serious adverse effects. A survey of studies concluded that omega-3 are generally recognized as safe and well tolerated at doses up to $5 \mathrm{~g} /$ day for a maximum period of 12-16 weeks. Several authors have assessed the effects of omega-3 HUFA administration in terms of bleeding risk, glucose metabolism, lipid profile, infection risk and cardiovascular function with a general consensus about their harmlessness.

In summary, preliminary findings on omega-3 fatty acids in psychiatric populations allow us to consider these naturally-derived and well-tolerated psychotropic agents as a promising therapeutic tool. However, their efficacy in treating specific mental disorders or clusters of psychiatric symptoms is not sufficiently proven, as the findings from studies and reviews are too divergent to draw any conclusion.

Acknowledgments: No funds or grants were received by the authors to support or cover the costs of publishing this work.

Conflicts of Interest: The authors declare no conflict of interest.

\section{References}

1. Ergas, D.; Eilat, E.; Mendlovic, S.; Sthoeger, Z.M. $n$-3 fatty acids and the immune system in autoimmunity. Isr. Med. Assoc. J. 2002, 4, 34-38. 
2. Simopoulos, A.P. Essential fatty acids in health and chronic disease. Am. J. Clin. Nutr. 1999, 70 (Suppl. 3), 560-569.

3. Lee, S.; Gura, K.M.; Kim, S.; Arsenault, D.A.; Bistrian, B.R.; Puder, M. Current clinical applications of omega-6 and omega-3 fatty acids. Nutr. Clin. Pract. 2006, 21, 323-341.

4. Milte, C.M.; Sinn, N.; Buckley, J.D.; Coates, A.M.; Young, R.M.; Howe, P.R. Polyunsaturated fatty acids, cognition and literacy in children with ADHD with and without learning difficulties. J. Child Health Care 2011, 15, 299-311.

5. Garland, M.R.; Hallahan, B. Essential fatty acids and their role in conditions characterised by impulsivity. Int. Rev. Psychiatry 2006, 18, 99-105.

6. Ross, B.M.; Seguin, J.; Sieswerda, L.E. Omega-3 fatty acids as treatments for mental illness: Which disorder and which fatty acid? Lipids Health Dis. 2007, 6, 21.

7. Hallahan, B.; Garland, M.R. Essential fatty acids and mental health. Br. J. Psychiatry 2005, 186, 275-277.

8. Sinn, N.; Milte, C.; Howe, P.R. Oiling the brain: A review of randomized controlled trials of omega-3 fatty acids in psychopathology across the lifespan. Nutrients 2010, 2, 128-170.

9. Assisi, A.; Banzi, R.; Buonocore, C.; Capasso, F.; Di Muzio, V.; Michelacci, F.; Renzo, D.; Tafuri, G.; Trotta, F.; Vitocolonna, M.; et al. Fish oil and mental health: The role of $n-3$ long-chain polyunsaturated fatty acids in cognitive development and neurological disorders. Int. Clin. Psychopharmacol. 2006, 21, 319-336.

10. De la Pressa, O.S.; Innis, S.M. Docosahexanoic and arachidonic acid prevent a decrease in dopaminergic and serotoninergic neurotrasmitters in frontal cortex caused by a linoleic and alpha-linoleic acid deficient diet in formula-fed piglets. J. Nutr. 1999, 129, 2088-2093.

11. Hamazaki, K.; Maekawa, M. Fatty acid composition of the postmortem prefrontal cortex of patients with schizophrenia, bipolar disorder, and major depressive disorder. Psychiatry Res. 2015, 227, 353-359.

12. Glen, A.I.; Glen, E.M.; Horrobin, D.F.; Vaddadi, K.S.; Spellman, M.; Morse-Fisher, N.; Ellis, K.; Skinner, F.S. A red cell membrane abnormality in a subgroup of schizophrenic patients: Evidence for two diseases. Schizophr. Res. 1994, 12, 53-61.

13. Horrobin, D.F. The membrane phospholipid hypothesis as a biochemical basis for the neurodevelopmental concept of schizophrenia. Schizophr. Res. 1998, 30, 193-208.

14. Schlögelhofer, M.; Amminger, G.P.; Schaefer, M.R.; Fusar-Poli, P.; Smesny, S.; McGorry, P.; Berger, G.; Mossaheb, N. Polyunsaturated fatty acids in emerging psychosis: A safer alternative? Early Interv. Psychiatry 2014, 8, 199-208.

15. Amminger, G.P.; Schäfer, M.R.; Papageorgiou, K.; Klier, C.M.; Cotton, S.M.; Harrigan, S.M.; Mackinnon, A.; McGorry, P.D.; Berger, G.E. Long-chain omega-3 fatty acids for indicated prevention of psychotic disorders: A randomized, placebo-controlled trial. Arch. Gen. Psychiatry 2010, 67, 146-154. 
16. Markulev, C.; McGorry, P.D.; Nelson, B.; Yuen, H.P.; Schaefer, M.; Yung, A.R.; Thompson, A.; Berger, G.; Mossaheb, N.; Schlögelhofer, M.; et al. NEURAPRO-E study protocol: A multicentre randomized controlled trial of omega-3 fatty acids and cognitive-behavioural case management for patients at ultra high risk of schizophrenia and other psychotic disorders. Early Interv. Psychiatry 2015.

17. Peet, M.; Brind, J.; Ramchand, C.N.; Shah, S.; Vankar, G.K. Two double-blind placebo-controlled pilot studies of eicosapentaenoic acid in the treatment of schizophrenia. Schizophr. Res. 2001, 49, 243-251.

18. Emsley, R.; Chiliza, B.; Asmal, L.; Emsley, R.; Chiliza, B.; Asmal, L.; du Plessis, S.; Phahladira, L.; van Niekerk, E.; van Rensburg, S.J.; et al. A randomized, controlled trial of omega-3 fatty acids plus an antioxidant for relapse prevention after antipsychotic discontinuation in first-episode schizophrenia. Schizophr. Res. 2014, 158, 230-235.

19. Peet, M.; Horrobin, D.F.; Study Group. A dose-ranging exploratory study of the effects of ethyl-eicosapentaenoate in patients with persistent schizophrenic symptoms. J. Psychiat. Res. 2002, 36, 7-18.

20. Jamilian, H.; Solhi, H.; Jamilian, M. Randomized, placebo-controlled clinical trial of omega-3 as supplemental treatment in schizophrenia. Glob. J. Health Sci. 2014, 18, 103-108.

21. Pawełczyk, T.; Grancow-Grabka, M.; Kotlicka-Antczak, M.; Trafalska, E.; Pawełczyk, A. A randomized controlled study of the efficacy of six-month supplementation with concentrated fish oil rich in omega-3 polyunsaturated fatty acids in first episode schizophrenia. J. Psychiat. Res. 2016, 73, 34-44.

22. Bentsen, H.; Osnes, K.; Refsum, H. A randomized placebo-controlled trial of an omega-3 fatty acid and vitamins E + C in schizophrenia. Transl. Psychiatry 2013.

23. Fenton, W.S.; Dickerson, F.; Boronow, J.; Hibbeln, J.R.; Knable, M. A placebo-controlled trial of omega-3 fatty acid (ethyl eicosapentaenoic acid) supplementation for residual symptoms and cognitive impairment in schizophrenia. Am. J. Psychiatry 2001, 158, 2071-2074.

24. Berger, G.E.; Wood, S.J.; Wellard, R.M.; Proffitt, T.M.; McConchie, M.; Amminger, G.P.; Jackson, G.D.; Velakoulis, D.; Pantelis, C.; McGorry, P.D. Ethyl-eicosapentaenoic acid in first-episode psychosis. A 1H-MRS study. Neuropsychopharmacology 2007, 33, 2467-2473.

25. Joy, C.B.; Mumby-Croft, R.; Joy, L.A. Polyunsaturated fatty acid supplementation for schizophrenia. Cochrane Database Syst. Rev. 2006, 19, CD001257.

26. Freeman, M.P.; Hibbeln, J.R.; Wisner, K.L.; Davis, J.M.; Mischoulon, D.; Peet, M.; Keck, P.E., Jr.; Marangell, L.B.; Richardson, A.J.; Lake, J.; et al. Omega-3 fatty acids: Evidence basis for treatment and future research in psychiatry. J. Clin. Psychiatry 2006, 67, 1954-1967.

27. Fusar-Poli, P.; Berger, G. Eicosapentaenoic acid interventions in schizophrenia: Meta-analysis of randomized, placebo-controlled studies. J. Clin. Psychopharmacol. 2012, $32,179-185$. 
28. Akter, K.; Gallo, D.A.; Martin, S.A.; Myronyuk, N.; Roberts, R.T.; Stercula, K.; Raffa, R.B. A review of the possible role of the essential fatty acids and fish oils in the aetiology, prevention or pharmacotherapy of schizophrenia. J. Clin. Pharm. Ther. 2012, 37, 132-139.

29. Emsley, R.; Myburgh, C.; Oosthuizen, P.; van Rensburg, S.J. Randomized, placebo-controlled study of ethyl-eicosapentaenoic acid as supplemental treatment in schizophrenia. Am. J. Psychiatry 2002, 159, 1596-1598.

30. Emsley, R.; Niehaus, D.J.; Koen, L.; Oosthuizen, P.P.; Turner, H.J.; Carey, P.; Murck, H. The effects of eicosapentaenoic acid in tardive dyskinesia: A randomized, placebo-controlled trial. Schizophr. Res. 2006, 84, 112-120.

31. Hibbeln, J.R.; Umhau, J.C.; Linnoila, M.; George, D.T.; Ragan, P.W.; Shoaf, S.E.; Vaughan, M.R.; Rawlings, R.; Salem, N., Jr. A replication study of violent and non-violent subjects: CSF metabolites of serotonin and dopamine are predicted by plasma essential fatty acids. Biol. Psychiatry 1998, 44, 243-249.

32. Horrobin, D.F.; Bennett, C.N. Depression and bipolar disorder: Relationships to impaired fatty acid and phospholipid metabolism and to diabetes, cardiovascular disease, immunological abnormalities, cancer, ageing and osteoporosis. Possible candidate genes. Prostaglandins Leukort. Essent. Fatty Acids 1999, 60, 217-234.

33. Tanskanen, A.; Hibbeln, J.R.; Hintikka, J.; Haatainen, K.; Honkalampi, K.; Viinamäki, H. Fish consumption, depression, and suicidality in a general population. Arch. Gen. Psychiatry 2001, 58, 512-513.

34. Lin, P.Y.; Huang, S.Y.; Su, K.P. A meta-analytic review of polyunsaturated fatty acid compositions in patients with depression. Biol. Psychiatry 2010, 68, 140-147.

35. Peet, M.; Horrobin, D.F. A dose-ranging study of the effects of ethyl-eicosapentaenoate in patients with ongoing depression despite apparently adequate treatment with standard drugs. Arch. Gen. Psychiatry 2002, 59, 913-919.

36. Su, K.P.; Huang, S.Y.; Chiu, C.C.; Shen, W.W. Omega-3 fatty acids in major depressive disorder. A preliminary double-blind, placebo-controlled trial. Eur. Neuropsychopharmacol. 2003, 13, 267-271.

37. Lespérance, F.; Frasure-Smith, N.; St-André, E.; Lespérance, F.; Frasure-Smith, N.; St-André, E.; Turecki, G.; Lespérance, P.; Wisniewski, S.R. The efficacy of omega-3 supplementation for major depression: A randomized controlled trial. J. Clin. Psychiatry 2011, 72, 1054-1062.

38. Jazayeri, S.; Tehrani-Doost, M.; Keshavarz, S.A.; Hosseini, M.; Djazayery, A.; Amini, H.; Jalali, M.; Peet, M. Comparison of therapeutic effects of omega-3 fatty acid eicosapentaenoic acid and fluoxetine, separately and in combination, in major depressive disorder. Aust. N. Z. J. Psychiatry 2008, 42, 192-198.

39. Gertsik, L.; Poland, R.E.; Bresee, C.; Rapaport, M.H. Omega-3 fatty acid augmentation of citalopram treatment for patients with major depressive disorder. J. Clin. Psychopharmacol. 2012, 32, 61-64.

40. Nemets, B.; Stahl, Z.; Belmaker, R.H. Addition of omega-3 fatty acid to maintenance medication treatment for recurrent unipolar depressive disorder. Am. J. Psychiatry 2002, 159, 477-479. 
41. Nemets, H.; Nemets, B.; Apter, A.; Bracha, Z.; Belmaker, R.H. Omega-3 treatment of childhood depression: A controlled, double-blind pilot study. Am. J. Psychiatry 2006, 163, 1098-1100.

42. Su, K.P.; Huang, S.Y.; Chiu, T.H.; Huang, K.C.; Huang, C.L.; Chang, H.C.; Pariante, C.M. Omega-3 fatty acids for major depressive disorder during pregnancy: Results from a randomized, double-blind, placebo-controlled trial. J. Clin. Psychiatry 2008, 69, 644-651.

43. Rondanelli, M.; Giacosa, A.; Opizzi, A.; Pelucchi, C.; La Vecchia, C.; Montorfano, G.; Negroni, M.; Berra, B.; Politi, P.; Rizzo, A.M. Long chain omega 3 polyunsaturated fatty acids supplementation in the treatment of elderly depression: Effects on depressive symptoms, on phospholipids fatty acids profile and on health-related quality of life. J. Nutr. Health Aging 2011, 15, 37-44.

44. Rondanelli, M.; Giacosa, A.; Opizzi, A.; Pelucchi, C.; La Vecchia, C.; Montorfano, G.; Negroni, M.; Berra, B.; Politi, P.; Rizzo, A.M. Effect of omega-3 fatty acids supplementation on depressive symptoms and on health-related quality of life in the treatment of elderly women with depression: A double-blind, placebo-controlled, randomized clinical trial. J. Am. Coll. Nutr. 2010, 29, 55-64.

45. Rizzo, A.M.; Corsetto, P.A.; Montorfano, G.; Opizzi, A.; Faliva, M.; Giacosa, A.; Ricevuti, G.; Pelucchi, C.; Berra, B.; Rondanelli, M. Comparison between the AA/EPA ratio in depressed and non depressed elderly females: Omega-3 fatty acid supplementation correlates with improved symptoms but does not change immunological parameters. Nutr. J. 2012, 11, 82.

46. Rees, A.M.; Austin, M.P.; Parker, G.B. Omega-3 fatty acids as a treatment for perinatal depression: Randomized double-blind placebo-controlled trial. Aust. N. Z. J. Psychiatry 2008, 42, 199-205.

47. Freeman, M.P.; Davis, M.; Sinha, P.; Wisner, K.L.; Hibbeln, J.R.; Gelenberg, A.J. Omega-3 fatty acids and supportive psychotherapy for perinatal depression: A randomized placebo-controlled study. J. Affect Disord. 2008, 110, 142-148.

48. Marangell, L.B.; Martinez, J.M.; Zboyan, H.A.; Kertz, B.; Kim, H.F.; Puryear, L.J. A double-blind, placebo-controlled study of the omega-3 fatty acid docosahexaenoic acid in the treatment of major depression. Am. J. Psychiatry 2003, 160, 996-998.

49. Silvers, K.M.; Woolley, C.C.; Hamilton, F.C.; Watts, P.M.; Watson, R.A. Randomised double-blind placebo-controlled trial of fish oil in the treatment of depression. Prostaglandins Leukort. Essent. Fatty Acids 2005, 72, 211-218.

50. Grenyer, B.F.; Crowe, T.; Meyer, B.; Owen, A.J.; Grigonis-Deane, E.M.; Caputi, P.; Howe, P.R. Fish oil supplementation in the treatment of major depression: A randomised double-blind placebo-controlled trial. Prog. Neuropsychopharmacol. Biol. Psychiatry 2007, 31, 1393-1396.

51. Rogers, P.J.; Appleton, K.M.; Kessler, D.; Peters, T.J.; Gunnell, D.; Hayward, R.C.; Heatherley, S.V.; Christian, L.M.; McNaughton, S.A.; Ness, A.R. No effect of $n-3$ long-chain polyunsaturated fatty acid (EPA and DHA) supplementation on depressed mood and cognitive function: A randomised controlled trial. Br. J. Nutr. 2008, 99, 421-431. 
52. Antypa, N.; Smelt, A.H.; Strengholt, A.; Van der Does, A.J. Effects of omega-3 fatty acid supplementation on mood and emotional information processing in recovered depressed individuals. J. Psychopharmacol. 2012, 26, 738-743.

53. Mischoulon, D.; Best-Popescu, C.; Laposata, M.; Merens, W.; Murakami, J.L.; Wu, S.L.; Papakostas, G.I.; Dording, C.M.; Sonawalla, S.B.; Nierenberg, A.A.; et al. A double-blind dose-finding pilot study of docosahexaenoic acid (DHA) for major depressive disorder. Eur. Neuropsychopharmacol. 2008, 18, 639-645.

54. Mischoulon, D.; Nierenberg, A.A.; Schettler, P.J.; Kinkead, B.L.; Fehling, K.; Martinson, M.A.; Hyman Rapaport, M. A double-blind, randomized controlled clinical trial comparing eicosapentaenoic acid versus docosahexaenoic acid for depression. J. Clin. Psychiat. 2015, 76, 54-61.

55. Carney, R.M.; Freedland, K.E.; Rubin, E.H.; Rich, M.W.; Steinmeyer, B.C.; Harris, W.S. Omega-3 augmentation of sertraline in treatment of depression in patients with coronary heart disease: A randomized controlled trial. JAMA 2009, 302, 1651-1657.

56. Appleton, K.M.; Rogers, P.J.; Ness, A.R. Updated systematic review and meta-analysis of the effects of $n-3$ long-chain polyunsaturated fatty acids on depressed mood. Am. J. Clin. Nutr. 2010, 91, 757-770.

57. Bloch, M.H.; Hannestad, J. Omega-3 fatty acids for the treatment of depression: Systematic review and meta-analysis. Mol. Psychiatry 2012, 17, 1272-1282.

58. Lin, P.Y.; Su, K.P. A meta-analytic review of double-blind, placebo-controlled trials of antidepressant efficacy of omega-3 fatty acids. J. Clin. Psychiatry 2007, 68, 1056-1061.

59. Martins, J.G. EPA but not DHA appears to be responsible for the efficacy of omega-3 long chain polyunsaturated fatty acid supplementation in depression: Evidence from a meta-analysis of randomized controlled trials. J. Am. Coll. Nutr. 2009, 28, 525-542.

60. Sublette, M.E.; Ellis, S.P.; Geant, A.L.; Mann, J.J. Meta-analysis of the effects of eicosapentaenoic acid (EPA) in clinical trials in depression. J. Clin. Psychiatry 2011, 72, 1577-1584.

61. Grosso, G.; Pajak, A.; Marventano, S.; Castellano, S.; Galvano, F.; Bucolo, C.; Caraci, F. Role of omega-3 fatty acids in the treatment of depressive disorders: A comprehensive meta-analysis of randomized clinical trials. PLoS ONE 2014, 9, e96905.

62. Chiu, C.C.; Huang, S.Y.; Su, K.P.; Lu, M.L.; Huang, M.C.; Chen, C.C.; Shen, W.W. Polyunsaturated fatty acid deficit in patients with bipolar mania. Eur. Neuropsychopharmacol. 2003, 13, 99-103.

63. Frangou, S.; Lewis, M.; McCrone, P. Efficacy of ethyl-eicosapentaenoic acid in bipolar depression: Randomised double-blind placebo-controlled study. Br. J. Psychiatry 2006, $188,46-50$.

64. Frangou, S.; Lewis, M.; Wollard, J.; Simmons, A. Preliminary in vivo evidence of increased $\mathrm{N}$-acetyl-aspartate following eicosapentanoic acid treatment in patients with bipolar disorder. J. Psychopharmacol. 2007, 21, 435-439. 
65. Keck, P.E., Jr.; Mintz, J.; McElroy, S.L.; Freeman, M.P.; Suppes, T.; Frye, M.A.; Altshuler, L.L.; Kupka, R.; Nolen, W.A.; Leverich, G.S.; et al. Double-blind, randomized, placebo-controlled trials of ethyl-eicosapentanoate in the treatment of bipolar depression and rapid cycling bipolar disorder. Biol. Psychiatry 2006, 60, 1020-1022.

66. Stoll, A.L.; Severus, W.E.; Freeman, M.P.; Rueter, S.; Zboyan, H.A.; Diamond, E.; Cress, K.K.; Marangell, L.B. Omega 3 fatty acids in bipolar disorder: A preliminary double-blind, placebo-controlled trial. Arch. Gen. Psychiatry 1999, 56, 407-412.

67. Chiu, C.C.; Huang, S.Y.; Chen, C.C.; Su, K.P. Omega-3 fatty acids are more beneficial in the depressive phase than in the manic phase in patients with bipolar I disorder. J. Clin. Psychiatry 2005, 66, 1613-1614.

68. Gracious, B.L.; Chirieac, M.C.; Costescu, S.; Finucane, T.L.; Youngstrom, E.A.; Hibbeln, J.R. Randomized, placebo-controlled trial of flax oil in pediatric bipolar disorder. Bipolar Disord. 2010, 12, 142-154.

69. Murphy, B.L.; Stoll, A.L.; Harris, P.Q.; Ravichandran, C.; Babb, S.M.; Carlezon, W.A., Jr.; Cohen, B.M. Omega-3 fatty acid treatment, with or without cytidine, fails to show therapeutic properties in bipolar disorder: A double-blind, randomized add-on clinical trial. J. Clin. Psychopharmacol. 2012, 32, 699-703.

70. Turnbull, T.; Cullen-Drill, M.; Smaldone, A. Efficacy of omega-3 fatty acid supplementation on improvement of bipolar symptoms: A systematic review. Arch. Psychiat. Nurs. 2008, 22, 305-311.

71. Montgomery, P.; Richardson, A.J. Omega-3 fatty acids for bipolar disorder. Cochrane Database Syst. Rev. 2008, 2, CD005169.

72. Kraguljac, N.V.; Montori, V.M.; Pavuluri, M.; Chai, H.S.; Wilson, B.S.; Unal, S.S. Efficacy of omega-3 fatty acids in mood disorders-A systematic review and metaanalysis. Psychopharmacol. Bull. 2009, 42,39-54.

73. Sarris, J.; Mischoulon, D.; Schweitzer, I. Omega-3 for bipolar disorder: Meta-analyses of use in mania and bipolar depression. J. Clin. Psychiatry 2012, 73, 81-86.

74. Ross, B.M. Omega-3 polyunsaturated fatty acids and anxiety disorders. Prostaglandins Leukort. Essent. Fatty Acids 2009, 81, 309-312.

75. Green, P. Red cell membrane omega-3 fatty acids are decreased in nondepressed patients with social anxiety disorder. Eur. Neuropsychopharmacol. 2006, 16, 107-113.

76. Liu, J.J. Omega-3 Polyunsaturated fatty acid status in major depression with comorbid anxiety disorders. J. Clin. Psychiatry 2013, 74, 732-738.

77. Buydens-Branchey, L.; Branchey, M. $n-3$ polyunsaturated fatty acids decrease anxiety feelings in a population of substance abusers. J. Clin. Psychopharmacol. 2006, 26, 661-665.

78. Ravindran, A.V.; da Silva, T.L. Complementary and alternative therapies as add-on to pharmacotherapy for mood and anxiety disorders: A systematic review. J. Affect Disord. 2013, 150, 707-719.

79. Fux, M.; Benjamin, J.; Nemets, B. A placebo-controlled cross-over trial of adjunctive EPA in OCD. J. Psychiat. Res. 2004, 38, 323-325.

80. Mitchell, E.A.; Aman, M.G.; Turbott, S.H.; Manku, M. Clinical characteristics and serum essential fatty acid levels in hyperactive children. Clin. Pediatr. (Phila.) 1987, 26, 406-411. 
81. Stevens, L.; Zhang, W.; Peck, L.; Kuczek, T.; Grevstad, N.; Mahon, A.; Zentall, S.S.; Arnold, L.E.; Burgess, J.R. EFA supplementation in children with inattention, hyperactivity, and other disruptive behaviors. Lipids 2003, 38, 1007-1021.

82. Burgess, J.R.; Stevens, L.; Zhang, W.; Peck, L. Long-chain polyunsaturated fatty acids in children with attention-deficit hyperactivity disorder. Am. J. Clin. Nutr. 2000, 71 (Suppl. S1), 327S-330S.

83. Hibbelna, J.R.; Gowb, R.V. Omega-3 fatty acid and nutrient deficits in adverse neurodevelopment and childhood behaviors. Child Adolesc. Psychiatr. Clin. N. Am. 2014, 23, 555-590.

84. Richardson, A.J.; Puri, B.K. A randomized double-blind, placebo-controlled study of the effects of supplementation with highly unsaturated fatty acids on ADHD-related symptoms in children with specific learning difficulties. Prog. Neuropsychopharmacol. Biol. Psychiatry 2002, 26, 233-239.

85. Richardson, A.J.; Montgomery, P. The Oxford-Durham study: A randomized, controlled trial of dietary supplementation with fatty acids in children with developmental coordination disorder. Pediatrics 2005, 115, 1360-1366.

86. Sinn, N.; Bryan, J. Effect of supplementation with polyunsaturated fatty acids and micronutrients on learning and behavior problems associated with child ADHD. J. Dev. Behav. Pediatr. 2007, 28, 82-91.

87. Sinn, N.; Bryan, J.; Wilson, C. Cognitive effects of polyunsaturated fatty acids in children with attention deficit hyperactivity disorder symptoms: A randomised controlled trial. Prostaglandins Leukort. Essent. Fatty Acids 2008, 78, 311-326.

88. Bélanger, S.A.; Vanasse, M.; Spahis, S.; Sylvestre, M.P.; Lippé, S.; L'heureux, F.; Ghadirian, P.; Vanasse, C.M.; Levy, E. Omega-3 fatty acid treatment of children with attention-deficit hyperactivity disorder: A randomized, double-blind, placebo-controlled study. Paediatr. Child Health 2009, 14, 89-98.

89. Kirby, A.; Woodward, A.; Jackson, S.; Wang, Y.; Crawford, M.A. A double-blind, placebo-controlled study investigating the effects of omega-3 supplementation in children aged 8-10 years from a mainstream school population. Res. Dev. Disabil. 2010, 31, 718-730.

90. Perera, H.; Jeewandara, K.C.; Seneviratne, S.; Guruge, C. Combined $\omega 3$ and $\omega 6$ supplementation in children with attention-deficit hyperactivity disorder (ADHD) refractory to methylphenidate treatment: A double-blind, placebo-controlled study. J. Child Neurol. 2012, 27, 747-753.

91. Voigt, R.G.; Llorente, A.M.; Jensen, C.L.; Fraley, J.K.; Berretta, M.C.; Heird, W.C. A randomized, double-blind, placebo-controlled trial of docosahexaenoic acid supplementation in children with attention-deficit/hyperactivity disorder. J. Pediatr. 2001, 139, 189-196.

92. Hirayama, S.; Hamazaki, T.; Terasawa, K. Effect of docosahexaenoic acid-containing food administration on symptoms of attention-deficit/hyperactivity disorder-A placebo-controlled double-blind study. Eur. J. Clin. Nutr. 2004, 58, 467-473. 
93. Johnson, M.; Ostlund, S.; Fransson, G.; Kadesjö, B.; Gillberg, C. Omega-3/omega-6 fatty acids for attention deficit hyperactivity disorder: A randomized placebo-controlled trial in children and adolescents. J. Atten. Disord. 2009, 12, 394-401.

94. Milte, C.M.; Parletta, N.; Buckley, J.D.; Coates, A.M.; Young, R.M.; Howe, P.R. Eicosapentaenoic and docosahexaenoic acids, cognition, and behavior in children with attention-deficit/hyperactivity disorder: A randomized controlled trial. Nutrition 2012, 28, 670-677.

95. Widenhorn-Müller, K.; Schwanda, S.; Scholz, E.; Spitzer, M.; Bode, H. Effect of supplementation with long-chain $\omega-3$ polyunsaturated fatty acids on behavior and cognition in children with attention deficit/hyperactivity disorder (ADHD): A randomized placebo-controlled intervention trial. Prostaglandins Leukort. Essent. Fatty Acids 2014, 91, 49-60.

96. Bloch, M.H.; Qawasmi, A. Omega-3 fatty acid supplementation for the treatment of children with attention-deficit/hyperactivity disorder symptomatology: Systematic review and meta-analysis. J. Am. Acad. Child Adolesc. Psychiatry 2011, 50, 991-1000.

97. Sonuga-Barke, E.J.S.; Brandeis, D.; Cortese, S.; Daley, D.; Ferrin, M.; Holtmann, M.; Stevenson, J.; Danckaerts, M.; van der Oord, S.; Döpfner, M.; et al. Nonpharmacological interventions for ADHD: Systematic review and metaanalyses of randomized controlled trials of dietary and psychological treatments. Am. J. Psychiatry 2013, 170, 275-289.

98. Gillies, D.; Sinn, J.K.h.; Lad, S.S.; Leach, M.J.; Ross, M.J. Polyunsaturated fatty acids (PUFA) for attention deficit hyperactivity disorder (ADHD) in children and adolescents. Cochrane Database Syst. Rev. 2012, 7, CD007986.

99. Gow, R.V.; Hibbeln, J.R.; Parletta, N. Current evidence and future directions for research with omega-3 fatty acids and attention deficit hyperactivity disorder. Curr. Opin. Clin. Nutr. Metab. Care 2015, 18, 133-138.

100. Bell, J.G.; Sargent, J.R.; Tocher, D.R.; Dick, J.R. Red blood cell fatty acid compositions in a patient with autistic spectrum disorder: A characteristic abnormality in neurodevelopmental disorders? Prostaglandins Leukort. Essent. Fatty Acids 2000, 63, 21-25.

101. Bell, J.G.; MacKinlay, E.E.; Dick, J.R.; MacDonald, D.J.; Boyle, R.M.; Glen, A.C.A. Essential fatty acids and phospholipase A2 in autistic spectrum disorders. Prostaglandins Leukort. Essent. Fatty Acids 2004, 71, 201-204.

102. Vancassel, S.; Durand, G.; Barthelemy, C. Plasma fatty acid levels in autistic children. Prostaglandins Leukort. Essent. Fatty Acids 2001, 65, 1-7.

103. Belluzzi, A. $n-3$ fatty acids for the treatment of inflammatory bowel diseases. Proc. Nutr. Soc. 2002, 61, 391-395.

104. Horvath, K.; Perman, J.A. Autism and gastrointestinal symptoms. Curr. Gastroenterol. Rep. 2002, 4, 251-258.

105. Amminger, G.P.; Berger, G.E.; Schäfer, M.R.; Klier, C.; Friedrich, M.H.; Feucht, M. Omega-3 fatty acids supplementation in children with autism: A double-blind randomized, placebo controlled pilot study. Biol. Psychiatry 2007, 61, 551-553. 
106. Voigt, R.G.; Mellon, M.W.; Katusic, S.K.; Weaver, A.L.; Matern, D.; Mellon, B.; Jensen, C.L.; Barbaresi, W.J. Dietary docosahexaenoic acid supplementation in children with autism. J. Pediatr. Gastroenterol. Nutr. 2014, 58, 715-722.

107. Mankad, D.; Dupuis, A.; Smile, S.; Roberts, W.; Brian, J.; Lui, T.; Genore, L.; Zaghloul, D.; Iaboni, A.; Marcon, P.M.; et al. A randomized, placebo controlled trial of omega-3 fatty acids in the treatment of young children with autism. Mol. Autism 2015.

108. Johnson, S.M.; Hollander, E. Evidence that eicosapentaenoic acid is effective in treating autism. J. Clin. Psychiatry 2003, 64, 848-849.

109. Patrick, L.; Salik, R. The effect of essential fatty acid supplementation on language development and learning skills in autism and Asperger's syndrome. Autism Asperger's Dig. Available online: http:/ / omega-research.com/researchview.php?ID=672\&catid=2 (accesssed on 21 June 2016).

110. Van Elst, K.; Bruining, H.; Birtoli, B.; Terreaux, C.; Buitelaar, J.K.; Kas, M.J. Food for thought: Dietary changes in essential fatty acid ratios and the increase in autism spectrum disorders. Neurosci. Biobehav. Rev. 2014, 45, 369-378.

111. Stevens, L.J.; Zentall, S.S.; Abate, M.L.; Kuczek, T.; Burgess, J.R. Omega-3 fatty acids in boys with behavior, learning, and health problems. Physiol. Behav. 1996, 59, 915-920.

112. Buydens-Branchey, L.; Branchey, M. Association between low plasma levels of cholesterol and relapse in cocaine addicts. Psychosom. Med. 2003, 65, 86-91.

113. Hamazaki, T.; Sawazaki, S.; Itomura, M.; Asaoka, E.; Nagao, Y.; Nishimura, N.; Yazawa, K.; Kuwamori, T.; Kobayashi, M. The effect of docosahexaenoic acid on aggression in young adults. A placebo-controlled double-blind study. J. Clin. Investig. 1996, 97, 1129-1133.

114. Itomura, M.; Hamazaki, K.; Sawazaki, S.; Kobayashi, M.; Terasawa, K.; Watanabe, S.; Hamazaki, T. The effect of fish oil on physical aggression in schoolchildren-A randomized, double-blind, placebo-controlled trial. J. Nutr. Biochem. 2005, 16, 163-171.

115. Bradbury, J.; Myers, S.P.; Oliver, C. An adaptogenic role for omega-3 fatty acids in stress; a randomised placebo controlled double blind intervention study (pilot). Nutr. J. 2004, $3,20$.

116. Zanarini, M.C.; Frankenburg, F.R. Omega-3 fatty acid treatment of women with borderline personality disorder: A double blind, placebo-controlled pilot study. Am. J. Psychiatry 2003, 160, 167-169.

117. Hallahan, B.; Hibblen, J.R.; Davis, J.M.; Garland, M.R. Omega-3 fatty acids supplementation in patients with recurrent self-harm: Single center double bind randomized controlled trial. Br. J. Psychiatry 2007, 190, 118-122.

118. Bellino, S.; Bozzatello, P.; Rocca, G.; Bogetto, F. Efficacy of omega-3 fatty acids in the treatment of borderline personality disorder: A study of the association with valproic acid. J. Psychopharmacol. 2014, 28, 125-132.

119. Song, C.; Li, X.; Leonard, B.E.; Horrobin, D.F. Effects of dietary $n-3$ or $n-6$ fatty acids on interleukin-1beta-induced anxiety, stress, and inflammatory responses in rats. J. Lipid Res. 2003, 44, 1984-1991. 
120. Buydens-Branchey, L.; Branchey, M.; Hibbeln, J.R. Associations between increases in plasma $n-3$ polyunsaturated fatty acids following supplementation and decreases in anger and anxiety in substance abusers. Prog. Neuropsychopharmacol. Biol. Psychiatry 2008, 32, 568-575.

121. Holman, R.T.; Adams, C.E.; Nelson, R.A.; Grater, S.J.; Jaskiewicz, J.A.; Johnson, S.B.; Erdman, J.W., Jr. Patients with anorexia nervosa demonstrate deficiencies of selected essential fatty acids, compensatory changes in nonessential fatty acids and decreased fluidity of plasma lipids. J. Nutr. 1995, 125, 901-907.

122. Langan, S.M.; Farrell, P.M. Vitamin E, vitamin A and essential fatty acid status of patients hospitalized for anorexia nervosa. Am. J. Clin. Nutr. 1985, 41, 1054-1056.

123. Ayton, A.K.; Azaz, A.; Horrobin, D.F. A pilot open case series of ethyl-EPA supplementation in the treatment of anorexia nervosa. Prostaglandins Leukort. Essent. Fatty Acids 2004, 71, 205-209.

124. Barbarich, N.C.; McConaha, C.W.; Halmi, K.A.; Gendall, K.; Sunday, S.R.; Gaskill, J.; la Via, M.; Frank, G.K.; Brooks, S.; Plotnicov, K.H.; et al. Use of nutritional supplements to increase the efficacy of fluoxetine in the treatment of anorexia nervosa. Int. J. Eat. Disord. 2004, 35, 10-15.

125. Freeman, M.P.; Fava, M.; Lake, J.; Trivedi, M.H.; Wisner, K.L.; Mischoulon, D. Complementary and alternative medicine in major depressive disorder: The American Psychiatric Association Task Force report. J. Clin. Psychiatry 2010, 71, 669-681.

126. European Food Safety Authority (EFSA) Panel on Dietetic Products, Nutrition and Allergies (NDA). Scientific Opinion on the Tolerable Upper Intake Level of eicosapentaenoic acid (EPA), docosahexaenoic acid (DHA) and docosapentaenoic acid (DPA). EFSA J. 2012, 10, 2815.

127. Yokoyama, M.; Origasa, H.; Matsuzaki, M.; Matsuzawa, Y.; Saito, Y.; Ishikawa, Y.; Oikawa, S.; Sasaki, J.; Hishida, H.; Itakura, H.; et al. Effects of eicosapentaenoic acid on major coronary events in hypercholesterolaemic patients (JELIS): A randomised open-label, blinded endpoint analysis. Lancet 2007, 369, 1090-1098.

128. Tanaka, K.; Ishikawa, Y.; Yokoyama, M.; Origasa, H.; Matsuzaki, M.; Saito, Y.; Matsuzawa, Y.; Sasaki, J.; Oikawa, S.; Hishida, H.; et al. Reduction in the recurrence of stroke by eicosapentaenoic acid for hypercholesterolemic patients: Subanalysis of the JELIS trial. Stroke 2008, 39, 2052-2058.

129. Hartweg, J.; Perera, R.; Montori, V.; Dinneen, S.; Neil HA and Farmer, A. Omega-3 polyunsaturated fatty acids (HUFA) for type 2 diabetes mellitus. Cochrane Database Syst. Rev. 2008.

130. Hartweg, J.; Farmer, A.J.; Holman, R.R.; Neil, A. Potential impact of omega-3 treatment on cardiovascular disease in type 2 diabetes. Curr. Opin. Lipidol. 2009, 20, 30-38.

131. MacLean, C.H.; Mojica, W.A.; Morton, S.C.; Pencharz, J.; Hasenfeld Garland, R. Effects of omega-3 fatty acids on lipids and glycemic control in type II diabetes and the metabolic syndrome and on inflammatory bowel disease, rheumatoid arthritis, renal disease, systemic lupus erythematosus, and osteoporosis. Evid. Rep. Technol. Assess. (Summ.) 2004, 89, 1-4. 
132. Bloomer, R.J.; Larson, D.E.; Fisher-Wellman, K.H.; Galpin, A.J.; Schilling, B.K. Effect of eicosapentaenoic and docosahexaenoic acid on resting and exercise-induced inflammatory and oxidative stress biomarkers: A randomized, placebo controlled, cross-over study. Lipids Health Dis. 2009.

133. VKM (Norwegian Scientific Committee for Food Safety). Opinion of the Steering Committee of the Norwegian Scientific Committee for Food Safety: Evaluation of Negative and Positive Health Effects of n-3 Fatty Acids as Constituents of Food Supplements and Fortified Foods. Available online: http:/ / english.vkm.no/dav/031c000d1a.pdf (accessed on 20 June 2016).

134. Farmer, A.; Montori, V.; Dinneen, S.; Clar, C. Fish oil in people with type 2 diabetes mellitus. Cochrane Database Syst. Rev. 2001. 


\section{Comments on Bozzatello et al. Supplementation with Omega-3 Fatty Acids in Psychiatric Disorders: A Review of Literature Data. J. Clin. Med. 2016, 5, 67}

\section{Gregor Berger}

Reprinted from J. Clin. Med. Cite as: Berger, G. Comments on Bozzatello et al. Supplementation with Omega-3 Fatty Acids in Psychiatric Disorders: A Review of Literature Data. J. Clin. Med. 2016, 5, 67. J. Clin. Med. 2016, 5, 69.

Paola Bozzatello et al. [1] have done a comprehensive qualitative review of the potential use of long-chain polyunsaturated fatty acids in the prevention and treatment of mental disorders. The number of placebo-controlled trials across a range of mental disorders has surged substantially over the last two decades; however, only very few well-designed large scale trials have been performed. Therefore, even in conditions like schizophrenia, depression or attention deficit and hyperactivity disorders (ADHD) where most omega-3 fatty acid RCTs have been performed, no final conclusions regarding the use of omega- 3 fatty acids can be drawn yet. One of the key problems of systematic reviews investigating the use of omega- 3 fatty acids in mental disorders is that they have integrated quite diverse phenotypic groups, e.g., the use of omega-3 fatty acids in controlled treatment trials that also assessed depressive symptoms included the following different conditions:

Primary diagnosis of adult major depressive disorders (MDD) [2-16]

Depressive episodes in bipolar affective disorders [17-23]

Depression during or post pregnancy (postpartum depression) [24-26]

Depression in non-MDD mood disorders (e.g., premenstrual syndrome, dysthymia) [27-32]

Depression in other psychiatric conditions (e.g., borderline PD, self-harm, OCD) [33-37]

Depression in established schizophrenia [2,38]

Depression in Alzheimer's dementia/mild cognitive impairment [13,39,40]

Depression in Parkinson disease [41]

Depression in medical conditions (cerebro-vascular and metabolic diseases or cancer) [42-46]

Depressive symptoms in healthy individuals [47-52]

Several meta-analytic reviews have tried to integrate the above-mentioned very heterogeneous controlled treatment trials investigating the effects of omega- 3 fatty 
acids on mood symptoms [53-59]. Most meta-analysis including RCTs investigating depressive syndromes confirmed a statistical significant effect in favour of omega-3 fatty acids with minimal to moderate effect sizes depending on the selection of studies (except of one meta-analysis [57]). Effect sizes in favour of omega-3 fatty acids [60] are larger if RCTs are selected based on (1) a EPA/DHA ratio $>60 \%$ of the overall omega- 3 fatty acid content $[54,56]$ and (2) only RCTs with a primary diagnosis of MDD are included [54,59]. To our knowledge, only one pilot RCT $(n=20)$ in children with a mean age of 10 was performed [61]. Martins et al.'s [54] meta-analysis including RCTs with primary and secondary MDD found a significant overall SMD $=-0.291$ in favour of omega-3 fatty acids, but also detected a marked study heterogeneity and evidence for publication bias. A more recent meta-analysis by Sublette et al. [56] only including primary MDD RCTs dichotomized according to a EPA/DHA ratio $>60 \%$ of the overall omega-3 fatty acids content found a moderate effect size (SMD $=0.558$ ) with negligible contribution of random effects or heteroscedasticity. Bloch and Hannestadl's meta-analysis [57] including studies with mildly depressed individuals not meeting criteria for clinical depression could not replicate previous meta-analyses; however, a sub-analysis restricted to moderate to marked depression confirmed an SMD of 0.42 in favour of omega-3 fatty acid treatment. It is likely that a single study by Rogers et al. [27] investigating the effects of omega-3 fatty acids on mild depressive symptoms in a large non-clinical population was responsible for the negative overall outcome as the Rogers study accounted for $31.7 \%$ of the overall weight in this particular meta-analysis [62]. Grosso et al. [59] found a SMD $=0.56$ for primary MDD, an SMD $=0.22$ for non-primary MDD, and an overall SMD $=0.38$ in favour of omega-3 fatty acid compared to placebo treatment. The above mentioned meta-analyses suggest that mainly the use of EPA rather than DHA rich formulations are responsible for the clinical efficacy of omega-3 fatty acids. Unexpected is the finding that the use of purified or DHA-enriched oils is not successful in treating depression, postnatal depression or OCD $[4,63,64]$. This finding is in contrast to the greater face validity of DHA, which is the major brain omega- 3 fatty acids and which is lower in brain tissue of depressed suicide victims [65].

Two RCTs encompassing a large proportion of patients with refractory depression highlight the potential use of EPA-enriched omega-3 fatty acids as an augmentation treatment of antidepressants (potentially via an increase in membrane fluidity) [2,3]. Two RCTs in populations without a primary MDD provide evidence of an association between inflammation and omega-3 fatty acids response: (1) A placebo-controlled trial investigating the positive effects of omega-3 fatty acids on depressive symptoms and chronic inflammation in haemodialysis patients [66]; and (2) a study [67] that found a preventive effect of EPA against the development of depressive symptoms in IFN-alpha-treated hepatitis $C$ virus carriers (associated with a very high risk of drug-induced depressive symptoms). The latter two studies 
suggest that omega-3 fatty acids rich in EPA may modulate its antidepressant properties via immune-modulatory strategies, which is of interest in the light of more recent models of the underlying pathophysiology of a range of mental disorders [68].

A whole range of RCTs have also been performed in schizophrenia and related disorders. A study in first episode psychosis adolescents demonstrates that omega-3 fatty acids augmentation treatment of antipsychotic medication may result in a better tolerability (less EPS, less sexual side effects) and faster response to antipsychotic medication; however, at the end of the three month treatment period, there was no difference in treatment effects on all primary outcome measures between active and placebo [69]. The final outcome of this study is in line with a meta-analysis of Dr. Fusar-Poli and the author of this commentary coming to the conclusion that omega-3 fatty acids in established (but not prodromal) schizophrenia have no or only minor additional efficacy compared to currently available treatments (also not on depressive symptoms in schizophrenia) [70], but may have some beneficial effects in tertiary prevention.

The use of omega-3 fatty acids in primary (indicated) prevention of mental disorders in general may be a separate important area of omega-3 fatty acid research that goes beyond the schizophrenia prodrome, and is an avenue yet to be further explored. A pilot RCT in 81 adolescents at ultra-high risk (UHR) for developing a psychotic disorder (mean age 16.4) compared $1.2 \mathrm{~g}$ of an EPA-enriched omega-3 fatty acids oil as a sole agent with a placebo oil in a double blind fashion [71]. A total of $27.5 \%$ in the placebo group progressed towards a first psychotic episode compared to only $4.9 \%$ in the omega- 3 fatty acids group. A recent multinational multicentre study (the NEURAPRO study [72]) including over 300 UHR adolescents tried to replicate this promising pilot study. However, the key problem of the replication study is that the overall transition rate of the study after one year was as low as $10.5 \%$ and the compliance rate of $43 \%$ was very poor so that a reasonable conclusion at this stage of data analysis is not really appropriate [73]. Another multinational multi-centre omega-3 RCT in prodromal schizophrenia will start in the near future (the PURPOSE trial). Bozzatello et al. [1] discussed the latter studies in the schizophrenia section. However, as only $10 \%$ to $20 \%$ of URH adolescents will progress to a first psychotic episode within one year and only about half these first episode cases actually meet criteria for core schizophrenia, it is probably not correct to discuss URH studies within the schizophrenia section. It may be much more appropriate to investigate neuroprotective interventions like omega-3 fatty acids not solely within a schizophrenia concept, but much more in the light of brain developmental factors and biological relevant markers, such as the inflammatory markers, the omega-3 index or markers of neuronal damage (e.g., TNF-beta).

The above mentioned small pilot omega-3 RCT in prepubertal children with childhood-onset depression shows a very large effect size (SMD =1.2) [61]. Also the 
pilot study in UHR adolescents for psychosis [71] with a mean age of 16.1 showed large effect sizes. Furthermore, most omega-3 fatty acids RCTs in children were done in ADHD and show a beneficial effect. Bloch et al.'s meta-analysis [74] including 699 ADHD children of ten RCTs between 7 and 12 years found a beneficial effect in favour of omega- 3 fatty acids with a SMD of 0.31 with no evidence of publication bias and a significant dose dependency; RCTs using a daily dose of 500 to $750 \mathrm{mg}$ EPA were the most effective ones [75]. Even so, the effect size of stimulant treatment with methylphenidate, dexamphetamines or atomotexine is still two to four times stronger compared to omega-3 fatty acids alone, the positive findings of omega- 3 fatty acid studies in children is suggestive that in particular the developing brain may benefit from omega-3 fatty acids. Future research has to address the question if subgroups of children with mental disorders may benefit more from benign interventions like omega-3 fatty acids compared to adults with established (end stage) mental disorders.

For all other mental disorders and associated conditions only limited evidence exists to promote or refute the use of Omega-3 fatty acids in daily clinical care. As outlined by Bozzatello et al. [1], there is some evidence that omega-3 fatty acids augmentation may have some beneficial effects in bipolar affective disorders [17], in particular against depressive symptoms [19,76]. Furthermore, EPA-enriched omega-3 fatty acids may also attenuate impulsivity in patients with Borderline Personality Disorder [35,77] and incarcerated young males [78]. The latter findings may be of particular importance for male pediatric MDD individuals that sometimes present with impulsive and aggressive behaviour rather than sadness [25]. A recently published trial in adolescents with conduct disorders highlights the importance to implement long study durations (e.g., one year) to be able to demonstrate potential positive effects of omega-3 fatty acids on difficult to treat behavioural traits [79]. Worth mentioning is a recent RCT in premenstrual syndrome (PMS) showing some beneficial effects on depression, nervousness, anxiety, lack of concentration and a reduction of somatic symptoms such as bloating, headaches and breast tenderness [32]. These studies need replication, but point towards important phenotypic features that may benefit from omega-3 fatty acids, such as impulsivity. We may consider more complex trial designs to address the questions if omega- 3 fatty acids may be of importance across a whole range of mental disorders, in particular in childhood and adolescents and for certain phenotypic features.

The underlying mechanisms of the potential preventive and therapeutic actions of omega-3 fatty acids against mental disorder are still unclear. Preclinical and clinical data point towards several mechanisms most likely acting in concert [80]. Some of them might be more responsible for short-term, other for postulated long-term effects of omega-3 fatty acids. There is some preclinical evidence that Omega-3 fatty acids may modulate the HPA-axis that is suggested to play a role in a range of mental disorders [81]. Omega-3 fatty acids have shown to attenuate 
stress-related changes in animal models with depressive features [82-85] as well as in humans [86-88]. Furthermore, Omega-3 fatty acids may influence myelination and synaptic pruning, important processes for normal pubertal brain development. The regulation of PUFA metabolism is crucial for both processes [89,90]. Of particular interest is a preclinical study investigating cognition and behaviour across different developmental stages. Omega-3 fatty acids deficient diets across consecutive generations produced a modality-selective and task-dependent impairment in cognitive and motivated behaviour in adolescence distinct from the deficits observed in adults [91,92]. Omega-3 fatty acids attenuate such depression-like animal behaviours during critical periods of brain development [93]. Furthermore, the FADS haplotype determining LC-PUFAs availability and concentrations in white matter (WM) showed age-related WM differences in humans (significant age $\times$ genotype interactions, $p$ (corrected) $<0.05)$. PUFA metabolism is therefore likely to play a role in disorders of neurodevelopmental origin [94]. Animal models with structural hippocampal alterations with depression-like and anxiety-like behaviours $[95,96]$ provide evidence that omega-3 fatty acids have a preventive and neurotrophic effect against hippocampal changes $[97,98]$. Omega-3 fatty acids enhance hippocampal cell viability and are able to protect hippocampal cells from stress-related damage [99]. Monoaminergic transmitter systems are proposed to be involved in the pathogenesis of many mental disorders. Animal experiments of omega-3 fatty acids deprived rats provide evidence for an increase in serotonin 2 (5-HT2) and a decrease in dopamine 2 (D2) receptor density in the frontal cortex, as well as an increased serotonin turnover in the prefrontal cortex and decreased midbrain tryptophan hydroxylase-2 expression [100-106]. In humans, omege-3 intake is associated with an increase in cerebrospinal fluid 5-HIAA release $[107,108]$. Several lines of evidence support that Omega-3 fatty acids have immune-modulatory, anti-inflammatory and pro-resolving properties [109], e.g., via the modulation of pro-inflammatory omega- 6 , the promotion of proresolvins, neuroprotectins and anti-inflammatory mediators [110-112]. Omega-3 fatty acids seem to induce protective in vivo brain mechanisms against oxidative stress. Ethyl-EPA supplementation is associated with a marked increase of glutathione, a strong intracellular antioxidant using proton magnetic resonance spectroscopy in patients with a first-episode psychosis [113]. Another group found similar effects in older patients at risk for depression [114]. Some evidence regarding the measurement of glutathione in peripheral blood is also suggestive that omega-3 fatty acids may support the antioxidative defence system in individuals at ultra-high risk for psychosis [115]. Finally, a decrease in membrane fluidity can affect the rotation and diffusion of proteins and other bio-molecules within the membrane, thereby affecting the functions of these molecules and processes. An increase in membrane fluidity results in a more flexible membrane and facilitates transmission (e.g., in the retina) [116]. In vivo imaging techniques such 
as diffusion tensor imaging could demonstrate that omega-3 fatty acids are closely linked to PUFA metabolism [94]. The effect of omega-3 fatty acids on membrane structure [117] may contribute to its clinical effects, in particular in augmentation studies. T2-relaxation time normalizes under the influence omega-3 fatty acids potentially being a signifier of normalization in membrane structure [118]. Future studies should therefore address if particular markers, such as low baseline levels of omega-3 fatty acids, increased inflammatory mediators, markers of intact myelination (e.g., measured with DTI), or a functional glia-neuronal interface (e.g., measured with MRS) may serve as predictors of omega-3 fatty acid response, in particular in children and adolescents in the early course of disorders [119]. Bozzatello et al.'s [1] qualitative and comprehensive review is a contribution to this endeavour and highly recommended to interested readers.

Conflicts of Interest: The author declare no conflict of interest.

\section{References}

1. Bozzatello, P.; Brignolo, E.; De Grandi, E.; Bellino, S. Supplementation with Omega-3 Fatty Acids in Psychiatric Disorders: A Review of Literature Data. J. Clin. Med. 2016, 5,67 .

2. Peet, M.; Horrobin, D.F. A dose-ranging study of the effects of ethyl-eicosapentaenoate in patients with ongoing depression despite apparently adequate treatment with standard drugs. Arch. Gen. Psychiatry 2002, 59, 913-919.

3. Nemets, B.; Stahl, Z.; Belmaker, R.H. Addition of omega-3 fatty acid to maintenance medication treatment for recurrent unipolar depressive disorder. Am. J. Psychiatry 2002, 159, 477-479.

4. Marangell, L.B.; Martinez, J.M.; Zboyan, H.A.; Kertz, B.; Kim, H.F.; Puryear, L.J. A double-blind, placebo-controlled study of the omega-3 fatty acid docosahexaenoic acid in the treatment of major depression. Am. J. Psychiatry 2003, 160, 996-998.

5. Su, K.P.; Huang, S.Y.; Chiu, C.C.; Shen, W.W. Omega-3 fatty acids in major depressive disorder. A preliminary double-blind, placebo-controlled trial. Eur. Neuropsychopharmacol. 2003, 13, 267-271.

6. Silvers, K.M.; Woolley, C.C.; Hamilton, F.C.; Watts, P.M.; Watson, R.A. Randomised double-blind placebo-controlled trial of fish oil in the treatment of depression. Prostaglandins Leukot. Essent. Fat. Acids 2005, 72, 211-218.

7. Grenyer, B.F.; Crowe, T.; Meyer, B.; Owen, A.J.; Grigonis-Deane, E.M.; Caputi, P.; Howe, P.R. Fish oil supplementation in the treatment of major depression: A randomised double-blind placebo-controlled trial. Prog. Neuro-Psychopharmacol. Biol. Psychiatry 2007, 31, 1393-1396. 
8. Jazayeri, S.; Tehrani-Doost, M.; Keshavarz, S.A.; Hosseini, M.; Djazayery, A.; Amini, H.; Jalali, M.; Peet, M. Comparison of therapeutic effects of omega-3 fatty acid eicosapentaenoic acid and fluoxetine, separately and in combination, in major depressive disorder. Aust. N. Z. J. Psychiatry 2008, 42, 192-198.

9. Mischoulon, D.; Papakostas, G.I.; Dording, C.M.; Farabaugh, A.H.; Sonawalla, S.B.; Agoston, A.M.; Smith, J.; Beaumont, E.C.; Dahan, L.E.; Alpert, J.E.; et al. A double-blind, randomized controlled trial of ethyl-eicosapentaenoate for major depressive disorder. J. Clin. Psychiatry 2009, 70, 1636-1644.

10. Rondanelli, M.; Giacosa, A.; Opizzi, A.; Pelucchi, C.; La Vecchia, C.; Montorfano, G.; Negroni, M.; Berra, B.; Politi, P.; Rizzo, A.M. Effect of omega-3 fatty acids supplementation on depressive symptoms and on health-related quality of life in the treatment of elderly women with depression: A double-blind, placebo-controlled, randomized clinical trial. J. Am. Coll. Nutr. 2010, 29, 55-64.

11. Rondanelli, M.; Giacosa, A.; Opizzi, A.; Pelucchi, C.; La Vecchia, C.; Montorfano, G.; Negroni, M.; Berra, B.; Politi, P.; Rizzo, A.M. Long chain omega 3 polyunsaturated fatty acids supplementation in the treatment of elderly depression: Effects on depressive symptoms, on phospholipids fatty acids profile and on health-related quality of life. J. Nutr. Health Aging 2011, 15, 37-44.

12. Lesperance, F.; Frasure-Smith, N.; St-Andre, E.; Turecki, G.; Lesperance, P.; Wisniewski, S.R. The efficacy of omega-3 supplementation for major depression: A randomized controlled trial. J. Clin. Psychiatry 2011, 72, 1054-1062.

13. Sinn, N.; Milte, C.M.; Street, S.J.; Buckley, J.D.; Coates, A.M.; Petkov, J.; Howe, P.R. Effects of $n-3$ fatty acids, epa $\mathrm{v}$. Dha, on depressive symptoms, quality of life, memory and executive function in older adults with mild cognitive impairment: A 6-month randomised controlled trial. Br. J. Nutr. 2012, 107, 1682-1693.

14. Gertsik, L.; Poland, R.E.; Bresee, C.; Rapaport, M.H. Omega-3 fatty acid augmentation of citalopram treatment for patients with major depressive disorder. J. Clin. Psychopharmacol. 2012, 32, 61-64.

15. Rizzo, A.M.; Corsetto, P.A.; Montorfano, G.; Opizzi, A.; Faliva, M.; Giacosa, A.; Ricevuti, G.; Pelucchi, C.; Berra, B.; Rondanelli, M. Comparison between the AA/EPA ratio in depressed and non depressed elderly females: Omega-3 fatty acid supplementation correlates with improved symptoms but does not change immunological parameters. Nutr. J. 2012, 11, 82.

16. Rapaport, M.H.; Nierenberg, A.A.; Schettler, P.J.; Kinkead, B.; Cardoos, A.; Walker, R.; Mischoulon, D. Inflammation as a predictive biomarker for response to omega-3 fatty acids in major depressive disorder: A proof-of-concept study. Mol. Psychiatry 2016, 21, 71-79.

17. Stoll, A.L.; Severus, W.E.; Freeman, M.P.; Rueter, S.; Zboyan, H.A.; Diamond, E.; Cress, K.K.; Marangell, L.B. Omega 3 fatty acids in bipolar disorder: A preliminary double-blind, placebo-controlled trial [see comments]. Arch. Gen. Psychiatry 1999, 56, $407-412$. 
18. Hirashima, F.; Parow, A.M.; Stoll, A.L.; Demopulos, C.M.; Damico, K.E.; Rohan, M.L.; Eskesen, J.G.; Zuo, C.S.; Cohen, B.M.; Renshaw, P.F. Omega-3 fatty acid treatment and t(2) whole brain relaxation times in bipolar disorder. Am. J. Psychiatry 2004, 161, 1922-1924.

19. Chiu, C.C.; Huang, S.Y.; Chen, C.C.; Su, K.P. Omega-3 fatty acids are more beneficial in the depressive phase than in the manic phase in patients with bipolar i disorder. J. Clin. Psychiatry 2005, 66, 1613-1614.

20. Frangou, S.; Lewis, M.; McCrone, P. Efficacy of ethyl-eicosapentaenoic acid in bipolar depression: Randomised double-blind placebo-controlled study. Br. J. Psychiatry 2006, 188, 46-50.

21. Keck, P.E., Jr.; Mintz, J.; McElroy, S.L.; Freeman, M.P.; Suppes, T.; Frye, M.A.; Altshuler, L.L.; Kupka, R.; Nolen, W.A.; Leverich, G.S.; et al. Double-blind, randomized, placebo-controlled trials of ethyl-eicosapentanoate in the treatment of bipolar depression and rapid cycling bipolar disorder. Biol. Psychiatry 2006, 60, 1020-1022.

22. Frangou, S.; Lewis, M.; Wollard, J.; Simmons, A. Preliminary in vivo evidence of increased $n$-acetyl-aspartate following eicosapentanoic acid treatment in patients with bipolar disorder. J. Psychopharmacol. 2007, 21, 435-439.

23. Murphy, B.L.; Stoll, A.L.; Harris, P.Q.; Ravichandran, C.; Babb, S.M.; Carlezon, W.A., Jr.; Cohen, B.M. Omega-3 fatty acid treatment, with or without cytidine, fails to show therapeutic properties in bipolar disorder: A double-blind, randomized add-on clinical trial. J. Clin. Psychopharmacol. 2012, 32, 699-703.

24. Freeman, M.P.; Davis, M.; Sinha, P.; Wisner, K.L.; Hibbeln, J.R.; Gelenberg, A.J. Omega-3 fatty acids and supportive psychotherapy for perinatal depression: A randomized placebo-controlled study. J. Affect. Disord. 2008, 110, 142-148.

25. Breland, D.J.; Park, M.J. Depression: Focus on the adolescent male. Am. J. Mens Health 2008, 2, 87-93.

26. Rees, A.M.; Austin, M.P.; Parker, G.B. Omega-3 fatty acids as a treatment for perinatal depression: Randomized double-blind placebo-controlled trial. Aust. N. Z. J. Psychiatry 2008, 42, 199-205.

27. Rogers, P.J.; Appleton, K.M.; Kessler, D.; Peters, T.J.; Gunnell, D.; Hayward, R.C.; Heatherley, S.V.; Christian, L.M.; McNaughton, S.A.; Ness, A.R. No effect of $n-3$ long-chain polyunsaturated fatty acid (epa and dha) supplementation on depressed mood and cognitive function: A randomised controlled trial. Br. J. Nutr. 2008, 99, 421-431.

28. Lucas, M.; Asselin, G.; Merette, C.; Poulin, M.J.; Dodin, S. Ethyl-eicosapentaenoic acid for the treatment of psychological distress and depressive symptoms in middle-aged women: A double-blind, placebo-controlled, randomized clinical trial. Am. J. Clin. Nutr. 2009, 89, 641-651.

29. Tajalizadekhoob, Y.; Sharifi, F.; Fakhrzadeh, H.; Mirarefin, M.; Ghaderpanahi, M.; Badamchizade, Z.; Azimipour, S. The effect of low-dose omega 3 fatty acids on the treatment of mild to moderate depression in the elderly: A double-blind, randomized, placebo-controlled study. Eur. Arch. Psychiatry Clin. Neurosci. 2011, 261, 539-549. 
30. Antypa, N.; Smelt, A.H.; Strengholt, A.; Van der Does, A.J. Effects of omega-3 fatty acid supplementation on mood and emotional information processing in recovered depressed individuals. J. Psychopharmacol. 2012, 26, 738-743.

31. Mozaffari-Khosravi, H.; Yassini-Ardakani, M.; Karamati, M.; Shariati-Bafghi, S.E. Eicosapentaenoic acid versus docosahexaenoic acid in mild-to-moderate depression: A randomized, double-blind, placebo-controlled trial. Eur. Neuropsychopharmacol. 2013, 23, 636-644.

32. Sohrabi, N.; Kashanian, M.; Ghafoori, S.S.; Malakouti, S.K. Evaluation of the effect of omega-3 fatty acids in the treatment of premenstrual syndrome: "A pilot trial". Complement. Ther. Med. 2013, 21, 141-146.

33. Behan, P.O.; Behan, W.M.; Horrobin, D.F. Effect of high doses of essential fatty acids on the postviral fatigue syndrome. Acta Neurol. Scand. 1990, 82, 209-216.

34. Warren, G.; McKendrick, M.; Peet, M. The role of essential fatty acids in chronic fatigue syndrome. A case-controlled study of red-cell membrane essential fatty acids (EFA) and a placebo-controlled treatment study with high dose of efa. Acta Neurol. Scand. 1999, 99, 112-116.

35. Zanarini, M.C.; Frankenburg, F.R. Omega-3 fatty acid treatment of women with borderline personality disorder: A double-blind, placebo-controlled pilot study. Am. J. Psychiatry 2003, 160, 167-169.

36. Fux, M.; Benjamin, J.; Nemets, B. A placebo-controlled cross-over trial of adjunctive epa in ocd. J. Psychiatry Res. 2004, 38, 323-325.

37. Hallahan, B.; Hibbeln, J.R.; Davis, J.M.; Garland, M.R. Omega-3 fatty acid supplementation in patients with recurrent self-harm. Single-centre double-blind randomised controlled trial. Br. J. Psychiatry 2007, 190, 118-122.

38. Fenton, W.S.; Dickerson, F.; Boronow, J.; Hibbeln, J.R.; Knable, M. A placebo-controlled trial of omega-3 fatty acid (ethyl eicosapentaenoic acid) supplementation for residual symptoms and cognitive impairment in schizophrenia. Am. J. Psychiatry 2001, 158, 2071-2074.

39. Chiu, C.C.; Su, K.P.; Cheng, T.C.; Liu, H.C.; Chang, C.J.; Dewey, M.E.; Stewart, R.; Huang, S.Y. The effects of omega-3 fatty acids monotherapy in Alzheimer's disease and mild cognitive impairment: A preliminary randomized double-blind placebo-controlled study. Prog. Neuro-psychopharmacol. Biol. Psychiatry 2008, 32, 1538-1544.

40. Freund-Levi, Y.; Basun, H.; Cederholm, T.; Faxen-Irving, G.; Garlind, A.; Grut, M.; Vedin, I.; Palmblad, J.; Wahlund, L.O.; Eriksdotter-Jonhagen, M. Omega-3 supplementation in mild to moderate alzheimer's disease: Effects on neuropsychiatric symptoms. Int. J. Geriatr. Psychiatry 2008, 23, 161-169.

41. Da Silva, T.M.; Munhoz, R.P.; Alvarez, C.; Naliwaiko, K.; Kiss, A.; Andreatini, R.; Ferraz, A.C. Depression in Parkinson's disease: A double-blind, randomized, placebo-controlled pilot study of omega-3 fatty-acid supplementation. J. Affect. Disord. 2008, 111, 351-359. 
42. Carney, R.M.; Freedland, K.E.; Rubin, E.H.; Rich, M.W.; Steinmeyer, B.C.; Harris, W.S. Omega-3 augmentation of sertraline in treatment of depression in patients with coronary heart disease: A randomized controlled trial. J. Am. Med. Assoc. (JAMA) 2009, 302, 1651-1657.

43. Bot, M.; Pouwer, F.; Assies, J.; Jansen, E.H.; Diamant, M.; Snoek, F.J.; Beekman, A.T.; de Jonge, P. Eicosapentaenoic acid as an add-on to antidepressant medication for co-morbid major depression in patients with diabetes mellitus: A randomized, double-blind placebo-controlled study. J. Affect. Disord. 2010, 126, 282-286.

44. Giltay, E.J.; Geleijnse, J.M.; Kromhout, D. Effects of $n-3$ fatty acids on depressive symptoms and dispositional optimism after myocardial infarction. Am. J. Clin. Nutr. 2011, 94, 1442-1450.

45. Bot, M.; Pouwer, F.; Assies, J.; Jansen, E.H.; Beekman, A.T.; de Jonge, P. Supplementation with eicosapentaenoic omega-3 fatty acid does not influence serum brain-derived neurotrophic factor in diabetes mellitus patients with major depression: A randomized controlled pilot study. Neuropsychobiology 2011, 63, 219-223.

46. Andreeva, V.A.; Galan, P.; Torres, M.; Julia, C.; Hercberg, S.; Kesse-Guyot, E. Supplementation with $\mathrm{b}$ vitamins or $n-3$ fatty acids and depressive symptoms in cardiovascular disease survivors: Ancillary findings from the supplementation with folate, vitamins b-6 and b-12 and/or omega-3 fatty acids (su.Fol.Om3) randomized trial. Am. J. Clin. Nutr. 2012, 96, 208-214.

47. Fontani, G.; Corradeschi, F.; Felici, A.; Alfatti, F.; Migliorini, S.; Lodi, L. Cognitive and physiological effects of omega-3 polyunsaturated fatty acid supplementation in healthy subjects. Eur. J. Clin. Investig. 2005, 35, 691-699.

48. Van de Rest, O.; Geleijnse, J.M.; Kok, F.J.; van Staveren, W.A.; Hoefnagels, W.H.; Beekman, A.T.; de Groot, L.C. Effect of fish-oil supplementation on mental well-being in older subjects: A randomized, double-blind, placebo-controlled trial. Am. J. Clin. Nutr. 2008, 88, 706-713.

49. Antypa, N.; Van der Does, A.J.; Smelt, A.H.; Rogers, R.D. Omega-3 fatty acids (fish-oil) and depression-related cognition in healthy volunteers. J. Psychopharmacol. 2009, 23, 831-840.

50. Kiecolt-Glaser, J.K.; Belury, M.A.; Andridge, R.; Malarkey, W.B.; Glaser, R. Omega-3 supplementation lowers inflammation and anxiety in medical students: A randomized controlled trial. Brain Behav. Immun. 2011, 25, 1725-1734.

51. DeFina, L.F.; Marcoux, L.G.; Devers, S.M.; Cleaver, J.P.; Willis, B.L. Effects of omega-3 supplementation in combination with diet and exercise on weight loss and body composition. Am. J. Clin. Nutr. 2011, 93, 455-462.

52. Kiecolt-Glaser, J.K.; Belury, M.A.; Andridge, R.; Malarkey, W.B.; Hwang, B.S.; Glaser, R. Omega-3 supplementation lowers inflammation in healthy middle-aged and older adults: A randomized controlled trial. Brain Behav. Immun. 2012, 26, 988-995.

53. Parker, G.; Gibson, N.A.; Brotchie, H.; Heruc, G.; Rees, A.M.; Hadzi-Pavlovic, D. Omega-3 fatty acids and mood disorders. Am. J. Psychiatry 2006, 163, 969-978. 
54. Martins, J.G. Epa but not dha appears to be responsible for the efficacy of omega-3 long chain polyunsaturated fatty acid supplementation in depression: Evidence from a meta-analysis of randomized controlled trials. J. Am. Coll. Nutr. 2009, 28, 525-542.

55. Freeman, M.P.; Fava, M.; Lake, J.; Trivedi, M.H.; Wisner, K.L.; Mischoulon, D. Complementary and alternative medicine in major depressive disorder: The american psychiatric association task force report. J. Clin. Psychiatry 2010, 71, 669-681.

56. Sublette, M.E.; Ellis, S.P.; Geant, A.L.; Mann, J.J. Meta-analysis of the effects of eicosapentaenoic acid (EPA) in clinical trials in depression. J. Clin. Psychiatry 2011, 72, 1577-1584.

57. Bloch, M.H.; Hannestad, J. Omega-3 fatty acids for the treatment of depression: Systematic review and meta-analysis. Mol. Psychiatry 2012, 17, 1272-1282.

58. Lin, P.Y.; Mischoulon, D.; Freeman, M.P.; Matsuoka, Y.; Hibbeln, J.; Belmaker, R.H.; $\mathrm{Su}, \mathrm{K} . \mathrm{P}$. Are omega-3 fatty acids antidepressants or just mood-improving agents? The effect depends upon diagnosis, supplement preparation, and severity of depression. Mol. Psychiatry 2012, 17, 1161-1163.

59. Grosso, G.; Pajak, A.; Marventano, S.; Castellano, S.; Galvano, F.; Bucolo, C.; Drago, F.; Caraci, F. Role of omega-3 fatty acids in the treatment of depressive disorders: A comprehensive meta-analysis of randomized clinical trials. PLoS ONE 2014, 9, e96905.

60. Li, F.; Liu, X.; Zhang, D. Fish consumption and risk of depression: A meta-analysis. J. Epidemiol. Commun. Health 2015.

61. Nemets, H.; Nemets, B.; Apter, A.; Bracha, Z.; Belmaker, R.H. Omega-3 treatment of childhood depression: A controlled, double-blind pilot study. Am. J. Psychiatry 2006, 163, 1098-1100.

62. Martins, J.G.; Bentsen, H.; Puri, B.K. Eicosapentaenoic acid appears to be the key omega-3 fatty acid component associated with efficacy in major depressive disorder: A critique of bloch and hannestad and updated meta-analysis. Mol. Psychiatry 2012, 17, 1144-1149.

63. Chiu, C.C.; Huang, S.Y.; Su, K.P. Omega-3 polyunsaturated fatty acids for postpartum depression. Am. J. Obstet. Gynecol. 2004, 190, 582-583.

64. Chiu, C.C.; Huang, S.Y.; Shen, W.W.; Su, K.P. Omega-3 fatty acids for depression in pregnancy. Am. J. Psychiatry 2003, 160, 385.

65. McNamara, R.K.; Jandacek, R.; Tso, P.; Dwivedi, Y.; Ren, X.; Pandey, G.N. Lower docosahexaenoic acid concentrations in the postmortem prefrontal cortex of adult depressed suicide victims compared with controls without cardiovascular disease. J. Psychiatry Res. 2013, 47, 1187-1191.

66. Gharekhani, A.; Khatami, M.R.; Dashti-Khavidaki, S.; Razeghi, E.; Noorbala, A.A.; Hashemi-Nazari, S.S.; Mansournia, M.A. The effect of omega-3 fatty acids on depressive symptoms and inflammatory markers in maintenance hemodialysis patients: A randomized, placebo-controlled clinical trial. Eur. J. Clin. Pharmacol. 2014, 70, 655-665.

67. Su, K.P.; Lai, H.C.; Yang, H.T.; Su, W.P.; Peng, C.Y.; Chang, J.P.; Chang, H.C.; Pariante, C.M. Omega-3 fatty acids in the prevention of interferon-alpha-induced depression: Results from a randomized, controlled trial. Biol. Psychiatry 2014, 76, 559-566. 
68. Lang, U.E.; Borgwardt, S. Molecular mechanisms of depression: Perspectives on new treatment strategies. Cell. Physiol. Biochem. 2013, 31, 761-777.

69. Berger, G.E.; Proffitt, T.M.; McConchie, M.; Yuen, H.; Wood, S.J.; Amminger, G.P.; Brewer, W.; McGorry, P.D. Ethyl-eicosapentaenoic acid in first-episode psychosis: A randomized, placebo-controlled trial. J. Clin. Psychiatry 2007, 68, 1867-1875.

70. Fusar-Poli, P.; Berger, G. Eicosapentaenoic acid interventions in schizophrenia: Meta-analysis of randomized, placebo-controlled studies. J. Clin. Psychopharmacol. 2012, 32, 179-185.

71. Amminger, G.P.; Schafer, M.R.; Papageorgiou, K.; Klier, C.M.; Cotton, S.M.; Harrigan, S.M.; Mackinnon, A.; McGorry, P.D.; Berger, G.E. Long-chain omega-3 fatty acids for indicated prevention of psychotic disorders: A randomized, placebo-controlled trial. Arch. Gen. Psychiatry 2010, 67, 146-154.

72. Markulev, C.; McGorry, P.D.; Nelson, B.; Yuen, H.P.; Schaefer, M.; Yung, A.R.; Thompson, A.; Berger, G.; Mossaheb, N.; Schlogelhofer, M.; et al. Neurapro-e study protocol: A multicentre randomized controlled trial of omega-3 fatty acids and cognitive-behavioural case management for patients at ultra high risk of schizophrenia and other psychotic disorders. Early Interv. Psychiatry 2015.

73. McGorry, P.D.; Goldstone, S.; Berger, G.E.; Chen, E.; de Haan, L.; Hickie, I.; Markulev, C.; Mossaheb, N.; Nelson, B.; Nieman, D.; et al. The neurapro-e study: A multicentre rct of omega-3 fatty acids and cognitive-behavioral case management for patients at ultra-high risk of psychosis (o8.2). In Proceedings of the 5th Biennial SIRS Conference, Florence, Italy, 2-6 April 2016; Schizophrenia International Research Society/Nature Publishing Group: Florence, Italy, 2016; Volume 2, p. 24.

74. Bloch, M.H.; Qawasmi, A. Omega-3 fatty acid supplementation for the treatment of children with attention-deficit/hyperactivity disorder symptomatology: Systematic review and meta-analysis. J. Am. Acad. Child Adolesc. Psychiatry 2011, 50, 991-1000.

75. Sonuga-Barke, E.J.; Brandeis, D.; Cortese, S.; Daley, D.; Ferrin, M.; Holtmann, M.; Stevenson, J.; Danckaerts, M.; van der Oord, S.; Dopfner, M.; et al. Nonpharmacological interventions for adhd: Systematic review and meta-analyses of randomized controlled trials of dietary and psychological treatments. Am. J. Psychiatry 2013, 170, 275-289.

76. Su, K.P.; Shen, W.W.; Huang, S.Y. Are omega3 fatty acids beneficial in depression but not mania? Arch. Gen. Psychiatry 2000, 57, 716-717.

77. Bellino, S.; Bozzatello, P.; Rocca, G.; Bogetto, F. Efficacy of omega-3 fatty acids in the treatment of borderline personality disorder: A study of the association with valproic acid. J. Psychopharmacol. 2014, 28, 125-132.

78. Gesch, C.B.; Hammond, S.M.; Hampson, S.E.; Eves, A.; Crowder, M.J. Influence of supplementary vitamins, minerals and essential fatty acids on the antisocial behaviour of young adult prisoners. Randomised, placebo-controlled trial. Br. J. Psychiatry 2002, 181, 22-28. 
79. Raine, A.; Portnoy, J.; Liu, J.; Mahoomed, T.; Hibbeln, J.R. Reduction in behavior problems with omega-3 supplementation in children aged 8-16 years: A randomized, double-blind, placebo-controlled, stratified, parallel-group trial. J. Child Psychol. Psychiatry 2015, 56, 509-520.

80. Grosso, G.; Galvano, F.; Marventano, S.; Malaguarnera, M.; Bucolo, C.; Drago, F.; Caraci, F. Omega-3 fatty acids and depression: Scientific evidence and biological mechanisms. Oxid. Med. Cell. Longev. 2014, 2014, 313570.

81. Gold, P.W. The organization of the stress system and its dysregulation in depressive illness. Mol. Psychiatry 2015, 20, 32-47.

82. El-Ansary, A.K.; Al-Daihan, S.K.; El-Gezeery, A.R. On the protective effect of omega-3 against propionic acid-induced neurotoxicity in rat pups. Lipids Health Dis. 2011, 10, 142.

83. Song, C.; Li, X.; Kang, Z.; Kadotomi, Y. Omega-3 fatty acid ethyl-eicosapentaenoate attenuates il-1beta-induced changes in dopamine and metabolites in the shell of the nucleus accumbens: Involved with pla2 activity and corticosterone secretion. Neuropsychopharmacology 2007, 32, 736-744.

84. Goshen, I.; Kreisel, T.; Ben-Menachem-Zidon, O.; Licht, T.; Weidenfeld, J.; Ben-Hur, T.; Yirmiya, R. Brain interleukin-1 mediates chronic stress-induced depression in mice via adrenocortical activation and hippocampal neurogenesis suppression. Mol. Psychiatry 2008, 13, 717-728.

85. Larrieu, T.; Hilal, M.L.; Fourrier, C.; De Smedt-Peyrusse, V.; Sans, N.; Capuron, L.; Laye, S. Nutritional omega-3 modulates neuronal morphology in the prefrontal cortex along with depression-related behaviour through corticosterone secretion. Transl. Psychiatry 2014, 4, e437.

86. Mocking, R.J.; Ruhe, H.G.; Assies, J.; Lok, A.; Koeter, M.W.; Visser, I.; Bockting, C.L.; Schene, A.H. Relationship between the hypothalamic-pituitary-adrenal-axis and fatty acid metabolism in recurrent depression. Psychoneuroendocrinology 2013, 38, 1607-1617.

87. Assies, J.; Mocking, R.J.; Lok, A.; Ruhe, H.G.; Pouwer, F.; Schene, A.H. Effects of oxidative stress on fatty acid- and one-carbon-metabolism in psychiatric and cardiovascular disease comorbidity. Acta Psychiatry Scand. 2014, 130, 163-180.

88. Mocking, R.J.; Verburg, H.F.; Westerink, A.M.; Assies, J.; Vaz, F.M.; Koeter, M.W.; Ruhe, H.G.; Schene, A.H. Fatty acid metabolism and its longitudinal relationship with the hypothalamic-pituitary-adrenal axis in major depression: Associations with prospective antidepressant response. Psychoneuroendocrinology 2015, 59, 1-13.

89. McNamara, R.K.; Vannest, J.J.; Valentine, C.J. Role of perinatal long-chain omega-3 fatty acids in cortical circuit maturation: Mechanisms and implications for psychopathology. World J. Psychiatry 2015, 5, 15-34.

90. McNamara, R.K.; Carlson, S.E. Role of omega-3 fatty acids in brain development and function: Potential implications for the pathogenesis and prevention of psychopathology. Prostaglandins Leukot. Essent. Fat. Acids 2006, 75, 329-349.

91. Bourre, J.M. Roles of unsaturated fatty acids (especially omega-3 fatty acids) in the brain at various ages and during ageing. J. Nutr. Health Aging 2004, 8, 163-174. 
92. Janssen, C.I.; Kiliaan, A.J. Long-chain polyunsaturated fatty acids (LCPUFA) from genesis to senescence: The influence of lcpufa on neural development, aging, and neurodegeneration. Prog. Lipid Res. 2013, 53, 1-17.

93. Pudell, C.; Vicente, B.A.; Delattre, A.M.; Carabelli, B.; Mori, M.A.; Suchecki, D.; Machado, R.B.; Zanata, S.M.; Visentainer, J.V.; de Oliveira Santos Junior, O.; et al. Fish oil improves anxiety-like, depressive-like and cognitive behaviors in olfactory bulbectomised rats. Eur. J. Neurosci. 2014, 39, 266-274.

94. Peters, B.D.; Voineskos, A.N.; Szeszko, P.R.; Lett, T.A.; DeRosse, P.; Guha, S.; Karlsgodt, K.H.; Ikuta, T.; Felsky, D.; John, M.; et al. Brain white matter development is associated with a human-specific haplotype increasing the synthesis of long chain fatty acids. J. Neurosci. 2014, 34, 6367-6376.

95. Song, C.; Wang, H. Cytokines mediated inflammation and decreased neurogenesis in animal models of depression. Prog. Neuro-psychopharmacol. Biol. Psychiatry 2011, 35, 760-768.

96. Song, C.; Zhang, X.Y.; Manku, M. Increased phospholipase a2 activity and inflammatory response but decreased nerve growth factor expression in the olfactory bulbectomized rat model of depression: Effects of chronic ethyl-eicosapentaenoate treatment. J. Neurosci. 2009, 29, 14-22.

97. Carlezon, W.A., Jr.; Mague, S.D.; Parow, A.M.; Stoll, A.L.; Cohen, B.M.; Renshaw, P.F. Antidepressant-like effects of uridine and omega-3 fatty acids are potentiated by combined treatment in rats. Biol. Psychiatry 2005, 57, 343-350.

98. Huang, S.Y.; Yang, H.T.; Chiu, C.C.; Pariante, C.M.; Su, K.P. Omega-3 fatty acids on the forced-swimming test. J. Psychiatry Res. 2008, 42, 58-63.

99. Bartl, J.; Walitza, S.; Grunblatt, E. Enhancement of cell viability after treatment with polyunsaturated fatty acids. Neurosci. Lett. 2014, 559, 56-60.

100. Delion, S.; Chalon, S.; Guilloteau, D.; Besnard, J.C.; Durand, G. Alpha-linolenic acid dietary deficiency alters age-related changes of dopaminergic and serotoninergic neurotransmission in the rat frontal cortex. J. Neurochem. 1996, 66, 1582-1591.

101. Delion, S.; Chalon, S.; Guilloteau, D.; Lejeune, B.; Besnard, J.C.; Durand, G. Age-related changes in phospholipid fatty acid composition and monoaminergic neurotransmission in the hippocampus of rats fed a balanced or an $n-3$ polyunsaturated fatty acid-deficient diet. J. Lipid Res. 1997, 38, 680-689.

102. Zimmer, L.; Delion-Vancassel, S.; Durand, G.; Guilloteau, D.; Bodard, S.; Besnard, J.C.; Chalon, S. Modification of dopamine neurotransmission in the nucleus accumbens of rats deficient in $n-3$ polyunsaturated fatty acids. J. Lipid Res. 2000, 41, 32-40.

103. Yao, J.K.; Magan, S.; Sonel, A.F.; Gurklis, J.A.; Sanders, R.; Reddy, R.D. Effects of omega-3 fatty acid on platelet serotonin responsivity in patients with schizophrenia. Prostaglandins Leukot. Essent. Fat. Acids 2004, 71, 171-176.

104. Song, C.; Horrobin, D. Omega-3 fatty acid ethyl-eicosapentaenoate, but not soybean oil, attenuates memory impairment induced by central il-1beta administration. J. Lipid Res. 2004, 45, 1112-1121. 
105. Chalon, S. Omega-3 fatty acids and monoamine neurotransmission. Prostaglandins Leukot. Essent. Fat. Acids 2006, 75, 259-269.

106. McNamara, R.K.; Able, J.; Liu, Y.; Jandacek, R.; Rider, T.; Tso, P.; Lipton, J.W. Omega-3 fatty acid deficiency during perinatal development increases serotonin turnover in the prefrontal cortex and decreases midbrain tryptophan hydroxylase- 2 expression in adult female rats: Dissociation from estrogenic effects. J. Psychiatry Res. 2009, 43, 656-663.

107. Hibbeln, J.R.; Linnoila, M.; Umhau, J.C.; Rawlings, R.; George, D.T.; Salem, N., Jr. Essential fatty acids predict metabolites of serotonin and dopamine in cerebrospinal fluid among healthy control subjects, and early- and late-onset alcoholics. Biol. Psychiatry 1998, $44,235-242$.

108. Hibbeln, J.R.; Umhau, J.C.; Linnoila, M.; George, D.T.; Ragan, P.W.; Shoaf, S.E.; Vaughan, M.R.; Rawlings, R.; Salem, N., Jr. A replication study of violent and nonviolent subjects: Cerebrospinal fluid metabolites of serotonin and dopamine are predicted by plasma essential fatty acids. Biol. Psychiatry 1998, 44, 243-249.

109. Serhan, C.N. Pro-resolving lipid mediators are leads for resolution physiology. Nature 2014, 510, 92-101.

110. Freeman, M.P.; Rapaport, M.H. Omega-3 fatty acids and depression: From cellular mechanisms to clinical care. J. Clin. Psychiatry 2011, 72, 258-259.

111. Maes, M.; Mihaylova, I.; Kubera, M.; Bosmans, E. Why fish oils may not always be adequate treatments for depression or other inflammatory illnesses: Docosahexaenoic acid, an omega-3 polyunsaturated fatty acid, induces a th-1-like immune response. Neuro Endocrinol. Lett. 2007, 28, 875-880.

112. Muller, N.; Myint, A.M.; Schwarz, M.J. The impact of neuroimmune dysregulation on neuroprotection and neurotoxicity in psychiatric disorders-Relation to drug treatment. Dialog. Clin. Neurosci. 2009, 11, 319-332.

113. Berger, G.E.; Wood, S.J.; Wellard, R.M.; Proffitt, T.M.; McConchie, M.; Amminger, G.P.; Jackson, G.D.; Velakoulis, D.; Pantelis, C.; McGorry, P.D. Ethyl-eicosapentaenoic acid in first-episode psychosis. A 1 h-mrs study. Neuropsychopharmacology 2008, 33, 2467-2473.

114. Duffy, S.L.; Lagopoulos, J.; Cockayne, N.; Lewis, S.J.; Hickie, I.B.; Hermens, D.F.; Naismith, S.L. The effect of 12-wk omega-3 fatty acid supplementation on in vivo thalamus glutathione concentration in patients "at risk" for major depression. Nutrition 2015, 31, 1247-1254.

115. Smesny, S.; Milleit, B.; Schaefer, M.R.; Hipler, U.C.; Milleit, C.; Wiegand, C.; Hesse, J.; Klier, C.M.; Holub, M.; Holzer, I.; et al. Effects of omega-3 pufa on the vitamin e and glutathione antioxidant defense system in individuals at ultra-high risk of psychosis. Prostaglandins Leukot. Essent. Fat. Acids 2015, 101, 15-21.

116. Said, T.; Tremblay-Mercier, J.; Berrougui, H.; Rat, P.; Khalil, A. Effects of vegetable oils on biochemical and biophysical properties of membrane retinal pigment epithelium cells. Can. J. Physiol. Pharmacol. 2013, 91, 812-817.

117. Onuki, Y.; Morishita, M.; Chiba, Y.; Tokiwa, S.; Takayama, K. Docosahexaenoic acid and eicosapentaenoic acid induce changes in the physical properties of a lipid bilayer model membrane. Chem. Pharm. Bull. 2006, 54, 68-71. 
118. Wood, S.J.; Cocchi, L.; Proffitt, T.M.; McConchie, M.; Jackson, G.D.; Takahashi, T.; Pantelis, C.; McGorry, P.D.; Berger, G.E. Neuroprotective effects of ethyl-eicosapentaenoic acid in first episode psychosis: A longitudinal t 2 relaxometry pilot study. Psychiatry Res. 2010, 182, 180-182.

119. Gillies, D.; Sinn, J.; Lad, S.S.; Leach, M.J.; Ross, M.J. Polyunsaturated fatty acids (PUFA) for attention deficit hyperactivity disorder (ADHD) in children and adolescents. Cochrane Database Syst. Rev. 2012, 7, CD007986. 



\section{Chapter 3: \\ New Aspects in Nutrition}





\title{
Omega-3 Fatty Acids in Modern Parenteral Nutrition: A Review of the Current Evidence
}

\section{Stanislaw Klek}

\begin{abstract}
Intravenous lipid emulsions are an essential component of parenteral nutrition regimens. Originally employed as an efficient non-glucose energy source to reduce the adverse effects of high glucose intake and provide essential fatty acids, lipid emulsions have assumed a larger therapeutic role due to research demonstrating the effects of omega-3 and omega-6 polyunsaturated fatty acids (PUFA) on key metabolic functions, including inflammatory and immune response, coagulation, and cell signaling. Indeed, emerging evidence suggests that the effects of omega-3 PUFA on inflammation and immune response result in meaningful therapeutic benefits in surgical, cancer, and critically ill patients as well as patients requiring long-term parenteral nutrition. The present review provides an overview of the mechanisms of action through which omega-3 and omega-6 PUFA modulate the immune-inflammatory response and summarizes the current body of evidence regarding the clinical and pharmacoeconomic benefits of intravenous $n-3$ fatty acid-containing lipid emulsions in patients requiring parenteral nutrition.
\end{abstract}

Reprinted from J. Clin. Med. Cite as: Klek, S. Omega-3 Fatty Acids in Modern Parenteral Nutrition: A Review of the Current Evidence. J. Clin. Med. 2016, 5, 34.

\section{Introduction}

Intravenous (IV) lipid emulsions (LE) are an integral component of parenteral nutrition (PN) regimens. The earliest LE, subsequently referred to as "first generation" LE, were derived from soybean or cottonseed oil. Soybean oil is rich in omega-6 (n-6) polyunsaturated fatty acids (PUFA) and provides high amounts of linoleic acid (LA) and moderate amounts of $\alpha$-linolenic acid (ALA), with an $n-6 / n-3$ ratio of approximately 7:1 [1-5]. These early emulsions, which were primarily used as an efficient non-glucose energy source to reduce the adverse effects of high dextrose intake, have two main functions: to provide a source of energy and supply essential fatty acids [6].

While the cottonseed oil-based LE (Lipomul, USA) has been permanently removed from the market [6], the soybean oil-based LE Intralipid ${ }^{\circledR}$ (Fresenius Kabi, Germany) has been used worldwide since its introduction in 1962 and has been proven to be a safe and well tolerated emulsion [3,7-10]. However, a potential disadvantage with existing soybean oil emulsions is their relatively high content of n-6 PUFA (polyunsaturated fatty acids), in particular LA [2,11,12]. The emergence of evidence suggesting that $n-6$ polyunsaturated fatty acids might be pro-inflammatory 
and immunosuppressive led to the development of more complex lipid emulsions consisting of a mixture of different oils [13]. As a result, the latest generation of $n-3$ fatty acid-containing LE provide a more balanced combination of $n-6$ and $n-3$ fatty acids, with an $n-6 / n-3$ ratio in the range of 2:1-4:1 [14,15]. For this reason, second and third generation IVLE contain not only soybean oil but alternative lipid sources such as medium-chain triglycerides (MCT), olive oil and/or fish oil/n-3 fatty acids [2,5]. In particular, $n$ - 3 fatty acid-containing IVLE have received considerable attention due to their ability to modulate key metabolic functions, including inflammatory response, coagulation, and cell signaling [5].

The present review provides a brief overview of the mechanisms of action and the role of $n-6$ and $n-3$ PUFA in modulating the immune-inflammatory response. The subsequent sections summarize the health benefits associated with $n-3$ long-chain (LC)-PUFA as well as the available evidence regarding the clinical and economic advantages of $\mathrm{PN}$ with $n-3$ fatty acids.

\section{Classification and Mechanisms of Action}

Fatty acids are classified according to their structure, carbon chain length (short, medium, or long), degree of saturation (number of double bonds), and the location of double bonds (counted from the methyl carbon of the hydrocarbon chain) [2-6]. As a common structural feature of all $n$-3 PUFAs, the double bond closest to the methyl terminus of the acyl chain of the fatty acid is located on carbon 3 [16]. The parent fatty acid of the $n-3$ family is ALA. The active ingredients in fish oil are the $n-3$ LC-PUFAs, eicosapentaenoic acid (EPA, C20:5n-3) and docosahexaenoic acid (DHA, C22:6n-3), as well as the n-6 LC-PUFA arachidonic acid (AA, C20:4n-6). All are involved in the generation of pro- and anti-inflammatory lipid mediators. While AA exerts a pro-inflammatory effect, EPA and DHA have the ability to reduce inflammation through anti-inflammatory and immunomodulatory mechanisms [13,17-19].

The oil obtained from the flesh of oily fish or livers of lean fish is commonly referred to as "fish oil" and it has the distinctive characteristic of being rich in n-3 LC-PUFAs [1]. The effects of emulsions described as "fish oil rich" or "n-3-rich/containing" are attributable to the LC-PUFAs EPA, DHA, and in some cases ALA. Various oily fish contain different amounts of $n-3$ fatty acids, as do the various fish oils. This also applies to the content of EPA and DHA in enteral and parenteral products; hence, it is important to analyze the ingredients of PN admixtures to ensure they provide the appropriate amount of EPA and DHA.

In Europe there are currently three available IVLE products containing $n-3$ LC-PUFAs: Omegaven ${ }^{\circledR}$ (Fresenius Kabi, Germany), Lipoplus ${ }^{\circledR} /$ Lipidem $^{\circledR}$ (B. Braun, Germany), and SMOFlipid ${ }^{\circledR}$ (Fresenius Kabi, Germany). Omegaven ${ }^{\circledR}$ is a $10 \%$ fish oil emulsion supplement. Lipoplus ${ }^{\circledR}$ contains a mix of $50 \%$ MCT, $40 \%$ soybean oil, 
and $10 \%$ fish oil, and SMOFlipid ${ }^{\circledR}$ is a 4 -oil mixture of $30 \%$ soybean oil, $30 \%$ MCT, $25 \%$ olive oil, and $15 \%$ fish oil.

\section{Fatty Acids: An Overview of Clinical Impact}

Fatty acids represent not only a significant energy source and a human body energy store, but they are also necessary for proper biologic function. Fatty acids are key structural components of cell membranes (phospholipids), assuring membrane integrity and fluidity. They also serve as precursors of bioactive mediators such as eicosanoids (prostaglandins, leukotrienes, and thromboxanes) and steroid hormones (cholesterol) [1]. Finally, lipids regulate the expression of a variety of genes and modulate cell signaling pathways (apoptosis, inflammation, and cell-mediated immune responses). Therefore, lipids can modulate metabolic processes at local, regional, and distant sites [1].

\subsection{Fatty Acids and Immune-Inflammatory Response}

Inflammation is part of the complex physiological response to harmful stimuli such as pathogens, damaged cells, toxins, and irritants [19]. The primary functions of inflammation are to eliminate the initial cause of injury, launch defense mechanisms, remove necrotic cells and tissues, and initiate tissue repair [1,19]. Properly regulated inflammation represents an efficient physiological mechanism that protects the host from infection and other insults and is thus essential to health. Conversely, excessive pathological inflammation may cause irreparable damage to host tissues and ultimately lead to sepsis-the complex systemic inflammatory host response to an infection [20].

Inflammation can be classified as either acute or chronic according to the duration of the response. It can also be categorized according to the intensity of the process. In 1992, the American College of Chest Physicians and the Society of Critical Care Medicine introduced definitions for systemic inflammatory response syndrome (SIRS), sepsis, severe sepsis, septic shock, and multiple organ dysfunction syndrome [21]. SIRS is nonspecific and can be caused by ischemia, inflammation, trauma, infection, or multiple combined insults. It is defined as two or more of the following: temperature $>38^{\circ} \mathrm{C}\left(100.4^{\circ} \mathrm{F}\right)$ or $<36{ }^{\circ} \mathrm{C}\left(96.8^{\circ} \mathrm{F}\right)$, heart rate $>90$ beats per minute and/or respiratory rate $>20$ breaths per minute or arterial carbon dioxide tension $(\mathrm{PaCO} 2)<32 \mathrm{~mm} \mathrm{Hg}$ [21]. It is important to recognize that SIRS is not always related to infection; accordingly, it cannot be treated the same way. Sepsis, by contrast, always stems from infection; indeed, it is defined as the presence of harmful bacteria and bacterial toxins in blood and tissues (sepsis = SIRS + infection). Severe sepsis is a condition in which there is organ dysfunction, hypotension, or hypoperfusion. If it progresses, septic shock may develop, potentially leading to multiple organ dysfunction syndrome (formerly known as multiple organ failure) and death. 
During critical illness, increased production of reactive oxygen species and inflammatory mediators occurs along with a reduction in antioxidant activity, which is partly due to preexisting nutritional deficiencies and/or suboptimal provision of clinical nutrition [6]. This state of imbalance can cause tissue damage and may play an important role in the development of sepsis and multiple organ failure [6]. Therefore, the primary goals of treatment in critically ill patients should be to decrease the pro-inflammatory response during the catabolic phase of illness and enhance immune defense mechanisms. PN should always be considered as part of any such intervention if enteral intervention is impossible or insufficient [22]. The $n-3$ fatty acids EPA and DHA exhibit strong anti-hyperinflammatory and immunomodulatory effects via direct and indirect mechanisms and may thus be of benefit in patients at risk of hyperinflammation and sepsis $[2,13,16]$.

The incorporation of PUFA into the phospholipids of cell membranes ensures the maintenance of membrane fluidity and the adequate function of membrane proteins. Through the formation of membrane rafts, $n-3$ PUFA are also involved in the inhibition of tumor growth $[23,24]$. The ratio of $n-6 / n-3$ PUFA released from the hydrolysis of membrane phospholipids influences the synthesis of eicosanoid mediators such as prostaglandins (PGs), thromboxanes (TXs), and leukotrienes (LTs). The n-6 PUFA AA gives rise to 2-series PGs, TXs, 5-hydroxy-eicosatetraenoic acid (HETE), and 4-series LTs, thereby contributing to the inflammatory process and suppressing cell-mediated immunity [25-28]. Conversely, enzymatic conversion of the $n-3$ fatty acid EPA results in the production of 3-series PGs and TXs and 5-series LTs, all of which are less potent than the AA-derived mediators [16,24,28].

The recent discovery of families of novel pro-resolving lipid mediators such as resolvins, protectins, maresins, and lipoxins has shed new light on the role of n-3 PUFAs in the inflammatory process [29-32]. Pro-resolving lipid mediators derived from EPA are designated resolvins of the E series (RvE), while those derived from DHA are designated resolvins of the D series (RvD) [33]. The latter include the neuroprotectins/protectins (NPD1/PD1) and the maresins (MaR). These mediators have been shown to limit neutrophilic infiltration and enhance macrophage resolution responses, thus playing a role in diseases characterized by excessive uncontrolled inflammation [34-41]. Notably, their generation is linked to the fatty acid composition of cellular membranes and can therefore be effectively increased with $n-3$ fatty acid supplementation [37].

Exposure of inflammatory cells to $n$-3 PUFA in vitro attenuates chemotaxis of human neutrophils and monocytes and decreases expression of certain adhesion molecules on the surface of monocytes, macrophages, lymphocytes, and endothelial cells [20]. By coupling with surface or intracellular "fatty acid receptors", the LC-PUFA or their oxidized derivatives can regulate gene expression via the activation of transcription factors such as peroxisome proliferator-activated receptor (PPAR)- $\gamma$ 
and nuclear factor-kappa B (NF-kB). N-3 LC-PUFA also modulate the inflammatory processes by inhibiting the production of pro-inflammatory cytokines and other pro-inflammatory proteins induced via activation of NF- $\mathrm{KB}$ in response to exogenous inflammatory stimuli. It appears that this effect is at least partly mediated by G-protein coupled cell surface receptors [20,24]. Consequently, the use of EPA and DHA leads to a more balanced immune response, which may result in faster resolution of inflammation $[4,13,17,18]$.

\section{Physiological Effects and Associated Health Benefits of $n-3$ LC-PUFA}

\subsection{Cardioprotective, Antihypertensive, and Antithrombotic Effects}

In animal models of atherogenesis, $n-3$ fatty acids inhibit the hepatic synthesis of triglycerides [1]. EPA and DHA partly replace AA in membrane phospholipids; therefore, $n-3$ fatty acids may improve membrane structure, ligand/receptor binding, enzyme secretion, antigen presentation, and activation of intracellular signaling pathways [1]. By reducing the availability of AA-derived fatty acids as a substrate for eicosanoid synthesis by cyclooxygenase and lipoxygenase in platelets, monocytes, and macrophages, EPA and DHA delay platelet aggregation and progression of atherogenesis [10].

Consuming n-3 PUFA from fish oil is beneficial in the prevention of cardiovascular disease (CVD) and associated complications, as demonstrated by randomized control trials (RCTs) investigating secondary prevention in heart disease patients as well as epidemiological studies in populations that traditionally consume large quantities of sea fish as a part of their daily diet (e.g., Inuit) [42,43]. Potential underlying mechanisms include the inhibition of thrombogenesis and cytokine-dependent inflammation [42]. At total doses $>3$ g/day, EPA + DHA reduces CVD risk factors by decreasing plasma triglycerides, blood pressure, platelet aggregation, and inflammation and improving vascular reactivity [43].

\subsection{Anticancer and Anti-Cachectic Effects and Inhibition of Tumor Growth}

$N-3$ PUFA have been shown to attenuate cell growth and induce apoptosis in a variety of human cancer cell lines such as colonic, pancreatic, prostate, and breast cancer [44]. Through their effects on eicosanoid metabolism, n-3 PUFA may also decrease "sprouting angiogenesis", suppress endothelial cell proliferation, decrease tumor micro-vessel density, and even decrease tumor growth [45-49]. Research indicates that $n-3$ fatty acids act synergistically with chemotherapeutic agents and may also be used to enhance tumor radiosensitivity [50-52]. In patients with cancer-related cachexia, the pro-inflammatory state can be modulated by suppressing the inflammatory milieu and the release of pro-inflammatory mediators such as cytokines and prostaglandins by means of fish oil-based preparations, thus allowing 
nutrition to have an anabolic effect [53]. The underlying mechanisms for these effects include the incorporation of $n-3$ fatty acids into biological membranes as well as the modulation of the expression of proteins involved in the regulation of cell cycle and apoptosis, such as Bcl-2, Bax, and c-Myc [23,45,54]. More studies are needed, however, to fully assess the effects of $n-3$ PUFA in cancer treatment and prevention.

\subsection{Visual and Cognitive Development}

N-3 PUFA may reduce the risk of neurological disorders by influencing neuronal membranes and the activities of membrane-bound enzymes, receptors, and transporters [55]. Moreover, EPA and DHA can affect neurotransmission, including dopaminergic, noradrenergic, serotoninergic, and GABAergic neurotransmission in specific brain regions [56,57]. DHA, together with AA, is crucial for the development and maintenance of normal structure and function of the central nervous system (CNS). During fetal development DHA is taken up via the placenta and accumulates in the brain where it is required for the proper function of cholinergic neurotransmission [57-60]. Additionally, DHA may protect the brain from free radical and reactive oxygen species by enhancing the activity of cerebral catalase and glutathione peroxidase [61]. DHA may also be important for the efficient regeneration of axons and dendrites following neuronal injury [55].

The putative effects of $n-3$ PUFA on CNS function are mediated not only through an effect on the physicochemical properties of neural membranes but also through activation of transcription factors such as PPAR- $\gamma$ [62]. AA, EPA, and DHA play a major role in protecting neuronal cells in the brain via inhibition of tumor necrosis factor (TNF)- $\alpha$ synthesis, thereby augmenting acetylcholine and endothelial nitric oxide (NO) formation and enhancing glucose uptake by neuronal cells, resulting in improved memory [53]. Epidemiological data suggest that a low dietary intake of n-3 LC-PUFA is a risk factor for Alzheimer disease [63]. Notably, studies have shown that the intake of fish oil lowers the risk of dementia by reducing the synthesis of proinflammatory cytokines and inhibiting the activities of phospholipase A2 and caspase A1 [64,65].

The effects of EPA and ethyl-EPA intake in schizophrenic patients have been investigated in a number of RCTs [63]. Overall, the results suggest a potential benefit; however, due to the limited size and duration of the studies it remains unknown whether the benefit is clinically meaningful. Research also suggests that $n$-3 LC-PUFA may confer a benefit in certain cases of depression; however, the routine use of $n-3$ LC-PUFA for the treatment of major depression cannot yet be recommended. Further research is necessary to establish the efficacy, appropriate dosing, and active components (EPA, DHA, or both) of $n-3$ LC-PUFA in patients with major depression [55]. 


\subsection{Lipid Metabolism and Insulin Sensitivity}

Fish oil supplementation has been shown to produce a clinically significant, dose-dependent reduction in fasting blood triglycerides and normalize serum lipid concentrations, including high density lipoproteins (HDL) and low density lipoproteins (LDL), in patients with hyperlipidemia [66]. Fish oil may also be beneficial in individuals with diabetes mellitus. In patients with type 2 diabetes, fish oil lowers triglycerides and raises LDL cholesterol [67]. However, additional studies are needed to gain a more comprehensive understanding of the role of fish oil supplementation in type 2 diabetes.

\subsection{Inflammatory Disease}

Due to their ability to regulate inflammatory processes and cellular responses, $n-3$ fatty acids have the potential to affect the development and progression of a multitude of diseases associated with an inflammatory state, including rheumatoid arthritis, Crohn's disease, ulcerative colitis, type- 1 diabetes, cystic fibrosis, asthma, allergic disease, chronic obstructive pulmonary disease, psoriasis, and multiple sclerosis (reviewed in $[20,68,69]$ ). In a rat model of experimental colitis [70], administration of an n-3 PUFA-enriched parenteral LE decreased colonic concentrations of pro-inflammatory mediators and attenuated the morphological and inflammatory consequences of colitis. Although the current state of knowledge is insufficient to support a clear recommendation for the use of $n-3$ PUFA in patients with inflammatory bowel disease, emerging evidence suggests a potential benefit. Clinical decisions regarding the use of fish oil in such patients should be informed by due consideration of the available evidence.

\subsection{Immune Function}

Among other effects, $n-3$ fatty acids inhibit immune and inflammatory functions by decreasing lymphocyte proliferation, cytokine production, natural killer (NK) cell cytotoxicity, and antibody production [71]. Additionally, $n-3$ fatty acids suppress neutrophil chemotactic responsiveness to leukotriene B4 [71,72], reduce antigen-presenting capability, and decrease expression of major histocompatibility complex II (MHC II) molecules of mononuclear phagocytes [73]. DHA has been shown to partly restore the oxygen-dependent bactericidal mechanisms of monocytes [74], and neutrophils treated with EPA and DHA showed enhanced antiparasitic activity against Plasmodium falciparum [75].

Reports on the effects of different $n-3$ fatty acids on cytokine secretion and immune cell activity are somewhat inconsistent. In mice fed a diet rich in EPA, plasma concentrations of TNF- $\alpha$ were increased [76], whereas others have demonstrated decreased TNF- $\alpha$ secretion by neutrophils in DHA-fed human volunteers [77]. 
Purasiri et al. [78] observed decreased production of interleukin-1 (IL)-1, IL-2, TNF- $\alpha$, and interferon- $\gamma$ by human neutrophils treated with $n-3$ fatty acids, while Chavali et al. [76] reported decreased expression of IL-6 and IL-10 by endothelial cells and monocytes in mice fed an EPA-enriched diet [76]. Diets rich in $n$ - 3 fatty acids have been shown to inhibit lymphocyte activation and modulate antibody production by B-lymphocytes [71]. Conversely, the proliferative response to T-cell mitogens was increased by $n-3$ fatty acids in an animal model of autoimmune disease [79]. In a rat model of antibody-mediated autoimmune disease, diets low in fat, deficient in essential fatty acids, or rich in fish omega-3 fatty acids were associated with a better prognosis and higher survival [80]. In general, n-3 PUFA inhibit NK cell and lymphokine-activated killer cell activities. DHA feeding inhibited NK cell activity in healthy men [77]; however, a diet rich in $n-3$ fatty acids augmented NK cell cytotoxicity in healthy rats [81].

N-3 and n-6 PUFA differentially influence the plasma free fatty acid profile, with consequent effects on neutrophil functions [82]. In an open-label randomized study of septic patients with markedly reduced neutrophil function, patients receiving n-6 lipid infusions experienced persistent or worsening abnormalities in plasma free fatty acids and impaired neutrophil function, while patients receiving $n-3$ lipid infusions showed a rapid switch in the plasma free fatty acid fraction to a predominance of EPA and DHA over AA, with rapid incorporation of $n-3$ fatty acids into mononuclear leukocyte membranes and subsequent suppression of pro-inflammatory cytokine generation. Additionally, neutrophil function was significantly improved following administration of $n-3$ LE [82].

Collectively, these findings suggest that $n$-3 PUFA have a favorable effect on immunocompetence and inflammation and may therefore reduce the risk of clinical sequelae in critically ill septic patients.

\section{N-3 Fatty Acids in Parenteral Nutrition-Clinical Benefits}

\subsection{Preservation of Hepatocellular Integrity During Long-Term PN in Adults and Children}

Patients with critical illness, compromised gastrointestinal tract, sepsis, or recurrent infection who require prolonged $\mathrm{PN}$ therapy are at high risk of developing hepatic complications [83-85]. The long-term use of IVLE, especially at doses exceeding $1 \mathrm{~g} / \mathrm{kg}$ of body weight per day, is thought to play a role in the etiology of intestinal failure-associated liver disease (IFALD) $[7,86]$. Emerging evidence suggests that reducing the amount of $n-6$ fatty acids from soybean oil by partial replacement with $n$ - 3 PUFA from fish oil may improve parameters of liver function in parenterally fed adult and pediatric patients [87-93]. Thus, replacing a pure soybean oil emulsion by an LE containing soybean oil, MCT, olive oil, and fish oil (resulting in a lower $n-6 / n-3$ fatty acid ratio of $\sim 2.5: 1$ ) appears to be a promising approach [7]. 
Indeed, the available evidence supports the use of $n-3$ PUFA in PN, infused either as a part of the basic LE or added separately (as a fish oil emulsion supplement). Studies evaluating both short- and long-term use of $n$-3 PUFA-containing LE have demonstrated preserved hepatic integrity and improvements in parameters of liver function in adult and pediatric patients $[7,89,90,94]$. Klek et al. investigated the safety and tolerability of a soybean/MCT/olive oil/fish oil emulsion in patients with intestinal failure receiving long-term PN. After four weeks of treatment, measures of liver function (alanine transaminase [ALT], aspartate transaminase [AST], and total bilirubin) were significantly improved in patients who received the soybean/MCT/olive oil/fish oil emulsion compared with controls who received a conventional soybean oil emulsion [7]. Parenteral infusion of $n-3$ PUFA from fish oil has also been repeatedly shown to be effective in reversing $\mathrm{PN}$-associated cholestasis in children when administered alone or in combination with a soybean oil-based lipid emulsion [95-99].

\subsection{Critical Illness}

According to the European Society for Clinical Nutrition and Metabolism (ESPEN) definition, a critically ill patient is a patient developing an intensive inflammatory response with failure of at least one organ (Sequential Organ Failure Assessment (SOFA) score $>4$ ) necessitating support of organ function during an ICU episode expected to be longer than three days [100]. Patients admitted to the intensive care unit (ICU) only for monitoring (typical ICU stay $<3$ days) are therefore not representative ICU patients. The aforementioned acute inflammatory conditions, SIRS, sepsis, and septic shock represent the range of abnormalities during critical illness.

Numerous studies in ICU patients confirm the clinical value of $n-3$ PUFA in critically ill patients. In a multicenter study in 661 critically ill patients with a Simplified Acute Physiology II Score (SAPS II) $>32$, Heller et al. demonstrated that IV fish oil improved survival compared with that predicted by the SAPS II score and reduced infection rates, antibiotic requirements, and length of stay in a dose-dependent manner [101]. Consistent with these findings, Mayer et al. concluded, based on a review of the available evidence, that inclusion of $n-3$ fatty acids in PN improves immunologic parameters and length of stay in surgical patients [102]. Finally, in a recent comprehensive meta-analysis of studies evaluating the use of $n-3$ PUFA in ICU patients, Manzanares et al. analyzed data from 10 RCTs involving 733 patients and showed that the use of fish oil-containing LE was associated with significantly fewer infectious complications (RR $0.64 ; 95 \%$ CI, $0.44-0.92 ; p=0.02$ ) [103]. Trends toward a reduction in the number of days on mechanical ventilation (weighted mean difference $[\mathrm{WMD}]-1.14 ; 95 \% \mathrm{CI},-2.67$ to $0.38 ; p=0.14$; heterogeneity $\mathrm{I} 2=0 \%$ ) and the length of hospital stay (WMD $-3.71 ; 95 \% \mathrm{CI},-9.31$ to $1.88 ; p=0.19$ ) were 
also reported. However, there was no significant effect on ICU length of stay (WMD $-1.42 ; 95 \% \mathrm{CI},-4.53$ to $1.69 ; p=0.37$ ) or mortality (RR $0.90 ; 95 \% \mathrm{CI}, 0.67-1.20$; $p=0.46$; heterogeneity $\mathrm{I} 2=0 \%$ ). In a subgroup analysis of trials including patients who received IV n-3 PUFA in combination with enteral nutrition (EN) [104-107], a trend toward a reduction in mortality was observed (RR $0.69 ; 95 \%$ CI, $0.40-1.18$; $p=0.18$; heterogeneity $\mathrm{I} 2=35 \%$ ) [103].

According to the ESPEN Guidelines on Parenteral Nutrition in Intensive Care, the addition of EPA and DHA to lipid emulsions has demonstrable effects on cell membranes and inflammatory processes, and fish oil-enriched LEs likely decrease the length of hospital stay in critically ill patients (grade B) [100]. Canadian recommendations endorse the use of second generation LE when PN is indicated [108].

\subsection{Oncology}

Preclinical studies have demonstrated that $n-3$ PUFA can be effective in preventing the initiation and progression of cancer cells [45]. In the clinical setting, it was recently shown that fish oil-supplemented PN may help to reduce inflammation and stabilize energy expenditure in patients with pancreatic cancer [49]. In the same study, fish oil also improved patients' quality of life (QOL) by reducing cachexia, increasing appetite, and increasing performance status. In a separate study, fish oil-containing LE combined with gemcitabine improved the effectiveness of anti-cancer therapy in patients with advanced pancreatic cancer by decreasing gemcitabine resistance and improving QOL [49]. However, referring to a systematic review of the published studies evaluating EPA in cancer patients by Dewey et al. [109], ESPEN concluded that there was no benefit associated with the oral administration of EPA in patients with consolidated cachexia [110].

In recent years several well-designed clinical studies assessing the effect of nutritional intervention with EPA/ fish oil in conjunction with antineoplastic therapy have been published. Positive results have be shown with regard to increased energy/protein intake and gain/maintenance of body weight/lean body mass (LBM) [111-113], with evidence to suggest a potential effect on several domains of health-related quality of life (HRQOL) [113]. Ma et al. recently published a systematic review examining the effects of $n-3$ fatty acids in patients with unresectable pancreatic cancer and reported a significant increase in body weight and LBM as well as a significant decrease in resting energy expenditure and improved overall survival (130-259 days vs. 63-130 days) in patients who consumed an oral nutrition supplement enriched with $n-3$ fatty acids compared to those who consumed conventional nutrition [114]. The review concluded that $n-3$ fatty acids are safe and appear to exert a positive effect on clinical outcomes and survival in pancreatic cancer patients. 


\subsection{Surgery}

Recent studies have shown that the use of LE containing an increased dosage of n-3 PUFA may improve outcomes in surgical patients [115-120]. Among the clinical benefits observed in these studies were improvements in liver function tests $[115,116]$, improvements in measures of immunologic function and inflammatory response $[117,118]$, decreased length of hospitalization $[116,119,120]$, and a reduced risk of clinical complications [116]. Moreover, the benefits of $n$-3 PUFA were observed when administered preoperatively (three days) [118], postoperatively (seven days) [116,117,119], or during the entire perioperative period [121]. Based on these findings, $n$-3 PUFA-enriched LE administered as a part of PN appear to confer benefits to selected groups of surgical patients, including those with major trauma or undergoing extended digestive tract resections or liver transplantation [122]. A summary of recent studies evaluating various IVLE formulations in surgical patients is presented in Table 1.

Table 1. Latest studies/meta-analyses evaluating the benefits of $n$-3 PUFA (polyunsaturated fatty acids) in parenteral nutrition in surgical populations *.

\begin{tabular}{|c|c|c|c|c|c|}
\hline Author & Year & Population & Intervention & Duration & Result \\
\hline Jiang [117] & 2010 & $\begin{array}{l}\text { Colectomy and } \\
\text { rectotomy }(n=206)\end{array}$ & $\begin{array}{c}\text { LCT vs } \\
\text { LCT+fish oil }\end{array}$ & $\begin{array}{c}7 \text { days } \\
\text { post-surgery }\end{array}$ & $\begin{array}{l}\text { Significant reduction in } \\
\text { LOS and SIRS }\end{array}$ \\
\hline Wang [115] & 2012 & $\begin{array}{l}\text { Gastrointestinal } \\
\text { surgery }(n=64)\end{array}$ & $\begin{array}{l}\mathrm{MCT} / \mathrm{LCT} \text { vs. } \\
\mathrm{LCT}+\text { fish oil }\end{array}$ & $\begin{array}{l}5 \text { days } \\
\text { post-surgery }\end{array}$ & $\begin{array}{l}\text { Amelioration of liver } \\
\text { function and immune } \\
\text { status }\end{array}$ \\
\hline Han [116] & 2012 & $\begin{array}{l}\text { Major surgery } \\
\quad(n=38)\end{array}$ & $\begin{array}{l}\mathrm{MCT} / \mathrm{LCT} v s . \\
\mathrm{LCT}+\text { fish oil }\end{array}$ & $\begin{array}{c}7 \text { days } \\
\text { post-surgery }\end{array}$ & $\begin{array}{c}\text { Reduced postoperative } \\
\text { liver dysfunction and } \\
\text { infection rate }\end{array}$ \\
\hline Zhu [119] & 2012 & $\begin{array}{l}\text { Colectomy and } \\
\text { rectotomy }(n=57)\end{array}$ & LCT vs. fish oil & $\begin{array}{c}7 \text { days } \\
\text { post-surgery }\end{array}$ & Reduced LOS \\
\hline Zhu [120] & 2012 & $\begin{array}{c}\text { Liver } \\
\text { transplantation } \\
(n=98)\end{array}$ & $\begin{array}{l}\text { Oral diet } v s . \\
\text { standard PN } \\
\text { vs fish oil PN }\end{array}$ & $\begin{array}{c}7 \text { days } \\
\text { post-surgery }\end{array}$ & $\begin{array}{l}\text { Reduced incidence of } \\
\text { liver injury, decreased } \\
\text { LOS and infectious } \\
\text { complications }\end{array}$ \\
\hline Berger [121] & 2013 & $\begin{array}{l}\text { Cardiopulmonary } \\
\text { bypass surgery } \\
(n=28)\end{array}$ & $\begin{array}{l}\text { Fish oils vs } \\
\text { saline }\end{array}$ & $\begin{array}{l}12 \text { and } 2 \mathrm{~h} \\
\text { before surgery } \\
\text { and after } \\
\text { surgery }\end{array}$ & $\begin{array}{l}\text { Decreased biological } \\
\text { and clinical signs of } \\
\text { inflammation }\end{array}$ \\
\hline $\begin{array}{c}\text { De Miranda } \\
\text { Torrinhas } \\
\text { [118] }\end{array}$ & 2013 & $\begin{array}{c}\text { Surgery for } \\
\text { gastrointestinal } \\
\text { cancer }(n=63)\end{array}$ & $\begin{array}{l}\mathrm{MCT} / \mathrm{LCT} \text { vs. } \\
\quad \text { fish oil }\end{array}$ & $\begin{array}{c}3 \text { days } \\
\text { post-surgery }\end{array}$ & $\begin{array}{l}\text { Significant increase in } \\
\text { IL-10 levels (day 3), } \\
\text { decrease in IL-6 and } \\
\text { IL-10 levels (day 6), less } \\
\text { decline in leukocyte } \\
\text { oxidative burst }\end{array}$ \\
\hline
\end{tabular}


Table 1. Cont.

\begin{tabular}{|c|c|c|c|c|c|}
\hline Author & Year & Population & Intervention & Duration & Result \\
\hline Chen [123] & 2010 & $\begin{array}{l}\text { Major abdominal } \\
\text { surgery, } \\
\text { meta-analysis } \\
(n=892)\end{array}$ & $\begin{array}{l}\text { Fish oil } v s . \\
\text { various control } \\
\text { emulsions }\end{array}$ & Various & $\begin{array}{c}\text { Decreased LOS in the } \\
\text { hospital and ICU, } \\
\text { reduced postoperative } \\
\text { infection rate, improved } \\
\text { liver function }\end{array}$ \\
\hline Li [124] & 2013 & $\begin{array}{l}\text { Major surgery, } \\
\text { meta-analysis } \\
\quad(n=1487)\end{array}$ & Fish oil & Various & $\begin{array}{l}\text { Decreased infection rate, } \\
\text { LOS, and liver } \\
\text { dysfunction; no effect } \\
\text { on mortality }\end{array}$ \\
\hline Pradelli [125] & 2012 & $\begin{array}{l}\text { Subgroup analysis } \\
\text { in patients } \\
\text { undergoing major } \\
\text { abdominal surgery } \\
\text { and not admitted to } \\
\text { ICU }(n=740)\end{array}$ & $\begin{array}{c}n-3 \\
\text { PUFA-enriched } \\
\text { vs. standard } \\
\text { lipid } \\
\text { emulsions }\end{array}$ & Various & $\begin{array}{c}\text { Significant reduction in } \\
\text { the infection rate } \\
\text { and LOS }\end{array}$ \\
\hline Tian [126] & 2013 & $\begin{array}{l}\text { Surgical patients, } \\
\text { meta-analysis } \\
\quad(n=306)\end{array}$ & $\begin{array}{l}\text { Fish oil/LCT/ } \\
\text { MCT vs. } \\
\text { LCT/olive oil }\end{array}$ & Various & $\begin{array}{l}\text { No significant difference, } \\
\text { fish oil less toxic to liver } \\
\text { when compared to } \\
\text { LCT or olive oil }\end{array}$ \\
\hline
\end{tabular}

* Adapted from Klek et al. 2015 [122]. Abbreviations: ICU = intensive care unit; LCT = long-chain triglycerides; LOS = length of stay; $\mathrm{MCT}=$ medium-chain triglycerides; $\mathrm{PN}=$ parenteral nutrition; $\mathrm{RCT}=$ randomized clinical trial; $\mathrm{SIRS}=$ systemic inflammatory response syndrome.

Several recent meta-analyses evaluating the clinical benefit of $n-3$ PUFA-enriched PN in surgical patients showed significant reductions in infectious complications [123-127], with three of these meta-analyses reporting a significant reduction in the length of hospital stay in surgical patients who received $n-3$ fatty acid-containing LE compared with controls [125-127].

\subsection{Safety and Tolerability during Long-Term Use}

Data from available studies confirm the safety and tolerability of $n-3$ PUFA-containing LE during long-term use, regardless of indication [7,91]. In adult patients with stable intestinal failure requiring long-term PN, beneficial effects were observed on parameters of liver function and Vitamin E profile following the administration of a mixed-type LE containing $15 \%$ fish oil for four weeks [7]. Safety and tolerability were also demonstrated in 28 children (age, five months to 11 years) with short bowel syndrome, chronic intestinal pseudo-obstruction, or congenital disease of the intestinal mucosa who required home PN for at least four weeks [91]. Finally, a meta-analysis of 23 trials involving 1503 patients found no evidence of deleterious effects or symptoms of intolerance during long-term use of fish oil-containing LE during ICU stay or surgical hospitalization [126]. 


\subsection{Recommended Dosage and Duration of Intervention}

According to ESPEN Guidelines on Parenteral Nutrition in Intensive Care, lipids should be an integral part of the regimen to provide energy and essential fatty acids in all patients requiring PN [100]. IVLE (LCT, MCT, or mixed emulsions) can be administered safely at a rate of $0.7-1.5 \mathrm{~g} / \mathrm{kg}$ over 12 to $24 \mathrm{~h}$ [100]. In the previously mentioned multicenter trial by Heller et al., IV fish oil was safe and conferred significant clinical benefits when administered to critically ill adults in doses between 0.1 and $0.2 \mathrm{~g} / \mathrm{kg} /$ day [101]. Therefore, it seems reasonable to recommend the administration of fish oil in doses higher than $0.1 \mathrm{~g} / \mathrm{kg} / \mathrm{day}$. Studies in surgical patients showed that while short-term ( $<5$ days) administration of n-3 PUFA influences immune parameters and liver function, postoperative administration also reduces complication rates and length of stay $[118,119]$. While it takes several days for $n-3$ PUFA to be incorporated in host tissues and influence the generation of inflammatory mediators, impairment of host defense mechanisms occurs immediately after surgery. Braga et al. [128] thus concluded based on a literature review that $n$-3 PUFA should be given prior to surgery to achieve an optimum effect. In premature infants, a mixed LE with $15 \%$ fish oil administered for seven to 14 days showed a potential beneficial effect on cholestasis, $n-3$ fatty acids, and Vitamin E status [90,92,129].

\subsection{Cost-Effectiveness}

The cost-effectiveness of omega-3 PUFA-enriched LE is an important consideration, as acquisition costs for fish oil-containing LE are comparatively higher than those for pure soybean or MCT/LCT-based emulsions [130]. Nonetheless, pharmacoeconomic analyses support the supplementation of LE with $n-3$ PUFA. $\mathrm{Wu}$ et al. demonstrated that fish oil-based LE improves clinical outcomes and decreases the overall costs of ICU stay compared with standard LE in Chinese ICU patients (mean savings, $¥ 10,000$ ) [131]. Additionally, in the most comprehensive analysis of the cost-effectiveness of LE published to date, Pradelli et al. analyzed patient outcomes and hospital economic data from Italian, French, German, and UK hospitals using a discrete event simulation scheme and demonstrated that treatment costs were entirely offset by reductions in antibiotic use and the length of hospital stay [132]. Cost savings ranged from $€ 3972$ to $€ 4897$ per ICU patient and from $€ 561$ to $€ 1762$ per non-ICU patient [126].

\section{Conclusions}

Lipids are not only an important energy source, but they also modulate metabolic processes at local, regional, and distant sites. IVLE are undoubtedly an indispensable element of PN regimens. Indeed, there is a strong scientific rationale 
for $n$-3 PUFA in PN; they improve clinical outcomes in pediatric, surgical, cancer, and critically ill patients as well as patients requiring long-term PN. Finally, LE-containing fish oils have a proven safety and tolerability profile and represent a cost-effective component of $\mathrm{PN}$ regimens.

Author Contributions: Stanislaw Klek was responsible for critical data analysis, evaluation of the outcomes, and writing of the manuscript.

Conflicts of Interest: Stanislaw Klek is consultant of Fresenius Kabi Deutschland GmbH and Asculap Chifa B Braun, Deuschland.

\section{References}

1. Sobotka, L. Basics in Clinical Nutrition, 4th ed.; Galen: Prague, Czech Republic, 2011.

2. Waitzberg, D.L.; Torrinhas, R.S.; Jacintho, T.M. New parenteral lipid emulsions for clinical use. JPEN J. Parenter. Enteral Nutr. 2006, 30, 351-367.

3. Wanten, G.J.; Calder, P.C. Immune modulation by parenteral lipid emulsions. Am. J. Clin. Nutr. 2007, 85, 1171-1184.

4. Calder, P.C. Long-chain $n-3$ fatty acids and inflammation: Potential Application in Surgical and Trauma Patients. Braz. J. Med. Biol. Res. 2003, 36, 433-446.

5. Carpentier, Y.A.; Dupont, I.E. Advances in intravenous lipid emulsions. World J. Surg. 2000, 24, 1493-1497.

6. Calder, P.C.; Jensen, G.L.; Koletzko, B.V.; Singer, P.; Wanten, G.J. Lipid emulsions in parenteral nutrition of intensive care patients: Current Thinking and Future Directions. Intensive Care Med. 2010, 36, 735-749.

7. Klek, S.; Chambrier, C.; Singer, P.; Rubin, M.; Bowling, T.; Staun, M.; Joly, F.; Rasmussen, H.; Strauss, B.J.; Wanten, G.; et al. Four-week parenteral nutrition using a third generation lipid emulsion (smoflipid)—A double-blind, randomised, multicentre study in adults. Clin. Nutr. 2013, 32, 224-231.

8. Carpentier, Y.A. Intravascular metabolism of fat emulsions: The Arvid Wretlind Lecture, Espen 1988. Clin. Nutr. 1989, 8, 115-125.

9. Carneheim, C.; Larsson-Backström, C.; Ekman, L. New fatty acids in the emulsions of the nineties. possibilities and implications. In Essential Fatty Acids and Total Parenteral Nutrition; Ghisolfi, J., Ed.; John Libbey Eurotext: Paris, France, 1990; pp. 171-180.

10. Calder, P.C. Hot topics in parenteral nutrition. Rationale for using new lipid emulsions in parenteral nutrition and a review of the trials performed in adults. Proc. Nutr. Soc. 2009, 68, 252-260.

11. Krohn, K.; Koletzko, B. Parenteral lipid emulsions in paediatrics. Curr. Opin. Clin. Nutr. Metab. Care 2006, 9, 319-323.

12. Hamilton, C.; Austin, T.; Seidner, D.L. Essential fatty acid deficiency in human adults during parenteral nutrition. Nutr. Clin. Pract. 2006, 21, 387-394.

13. Calder, P.C. Use of fish oil in parenteral nutrition: Rationale and reality. Proc. Nutr. Soc. 2006, 65, 264-277. 
14. Mayer, K.; Schaefer, M.B.; Seeger, W. Fish oil in the critically ill: From experimental to clinical data. Curr. Opin. Clin. Nutr. Metab. Care 2006, 9, 140-148.

15. Fürst, P.; Kuhn, K.S. Fish oil emulsions: What benefits can they bring? Clin. Nutr. 2000, 19, 7-14.

16. Calder, P.C. Omega-3 polyunsaturated fatty acids and inflammatory processes: Nutrition or pharmacology? Br. J. Clin. Pharmacol. 2013, 75, 645-662.

17. Mayer, K.; Gokorsch, S.; Fegbeutel, C.; Hattar, K.; Rosseau, S.; Walmrath, D.; Seeger, W.; Grimminger, F. Parenteral nutrition with fish oil modulates cytokine response in patients with sepsis. Am. J. Respir. Crit Care Med. 2003, 167, 1321-1328.

18. Calder, P.C. Rationale and use of $n-3$ fatty acids in artificial nutrition. Proc. Nutr. Soc. 2010, 69, 565-573.

19. Ferrero-Miliani, L.; Nielsen, O.H.; Andersen, P.S.; Girardin, S.E. Chronic inflammation: Importance of Nod2 and Nalp3 in interleukin-1beta generation. Clin. Exp. Immunol. 2007, 147, 227-235.

20. Calder, P.C. Omega-3 fatty acids and inflammatory processes. Nutrients 2010, 2, 355-374.

21. Bone, R.C.; Balk, R.A.; Cerra, F.B.; Dellinger, R.P.; Fein, A.M.; Knaus, W.A.; Schein, R.M.; Sibbald, W.J. Definitions for sepsis and organ failure and guidelines for the use of innovative therapies in sepsis. The ACCP/SCCM consensus conference committee. American college of chest physicians/society of critical care medicine. Chest 1992, 101, 1644-1655.

22. Singer, P.; Doig, G.; Pichard, C. The truth about nutrition in the icu. Intensive Care Med. 2014, 40, 252-255.

23. Calviello, G.; Serini, S.; Piccioni, E. N-3 polyunsaturated fatty acids and the prevention of colorectal cancer: Molecular mechanisms involved. Curr. Med. Chem. 2007, 14, 3059-3069.

24. Calder, P.C. Mechanisms of action of (n-3) fatty acids. J. Nutr. 2012, 142, 592S-599S.

25. Grbic, J.T.; Mannick, J.A.; Gough, D.B.; Rodrick, M.L. The role of prostaglandin E2 in immune suppression following injury. Ann. Surg. 1991, 214, 253-262.

26. Ertel, W.; Morrison, M.H.; Meldrum, D.R.; Ayala, A.; Chaudry, I.H. Ibuprofen restores cellular immunity and decreases susceptibility to sepsis following hemorrhage. J. Surg Res. 1992, 53, 55-61.

27. Kollef, M.H.; Schuster, D.P. The acute respiratory distress syndrome. N. Engl. J. Med. 1995, 332, 27-37.

28. Calder, P.C. The 2008 espen sir david cuthbertson lecture: Fatty acids and inflammation-From the membrane to the nucleus and from the laboratory bench to the clinic. Clin. Nutr. 2010, 29, 5-12.

29. Bannenberg, G.L.; Chiang, N.; Ariel, A.; Arita, M.; Tjonahen, E.; Gotlinger, K.H.; Hong, S.; Serhan, C.N. Molecular circuits of resolution: Formation and actions of resolvins and protectins. J. Immunol. 2005, 174, 4345-4355.

30. Bannenberg, G.; Arita, M.; Serhan, C.N. Endogenous receptor agonists: Resolving inflammation. Sci. World J. 2007, 7, 1440-1462. 
31. Bannenberg, G.; Serhan, C.N. Specialized pro-resolving lipid mediators in the inflammatory response: An update. Biochim. Biophys. Acta 2010, 1801, 1260-1273.

32. Serhan, C.N. Pro-resolving lipid mediators are leads for resolution physiology. Nature 2014, 510, 92-101.

33. Hong, S.; Lu, Y. Omega-3 fatty acid-derived resolvins and protectins in inflammation resolution and leukocyte functions: Targeting novel lipid mediator pathways in mitigation of acute kidney injury. Front. Immunol. 2013, 4, 13.

34. Hong, S.; Gronert, K.; Devchand, P.R.; Moussignac, R.L.; Serhan, C.N. Novel docosatrienes and 17s-resolvins generated from docosahexaenoic acid in murine brain, human blood, and glial cells autacoids in anti-inflammation. J. Biol. Chem. 2003, 278, 14677-14687.

35. Serhan, C.N.; Hong, S.; Gronert, K.; Colgan, S.P.; Devchand, P.R.; Mirick, G.; Moussignac, R.L. Resolvins: A Family of Bioactive Products of Omega-3 Fatty Acid Transformation Circuits Initiated by Aspirin Treatment that Counter Proinflammation Signals. J. Exp. Med. 2002, 196, 1025-1037.

36. Marcheselli, V.L.; Hong, S.; Lukiw, W.J.; Tian, X.H.; Gronert, K.; Musto, A.; Hardy, M.; Gimenez, J.M.; Chiang, N.; Serhan, C.N.; et al. Novel docosanoids inhibit brain ischemia-reperfusion-mediated leukocyte infiltration and pro-inflammatory gene expression. J. Biol. Chem. 2003, 278, 43807-43817.

37. Serhan, C.N.; Petasis, N.A. Resolvins and protectins in inflammation resolution. Chem. Rev. 2011, 111, 5922-5943.

38. Tian, H.; Lu, Y.; Shah, S.P.; Hong, S. Autacoid 14S, 21R-dihydroxy-docosahexaenoic acid counteracts diabetic impairment of macrophage prohealing functions. Am. J. Pathol. 2011, 179, 1780-1791.

39. Hisada, T.; Ishizuka, T.; Aoki, H.; Mori, M. Resolvin E1 as a novel agent for the treatment of asthma. Expert Opin. Ther. Targets 2009, 13, 513-522.

40. Morita, M.; Kuba, K.; Ichikawa, A.; Nakayama, M.; Katahira, J.; Iwamoto, R.; Watanebe, T.; Sakabe, S.; Daidoji, T.; Nakamura, S.; et al. The lipid mediator protectin D1 inhibits influenza virus replication and improves severe influenza. Cell 2013, 153, 112-125.

41. Ortigoza, M.B.; Dibben, O.; Maamary, J.; Martinez-Gil, L.; Leyva-Grado, V.H.; Abreu, P., Jr.; Ayllon, J.; Palese, P.; Shaw, M.L. A novel small molecule inhibitor of influenza a viruses that targets polymerase function and indirectly induces interferon. PLoS Pathog. 2012, 8, e1002668.

42. Von Schacky, C. Cardiovascular disease prevention and treatment. Prostaglandins Leukot. Essent. Fatty Acids 2009, 81, 193-198.

43. Breslow, J.L. N-3 fatty acids and cardiovascular disease. Am. J. Clin. Nutr. 2006, 83, 1477S-1482S.

44. Wendel, M.; Heller, A.R. Anticancer actions of omega-3 fatty acids-Current state and future perspectives. Anticancer Agents Med. Chem. 2009, 9, 457-470.

45. Yang, P.; Jiang, Y.; Fischer, S.M. Prostaglandin E3 metabolism and cancer. Cancer Lett. 2014, 348, 1-11. 
46. Jing, K.; Wu, T.; Lim, K. Omega-3 polyunsaturated fatty acids and cancer. Anticancer Agents Med. Chem. 2013, 13, 1162-1177.

47. Tsuji, M.; Murota, S.I.; Morita, I. Docosapentaenoic acid (22:5, n-3) suppressed tube-forming activity in endothelial cells induced by vascular endothelial growth factor. Prostaglandins Leukot. Essent. Fatty Acids 2003, 68, 337-342.

48. Calviello, G.; di Nicuolo, F.; Gragnoli, S.; Piccioni, E.; Serini, S.; Maggiano, N.; Tringali, G.; Navarra, P.; Ranelletti, F.O.; Palozza, P. N-3 pufas reduce vegf expression in human colon cancer cells modulating the COX-2/PGE2 induced ERK-1 and -2 and HIF-1alpha induction pathway. Carcinogenesis 2004, 25, 2303-2310.

49. Arshad, A.; Isherwood, J.; Mann, C.; Cooke, J.; Pollard, C.; Runau, F.; Morgan, B.; Steward, W.; Metcalfe, M.; Dennison, A. Intravenous omega-3 fatty acids plus gemcitabine: Potential to improve response and quality of life in advanced pancreatic cancer. J. Parenter Enteral Nutr. 2015.

50. Baracos, V.E.; Mazurak, V.C.; Ma, D.W. N-3 polyunsaturated fatty acids throughout the cancer trajectory: Influence on disease incidence, progression, response to therapy and cancer-associated cachexia. Nutr. Res. Rev. 2004, 17, 177-192.

51. Xue, H.; Sawyer, M.B.; Field, C.J.; Dieleman, L.A.; Baracos, V.E. Nutritional modulation of antitumor efficacy and diarrhea toxicity related to irinotecan chemotherapy in rats bearing the ward colon tumor. Clin. Cancer Res. 2007, 13, 7146-7154.

52. Xue, H.; Ren, W.; Denkinger, M.; Schlotzer, E.; Wischmeyer, P.E. Nutrition modulation of cardiotoxicity and anticancer efficacy related to doxorubicin chemotherapy by glutamine and omega-3 polyunsaturated fatty acids. J. Parenter. Enteral Nutr. 2015.

53. Barber, M.D. The pathophysiology and treatment of cancer cachexia. Nutr. Clin. Pract. 2002, 17, 203-209.

54. Chang, W.L.; Chapkin, R.S.; Lupton, J.R. Fish oil blocks azoxymethane-induced rat colon tumorigenesis by increasing cell differentiation and apoptosis rather than decreasing cell proliferation. J. Nutr. 1998, 128, 491-497.

55. Assisi, A.; Banzi, R.; Buonocore, C.; Capasso, F.; Di Muzio, V.; Michelacci, F.; Renzo, D.; Tafuri, G.; Trotta, F.; Vitocolonna, M.; et al. Fish oil and mental health: The role of $n-3$ long-chain polyunsaturated fatty acids in cognitive development and neurological disorders. Int. Clin. Psychopharmacol. 2006, 21, 319-336.

56. Chalon, S.; Delion-Vancassel, S.; Belzung, C.; Guilloteau, D.; Leguisquet, A.M.; Besnard, J.C.; Durand, G. Dietary fish oil affects monoaminergic neurotransmission and behavior in rats. J. Nutr. 1998, 128, 2512-2519.

57. Chalon, S.; Vancassel, S.; Zimmer, L.; Guilloteau, D.; Durand, G. Polyunsaturated fatty acids and cerebral function: Focus on monoaminergic neurotransmission. Lipids 2001, 36, 937-944.

58. Hogyes, E.; Nyakas, C.; Kiliaan, A.; Farkas, T.; Penke, B.; Luiten, P.G. Neuroprotective effect of developmental docosahexaenoic acid supplement against excitotoxic brain damage in infant rats. Neuroscience 2003, 119, 999-1012. 
59. Minami, M.; Kimura, S.; Endo, T.; Hamaue, N.; Hirafuji, M.; Togashi, H.; Matsumoto, M.; Yoshioka, M.; Saito, H.; Watanabe, S.; et al. Dietary docosahexaenoic acid increases cerebral acetylcholine levels and improves passive avoidance performance in stroke-prone spontaneously hypertensive rats. Pharmacol. Biochem. Behav. 1997, 58, 1123-1129.

60. Xiao, Y.; Li, X. Polyunsaturated fatty acids modify mouse hippocampal neuronal excitability during excitotoxic or convulsant stimulation. Brain Res. 1999, 846, 112-121.

61. Hossain, M.S.; Hashimoto, M.; Gamoh, S.; Masumura, S. Antioxidative effects of docosahexaenoic acid in the cerebrum versus cerebellum and brainstem of aged hypercholesterolemic rats. J. Neurochem. 1999, 72, 1133-1138.

62. Issemann, I.; Prince, R.A.; Tugwood, J.D.; Green, S. The peroxisome proliferator-activated receptor:Retinoid $\mathrm{x}$ receptor heterodimer is activated by fatty acids and fibrate hypolipidaemic drugs. J. Mol. Endocrinol. 1993, 11, 37-47.

63. Kyle, D.J.; Schaefer, E.; Patton, G.; Beiser, A. Low serum docosahexaenoic acid is a significant risk factor for alzheimer's dementia. Lipids 1999, 34, S245.

64. Akiyama, H.; Barger, S.; Barnum, S.; Bradt, B.; Bauer, J.; Cole, G.M.; Cooper, N.R.; Eikelenboom, P.; Emmerling, M.; Fiebich, B.L.; et al. Inflammation and Alzheimer's disease. Neurobiol. Aging 2000, 21, 383-421.

65. Boston, P.F.; Bennett, A.; Horrobin, D.F.; Bennett, C.N. Ethyl-EPA in alzheimer's disease-A pilot study. Prostaglandins Leukot. Essent. Fatty Acids 2004, 71, 341-346.

66. Eslick, G.D.; Howe, P.R.; Smith, C.; Priest, R.; Bensoussan, A. Benefits of fish oil supplementation in hyperlipidemia: A systematic review and meta-analysis. Int. J. Cardiol. 2009, 136, 4-16.

67. Montori, V.M.; Farmer, A.; Wollan, P.C.; Dinneen, S.F. Fish oil supplementation in type 2 diabetes: A quantitative systematic review. Diabetes Care 2000, 23, 1407-1415.

68. Spite, M. Deciphering the role of $n-3$ polyunsaturated fatty acid-derived lipid mediators in health and disease. Proc. Nutr. Soc. 2013, 72, 441-450.

69. Miles, E.A.; Calder, P.C. Influence of marine $n-3$ polyunsaturated fatty acids on immune function and a systematic review of their effects on clinical outcomes in rheumatoid arthritis. Br. J. Nutr. 2012, 107, S171-S184.

70. Campos, F.G.; Waitzberg, D.L.; Logulo, A.F.; Torrinhas, R.S.; Teixeira, W.G.; Habr-Gama, A. Immunonutrition in experimental colitis: Beneficial effects of omega-3 fatty acids. Arq. Gastroenterol. 2002, 39, 48-54.

71. Pompeia, C.; Lopes, L.R.; Miyasaka, C.K.; Procopio, J.; Sannomiya, P.; Curi, R. Effect of fatty acids on leukocyte function. Braz.J. Med. Biol. Res. 2000, 33, 1255-1268.

72. Calder, P.C. Immunoregulatory and anti-inflammatory effects of $n$ - 3 polyunsaturated fatty acids. Braz. J. Med. Biol. Res. 1998, 31, 467-490.

73. Hughes, D.A.; Pinder, A.C. N-3 polyunsaturated fatty acids modulate the expression of functionally associated molecules on human monocytes and inhibit antigen-presentation in vitro. Clin. Exp. Immunol. 1997, 110, 516-523. 
74. Zheng, L.; Zomerdijk, T.P.; van den Barselaar, M.T.; Geertsma, M.F.; Van Furth, R.; Nibbering, P.H. Arachidonic acid, but not its metabolites, is essential for fcgammar-stimulated intracellular killing of staphylococcus aureus by human monocytes. Immunology 1999, 96, 90-97.

75. Kumaratilake, L.M.; Ferrante, A.; Robinson, B.S.; Jaeger, T.; Poulos, A. Enhancement of neutrophil-mediated killing of plasmodium falciparum asexual blood forms by fatty acids: Importance of fatty acid structure. Infect. Immun. 1997, 65, 4152-4157.

76. Chavali, S.R.; Weeks, C.E.; Zhong, W.W.; Forse, R.A. Increased production of tnf-alpha and decreased levels of dienoic eicosanoids, IL-6 and IL-10 in mice fed menhaden oil and juniper oil diets in response to an intraperitoneal lethal dose of lps. Prostaglandins Leukot. Essent. Fatty Acids 1998, 59, 89-93.

77. Kelley, D.S.; Taylor, P.C.; Nelson, G.J.; Schmidt, P.C.; Ferretti, A.; Erickson, K.L.; Yu, R.; Chandra, R.K.; Mackey, B.E. Docosahexaenoic acid ingestion inhibits natural killer cell activity and production of inflammatory mediators in young healthy men. Lipids 1999, 34, 317-324.

78. Purasiri, P.; McKechnie, A.; Heys, S.D.; Eremin, O. Modulation in vitro of human natural cytotoxicity, lymphocyte proliferative response to mitogens and cytokine production by essential fatty acids. Immunology 1997, 92, 166-172.

79. Fernandes, G.; Bysani, C.; Venkatraman, J.T.; Tomar, V.; Zhao, W. Increased tgf-beta and decreased oncogene expression by omega-3 fatty acids in the spleen delays onset of autoimmune disease in b/w mice. J. Immunol. 1994, 152, 5979-5987.

80. Miyasaka, C.K.; Mendonca, J.R.; Silva, Z.L.; de Sousa, J.A.; Tavares de Lima, W.; Curi, R. Modulation of hypersensitivity reaction by lipids given orally. Gen. Pharmacol. 1999, 32, 597-602.

81. Robinson, L.E.; Field, C.J. Dietary long-chain (n-3) fatty acids facilitate immune cell activation in sedentary, but not exercise-trained rats. J. Nutr. 1998, 128, 498-504.

82. Mayer, K.; Fegbeutel, C.; Hattar, K.; Sibelius, U.; Kramer, H.J.; Heuer, K.U.; Temmesfeld-Wollbruck, B.; Gokorsch, S.; Grimminger, F.; Seeger, W. Omega-3 vs. Omega-6 lipid emulsions exert differential influence on neutrophils in septic shock patients: Impact on plasma fatty acids and lipid mediator generation. Intensive Care Med. 2003, 29, 1472-1481.

83. Gabe, S.; Culkin, A. Abnormal liver function tests in the parenteral nutrition fed patient. Frontline Gastroenterol. 2010, 1, 98-104.

84. Lindor, K.D.; Fleming, C.R.; Abrams, A.; Hirschkorn, M.A. Liver function values in adults receiving total parenteral nutrition. JAMA 1979, 241, 2398-2400.

85. Staun, M.; Pironi, L.; Bozzetti, F.; Baxter, J.; Forbes, A.; Joly, F.; Jeppesen, P.; Moreno, J.; Hebuterne, X.; Pertkiewicz, M.; et al. Espen guidelines on parenteral nutrition: Home parenteral nutrition (HPN) in adult patients. Clin. Nutr. 2009, 28, 467-479.

86. Goulet, O.; Joly, F.; Corriol, O.; Colomb-Jung, V. Some new insights in intestinal failure-associated liver disease. Curr. Opin. Organ. Transplant. 2009, 14, 256-261. 
87. Antebi, H.; Mansoor, O.; Ferrier, C.; Tetegan, M.; Morvan, C.; Rangaraj, J.; Alcindor, L.G. Liver function and plasma antioxidant status in intensive care unit patients requiring total parenteral nutrition: Comparison of 2 fat emulsions. J. Parenter. Enteral Nutr. 2004, $28,142-148$.

88. Heller, A.R.; Rossel, T.; Gottschlich, B.; Tiebel, O.; Menschikowski, M.; Litz, R.J.; Zimmermann, T.; Koch, T. Omega-3 fatty acids improve liver and pancreas function in postoperative cancer patients. Int. J. Cancer 2004, 111, 611-616.

89. Piper, S.N.; Schade, I.; Beschmann, R.B.; Maleck, W.H.; Boldt, J.; Rohm, K.D. Hepatocellular integrity after parenteral nutrition: Comparison of a fish-oil-containing lipid emulsion with an olive-soybean oil-based lipid emulsion. Eur. J. Anaesthesiol. 2009, 26, 1076-1082.

90. Tomsits, E.; Pataki, M.; Tolgyesi, A.; Fekete, G.; Rischak, K.; Szollar, L. Safety and efficacy of a lipid emulsion containing a mixture of soybean oil, medium-chain triglycerides, olive oil, and fish oil: A randomised, double-blind clinical trial in premature infants requiring parenteral nutrition. J. Pediatr. Gastroenterol. Nutr. 2010, 51, 514-521.

91. Goulet, O.; Antebi, H.; Wolf, C.; Talbotec, C.; Alcindor, L.G.; Corriol, O.; Lamor, M.; Colomb-Jung, V. A new intravenous fat emulsion containing soybean oil, medium-chain triglycerides, olive oil, and fish oil: A single-center, double-blind randomized study on efficacy and safety in pediatric patients receiving home parenteral nutrition. J. Parenter. Enteral Nutr. 2010, 34, 485-495.

92. Rayyan, M.; Devlieger, H.; Jochum, F.; Allegaert, K. Short-term use of parenteral nutrition with a lipid emulsion containing a mixture of soybean oil, olive oil, medium-chain triglycerides, and fish oil: A randomized, double-blind study in preterm infants. JPEN 2012, 36, 81S-94S.

93. D'Ascenzo, R.; Savini, S.; Biagetti, C.; Bellagamba, M.P.; Marchionni, P.; Pompilio, A.; Cogo, P.E.; Carnielli, V.P. Higher docosahexaenoic acid, lower arachidonic acid and reduced lipid tolerance with high doses of a lipid emulsion containing 15\% fish oil: A randomized clinical trial. Clin. Nutr. 2014, 33, 1002-1009.

94. De Meijer, V.E.; Gura, K.M.; Meisel, J.A.; Le, H.D.; Puder, M. Parenteral fish oil monotherapy in the management of patients with parenteral nutrition-associated liver disease. Arch. Surg. 2010, 145, 547-551.

95. Diamond, I.R.; Sterescu, A.; Pencharz, P.B.; Kim, J.H.; Wales, P.W. Changing the paradigm: Omegaven for the treatment of liver failure in pediatric short bowel syndrome. J. Pediatr. Gastroenterol. Nutr. 2009, 48, 209-215.

96. Gura, K.M.; Duggan, C.P.; Collier, S.B.; Jennings, R.W.; Folkman, J.; Bistrian, B.R.; Puder, M. Reversal of parenteral nutrition-associated liver disease in two infants with short bowel syndrome using parenteral fish oil: Implications for future management. Pediatrics 2006, 118, e197-e201.

97. Gura, K.M.; Lee, S.; Valim, C.; Zhou, J.; Kim, S.; Modi, B.P.; Arsenault, D.A.; Strijbosch, R.A.; Lopes, S.; Duggan, C.; et al. Safety and efficacy of a fish-oil-based fat emulsion in the treatment of parenteral nutrition-associated liver disease. Pediatrics 2008, 121, e678-e686. 
98. Ekema, G.; Falchetti, D.; Boroni, G.; Tanca, A.R.; Altana, C.; Righetti, L.; Ridella, M.; Gambarotti, M.; Berchich, L. Reversal of severe parenteral nutrition-associated liver disease in an infant with short bowel syndrome using parenteral fish oil (omega-3 fatty acids). J. Pediatr. Surg. 2008, 43, 1191-1195.

99. Beken, S.; Dilli, D.; Fettah, N.D.; Kabatas, E.U.; Zenciroglu, A.; Okumus, N. The influence of fish-oil lipid emulsions on retinopathy of prematurity in very low birth weight infants: A randomized controlled trial. Early Hum. Dev. 2014, 90, 27-31.

100. Singer, P.; Berger, M.M.; Van den, B.G.; Biolo, G.; Calder, P.; Forbes, A.; Griffiths, R.; Kreyman, G.; Leverve, X.; Pichard, C.; et al. Espen guidelines on parenteral nutrition: Intensive care. Clin. Nutr. 2009, 28, 387-400.

101. Heller, A.R.; Rossler, S.; Litz, R.J.; Stehr, S.N.; Heller, S.C.; Koch, R.; Koch, T. Omega-3 fatty acids improve the diagnosis-related clinical outcome. Crit. Care Med. 2006, 34, 972-979.

102. Mayer, K.; Seeger, W. Fish oil in critical illness. Curr. Opin. Clin. Nutr. Metab. Care 2008, 11, 121-127.

103. Manzanares, W.; Langlois, P.L.; Dhaliwal, R.; Lemieux, M.; Heyland, D.K. Intravenous fish oil lipid emulsions in critically ill patients: An updated systematic review and meta-analysis. Crit. Care 2015, 19, 167.

104. Gupta, A.; Govil, D.; Bhatnagar, S.; Gupta, S.; Goyal, J.; Patel, S.; Baweja, H. Efficacy and safety of parenteral omega 3 fatty acids in ventilated patients with acute lung injury. Indian J. Crit. Care Med. 2011, 15, 108.

105. Khor, B.S.; Liaw, S.J.; Shih, H.C.; Wang, L.S. Randomized, double blind, placebo-controlled trial of fish-oil-based lipid emulsion infusion for treatment of critically ill patients with severe sepsis. Asian J. Surg. 2011, 34, 1-10.

106. Hall, T.C.; Bilku, D.K.; Al-Leswas, D.; Neal, C.P.; Horst, C.; Cooke, J.; Metcalfe, M.S.; Dennison, A.R. A randomized controlled trial investigating the effects of parenteral fish oil on survival outcomes in critically ill patients with sepsis: A pilot study. J. Parenter. Enteral Nutr. 2015, 39, 301-312.

107. Burkhart, C.S.; Dell-Kuster, S.; Siegemund, M.; Pargger, H.; Marsch, S.; Strebel, S.P.; Steiner, L.A. Effect of $n-3$ fatty acids on markers of brain injury and incidence of sepsis-associated delirium in septic patients. Acta Anaesthesiol. Scand. 2014, 58, 689-700.

108. Dhaliwal, R.; Cahill, N.; Lemieux, M.; Heyland, D.K. The canadian critical care nutrition guidelines in 2013: An update on current recommendations and implementation strategies. Nutr. Clin. Pract. 2014, 29, 29-43.

109. Dewey, A.; Baughan, C.; Dean, T.; Higgins, B.; Johnson, I. Eicospentaenoic acid (EPA, an omega-3 fatty acid from fish oils) for the treatment of cancer cachexia (review). Cochrane Database Syst. Rev. 2010.

110. Bozzetti, F.; Arends, J.; Lundholm, K.; Micklewright, A.; Zurcher, G.; Muscaritoli, M. Espen guidelines on parenteral nutrition: Non-surgical oncology. Clin. Nutr. 2009, 28, 445-454. 
111. Sanchez-Lara, K.; Turcott, J.G.; Juarez-Hernandez, E.; Nunez-Valencia, C.; Villanueva, G.; Guevara, P.; de la Torre-Vallejo, M.; Mohar, A.; Arrieta, O. Effects of an oral nutritional supplement containing eicosapentaenoic acid on nutritional and clinical outcomes in patients with advanced non-small cell lung cancer: Randomised trial. Clin. Nutr. 2014, 33, 1017-1023.

112. van der Meij, B.S.; Langius, J.A.; Spreeuwenberg, M.D.; Slootmaker, S.M.; Paul, M.A.; Smit, E.F.; van Leeuwen, P.A. Oral nutritional supplements containing $n-3$ polyunsaturated fatty acids affect quality of life and functional status in lung cancer patients during multimodality treatment: An RCT. Eur. J. Clin. Nutr. 2012, 66, 399-404.

113. Trabal, J.; Leyes, P.; Forga, M.; Maurel, J. Potential usefulness of an EPA-enriched nutritional supplement on chemotherapy tolerability in cancer patients without overt malnutrition. Nutr. Hosp. 2010, 25, 736-740.

114. Ma, Y.J.; Yu, J.; Xiao, J.; Cao, B.W. The consumption of omega-3 polyunsaturated fatty acids improves clinical outcomes and prognosis in pancreatic cancer patients: A systematic evaluation. Nutr. Cancer 2015, 67, 112-118.

115. Wang, J.; Yu, J.C.; Kang, W.M.; Ma, Z.Q. Superiority of a fish oil-enriched emulsion to medium-chain triacylglycerols/long-chain triacylglycerols in gastrointestinal surgery patients: A randomized clinical trial. Nutrition 2012, 28, 623-629.

116. Han, Y.Y.; Lai, S.L.; Ko, W.J.; Chou, C.H.; Lai, H.S. Effects of fish oil on inflammatory modulation in surgical intensive care unit patients. Nutr. Clin. Pract. 2012, 27, 91-98.

117. Jiang, Z.M.; Wilmore, D.W.; Wang, X.R.; Wei, J.M.; Zhang, Z.T.; Gu, Z.Y.; Wang, S.; Han, S.M.; Jiang, H.; Yu, K. Randomized clinical trial of intravenous soybean oil alone versus soybean oil plus fish oil emulsion after gastrointestinal cancer surgery. Br. J. Surg. 2010, 97, 804-809.

118. de Miranda Torrinhas, R.S.; Santana, R.; Garcia, T.; Cury-Boaventura, M.F.; Sales, M.M.; Curi, R.; Waitzberg, D.L. Parenteral fish oil as a pharmacological agent to modulate post-operative immune response: A randomized, double-blind, and controlled clinical trial in patients with gastrointestinal cancer. Clin. Nutr. 2013, 32, 503-510.

119. Zhu, M.W.; Tang, D.N.; Hou, J.; Wei, J.M.; Hua, B.; Sun, J.H.; Cui, H.Y. Impact of fish oil enriched total parenteral nutrition on elderly patients after colorectal cancer surgery. Chin. Med. J. (Engl.) 2012, 125, 178-181.

120. Zhu, X.; Wu, Y.; Qiu, Y.; Jiang, C.; Ding, Y. Effects of omega-3 fish oil lipid emulsion combined with parenteral nutrition on patients undergoing liver transplantation. J. Parenter. Enteral Nutr. 2013, 37, 68-74.

121. Berger, M.M.; Delodder, F.; Liaudet, L.; Tozzi, P.; Schlaepfer, J.; Chiolero, R.L.; Tappy, L. Three short perioperative infusions of $n-3$ pufas reduce systemic inflammation induced by cardiopulmonary bypass surgery: A Randomized Controlled Trial. Am. J. Clin. Nutr. 2013, 97, 246-254.

122. Klek, S.; Waitzberg, D.L. Intravenous lipids in adult surgical patients. In Intravenous Lipid Emulsions; Calder, P.C., Waitzberg, D.L., Koletzko, B., Eds.; Karger: Basel, Switzerland, 2015; Volume 112, pp. 115-119. 
123. Chen, B.; Zhou, Y.; Yang, P.; Wan, H.W.; Wu, X.T. Safety and efficacy of fish oil-enriched parenteral nutrition regimen on postoperative patients undergoing major abdominal surgery: A Meta-Analysis of Randomized Controlled Trials. J. Parenter. Enteral Nutr. 2010, 34, 387-394.

124. Li, N.N.; Zhou, Y.; Qin, X.P.; Chen, Y.; He, D.; Feng, J.Y.; Wu, X.T. Does intravenous fish oil benefit patients post-surgery? A meta-analysis of randomised controlled trials. Clin. Nutr. 2014, 33, 226-239.

125. Pradelli, L.; Mayer, K.; Muscaritoli, M.; Heller, A.R. N-3 fatty acid-enriched parenteral nutrition regimens in elective surgical and icu patients: A Meta-Analysis. Crit. Care 2012, 16, R184.

126. Tian, H.; Yao, X.; Zeng, R.; Sun, R.; Tian, H.; Shi, C.; Li, L.; Tian, J.; Yang, K. Safety and efficacy of a new parenteral lipid emulsion (SMOF) for surgical patients: A systematic review and meta-analysis of randomized controlled trials. Nutr. Rev. 2013, 71, 815-821.

127. Wei, C.; Hua, J.; Bin, C.; Klassen, K. Impact of lipid emulsion containing fish oil on outcomes of surgical patients: Systematic Review of Randomized Controlled Trials from Europe and Asia. Nutrition 2010, 26, 474-481.

128. Braga, M.; Wischmeyer, P.E.; Drover, J.; Heyland, D.K. Clinical evidence for pharmaconutrition in major elective surgery. J. Parenter. Enteral Nutr. 2013, 37, 66S-72S.

129. Skouroliakou, M.; Konstantinou, D.; Agakidis, C.; Delikou, N.; Koutri, K.; Antoniadi, M.; Karagiozoglou-Lampoudi, T. Cholestasis, bronchopulmonary dysplasia, and lipid profile in preterm infants receiving MCT/omega-3-PUFA-containing or soybean-based lipid emulsions. Nutr. Clin. Pract. 2012, 27, 817-824.

130. Klek, S.; Kulig, J.; Szczepanik, A.M.; Jedrys, J.; Kolodziejczyk, P. The clinical value of parenteral immunonutrition in surgical patients. Acta Chir. Belg. 2005, 105, 175-179.

131. Wu, G.H.; Gao, J.; Ji, C.Y.; Pradelli, L.; Xi, Q.L.; Zhuang, Q.L. Cost and effectiveness of omega-3 fatty acid supplementation in chinese iCU patients receiving parenteral nutrition. Clinicoecon. Outcomes Res. 2015, 7, 369-375.

132. Pradelli, L.; Eandi, M.; Povero, M.; Mayer, K.; Muscaritoli, M.; Heller, A.R.; Fries-Schaffner, E. Cost-effectiveness of omega-3 fatty acid supplements in parenteral nutrition therapy in hospitals: A Discrete Event Simulation Model. Clin. Nutr. 2014, 33, 785-792. 


\title{
Is There A Role for Alpha-Linolenic Acid in the Fetal Programming of Health?
}

\author{
Alicia I. Leikin-Frenkel
}

Abstract: The role of $\omega 3$ alpha linolenic acid (ALA) in the maternal diet during pregnancy and lactation, and its effect on the prevention of disease and programming of health in offspring, is largely unknown. Compared to ALA, w3 docosahexaenoic (DHA) and eicosapentaenoic (EPA) acids have been more widely researched due to their direct implication in fetal neural development. In this literature search we found that ALA, the essential $\omega 3$ fatty acid and metabolic precursor of DHA and EPA has been, paradoxically, almost unexplored. In light of new and evolving findings, this review proposes that ALA may have an intrinsic role, beyond the role as metabolic parent of DHA and EPA, during fetal development as a regulator of gene programming for the prevention of metabolic disease and promotion of health in offspring.

Reprinted from J. Clin. Med. Cite as: Leikin-Frenkel, A.I. Is There A Role for Alpha-Linolenic Acid in the Fetal Programming of Health?. J. Clin. Med. 2016, 5,40 .

\section{Introduction}

More than 80 years ago, Burr et al. [1] described the essential fatty acids (EFA) linoleic acid ( $\omega 6$ LA) and alpha linolenic acid ( $\omega 3$ ALA) in animals. Their research contributed to the knowledge and concept that normal growth, development and health in mammals depends on EFA nutritional supply. Both EFA share enzymes like the $\Delta 6$ desaturase and elongases, and, thus, compete as substrates in this metabolic pathway (Figure 1). The genetic variants of desaturases determine the nutritional requirements for the supply of fatty acids and consequently, health and/or disease, as previously reviewed $[2,3]$.

During the course of mammalian development, fatty acids (FA) are transferred to the fetus through the placenta [4] and their composition depends, to a great extent, on the maternal diet [5]. Maternal nutrition during pregnancy-lactation can induce significant changes in body composition, physiology and metabolism in offspring. Thus, the Fetal Origins Hypothesis was inspired by evidence showing that adult cardiovascular disease begins through developmental activation of a set of genes and metabolic pathways in the offspring in response to in utero under- or over-nutrition [6]. FAs play a primary role in growth and development and it is now accepted that imbalances in their intake during pregnancy and lactation may result in permanent changes that affect appetite control, neuroendocrine function and energy 
metabolism in the fetus; thus, influencing the metabolic programming [7]. However, the roles of EFAs and the mechanisms by which they impact the long-term health of the offspring remains to be determined [8]. The importance of the FA composition in the diet and the significant differences between ALA and its metabolic products DHA and EPA has been spotted both in adults [9] and in maternal diets during pregnancy [10]. Some recent reviews even reinforce the importance of maternal dietary FA quality for the health outcomes in offspring [7,8,11,12].

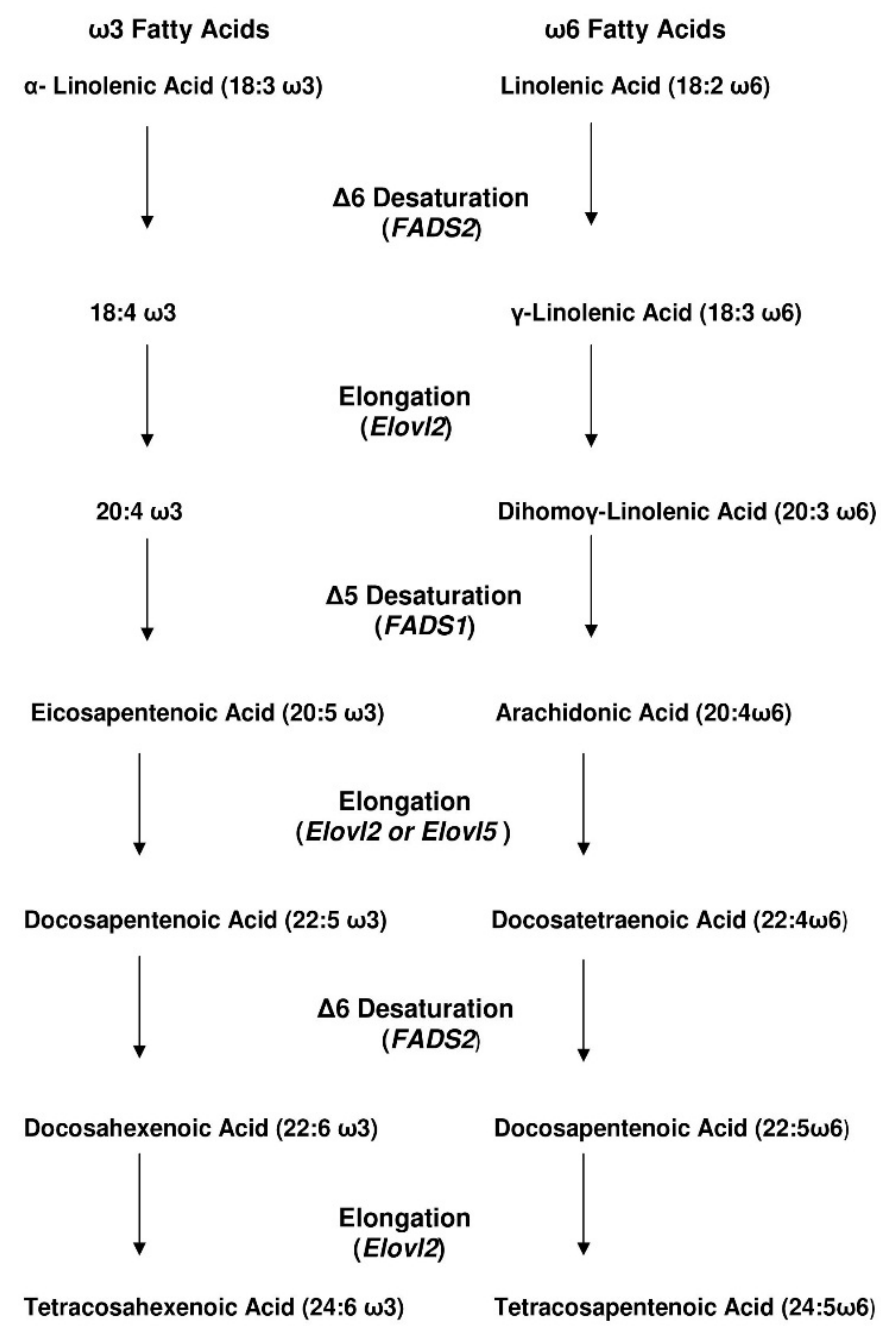

Figure 1. Linoleic (C18:2n-6) and $\alpha$-Linolenic (C18:3n-3) acid metabolism and elongation. 
Beyond the existing differences between human and animals, the basic tissue, physiological and morphological placental developments are conserved between species. Therefore, animal models can be considered suitable when researching the impact of nutritional FAs on development and their long-term influence on the offspring's susceptibility to metabolic diseases, including obesity, insulin resistance (IR), and cardiovascular risk [13]. Importantly, when looking for a comprehensive understanding of the role of nutritional FAs on development for the prevention of adult disease, a nutrigenetics approach is recommended [14,15].

\section{Dietary $\omega 3$ Fatty Acids and Health}

DHA and EPA: As defined by Burr [1], ALA is the only $\omega 3$ essential FA for mammals, while DHA and EPA are its downstream metabolic products. The benefits of consumption or supplementation of $\omega 3$ polyunsaturated fatty acids (PUFAs) by adults on the prevention and treatment of obesity, metabolic syndrome (MS), and cardiovascular disease (CVD) have been reported [16,17]. Most of the reviewed studies were randomized-controlled intervention trials suggesting that supplementation with $\omega 3$ PUFA might improve some obesity-associated metabolic syndrome features such as insulin resistance, hypertension and dyslipidemia by decreasing plasma triglycerides [18]. Similarly, they also confer cardio protection by lowering blood pressure and through their benefits on vascular and anti-inflammatory properties [19]. The efficacy of $\omega 3$ PUFA on reducing myocardial infarction, arrhythmia, cardiac and sudden death, or stroke is, however, controversial [20]. Although it is now widely accepted that DHA and EPA have beneficial effects on CVD, it is not yet clear if these benefits are directly or exclusively related to DHA and EPA [21,22]. Other benefits of DHA and EPA, such as reversion of neuropathies, have also been described $[23,24]$ for both plant and marine $\omega-3$ FAs.

ALA: The relationship between ALA and chronic disease is unclear. As supported by human studies [22-24], high intake of ALA is protective against fatal ischemic heart disease. In later years, the use of ALA-rich oils deserved attention in the search for nutritional ways of preventing or ameliorating cardiovascular disease and metabolic syndrome. Rodriguez-Leyva et al. reported that epidemiological randomized studies using flaxseed oil as a preventive intervention in a healthy population or in subjects identified as "at risk" for CVD are missing [25]. Baxheinrich et al. reported the beneficial effects of ALA rich rapeseed oil on body weight, systemic inflammation and endothelial function in patients with MS traits $[26,27]$. These past works showed that providing ALA significantly contributed to reductions in systolic blood pressure, total cholesterol, LDL-cholesterol and insulin levels after six months. Moreover, ALA was shown to significantly decrease body fat mass, as well as improve both vascular function and inflammation. 
The effects of ALA, as well as those of EPA and DHA, on the metabolic syndrome have been further reviewed by Poudyal et al. [28]. They addressed ALA, DHA and EPA as individual entities, and provided evidence of potentially independent effects for each of the $\omega 3$ FAs on cardiovascular health. These same authors also reported that the three $\omega 3$ FA could each reduce inflammation in cardiac fibrosis and hepatic steatosis in a high-fat diet induced model of metabolic syndrome in rats. Those effects were associated to a complete suppression of Stearoyl CoA desaturase (SCD1) function. In those studies, ALA induced comparatively different FA redistribution of retroperitoneal fat, skeletal muscle and liver. Furthermore, it was suggested that the accumulation of the $\omega 3$ FA in adipose tissue, as well as in skeletal muscle, may account for their crucial role in the reduction of abdominal fat, inflammation, dyslipidemia and IR [28]. Conversely, recommendations for ALA intake in pregnant women for the prevention of metabolic diseases in offspring are limited [29-33].

\section{How Efficient Is ALA Conversion to EPA and DHA in Humans?}

Dietary ALA is metabolically converted into acetate or $\mathrm{CO}_{2}$ through $\beta$-oxidation, or desaturated and elongated into EPA, w3 DPA and DHA [34]. ALA conversion to longer products in tracer studies has been observed in nearly all humans studied from birth through late middle-aged men and women [34,35].

It is clear that the metabolism of $\omega 3$ FA depends on other nutrients, in particular $\omega 6$ FAs, due to their competition for the same enzymes and transport systems [2]. They also compete for incorporation into more complex lipids that comprise mammalian tissues, where high levels of $\omega 6$ PUFA replace and reduce $\omega 3$ PUFA levels. Early studies of rat liver microsomes showed that the $\Delta-6$ desaturase activity measured in vitro was subject to competitive inhibition by other substrates. In particular, desaturation of ALA to $\omega 3$ eicosatrienoic acid was shown to be inhibited by LA and, conversely, LA conversion to $\omega 6$ gamma linolenic acid was inhibited by ALA [36,37].

When studying the impact of ALA on the improvement of the metabolic syndrome, the following question arises: is the conversion of ALA into EPA and DHA responsible for the observed effects? This question was addressed by Truong et al. [32] in relation to genetic variants in the $\Delta 6$ desaturase gene (Fads2). Adipose tissue was used as a biomarker of ALA intake in adult humans, and the authors showed that high concentrations of ALA in adipose tissue were associated with lower prevalence ratios of the metabolic syndrome compared to low ALA. Although an interaction between ALA and Fads2 genotype (T-del) was borderline significant, it nevertheless suggested that genetic variation may play an important role along with diet in the development of metabolic syndrome, at least in the studied population. A variety of models have confirmed that ALA accumulates significantly and is converted into longer $\omega 3$ FA in humans [34]. According to the ISSFAL official 
statement \#5, studies in healthy adults showed that supplementing ALA to Western diets containing LA raises DHA and EPA levels in blood and breast milk [38]. In addition, these and other studies have provided evidence indicating that FA accumulation is tissue-dependent [38] suggesting that metabolism may be based upon a tissue-selective need for longer $\omega 3$ FA, such as DHA.

As claimed by Anderson et al. [39], clarification of ALA's involvement in health and disease is essential. Indeed, it is insufficient to assume that ALA exerts its beneficial effects through conversion to EPA and DHA. More thorough research is required to identify the differential effects of ALA on metabolic disease like IR and CVD and to differentiate the possible heterogeneous effects of ALA versus DHA and EPA. The use of developed animal models such as the $\Delta 6$ knockout mouse [40] that inhibits the conversion of ALA into EPA, and EPA into DHA, could be highly useful in this regard. No such work has been performed to date.

\section{Adults}

It has been observed that humans of all ages, from preterm and even fetuses to adults, convert ALA to DHA. However, the efficiency of conversion seems to decrease as infants mature [35]. ALA is partitioned to $\beta$-oxidation as energy source, for metabolic conversion to longer PUFA and for incorporation into tissues [39,41,42]. Importantly, studies were also reported in which a significant increase in plasma DHA levels was achieved by altering the oils in the diet and changing both ALA and LA content [43].

There seems to be an agreement that the partitioning of ALA towards $\beta$-oxidation in humans is lower in women than in men $[44,45]$, an effect attributed to estrogen [34], which may explain the higher conversion of ALA to longer-chain PUFA in women [32,41]. The explanation for the preferred use of ALA for $\beta$-oxidation seems to be the greater affinity of carnitine palmitoyl transferase-1, the rate limiting enzyme in mitochondrial $\beta$-oxidation, for this EFA compared to other PUFA [41]. Most studies examining ALA have been performed in young men. The few studies focused specifically on women of reproductive age showed that conversion of ALA to EPA and DHA is $2.5 \times$ greater in women compared to age-matched men [43-45]. This is ascribed not only to the differential partitioning towards $\beta$-oxidation, but also to an up-regulation of the translocation of very long metabolic products towards the peroxisome in women [34]. It has been suggested that ALA conversion increases in pregnancy, which is supported by data in pregnant rats [44-46].

Clearly, gender differences exist and are of importance when recommending w3 FA to men or women and, in this case of the latter, whether they are pregnant or not [41]. Work performed by Childs and others [46,47] have provided further support for this concept. The examination of whether there are sex differences in the long-chain w3 FA response to increased ALA intake in humans showed that women 
have a higher increase than men in the EPA content of plasma phospholipids after six months. The gender differences in ALA use, metabolism and destination, warrant further investigation.

\section{Fatty Acids Quality and Early Life}

Pregnancy is supposed to be a period of high requirement for DHA in humans due to the fetus' need for rapid growth and neural development [34]. FA levels in the embryo and newborn babies are directly associated with maternal FA levels and composition; therefore, any variation in the maternal intake of FAs susceptible to genetic variability is pivotal for fetal growth, development and health [47]. There is some evidence suggesting that the influence of genetic variation in FADS genes on both circulating and tissue FA profiles, which can influence disease risk, may have a trans-generational effect $[48,49]$. Moreover, it has been shown that breastfeeding also exposes babies to the maternal genetic Fads variations through their effects on milk quality and quantity of fatty acids that, in turn, affect the baby health and intelligence quotient [50]. Consequently, these studies show that the influence of FADS2 polymorphisms in the mother is of uttermost importance for the array of FAs transferred from mother to child during uterine development and breastfeeding. This knowledge reinforces the importance of a nutritional adjustment during the critical perinatal period.

DHA and EPA: The effects of different qualities of dietary FAs during pregnancy and/or lactation on fetal development and offspring metabolism have been recently reviewed [7]. Maternal consumption of diets rich in $\omega 3$ PUFA in particular showed benefits for the development of offspring and it was even suggested that they exert epigenetic regulation for the prevention of obesity, insulin resistance and cardiovascular diseases [51,52]. The consumption of DHA and EPA during crucial periods of fetal development has beneficial physiologic and metabolic effects on the health of offspring by protecting them from the onset of metabolic diseases [7,11]. Nevertheless, results in the field are controversial, and the independent effects of ALA have not been studied enough.

ALA: During pregnancy there is a reduction of DHA in maternal serum while, at the same time, an increased requirement of DHA and EPA for fetal brain development $[38,49]$. The solution to compensate for this supposed imbalance can be to either provide ALA, the precursor for DHA and EPA, or these end products directly. Not enough scientific information exists comparing for the benefits of either supplementation. From the clinical aspect, criticism is raised regarding the claim of support for fetal cognitive health and brain function improvement associated with DHA and EPA supplementation [53].

Larque et al. [12] reported a somewhat positive relationship between maternal or cord serum DHA percentages and cognitive skills in young children. Unfortunately, 
valuable information was missing from this study: ALA is not mentioned amongst $\omega 3$ FAs and the blood FA composition of mothers and/or children was not described.

During the literature search described here, only one study was found indicating a negative effect of maternal over-consumption of $\omega 3$ FAs on life span and auditory brainstem response in older adult offspring. As in several papers, the term $\omega 3$ FA was broadly used, without specifying the dietary FA composition and without mentioning the specific effects of ALA [54].

\section{Perinatal Manipulation of ALA}

In relation to perinatal metabolism, significant FA desaturase activities have been detected in fetuses and preterm infants, indicating the existence of the molecular pathways at early stages of life $[55,56]$.

Published research regarding the positive effects of DHA and EPA has been reviewed showing that they are essential for proper fetal development [57]. Supplementation of $\omega 3$ PUFA during pregnancy has been linked to decreased incidence of allergies [58]. An interesting study using flaxseed oil in mice questions whether ALA provision during gestation and lactation could induce epigenetic changes in maternal and offspring livers [58]. In this study, the authors described an interaction between ALA dietary content and the FA metabolism, through down-regulation of the expression of enzymes involved in the elongation (Elovl2) and desaturation (Fads1) of FAs in maternal livers. A positive correlation between Fads2 promoter methylation in maternal livers and offspring livers with changes in the expression of DNA methyltransferases at day 19 of pregnancy was described. Even though the work was inconclusive, the authors suggested that the maternal availability of ALA during gestation-lactation could differentially alter the metabolism of $\omega 3$ and $\omega 6$ FAs, as well as the epigenetic status of Fads2 in maternal and offspring' livers.

Research focusing on ALA content in the maternal diet and the long-term side effects on metabolic syndrome in the offspring is scarce. Our laboratory has begun focusing on those effects and showed that maternal dietary enrichment of ALA, compared to SFA, led to lower body weight gain, liver fat accumulation, and homeostasis model assessment (HOMA) index, as well as reduced SCD1 in the adult offspring exposed to a high fat diet [59]. Those results suggested that the relative increase in dietary ALA during pregnancy and lactation may have the potential to prevent obesity and insulin resistance in the offspring. Furthermore, Shomonov-Wagner et al. [60] compared the effects of dietary enrichment in $\omega 3$ ALA, DHA or EPA, compared to saturated fatty acids (SFA). That work showed that SFA, independent of total fat amount or calories, induced liver lipid accumulation and IR in offspring at weaning, while ALA was the most efficient $\omega 3$ FA to prevent the induced metabolic alterations. That work proposed that not only ALA and SFA have 
divergent effects on IR and liver lipid levels, but also that each of ALA, DHA and EPA behaved differently. Furthermore, ALA preventive effects were, apparently, unrelated to its conversion into DHA and EPA. Consequently, we conclude that w3 ALA, DHA and EPA should be studied and referred to individually and not as a group.

Recently published results seem to indicate that ALA, as opposed to SFA, up-regulates the expression of genes involved in lipid oxidation and in the circadian rhythm [61].

\section{Regulatory Mechanisms}

The $\omega 3$ FA family seems to have distinct abilities to modulate metabolic functions and gene expression [62]. Deckelman et al. [63] mentioned that the longer chain length and higher number of double bonds give these FAs unique properties and suggested that they relate to modulation of enzymes associated with signaling pathways/incorporation of EPA and DHA into membrane phospholipids and direct effects on gene expression, amongst others. However, ALA has not been studied enough, and the molecular interactions between ALA and genes, are only now beginning to be described [61].

Maternal dietary enrichment with ALA and the long terms effects on offspring susceptibility to obesity and metabolic syndrome are an important and urgent subject waiting to be investigated. Also important to consider are FA-gene interactions. Based on the literature found, we may assume that ALA has beneficial effects during gestation, presumably on the prevention of obesity-associated disease in offspring [61]. Ahmed [64] described a proteomics approach to examine the regulatory roles of $\omega 3$ FAs. By using mice fed high or low $\omega 3$ FA containing diets, some affected proteins were identified related to lipid, glucose and protein metabolism. Unfortunately their work excluded ALA, but their information hints at the interaction between FAs and protein targets for the regulation of metabolic pathways. These results, together with preliminary evolving information, warrant more studies that would benefit from testing ALA, DHA and EPA separately.

\section{Epigenetics}

Environmental factors such as diet during fetal development can induce long-term modifications in the genes of the fetus. Human epidemiological data and controlled animal studies corroborate the impact of diet in the perinatal period and its lasting effect on gene expression and metabolism [65]. The question, "how do FAs influence the establishment of an epigenotype" [66], is intriguing and stimulating. It is proposed that the peroxisome proliferator-activated receptor alpha (PPARa), an abundant nuclear receptor transcription factor, may be a candidate to be regulated by maternal dietary FAs in embryonic life. Wang et al. [67] described the way maternal 
and, possibly, paternal imbalanced over- and under-nutrition may induce epigenetic modifications. Thus, DNA methylation, histone modification and miRNAs, may regulate genome activity and gene expression leading to proteins that affect fetal programming and organ physiology with lifelong consequences, sex-dependent in some cases. The developmental adaptations that permanently change structure, physiology and metabolism in offspring would thereby predispose for metabolic and cardiovascular risk later in adult life. Vickers's [68] analyzed not only the in utero, but also the trans-generational, possibilities for epigenetic modifications and its consequences. Niculescu et al. [58] showed epigenetic modifications induced specifically by ALA in an in vitro study. ALA was shown to alter the distribution of cells by influencing cell cycle phases, apoptosis and gene and protein expression of DNA methyl transferases 1 and 3. However, no modifications in global DNA methylation were found.

Burdge et al. [69] reviewed the interaction between FAs and the epigenome to conclude that it is not known, at present, how FAs modify the epigenome. Some of the existing limitations include the lack of definition of the experimental diets and the susceptibility of histone deacetylases to inhibition by short chain FAs present in most experimental diets. Nevertheless, the mechanisms underpinning transmission of developmental programming urgently require further research.

\section{Final Thoughts and Recommendations}

The importance of $\omega 3$ FAs for the development and long-term health of offspring is widely recognized. The importance of ALA, specifically, is only now beginning to be recognized; however, more thorough research is necessary in order to better understand its independent role in developmental programming.

Based on ongoing research $[28,61]$ we propose here the concept that ALA may have a role in the programming of health. Specifically, it may have intrinsic regulatory properties on gene expression during fetal development that extend beyond its simple metabolic conversion to DHA and EPA.

Although the human conversion of ALA to DHA and EPA is gender-related and relatively low (up to $4 \%$ ) [41], a higher consumption of ALA related to LA may increase it. Besides, individual DHA and EPA are not easily available and expensive. Moreover, DHA- and EPA-rich fish oil has some health disadvantages [22] due to contaminating factors such as heavy metals, teratogens, and others.

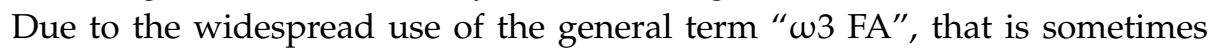
misleading, we propose that scientific publications apply a more precise nomenclature to identify the specific FAs tested (i.e., ALA, DHA, and EPA).

In order to enlarge the understanding of ALA's role in fetal development and programming, we recommend to analyze the effects of a maternal diet enriched in 
each of the individual $\omega 3$ FAs, in simple nutritional animal models that examine the tissue distribution, as well as gene expression and metabolic outcomes, in offspring.

Acknowledgments: Alicia I. Leikin-Frenkel is grateful to Dror Harats, Director of the Bert Strassburger Lipid Center, for providing the infrastructure and conditions for working on this manuscript. She is also grateful to David M. Mutch for carefully reading the manuscript.

Conflicts of Interest: The author declares no conflict of Interest.

\section{Abbreviations}

$\begin{array}{ll}\text { ALA } & \omega 3 \text { alpha linolenic acid } \\ \text { FA } & \text { fatty acid } \\ \text { CVD } & \text { cardiovascular risk disease } \\ \text { docosahexenoic acid } \\ \text { DHA } & \text { docosapentenoic acid } \\ \text { DPA } & \text { essential fatty acid } \\ \text { EFA } & \text { eicosapentenoic acid } \\ \text { EPA } & \text { gene of } \Delta 6 \text { desaturase enzyme } \\ \text { Fads2 } & \text { insulin resistance } \\ \text { IR } & \text { linoleic acid } \\ \text { LA } & \text { low density lipoproteins } \\ \text { LDL } & \text { peroxisome proliferative activated receptor gamma } \\ \text { Ppargc1a/Ppargc1a } & \text { coactivator1a enzyle and gene, respectively } \\ \text { PUFA } & \text { polyunsaturated fatty acid } \\ \text { SCD1/Scd1 } & \text { stearoyl-CoA desaturase } 1 \text { enzyme and gene, respectively } \\ \text { SFA } & \text { saturated fatty acid }\end{array}$

\section{References}

1. Burr, G.O.; Burr, M.M. The nature and role of the fatty acids essential in nutrition. J. Biol. Chem. 1930, 86, 587-621.

2. Sinopolus, A. Genetic variants in the metabolism of omega- 6 and omega- 3 fatty acids: Their role in the determination of nutritional requirements and chronic disease risk. Exp. Biol. Med. 2010, 235, 785-795.

3. Merino, D.M.; Ma, D.W.L.; Mutch, D.M. Genetic variation in lipid desaturases and its impact on the development of human disease. Lipids Health Dis. 2010, 9, 63.

4. Innis, S.M. Essential fatty acid transfer and fetal development. Placenta 2005, 26, 570-575.

5. Hornstra, G. Essential fatty acids in mothers and their neonates. Am. J. Clin. Nutr. 2000, 71, 1262S-1269S.

6. Barker, D.J.P. Fetal origins of coronary heart disease. BJM 1995, 311, 171-174. 
7. Mennitti, L.V.; Oliveira, J.L.; Morais, C.A.; Estadella, D.; Oyama, L.M.; Nascimento, C.M.D.; Pisani, L.P. Type of fatty acids in maternal diets during pregnancy and/or lactation and metabolic consequences of the offspring. J. Nutr. Biochem. 2015, 26, 99-111.

8. Kabaran, S.; TanjuBesler, H.T. Do fatty acids affect fetal programming? J. Health Popul. Nutr. 2015, 33, 14.

9. Alfaradhi, M.; Ozanne, S.E. Developmental programming in response to maternal overnutrition. Front. Genet. 2011, 27, 1-13.

10. Valenzuela, R.; Bascuñán, K.A.; Chamorro, R.; Barrera, C.; Sandoval, J.; Puigrredon, C.; Parraguez, G.; Orellana, P.; Gonzalez, V.; Valenzuela, A. Modification of Docosahexaenoic Acid Composition of Milk from Nursing Women Who Received Alpha Linolenic Acid from Chia Oil during Gestation and Nursing. Nutrients 2015, 7, 6405-6424.

11. Swanson, D.; Block, R.; Mousa, S.A. Omega-3 fatty acids EPA and DHA: Health benefits throughout life. Adv. Nutr. 2012, 3, 1-7.

12. Larque, E.; Gil-Sanchez, A.; Prieto-Sanchez, M.T.; Koletzko, B. Omega 3 fatty acids, gestation and pregnancy outcomes. Br. J. Nutr. 2012, 107, S77-S84.

13. Fonseca, B.M.; Correia-da-Silva, G.; Teixeira, N.A. The rat as animal model for fetoplacental development: A reappraisal of the post-implantation period. Reprod. Biol. 2012, 12, 97-118.

14. Brennan, R.O.; Mulligan, W.C. Nutrigenetics: New Concepts for Relieving Hypoglycemia; M Evans \& Co.: New York, NY, USA, 1975; p. 258.

15. Mutch, D.M.; Wahli, W.; Williamson, G. Nutrigenomics and nutrigenetics: The emerging faces of Nutrition. FASEB. 2005, 19, 1602-1616.

16. Dayton, S.; Pearce, M.L.; Goldman, H. Controlled trial of a diet high in unsaturated fat for prevention of atherosclerotic complications. Lancet 1968, 16, 1060-1062.

17. Lorente-Cebrián, S.; Costa, A.G.; Navas-Carretero, S.; Zabala, M.; Martínez, J.A.; Moreno-Aliaga, M.J. Role of omega-3 fatty acids in obesity, metabolic syndrome, and cardiovascular diseases: A review of the evidence. Physiol. Biochem. 2013, 69, 633-651.

18. Gebauer, S.K.; Psota, T.L.; Harris, W.S.; Kris-Etherton, P.M. n-3 Fatty acid dietary recommendations and food sources to achieve essentiality and cardiovascular benefits. Am. J. Clin. Nutr. 2006, 83, S1526-S1535.

19. Poudyal, H.; Panchal, S.K.; Diwan, V.; Brown, L. Omega-3 fatty acids and metabolic syndrome: Effects and emerging mechanisms of action. Prog. Lipid Res. 2011, 50, 372-387.

20. Abeywardena, M.W.; Patten, G.S. Role of $w 3$ Long chain Polyunsaturated Fatty Acids in Reducing Cardio-Metabolic Risk Factors. Endocr. Metab. Immune Disord. Drug Targets 2011, 11, 232-246.

21. Larsson, S.C.; Orsini, N.; Wolk, A. Long-chain omega-3 polyunsaturated fatty acids and risk of stroke: A meta-analysis. Eur. J. Epidemiol. 2012, 27, 895-901.

22. Kris-Etherton, P.M.; Harris, W.S.; Appel, L.J. Fish Consumption, Fish Oil, Omega-3 Fatty Acids, and Cardiovascular Disease. Circulation 2002, 106, 2747-2757. 
23. Coste, T.C.; Gerbi, A.; Vague, P.; Pieroni, G.; Raccah, D. Neuroprotective Effect of Docosahexaenoic Acid-Enriched Phospholipids in Experimental Diabetic Neuropathy. Diabetes 2003, 52, 2578-2585.

24. Barceló-Coblijn, G.; Murphy, E.J. Alpha-linolenic acid and its conversion to longer chain $n-3$ fatty acids: Benefits for human health and a role in maintaining tissue $n-3$ fatty acid levels. Prog. Lipid Res. 2009, 48, 355-374.

25. Rodriguez-Leyva, D.; Bassett, C.M.C.; McCullough, R.; Pierce, P. The cardiovascular effects of flaxseed and its omega-3 fatty acid, alpha-linolenic acid. Can. J. Cardiol. 2010, 26, 489-496.

26. Baxheinrich, A.; Stratmann, B.; Lee-Barkey, Y.H.; Tschoepe, D.; Wahrburg, U. Effects of an energy-restricted diet rich in plant-derived a-linolenic acid on systemic inflammation and endothelial function in overweight-to-obese patients with metabolic syndrome traits. Br. J. Nutr. 2014, 112, 1315-1322.

27. Egert, S.; Baxheinrich, A.; Lee-Barkey, Y.H.; Tschoepe, D.; Wahrburg, U.; Stratmann, B. Effects of a rapeseed oil-enriched hypoenergetic diet with a high content of $\alpha$-linolenic acid on body weight and cardiovascular risk profile in patients with the metabolic syndrome. Br. J. Nutr. 2012, 108, 682-691.

28. Poudyal, H.; Panchal, S.K.; Ward, L.C.; Brown, L. Effects of ALA, EPA and DHA in high-carbohydrate, high-fat diet-induced metabolic syndrome in rats. J. Nutr. Biochem. 2013, 24, 1041-1052.

29. Commor, W.E.; Bendich, A. Highly unsaturated fatty acids in nutrition and disease prevention. Am. J. Clin. Nutr. 2000, 71 (Suppl. 1), 169S-398S.

30. Hu, F.B.; Stampfer, M.J.; Manson, J.A.E.; Rimm, E.B.; Wolk, A.; Colditz, G.A.; Hennekens, C.H.; Willett, W.C. Dietary intake of a-linolenic acid and risk of fatal ischemic heart disease among women. Am. J. Clin. Nutr. 1999, 69, 890-897.

31. Truong, H.; di Bello, J.R.; Ruiz-Narvaez, E.; Kraft, P.; Campos, H.; Baylin, A. Does genetic variation in the $\Delta 6$-desaturase promoter modify the association between $\alpha$-linolenic acid and the prevalence of metabolic syndrome? Am. J. Clin. Nutr. 2009, 89, 920-925.

32. Fleming, J.A.; Kris-Etherton, P.M. The evidence for $\alpha$-linolenic acid and cardiovascular disease benefits: Comparisons with eicosapentaenoic acid and docosahexaenoic acid. Adv. Nutr. 2014, 5, 863S-876S.

33. Decsi, T.; Koletzko, B. n-3 Fatty acids and pregnancy outcomes. Curr. Opin. Clin. Nutr. Metab. Care 2005, 8, 161-166.

34. Brenna, J.T. Efficiency of conversion of $\alpha$-linolenic acid to long chain $n-3$ fatty acids in man. Curr. Opin. Clin. Nutr. Metab. Care 2002, 5, 127-132.

35. Burdge, G.C. Metabolism of $\alpha$-linolenic acid in humans. Prostaglandins Leukot. Essent. Fat. Acids 2006, 75, 161-168.

36. Mohrhauer, H.; Holman, R.T. Effect of linolenic acid upon the metabolism of linoleic acid. J. Nutr. 1963, 81, 67-74.

37. Brenner, R.R.; Peluffo, R.O. Effect of saturated and unsaturated fatty acids on the desatu-ration in vitro of palmitic, stearic, oleic, linoleic, and linolenic acids. J. Biol. Chem. 1966, 241, 5213-5219. 
38. Brenna, J.T.; Salem, N., Jr.; Sinclair, A.J.; Cunnane, S.C. Alpha-Linolenic acid supplementation and conversion to n-3 long-chain polyunsaturated fatty acids in humans. Prostaglandins Leukot Essent Fatty Acids. 2009, 80, 85-91.

39. Anderson, B.M.; Ma, D.W. Are all n-3 polyunsaturated fatty acids created equal? Lipids Health Dis. 2009, 10, 33.

40. Stoffel, W.; Holz, B.; Jenke, B.; Binczek, E.; Gunter, R.H.; Kiss, C.; Karakesisoglou, I.; Thevis, M.; Weber, A.A.; Arnhold, S.; et al. Delta6-desaturase (FADS2) deficiency unveils the role of omega3- and omega6-polyunsaturated fatty acids. $E M B O$ J. 2008, 27, 2281-2292.

41. Burdge, G. Alpha-linolenic acid metabolism in men and women: Nutritional and biological implications. Curr. Opin. Clin. Nutr. Metab. Care 2004, 7, 137-144.

42. DeLany, J.P.; Windhauser, M.M.; Champagne, C.M.; Bray, G.A. Differential oxidation of individual dietary fatty acids in humans. Am. J. Clin. Nutr. 2000, 72, 905-911.

43. Burdge, G.C.; Jones, A.E.; Wootton, S.A. Eicosapentaenoic and docosapentaenoic acids are the principal products of $\alpha$-linolenic acid metabolism in young men. Br. J. Nutr. 2002, 88, 355-363.

44. Burdge, G.C.; Wootton, S.A. Conversion of $\alpha$-linolenic acid to eicosapentaenoic, docosapentaenoic and docosahexaenoic acids in young women. Br. J. Nutr. 2002, $88,411-420$.

45. Burdge, G.C.; Calder, P.C. Conversion of alpha-linolenic acid to longer-chain polyunsaturated fatty acids in human adults. Reprod. Nutr. Dev. 2005, 45, 581-597.

46. Childs, C.; Kew, E.S.; Finnegan, Y.E.; Minihane, A.M.; Leigh-Firban, E.C.; Williams, C.M.; Calder, P.C. Increased dietary $\alpha$-linolenic acid has sex-specific effects upon eicosapentaenoic acid status in humans: Re-examination of data from a randomized, placebo-controlled, parallel study. Nutr. J. 2014, 13, 113-117.

47. Molto-Puigmarti, C.; Plat, J.; Mensink, R.P.; Muller, A.; Jansen, E.; Zeegers, M.P.; Thijs, C. FADS1 FADS2 gene variants modify the association between fishintake and the docosahexaenoic acid proportions in human milk. Am. J. Clin. Nutr. 2010, 91, 1368-1376.

48. Xie, L.; Innis, S. Genetic variants of the FADS1 FADS2 gene cluster are associated with altered $(n-6)$ and $(n-3)$ essential fatty acids in plasma and erythrocyte phospholipids in women during pregnancy and in breast milk during lactation. J. Nutr. 2008, 138, 2222-2228.

49. Caspi, A.; Williams, B.; Kim-Cohen, J.; Craig, I.W.; Milne, B.J.; Poulton, R.; Schalkwyk, L.C.; Taylor, A.; Werts, H.; Moffitt, T.E. Moderation of breastfeeding effects on the IQ by genetic variation in fatty acid metabolism. Proc. Natl. Acad. Sci. USA 2007, 104, 18860-18865.

50. Burdge, G.; Calder, P.C. Dietary $\alpha$-linolenic acid and health-related outcomes: A metabolic perspective. Nutr. Res. Rev. 2006, 19, 26-52.

51. Arterburn, L.M.; Hall, E.B.; Oken, H. Distribution, interconversion, and dose response of n-3 fatty acids in humans. Am. J. Clin. Nutr. 2006, 83, S1467-1476S. 
52. Niculescu, M.D.; Lupu, D.S.; Craciunescu, C.N. Perinatal manipulation of $\alpha$-linolenic acid intake induces epigenetic changes in maternal and offspring livers. FASEB J. 2013, 27, 350-358.

53. Koren, G. Polyunsaturated fatty acids and fetal brain development Unfulfilled promises. Can. Fam. Phys. 2015, 61, 41-42.

54. Church, M.W.; Jen, K.-L.C.; Anumba, J.I.; Jackson, D.A.; Adams, B.R.; Hotra, J.W. Excess Omega-3 Fatty Acid Consumption by Mothers during Pregnancy and Lactation Caused Shorter Life Span and Abnormal ABRs in Old Adult Offspring. Neurotoxicol. Teratol. 2010, 32, 171-181.

55. Uauy, R.; Mena, P.; Wegher, B.; Nieto, S.; Salem, N. Long chain polyunsaturated fatty acid formation in neonates: Effect of gestational age and intrauterine growth. Pediatr. Res. 2000, 47, 127.

56. Rodriguez, A.; Sarda, P.; Nessmann, C.; Boulot, P.; Poisson, J.P.; Leger, C.L.; Descomps, B. Fatty acid desaturase activities and polyunsaturated fatty acid composition in human liver between the seventeenth and thirty-sixth gestational weeks. Am. J. Obstet. Gynecol. 1998, 179, 1063-1070.

57. Miles, E.A.; Calder, P.C. Omega- 6 and omega-3 polyunsaturated fatty acids and allergic diseases in infancy and childhood. Curr. Pharm. Des. 2014, 20, 946-953.

58. Niculescu, M.D. Alpha-Linolenic Acid Alters Cell Cycle, Apoptosis, and DNA Methyltransferase Expression in Mouse Neural Stem Cells, but Not Global DNA Methylation. J. Hum. Nutr. Food Sci. 2014, 2, 1026.

59. Hollander, K.S.; TempelBrami, C.; Konikoff, F.M.; Fainaru, M.; Leikin-Frenkel, A. Dietary enrichment with alpha-linolenic acid during pregnancy attenuates insulin resistance in adult offspring in mice. Arch. Physiol. Biochem. 2014, 120, 99-111.

60. Shomonov-Wagner, L.; Raz, A.; Leikin-Frenkel, A. Alpha linolenic acid in maternal diet halts the lipid disarray due to saturated fatty acids in the liver of mice offspring at weaning. Lipids Health Dis. 2015, 14, 14.

61. Leikin-Frenkel, A.; Shomonov-Wagner, L.; Juknat, A.; Pasmanik-Chor, M. Maternal Diet Enriched with alpha-Linolenic or Saturated Fatty Acids Differentially Regulates Gene Expression in the Liver of Mouse Offspring. J. Nutrigenet. Nutrigenom. 2015, 8, 185-194.

62. Clarke, S.D. Polyunsaturated Fatty Acid Regulation of Gene Transcription: A Molecular Mechanism to Improve the Metabolic Syndrome. J. Nutr. 2001, 131, 1129-1132.

63. Deckelbaum, R.J.; Worgall, T.S.; Seo, T. n-3 Fatty acids and gene expression. J. Clin. Nutr. 2006, 83 (Suppl. 6), 1520S-1525S.

64. Ahmed, A.A.; Balogun, K.A.; Bykova, N.V.; Cheema, S.K. Novel regulatory roles of omega-3 fatty acids in metabolic pathways: A proteomics approach. Nutr. Metab. 2014, 11,6 .

65. Ong, T.P.; Ozanne, S.E. Developmental programming of type 2 diabetes: Early nutrition and epigenetic mechanisms. Curr. Opin. Clin. Nutr. Metab. Care 2015, 18, 354-360.

66. Waterland, R.A.; Rached, M.-T. Developmental establishment of epigenotype: A role for dietary fatty acids? Scand. J. Food Nutr. 2006, 50 (Suppl. 2), 21-26. 
67. Wang, J.; Wu, Z.; Li, D.; Li, N.; Dindot, S.V.; Satterfield, M.C.; Bazer, F.W.; Wu, G. Nutrition Epigenetics and Metabolic Syndrome. Antioxid. Redox Signal. 2012, 17, $282-301$.

68. Vickers, M.H. Early Life Nutrition, Epigenetics and Programming of Later Life Disease. Nutrients 2004, 6, 2165-2178.

69. Burdge, G.C.; Lillycrop, K.A. Fatty acids and epigenetics. Curr. Opin. Clin. Nutr. Metab. Care 2014, 17, 156-161. 


\title{
Pork as a Source of Omega-3 (n-3) Fatty Acids
}

\author{
Michael E.R. Dugan, Payam Vahmani, Tyler D. Turner, Cletos Mapiye, \\ Manuel Juárez, Nuria Prieto, Angela D. Beaulieu, Ruurd T. Zijlstra, \\ John F. Patience and Jennifer L. Aalhus
}

\begin{abstract}
Pork is the most widely eaten meat in the world, but typical feeding practices give it a high omega- $6(n-6)$ to omega-3 (n-3) fatty acid ratio and make it a poor source of $n-3$ fatty acids. Feeding pigs $n-3$ fatty acids can increase their contents in pork, and in countries where label claims are permitted, claims can be met with limited feeding of $n-3$ fatty acid enrich feedstuffs, provided contributions of both fat and muscle are included in pork servings. Pork enriched with $n-3$ fatty acids is, however, not widely available. Producing and marketing $n-3$ fatty acid enriched pork requires regulatory approval, development costs, quality control costs, may increase production costs, and enriched pork has to be tracked to retail and sold for a premium. Mandatory labelling of the $n-6 / n-3$ ratio and the $n-3$ fatty acid content of pork may help drive production of $n-3$ fatty acid enriched pork, and open the door to population-based disease prevention polices (i.e., food tax to provide incentives to improve production practices). A shift from the status-quo, however, will require stronger signals along the value chain indicating production of $n-3$ fatty acid enriched pork is an industry priority.
\end{abstract}

Reprinted from J. Clin. Med. Cite as: Dugan, M.E.R.; Vahmani, P.; Turner, T.D.; Mapiye, C.; Juárez, M.; Prieto, N.; Beaulieu, A.D.; Zijlstra, R.T.; Patience, J.F.; Aalhus, J.L. Pork as a Source of Omega-3 (n-3) Fatty Acids. J. Clin. Med. 2015, 4, 1999-2011.

\section{Introduction}

Over the last century, changes in agricultural production and patterns of food consumption have led to an increase in the omega-6 (n-6) to omega-3 (n-3) fatty acid ratio in human diets. The imbalance in the $n-6$ to $n-3$ ratio has been associated with numerous diseases, from cardiovascular and inflammatory diseases to diabetes and autoimmune disorders, and led to calls for rebalancing the ratio in the food supply [1]. Pork is the most widely eaten meat world-wide, accounting for $38 \%$ of meat production and over $36 \%$ of meat intake in the world [2]. Enriching pork among other meats represents a viable means to increase $n-3$ fatty acid consumption in humans, while leading to concomitant reductions in fatty acids associated with adverse health outcomes (e.g., saturated fatty acids (SFA)). This may be of particular importance in populations that do not consume fish or marine products, where red 
meat may contribute up to $30 \%$ of dietary long chain $n-3$ fatty acids [3]. The objectives of the present review are to examine where pork fits in the human diet, the fatty acid composition of pork, efforts to enrich pork with $n$ - 3 fatty acids when feeding flaxseed, and examination of practical barriers and possible strategies to help drive $n-3$ pork development and entry into the food supply.

\section{Pork in Human Diets}

The apparent link between plasma cholesterol and cardiovascular disease, and its association with SFA intake, led to recommendations to reduce the intake of SFA rich foods including red meat [4]. In response, swine industries in several countries adopted feeding and breeding strategies to reduce the fat content of pork. Results of these efforts were dramatic, leading to as little as $0.8 \%-1 \%$ intramuscular (marbling) fat, and increasing to a minimum of $1.5 \%$ marbling fat has since been recommended to ensure palatability [5]. Even at 2\% marbling fat, lean pork contains 120-130 calories per $100 \mathrm{~g}$ serving, and only $15 \%$ of the calories are from fat. It would take pork marbling levels of $3 \%-6 \%$ to fall into the maximum recommended range of fat intakes for humans (20\%-35\% of dietary energy, Figure 1) [6]. In addition, if retail pork contains $2 \%$ intramuscular fat, the fat typically contains $\sim 35 \%-40 \%$ SFA, equaling $5 \%-6 \%$ of total energy, which is again less than the maximum recommendation of $10 \%$ [6]. In light of these facts, and results of a meta-analyses of observational and epidemiological studies indicating SFA intake is not associated with cardiovascular disease risk $[7,8]$, recommendations to substitute other protein sources for lean red meat in the human diet have been questioned $[9,10]$. On the other hand, even though the low fat and high protein contents of lean pork make it an excellent whole food for inclusion in human diets, complete pork carcasses contain $\sim 47 \%$ fat [11], and this fat enters the food supply in the form of sausages, bacon, processed meats, and as lard in baked goods.

Common indicators of the healthfulness of fatty acid profiles (polyunsaturated fatty acid (PUFA)/SFA, and $n-6 / n-3$ ratios) indicate there may be existing opportunities for their rebalancing in pork. In a survey of retail pork in England, the PUFA/SFA ratio of lean pork was 0.58 [12], which is greater than the 0.4 minimum recommended by the UK Department of Health [13], but an $n-6 / n-3$ ratio of 7.2:1 was found, exceeding the recommended ratio of $4: 1$ [13]. Swine diets used in commercial intensive production are typically grain based, with the type of grain fed dependent on local availability and economics. Swine in the US and Canada are typically fed diets based on corn or small grains (barley/wheat). Juárez et al. [14] fed pigs on a barley/wheat/soybean based diet for 11 weeks prior to market and found a longissimus muscle (i.e., the largest steak/chop muscle running the length of the spine) PUFA/SFA ratio of 0.32 and a $n-6 / n-3$ ratio of 4.5:1. Romans et al. [15] finished pigs using a typical corn/soybean based diet and found a longissimus muscle PUFA/SFA 
ratio of 0.24 and an $n-6 / n-3$ ratio of 14.3:1. Thus, although lean pork has a desirable nutrient profile with high protein and low fat, there may still be some health benefit to adjusting the fatty acid composition (i.e., reducing the $n-6 / n-3$ ratio and increasing the PUFA/SFA ratio).

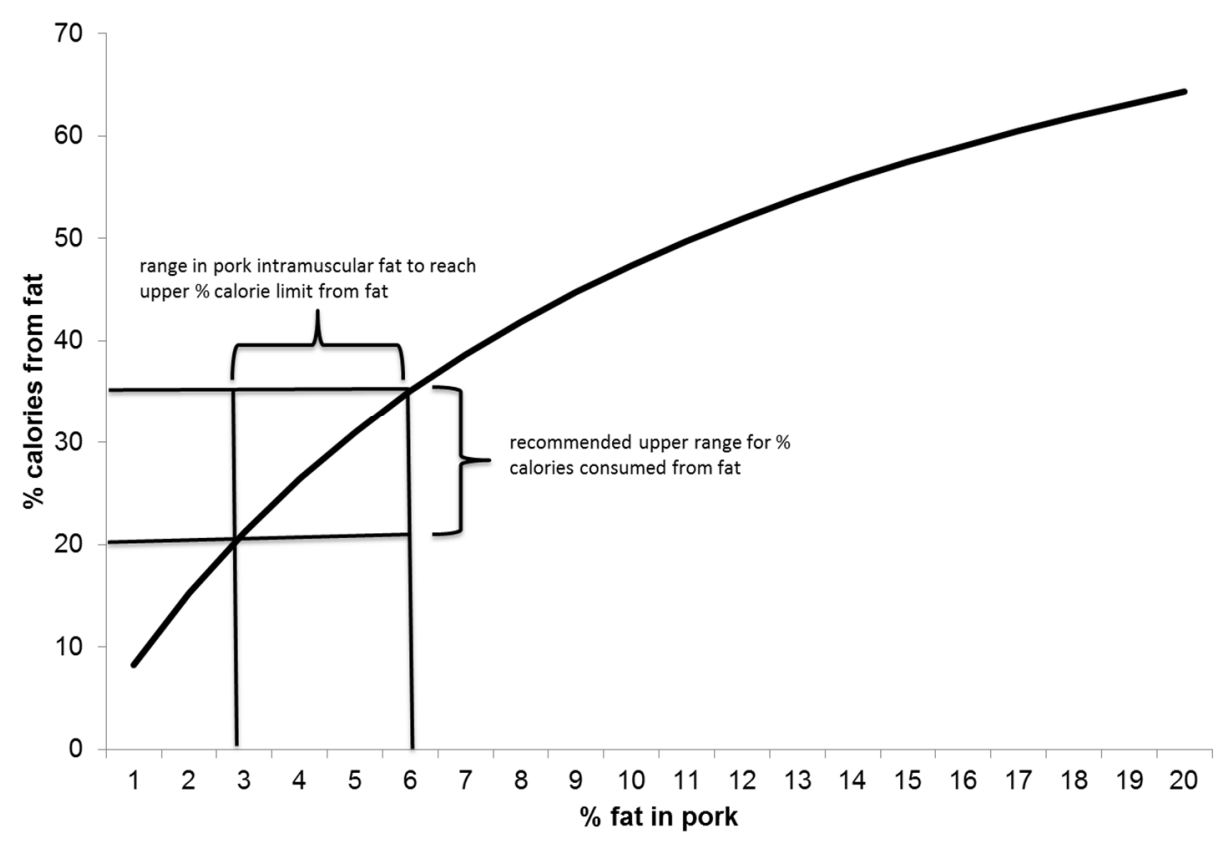

Figure 1. Calories in pork fat relative to recommendations for fat consumption.

\section{Pork Fatty Acid Composition}

Lean pork contains a fairly constant proportion of cell membrane phospholipids, which are relatively rich in PUFA [16]. Lean pork also contains variable amounts of neutral lipid, composed mainly of SFA-rich triacyglycerol. The fatty acid composition of pork can thus be improved by reducing its total fat content, but if the fat content is too low, it can lead to palatability issues [17]. The composition of pork can also be influenced by diet, and diet adjustments can be used to improve pork fatty acid profiles without reducing total fat content $[18,19]$. Opportunities to use diet to change the fatty acid composition of meat are species specific, with major differences found between monogastric and ruminant livestock [20]. Pigs are monogastrics and categorized as homolipoid organisms [21], meaning their fatty acid composition closely reflects the fatty acid composition of their diet. In contrast to ruminant animals, in pigs, fatty acids are not metabolized to any great extent by microbes in the digestive tract prior to lipid digestion and absorption [20]. This makes pork a good candidate for enrichment with $n$-3 fatty acids. 
Contrary to popular assumption, fat in red meats, including pork, is not solely composed of SFA (Table 1). When Juárez et al. [14] and Turner et al. [22] fed pigs a barley/wheat/soybean meal based diet, the longissimus muscle (denuded of epimysium and closely associated adipose tissue) was found to contain $2.9 \%$ total fat, and the fat contained 39\% SFA, 47\% monounsaturated fatty acids (MUFA), $11.5 \%$ PUFA, and the PUFA were comprised of $9.4 \% n-6$ and $2.0 \% n-3$ fatty acids. Within the $n-3$ fatty acids, $\alpha$-linolenic acid (LNA, C18:3n-3) was the most concentrated at $22.7 \mathrm{mg} / 100 \mathrm{~g}$ of fresh tissue, and the most abundant long chain (LC) $n-3$ fatty acid was docosapentaenoic acid (DPA, C22:5n-3) at $11 \mathrm{mg} / 100 \mathrm{~g}$ of fresh tissue. The LC $n-3$ fatty acids most widely studied for their health promoting properties include eicosapentaenoic acid (EPA, C20:5n-3) and docosahexaenoic acid (DHA, C22:6n-3) [23,24], and are concentrated in oily fish and marine products (e.g., microalgae). On the other hand, DPA is an intermediate in the pathway during DHA synthesis from EPA (Figure 2), and it is the most abundant LC $n-3$ fatty acid in meat and adipose tissue of terrestrial animals [25]. Docosapentaenoic acid which is freely converted between EPA and DHA, may have beneficial health effects on its own [26]. Consequently, when considering $n-3$ fatty acid nutrition, particularly in populations where oily fish or other LC $n-3$ fatty acid enriched marine products are not consumed, contributions of DPA made by terrestrial animals should be taken into consideration [27].

Table 1. Typical fatty acid composition of longissimus muscle and associated tissues in pork from pigs fed a barley/wheat/soybean meal diet.

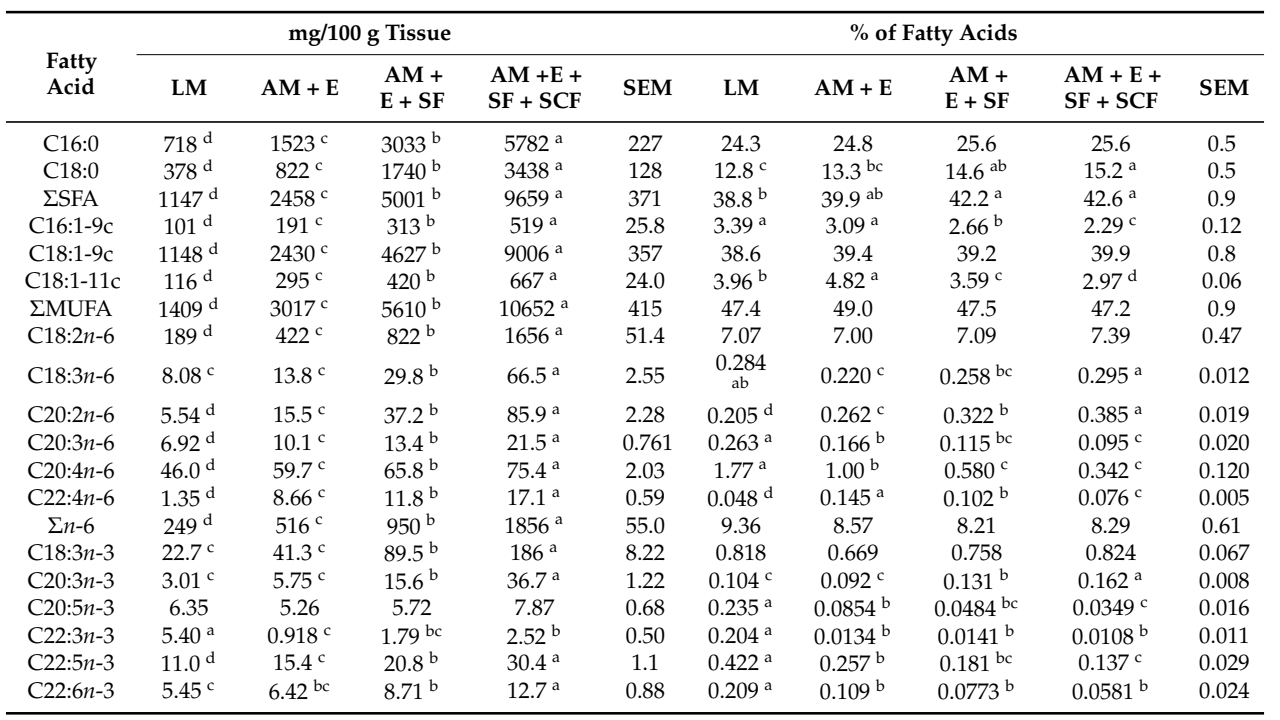


Table 1. Cont.

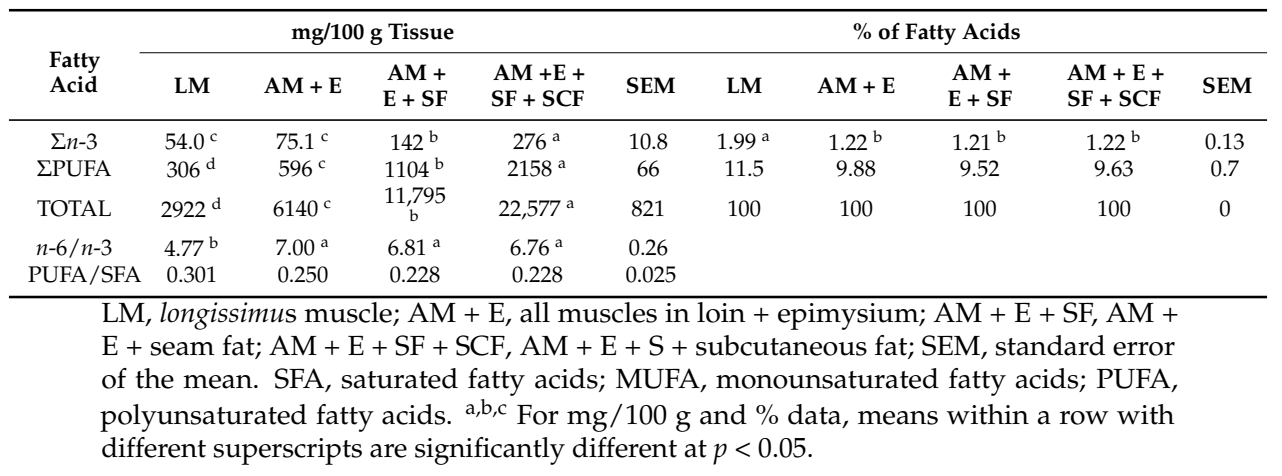

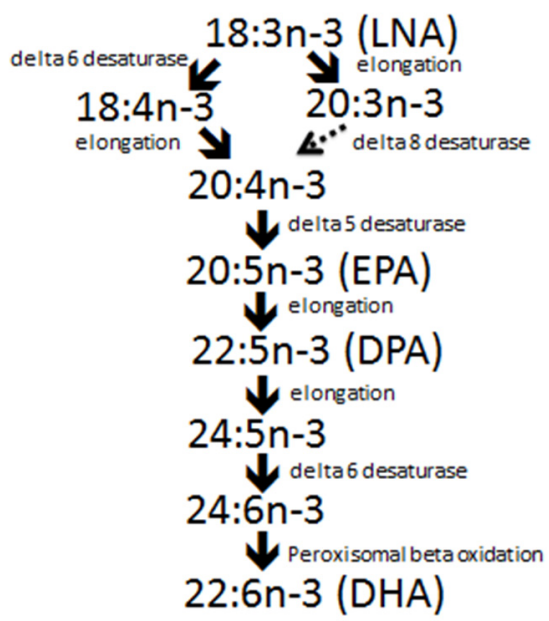

Figure 2. Pathways for $n-3$ fatty acid synthesis. LNA, alpha-linolenic acid; EPA, eicosapentaenoic acid; DPA, docosapentaenoic acid; DHA, docosahexaenoic acid.

The fatty acid composition of pig longissimus is the muscle most reported in the literature. Scientifically it is expedient to report fatty acid compositions on pure muscle, but when examining servings of pork, it is important to consider contributions made by various tissues. When Juárez et al. [14] and Turner et al. [22] combined longissimus and other muscles in the primal loin cut, including epimysium and closely associated adipose tissues, the fat content was $6.1 \%$ (i.e., increased from $2.9 \%$ fat in pure longissimus muscle). When intermuscular (seam) fat was further included, the fat content of pork increased to $11.8 \%$, and when $5 \mathrm{~mm}$ of overlying backfat was added to complete a commercially trimmed pork chop, the total fat content increased to $22.6 \%$. The addition of fatty tissues to pure muscle also increased 
the percentage of SFA in total fat, and increased the $n-6 / n-3$ ratio. In addition, when fatty tissues were included with pure muscle, LNA remained as the most abundant $n-3$ fatty acid, but the second most abundant $n-3$ fatty acid changed from DPA to eicosatrienoic acid (ETA, C20:3n-3). Despite the reduction in the \%PUFA in the fat because the total fat increased, the actual amount of PUFA on a mg/100 $\mathrm{g}$ of fresh tissue basis increased from $306 \mathrm{mg}$ to $2158 \mathrm{mg}$, and the $n$ - 3 fatty acids increase from 54 to $276 \mathrm{mg}$ per $100 \mathrm{~g}$. Knowing the content of $n-3$ fatty acids in various cuts of pork is therefore of considerable importance as these help define the pig feeding practices required to meet label (enrichment) claims for $n-3$ fatty acids. For example, in Canada to make a retail label claim for $n-3$ fatty acid enrichment, a serving of food must contain at least $300 \mathrm{mg} n-3$ fatty acids [28]. If the serving only contains longissimus muscle, the $n-3$ fatty acid content would need to be increased 5-6 fold to reach enrichment status. On the other hand, a commercial loin chop (all muscles, seam and $5 \mathrm{~mm}$ of back fat) would likely need limited $n-3$ fatty acid supplementation in pig diets to reach $300 \mathrm{mg} \mathrm{n-3}$ fatty acids per serving. Similarly, other higher fat cuts such as back or side ribs and bacon could also meet this label claim with limited supplementation.

As previously mentioned, reducing the marbling fat content of lean pork improves the fatty acid composition by increasing the proportion of PUFA and the PUFA/SFA ratio. If extra-muscular fatty tissues are then added to pork cuts, the proportion of SFA goes up (Table 1). As illustrated in Figure 3, however, when extra-muscular sources of fatty tissue are included in pork cuts, changes in fatty acid composition are not striking. This is because, even when as little as $2.9 \%$ intramuscular fat is present, the content of SFA rich triacylglycerols are still enough to overwhelm the contribution made by PUFA rich phospholipids. In practical terms, the fatty acid profile of pork with enough intramuscular fat to ensure palatability is not very different from higher fat products such as pork sausage. The difference in healthfulness is more related to the total fat content rather than the fatty acid profile. It is also worth re-emphasizing that the most abundant class of fatty acids in pork are MUFA, and these are the main fatty acids in the heart healthy Mediterranean diet [29]. Moreover, approximately $\sim 25 \%$ of the SFA in pork is stearic acid (C18:0), which is noted to have neutral effects on plasma cholesterol [30]. 


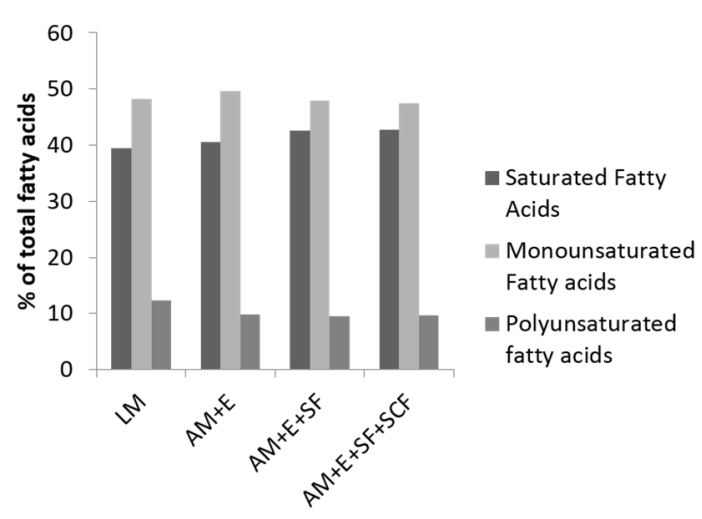

Figure 3. The percentage of saturated fatty acid (SFA), monounsaturated fatty acid (MUFA), and polyunsaturated fatty acid (PUFA) in total fatty acids in cuts of pork loin (LM, longissimus muscle; $\mathrm{AM}+\mathrm{E}$ all loin muscles + epimysium; $\mathrm{AM}+\mathrm{E}+$ seam fat; $\mathrm{AM}+\mathrm{E}+\mathrm{SF}+$ subcutaneous fat).

\section{Enriching Pork with $n$-3 Fatty Acids}

\subsection{Initial Efforts to Improve Pork Fatty Acid Composition}

The idea of modifying the fatty acid composition of pork to make it more healthful is not new. To improve the healthfulness of pork fatty acid profiles, Koch et al. [31] fed pigs safflower oil and achieved large increases in pork PUFA in the form of linoleic acid (LA, C18:2n-6). Stewart et al. [32] also fed a diet rich in LA, and achieved increased PUFA levels in pork and lard. When the LA enriched pork and foods made with LA enriched lard were fed to young women (aged 19-24) they significantly lowered total plasma and low-density lipoprotein (LDL) cholesterol, and increased PUFA and reduced SFA and MUFA in plasma lipids and erythrocytes. The pig diet, however, had $n-6 / n-3$ fatty acid ratios of $\sim 10: 1$, and with today's understanding that lower ratios may impart even further benefits, there have been a number of studies conducted attempting to increase pork PUFA by increasing the $n-3$ fatty acid content.

\subsection{Efforts to Increase n-3 Fatty Acids in Pork}

The ability to substantially alter the $n-3$ fatty acid content of pork was demonstrated in 1972 by Anderson et al. [33] when studying LNA turnover. Feeding $20 \%$ flaxseed oil for two months to six month old pigs increased fat depot concentrations of LNA from $1 \%$ to $15 \%$. Given the understanding that increasing the $n-3$ fatty acid content of pork may benefit consumers, and that feeding flaxseed instead of extracted flaxseed oil may reduce input costs, Cunnane et al. [34] fed 5\% ground flaxseed to weaned pigs for 8 weeks and found several fold increases in 
LNA and its elongation and desaturation products in a number of tissues. Since this time, a number of studies have been conducted feeding flaxseed, flaxseed oil or sources of LC n-3 fatty acids (i.e., fish meal, fish oil, and marine algae) and results have been extensively reviewed [16,18,19,35,36]. Cherian et al. [37], Romans et al. [15], Riley et al. [38] and Ahn et al. [39] conducted intensive studies on the effects of feeding 0 to $\sim 15 \%$ flaxseed on pork quality and fatty acid composition, Romans et al. [40] and Juárez et al. [41] investigated feeding flaxseed for different durations, and Fontanillas et al. [42] and Huang et al. [43] investigated the evolution of $n-3$ fatty acids in pig tissues over the feeding period. Maximum levels of $n-3$ fatty acid deposition were comparable with enrichments achieved by Anderson et al. [33] when feeding flaxseed oil. To date, most studies feeding higher levels of flaxseed or flaxseed oil have found increased tissue contents of LNA, EPA and DPA, but not DHA. For example, Martínez-Ramírez et al. [44] found no increase in DHA when feeding pigs diets containing $7 \%-10 \%$ flaxseed, but interestingly, the amounts of other LC n-3 fatty acids deposited in pork were independent of whether the flaxseed was fed early or late in the feeding period. When feeding a limited amount of flaxseed $(\sim 2 \%)$, however, Enser et al. [45] found a reduction in the $n-6 / n-3$ ratio from 9:1 to 5:1 and an increase in DHA. The pathway for $n-3$ fatty acid synthesis from LNA to DHA requires delta-6 desaturation in two places (Figure 2), and the increase in DHA was attributed to the low level of flaxseed supplementation. This means enough LNA was supplied to provide substrate for delta- 6 desaturation to C18:4n-3, but not enough to out complete for delta- 6 desaturase activity later in the pathway (i.e., conversion of C24:5n-3 to C24:6n-3). Overall, feeding sources of $n-3$ fatty acids to pigs increased their content in pork, but results have been variable, and the differences attributed to the source, amount and type of $n-3$ fatty acids fed, the duration of feeding, the type of feed processing, the weight or age of pigs fed and their gender. Nevertheless, Nguyen et al. [46] found the mathematical relationship between the amount of LNA fed and deposited in pork was strong within a study $\left(R^{2}=0.98\right)$, but lower when data from several studies are incorporated into a regression $\left(R^{2}=0.68\right)$. Consequently, producers seeking to develop a strategy to produce $n-3$ enriched pork will likely be able to achieve consistency, but a standardized feeding program will be required based on in-house development rather than solely on strategies reported in the literature.

\subsection{An Example of n-3 Enriched Pork and Post-Production Considerations}

Results from Juárez et al. [14] and Turner et al. [22] provide an example of $n-3$ fatty acid enrichments in pork that might be attained when feeding optimally processed flaxseed to pigs, and factors that need to be considered when developing feeding strategies to meet $n-3$ fatty acid enrichment goals (Table 2). Pigs were fed a diet containing 10\% flaxseed co-extruded 50:50 with field peas to optimize LNA 
digestibility [47]. The diet was fed from 48 to $121 \mathrm{~kg}$ body weight over an 11 week period resulting in increased LNA and total $n-3$ fatty acids from 0.82 and $1.99 \%$ of total longissimus muscle fatty acids to 5.76 and $8.94 \%$ respectively. This translated into an increase of LNA and total $n-3$ fatty acids from 22.7 and $54.0 \mathrm{mg}$ per $100 \mathrm{~g}$ serving of pork to 145 and $217 \mathrm{mg}$ respectively. These amounts of $n-3$ fatty acids would, however, not qualify for a source claim in a number of countries including Canada and the European Union, but may have potential in the United States. As mentioned previously, to be labelled as a source of $n-3$ fatty acids in Canada, a serving of food serving has to have $300 \mathrm{mg}$ of total $n-3$ fatty acids [28]. In the USA, food servings with $\geqslant 160 \mathrm{mg}$ or $\geqslant 320 \mathrm{mg}$ LNA can be referred to as a "source" or "rich" in LNA respectively, and claims cannot be made for EPA and DHA [48]. In the European Union, foods with $300 \mathrm{mg}$ LNA or $40 \mathrm{mg}$ combined EPA and DHA per serving can be labeled as a source of $n-3$ fatty acids, and foods with $600 \mathrm{mg}$ LNA or $80 \mathrm{mg}$ combined EPA and DHA can be labeled as rich in $n-3$ fatty acids [49]. Source claims in all countries would, however, be possible when all muscles and fatty tissues were included in a retail pork chop. In fact, the amount of $n-3$ fatty acids in commercially trimmed pork chops was 10 times more than required for a source claim in Canada (3360 mg per $100 \mathrm{~g}$ serving) [22]. A source claim for combined EPA and DHA could also be made in the European Union $(71.5 \mathrm{mg})$, but could only be considered a rich source if DPA was included in LC $n-3$ fatty acids (174 mg). The ability to make a source claim is, therefore, dependent on the country, what tissues are included in a serving of pork, the type of cut and the $n-3$ fatty acids considered to be LC. Consideration should, however, also be given to what consumers actually eat, as often some external fat may be trimmed before consumption. Therefore, ensuring consistent $n-3$ fatty acid enrichment and consumption might be most easily attained through development of further processed $n-3$ enriched pork products (e.g., sausages) and secondary products prepared with enriched lard (e.g., baked goods). Notably, when fatty tissues are added to pure muscle, the second most abundant $n-3$ fatty acid in pork changes from DPA to ETA (up to $381 \mathrm{mg}$ per $100 \mathrm{~g}$ serving), and ETA has been shown to have a photo-protective effect in human skin [50]. There is also some limited evidence for delta-8 desaturase activity [51], converting ETA to C20:4n-3 in liver, which may gain importance in LC $n-3$ fatty acid synthesis as ETA concentrations increase (Figure 2). 
Table 2. The fatty acid composition of longissimus muscle and associated tissues in pork from pigs fed a diet supplemented with $10 \%$ flaxseed.

\begin{tabular}{|c|c|c|c|c|c|c|c|c|c|c|}
\hline \multirow[b]{2}{*}{$\begin{array}{l}\text { Fatty } \\
\text { Acid }\end{array}$} & \multicolumn{4}{|c|}{ mg/100 g Tissue } & \multicolumn{6}{|c|}{$\%$ of Fatty Acids } \\
\hline & LM & $\mathbf{A M}+\mathbf{E}$ & $\begin{array}{c}\mathrm{AM}+ \\
\mathrm{E}+\mathrm{SF}\end{array}$ & $\begin{array}{c}\mathrm{AM}+ \\
\mathrm{E}+\mathrm{SF}+ \\
\mathrm{SCF}\end{array}$ & SEM & LM & $\mathrm{AM}+\mathrm{E}$ & $\underset{\mathrm{E}+\mathrm{SF}}{\mathrm{AM}+}$ & $\begin{array}{c}\mathrm{AM}+ \\
\mathrm{E}+\mathrm{SF}+ \\
\mathrm{SCF}\end{array}$ & SEM \\
\hline C16:0 & $571^{d}$ & $1180^{c}$ & $2227^{b}$ & $4169^{a}$ & 152 & $22.1^{\mathrm{a}}$ & $20.1^{b}$ & $20.5^{b}$ & $19.8^{b}$ & 0.3 \\
\hline C18:0 & $318^{d}$ & $678^{c}$ & $1326^{\mathrm{b}}$ & $2545^{a}$ & 102 & 12.4 & 11.7 & 12.2 & 12.1 & 0.3 \\
\hline$\Sigma S F A$ & $931^{d}$ & $1953^{c}$ & $3731^{b}$ & $7040^{a}$ & 265 & $36.0^{\mathrm{a}}$ & $33.4^{\mathrm{b}}$ & $34.3^{b}$ & $33.5^{b}$ & 0.6 \\
\hline C16:1-9c & $66.6^{d}$ & $113^{c}$ & $177^{\mathrm{b}}$ & $295^{a}$ & 12.0 & $2.59^{\mathrm{a}}$ & $1.94^{\mathrm{b}}$ & $1.68^{b c}$ & $1.41^{\mathrm{c}}$ & 0.10 \\
\hline C18:1-9c & $873^{d}$ & $1871^{\mathrm{c}}$ & $3380^{b}$ & $6727^{a}$ & 218 & $33.8^{\mathrm{a}}$ & $32.2^{b}$ & $31.7^{\mathrm{b}}$ & $32.2^{b}$ & 0.5 \\
\hline C18:1-11c & $76.7^{\mathrm{d}}$ & $196^{c}$ & $265^{b}$ & $419^{a}$ & 14.3 & $3.06^{b}$ & $3.39^{\mathrm{a}}$ & $2.53^{c}$ & $2.01^{\mathrm{d}}$ & 0.08 \\
\hline इMUFA & $1045^{\mathrm{d}}$ & $2259^{c}$ & $3991^{b}$ & $7724^{a}$ & 256 & $40.7^{\mathrm{a}}$ & $38.9^{\mathrm{ab}}$ & $37.4 \mathrm{bc}$ & $36.9^{c}$ & 0.6 \\
\hline $\mathrm{C} 18: 2 n-6$ & $225^{d}$ & $652^{c}$ & $1207^{\mathrm{b}}$ & $2470^{a}$ & 55.0 & $9.68^{b}$ & $11.4^{\mathrm{a}}$ & $11.5^{\mathrm{a}}$ & $11.9^{\mathrm{a}}$ & 0.34 \\
\hline $\mathrm{C} 18: 3 n-6$ & $6.38^{\mathrm{d}}$ & $12.8^{c}$ & $25.1^{b}$ & $53.0^{\mathrm{a}}$ & 1.54 & 0.26 & 0.223 & 0.236 & 0.253 & 0.011 \\
\hline C20:2n-6 & $6.40^{\mathrm{d}}$ & $24.7^{c}$ & $47.1^{\mathrm{b}}$ & $106^{\mathrm{a}}$ & 2.75 & $0.27^{b}$ & $0.437^{\mathrm{a}}$ & $0.454^{\mathrm{a}}$ & $0.509^{a}$ & 0.025 \\
\hline C20:3n-6 & $5.86^{\mathrm{c}}$ & $7.83^{b c}$ & $10.0^{\mathrm{b}}$ & $15.9^{a}$ & 0.79 & $0.26^{\mathrm{a}}$ & $0.141^{b}$ & $0.099^{c}$ & $0.076^{c}$ & 0.012 \\
\hline C20:4n-6 & $28.2^{c}$ & $34.3^{b}$ & $36.6^{b}$ & $42.3^{a}$ & 1.9 & $1.35^{\mathrm{a}}$ & $0.626^{b}$ & $0.372^{c}$ & $0.205^{c}$ & 0.06 \\
\hline $\mathrm{C} 22: 4 n-6$ & $1.23^{c}$ & $3.87^{b}$ & $5.27^{b}$ & $7.95^{a}$ & 0.56 & $\underset{\mathrm{ab}}{0.05}$ & $0.067^{\mathrm{a}}$ & $0.049^{a b}$ & $0.037^{b}$ & 0.007 \\
\hline$\Sigma n-6$ & $266^{d}$ & $723^{c}$ & $1306^{\mathrm{b}}$ & $2643^{a}$ & 59 & 11.6 & 12.7 & 12.5 & 12.7 & 0.4 \\
\hline $\mathrm{C} 18: 3 n-3$ & $145^{\mathrm{d}}$ & $614^{\mathrm{c}}$ & $1290^{\mathrm{b}}$ & $2800^{a}$ & 71 & $5.76^{\mathrm{d}}$ & $10.6^{c}$ & $12.1^{\mathrm{b}}$ & $13.4^{\mathrm{a}}$ & 0.30 \\
\hline $\mathrm{C} 20: 3 n-3$ & $21.5^{\mathrm{d}}$ & $82.2^{c}$ & $166^{\mathrm{b}}$ & $381^{a}$ & 11.6 & $0.85^{c}$ & $1.42^{b}$ & $1.55^{\mathrm{b}}$ & $1.82^{\mathrm{a}}$ & 0.053 \\
\hline $\mathrm{C} 20: 5 n-3$ & $23.3^{d}$ & $31.9^{c}$ & $38.8^{\mathrm{b}}$ & $55.3^{a}$ & 1.7 & $1.08^{\mathrm{a}}$ & $0.573^{b}$ & $0.385^{c}$ & $0.268^{c}$ & 0.05 \\
\hline $\mathrm{C} 22: 3 n-3$ & $2.48^{b}$ & $2.27^{b}$ & $3.06^{b}$ & $4.75^{\mathrm{a}}$ & 0.40 & $0.10^{\mathrm{a}}$ & $0.038^{\mathrm{b}}$ & $0.029^{b}$ & $0.023^{b}$ & 0.005 \\
\hline $\mathrm{C} 22: 5 n-3$ & $21.1^{\mathrm{d}}$ & $39.5^{\mathrm{c}}$ & $59.7^{b}$ & $102^{a}$ & 3.0 & $0.95^{\mathrm{a}}$ & $0.705^{\mathrm{b}}$ & $0.575^{c}$ & $0.491^{\mathrm{c}}$ & 0.038 \\
\hline $\mathrm{C} 22: 6 n-3$ & $3.85^{\mathrm{d}}$ & $8.13^{c}$ & $10.6^{b}$ & $16.2^{\mathrm{a}}$ & 0.77 & $0.18^{a}$ & $0.146^{\mathrm{b}}$ & $0.105^{c}$ & $0.079^{c}$ & 0.011 \\
\hline$\Sigma n-3$ & $217^{\mathrm{d}}$ & $778^{c}$ & $1569^{b}$ & $3360^{a}$ & 85 & $8.94^{\mathrm{d}}$ & $13.5^{\mathrm{c}}$ & $14.7^{\mathrm{b}}$ & $16.1^{\mathrm{a}}$ & 0.39 \\
\hline$\Sigma \overline{P U F A}$ & $486^{d}$ & $1506^{c}$ & $2883^{b}$ & $6018^{a}$ & 143 & $20.6^{c}$ & $26.3^{b}$ & $27.4^{\mathrm{ab}}$ & $28.9^{a}$ & 0.7 \\
\hline TOTAL & $2519^{d}$ & $5788^{c}$ & $10,683^{b}$ & $20,881^{a}$ & 627 & 100 & 100 & 100 & 100 & 0 \\
\hline$n-6 / n-3$ & $1.28^{\mathrm{a}}$ & $0.935^{b}$ & $0.850^{\mathrm{c}}$ & $0.788^{c}$ & & & & & & \\
\hline PUFA/SFA & $0.581^{b}$ & $0.796^{\mathrm{a}}$ & $0.807^{\mathrm{a}}$ & $0.869^{\mathrm{a}}$ & & & & & & \\
\hline
\end{tabular}

$\mathrm{LM}$, longissimus muscle; $\mathrm{AM}+\mathrm{E}$, all muscles in loin + epimysium; $\mathrm{AM}+\mathrm{E}+\mathrm{SF}, \mathrm{AM}+\mathrm{E}+$ seam fat; $A M+E+S F+S C F, A M+E+S+$ subcutaneous fat; SEM, standard error of the mean. SFA, saturated fatty acids; MUFA, monounsaturated fatty acids; PUFA, polyunsaturated fatty acids. ${ }^{a, b, c}$ For $\mathrm{mg} / 100 \mathrm{~g}$ and \% data, means within a row with different superscripts are significantly different at $p<0.05$.

Currently, production of pork enriched with $n-3$ fatty acids is possible, but it is not clear which $n-3$ fatty acid should be enriched, to what extent they should be enriched and in what tissues. Feeding pigs a limited amount of flaxseed can rebalance the $n-6 / n-3$ ratio in pork [45], but a healthier $n-6 / n-3$ ratio is not something that can be advertised or put on a label in most countries. Feeding increased amounts of flaxseed can yield pork that can be labelled as a source, or rich source of $n-3$ fatty acids, but if consumers trim visible fat or the fat is lost during cooking, purchased pork may differ from pork consumed. Even when pork is enriched with enough $n-3$ fatty acids to allow for a source claim, beneficial effects of consuming such pork have not been extensively investigated. Coats et al. [52] found regular consumption of pork enriched with LC $n-3$ fatty acids by feeding fish meal increased erythrocyte DHA by $15 \%$, and compared to a control group, serum triacylglycerol decreased to a greater extent and thromboxane production increased to a lesser extent. Using 
a rabbit model, Vossen et al. [53] fed pork enriched with LNA or LNA plus LC n-3, and found only pork enriched with LNA plus LC n-3 fatty acids reduced the total plasma cholesterol to high-density lipoprotein cholesterol (HDL-C) ratio. Clearly, it would be of benefit to conduct additional clinical trials to establish the health effects of consuming commercial pork compared to pork enriched with $n-3$ fatty acids, and factors considered should include the $n-6 / n-3$ ratio, the amount and composition of $n-3$ fatty acids and the overall fat content of the pork in different meat cuts.

\section{Practical Barriers Limiting $n-3$ Pork Development and Entry into the Food Supply}

\subsection{The Call for $n-3$ Enriched Meat Unfulfilled to Date}

Simopoulos [54] indicated that it is essential in the process of returning the $n-3$ fatty acids into the food supply, that the balance of $n-6 / n-3$ fatty acids in the diet that existed during human evolution is maintained. To date fish-meal, flaxseed, and marine algae in poultry feeds have increased the $n-3$ fatty acid content of egg yolks and led to the supply of $n-3$ fatty acid-enriched eggs in the marketplace [55]. Simopoulos [54] noted research on the production of $n-3$ fatty acid-enriched products from poultry, beef, lamb, pork, milk and bakery products was ongoing. Taking advancements in the ability to enrich animal products with $n-3$ fatty acids into account, Givens and Gibbs [56] estimated potential dietary intakes of EPA+DHA from foods derived from animals fed enriched diets would be $\sim 231 \mathrm{mg} /$ day, which would double current intakes in the UK, and help meet the recommend intake of $450 \mathrm{mg} /$ day. Currently, however, in most countries, despite the technological capability to do so, the availability of $n-3$ enriched meats, including pork, is not widespread.

\subsection{Why Production of n-3 Enriched Pork Has Not Been Adopted}

\subsubsection{Visibility}

When the SFA content of red meat was associated with increased plasma cholesterol and cardiovascular disease in the 1960s, consumers could visually identify and select meats with lower fat contents, and animal producers selected and fed animals to meet consumer demands for leaner meat. In developed countries, sweeping changes in pork production took place within a value chain geared to produce commodity pork for mass markets. The cost of pork production and the retail price of pork were not increased due to changes in production strategies. Currently, even though the production of $n-3$ fatty acid enriched meats, including pork, has been encouraged, changing fat composition in retail pork has not been as successful as previous efforts to reduce the total fat content of pork. As opposed to the total fat content, the fatty acid composition of retail pork is not visible to consumers. 


\subsubsection{Challenges along the Value Chain}

There are several challenges to producing $n-3$ enriched pork along the value chain. Increasing the $n-3$ fatty acid content of pork can lead to increased input costs depending on feedstuffs available and requirements for processing. Entry into the $n-3$ pork market may also have lagged because feeding high levels of $n-3$ fatty acids can lead to fat softness and palatability problems [14]. Effects on freshly cooked pork chops have, however, been limited [14,39,57] and most negative effects have been found in cooked/reheated pork chops and freshly cooked ground pork with excessive $n-3$ fatty acid enrichments. Once $n-3$ fatty acid enriched pork is produced, it also requires vertical integration from production to retail, along with differentiated marketing and higher prices to cover input costs, distribution costs and profits for producers. Producing n-3 fatty acid enriched pork to meet source claims also requires regulatory approval for package labelling, defining what will be included in portions, what production strategies are needed to meet enrichment requirements, and also added costs for fatty acid analysis of feeds and pork during product development and for quality control. Strategies to drive an industry-wide shift towards $n-3$ fatty acid enriched pork must, therefore, be developed if a clear goal for producers is widespread production and marketing of $n-3$ enriched pork.

\section{Strategies to Encourage Production and Market Availability of $n-3$ Fatty Acid Enriched Pork}

Strategies to encourage production and market availability of $n-3$ fatty acid enriched pork will likely require concerted efforts along the value chain. Producer entry into the $n-3$ fatty acid enriched pork market may be enhanced with the understanding that only limited supplementation of $n-3$ fatty acids in diets is required to meet label claims when contributions of all tissues in a serving are included [22]. This also opens possibilities for feeding oils or oilseeds that may not be as highly enriched with LNA as flaxseed (e.g., whole canola or canola oil). An industry-wide shift in pork production practices might also be driven by mandatory labelling of $n-3$ fatty acids and the $n-6 / n-3$ ratio in meat, making these visible to consumers. When consumers know the $n-3$ fatty acid and $n-6 / n-3$ ratio in foods, it provides the opportunity to select more healthful foods, and impetus to the industry to find lower cost production strategies. In this way, pork may not have to reach specified amounts of $n-3$ fatty acids to meet regulatory approval as a source of $n-3$ fatty acids, but could contribute a greater quantity of $n-3$ fatty acids to the human diet, and at the very least, not further imbalance in the $n-6 / n-3$ ratio. Analyzing the fatty acid composition of pork in the packing house or at retail by traditional means (i.e., gas chromatography) would not be cost effective or practical, but newer non-invasive technologies including near infra-red reflectance spectrophotometry (NIRS), NIRS hyperspectral imaging, or Raman spectroscopy [58-61] may hold promise to deliver 
analyses in seconds versus days, and coupled to new tracking systems, such as radio frequency identifier tags, may be able to deliver this information to the consumer at retail. Mandatory labelling of $n-3$ fatty acids and the $n-6 / n-3$ ratio might also open the door to population-based prevention policies (i.e., food tax to drive nutritional improvements through changes in production practices), which could generate health gains while paying for themselves through future reductions of health-care expenditures [62], or by providing incentives to producers with specified amounts and types of $n-3$ fatty acids in their pig feed. Market pull could also be generated through inclusion of healthier sources of fatty acids (i.e., $n-3$ enriched lard) in baked goods, and further processed products that already qualify for nutritional labels, and this might in turn result in healthier pork meat as a byproduct. For all the strategies, however, it is clear that there needs to be more defined incentives provided to producers, and a stronger signal that changes are required along the value chain.

\section{Conclusions}

Currently retail pork is not considered a source of $n-3$ fatty acids, and in fact suffers from an imbalanced $n-6 / n-3$ fatty acid ratio related to modern feeding practices. Pork is the most consumed meat in the world, and its fatty acid content and composition is directly influenced by diet. There have been calls to correct the imbalanced $n-6 / n-3$ ratio in foods, including pork, and although this correction would seem to be a simple fix by modifying pig diets, adoption of such practices is not widespread. Producing $n-3$ enriched pork may increase production costs, enriched pork has to be tracked to retail, and the pork must be sold at a premium to recover added costs and provide profit for the effort. Labelling pork as a source of $n-3$ fatty acids also requires regulatory approval, development costs and costs for quality control to maintain enrichment status. As a result, $n-3$ enriched pork will likely continue to command a limited market share, and only be available to those willing to pay a premium. Several strategies to drive an industry wide shift towards $n-3$ fatty acid enriched pork production are possible including mandatory labelling of the $n-3$ fatty acid content and $n-6 / n-3$ ratio, and development of population based prevention polices. This would allow consumers to make choices based on valued attributes, and provide for natural market segmentation without having to reach specific amounts of $n-3$ fatty acids per serving. When coupled with improvements in the speed of non-invasive fatty acid analyses and tracking technologies, we could be on the verge of meeting health conscious consumers growing demand for nutritional information, while providing impetus to pork value chain to make producing pork with a higher $n-3$ fatty acid content and lower $n-6 / n-3$ ratio an industry wide priority. 
Author Contributions: The authors are all part of a collaborative group which has been involved in several research trials developing flaxseed feeding strategies to enrich $n-3$ fatty acids in pork. M.E.R.D. is the lead author on the review, and all co-authors contributed by providing comments and suggested revisions to the manuscript.

Conflicts of Interest: The authors declare no conflict of interest.

\section{References}

1. Simopoulos, A.P. Importance of the omega-6/omega-3 balance in health and disease: Evolutionary aspects of diet. World Rev. Nutr. Diet. 2011, 102, 10-21.

2. FAO Sources of Meat. Available online: http://www.fao.org/ag/againfo/themes/en/ meat/backgr_sources.html (accessed on 2 September 2015).

3. Ollis, T.E.; Meyer, B.J.; Howe, P.R. Australian food sources and intakes of omega-6 and omega-3 polyunsaturated fatty acids. Ann. Nutr. Metab. 1999, 43, 346-355.

4. Teicholz, N. The Big Fat Surprise: Why Butter, Meat and Cheese belong in a Healthy Diet; Simon and Schuster: New York, NY, USA, 2014.

5. Fortin, A.; Robertson, W.; Tong, A. The eating quality of canadian pork and its relationship with intramuscular fat. Meat Sci. 2005, 69, 297-305.

6. U.S. Department of Health and Human Services; U.S. Department of Agriculture. Dietary Guidelines for Americans; US Government Printing Office: Washington, DC, USA, 2010.

7. Siri-Tarino, P.W.; Sun, Q.; Hu, F.B.; Krauss, R.M. Meta-analysis of prospective cohort studies evaluating the association of saturated fat with cardiovascular disease. Am. J. Clin. Nutr. 2010, 98, 535-546.

8. Chowdhury, R.; Warnakula, S.; Kunutsor, S.; Crowe, F.; Ward, H.A.; Johnson, L.; Franco, O.H.; Butterworth, A.S.; Forouhi, N.G.; Thompson, S.G.; et al. Association of dietary, circulating, and supplement fatty acids with coronary risk: A systematic review and meta-analysis. Ann. Intern. Med. 2014, 160, 398-406.

9. Barendse, W. Should animal fats be back on the table? A critical review of the human health effects of animal fat. Anim. Prod. Sci. 2014, 54, 831-855.

10. Binnie, M.A.; Barlow, K.; Johnson, V.; Harrison, C. Red meats: Time for a paradigm shift in dietary advice. Meat Sci. 2014, 98, 445-451.

11. Heinz, G.; Hautzinger, P. Meat Processing Technology for Small to Medium Scale Producers. Available online: http://www.fao.org/docrep/010/ai407e/ai407e00.htm (accessed on 2 September 2015).

12. Enser, M.; Hallett, K.; Hewitt, B.; Fursey, G.A.J.; Wood, J.D. Fatty acid content and composition of english beef, lamb and pork at retail. Meat Sci. 1996, 42, 443-456.

13. Cardiovascular Review Group, Great Britain-Department of Health. Nutritional Aspects of Cardiovascular Disease; HMSO: Richmond, UK, 1994.

14. Juárez, M.; Dugan, M.E.R.; Aldai, N.; Aalhus, J.L.; Patience, J.F.; Zijlstra, R.T.; Beaulieu, A.D. Increasing omega-3 levels through dietary co-extruded flaxseed supplementation negatively affects pork palatability. Food Chem. 2011, 126, 1716-1723. 
15. Romans, J.R.; Johnson, R.C.; Wulf, D.M.; Libal, G.W.; Costello, W.J. Effects of ground flaxseed in swine diets on pig performance and on physical and sensory characteristics and omega-3 fatty acid content of pork: I. Dietary level of flaxseed. J. Anim. Sci. 1995, 73, 1982-1986.

16. Wood, J.D.; Enser, M.; Fisher, A.V.; Nute, G.R.; Sheard, P.R.; Richardson, R.I.; Hughes, S.I.; Whittington, F.M. Fat deposition, fatty acid composition and meat quality: A review. Meat Sci. 2008, 78, 343-358.

17. Savell, J.; Cross, H. The role of fat in the palatability of beef, pork, and lamb. In Designing Foods: Animal Product Options in the Marketplace; National Academy Press: Washington, DC, USA, 1988; pp. 345-355.

18. Raes, K.; de Smet, S.; Demeyer, D. Effect of dietary fatty acids on incorporation of long chain polyunsaturated fatty acids and conjugated linoleic acid in lamb, beef and pork meat: A review. Anim. Feed Sci. Technol. 2004, 113, 199-221.

19. Woods, V.B.; Fearon, A.M. Dietary sources of unsaturated fatty acids for animals and their transfer into meat, milk and eggs: A review. Livest. Sci. 2009, 126.

20. Doreau, M.; Chilliard, Y. Digestion and metabolism of dietary fat in farm animals. Br. J. Nutr. 1997, 78, S15-S35.

21. Shorland, F.B. Effect of the dietary fat on the composition of depot fats of animals. Nature 1950, 165.

22. Turner, T.D.; Mapiye, C.; Aalhus, J.L.; Beaulieu, A.D.; Patience, J.F.; Zijlstra, R.T.; Dugan, M.E.R. Flaxseed fed pork: N-3 fatty acid enrichment and contribution to dietary recommendations. Meat Sci. 2014, 96, 541-547.

23. Narayan, B.; Miyashita, K.; Hosakawa, M. Physiological effects of eicosapentaenoic acid (EPA) and docosahexaenoic acid (DHA) - A review. Food Rev. Int. 2006, 22, 291-307.

24. Swanson, D.; Block, R.; Mousa, S.A. Omega-3 fatty acids EPA and DHA: Health benefits throughout life. Adv. Nutr. 2012, 3.

25. Howe, P.; Meyer, B.; Record, S.; Baghurst, K. Dietary intake of long-chain omega-3 polyunsaturated fatty acids: Contribution of meat sources. Nutrition 2006, 22, 47-53.

26. Miller, E.; Kaur, G.; Larsen, A.; Loh, S.P.; Linderborg, K.; Weisinger, H.S.; Turchini, G.M.; Cameron-Smith, D.; Sinclair, A.J. A short-term $n$-3 DPA supplementation study in humans. Eur. J. Nutr. 2013, 52, 895-904.

27. Vahmani, P.; Mapiye, C.; Prieto, N.; Rolland, D.C.; McAllister, T.A.; Aalhus, J.L.; Dugan, M.E. The scope for manipulating the polyunsaturated fatty acid content of beef: A review. J. Anim. Sci. Biotechnol. 2015, 6.

28. Canadian Food Inspection Agency (CFIA): Omega-3 and Omega-6 Polyunsaturated Fatty Acid Claims. Available online: http://www.inspection.gc.ca/food/labelling/ food-labelling-for-industry/nutrient-content/specific-claim-requirements/eng/ 1389907770176/1389907817577?chap=7 (accessed on 9 September 2015).

29. Kris-Etherton, P.M. Monounsaturated fatty acids and risk of cardiovascular disease. Circulation 1999, 100, 1253-1258. 
30. Yu, S.; Derr, J.; Etherton, T.D.; Kris-Etherton, P. Plasma cholesterol-predictive equations demonstrate that stearic acid is neutral and monounsaturated fatty acids are hypocholesterolemic. Am. J. Clin. Nutr. 1995, 61, 1129-1139.

31. Koch, D.E.; Pearson, A.M.; Magee, W.T.; Hoefer, J.A.; Schweigert, B.S. Effect of diet on the fatty acid composition of pork fat. J. Anim. Sci. 1968, 27, 360-365.

32. Stewart, J.W.; Kaplan, M.L.; Beitz, D.C. Pork with a high content of polyunsaturated fatty acids lowers LDL cholesterol in women. Am. J. Clin. Nutr. 2001, 74, 179-187.

33. Anderson, D.B.; Kauffman, R.G.; Benevenga, N.J. Estimate of fatty acid turnover in porcine adipose tissue. Lipids 1972, 7, 488-489.

34. Cunnane, S.C.; Stitt, P.A.; Sujata, G.; Armstrong, J.K. Raised omega-3 fatty acid levels in pigs fed flax. Can. J. Anim. Sci. 1990, 70, 251-254.

35. Kouba, M.; Enser, M.; Whittington, F.M.; Nute, G.R.; Wood, J.D. Effect of a high-linolenic acid diet on lipogenic enzyme activities, fatty acid composition, and meat quality in the growing pig. J. Anim. Sci. 2003, 81, 1967-1979.

36. Corino, C.; Rossi, R.; Cannata, S.; Ratti, S. Effect of dietary linseed on the nutritional value and quality of pork and pork products: Systematic review and meta-analysis. Meat Sci. 2014, 98, 679-688.

37. Cherian, G.; Sim, J.S. Dietary alpha-linolenic acid alters the fatty acid composition of lipid classes in swine tissues. J. Agric. Food Chem. 1995, 43, 2911-2916.

38. Riley, P.A.; Enser, M.; Nute, G.R.; Wood, J.D. Effects of dietary linseed on nutritional value and other quality aspects of pig muscle and adipose tissue. Anim. Sci. 2000, 71, 483-500.

39. Ahn, D.U.; Lutz, S.; Sim, J.S. Effects of dietary alpha-linolenic acid on the fatty acid composition, storage stability and sensory characteristics of pork loin. Meat Sci. 1996, 43, 291-299.

40. Romans, J.R.; Wulf, D.M.; Johnson, R.C.; Libal, G.W.; Costello, W.J. Effects of ground flaxseed in swine diets on pig performance and on physical and sensory characteristics and omega-3 fatty acid content of pork: II. Duration of 15\% dietary flaxseed. J. Anim. Sci. 1995, 73, 1987-1999.

41. Juárez, M.; Dugan, M.; Aldai, N.; Aalhus, J.; Patience, J.; Zijlstra, R.; Beaulieu, A. Feeding co-extruded flaxseed to pigs: Effects of duration and feeding level on growth performance and backfat fatty acid composition of grower-finisher pigs. Meat Sci. 2010, 84, 578-584.

42. Fontanillas, R.; Barroeta, A.; Baucells, M.D.; Guardiola, F. Backfat fatty acid evolution in swine fed diets high in either cis-monounsaturated, trans, or (n-3) fats. J. Anim. Sci. 1998, 76, 1045-1055.

43. Huang, F.R.; Zhan, Z.P.; Luo, J.; Liu, Z.X.; Peng, J. Duration of dietary linseed feeding affects the intramuscular fat, muscle mass and fatty acid composition in pig muscle. Livest. Sci. 2008, 118, 132-139.

44. Martínez-Ramírez, H.R.; Kramer, J.K.G.; de Lange, C.F.M. Retention of n-3 polyunsaturated fatty acids in trimmed loin and belly is independent of timing of feeding ground flaxseed to growing-finishing female pigs. J. Anim. Sci. 2014, 92, 238-249. 
45. Enser, M.; Richardson, R.I.; Wood, J.D.; Gill, B.P.; Sheard, P.R. Feeding linseed to increase the $n-3$ pufa of pork: Fatty acid composition of muscle, adipose tissue, liver and sausages. Meat Sci. 2000, 55, 201-212.

46. Nguyen, L.Q.; Nuijens, M.C.G.A.; Everts, H.; Salden, N.; Beynen, A.C. Mathematical relationships between the intake of $n-6$ and $n-3$ polyunsaturated fatty acids and their contents in adipose tissue of growing pigs. Meat Sci. 2003, 65, 1399-1406.

47. Htoo, J.K.; Meng, X.; Patience, J.F.; Dugan, M.E.R.; Zijlstra, R.T. Effects of coextrusion of flaxseed and field pea on the digestibility of energy, ether extract, fatty acids, protein, and amino acids in grower-finisher pigs. J. Anim. Sci. 2008, 86, 2942-2951.

48. U.S. Department of Health and Human Services (HHS). Food labeling: Nutrient content claims; alpha-linolenic acid, eicosapentaenoic acid, and docosahexaenoic acid omega-3 fatty acids. Fed. Regist. 2014, 79, 23262-23273.

49. European Food Safety Authority (EFSA). Scientific opinion: Labelling reference intake values for $n-3$ and $n-6$ polyunsaturated fatty acids. EPSA J. 2009, 1176, 1-11.

50. Kim, E.J.; Kim, M.-K.; Jin, X.-J.; Oh, J.-H.; Kim, J.E.; Chung, J.H. Skin aging and photoaging alter fatty acids composition, including 11,14,17-eicosatrienoic acid, in the epidermis of human skin. J. Korean Med. Sci. 2010, 25, 980-983.

51. Schenck, P.A.; Rakoff, H.; Emken, E.A. $\delta 8$ desaturation in vivo of deuterated eicosatrienoic acid by mouse liver. Lipids 1996, 31, 593-600.

52. Coates, A.M.; Sioutis, S.; Buckley, J.D.; Howe, P.R. Regular consumption of $n-3$ fatty acid-enriched pork modifies cardiovascular risk factors. Br. J. Nutr. 2009, 101, 592-597.

53. Vossen, E.; Raes, K.; Maertens, L.; Vandenberge, V.; Haak, L.; Chiers, K.; Ducatelle, R.; de Smet, S. Diets containing $n-3$ fatty acids-enriched pork: Effect on blood lipids, oxidative status and atherosclerosis in rabbits. J. Food Biochem. 2012, 36, 359-368.

54. Simopoulos, A.P. New products from the agri-food industry: The return of $n-3$ fatty acids into the food supply. Lipids 1999, 34, S297-S301.

55. Lewis, N.M.; Seburg, S.; Flanagan, N.L. Enriched eggs as a source of $n-3$ polyunsaturated fatty acids for humans. Poult. Sci. 2000, 79, 971-974.

56. Givens, D.; Gibbs, R. Very long chain $n-3$ polyunsaturated fatty acids in the food chain in the uk and the potential of animal-derived foods to increase intake. Nutr. Bull. 2006, 31, 104-110.

57. Bryhni, E.A.; Kjos, N.P.; Ofstad, R.; Hunt, M. Polyunsaturated fat and fish oil in diets for growing-finishing pigs: Effects on fatty acid composition and meat, fat, and sausage quality. Meat Sci. 2002, 62.

58. Damez, J.-L.; Clerjon, S. Quantifying and predicting meat and meat products quality attributes using electromagnetic waves: An overview. Meat Sci. 2013, 95, 879-896.

59. Prieto, N.; Dugan, M.E.R.; López-Campos, O.; McAllister, T.A.; Aalhus, J.L.; Uttaro, B. Near infrared reflectance spectroscopy predicts the content of polyunsaturated fatty acids and biohydrogenation products in the subcutaneous fat of beef cows fed flaxseed. Meat Sci. 2012, 90, 43-51. 
60. Prieto, N.; Uttaro, B.; Mapiye, C.; Turner, T.; Dugan, M.; Zamora, V.; Young, M.; Beltranena, E. Predicting fat quality from pigs fed reduced-oil corn dried distillers grains with solubles by near infrared reflectance spectroscopy: Fatty acid composition and iodine value. Meat Sci. 2014, 98, 585-590.

61. Berhe, D.T.; Eskildsen, C.E.; Lametsch, R.; Hviid, M.S.; van den Berg, F.; Engelsen, S.B. Prediction of total fatty acid parameters and individual fatty acids in pork backfat using raman spectroscopy and chemometrics: Understanding the cage of covariance between highly correlated fat parameters. Meat Sci. 2016, 111, 18-26.

62. Cecchini, M.; Sassi, F.; Lauer, J.A.; Lee, Y.Y.; Guajardo-Barron, V.; Chisholm, D. Tackling of unhealthy diets, physical inactivity, and obesity: Health effects and cost-effectiveness. Lancet 2010, 376, 1775-1784. 


\title{
Mechanisms Involved in the Improvement of Lipotoxicity and Impaired Lipid Metabolism by Dietary $\alpha$-Linolenic Acid Rich Salvia hispanica L (Salba) Seed in the Heart of Dyslipemic Insulin-Resistant Rats
}

\author{
Agustina Creus, María R. Ferreira, María E. Oliva and Yolanda B. Lombardo
}

Abstract: This study explores the mechanisms underlying the altered lipid metabolism in the heart of dyslipemic insulin-resistant (IR) rats fed a sucrose-rich diet (SRD) and investigates if chia seeds (rich in $\alpha$-linolenic acid 18:3, n-3 ALA) improve/reverse cardiac lipotoxicity. Wistar rats received an SRD-diet for three months. Half of the animals continued with the SRD up to month 6 . The other half was fed an SRD in which the fat source, corn oil (CO), was replaced by chia seeds from month 3 to 6 (SRD+chia). A reference group consumed a control diet (CD) all the time. Triglyceride, long-chain acyl CoA (LC ACoA) and diacylglycerol (DAG) contents, pyruvate dehydrogenase complex (PDHc) and muscle-type carnitine palmitoyltransferase 1 (M-CPT1) activities and protein mass levels of M-CPT1, membrane fatty acid transporter (FAT/CD36), peroxisome proliferator activated receptor $\alpha(\operatorname{PPAR} \alpha)$ and uncoupling protein 2 (UCP2) were analyzed. Results show that: (a) the hearts of SRD-fed rats display lipotoxicity suggesting impaired myocardial lipid utilization; (b) Compared with the SRD group, dietary chia normalizes blood pressure; reverses/improves heart lipotoxicity, glucose oxidation, the increased protein mass level of FAT/CD36, and the impaired insulin stimulated FAT/CD36 translocation to the plasma membrane. The enhanced M-CPT1 activity is markedly reduced without similar changes in protein mass. PPAR $\alpha$ slightly decreases, while the UCP2 protein level remains unchanged in all groups. Normalization of dyslipidemia and IR by chia reduces plasma fatty acids (FAs) availability, suggesting that a different milieu prevents the robust translocation of FAT/CD36. This could reduce the influx of FAs, decreasing the elevated M-CPT1 activity and lipid storage and improving glucose oxidation in cardiac muscles of SRD-fed rats.

Reprinted from J. Clin. Med. Cite as: Creus, A.; Ferreira, M.R.; Oliva, M.E.; Lombardo, Y.B. Mechanisms Involved in the Improvement of Lipotoxicity and Impaired Lipid Metabolism by Dietary $\alpha$-Linolenic Acid Rich Salvia hispanica L (Salba) Seed in the Heart of Dyslipemic Insulin-Resistant Rats. J. Clin. Med. 2016, 5,18 . 


\section{Introduction}

Metabolic syndrome (MS) is a complex metabolic disorder influenced by genetic and environmental factors [1]. In Western societies, the high increase of MS, including cardiovascular disease (CVD), seems to be due to changes in lifestyle (e.g., increased consumption of food high in refined sugar and decreased physical activities). CVD represents a major cause of premature death in Western countries [2]. Therefore, there is growing interest in identifying novel therapeutic approaches including a particular focus on nutrition and dietary interventions.

Cardiac energy metabolic shifts occur as a normal response to diverse physiological and dietary conditions and as a component of the pathophysiological processes that accompany heart disease. It is well established that insulin and fatty acids (FAs) are important modulators of cardiac substrate utilization [3]. In the heart, there is a fine-tuning of high rates of myocellular FAs uptake and of mitochondrial fatty acid oxidation. When the rate of FA delivery to the heart increases (e.g., diabetes, high fat feeding) it may cause a mismatch between FA uptake and oxidation leading to excessive intracellular storage of the bio-active lipid intermediates within the cardiomyocytes that could subsequently lead to cardiac dysfunction [4,5]. Accordingly, several studies have shown that lipids accumulate in the heart of diabetic animals [6,7]. Myocardial FA uptake is largely regulated by the membrane fatty acid transporter (FAT/CD36) [8]. Chiu et al. [9] showed that a myocardial lipid accretion due to an increase of fat uptake leads to myocyte apoptosis and cardiomyopathy. An enhanced long-chain acyl CoA (LC ACoA) uptake and channeling into triglycerides was observed in the heart of obese Zucker rats [10]. The accumulation of triglyceride is likely toxic to the myocardium and has been linked with insulin resistance (IR) and cardiac dysfunction [6,11]. Besides, it is generally acknowledged that dietary factors, among them FAs, up-regulated the transcription of genes encoding for proteins involved in cardiac FA transport and metabolism, most likely through the activation of peroxisome proliferator activated receptor $\alpha$ (PPAR $\alpha)$ (e.g., expression of muscle-type carnitine palmitoyltransferase 1 (M-CPT1) [12,13].

On the other hand, high sucrose, high fructose and/or high fat diets have been used to induce metabolic and physiological alterations in rodents, mimicking several aspects of the MS in humans such as dyslipidemia, IR and adiposity [14]. Furthermore, we have previously demonstrated that the cardiac muscle of rats chronically fed a sucrose-rich diet (SRD) showed a significant increase of lipid storage accompanied by a significant reduction of basal and insulin stimulated glucose uptake and metabolism (isolated perfusion according to Langendorff's recirculating mode), as well as in the activities of key enzymes involved in glucose metabolism $[15,16]$.

Epidemiological data show that a high intake of $n-3$ polyunsaturated fatty acids ( $n-3$ PUFAs) from fish is associated with a lower incidence of heart failure 
and cardio protective function [17]. Moreover, different epidemiological and clinical studies have suggested that a high concentration of dietary $\alpha$-linolenic acid 18:3 $n-3,($ ALA) is associated with a decreased risk of CVD [18,19]. Recent studies carried out in rats by Folino et al. [20] showed that ALA protects against cardiac injury and remodeling induced by beta-adrenergic over stimulation, and that a protective role is played by $\beta_{2}$ adrenergic receptors which mediate the activation of the Src kinase-phosphatidylinositol-3-kinase protective pathway. The seeds of Salvia hispanica L, commonly known as chia seeds, contain the richest botanical oil source of ALA and high amounts of fiber and minerals. Poudyal et al. [21,22] have recently shown that the administration of chia oil improved heart left ventricular dimensions, contractility, volume and stiffness as well as hypertension, glucose tolerance and insulin sensitivity in rats fed a high fat-high fructose diet. In this line, recent studies of our group have demonstrated that the administration of chia seeds as a dietary source of fat in rats fed an SRD reversed dyslipidemia and IR, improved adipose tissue dysfunction and glucose and lipid metabolism in the skeletal muscle [23-25]. However, the effect of chia seeds on myocardial substrate utilization has been only partially investigated in this experimental model [21,22].

Thus, the aims of the present study were the following: (i) to further explore the mechanisms underlying the impaired lipid metabolism in the heart muscle of dyslipidemic insulin-resistant rats fed an SRD; (ii) to investigate if chia seeds as a dietary intervention could improve or even revert cardiac lipotoxicity. To achieve these goals: (a) we analyzed the protein mass levels of FAT/CD36 both at basal conditions and under insulin stimulation and the mitochondrial oxidation of LC ACoA by the activity and protein mass levels of the enzyme M-CPT1; (b) since the effect of FAs or FA derivatives in cardiac myocytes are considered to be PPAR $\alpha$ mediated, we measured the protein mass level of this receptor; (c) we evaluated the protein mass levels of uncoupling protein 2 (UCP2), which plays a major role in the mitochondrial FAs flux. Additionally, the activities of the pyruvate dehydrogenase complex (PDHc) and lipid storage were assessed. The study was conducted in rats fed an SRD during 6 months, during which permanent dyslipidemia, IR, abnormal glucose homeostasis and visceral adiposity were present before the source of dietary fat, corn oil (CO), was replaced by an isocaloric amount of chia seeds for the last three months of the experimental period in half of the animals.

\section{Materials and Methods}

\subsection{Animals}

Male Wistar rats initially weighing 180-190 g purchased from the National Institute of Pharmacology (Buenos Aires, Argentina) were housed in an animal room under controlled temperature $\left(22 \pm 1{ }^{\circ} \mathrm{C}\right)$, humidity and airflow, with a 
fixed (12 h) light-dark cycle (lights on from 07:00 to 19:00 h) and with free access to water and food. Adequate measures were taken to minimize the pain or discomfort of the rats and we used the smallest number of animals as possible. The animal protocols were evaluated and approved by the Human and Animal Research Investigation Committee of the School of Biochemistry, University of Litoral, Argentina (FONCyT-PICT \#945/2012).

\subsection{Experimental Design}

The rats $(n=60)$ were initially fed with a standard non-purified diet (Ralston Purina, St Louis, MO, USA). After one week of acclimation, the rats were randomly divided into two separate groups and were fed a semi-synthetic diet. The control group $(n=20)$ received a control diet (CD) containing corn starch $(60 \%$ energy), protein (17\% energy) and $\mathrm{CO}$ as sources of fat $(23 \%$ energy) throughout the experimental period (6 months). The other group $(n=40)$ received the same semi-synthetic diet with the sucrose as the carbohydrate source (SRD). After 3 months, the animals in the SRD group were randomly divided into two subgroups. The rats in the first subgroup continued with the SRD diet for up to 6 months of feeding. The second subgroup received the Salba seed (chia) as the source of dietary fat (SRD + chia) for the next 3 months. The carbohydrates, proteins, fibers, vitamins and mineral contents in the chia seed of SRD + chia group were balanced with the CD and SRD groups, according to the amount of these nutrients present in the chia seeds. A detailed composition of each diet is described in Table 1 . The fatty acid composition of each experimental diet is shown in Table 2. The preparation and handling of diets have been reported elsewhere $[23,24]$. All diets provided approximately $17 \mathrm{~kJ} / \mathrm{g}$ of food.

Table 1. Composition of experimental diets ${ }^{1}$.

\begin{tabular}{|c|c|c|c|c|c|c|}
\hline \multirow[t]{2}{*}{ Diet Ingredients } & \multicolumn{2}{|c|}{ Control Diet (CD) } & \multicolumn{2}{|c|}{$\begin{array}{l}\text { Sucrose-Rich Diet } \\
\text { (SRD) }\end{array}$} & \multicolumn{2}{|c|}{$\begin{array}{l}\text { SRD+chia Seed } \\
\text { (SRD+chia) }\end{array}$} \\
\hline & $\% w / w$ & \% Energy & $\% w / w$ & \% Energy & $\% w / w$ & \% Energy \\
\hline \multicolumn{7}{|l|}{ Carbohydrates } \\
\hline Corn starch & 58.0 & 60.0 & 2.5 & 2.6 & - & - \\
\hline Sucrose & - & - & 55.5 & 57.4 & 55.5 & 57.4 \\
\hline $\begin{array}{c}\text { Chia seed }^{2} \\
\text { Fat }\end{array}$ & - & - & - & - & 2.5 & 2.6 \\
\hline Corn oil & 10.5 & 23.0 & 10.5 & 23.0 & 0.1 & 0.2 \\
\hline $\begin{array}{l}\text { Chia seed } \\
\text { Protein }\end{array}$ & - & - & - & - & 10.4 & 22.8 \\
\hline Casein (vitamin free) & 16.3 & 17.0 & 16.3 & 17.0 & 8.6 & 9.0 \\
\hline Chia seed & - & - & - & - & 7.7 & 8.0 \\
\hline
\end{tabular}

${ }^{1}$ The compositions of experimental diets are based on the AIN-93M diet. All diets contain by weight: salt mix 3.5\% (AIN-93Mx); vitamin mix $1 \%$ (AIN-93Vx); choline chloride $0.2 \%$; methionine $0.3 \%$; fiber $10 \%-11 \%{ }^{2}$ Chia seed (variety Salba; Salvia hispánica L.): $362 \mathrm{~g} / \mathrm{Kg}$ diet. Chia composition (g/100 g chia seed): carbohydrate 37.45 ; insoluble fiber $81 \%$ of total of carbohydrates; fat 30.23; protein 21.19. Mineral composition (mg/100 g chia seed): Na 103.15; K 826.15; Ca 589.60; Fe 11.90; Mg 77.0; P 604.0; Zn 5.32; Cu 1.66; Mn 1.36. 
Table 2. Total fatty acid composition of experimental diets ( $\mathrm{g} / \mathrm{kg}$ diet).

\begin{tabular}{ccc}
\hline Fatty Acids $^{\mathbf{1}}$ & CD and SRD & SRD+chia Seed \\
\hline $16: 0$ & 10.92 & 6.96 \\
$18: 0$ & 2.73 & 2.42 \\
$18: 1 n-9$ & 33.71 & 7.39 \\
$18: 2 n-6$ & 54.10 & 19.85 \\
$18: 3 n-3$ & 0.80 & 67.26 \\
$20: 1 n-9$ & 0.47 & 0.36 \\
Total saturated & 13.65 & 9.38 \\
Monounsaturated & 34.18 & 7.75 \\
Polyunsaturated & & \\
$n-6$ & 54.10 & 19.85 \\
$n-3$ & 0.80 & 67.26 \\
$n-6 / n-3$ & 67.62 & 0.295 \\
\hline
\end{tabular}

${ }^{1}$ Other minor fatty acids have been excluded.

The body weight and energy intake of each animal were recorded twice per week throughout the experimental period in all groups and subgroups of rats. In a separate experiment, the individual caloric intake and weight gain of eight animals in each group and subgroup were assessed twice a week. At the end of the experimental period, food was removed at 07:00 h (end of the dark period) and unless otherwise indicated, experiments were performed under feed conditions.

\subsection{Analytical Methods}

Rats from the three dietary groups were anaesthetized with intraperitoneal sodium pentobarbital (60 mg/kg body weight). Blood samples were obtained from the jugular vein, collected in tubes containing sodium EDTA as anticoagulant and rapidly centrifuged. Plasma was either immediately assayed or stored at $-20{ }^{\circ} \mathrm{C}$. Plasma triglycerides, cholesterol, glucose and free fatty acids and immunoreactive insulin were determined as previously described [25]. Epididymal, retroperitoneal and omental adipose tissues were removed and weighed. The visceral adiposity index (\%) was calculated as shown elsewhere [25]. The heart muscle was totally removed; then, it was weighed and the left ventricle was separated. The heart tissue was immediately frozen and stored at temperature of liquid $\mathrm{N}_{2}$. Heart weight was normalized relative to the tibia length at the time of removal. The homogenate of frozen muscle powder was used to determine triglyceride [26], LC ACoA [27] and diacylglycerol (DAG) content [28].

\subsection{Determination of Blood Pressure}

Blood pressure was measured in the three dietary groups in conscious animals maintained at $28^{\circ} \mathrm{C}$ in a restraining tube, at the beginning, at 3 months and at the end 
of the experimental period using a CODA ${ }^{\mathrm{TM}}$ Monitor of tail-cuff non-invasive blood pressure system (Kent Scientific Corporation, Torrington, CT, USA). The values are reported as the average of 8 individual measurements.

\subsection{Enzymatic Activity Assays of CPT and PDHc}

Heart M-CPT1 enzyme activities were measured as reported by Ling et al. [29] with some modifications. Frozen tissue was homogenized in buffer $(20 \mathrm{mM}$ HEPES $\mathrm{pH} 7.4,140 \mathrm{mM} \mathrm{KCl}, 10 \mathrm{mM}$ EDTA and $5 \mathrm{mM} \mathrm{MgCl}_{2}$ ) and after centrifugation; the mitochondrial pellet was resuspended in a homogenization buffer. Protein concentration was measured by the Bradford assay (Bio-Rad reagent). To determine the total CPT activity, $100 \mu \mathrm{g}$ of protein were added in a reaction buffer (20 mM HEPES pH 7.4, 220 mM sucrose, 40 mM KCl, 1 mM EGTA, $0.1 \mathrm{mM}$ 5,5'-dithio-bis(2-nitrobenzoic acid) (DTNB) and $40 \mu \mathrm{M}$ palmitoyl CoA). In addition, $1 \mathrm{mM}$ of L-carnitine was added to start the reaction. The rate of appearance of CoASH-DTNB was monitored at $412 \mathrm{~nm}$ at $37^{\circ} \mathrm{C}$. CPT2 activity was measured under the same conditions in the presence of $10 \mu \mathrm{M}$ of malonyl CoA (M-CPT1 inhibitor). The M-CPT1 activity was calculated as the difference between total CPT and CPT2 activities. The M-CPT1 activity was expressed as the amount of CoASH released per min per mg of protein. The extract and assay of the PDHc activity from the heart muscle were measured as previously described [15].

\subsection{Determination of FAT/CD36 Protein Mass Level (Euglycemic-Hyperinsulinemic Clamp Studies)}

The heart muscles of six rats from the CD, SRD and SRD+chia groups were rapidly removed (time 0 of clamp study) and stored at $-80^{\circ} \mathrm{C}$ for the determination of the FAT/CD36 protein levels. Immediately, in the other six rats from each dietary group, an infusion of highly purified porcine neutral insulin (Actrapid, Novo Nordisk, ) was administered at 0.8 units $/(\mathrm{kg} \times \mathrm{h})$ for $120 \mathrm{~min}$. Glycemia was maintained at a euglycemic level by infusing glucose at a variable rate. The glucose infusion rate (GIR) during the second hour of the clamp study was taken as the net steady state of the whole body glucose. At the end of the clamp period, the heart muscle of each dietary group was rapidly removed for determination of the FAT/CD36 protein mass levels (for details see ref. [30]). Plasma membrane fractions from heart muscle were prepared according to the method of Rodnick et al. [31]. Briefly, the muscle was homogenized in ice-cold buffer $(20 \mathrm{mM} \mathrm{HEPES} \mathrm{pH} \mathrm{7.4,}$ $1 \mathrm{mM}$ EDTA, $250 \mathrm{mM}$ sucrose and $5 \mu \mathrm{L} / \mathrm{mL}$ protease inhibitor cocktail (Sigma, St. Louis, MO, USA)) and the homogenate was centrifuged at $3000 \times g$ at $4{ }^{\circ} \mathrm{C}$. The supernatant was centrifuged at $184,000 \times g$ at $4{ }^{\circ} \mathrm{C}$. The resulting pellet (membrane fraction) was resuspended in buffer and stored at $-80{ }^{\circ} \mathrm{C}$ until the assay. Protein concentrations were quantified by the Bradford assay (Bio-Rad reagent). Proteins 
were separated by SDS-PAGE and transferred to PVDF membranes. The membranes were probed with a specific antibody (rabbit polyclonal anti-CD36 from Santa Cruz Biotechnology, Inc., Santa Cruz, CA, USA). The blot was incubated with horseradish peroxidase-linked secondary antibody (Santa Cruz Biotechnology) followed by chemiluminescence detection according to the manufacturer's instruction (Pierce Biotechnology, Rockford, IL, USA). The protein levels were normalized to actin. The intensity of the bands was quantified by National Institutes of Health (NIH) imaging software (Bethesda, MD, USA). The relationship between the amount of the sample subjected to immunoblotting and the signal intensity observed was linear under the conditions described above.

\subsection{Determination of M-CPT1 and PPAR $\alpha$ Protein Mass Levels}

Frozen heart powder was homogenized in a lysis buffer $(10 \mathrm{mM}$ Tris- $\mathrm{HCl}$ pH 7.4, $150 \mathrm{mM} \mathrm{NaCl}, 1 \mathrm{mM}$ EGTA, $1 \mathrm{mM}$ EDTA, 1\% Triton X-100 and $5 \mu \mathrm{L} / \mathrm{mL}$ protease inhibitor cocktail (Sigma)) as described by Bogazzi et al. [32]. After $30 \mathrm{~min}$ incubation on ice, the lysates were centrifuged at $4{ }^{\circ} \mathrm{C}$ and supernatants were stored at $-80{ }^{\circ} \mathrm{C}$. The protein content was quantified by the Bradford assay. Total protein samples (100 $\mu \mathrm{g} /$ lane) were resolved on SDS-PAGE, transferred to PVDF membranes and probed with specific antibodies (rabbit polyclonal anti M-CPT1 or rabbit polyclonal anti-PPAR $\alpha$ from Santa Cruz Biotechnology, Inc.). The blots were incubated with horseradish peroxidase-linked secondary antibody (Santa Cruz Biotechnology) followed by chemiluminescence detection as described above. The protein levels were normalized to actin.

\subsection{Determination of UCP2 Protein Mass Level}

The mitochondrial fraction from the heart muscle was carried out according to the method described by Pecqueur et al. [33]. Briefly, the tissue was homogenized at $4{ }^{\circ} \mathrm{C}$ in a TES buffer (10 mM Tris pH 7.5, $1 \mathrm{mM}$ EDTA, $250 \mathrm{mM}$ sucrose and $5 \mu \mathrm{L} / \mathrm{mL}$ protease inhibitor cocktail (Sigma)). After centrifugation, the mitochondrial pellet was resuspended in TES buffer and stored at $-80^{\circ} \mathrm{C}$. The protein content was quantified by the Bradford assay. Protein samples (50 $\mu \mathrm{g}$ protein/lane) were resolved by SDS-PAGE and transferred to PVDF membranes. The membranes were probed with a specific antibody (goat polyclonal anti-UCP2 from Santa Cruz Biotechnology, Inc.). The blot was incubated with horseradish peroxidase-linked secondary antibody (Santa Cruz Biotechnology) followed by chemiluminescence detection as described above. The protein levels were normalized to actin.

\subsection{Statistical Analysis}

Sample sizes were calculated on the basis of measurements previously made with rats fed either a CD or SRD $[16,25,30]$ considering an $80 \%$ power as described 
by Glantz [34]. Results were expressed as mean with their standard errors. The homogeneity of variances were tested using Barlett's test. The statistical significance between groups was determined by one way analysis of variance (ANOVA) with one factor (diet) followed by the Newman Keul's multiple comparison post hoc test [35]. Differences having $p$ values lower than 0.05 were considered to be statistically significant. Statistical analyses were performed using GraphPad Prism version 5.00 for Windows (San Diego, CA, USA). All reported $p$ values are 2-sided.

\section{Results}

\subsection{Body Weight, Energy Intake and Visceral Adiposity Index}

Body weight and energy intake were carefully monitored in all groups of rats through the experimental period. As previously shown [25], a significant increase in body weight and energy intake occurred in rats fed an SRD from month 3 to 6 compared to those fed a CD (Table 3). A similar energy intake was recorded in both SRD and SRD+chia groups during the last three months of the experimental period while body weight at month 6 was slightly lower without statistical differences in the latter group. As in our previous report [24], SRD-fed rats showed a significant increase of the visceral adiposity index compared to the CD group. The chia seed enriched diet significantly reduced the aforementioned index, which reached values similar to those of the CD group.

Table 3. Body weight, energy intake and adiposity index of rats fed a control diet (CD), sucrose-rich diet (SRD) or SRD + chia seed (SRD + chia) ${ }^{\mathbf{1}}$.

\begin{tabular}{|c|c|c|c|c|c|c|c|}
\hline \multirow[t]{2}{*}{ Diet } & \multicolumn{2}{|c|}{ Body Weight (g) } & \multirow{2}{*}{$\begin{array}{c}\text { Energy Intake } \\
\text { (kJ/Day) }\end{array}$} & \multirow[t]{2}{*}{ Diet } & \multirow{2}{*}{$\begin{array}{c}\begin{array}{c}\text { Body Weight } \\
(\mathrm{g})\end{array} \\
6 \text { Months }\end{array}$} & \multirow{2}{*}{$\begin{array}{c}\begin{array}{c}\text { Energy } \\
\text { Intake } \\
\text { (kJ/Day) }\end{array} \\
3 \text { to } 6 \\
\text { Months }\end{array}$} & \multirow{2}{*}{$\begin{array}{c}\text { Visceral } \\
\text { Adiposity } \\
\text { Index (\%) }\end{array}$} \\
\hline & Initial & 3 Months & & & & & \\
\hline $\mathrm{CD}(8)$ & $184.3 \pm 2.6$ & $414.5 \pm 5.5$ & $294.5 \pm 12.5$ & $\mathrm{CD}(8)$ & $476.3 \pm 7.6^{b}$ & $292.0 \pm 7.2^{b}$ & $4.1 \pm 0.3^{b}$ \\
\hline \multirow[t]{2}{*}{ SRD (16) } & $186.0 \pm 1.6$ & $428.0 \pm 6.0$ & $292.0 \pm 7.2$ & SRD (8) & $545.0 \pm 10.0^{\mathrm{a}}$ & $374.0 \pm 9.5^{\mathrm{a}}$ & $6.2 \pm 0.4^{\mathrm{a}}$ \\
\hline & & & & SRD+chia (8) & $524.0 \pm 7.3^{\mathrm{a}}$ & $356.5 \pm 13.0^{\mathrm{a}}$ & $4.5 \pm 0.2^{b}$ \\
\hline
\end{tabular}

${ }^{1}$ Values are expressed as mean \pm SEM, () number of rats. Values in a column that do not share the same superscript letter $(\mathrm{a}, \mathrm{b})$ are significantly different $p<0.05$ when one variable at a time was compared by the Newman Keul's test.

\subsection{Total and Relative Heart Weight and Systolic Blood Pressure}

Total heart weights recorded at the end of experimental period in each dietary group showed a significant increase in SRD-fed rats compared with the CD-fed rats. SRD + chia did not modify heart weight. Conversely, relative heart weights as $\mathrm{g} / 100 \mathrm{~g}$ body weight or by the length of the tibia $(\mathrm{mg} / \mathrm{mm})$ were similar in all dietary groups (Table 4). SRD feeding increased systolic blood pressure throughout the six-month feeding protocol with values at three and six months significantly 
higher compared to those observed in the CD-fed group. Chia seeds in the SRD group normalized systolic blood pressure after three months of treatment reaching values similar to those of the CD group (Table 4).

Table 4. Total and relative heart weight at the end of the experimental period and systolic blood pressure of rats fed a control diet (CD), sucrose-rich diet (SRD) or SRD + chia seed $\left(\right.$ SRD + chia) ${ }^{1}$.

\begin{tabular}{cccc}
\hline & CD & SRD & SRD+chia \\
\hline Heart tissue & & & \\
Total wet weight, g & $1.24 \pm 0.01^{\mathrm{b}}(8)$ & $1.31 \pm 0.03^{\mathrm{a}}(8)$ & $1.28 \pm 0.02^{\mathrm{a}}(8)$ \\
g wet weight/100 g body weight & $0.260 \pm 0.003(8)$ & $0.250 \pm 0.004(8)$ & $0.250 \pm 0.005(8)$ \\
mg wet weight/mm tibia length & $27.2 \pm 1.0(8)$ & $30.1 \pm 1.3(8)$ & $28.3 \pm 1.0(8)$ \\
Systolic blood pressure, mmHg & & & \\
Initial & $115.0 \pm 4.6(8)$ & $116.0 \pm 2.4(16)$ & \\
3 months & $123.3 \pm 1.0^{\mathrm{b}}(8)$ & $130.9 \pm 1.2^{\mathrm{a}}(16)$ & \\
6 months & $120.2 \pm 4.8^{\mathrm{b}}(8)$ & $138.4 \pm 1.6^{\mathrm{a}}(8)$ & $118.8 \pm 1.6^{\mathrm{b}}(8)$ \\
\hline
\end{tabular}

${ }^{1}$ Values are expressed as mean \pm SEM, () number of rats. Values in a line that do not share the same superscript letter $(\mathrm{a}, \mathrm{b})$ are significantly different $p<0.05$ when one variable at a time was compared by the Newman Keul's test.

\subsection{Plasma Metabolites, Insulin Levels and GIR}

Confirming previous studies [25], the changes observed after three or six months of SRD on plasma triglyceride, free fatty acids and glucose concentration were similar and both significantly different from those of the CD groups to which the data from SRD + chia were similar (Table 5). No statistical differences in plasma insulin levels were observed at the end of the experimental period between the three dietary groups. Furthermore, the significant decrease of the GIR recorded in the SRD-fed group returned to values similar to those obtained in the CD-fed rats in the SRD + chia group (Figure 1).

Table 5. Plasma metabolites of rats fed a control diet (CD), sucrose-rich diet (SRD) or SRD + chia seed (SRD+chia) ${ }^{\mathbf{1}}$.

\begin{tabular}{ccccccc}
\hline Diet & $\begin{array}{c}\text { Time on Diet } \\
(\text { Months) }\end{array}$ & $\begin{array}{c}\text { Triglyceride } \\
(\mathbf{m M})\end{array}$ & $\begin{array}{c}\text { Free Fatty } \\
\text { Acids }(\mu \mathbf{M})\end{array}$ & $\begin{array}{c}\text { Cholesterol } \\
(\mathbf{m M})\end{array}$ & $\begin{array}{c}\text { Glucose } \\
(\mathbf{m M})\end{array}$ & $\begin{array}{c}\text { Insulin } \\
(\mu \mathbf{U} / \mathbf{m L})\end{array}$ \\
\hline CD & 3 & $0.69 \pm 0.04^{\mathrm{b}}$ & $300.1 \pm 16.0^{\mathrm{b}}$ & $1.85 \pm 0.10^{\mathrm{b}}$ & $6.5 \pm 0.2^{\mathrm{b}}$ & $64.1 \pm 3.2$ \\
SRD & 3 & $1.98 \pm 0.08^{\mathrm{a}}$ & $716.0 \pm 8.1^{\mathrm{a}}$ & $3.21 \pm 0.14^{\mathrm{a}}$ & $7.9 \pm 0.1^{\mathrm{a}}$ & $60.1 \pm 4.2$ \\
CD & 6 & $0.72 \pm 0.03^{\mathrm{b}}$ & $335.0 \pm 13.0^{\mathrm{b}}$ & $1.92 \pm 0.11^{\mathrm{b}}$ & $6.6 \pm 0.1^{\mathrm{b}}$ & $62.0 \pm 2.9$ \\
SRD & 6 & $2.06 \pm 0.17^{\mathrm{a}}$ & $760.4 \pm 16.3^{\mathrm{a}}$ & $3.60 \pm 0.04^{\mathrm{a}}$ & $8.3 \pm 0.1^{\mathrm{a}}$ & $65.0 \pm 3.2$ \\
SRD+chia & 3 to 6 & $0.72 \pm 0.05^{\mathrm{b}}$ & $363.0 \pm 35.4^{\mathrm{b}}$ & $1.75 \pm 0.21^{\mathrm{b}}$ & $6.9 \pm 0.1^{\mathrm{b}}$ & $67.4 \pm 6.5$ \\
\hline
\end{tabular}

${ }^{1}$ Values are expressed as mean \pm SEM, $n=6$. Values in a column that do not share the same superscript letter $(\mathrm{a}, \mathrm{b})$ are significantly different $p<0.05$ when one variable at a time was compared by the Newman Keul's test. 


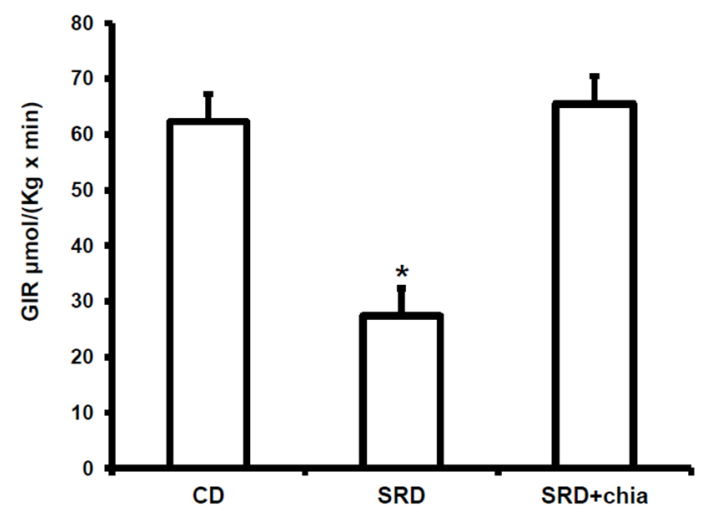

Values are mean, with their standard errors depicted by vertical bars (six animals per group). ${ }^{*} p<0.05$ SRD vs. CD and SRD + chia.

Figure 1. GIR during euglycemic-hyperinsulinemic clamp in rats fed a control $\operatorname{diet}(\mathrm{CD})$, sucrose-rich diet (SRD) or SRD + chia seed (SRD + chia).

\subsection{Heart Muscle Metabolites Concentrations and PDHc Activity}

Table 6 depicts significant increases of lipid storage (triglycerides, LC ACoA and DAG) levels within the cardiac muscle of SRD-fed rats. The present data show that in the SRD + chia seed group, neither parameter differed from those of the CD group at the end of the experimental period. Moreover, the administration of chia seed (SRD+chia) was able to revert the reduced active form of PDHc (PDHa) observed in the SRD-fed group reaching values similar to those of the control group (CD). The total PDHc activity expressed per gram of wet tissue did not differ between the groups (data not shown).

Table 6. Intramyocardial lipid accumulation and PDHc activity of rats fed a control diet (CD), sucrose-rich diet (SRD) or SRD + chia seed (SRD + chia) at the end of the experimental period ${ }^{\mathbf{1}}$.

\begin{tabular}{cccc}
\hline & CD & SRD & SRD+chia \\
\hline Triglyceride ( $\mu$ mol/g wet tissue) & $3.60 \pm 0.22^{\mathrm{b}}$ & $6.03 \pm 0.34^{\mathrm{a}}$ & $4.44 \pm 0.60^{\mathrm{b}}$ \\
\hline LC ACoA (nmol/g wet tissue) & $31.4 \pm 5.5^{\mathrm{b}}$ & $68.0 \pm 4.0^{\mathrm{a}}$ & $45.8 \pm 5.5^{\mathrm{b}}$ \\
\hline DAG (nmol/g wet tissue) & $250.8 \pm 19.5^{\mathrm{b}}$ & $355.3 \pm 15.4^{\mathrm{a}}$ & $276.6 \pm 24.0^{\mathrm{b}}$ \\
\hline PDHa (\% of total PDHc) & $59.7 \pm 5.8^{\mathrm{a}}$ & $26.6 \pm 4.6^{\mathrm{b}}$ & $45.1 \pm 4.9^{\mathrm{a}}$ \\
\hline
\end{tabular}

${ }^{1}$ Values are expressed as mean \pm SEM, $n=6$. Values in a line that do not share the same superscript letter $(\mathrm{a}, \mathrm{b})$ are significantly different $p<0.05$ when one variable at a time was compared by the Newman Keul's test. 
3.5. FAT/CD36 Protein Mass Level in Heart Muscle at the Beginning and at the End of the Euglycemic-Hyperinsulinemic Clamp Studies

Figure 2 shows the protein mass level of FAT/CD36 at the beginning ( 0 min) and at the end of the clamp. The immunoblottting of the heart muscle revealed a single $90 \mathrm{KDa}$ band consistent with FAT/CD36. Each gel contained an equal number of samples from rats fed a CD, SRD and SRD + chia at $0 \mathrm{~min}$ and $120 \mathrm{~min}$ of euglycemic hyperinsulinemic clamp (Figure 2, top panel). After the densitometry of immunoblots, FAT/CD36 at the beginning of the clamp was normalized to $100 \%$, and both the SRD and SRD + chia groups at the beginning as well as the three dietary groups at the end of the study were expressed relative to this. At the beginning of the clamp, the qualitative and quantitative analysis of the Western blot showed a significant increase $(p<0.05)$ in the relative abundance of FAT/CD36 in the SRD-fed rats compared to the $\mathrm{CD}$ and SRD+chia groups. The latter reached values similar to those of the CD group. At the end of the clamp study, insulin stimulated the translocation of FAT/CD36 to the sarcolemma in the CD-fed rats that showed a significant $(p<0.05)$ increase of their protein mass level, while in the heart of SRD-fed rats, insulin did not further recruit FAT/CD36 to the sarcolemma under the same experimental conditions. Moreover, changes induced by the SRD were reverted by the chia seed enriched diet (Figure 2 bottom panel).

\subsection{M-CPT1 Activity and Protein Mass Level}

Figure 3a shows the cardiac muscle activity of the M-CPT1 in the three dietary groups. Compared with the CD-fed group, a three-fold increase of the mitochondrial M-CPT1 activity was observed in the heart of rats fed an SRD. The M-CPT1 activity was significantly reduced under the administration of chia seed. However, values were still higher than those recorded in the CD-fed rats. The CPT2 activity remained similar in the three dietary groups. Immunoblotting of cardiac muscle M-CPT1 revealed a single $75 \mathrm{KDa}$ band consistent with $\mathrm{M}-\mathrm{CPT} 1$. Each gel contained an equal number of samples from rats fed a CD, SRD and SRD+chia (Figure $3 b$ top panel). After densitometry of immunoblots the M-CPT1 of the CD group was normalized to $100 \%$, and both the SRD and SRD+chia groups were expressed relative to this. The qualitative and quantitative abundance of the Western blot showed that M-CPT1 protein mass level significantly increased $(p<0.05)$ in the heart muscle of the SRD group when compared with rats fed a CD (Figure $3 b$ bottom panel). Interestingly, although the enzymatic activity of M-CPT1 decreased when chia seed replaced $\mathrm{CO}$ as a source of fat in the SRD, the relative abundance of M-CPT1 was still significantly higher. 

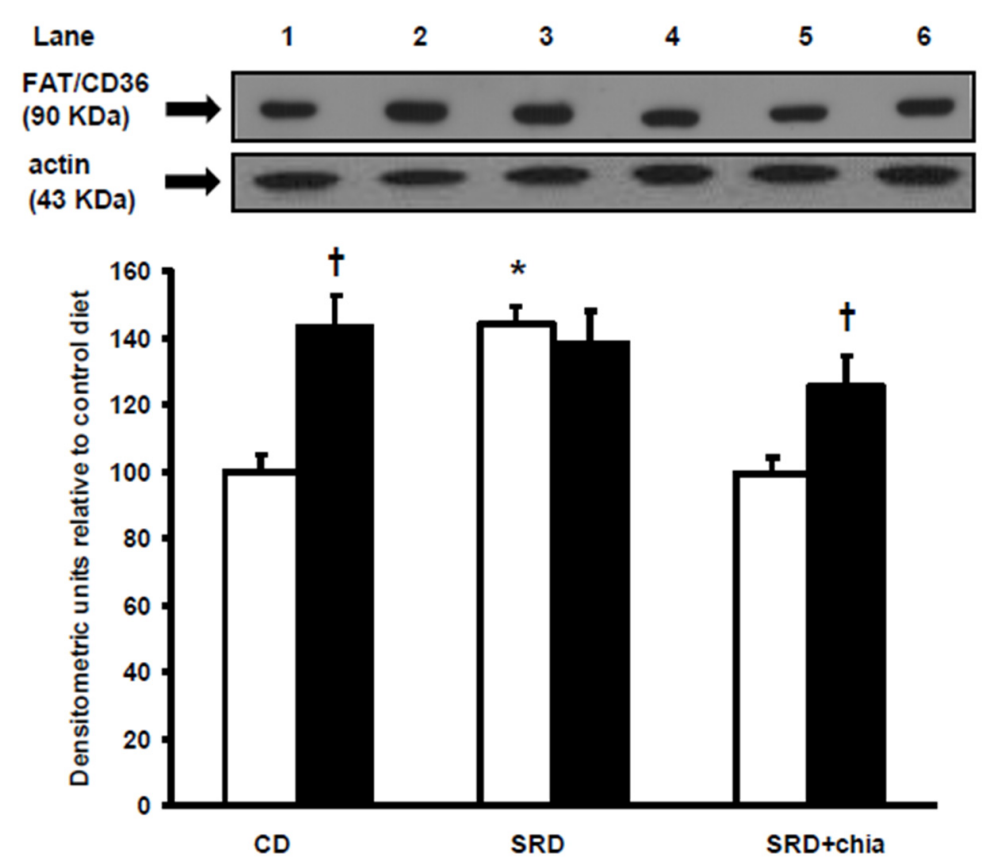

Values are mean, with their standard errors depicted by vertical bars (six animals per group) and expressed as percentage relative to the control diet at $0 \mathrm{~min}$ of the clamp. ${ }^{*} p<0.05 \mathrm{SRD}$ at $0 \mathrm{~min} v \mathrm{~s}$. CD and SRD+chia at $0 \mathrm{~min}$ of the clamp. $+p<0.05 \mathrm{CD}$ and SRD+chia at $120 \mathrm{~min} v s$. CD and SRD+chia at 0 min of the clamp.

Figure 2. Heart protein mass levels of FAT/CD36 at the beginning ( $0 \mathrm{~min})$ and under the insulin stimulation at the end $(120 \mathrm{~min})$ of the clamp studies in rats fed a control diet (CD), a sucrose-rich diet (SRD) or SRD + chia seed (SRD + chia). Top panel: a representative immunoblot of heart FAT/CD36 of rats fed CD, SRD and $\mathrm{SRD}+$ chia. Molecular marker is shown on the right. Lane 1, CD 0 min; lane 2, CD 120 min; lane 3, SRD 0 min; lane 4, SRD 120 min; lane 5, SRD + chia 0 min; lane 6, SRD + chia 120 min. Bottom panel: densitometric immunoblot analysis of heart FAT/CD36 protein mass level of rats fed CD, SRD or SRD + chia at the beginning (0 min $\square$ ) and at the end (120 min $\mathbf{\square})$ of clamp studies.

a

\begin{tabular}{cccc}
\hline $\begin{array}{c}\text { Oxidative enzyme activity } \\
{[\text { nmol/(mg protein x min) }]}\end{array}$ & CD & SRD & SRD +chia \\
\cline { 2 - 4 } M-CPT1 & $8.80 \pm 0.74^{\mathrm{c}}$ & $22.10 \pm 1.93^{\mathrm{a}}$ & $14.80 \pm 2.10^{\mathrm{b}}$ \\
CPT2 & $11.3 \pm 1.0$ & $12.8 \pm 1.0$ & $11.0 \pm 1.0$ \\
\hline
\end{tabular}

Figure 3. Cont. 


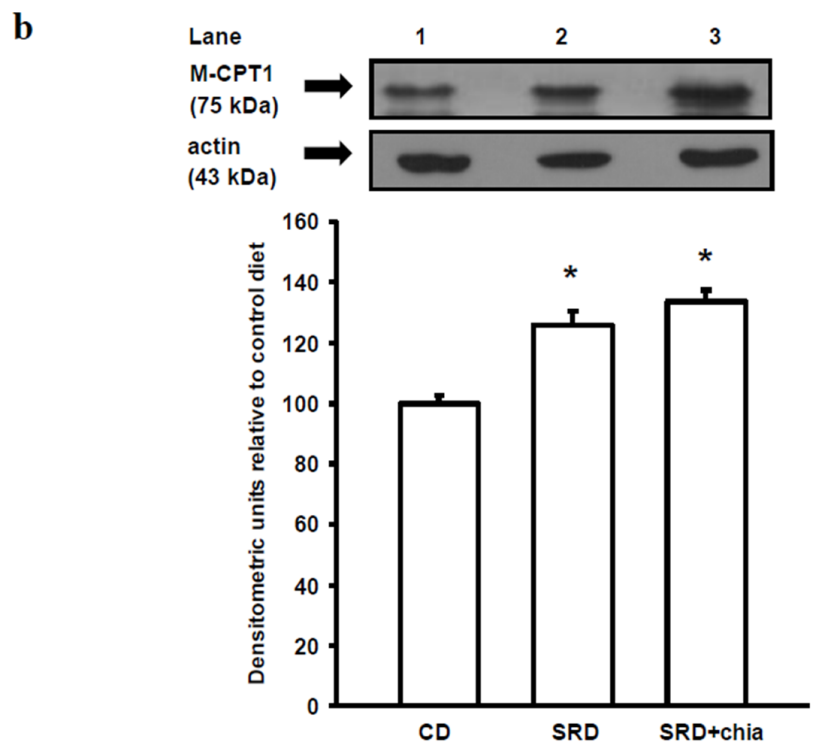

Values are mean, with their standard errors depicted by vertical bars (six animals per group) and expressed as percentage relative to the control diet. ${ }^{*} p<0.05$ SRD and SRD + chia vs. CD.

Figure 3. (a) Heart enzyme activities of M-CPT1 and CPT2 in rats fed a control diet (CD), sucrose-rich diet (SRD) or SRD+chia seed (SRD+chia). Values are expressed as mean \pm SEM (six animals per group). Values in a line that do not share the same superscript letter $(\mathbf{a}, \mathbf{b}, \mathbf{c})$ are significantly different $(p<0.05)$ when one variable at time was compared by the Newman Keul's test; (b) Top panel: a representative immunoblot of heart M-CPT1 protein mass level of rats fed CD, SRD and SRD + chia. Molecular marker is shown on the right. Lane 1, CD; lane 2, SRD; lane 3, SRD + chia. Bottom panel: densitometric immunoblot analysis of heart M-CPT1 protein mass level of rats fed CD, SRD or SRD + chia.

\subsection{PPAR $\alpha$ Protein Mass Level}

We examined the protein mass level of $\operatorname{PPAR} \alpha$, since this receptor is considered a master regulator of FAs metabolism in several organs including the heart. The immunoblotting of the cardiac muscle revealed a single 55KDa band consistent with PPAR $\alpha$. Each gel contained an equal number of samples from the CD, SRD and SRD + chia groups (Figure 4 top panel). After the densitometry of immunoblots, the PPAR $\alpha$ of the CD group was normalized to $100 \%$, and both the SRD and SRD + chia groups were expressed relative to this. The qualitative and quantitative abundance of the Western blot showed that the relative abundance of the PPAR $\alpha$ protein mass level significantly increased $(p<0.05)$ in the hearts of both the SRD and SRD + chia groups, although the protein mass level was slightly lower without statistical differences in 
the latter group (Figure 4 bottom panel). On the other hand, we measured the protein mass levels of UCP2 since this uncoupling protein could be regulated by increased FA concentration through PPAR $\alpha$ activation. The qualitative and quantitative analysis of the Western blots showed that the relative abundance of mitochondrial UCP2 protein mass level was similar in the hearts of the three dietary groups. Values were as follows: (mean \pm SEM, $n=6$ ); CD $100 \pm 3.2$; SRD $103.8 \pm 2.5$ and SRD + chia $95.7 \pm 5.3, \mathrm{p}$ NS.

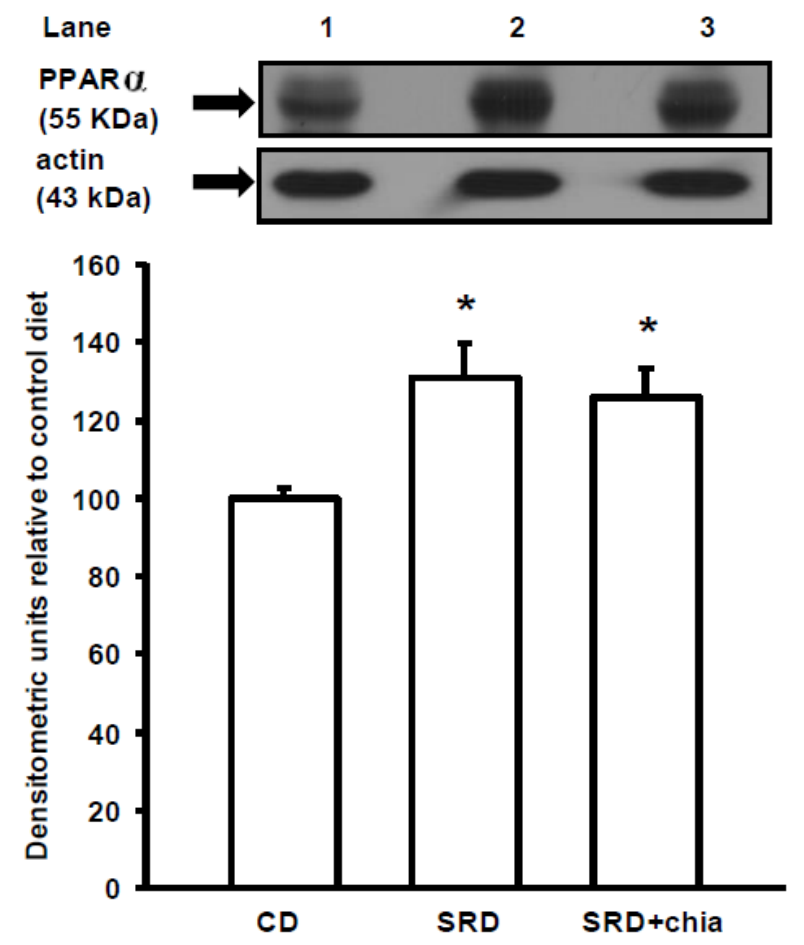

Figure 4. Heart protein mass level of PPAR $\alpha$ in rats fed a control diet (CD), sucrose-rich diet (SRD) or SRD + chia seed (SRD + chia). Top panel: a representative immunoblot of heart PPAR $\alpha$ of rats fed a CD, SRD and SRD + chia. Molecular marker is shown on the right. Lane 1 CD; lane 2 SRD; lane 3 SRD + chia. Bottom panel: densitometric immunoblot analysis of heart PPAR $\alpha$ protein mass level of rats fed CD, SRD or SRD + chia.. Values are mean, with their standard errors depicted by vertical bars ( 6 animals per group) and expressed as percentage relative to the control diet. ${ }^{*} p<0.05$ SRD and SRD + chia vs. CD. 


\section{Discussion}

The present study provides new information on the mechanisms involved in heart muscle lipotoxicity in dyslipemic insulin resistant rats fed an SRD and explores the possible beneficial effects of dietary chia supplementation on reversed or improved pre-exiting impaired cardiac lipid metabolism. Disruption of the sensitive balance between FAs and glucose in the heart and increased intramyocellular fat contents and fatty acid metabolites are likely to play a pivotal role in the development of insulin resistance, cardiac lipotoxicity and heart dysfunction [36]. FAT/CD36 plays a pivotal role in governing myocardial FAs uptake [8]. In the present work, the increased intracellular LC ACOA in the heart of SRD-fed rats is accompanied by a significant increase of protein mass level of FAT/CD36 in the plasma membrane, suggesting that the enhanced amount of FAT/CD36 on sarcolemma elicits an increased rate of FAs uptake. In this regard, an increased availability of plasma free fatty acids and triglyceride levels is recorded in the SRD-fed group. Moreover, despite a significant increase of both the M-CPT1 activity and its protein mass level, triglyceride accumulates in the heart of this dietary group. It is possible that the increased flux of FAs to the heart exceeds the mitochondrial oxidative capacity leading to an increase of FAs storage into the triglyceride pool. The dynamic equilibrium between triglyceride stores and their metabolites cause accumulation of DAG and ceramide during prolonged long-chain fatty acid (LCFA) influx. Although in the present study we did not measure the level of ceramide, our previous results [16] and the present data show an increase of DAG concentration in the heart of rats fed an SRD. Both metabolites are implicated in counteracting insulin signaling, reducing insulin responsiveness and altering its ability to regulate substrate handling [4]. In this regard, the present data show that insulin stimulated the cell surface recruitment of FAT/CD36 in the heart of CD-fed rats. However, insulin was unable to further recruit FAT/CD36 to the sarcolemma in the heart of SRD-fed rats that was completely insensitive to the stimulus of the hormone. Similarly, in cardiac myocytes from obese Zucker rats, Coort et al. [10] reported that insulin failed to alter the sub cellular localization of FAT/CD36 and the rate of LCFA uptake and triglyceride esterification. Besides, in cardiamyocytes of Wistar rats in which a high-fat diet induced cardiac contractile dysfunction, Ouwens et al. [5] demonstrated that a permanent presence of FAT/CD36 in the sarcolemma membrane resulted in the enhancement of LCFA uptake and myocardial triglyceride accumulation.

PPAR $\alpha$ and its co-activator PPAR $\gamma$ co-activator 1 alpha (PGC- $1 \alpha$ ) play an important role in the transcriptional regulation of cardiac energy metabolism, and the effect of FAs in cardiac myocytes is considered to be PPAR $\alpha$ mediated [37]. Several lines of evidence suggested that LCFAs that induce the gene expression of M-CPT1 and other enzymes in the cellular fatty acid utilization pathway are namely mediated by PPAR $\alpha$ transcriptional control [13]. LCFAs: linoleic acid 18:2 n-6, ALA 
and docosahexaenoic acid 22:6, n-3 (DHA) among them, and a variety of related compounds serve as PPAR $\alpha$ ligands [38]. In this context, the present study shows a significant increase of the relative abundance of the protein mass level of PPAR $\alpha$ and the mitochondrial M-CPT1 activity in the heart of SRD-fed rats. These results suggest that a chronic high exposure to Fas, which enhances their uptake, induces the activation of PPAR $\alpha$ protein expression that, in turn, encodes the proteins responsible for FAs oxidation, M-CPT1 among others. Since an increase of intramyocardial lipids is observed in the SRD heart, it is possible that a disruption of the balance between lipid oxidation-storage occurs in the heart muscle of this dyslipemic insulin-resistant model. In this vein, Buchanam et al. [39] have documented an increased PPAR $\alpha$ and PGC-1 $\alpha$ expression in murine insulin-resistant hearts. A high fat diet also activates PPAR $\alpha$ in the heart and stimulates expression of key proteins involved in fatty acid oxidation [40].

It is well known that dietary n-3 PUFAs, mainly eicosapentaenoic acid 20:5, n-3 (EPA) and DHA, improve cardiac function [17]. ALA could be a valuable source of $n-3$ long-chain FAs via elongase/desaturase activities. Dietary ALA exerts a protective effect on the CVD [18]. In this regard, and confirming previous results, the present work demonstrates a reversion of dyslipidemia, abnormal glucose homeostasis and whole body peripheral insulin insensitivity when dietary chia seed replaced $\mathrm{CO}$ in the SRD-fed rats. The reverse of dyslipidemia by chia seed led to a significant reduction of lipid storage in the heart of SRD-fed rats, reaching values similar to those observed in the heart of CD-fed rats. Moreover, at basal conditions (beginning of the clamp study) a decrease of FAT/CD36 protein mass level suggests that a different milieu (decreased plasma lipids levels) prevented the robust relocations of fatty acid translocase to the sarcolemma, which was otherwise seen in the cardiac muscle of SRD-fed rats and, therefore, reduced the influx of FAs. Furthermore, the heart of dietary chia-fed rats was sensitive to the stimulus of insulin. As in the CD-fed group, the hormone significantly induced the translocation of FAT/CD36 to the plasma membrane. Recently, in isolated rat cardiomyocytes incubated under insulin resistance evoking conditions, Franekova et al. [41] demonstrated that the inclusion of EPA and DHA to the medium prevented the persistent translocation of CD36 to the sarcolemma and protected the metabolic and functional properties of the cardiomyocytes. In this regard, we previously demonstrated that the administration of dietary fish oil to SRD-fed rats was able to reverse heart muscle lipotoxicity and benefit key enzyme activities involved in the glucose metabolism [16]. At present, we are unaware of other studies concerning the effect of the long-term consumption of dietary chia and/or ALA on lipid metabolism in the cardiac muscle of SRD-fed rats. However, the reversion of the impaired glucose oxidation, as well as the accretion of triglyceride and fatty acid derivatives, the normalization of the enhanced sarcolemmal FAT/CD36 and the significantly reduced mitochondrial oxidative flux 
suggests that dietary chia seeds could improve the altered balance of heart fuel utilization. Interestingly, compared with CD-fed rats the protein mass level of the nuclear receptor PPAR $\alpha$ and the activity of its target enzyme M-CPT1 were still higher in the heart of the SRD + chia group. Our results do not provide data on the mechanisms underlying the effect of chia seed on this nuclear receptor but it was shown that ALA and DHA, among others, are natural occurring ligands of PPAR $\alpha$ in the heart [38]. In this regard, it has been demonstrated by our group [23] and others $[21,42]$ that chia seeds change the plasma fatty acid profile increasing ALA, EPA, docosapentaenoic acid 22:5, n-3 (DPA) and DHA levels as well as the $n-3 / n-6$ FAs ratio in rats fed an SRD, a control diet, or a high fat, high fructose diet. Thus, we do not discard the possibility that the different plasma FA profiles to which the heart was exposed could contribute to this finding.

On the other hand, an increase of FAs could induce UCP2 expression through PPAR $\alpha$ activation in adult rat cardiomyocytes [43]. Besides, an increase of UCP2 and PPAR $\alpha$ was recorded in the heart of ob/ob mice [39]. However, under the present experimental protocol, our results showed that the mitochondrial protein mass levels of UCP2 were similar in the three experimental groups. Besides, we are unaware of any other studies that evaluated the potential role of UCP2 in altering myocardial substrate in the heart muscle of SRD rats, and the effect of either chia seeds or oil upon UCP2. Further studies will be needed to evaluate this matter.

Chia seeds were able to decrease the systolic blood pressure that developed in the SRD-fed rats. In this regard, Poudyal et al. [21], in rats fed a high fructose-high fat diet, recorded that chia seed normalized systolic blood pressure increasing DPA and DHA contents in FAs of the heart, and Rousseau et al. [44] showed a decrease in blood pressure and increased DHA and EPA in cardiac phospholipids in rats fed a high fructose diet supplemented with either DHA or EPA. Interestingly, we observed an increase of DHA and ALA in the FA phospholipids of cardiac membrane in the SRD + chia group (data not shown). Moreover, Vuksan et al. [45] showed that a long-term supplementation with Salba (S. hispanica L) attenuates systolic blood pressure and emerging cardiovascular risk factors, safely beyond conventional therapy, while maintaining good glycemic and lipid control in well-controlled type-2 diabetic patients.

\section{Conclusions}

In brief, this study demonstrated that the lipotoxicity present in the heart of SRD-fed rats, an experimental model of dyslipidemia and insulin resistance, is accompanied by changes involving lipid metabolism suggesting an impaired myocardial lipid utilization. In this scenario, and for the first time, this work also provides new information concerning the possible mechanisms underlying the beneficial effects of dietary chia seeds on lipid cardiac metabolism and glucose 
oxidation. Although caution is warranted before extrapolating results from rodents to humans, chia seeds may serve as an alternative dietary strategy in the management of these metabolic alterations susceptible to dietary manipulation.

Acknowledgments: The authors wish to thank Silvia Rodriguez and Walter Daru for their skillful technical assistance and Adriana Chicco for her valuable suggestions throughout the development of this study. The present study was carried out with the financial support of Agencia Nacional de Promoción Científica y Tecnológica (ANPCyT) (grants PICT 945 BID OC/AR 2011) and University of Litoral (CAI+D 50120110100058 LI-2012). The authors thank Agrisalba S. A. Buenos Aires, Argentina for providing the chia seeds Salba.

Author Contributions: Agustina Creus was involved in the design of the experimental protocol and performed the experiments. María R. Ferreira was involved in the analysis of protein mass levels and contributes of the analysis of the results. María E. Oliva was involved on the determination of plasma and tissues metabolites. Yolanda B. Lombardo designs the experimental protocol, wrote the manuscripts and discussed it with the whole group of authors.

Conflicts of Interest: The authors declare no conflict of interest.

\section{References}

1. Pollex, R.L.; Hegele, R.A. Genetic determinants of the metabolic syndrome. Nat. Clin. Pract. Cardiovasc. Med. 2006, 3, 482-489.

2. Gaziano, T.A.; Bitton, A.; Anand, S.; Abrahams-Gessel, S.; Murphy, A. Growing epidemic of coronary heart disease in low- and middle- income countries. Curr. Probl. Cardiol. 2010, 35, 72-115.

3. Mazumder, P.K.; O’Neill, B.T.; Roberts, M.W.; Buchanan, J.; Yun, U.J.; Cooksey, R.C.; Boudina, S.; Abel, E.D. Impaired cardiac efficiency and increased fatty acid oxidation in insulin-resistant $\mathrm{Ob} / \mathrm{Ob}$ mouse hearts. Diabetes 2004, 53, 2366-2374.

4. Glatz, J.F.; Angin, Y.; Steinbusch, L.K.; Schwenk, R.W.; Luiken, J.J. CD36 as a target to prevent cardiac lipotoxicity and insulin resistance. Prostaglandins Leukot. Essent. Fatty Acids 2013, 88, 71-77.

5. Ouwens, D.M.; Diamant, M.; Fodor, M.; Habets, D.D.; Pelsers, M.M.; El, H.M.; Dang, Z.C.; van den Brom, C.E.; Vlasblom, R.; Rietdijk, A.; et al. Cardiac contractile dysfunction in insulin-resistant rats fed a high-fat diet is associated with elevated CD36-mediated fatty acid uptake and esterification. Diabetologia 2007, 50, 1938-1948.

6. Zhou, Y.T.; Grayburn, P.; Karim, A.; Shimabukuro, M.; Higa, M.; Baetens, D.; Orci, L.; Unger, R.H. Lipotoxic heart disease in obese rats: Implications for human obesity. Proc. Natl. Acad. Sci. USA 2000, 97, 1784-1789.

7. Paulson, D.J.; Crass, M.F., III. Endogenous triacylglycerol metabolism in diabetic heart. Am. J. Physiol. 1982, 242, H1084-H1094.

8. Van Oort, M.M.; van Doorn, J.M.; Bonen, A.; Glatz, J.F.; van der Horst, D.J.; Rodenburg, K.W.; Luiken, J.J. Insulin-induced translocation of CD36 to the plasma membrane is reversible and shows similarity to that of GLUT4. Biochim. Biophys. Acta 2008, 1781, 61-71. 
9. Chiu, H.C.; Kovacs, A.; Ford, D.A.; Hsu, F.F.; Garcia, R.; Herrero, P.; Saffitz, J.E.; Schaffer, J.E. A novel mouse model of lipotoxic cardiomyopathy. J. Clin. Investig. 2001, $107,813-822$.

10. Coort, S.L.; Hasselbaink, D.M.; Koonen, D.P.; Willems, J.; Coumans, W.A.; Chabowski, A.; van der Vusse, G.J.; Bonen, A.; Glatz, J.F.; Luiken, J.J. Enhanced sarcolemmal FAT/CD36 content and triacylglycerol storage in cardiac myocytes from obese Zucker rats. Diabetes 2004, 53, 1655-1663.

11. Finck, B.N.; Han, X.; Courtois, M.; Aimond, F.; Nerbonne, J.M.; Kovacs, A.; Gross, R.W.; Kelly, D.P. A critical role for PPARalpha-mediated lipotoxicity in the pathogenesis of diabetic cardiomyopathy: Modulation by dietary fat content. Proc. Natl. Acad. Sci. USA 2003, 100, 1226-1231.

12. Barger, P.M.; Kelly, D.P. PPAR signaling in the control of cardiac energy metabolism. Trends Cardiovasc. Med. 2000, 10, 238-245.

13. Brandt, J.M.; Djouadi, F.; Kelly, D.P. Fatty acids activate transcription of the muscle carnitine palmitoyltransferase I gene in cardiac myocytes via the peroxisome proliferator-activated receptor alpha. J. Biol. Chem. 1998, 273, 23786-23792.

14. Lombardo, Y.B.; Chicco, A.G. Effects of dietary polyunsaturated n-3 fatty acids on dyslipidemia and insulin resistance in rodents and humans. A review. J. Nutr. Biochem. 2006, 17, 1-13.

15. Montes, M.; Chicco, A.; Lombardo, Y.B. The effect of insulin on the uptake and metabolic fate of glucose in isolated perfused hearts of dyslipemic rats. J. Nutr. Biochem. 2000, 11, 30-37.

16. D'Alessandro, M.E.; Chicco, A.; Lombardo, Y.B. Dietary fish oil reverses lipotoxicity, altered glucose metabolism, and nPKCepsilon translocation in the heart of dyslipemic insulin-resistant rats. Metabolism 2008, 57, 911-919.

17. Jump, D.B.; Depner, C.M.; Tripathy, S. Omega-3 fatty acid supplementation and cardiovascular disease. J. Lipid Res. 2012, 53, 2525-2545.

18. Djousse, L.; Arnett, D.K.; Carr, J.J.; Eckfeldt, J.H.; Hopkins, P.N.; Province, M.A.; Ellison, R.C. Dietary linolenic acid is inversely associated with calcified atherosclerotic plaque in the coronary arteries: The National Heart, Lung, and Blood Institute Family Heart Study. Circulation 2005, 111, 2921-2926.

19. Mozaffarian, D.; Ascherio, A.; Hu, F.B.; Stampfer, M.J.; Willett, W.C.; Siscovick, D.S.; Rimm, E.B. Interplay between different polyunsaturated fatty acids and risk of coronary heart disease in men. Circulation 2005, 111, 157-164.

20. Folino, A.; Sprio, A.E.; di Scipio, F.; Berta, G.N.; Rastaldo, R. Alpha-linolenic acid protects against cardiac injury and remodeling induced by beta-adrenergic overstimulation. Food Funct. 2015, 6, 2231-2239.

21. Poudyal, H.; Panchal, S.K.; Ward, L.C.; Waanders, J.; Brown, L. Chronic high-carbohydrate, high-fat feeding in rats induces reversible metabolic, cardiovascular, and liver changes. Am. J. Physiol. Endocrinol. Metab. 2012, 302, E1472-E1482. 
22. Poudyal, H.; Panchal, S.K.; Ward, L.C.; Brown, L. Effects of ALA, EPA and DHA in high-carbohydrate, high-fat diet-induced metabolic syndrome in rats. J. Nutr. Biochem. 2013, 24, 1041-1052.

23. Chicco, A.G.; D’Alessandro, M.E.; Hein, G.J.; Oliva, M.E.; Lombardo, Y.B. Dietary chia seed (Salvia hispanica L.) rich in alpha-linolenic acid improves adiposity and normalises hypertriacylglycerolaemia and insulin resistance in dyslipaemic rats. Br. J. Nutr. 2009, 101, 41-50.

24. Rossi, A.S.; Oliva, M.E.; Ferreira, M.R.; Chicco, A.; Lombardo, Y.B. Dietary chia seed induced changes in hepatic transcription factors and their target lipogenic and oxidative enzyme activities in dyslipidaemic insulin-resistant rats. Br. J. Nutr. 2013, 109, 1617-1627.

25. Oliva, M.E.; Ferreira, M.R.; Chicco, A.; Lombardo, Y.B. Dietary salba (Salvia hispanica L) seed rich in alpha-linolenic acid improves adipose tissue dysfunction and the altered skeletal muscle glucose and lipid metabolism in dyslipidemic insulin-resistant rats. Prostaglandins Leukot. Essent. Fatty Acids 2013, 89, 279-289.

26. Laurell, S. A method for routine determination of plasma triglycerides. Scan. J. Clin. Lab. Investig. 1966, 18, 668-672.

27. Lowenstein, J.M. Citric Acid cycle. In Methods in Enzymology; Academic Press: New York, NY, USA, 1969; pp. 450-468.

28. Schmitz-Peiffer, C.; Browne, C.L.; Oakes, N.D.; Watkinson, A.; Chisholm, D.J.; Kraegen, E.W.; Biden, T.J. Alterations in the expression and cellular localization of protein kinase $C$ isozymes $\varepsilon$ and $\theta$ are associated with insulin resistance in skeletal muscle of the high-fat-fed rat. Diabetes 1997, 46, 169-178.

29. Ling, B.; Aziz, C.; Alcorn, J. Systematic evaluation of key L-carnitine homeostasis mechanisms during postnatal development in rat. Nutr. Metab (Lond.) 2012, 9, 66.

30. Chicco, A.; D’Alessandro, M.E.; Karabatas, L.; Pastorale, C.; Basabe, J.C.; Lombardo, Y.B. Muscle lipid metabolism and insulin secretion are altered in insulin-resistant rats fed a high sucrose diet. J. Nutr. 2003, 133, 127-133.

31. Rodnick, K.J.; Slot, J.W.; Studelska, D.R.; Hanpeter, D.E.; Robinson, L.J.; Geuze, H.J.; James, D.E. Immunocytochemical and biochemical studies of GLUT4 in rat skeletal muscle. J. Biol. Chem. 1992, 267, 6278-6285.

32. Bogazzi, F.; Raggi, F.; Ultimieri, F.; Russo, D.; D'Alessio, A.; Manariti, A.; Brogioni, S.; Manetti, L.; Martino, E. Regulation of cardiac fatty acids metabolism in transgenic mice overexpressing bovine GH. J. Endocrinol. 2009, 201, 419-427.

33. Pecqueur, C.; Alves-Guerra, M.C.; Gelly, C.; Levi-Meyrueis, C.; Couplan, E.; Collins, S.; Ricquier, D.; Bouillaud, F.; Miroux, B. Uncoupling protein 2, in vivo distribution, induction upon oxidative stress, and evidence for translational regulation. J. Biol. Chem. 2001, 276, 8705-8712.

34. Glantz, S.A. Primer of Biostatistics; McGraw Hill: New York, NY, USA, 2005.

35. Snedecor, G.W.; Cochran, W.G. Factorial Experiments, in Statistical Methods Applied to Experimental in Agriculture and Biology; Iowa State University Press: Ames, IA, USA, 1967; pp. 339-350. 
36. Chess, D.J.; Stanley, W.C. Role of diet and fuel overabundance in the development and progression of heart failure. Cardiovasc. Res. 2008, 79, 269-278.

37. Duncan, J.G. Peroxisome proliferator activated receptor-alpha (PPARalpha) and PPAR gamma coactivator-1alpha (PGC-1alpha) regulation of cardiac metabolism in diabetes. Pediatr. Cardiol. 2011, 32, 323-328.

38. Georgiadi, A.; Boekschoten, M.V.; Muller, M.; Kersten, S. Detailed transcriptomics analysis of the effect of dietary fatty acids on gene expression in the heart. Physiol. Genom. 2012, 44, 352-361.

39. Buchanan, J.; Mazumder, P.K.; Hu, P.; Chakrabarti, G.; Roberts, M.W.; Yun, U.J.; Cooksey, R.C.; Litwin, S.E.; Abel, E.D. Reduced cardiac efficiency and altered substrate metabolism precedes the onset of hyperglycemia and contractile dysfunction in two mouse models of insulin resistance and obesity. Endocrinology 2005, 146, 5341-5349.

40. Stanley, W.C.; Dabkowski, E.R.; Ribeiro, R.F., Jr.; O'Connell, K.A. Dietary fat and heart failure: Moving from lipotoxicity to lipoprotection. Circ. Res. 2012, 110, 764-776.

41. Franekova, V.; Angin, Y.; Hoebers, N.T.; Coumans, W.A.; Simons, P.J.; Glatz, J.F.; Luiken, J.J.; Larsen, T.S. Marine omega-3 fatty acids prevent myocardial insulin resistance and metabolic remodeling as induced experimentally by high insulin exposure. Am. J. Physiol. Cell Physiol. 2015, 308, C297-C307.

42. Ayerza, R.; Coates, W. Ground chia seed and chia oil effects on plasma lipids and fatty acids in the rat. J. Endocrinol. 2005, 25, 995-1003.

43. Li, N.; Wang, J.; Gao, F.; Tian, Y.; Song, R.; Zhu, S.J. The role of uncoupling protein 2 in the apoptosis induced by free fatty acid in rat cardiomyocytes. J. Cardiovasc. Pharmacol. 2010, 55, 161-167.

44. Rousseau, D.; Helies-Toussaint, C.; Moreau, D.; Raederstorff, D.; Grynberg, A. Dietary $n-3$ PUFAs affect the blood pressure rise and cardiac impairments in a hyperinsulinemia rat model in vivo. Am. J. Physiol. Heart Circ. Physiol. 2003, 285, H1294-H1302.

45. Vuksan, V.; Whitham, D.; Sievenpiper, J.L.; Jenkins, A.L.; Rogovik, A.L.; Bazinet, R.P.; Vidgen, E.; Hanna, A. Supplementation of conventional therapy with the novel grain Salba (Salvia hispanica L.) improves major and emerging cardiovascular risk factors in Type 2 diabetes: Results of a randomized controlled trial. Diabetes Care 2007, 30, 2804-2810. 

Chapter 4:

Basic Research 



\section{Lipidomics to Assess Omega 3 Bioactivity}

\section{Francesco Visioli}

Abstract: How can we resolve the conflict between the strong epidemiological evidence pointing to the usefulness of fish-and, thus, omega 3-consumption with the debacle of supplementation trials? One potential explanation is that the null results obtained thus far are the consequences of ill-contrived investigations that do not allow us to conclude on the effects (or lack thereof) of omega 3 fatty acid supplementation. One potential solution is through the use of lipidomics, which should prove very useful to screen suitable patients and to correlate plasma (or red blood cells, or whole blood, or phospholipid) fatty acid profile with outcomes. This has never been done in omega 3 trials. The wise use of lipidomics should be essential part of future omega 3 trials and would help in untangling this current riddle.

Reprinted from J. Clin. Med. Cite as: Visioli, F. Lipidomics to Assess Omega 3 Bioactivity. J. Clin. Med. 2015, 4, 1753-1760.

\section{Introduction}

Fish consumption is directly associated with better cardiovascular prognosis [1]. From the first observations made in Greenland to the latest randomized controlled trials (RCT), long-chain omega 3 fatty acids, namely docosahexaenoic (DHA) and eicosapentaenoic (EPA) fatty acids have been held responsible for the health effects of fish [1]. One notable example is that of the omega 3 index, which is influenced by omega 3 consumption and is a strong predictor of cardiovascular risk [2]. Consequently, much research has been devoted to elucidating, in vitro and in vivo, the protective activities of EPA and DHA; indeed, the general perception is that supplementary intake of these fatty acids would protect from cardiovascular disease and other degenerative pathologies. Suggesting consumption of adequate amounts of omega 3 fatty acids actually makes biological sense; these fatty acids are important in that mammals cannot efficiently synthesize them and they are indispensable to a number of biological processes, especially those that involve excitable cells such as cardiomyocytes, neurons, retinal cells, etc. [1]. In addition, nearly every randomized clinical trial reported that omega 3 fatty acids positively modulate surrogate markers of cardiovascular disease and in vitro studies explored a wide range of mechanisms underlying the purported beneficial activities of EPA and DHA. The sad truth is that, when given as supplements, long chain omega 3 fatty acids are-very likely-ineffective in affording better health [3]. The big question is why [4]? What is wrong [5]? Indeed, this is probably the major current conundrum in pharma-nutrition research, with fish oils threatening to join the vitamin E, beta-carotene, resveratrol, 
etc. in the dustbin of promising nutritional therapies that failed to show benefit in RCTs [6-8].

\section{Omega 3 Fatty Acids in Cardiovascular Therapy}

How can we reconcile this apparent failure with strong epidemiological evidence pointing to the usefulness of fish-and, thus, omega 3-consumption? One potential explanation is that the null results obtained thus far are the consequences of ill-contrived trials that do not allow one to draw firm conclusions on the effects (or lack thereof) of omega 3 fatty acid supplementation [9].

In this respect, one paradigmatic example is that of folate in stroke prevention. In a well-designed trial recently published by Huo et al. [10], the highest risk of stroke and the greatest benefit of folic acid therapy were seen in patients with the lowest baseline folate levels. In addition, Huo et al. [10] suggest that individuals with the TT genotype may require a higher dosage of folic acid supplementation to overcome biologically-insufficient levels (as reflected in the relatively greater folate requirement for subjects with the TT genotype).

Unfortunately, neither basal plasma concentrations nor genotype/nutrigenomics issues have ever been taken into consideration in omega 3 supplementation studies [9]. The natural consequence is that the near totality of omega 3 trials could not really conclude that modulation of omega 3 status is ineffective. This situation is unheard of in classic drug research, where surrogate markers, e.g., blood pressure of cholesterol concentrations, are mandatorily assessed before and after interventions on these risk factors.

In this respect, the use of lipidomics in RCTs should prove very useful to screen patients first and to correlate plasma (or red blood cells, or whole blood, or phospholipid) fatty acid profile with outcomes.

\section{Lipidomics}

From a technical viewpoint, lipidomics can be conveniently classified into as two distinct approaches: A "shotgun", i.e., electrospray ionization coupled with single stage or tandem mass spectrometry approach and a liquid chromatography-mass spectrometry (LC-MS)-based one. The shotgun approach does not require separation prior to MS analyses. The shotgun approach is being considered as the best method for analysis of lipid extracts. Its major advantage over other methods is that mixtures of lipids are directly infused and sprayed into the mass spectrometer, therefore analyzing all the molecules collectively [11]. In this way, shotgun lipidomics, when applied to whole tissue lipid extracts, generates molecular ions without extensive fragmentation of the molecules. This type of lipidomics allows the identification and quantitation of the lipids in complex mixtures and provides helpful global information about cellular and tissue lipidomes. The procedures generate 
quasi-molecular ions in high yields. The major limitation of shotgun lipidomics is that the most abundant or most efficiently ionized molecules "quench" the signal of other ones, in turn causing ion suppression and hampering detection of lipids that are present in lower concentrations [12]. Therefore, intra-source separation is often employed to overcome this limitation. Mostly, the analytical setting of lipidomics involves a triple-quadrupole mass spectrometer with electrospray ionization (ESI, primarily negative mode) in selective reaction monitoring (SRM) or multiple reaction monitoring (MRM) to maximize sensitivity [12]. Whereas reversed-phase chromatography, i.e., $\mathrm{C} 18$, is the first separation choice, hydrophilic interaction liquid chromatography (HILIC) is best for phospholipids, sphingolipids, and phosphatidylcholines [12]. In summary, the most appropriate methodological approach should be based on the goals of the analysis and, in the future, we might witness a combination of techniques that would match ease of use with accuracy.

When mass spectral equipment is available in clinical laboratories, lipidomics can be applied to clinical studies. Current bottlenecks include sample handling methods and data reduction and analysis of the enormous volume of data generated by contemporary mass spectrometric analysis. However, this rapidly evolving field can rapidly elucidate the lipidome in medical samples of small size. Of note, current techniques can identify thousands of distinct lipid molecular species, of which the most biologically important ones are fatty acyls, glycerolipids (neutral), glycerophospholipids (polar), sphingolipids, sterols, and prenols [13,14].

\section{Lipidomics Applied to Fatty Acid Research}

As mentioned, the lipidome provides useful information on the basal omega 3 status of patients or healthy volunteers and can greatly help resolve the current omega 3 conundrum in cardiovascular research/clinic. To date, lipidomics have been applied to observational and preclinical models of cardiovascular disease, including mice, hamsters, rabbits, and pigs, and nonhuman primates.

Example of observational research include the use of lipid profiling in diabetes mellitus [15,16], cardiovascular disease [17,18], metabolic disorders [19], dietary habits, and obesity [20], as well as in determining response to drug therapy (elegantly reviewed by Hinterwirth et al. [13]). These techniques can provides useful information on the lipid composition and profile of whole blood or of tissues e.g., the retina [21], atherosclerotic plaques [22], muscles [23], or adipose tissue [24].

In terms of cardiovascular disease and lipid profiling, some examples are worth our attention. The use of olive oil as a predominant source of fat has been consistently associated with better cardiovascular prognosis. Most researchers are still attributing the purported healthful properties of olive oil to its relatively high monounsaturated fatty acids (MUFAs) content [25]. The theory behind this is that MUFAs, namely oleic acid, favorably modifies the high-density lipoprotein/low-density 
lipoprotein (HDL/LDL) ratio and, therefore, afford cardiovascular protection [25]. Yet, accumulated evidence clearly indicates that MUFAs only mildly modify the HDL/LDL ratio, to an extent that should minimally influence cardiovascular risk [26]. Moreover, at least two lipidomic studies $[27,28]$ show that the proportion of MUFAs is higher in red blood cells and whole blood, respectively, of myocardial infarct patients as compared with controls, confirming findings from the large dataset of Stegeman et al. [17]. In summary, the hypothesis that MUFAs are cardioprotective and that increasing their intake would benefit the cardiovascular system is still unproven.

Lipidomics have also been employed in pharmaceutical research [11], namely to assess the effects of selected drugs on hypertension [29] and cardiovascular disease, in particular when recombinant HDL [16] and statins [30] were employed.

Within the context of this paper, it is worth reiterating that lipidomics have never been applied to omega 3 intervention trials and that precisely this improper approach is leading to pallid conclusions on their therapeutic roles.

\section{Examples of Lipidomics Applied to Omega 3 Research}

As mentioned, the near totality of omega 3 trials did not include baseline or post-treatment evaluations of omega 3 status in patients of healthy volunteers. In rats, lipidomics have been successfully employed by Lamaziere and coworkers to assess the effects of omega 3 supplementation on hepatic lipogenesis [31] and brain composition [32]. Additionally, this technique allowed the identification of the in vitro concentrations to be employed in cardiac biochemistry studies [33]. It is worth noting that, even though rats are given identical feed, their tissutal omega 3 status varies greatly [31-33]. One can only speculate that these differences are enhanced in humans who consume varied diets. Additionally, omega 3 supplementation reduces such inter-individual variations (Figure 1) and renders the omega 3 composition of organs and tissues more homogeneous [31-33].

Another mechanistic application of lipidomics to omega 3 research concerns the differential distribution of DHA and EPA in the various lipid classes. As an example, the former mostly accumulates into phospholipids whereas the latter contributes to cholesterol esters [34]. Likely, the biological importance of phospholipids is higher than that of cholesterol esters because they originate from important lipid mediators; their compositional modulation by diet or supplement should result into more relevant biological actions.

Finally, we should not forget that the in vivo effects of omega 3 fatty acids likely depend on capsules' formulation, i.e., tryacylglycerols vs. ethyl esters vs. phospholipids [35] and on whether they are taken as supplements or as part of food items (either food as such [36] or functional food such as milk [37,38]). It is conceivable that omega 3 fatty acids' bioavailability and subsequent distribution in 
the body might be different depending on the matrix [35]. This can be explored by proper lipidomic approaches.

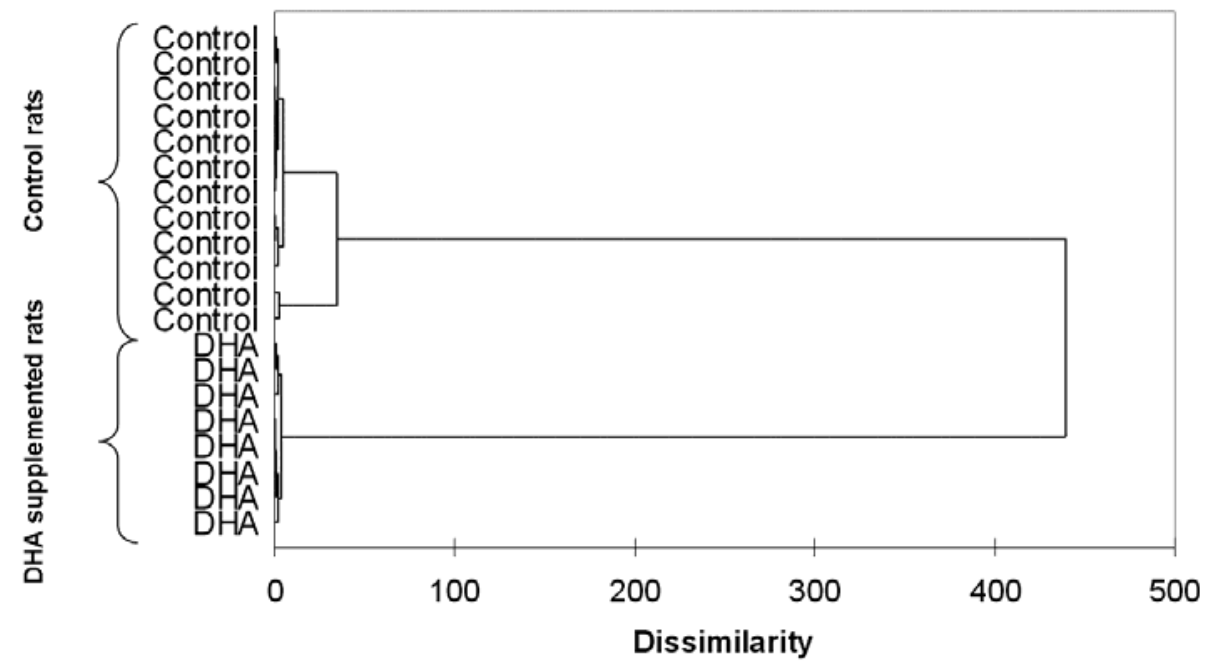

Figure 1. Example of lipidomics applied to omega 3 research: dendrogram resulting from the hierarchical cluster analysis of atrial rat cardiomyocytes isolated from control and docosahexaenoic (DHA)-treated rats. Unpublished data. Methodological details can be found in Lamaziere et al. [33].

In recent years, much investigation has been dedicated to bioactive small molecules that activate the resolution of acute inflammation [39]. These molecules are autacoids that include resolvins, protectins, and maresins, which are formed from EPA and DHA during acute inflammation. Interestingly, resolvins and protectins can currently be synthesized [40], which paves the way to their future use in therapy. Lipid-mediator lipidomic profiling of self-resolving exudates (after solid-phase extraction) allowed framing the time course of inflammation resolution and elucidating the roles played by these important omega 3-derived molecules [40]. Alas, the lipidomic methods that have been successfully employed to clarify the lipid mediator profiles of phagocytes and inflammatory exudates [41] have never been employed in omega 3 clinical trials, thus preventing drawing firm conclusions on their real contribution to the resolution of inflammation in humans.

\section{Conclusions}

While the assessment of relevant biomarkers, e.g., blood pressure, glycaemia, or cholesterol is habitual in pharmacological research, research in the omega 3 area has been hampered by the lack of baseline and post-treatment measurements of fatty 
acid levels. This prevents drawing solid conclusions on the true healthful role of these essential fatty acids. While it is tempting - based on cumulated evidence-to state that the omega 3 myth has been debunked, we need much more focused research before solid conclusions be reached and before we can educe evidence-based recommendations for or against using omega 3 supplements. This is even more relevant for cardiovascular patients who, as reviewed elsewhere [9], are treated with effective and potent drugs and might enjoy additional benefits from omega 3 supplementation only if their basal concentrations are low, i.e., with an omega 3 index $<4 \%$.

The wise use of lipidomics should be essential part of future omega 3 trials and would help untangle this current riddle.

Conflicts of Interest: The author declares no conflict of interest.

\section{References}

1. Richard, D.; Bausero, P.; Schneider, C.; Visioli, F. Polyunsaturated fatty acids and cardiovascular disease. Cell. Mol. Life Sci. 2009, 66, 3277-3288.

2. Harris, W.S.; von Schacky, C. The omega-3 index: A new risk factor for death from coronary heart disease? Prev. Med. 2004, 39, 212-220.

3. Messori, A.; Fadda, V.; Maratea, D.; Trippoli, S. Omega-3 fatty acid supplements for secondary prevention of cardiovascular disease: From "no proof of effectiveness" to "proof of no effectiveness". JAMA Intern. Med. 2013, 173, 1466-1468.

4. Shaikh, N.A.; Tappia, P.S. Why are there inconsistencies in the outcomes of some omega-3 fatty acid trials for the management of cardiovascular disease? Clin. Lipidol. 2015, 10, 27-32.

5. Visioli, F. Cover story: What is wrong with omega 3 fatty acids? PharmaNutrition 2014, 2, A1.

6. Moyer, V.A. Vitamin, mineral, and multivitamin supplements for the primary prevention of cardiovascular disease and cancer: U.S. preventive services task force recommendation statement. Ann. Intern. Med. 2014, 160, 558-564.

7. Bjelakovic, G.; Nikolova, D.; Gluud, C. Antioxidant supplements to prevent mortality. JAMA 2013, 310, 1178-1179.

8. Visioli, F. The resveratrol fiasco. Pharmacol. Res. 2014, 90, 87.

9. Von Schacky, C. Omega-3 fatty acids in cardiovascular disease-An uphill battle. Prostaglandins Leukot. Essent. Fatty Acids 2015, 92, 41-47.

10. Huo, Y.; Li, J.; Qin, X.; Huang, Y.; Wang, X.; Gottesman, R.F.; Tang, G.; Wang, B.; Chen, D.; He, M.; et al. Efficacy of folic acid therapy in primary prevention of stroke among adults with hypertension in china: The csppt randomized clinical trial. JAMA 2015, 313, 1325-1335.

11. Lamaziere, A.; Wolf, C.; Quinn, P.J. Application of lipidomics to assess lipogenesis in drug development and pre-clinical trials. Curr. Pharm. Biotechnol. 2012, 13, 736-745. 
12. Kasuga, K.; Suga, T.; Mano, N. Bioanalytical insights into mediator lipidomics. J. Pharm. Biomed. Anal. 2015, 113, 151-162.

13. Hinterwirth, H.; Stegemann, C.; Mayr, M. Lipidomics: Quest for molecular lipid biomarkers in cardiovascular disease. Circ. Cardiovasc. Genet. 2014, 7, 941-954.

14. Quehenberger, O.; Armando, A.M.; Brown, A.H.; Milne, S.B.; Myers, D.S.; Merrill, A.H.; Bandyopadhyay, S.; Jones, K.N.; Kelly, S.; Shaner, R.L.; et al. Lipidomics reveals a remarkable diversity of lipids in human plasma. J. Lipid Res. 2010, 51, 3299-3305.

15. Rhee, E.P.; Cheng, S.; Larson, M.G.; Walford, G.A.; Lewis, G.D.; McCabe, E.; Yang, E.; Farrell, L.; Fox, C.S.; O'Donnell, C.J.; et al. Lipid profiling identifies a triacylglycerol signature of insulin resistance and improves diabetes prediction in humans. J. Clin. Investig. 2011, 121, 1402-1411.

16. Drew, B.G.; Carey, A.L.; Natoli, A.K.; Formosa, M.F.; Vizi, D.; Reddy-Luthmoodoo, M.; Weir, J.M.; Barlow, C.K.; van Hall, G.; Meikle, P.J.; et al. Reconstituted high-density lipoprotein infusion modulates fatty acid metabolism in patients with type 2 diabetes mellitus. J. Lipid Res. 2011, 52, 572-581.

17. Stegemann, C.; Pechlaner, R.; Willeit, P.; Langley, S.R.; Mangino, M.; Mayr, U.; Menni, C.; Moayyeri, A.; Santer, P.; Rungger, G.; et al. Lipidomics profiling and risk of cardiovascular disease in the prospective population-based bruneck study. Circulation 2014, 129, 1821-1831.

18. Sysi-Aho, M.; Koikkalainen, J.; Seppanen-Laakso, T.; Kaartinen, M.; Kuusisto, J.; Peuhkurinen, K.; Karkkainen, S.; Antila, M.; Lauerma, K.; Reissell, E.; et al. Serum lipidomics meets cardiac magnetic resonance imaging: Profiling of subjects at risk of dilated cardiomyopathy. PLOS ONE 2011, 6, e15744.

19. Stubiger, G.; Aldover-Macasaet, E.; Bicker, W.; Sobal, G.; Willfort-Ehringer, A.; Pock, K.; Bochkov, V.; Widhalm, K.; Belgacem, O. Targeted profiling of atherogenic phospholipids in human plasma and lipoproteins of hyperlipidemic patients using MALDI-QIT-TOF-MS/MS. Atherosclerosis 2012, 224, 177-186.

20. Martin, F.P.; Montoliu, I.; Collino, S.; Scherer, M.; Guy, P.; Tavazzi, I.; Thorimbert, A.; Moco, S.; Rothney, M.P.; Ergun, D.L.; et al. Topographical body fat distribution links to amino acid and lipid metabolism in healthy obese women (corrected). PLOS ONE 2013, $8, \mathrm{e} 73445$.

21. Schwartzman, M.L.; Iserovich, P.; Gotlinger, K.; Bellner, L.; Dunn, M.W.; Sartore, M.; Grazia Pertile, M.; Leonardi, A.; Sathe, S.; Beaton, A.; et al. Profile of lipid and protein autacoids in diabetic vitreous correlates with the progression of diabetic retinopathy. Diabetes 2010, 59, 1780-1788.

22. Spijkers, L.J.; van den Akker, R.F.; Janssen, B.J.; Debets, J.J.; de Mey, J.G.; Stroes, E.S.; van den Born, B.J.; Wijesinghe, D.S.; Chalfant, C.E.; MacAleese, L.; et al. Hypertension is associated with marked alterations in sphingolipid biology: A potential role for ceramide. PLoS ONE 2011, 6, e21817. 
23. Kien, C.L.; Bunn, J.Y.; Poynter, M.E.; Stevens, R.; Bain, J.; Ikayeva, O.; Fukagawa, N.K.; Champagne, C.M.; Crain, K.I.; Koves, T.R.; et al. A lipidomics analysis of the relationship between dietary fatty acid composition and insulin sensitivity in young adults. Diabetes 2013, 62, 1054-1063.

24. Pietilainen, K.H.; Rog, T.; Seppanen-Laakso, T.; Virtue, S.; Gopalacharyulu, P.; Tang, J.; Rodriguez-Cuenca, S.; Maciejewski, A.; Naukkarinen, J.; Ruskeepaa, A.L.; et al. Association of lipidome remodeling in the adipocyte membrane with acquired obesity in humans. PLoS Biol. 2011, 9, e1000623.

25. Visioli, F.; Bernardini, E. Extra virgin olive oil's polyphenols: Biological activities. Curr. Pharm. Des. 2011, 17, 786-804.

26. Mozaffarian, D.; Micha, R.; Wallace, S. Effects on coronary heart disease of increasing polyunsaturated fat in place of saturated fat: A systematic review and meta-analysis of randomized controlled trials. PLoS Med. 2010, 7, e1000252.

27. Marangoni, F.; Novo, G.; Perna, G.; Perrone Filardi, P.; Pirelli, S.; Ceroti, M.; Querci, A.; Poli, A. Omega-6 and omega-3 polyunsaturated fatty acid levels are reduced in whole blood of italian patients with a recent myocardial infarction: The AGE-IM study. Atherosclerosis 2014, 232, 334-338.

28. Block, R.C.; Harris, W.S.; Reid, K.J.; Spertus, J.A. Omega-6 and trans fatty acids in blood cell membranes: A risk factor for acute coronary syndromes? Am. Heart J. 2008, 156, $1117-1123$.

29. Hu, C.; Kong, H.; Qu, F.; Li, Y.; Yu, Z.; Gao, P.; Peng, S.; Xu, G. Application of plasma lipidomics in studying the response of patients with essential hypertension to antihypertensive drug therapy. Mol. Biosyst. 2011, 7, 3271-3279.

30. Kaddurah-Daouk, R.; Baillie, R.A.; Zhu, H.; Zeng, Z.B.; Wiest, M.M.; Nguyen, U.T.; Watkins, S.M.; Krauss, R.M. Lipidomic analysis of variation in response to simvastatin in the cholesterol and pharmacogenetics study. Metabolomics 2010, 6, 191-201.

31. Lamaziere, A.; Wolf, C.; Barbe, U.; Bausero, P.; Visioli, F. Lipidomics of hepatic lipogenesis inhibition by omega 3 fatty acids. Prostaglandins Leukot. Essent. Fatty Acids 2013, 88, 149-154.

32. Lamaziere, A.; Richard, D.; Barbe, U.; Kefi, K.; Bausero, P.; Wolf, C.; Visioli, F. Differential distribution of dha-phospholipids in rat brain after feeding: A lipidomic approach. Prostaglandins Leukot. Essent. Fatty Acids 2011, 84, 7-11.

33. Lamaziere, A.; Richard, D.; Bausero, P.; Barbe, U.; Kefi, K.; Wolf, C.; Visioli, F. Comparison of docosahexaenoic acid uptake in murine cardiomyocyte culture and tissue: Significance to physiologically relevant studies. Prostaglandins Leukot. Essent. Fatty Acids 2015, 94, 49-54.

34. Vidgren, H.M.; Agren, J.J.; Schwab, U.; Rissanen, T.; Hanninen, O.; Uusitupa, M.I. Incorporation of $n-3$ fatty acids into plasma lipid fractions, and erythrocyte membranes and platelets during dietary supplementation with fish, fish oil, and docosahexaenoic acid-rich oil among healthy young men. Lipids 1997, 32, 697-705.

35. Schuchardt, J.P.; Hahn, A. Bioavailability of long-chain omega-3 fatty acids. Prostaglandins Leukot. Essent. Fatty Acids 2013, 89, 1-8. 
36. Visioli, F.; Rise, P.; Barassi, M.C.; Marangoni, F.; Galli, C. Dietary intake of fish vs. formulations leads to higher plasma concentrations of n-3 fatty acids. Lipids 2003, 38, 415-418.

37. Molinari, C.; Risé, P.; Guerra, C.; Mauro, N.; Piani, C.; Bosi, E.; Galli, C.; Scavini, M. Eight-week consumption of milk enriched with omega 3 fatty acids raises their blood concentrations yet does not affect lipids and cardiovascular disease risk factors in adult healthy volunteers. Pharmanutrition 2014, 2, 141-148.

38. Visioli, F.; Rise, P.; Plasmati, E.; Pazzucconi, F.; Sirtori, C.R.; Galli, C. Very low intakes of $n-3$ fatty acids incorporated into bovine milk reduce plasma triacylglycerol and increase HDL-cholesterol concentrations in healthy subjects. Pharmacol. Res. 2000, 41, 571-576.

39. Serhan, C.N.; Chiang, N.; Dalli, J. The resolution code of acute inflammation: Novel pro-resolving lipid mediators in resolution. Semin. Immunol. 2015, 27, 200-215.

40. Dalli, J.; Winkler, J.W.; Colas, R.A.; Arnardottir, H.; Cheng, C.Y.; Chiang, N.; Petasis, N.A.; Serhan, C.N. Resolvin D3 and aspirin-triggered resolvin D3 are potent immunoresolvents. Chem. Biol. 2013, 20, 188-201.

41. Yang, R.; Chiang, N.; Oh, S.F.; Serhan, C.N. Metabolomics-lipidomics of eicosanoids and docosanoids generated by phagocytes. Curr. Protoc. Immunol. 2011. 


\title{
Using Caenorhabditis elegans to Uncover Conserved Functions of Omega-3 and Omega-6 Fatty Acids
}

\author{
Jennifer L. Watts
}

Abstract: The nematode Caenorhabditis elegans is a powerful model organism to study functions of polyunsaturated fatty acids. The ability to alter fatty acid composition with genetic manipulation and dietary supplementation permits the dissection of the roles of omega- 3 and omega- 6 fatty acids in many biological process including reproduction, aging and neurobiology. Studies in C. elegans to date have mostly identified overlapping functions of 20-carbon omega- 6 and omega- 3 fatty acids in reproduction and in neurons, however, specific roles for either omega-3 or omega-6 fatty acids are beginning to emerge. Recent findings with importance to human health include the identification of a conserved Cox-independent prostaglandin synthesis pathway, critical functions for cytochrome P450 derivatives of polyunsaturated fatty acids, the requirements for omega- 6 and omega- 3 fatty acids in sensory neurons, and the importance of fatty acid desaturation for long lifespan. Furthermore, the ability of C. elegans to interconvert omega- 6 to omega-3 fatty acids using the FAT- 1 omega- 3 desaturase has been exploited in mammalian studies and biotechnology approaches to generate mammals capable of exogenous generation of omega-3 fatty acids.

Reprinted from J. Clin. Med. Cite as: Watts, J.L. Using Caenorhabditis elegans to Uncover Conserved Functions of Omega-3 and Omega-6 Fatty Acids. J. Clin. Med. 2016, 5, 19.

\section{Introduction}

The adage "you are what you eat" is especially true regarding fat. Specific types of fat in the human diet have important biological consequences on health and wellness. Long chain polyunsaturated fatty acids (PUFAs) are dietary requirements for humans and other mammals. Essential fatty acids can be classified as omega-6, or omega-3, depending on the position of the terminal double bond relative to the methyl end of the fatty acids. For example, the omega- 6 fatty acid linoleic acid (LA, 18:2) is an 18-carbon fatty acid with double bonds at carbons 9 and 12, while the omega-3 fatty acid alpha-linolenic acid (ALA, 18:3) is an 18-carbon fatty acid with double bonds at carbons 9,12 , and 15 . The essential fatty acids can be elongated and further desaturated to generate a range of 20- and 22-carbon omega-6 and omega-3 fatty acids [1]. Omega-6 fatty acids are prevalent in vegetable oils, especially corn, safflower, and soybean oil, while omega-3 fatty acids are found in 
fishes such as salmon and tuna. Because humans cannot interconvert omega- 6 and omega- 3 fatty acids, the ratio of omega- 6 to omega- 3 fatty acids is determined by dietary intake [2]. This is important because, in spite of their structural similarity, the biological functions of omega- 6 and omega-3 fatty acids can be quite divergent [3].

Long chain fatty acids from the omega- 3 and omega- 6 families play crucial roles in membrane structure and function [4]. For example, the cis double bonds influence lipid packing, membrane fluidity [5], and membrane protein activity [6]. Omega- 3 and omega- 6 fatty acids are precursors for potent signaling molecules, and signals produced from omega-3 versus omega-6 PUFAs can sometimes have opposing effects [3]. Upon stimulation, long chain omega-6 and omega-3 fatty acids are cleaved from membrane lipids by phospholipases and oxygenated by cyclooxygenase, lipoxygenase, or cytochrome P450 enzymes to form a wide range of prostaglandins, leukotrienes, lipoxins, as well as hydroxy-, epoxy-, and hydroperoxy-derivatives $[7,8]$. These molecules, collectively termed "eicosanoids" act as powerful short range hormones affecting inflammation, immune responses, and reproductive processes [9]. PUFAs are important components of endocannabinoids, which are ethanolamide derivatives of phospholipids that bind to endocannabinoid receptors to regulate memory, appetite, mood, and pain sensation [10,11]. Finally, both omega-3 and omega-6 PUFAs and their eicosanoid derivatives are ligands for transcription factors, and therefore they influence gene expression. In addition to known receptors for eicosanoid and ethanolamide derivatives, an omega- 3 fatty acid receptor, GPR120, has recently been identified [12,13]. Substantial evidence exists for opposing functions of omega-3 and omega- 6 fatty acids in the regulation of inflammation, primarily that eicosanoids derived from most omega- 6 fatty acids have pro-inflammatory effects, while those derived from omega-3 fatty acids do not (reviewed in [3]). However, opposing functions for omega-3 and omega-6 fatty acids in non-inflammatory processes are not well defined.

\section{Why Study Fatty Acid Functions Using C. elegans?}

An attractive animal model for studies of fatty acid function is the roundworm C. elegans. This popular model organism is easy and inexpensive to maintain in the lab, and its well-understood developmental programs, simple anatomy, short lifespan, well-annotated genome, and ease of genetic analysis allow for studies of diverse biological processes, including those related to human nutrition and disease [14]. In the lab, the nematodes grow on petri plates on lawns of Escherichia coli bacteria, which provide dietary nutrients, including proteins, carbohydrates, and saturated and mono-unsaturated fatty acids derived from digestion of bacterial membranes [15]. However, C. elegans are capable of synthesizing all necessary fatty acids de novo, and the core enzymes of fatty acid biosynthesis are conserved, including acetyl CoA carboxylase, fatty acid synthase, and a range of fatty acid 
desaturase and elongase activities, enabling $C$. elegans to synthesize long chain PUFAs including arachidonic acid (AA, 20:4) and eicosapentaenoic acid (EPA, 20:5) [16] (Figure 1A). Unlike most other animal species, the $C$. elegans genome encodes an omega-3 desaturase enzyme that can convert 18-carbon and 20-carbon omega-6 fatty acids into omega- 3 fatty acids [17], along with a $\Delta 12$ desaturase, which catalyzes the formation of LA from oleic acid (OA, 18:1) [18]. Thus, C. elegans does not have any dietary fatty acid requirements. Like most other animals, C. elegans also possesses $\Delta 6$ and $\Delta 5$ desaturase enzymes, which act, in conjunction with fatty acid elongases, on similar substrates used by mammals and other animals to form 20-carbon PUFAs [19]. However, C. elegans lacks the specific elongase activity to produce 22-carbon PUFAs. Strains containing mutations in genes of the fatty acid desaturation pathway facilitate functional studies of PUFAs, because fatty acid composition can be manipulated both genetically and through the diet [20-22].
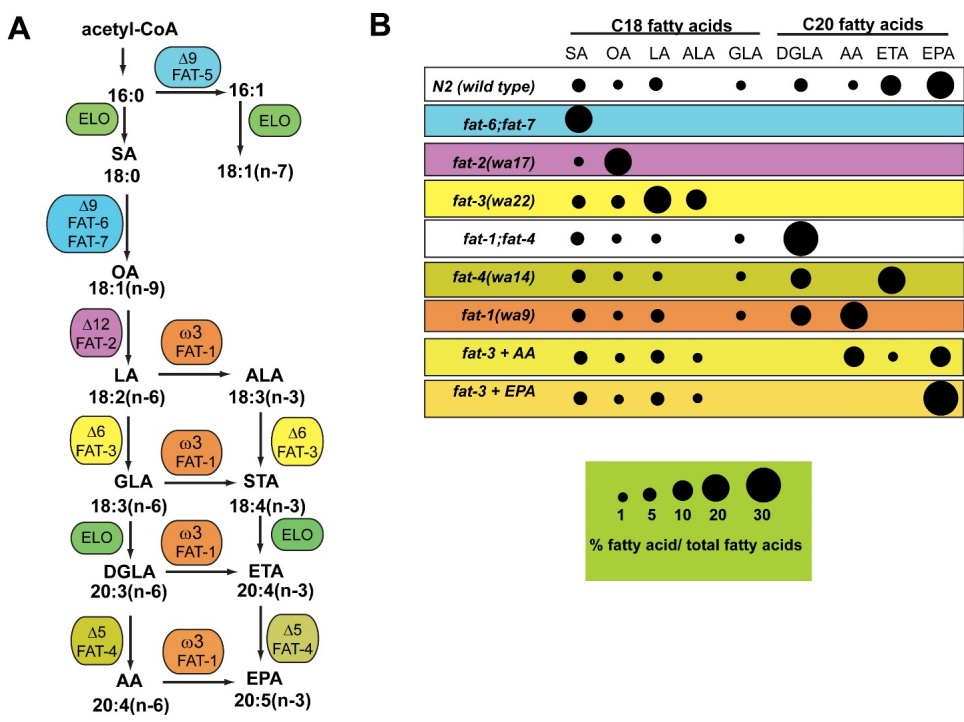

Figure 1. (A) Biosynthesis of omega-6 and omega-3 fatty acids in C. elegans. Unlike most other animals, $C$. elegans possesses $\Delta 12$ desaturase and $\omega 3$ desaturase enzymes, and therefore does not require essential dietary fatty acids to synthesize a range of omega- 6 and omega- 3 fatty acids; (B) Mutant strains lacking functional desaturase enzymes are depleted in specific polyunsaturated fatty acids. The area of each circle represents the ratio of specific fatty acids relative to total fatty acid content. Data shown are representative examples of multiple GC/MS measurements. Abbreviations: ELO, elongase; SA, stearic acid; OA, oleic acid; LA, linoleic acid; ALA, alpha-linolenic acid; GLA, gamma-linolenic acid; STA, stearidonic acid; DGLA, dihommo gamma-linolenic acid; ETA, eicosatrienoic acid; AA, arachidonic acid; EPA, eicosapentaenoic acid. Figure is modified from [23]. 


\section{C. elegans Mutants Lacking Fatty Acid Desaturase Activity Are Useful for Studies of Conserved Functions of Omega-6 and Omega-3 Fatty Acids}

The $C$. elegans genome contains single genes encoding the $\Delta 12, \Delta 5, \Delta 6$, and omega-3 desaturase enzymes necessary for the biosynthesis of a range of omega- 6 and omega-3 PUFAs. There are three genes encoding the $\Delta 9$ desaturases, which convert saturated fatty acids into monounsaturated fatty acids (MUFAs). One $\Delta 9$ desaturase, FAT-5, uses palmitic acid (16:0) as a substrate to produce palmitoleic acid (16:1), while the other two $\Delta 9$ desaturases, FAT-6 and FAT-7 use both 16:0 and stearic acid (18:0) as substrates to produce (16:1) and oleic acid (18:1) [24]. Mutant strains lacking functional desaturase enzymes have been isolated and used for many studies of functions of specific fatty acids in reproduction, longevity and neurobiology. Because roundworms do not have blood vessels, nor do they express inflammatory markers such as TNF $\alpha$ and NFK $\beta$, the roles of omega- 6 and omega-3 PUFAs can be studied independently of their inflammatory functions. As might be expected, strains carrying mutations in the desaturase genes acting early in the pathway, such as $\Delta 9$ desaturases (fat-6;fat-7 double mutants), $\Delta 12$ desaturase (fat-2 mutants), and $\Delta 6$ desaturase (fat-3 mutants) have more severe lipid composition changes, as well as more severe phenotypic consequences, such as growth, movement, and reproductive defects, than strains carrying mutations in desaturases acting later in the pathway, such as the $\Delta 5$ desaturase [20,21,25] (Figure 1B). C. elegans has been a powerful model for unraveling mechanisms of development, neurobiology, and longevity, and the studies reviewed below demonstrate how fatty acid function is involved in these fundamental biological processes.

\section{Functions of Omega-6 and Omega-3 Fatty Acids in C. elegans Reproduction: Sperm Guidance and Germ Cell Maintenance}

PUFAs are required for efficient reproduction in many animal species, although specific mechanisms in invertebrates are just starting to be elucidated [26]. Initial characterization of fat-2, fat-3 and fat-6;fat-7 mutants lacking 20-carbon PUFAs revealed slow growth and greatly reduced reproductive capacity in the mutant strains $[20,21,25]$. It is likely that PUFAs are required for multiple processes to ensure optimum reproductive output. One process characterized by the Miller lab involves signaling molecules derived from PUFAs that are required in the female germ line for sperm guidance toward oocytes [27]. When wild-type males were mated to fat-2 mutants, which are unable to synthesize PUFAs, the sperm failed to migrate toward the spermatheca, the region of the uterus where fertilization occurs. However, when fat-2 mutants were provided dietary 20-carbon PUFAs, either omega-3 or omega- 6 species, sperm migration greatly improved [27]. It is likely that the signaling is mediated by prostaglandin derivatives of omega- 3 and omega- 6 PUFAs, because directly injecting nanomolar concentrations of human F-series 
prostaglandins promoted sperm movement [28]. A range of F-series prostaglandins are synthesized in the $C$. elegans germ line, including those derived from both omega-3 and omega-6 PUFAs [29]. Interestingly, C. elegans does not encode a clear homolog of the mammalian cyclooxygenase enzyme that is the rate-limiting step of prostaglandin synthesis. Instead, metabolite analysis revealed an alternative prostaglandin synthesis pathway that does not involve prostaglandin-D or -E intermediates $[29,30]$. The Miller lab took full advantage of whole-organism genetic approaches to discover that the synthesis of F-series prostaglandins is regulated by both insulin signaling in the intestine and TGF- $\beta$ signaling in sensory neurons in order to finely control reproductive output [28,31]. Both of these signaling pathways respond to food availability, thus, during food scarcity, prostaglandin biosynthesis is reduced, leading to reduced efficiency of sperm locating the fertilization site, ultimately resulting in a lower fertilization rate. Interestingly, the alternative Cox-independent prostaglandin pathway may be conserved in mammals, because F-class prostaglandins have been detected in Cox double knockout mice [31].

While 20-carbon omega-6 and omega-3 PUFAs are redundant in their ability to promote proper sperm migration to oocytes, a role for divergent activities of omega- 6 and omega-3 PUFAs in reproduction is suggested by dietary studies. Watts and Browse discovered that dietary supplementation of the omega-6 PUFA dihomogamma linolenic acid (DGLA, 20:3n-6) resulted in sterility due to the destruction of germ cells [32]. Supplementation of arachidonic acid (AA, 20:4n-6) also led to germ cell death, although at a much higher concentration than DGLA. On the contrary, supplementation with omega-3 fatty acids, such as eicosapentaenoic acid (EPA, 20:5n-3) had no adverse effects on the nematodes [32]. Genetic analyses revealed a large number of gene mutations that altered the sensitivity to DGLA. For example, the fat-1 mutant strain, which cannot convert omega- 6 fatty acids to omega-3 fatty acids, was more sensitive to dietary DGLA than wild type [32]. On the other hand, stress resistant strains, such as the insulin-like growth factor receptor daf-2 mutants, did not become sterile when exposed to DGLA [33]. The daf-2 mutants showed increased transcription of genes involved in detoxification and stress resistance, suggesting that the negative effects of DGLA may be due to a toxic product derived from DGLA [33,34]. A recent study from the Watts lab showed that the negative effects of DGLA in the C. elegans germ line are likely due to the production of specific epoxide derivatives of DGLA, the synthesis of which depend on the cytochrome P450 (CYP) enzyme CYP-33E2 [35]. Direct injection of specific epoxides derived from DGLA, but not those derived from EPA, triggered germ cell abnormalities resembling cell fusion or failed cytokinesis, which ultimately lead to apoptosis and germ cell death. Interestingly, even though knockdown of CYP-33E2 in C. elegans led to reduced germ cell death and higher reproductive outputs during DGLA feeding, the CYP-33E2 knock-down worms had a lower brood size when fed a 
normal diet [35]. This suggests that CYP-33E2 may be producing beneficial epoxides from other PUFAs, such as EPA, that could be required for optimal reproduction.

\section{Functions of Omega-6 and Omega-3 Fatty Acids in Longevity: Critical Roles for $\Delta 9$ Desaturases}

Because of its small size, short lifespan, and ease of genetic manipulation, C. elegans has been a premier organism for discoveries of genetic and physiological mechanisms regulating aging and longevity [36,37]. Recent lipidomics and genetic studies suggest roles for specific fatty acids in promoting longevity in C. elegans [38]. PUFAs contain more double bonds than saturated or MUFAs, therefore they are more likely to undergo peroxidation, which in turn leads to propagation of reactive oxygen species (ROS), which cause further damage to proteins and nucleic acids. In many organisms the degree of membrane unsaturation negatively correlates with lifespan [39]. This concept is supported by a C. elegans study showing a correlation between long-lived mutants in the insulin signaling pathway and changes in unsaturated fatty acid composition [40]. The mutants examined had increased (MUFAs) with decreased PUFAs. Additionally, high concentrations of dietary fish oil was shown to lead to higher levels of lipid peroxidation products and shorter lifespan $[40,41]$. In contrast, another study showed that supplementation of omega-6 PUFAs activates autophagy and promotes long lifespan [42]. Furthermore, several long-lived mutant strains express higher levels of $\Delta 9$ desaturases than wild type [34]. The $\Delta 9$ desaturases synthesize MUFAs in mammals, but are the first step in the synthesis of PUFAs in C. elegans. Thus, the roles of PUFAs in aging are still not clear.

Importantly, the oxidative damage theory of aging, which states that the accumulation of molecular damage due to ROS is a key contributor to aging, is currently undergoing a paradigm shift based on experiments in C. elegans [43]. Studies in which worms were grown under oxygen levels ranging from $2 \%-40 \%$ did not show significant changes in lifespan [44]. In addition, growth of $\mathrm{m}$ in the presence of antioxidants did not always increase lifespan [43], and specific mutations in antioxidant genes did not always lead to reduced lifespan $[43,45,46]$. Furthermore, treatment with low levels of chemicals that induce ROS actually produced increased resistance to oxidative stress and increased lifespan through the process of stress-induced hormesis $[47,48]$. Thus, while it is clear that high levels of ROS cause cellular damage, regulated ROS release and fluctuations in ROS are important for inducing both signaling and protection pathways [49-52]. This might explain why lower concentrations of dietary fish oil led to slightly longer lifespans [41]. It is also crucial to consider that membranes undergo constant remodeling and turnover. A recent study demonstrated that PUFAs in C. elegans membranes turn over very rapidly, such that the majority of PUFAs and other membrane lipids are replaced each day, suggesting that oxidized fatty acids are 
rapidly removed [53]. Knock-down of $\Delta 9$ desaturase activity reduces membrane turn-over, which might lead to increased lipid peroxidation in spite of lower PUFA production [53]. It appears that the beneficial functions of omega- 6 and omage- 3 PUFAs might outweigh the potential for oxidative damage conferred by the high degree of unsaturation.

In animals, dietary resources must be allocated between reproduction and somatic maintenance, thus reproduction and life span are metabolically linked. In C. elegans, removal of the germ line results in worms with increased fat stores and longer lifespan (reviewed in $[54,55]$ ). Several recent studies implicate fatty acid metabolism in long lifespan in germ-line ablated animals. Two nuclear hormone receptor homologs, NHR-80 and NHR-49, regulate the expression of $\Delta 9$ desaturases [56,57], and these two nuclear receptor proteins, as well as the FAT-6 and FAT-7 $\Delta 9$ desaturases are required for long lifespan in animals lacking a germ line $[58,59]$. Additionally, the lysosomal lipase LIPL-4 and intact autophagy pathways are required for extended longevity in germ-line less nematodes [60,61]. A recent metabolomic analysis revealed that a specific lipid species, oleoylethanolamide, accumulates in worms over-expressing the lipase LIPL-4. This ethanolamide derivative of oleic acid then directly binds to the lysosomal lipid chaperone LPB-8, which is then translocated to the nucleus. Oleoylethanolamide also binds to and activates the nuclear receptor NHR-80, activating transcription of $\Delta 9$ desaturases and promoting longevity [62]. Thus, lipid signaling from the lysosome to the nucleus has long lasting physiological effects, including lifespan extension.

In humans, diets high in sugars lead to excess lipid storage and ultimately cause adverse health effects, including obesity, diabetes, and cardiovascular coronary heart disease $[63,64]$. In C. elegans, dietary glucose causes shortened lifespan $[65,66]$. Two transcriptional regulators of $\Delta 9$ desaturases, MDT-15 and SBP-1 were recently shown to protect $C$. elegans from glucose-induced accelerated aging by preventing the accumulation of saturated fat [67]. Glucose feeding increases the saturated fatty acid composition of C. elegans, and MDT-15 and SBP- 1 activate $\Delta 9$ and other fatty acid desaturases to prevent saturated fatty acid-induced lipotoxicity by converting saturated fatty acids into MUFAs and PUFAs [67-70].

A key transcription factor required for increased longevity in germ-line ablated worms is SKN-1/Nrf. This transcription factor is most studied for its roles in stress responses, especially to oxidative stress [71]. Interestingly, SKN-1 also regulates the transcription of lipid metabolism genes that are up-regulated in germ-line deficient animals, including fatty acid desaturases [71]. When SKN-1 is over-expressed, lipids are depleted from somatic, but not germ-line tissues [72]. This phenotype is similar to that seen in wild type worms upon nutrient depletion or exposure to pro-oxidants, and is also seen in fat-6;fat-7 double mutants lacking 20-carbon PUFAs, as well as in fat-1;fat-4 double mutants, which accumulate DGLA, but cannot synthesize EPA or 
AA. Supplementation with OA, AA, and EPA, but not other fatty acids, suppressed the somatic depletion of lipids, suggesting that specific fatty acid species may be involved in the allocation of germline versus somatic lipids, thereby influencing both reproduction and longevity [72].

\section{Functions of Omega-6 and Omega-3 Fatty Acids in C. elegans Neurons: Synaptic Vesicle Formation, Signal Transduction in Sensory Neurons, and Complex Behavioral Responses to Alcohol and Oxygen}

Other than adipose tissue, human brain tissue contains the highest proportion of lipids, with brain phospholipids containing high levels of both omega- 6 and omega-3 PUFAs. Diets deficient in omega-3 and other PUFAs lead to defective neural function (reviewed in $[73,74]$ ). C. elegans has a simplified nervous system consisting of 302 neurons, and the network of neurons and their connections has been thoroughly mapped [75]. The fat-3 mutant strain, which lacks 20-carbon omega-6 and omega-3 PUFAs, shows uncoordinated movement and defective egg laying behavior, phenotypes which are controlled by motor neurons and hermaphrodite-specific serotonergic vulva neurons [21,76]. Lesa et al. showed that fat-3 mutants display defects in neurotransmitter release, and that synaptic vesicles were depleted in neuronal termini at the neuromuscular junction, indicating that 20-carbon PUFAs are required for synaptic vesicle formation and accumulation [76].

Sensory neurons are also affected in fat-3 and fat-1;fat-4 double mutants, which exhibit defects in olfactory chemotaxis behavior to volatile odorants sensed by AWC neurons, but less or no defective chemotaxis behavior to odorants sensed by AWA neurons [23]. This genetic evidence implicates PUFAs in specific neuronal signal transduction pathways. The AWA neurons possess TRPV type channels to respond to stimulatory signals, whereas the AWC neurons, which are not affected by PUFA deficiency, use cyclic nucleotide gated channels. Similarly, the ASH sensory neurons use TRPV channels to respond to noxious stimuli, and stimulate rapid escape behavior to heavy metals, high osmolarity, and nose touch. The fat-3 and fat-1;fat-4 double mutants showed behavioral defects upon exposure to these stimuli, and were rescued by dietary supplementation of both omega- 6 and omega- 3 fatty acids, indicating that PUFAs are also necessary for function in ASH neurons [23]. Direct calcium imaging of ASH neuronal response in fat-3 mutants showed diminished calcium responses, while exogenous EPA elicited calcium responses and avoidance responses in fat-3 mutants, bypassing the PUFA biosynthesis defects, and providing evidence for 20-carbon omega-6 and omega-3 PUFAs as regulators of in vivo TRPV channel activity [23].

Olfactory adaptation is the process where sensory neurons reduce their response to prolonged stimulation. Even though sensory response to volatile odorants sensed by AWC neurons was nearly normal in PUFA-deficient mutants, adaptation to 
volatile odorants mediated by AWC neurons was abnormal in fat-3 and fat-1;fat-4 mutant strains, and this defect was rescued by dietary PUFAs [77]. Similar to the findings described above for AWA neurons, this study linked the roles of PUFAs in olfactory adaptation in AWC neurons to TRPV channels, which in AWC neurons function downstream of the nuclear accumulation of the cGMP-dependent protein kinase EGL-4 [77].

In the research described above, omega- 6 and omega- 3 fatty acids play redundant roles in their ability to rescue the neuronal defects, indicating that both omega- 6 and omega- 3 fatty acids perform the required cellular functions. Several recent studies suggest specific roles for omega- 6 and omega- 3 fatty acids in neuronal processes. A C. elegans mutant in fatty acid amide hydrolase activity (faah-1) is defective in the regeneration of axons after laser surgery [78]. Fatty acid amide hydrolase breaks down endocannabinoids, such as arachidonyl ethanolamide (AEA). In C. elegans, AEA appears to inhibit axon regeneration via the Go $\alpha$ subunit GOA-1 [78]. Surprisingly, eicosapentaenoyl ethanolamide (EPEA), derived from EPA, shows less inhibitory activity, even though EPEA is much more abundant in C. elegans tissues than AEA [79]. On the other hand, omega-3 fatty acids are specifically required for the process of alcohol tolerance [80], a neuroadaptive process that compensates for the effects of alcohol in humans and in C. elegans [81]. This study showed that unlike wild type nematodes, fat-3, fat-4, and fat-1 mutants did not recover movement after exposure to ethanol. EPA, but not AA supplementation was necessary and sufficient for the neuroplasticity required to compensate for the effects of alcohol intoxication in C. elegans [80]. Finally, AA in phospholipids, but not EPA, is required for neurons responding to light touch [82]. The Goodman lab found that fat-3 and fat-4 mutants, neither of which can synthesize AA, showed reduced response to touch. AA, but not EPA, rescued touch sensitivity in fat-3 and fat-4 mutants. Interestingly, eicosatetraynoic acid (EYTA), a non-metabolizable structural analog of AA, also rescued the touch response phenotype. The ability of EYTA to rescue touch response indicates that $\mathrm{AA}$ is not required to be oxidized into an eicosanoid for its activity. In addition to mutant and dietary supplementation analysis, the researchers used dynamic force spectroscopy to reveal that AA in phospholipids modulates biophysical properties of touch receptor neuron membranes to allow for optimal function [82]. Taken together, these recent studies reveal that omega-6 and omega-3 PUFAs can have distinct roles in neurological processes.

In addition to the alternative prostaglandin synthesis pathway described above, recent studies demonstrate that in C. elegans, omega- 3 and omega- 6 fatty acids are modified into eicosanoid-like molecules by the actions of CYP enzymes, and that these CYP-derived eicosanoids have important biological functions. Studies from Menzel's group revealed that CYP-33E2, which is most closely related to human CYP2J2, prefers EPA over AA as a substrate, and produces epoxyeicosatetraenoic 
acid $(17,18-\mathrm{EEQ})$ as its main product [83]. CYP-29A3 is most closely related to human CYP4, and it uses AA to produce the hydroxyl derivative 20-HETE [84]. These eicosanoids have opposing effects on pharyngeal pumping and food uptake, with 17,18-EEQ mimicking the effects of the neurohormone serotonin on fasted worms, where both the eicosanoid and serotonin stimulate pharyngeal pumping. In contrast, 20-HETE and octopamine reduced pharyngeal pumping and food intact in well-fed worms [85]. Furthermore, 17,18-EEQ synthesis is increased upon serotonin supplementation, while 20-HETE synthesis increases upon application of octopamine [85], implicating eicosanoids as mediators of neurohormones affecting food intake. Another CYP enzyme, CYP-13A12, acts on PUFAs to respond oxygen levels [86]. In humans, reoxygenzation after oxygen deprivation causes tissue damage due to inflammation [87]. The C. elegans study showed that CYP-13A12 responds to the oxygen-dependent enzyme EGL-9 and hypoxia-inducible factor (HIF-1) to facilitate a movement response to reoxygenation after oxygen deprivation [86]. CYP-13A12 generates epoxy and hydroxyl eicosanoids from AA and EPA, including 14,15-epoxyeicosatrienoic acid (14,15-EET) from AA and 17,18-EEQ from EPA [88]. This research implicates conserved roles for omega-6 and omega-3 PUFAs and eicosanoid formation in ischemia and reperfusion.

\section{Using the C. elegans Fat-1 Gene for Studies in Mammals: Endogenous Omega-3 Functions and Biotechnology Applications}

The discovery of the first animal omega-3 desaturase, the C. elegans FAT-1 desaturase [17], enabled Kang to construct the fat-1 transgenic mouse, which expresses the $C$. elegans omega- 3 desaturase and permits the conversion endogenous omega-6 to omega-3 fatty acids in mammalian tissues [89]. Lipidomic analysis revealed hundreds of specific lipid species that are altered between wild type and the fat-1 mouse, including EPA and DHA-containing phospholipids and cholesterol esters, as well as many species of EPA-derived epoxides and diols formed via CYP enzymes [90]. Studies using the fat-1 mouse model now appear in numerous publications in which researchers examined the effects of endogenously synthesized, as opposed to dietary, omega- 3 fatty acids on a range of processes in a mammalian model. This research provides evidence that increased production of omega-3 PUFAs, coupled with a reduction in omega-6 PUFAs, protects mammals from a range of diseases, including cancer, diabetes and inflammatory diseases (reviewed in [91-95]). Recent studies reveal new insights into molecular mechanisms in which omega-3 PUFAs protect against various disease outcomes as diverse as diabetic neuropathy [96], fatty liver disease [97], pancreatic beta cell death [98], and vascular inflammation [99]. Thus, the fat-1 mouse model promises to be central to unraveling the mechanisms of omega- 3 and omega- 6 fatty acids in health and disease. 
The success of expressing the C. elegans fat- 1 omega- 3 desaturase gene in mice led the way for expression of fat-1 in other mammals. Because modern diets are thought to be deficient in omega-3 fatty acids, increasing omega-3 fatty acids in human food is desirable [100]. Concerns regarding heavy metal contamination of marine fish [101], as well as depletion of ocean fish stocks due to overfishing, lead to a desire for alternative sources of omega-3 fatty acids in human diets. The creation of transgenic farm animals, such as pigs [102-104] and cattle [105,106], set the stage for using nematode fat-1 genes to someday provide milk and meat with higher omega- 3 content to human consumers. However, more studies are needed to ensure the safety of food produced from transgenic animals. In addition, altering the omega-3/omega-6 ratio in farm animals might have adverse effects on their reproduction, as lower brood size was observed in mice expressing fat-1 in their mammary glands [107].

\section{Conclusions and Future Studies}

Discoveries made using model organisms have had significant impact on human medicine. For example, C. elegans research has been crucial for the elucidation of genetic pathways underlying programmed cell death, longevity, and signal transduction pathways that occur during development as well as during carcinogenesis (reviewed in [14]). This research has led to the discovery of drug targets and novel therapeutics in humans. Studies in C. elegans regarding non-inflammatory functions of PUFAs clearly demonstrate that 20-carbon PUFAs play key roles in reproduction and in the nervous system. While it appears that many functions of 20-carbon omega- 6 and omega-3 fatty acids are redundant, examples of specific functions for DGLA, AA and EPA are beginning to emerge $[35,42,80,82,85]$. Genetic analysis and simple physiology render the C. elegans model especially useful for studies of PUFA functions in reproduction, development, and longevity, because vertebrate models are much more difficult, time consuming, and expensive to manipulate. Roles of PUFAs in longevity are just starting to be examined, and several studies suggest that $\Delta 9$ desaturase is a pro-longevity factor. Future studies are needed to determine if PUFA activity depends on being metabolized into eicosanoids or other signaling molecules, or whether their functions are derived from membrane biophysical properties or direct interactions with membrane proteins. Similarly, the identification of $C$. elegans receptors for PUFAs and eicosanoids, as well as the identification of specific signal transduction pathways that are affected by PUFA composition, will allow for more mechanistic studies. It is likely that mammalian studies using the fat- 1 transgenic mouse will continue to be fruitful because the transgene can be crossed into numerous genetic models of disease, thereby examining the effects of endogenous omega-3 fatty acid production on many different disease outcomes. In summary, C. elegans is a powerful model for the integration of dietary 
and genetic studies for PUFA function in reproduction, development, neuroscience and aging.

Acknowledgments: The author thanks Marshall Deline and Jason Watts for helpful comments on the manuscript. Research in the Watts lab is supported by the National Institute of Diabetes and Digestive and Kidney Diseases of the National Institutes of Health under award number R01DK074114.

Conflicts of Interest: The author declares no conflict of interest.

\section{References}

1. Wallis, J.G.; Watts, J.L.; Browse, J. Polyunsaturated fatty acid synthesis: What will they think of next? Trends Biochem. Sci. 2002, 27, 467.

2. Simopoulos, A.P. Genetic variants in the metabolism of omega- 6 and omega- 3 fatty acids: Their role in the determination of nutritional requirements and chronic disease risk. Exp. Biol. Med. 2010, 235, 785-795.

3. Schmitz, G.; Ecker, J. The opposing effects of $n-3$ and $n-6$ fatty acids. Prog. Lipid Res. 2008, 47, 147-155.

4. Antonny, B.; Vanni, S.; Shindou, H.; Ferreira, T. From zero to six double bonds: Phospholipid unsaturation and organelle function. Trends Cell Biol. 2015, 25, 427-436.

5. Bigay, J.; Antonny, B. Curvature, lipid packing, and electrostatics of membrane organelles: Defining cellular territories in determining specificity. Dev. Cell 2012, 23, 886-895.

6. Niu, S.L.; Mitchell, D.C.; Lim, S.Y.; Wen, Z.M.; Kim, H.Y.; Salem, N., Jr.; Litman, B.J. Reduced $G$ protein-coupled signaling efficiency in retinal rod outer segments in response to $n$-3 fatty acid deficiency. J. Biol. Chem. 2004, 279, 31098-31104.

7. Funk, C.D. Prostaglandins and leukotrienes: Advances in eicosanoid biology. Science 2001, 294, 1871-1875.

8. Spector, A.A.; Kim, H.Y. Cytochrome p450 epoxygenase pathway of polyunsaturated fatty acid metabolism. Biochim. Biophys. Acta 2015, 1851, 356-365.

9. Nebert, D.W.; Wikvall, K.; Miller, W.L. Human cytochromes P450 in health and disease. Philos. Trans. R. Soc. Lond. Ser. B Biol. Sci. 2013, 368, 20120431.

10. Xu, J.Y.; Chen, C. Endocannabinoids in synaptic plasticity and neuroprotection. Neuroscientist 2015, 21, 152-168.

11. Maccarrone, M.; Bab, R.; Biro, T.; Cabral, G.A.; Dey, S.K.; di Marzo, V.; Konje, J.C.; Kunos, G.; Mechoulam, R.; Pacher, P.; et al. Endocannabinoid signaling at the periphery: 50 years after THC. Trends Pharmacol. Sci. 2015, 36, 277-296.

12. Oh, D.Y.; Olefsky, J.M. Omega 3 fatty acids and GPR120. Cell Metab. 2012, 15, 564-565.

13. Im, D.S. Functions of omega-3 fatty acids and FFA4 (GPR120) in macrophages. Eur. J. Pharmacol. 2015.

14. Corsi, A.K.; Wightman, B.; Chalfie, M. A transparent window into biology: A primer on Caenorhabditis elegans. Genetics 2015, 200, 387-407.

15. Brooks, K.K.; Liang, B.; Watts, J.L. The influence of bacterial diet on fat storage in C. elegans. PLoS ONE 2009, 4, e7545. 
16. Watts, J.L. Fat synthesis and adiposity regulation in Caenorhabditis elegans. Trends Endocrinol. Metab. 2009, 20, 58-65.

17. Spychalla, J.P.; Kinney, A.J.; Browse, J. Identification of an animal omega-3 fatty acid desaturase by heterologous expression in arabidopsis. Proc. Natl. Acad. Sci. USA 1997, 94, 1142-1147.

18. Peyou-Ndi, M.M.; Watts, J.L.; Browse, J. Identification and characterization of an animal delta(12) fatty acid desaturase gene by heterologous expression in Saccharomyces cerevisiae. Arch. Biochem. Biophys. 2000, 376, 399-408.

19. Watts, J.L.; Browse, J. Isolation and characterization of a delta 5-fatty acid desaturase from Caenorhabditis elegans. Arch. Biochem. Biophys. 1999, 362, 175-182.

20. Watts, J.L.; Browse, J. Genetic dissection of polyunsaturated fatty acid synthesis in Caenorhabditis elegans. Proc. Natl. Acad. Sci. USA 2002, 99, 5854-5859.

21. Watts, J.L.; Phillips, E.; Griffing, K.R.; Browse, J. Deficiencies in C20 polyunsaturated fatty acids cause behavioral and developmental defects in Caenorhabditis elegans fat-3 mutants. Genetics 2003, 163, 581-589.

22. Deline, M.L.; Vrablik, T.L.; Watts, J.L. Dietary supplementation of polyunsaturated fatty acids in Caenorhabditis elegans. J. Vis. Exp. 2013.

23. Kahn-Kirby, A.H.; Dantzker, J.L.; Apicella, A.J.; Schafer, W.R.; Browse, J.; Bargmann, C.I.; Watts, J.L. Specific polyunsaturated fatty acids drive TRPV-dependent sensory signaling in vivo. Cell 2004, 119, 889-900.

24. Watts, J.L.; Browse, J. A palmitoyl-CoA-specific delta9 fatty acid desaturase from Caenorhabditis elegans. Biochem. Biophys. Res. Commun. 2000, 272, 263-269.

25. Brock, T.J.; Browse, J.; Watts, J.L. Fatty acid desaturation and the regulation of adiposity in Caenorhabditis elegans. Genetics 2007, 176, 865-875.

26. Vrablik, T.L.; Watts, J.L. Polyunsaturated fatty acid derived signaling in reproduction and development: Insights from Caenorhabditis elegans and Drosophila melanogaster. Mol. Reprod. Dev. 2013, 80, 244-259.

27. Kubagawa, H.M.; Watts, J.L.; Corrigan, C.; Edmonds, J.W.; Sztul, E.; Browse, J.; Miller, M.A. Oocyte signals derived from polyunsaturated fatty acids control sperm recruitment in vivo. Nat. Cell Biol. 2006, 8, 1143-1148.

28. Edmonds, J.W.; Prasain, J.K.; Dorand, D.; Yang, Y.; Hoang, H.D.; Vibbert, J.; Kubagawa, H.M.; Miller, M.A. Insulin/FOXO signaling regulates ovarian prostaglandins critical for reproduction. Dev. Cell 2010, 19, 858-871.

29. Hoang, H.D.; Prasain, J.K.; Dorand, D.; Miller, M.A. A heterogeneous mixture of F-series prostaglandins promotes sperm guidance in the Caenorhabditis elegans reproductive tract. PLoS Genet. 2013, 9, e1003271.

30. Prasain, J.K.; Wilson, L.; Hoang, H.D.; Moore, R.; Miller, M.A. Comparative lipidomics of Caenorhabditis elegans metabolic disease models by swath non-targeted tandem mass spectrometry. Metabolites 2015, 5, 677-696.

31. McKnight, K.; Hoang, H.D.; Prasain, J.K.; Brown, N.; Vibbert, J.; Hollister, K.A.; Moore, R.; Ragains, J.R.; Reese, J.; Miller, M.A. Neurosensory perception of environmental cues modulates sperm motility critical for fertilization. Science 2014, 344, 754-757. 
32. Watts, J.L.; Browse, J. Dietary manipulation implicates lipid signaling in the regulation of germ cell maintenance in C. elegans. Dev. Biol. 2006, 292, 381-392.

33. Webster, C.M.; Deline, M.L.; Watts, J.L. Stress response pathways protect germ cells from omega-6 polyunsaturated fatty acid-mediated toxicity in Caenorhabditis elegans. Dev. Biol. 2013, 373, 14-25.

34. Murphy, C.T.; McCarroll, S.A.; Bargmann, C.I.; Fraser, A.; Kamath, R.S.; Ahringer, J.; Li, H.; Kenyon, C. Genes that act downstream of DAF-16 to influence the lifespan of Caenorhabditis elegans. Nature 2003, 424, 277-283.

35. Deline, M.; Keller, J.; Rothe, M.; Schunck, W.H.; Menzel, R.; Watts, J.L. Epoxides derived from dietary dihomo-gamma-linolenic acid induce germ cell death in C. elegans. Sci. Rep. 2015, 5, 15417.

36. Amrit, F.R.; Ratnappan, R.; Keith, S.A.; Ghazi, A. The C. elegans lifespan assay toolkit. Methods 2014, 68, 465-475.

37. Kenyon, C.J. The genetics of ageing. Nature 2010, 464, 504-512.

38. Schroeder, E.A.; Brunet, A. Lipid profiles and signals for long life. Trends Endocrinol. Metab. 2015, 26, 589-592.

39. Hulbert, A.J.; Kelly, M.A.; Abbott, S.K. Polyunsaturated fats, membrane lipids and animal longevity. J. Comp. Physiol. B Biochem.Syst. Environ. Physiol. 2014, 184, 149-166.

40. Shmookler-Reis, R.J.; Xu, L.; Lee, H.; Chae, M.; Thaden, J.J.; Bharill, P.; Tazearslan, C.; Siegel, E.; Alla, R.; Zimniak, P.; et al. Modulation of lipid biosynthesis contributes to stress resistance and longevity of C. elegans mutants. Aging 2011, 3, 125-147.

41. Sugawara, S.; Honma, T.; Ito, J.; Kijima, R.; Tsuduki, T. Fish oil changes the lifespan of Caenorhabditis elegans via lipid peroxidation. J. Clin. Biochem. Nutr. 2013, 52, 139-145.

42. O'Rourke, E.J.; Kuballa, P.; Xavier, R.; Ruvkun, G. Omega-6 polyunsaturated fatty acids extend life span through the activation of autophagy. Genes Dev. 2013, 27, 429-440.

43. Gems, D.; Doonan, R. Antioxidant defense and aging in C. elegans: Is the oxidative damage theory of aging wrong? Cell Cycle 2009, 8, 1681-1687.

44. Honda, S.; Ishii, N.; Suzuki, K.; Matsuo, M. Oxygen-dependent perturbation of life span and aging rate in the nematode. J. Gerontol. 1993, 48, B57-B61.

45. Zhou, K.I.; Pincus, Z.; Slack, F.J. Longevity and stress in Caenorhabditis elegans. Aging 2011, 3, 733-753.

46. Van Raamsdonk, J.M.; Hekimi, S. Deletion of the mitochondrial superoxide dismutase SOD-2 extends lifespan in Caenorhabditis elegans. PLoS Genet. 2009, 5, e1000361.

47. Cypser, J.R.; Johnson, T.E. Multiple stressors in Caenorhabditis elegans induce stress hormesis and extended longevity. J. Gerontol. A Biol. Sci. Med. Sci. 2002, 57, B109-B114.

48. Cypser, J.R.; Tedesco, P.; Johnson, T.E. Hormesis and aging in Caenorhabditis elegans. Exp. Gerontol. 2006, 41, 935-939.

49. Schaar, C.E.; Dues, D.J.; Spielbauer, K.K.; Machiela, E.; Cooper, J.F.; Senchuk, M.; Hekimi, S.; van Raamsdonk, J.M. Mitochondrial and cytoplasmic ROS have opposing effects on lifespan. PLoS Genet. 2015, 11, e1004972.

50. Back, P.; Braeckman, B.P.; Matthijssens, F. ROS in aging Caenorhabditis elegans: Damage or signaling? Oxid. Med. Cell. Longev. 2012, 2012, 608478. 
51. Yee, C.; Yang, W.; Hekimi, S. The intrinsic apoptosis pathway mediates the pro-longevity response to mitochondrial ROS in C. elegans. Cell 2014, 157, 897-909.

52. Shadel, G.S.; Horvath, T.L. Mitochondrial ros signaling in organismal homeostasis. Cell 2015, 163, 560-569.

53. Dancy, B.C.; Chen, S.W.; Drechsler, R.; Gafken, P.R.; Olsen, C.P. 13C- and 15N-Labeling strategies combined with mass spectrometry comprehensively quantify phospholipid dynamics in C. elegans. PLoS ONE 2015, 10, e0141850.

54. Kenyon, C. A pathway that links reproductive status to lifespan in Caenorhabditis elegans. Ann. N. Y. Acad. Sci. 2010, 1204, 156-162.

55. Hansen, M.; Flatt, T.; Aguilaniu, H. Reproduction, fat metabolism, and life span: What is the connection? Cell Metab. 2013, 17, 10-19.

56. Brock, T.J.; Browse, J.; Watts, J.L. Genetic regulation of unsaturated fatty acid composition in C. elegans. PLoS Genet. 2006, 2, e108.

57. Van Gilst, M.R.; Hadjivassiliou, H.; Jolly, A.; Yamamoto, K.R. Nuclear hormone receptor NHR-49 controls fat consumption and fatty acid composition in C. elegans. PLoS Biol. 2005, 3, e53.

58. Ratnappan, R.; Amrit, F.R.; Chen, S.W.; Gill, H.; Holden, K.; Ward, J.; Yamamoto, K.R.; Olsen, C.P.; Ghazi, A. Germline signals deploy NHR-49 to modulate fatty-acid beta-oxidation and desaturation in somatic tissues of C. elegans. PLoS Genet. 2014, 10, e1004829.

59. Goudeau, J.; Bellemin, S.; Toselli-Mollereau, E.; Shamalnasab, M.; Chen, Y.; Aguilaniu, H. Fatty acid desaturation links germ cell loss to longevity through NHR-80/HNF4 in C. elegans. PLoS Biol. 2011, 9, e1000599.

60. Lapierre, L.R.; Gelino, S.; Melendez, A.; Hansen, M. Autophagy and lipid metabolism coordinately modulate life span in germline-less C. elegans. Curr. Biol. 2011, 21, 1507-1514.

61. Wang, M.C.; O’Rourke, E.J.; Ruvkun, G. Fat metabolism links germline stem cells and longevity in C. elegans. Science 2008, 322, 957-960.

62. Folick, A.; Oakley, H.D.; Yu, Y.; Armstrong, E.H.; Kumari, M.; Sanor, L.; Moore, D.D.; Ortlund, E.A.; Zechner, R.; Wang, M.C. Aging. Lysosomal signaling molecules regulate longevity in Caenorhabditis elegans. Science 2015, 347, 83-86.

63. DiNicolantonio, J.J.; Lucan, S.C.; O'Keefe, J.H. The evidence for saturated fat and for sugar related to coronary heart disease. Prog. Cardiovasc. Dis. 2015.

64. Kolderup, A.; Svihus, B. Fructose metabolism and relation to atherosclerosis, type 2 diabetes, and obesity. J. Nutr. Metab. 2015, 2015, 823081.

65. Lee, S.J.; Murphy, C.T.; Kenyon, C. Glucose shortens the life span of C. elegans by downregulating DAF-16/FOXO activity and aquaporin gene expression. Cell Metab. 2009, 10, 379-391.

66. Schulz, T.J.; Zarse, K.; Voigt, A.; Urban, N.; Birringer, M.; Ristow, M. Glucose restriction extends Caenorhabditis elegans life span by inducing mitochondrial respiration and increasing oxidative stress. Cell Metab. 2007, 6, 280-293. 
67. Lee, D.; Jeong, D.E.; Son, H.G.; Yamaoka, Y.; Kim, H.; Seo, K.; Khan, A.A.; Roh, T.Y.; Moon, D.W.; Lee, Y.; et al. SREBP and MDT-15 protect C. elegans from glucose-induced accelerated aging by preventing accumulation of saturated fat. Genes Dev. 2015, 29, 2490-2503.

68. Taubert, S.; van Gilst, M.R.; Hansen, M.; Yamamoto, K.R. A mediator subunit, MDT-15, integrates regulation of fatty acid metabolism by NHR-49-dependent and -independent pathways in C. elegans. Genes Dev. 2006, 20, 1137-1149.

69. Walker, A.K.; Jacobs, R.L.; Watts, J.L.; Rottiers, V.; Jiang, K.; Finnegan, D.M.; Shioda, T.; Hansen, M.; Yang, F.; Niebergall, L.J.; et al. A conserved SREBP-1/phosphatidylcholine feedback circuit regulates lipogenesis in metazoans. Cell 2011, 147, 840-852.

70. Yang, F.; Vought, B.W.; Satterlee, J.S.; Walker, A.K.; Jim Sun, Z.Y.; Watts, J.L.; DeBeaumont, R.; Saito, R.M.; Hyberts, S.G.; Yang, S.; et al. An ARC/mediator subunit required for SREBPP control of cholesterol and lipid homeostasis. Nature 2006, 442, 700-704.

71. Steinbaugh, M.J.; Narasimhan, S.D.; Robida-Stubbs, S.; Moronetti Mazzeo, L.E.; Dreyfuss, J.M.; Hourihan, J.M.; Raghavan, P.; Operana, T.N.; Esmaillie, R.; Blackwell, T.K. Lipid-mediated regulation of SKN-1/Nrf in response to germ cell absence. eLife 2015, 4.

72. Lynn, D.A.; Dalton, H.M.; Sowa, J.N.; Wang, M.C.; Soukas, A.A.; Curran, S.P. Omega-3 and -6 fatty acids allocate somatic and germline lipids to ensure fitness during nutrient and oxidative stress in Caenorhabditis elegans. Proc. Natl. Acad. Sci. USA 2015, 112, 15378-15383.

73. Bazinet, R.P.; Laye, S. Polyunsaturated fatty acids and their metabolites in brain function and disease. Nat. Rev. Neurosci. 2014, 15, 771-785.

74. Sinclair, A.J.; Begg, D.; Mathai, M.; Weisinger, R.S. Omega 3 fatty acids and the brain: Review of studies in depression. Asia Pac. J. Clin.Nutr. 2007, 16, 391-397.

75. White, J.G.; Southgate, E.; Thomson, J.N.; Brenner, S. The structure of the nervous system of the nematode Caenorhabditis elegans. Philos. Trans. R. Soc. Lond. Ser. B Biol. Sci. 1986, $314,1-340$.

76. Lesa, G.M.; Palfreyman, M.; Hall, D.H.; Clandinin, M.T.; Rudolph, C.; Jorgensen, E.M.; Schiavo, G. Long chain polyunsaturated fatty acids are required for efficient neurotransmission in C. elegans. J. Cell Sci. 2003, 116, 4965-4975.

77. O'Halloran, D.M.; Altshuler-Keylin, S.; Lee, J.I.; L'Etoile, N.D. Regulators of AWC-mediated olfactory plasticity in Caenorhabditis elegans. PLoS Genet. 2009, 5, e1000761.

78. Pastuhov, S.I.; Fujiki, K.; Nix, P.; Kanao, S.; Bastiani, M.; Matsumoto, K.; Hisamoto, N. Endocannabinoid-goalpha signalling inhibits axon regeneration in Caenorhabditis elegans by antagonizing gqalpha-PKC-JNK signalling. Nat. Commun. 2012, 3, 1136.

79. Lucanic, M.; Held, J.M.; Vantipalli, M.C.; Klang, I.M.; Graham, J.B.; Gibson, B.W.; Lithgow, G.J.; Gill, M.S. N-Acylethanolamine signalling mediates the effect of diet on lifespan in Caenorhabditis elegans. Nature 2011, 473, 226-229.

80. Raabe, R.C.; Mathies, L.D.; Davies, A.G.; Bettinger, J.C. The omega-3 fatty acid eicosapentaenoic acid is required for normal alcohol response behaviors in C. elegans. PLOS ONE 2014, 9. 
81. Davies, A.G.; Bettinger, J.C.; Thiele, T.R.; Judy, M.E.; McIntire, S.L. Natural variation in the NPR-1 gene modifies ethanol responses of wild strains of C. elegans. Neuron 2004, 42, 731-743.

82. Vasquez, V.; Krieg, M.; Lockhead, D.; Goodman, M.B. Phospholipids that contain polyunsaturated fatty acids enhance neuronal cell mechanics and touch sensation. Cell Rep. 2014, 6, 70-80.

83. Kosel, M.; Wild, W.; Bell, A.; Rothe, M.; Lindschau, C.; Steinberg, C.E.; Schunck, W.H.; Menzel, R. Eicosanoid formation by a cytochrome P450 isoform expressed in the pharynx of Caenorhabditis elegans. Biochem. J. 2011, 435, 689-700.

84. Kulas, J.; Schmidt, C.; Rothe, M.; Schunck, W.H.; Menzel, R. Cytochrome P450-dependent metabolism of eicosapentaenoic acid in the nematode Caenorhabditis elegans. Arch. Biochem. Biophys. 2008, 472, 65-75.

85. Zhou, Y.; Falck, J.R.; Rothe, M.; Schunck, W.H.; Menzel, R. Role of CYP eicosanoids in the regulation of pharyngeal pumping and food uptake in Caenorhabditis elegans. J. Lipid Res. 2015, 56, 2110-2123.

86. Ma, D.K.; Rothe, M.; Zheng, S.; Bhatla, N.; Pender, C.L.; Menzel, R.; Horvitz, H.R. Cytochrome $\mathrm{P} 450$ drives a HIF-regulated behavioral response to reoxygenation by $C$. elegans. Science 2013, 341, 554-558.

87. Eltzschig, H.K.; Eckle, T. Ischemia and reperfusion-from mechanism to translation. Nat. Med. 2011, 17, 1391-1401.

88. Keller, J.; Ellieva, A.; Ma, D.K.; Ju, J.J.; Nehk, E.; Konkel, A.; Falck, J.R.; Schunck, W.H.; Menzel, R. CYP-13A12 of the nematode Caenorhabditis elegans is a PUFA-epoxygenase involved in behavioural response to reoxygenation. Biochem. J. 2014, 464, 61-71.

89. Kang, J.X.; Wang, J.; Wu, L.; Kang, Z.B. Transgenic mice: Fat-1 mice convert $n-6$ to $n-3$ fatty acids. Nature 2004, 427, 504.

90. Astarita, G.; Kendall, A.C.; Dennis, E.A.; Nicolaou, A. Targeted lipidomic strategies for oxygenated metabolites of polyunsaturated fatty acids. Biochim. Biophys. Acta 2015, 1851, 456-468.

91. Kang, J.X. From fat to fat-1: A tale of omega-3 fatty acids. J. Membr. Biol. 2005, 206, 165-172.

92. Kang, J.X. Fat-1 transgenic mice: A new model for omega-3 research. Prostaglandins Leukot Essent Fat. Acids 2007, 77, 263-267.

93. Kang, J.X. The omega-6/omega-3 fatty acid ratio in chronic diseases: Animal models and molecular aspects. World Rev. Nutr. Diet. 2011, 102, 22-29.

94. Kang, J.X.; Liu, A. The role of the tissue omega-6/omega-3 fatty acid ratio in regulating tumor angiogenesis. Cancer Metastasis Rev. 2013, 32, 201-210.

95. Kang, J.X.; Weylandt, K.H. Modulation of inflammatory cytokines by omega-3 fatty acids. Sub-Cell. Biochem. 2008, 49, 133-143.

96. Bak, D.H.; Zhang, E.; Yi, M.H.; Kim, D.K.; Lim, K.; Kim, J.J.; Kim, D.W. High omega3-polyunsaturated fatty acids in fat-1 mice prevent streptozotocin-induced purkinje cell degeneration through bdnf-mediated autophagy. Sci. Rep. 2015, 5, 15465. 
97. Huang, W.; Wang, B.; Li, X.; Kang, J.X. Endogenously elevated n-3 polyunsaturated fatty acids alleviate acute ethanol-induced liver steatosis. BioFactors 2015, 41, 453-462.

98. Hwang, W.M.; Bak, D.H.; Kim, D.H.; Hong, J.Y.; Han, S.Y.; Park, K.Y.; Lim, K.; Lim, D.M. Attenuation of streptozotocin-induced pancreatic beta cell death in transgenic fat-1 mice via autophagy activation. Endocrinol. Metab. 2015. Available online: http:/ /e-enm.org/DOIx.php?id=10.3803/EnM.2015.30.e24 (accessed on 2 February 2016).

99. Li, X.; Ballantyne, L.L.; Che, X.; Mewburn, J.D.; Kang, J.X.; Barkley, R.M.; Murphy, R.C.; Yu, Y.; Funk, C.D. Endogenously generated omega-3 fatty acids attenuate vascular inflammation and neointimal hyperplasia by interaction with free fatty acid receptor 4 in mice. J. Am. Heart Assoc. 2015, 4, e001856.

100. Simopoulos, A.P. Human requirement for $n-3$ polyunsaturated fatty acids. Poult. Sci. 2000, 79, 961-970.

101. Rice, K.M.; Walker, E.M., Jr.; Wu, M.; Gillette, C.; Blough, E.R. Environmental mercury and its toxic effects. J. Prev. Med. Public Health 2014, 47, 74-83.

102. Lai, L.; Kang, J.X.; Li, R.; Wang, J.; Witt, W.T.; Yong, H.Y.; Hao, Y.; Wax, D.M.; Murphy, C.N.; Rieke, A.; et al. Generation of cloned transgenic pigs rich in omega-3 fatty acids. Nat. Biotechnol. 2006, 24, 435-436.

103. Zhang, P.; Zhang, Y.; Dou, H.; Yin, J.; Chen, Y.; Pang, X.; Vajta, G.; Bolund, L.; Du, Y.; Ma, R.Z. Handmade cloned transgenic piglets expressing the nematode fat-1 gene. Cell. Reprogram. 2012, 14, 258-266.

104. Zhou, Y.; Lin, Y.; Wu, X.; Feng, C.; Long, C.; Xiong, F.; Wang, N.; Pan, D.; Chen, H. The high-level accumulation of $n-3$ polyunsaturated fatty acids in transgenic pigs harboring the n-3 fatty acid desaturase gene from caenorhabditis briggsae. Transgenic Res. 2014, 23, 89-97.

105. Guo, T.; Liu, X.F.; Ding, X.B.; Yang, F.F.; Nie, Y.W.; An, Y.J.; Guo, H. Fat-1 transgenic cattle as a model to study the function of omega-3 fatty acids. Lipids Health Dis. 2011, 10, 244.

106. Wu, X.; Ouyang, H.; Duan, B.; Pang, D.; Zhang, L.; Yuan, T.; Xue, L.; Ni, D.; Cheng, L.; Dong, S.; et al. Production of cloned transgenic cow expressing omega-3 fatty acids. Transgenic Res. 2012, 21, 537-543.

107. Pohlmeier, W.E.; Hovey, R.C.; Van Eenennaam, A.L. Reproductive abnormalities in mice expressing omega-3 fatty acid desaturase in their mammary glands. Transgenic Res. 2011, 20, 283-292. 


\title{
Up-Regulation of Mitochondrial Antioxidant Superoxide Dismutase Underpins Persistent Cardiac Nutritional-Preconditioning by Long Chain n-3 Polyunsaturated Fatty Acids in the Rat
}

\author{
Grace G. Abdukeyum, Alice J. Owen, Theresa A. Larkin and Peter L. McLennan
}

Abstract: Reactive oxygen species paradoxically underpin both ischaemia/ reperfusion (I/R) damage and ischaemic preconditioning (IPC) cardioprotection. Long-chain omega-3 polyunsaturated fatty acids (LCn-3 PUFA) are highly susceptible to peroxidation, but are paradoxically cardioprotective. This study tested the hypothesis that LCn-3 PUFA cardioprotection is underpinned by peroxidation, upregulating antioxidant activity to reduce I/R-induced lipid oxidation, and the mechanisms of this nutritional preconditioning contrast to mechanisms of IPC. Rats were fed: fish oil (LCn-3 PUFA); sunflower seed oil (n-6 PUFA); or beef tallow (saturated fat, SF) enriched diets for six weeks. Isolated hearts were subject to: $180 \mathrm{~min}$ normoxic perfusion; a $30 \mathrm{~min}$ coronary occlusion ischaemia protocol then $120 \mathrm{~min}$ normoxic reperfusion; or a $3 \times 5 \mathrm{~min}$ global IPC protocol, $30 \mathrm{~min}$ ischaemia, then reperfusion. Dietary LCn-3 PUFA raised basal: membrane docosahexaenoic acid (22:6n-3 DHA); fatty acid peroxidisability index; concentrations of lipid oxidation products; and superoxide dismutase (MnSOD) activity (but not CuZnSOD or glutathione peroxidase). Infarct size correlated inversely with basal MnSOD activity $\left(r^{2}=0.85\right)$ in the ischaemia protocol and positively with I/R-induced lipid oxidation (lipid hydroperoxides (LPO), $r^{2}=0.475$; malondialdehyde (MDA), $r^{2}=0.583$ ) across ischaemia and IPC protocols. While both dietary fish oil and IPC infarct-reduction were associated with reduced I/R-induced lipid oxidation, fish oil produced nutritional preconditioning by prior LCn-3 PUFA incorporation and increased peroxidisability leading to up-regulated mitochondrial SOD antioxidant activity.

Reprinted from J. Clin. Med. Cite as: Abdukeyum, G.G.; Owen, A.J.; Larkin, T.A.; McLennan, P.L. Up-Regulation of Mitochondrial Antioxidant Superoxide Dismutase Underpins Persistent Cardiac Nutritional-Preconditioning by Long Chain n-3 Polyunsaturated Fatty Acids in the Rat. J. Clin. Med. 2016, 5, 32.

\section{Introduction}

Regular consumption of fish or fish oil reduces cardiovascular mortality [1], often without modifying classical risk factors. For example, sudden death is reduced 
in high-risk post-MI patients, without significant reductions in blood pressure, blood lipids or prevention of new cardiac events [2]. This cardioprotection, associated with omega-3 long-chain polyunsaturated fatty acid (LCn-3 PUFA) consumption, is observed independently of the prevention of ischaemic events [3], and therefore supports a cardiac origin related to incorporation of the fatty acids into myocardial membranes [4]. Ischaemic preconditioning (IPC) is a powerful cardioprotective process, wherein brief periods of ischaemia, insufficient to produce cellular damage, can protect the myocardium from the damaging effects of a subsequent more prolonged ischaemic insult. The protective envelope of IPC is twofold, categorised as: classical or early preconditioning, which provides cardioprotection for several hours after the IPC stimulus; with a second phase called late or delayed preconditioning which occurs 24-7 h after the stimulus. Experimentally, the LCn-3 PUFA confer their cardioprotection in part through nutritional preconditioning of the myocardium that in rat heart is at least as effective in reducing infarct size [5,6] and promoting post-ischaemic contractile recovery [5], and more effective in preventing ischaemia or reperfusion induced cardiac arrhythmias [5], as early ischaemic preconditioning (IPC). Classical, early IPC cardioprotection disappears within several hours of the initial preconditioning stimulus and repeated brief preconditioning episodes become ineffective in providing this early protection [7]. In contrast, the cardioprotection derived from dietary LCn-3 PUFA is obtained only after they are incorporated into and continuously present in the myocardium for at least seven days and it persists over weeks or months, for however long elevated membrane content is sustained $[5,8,9]$. There is no desensitisation apparent, with acute ischaemia or reperfusion arrhythmias prevented after five weeks [8] to 52 weeks [10] of continuous exposure to dietary LCn-3 PUFA. Therefore, the cardioprotective benefit of fish oil appears to mimic the more sustained, repeatable protection of late IPC and other persistent preconditioning stimuli [11-13]. Moreover, the LCn-3 PUFA [5] share with late IPC [14] the capacity to protect against both infarction and myocardial stunning.

Myocardial ischaemia and reperfusion (I/R) stimulates production of reactive oxygen species (ROS) and depletes antioxidants in the heart, creating oxidative stress, oxidation of biomolecules and cell damage. Paradoxically, these free radicals also act as triggers of IPC $[15,16]$. The highly unsaturated LCn-3 PUFA found in fish oil: 20:5n-3 eicosapentaenoic acid (EPA) and 22:6n-3 docosahexaenoic acid (DHA) with their numerous bisallylic carbon atoms, are more susceptible to peroxidation and generation of damaging reactive oxygen species than are shorter, less unsaturated fatty acids such as 18:2n-6 linoleic acid and 20:4n-6 arachidonic acid [17], which raises the prospect of adverse effects of fish oil supplementation. However, there is no clinical evidence to suggest that fish oil supplementation or high fish diets promote oxidative stress-related cardiovascular disease. In contrast, production of ROS is a mechanism invoked to explain the paradoxical effects of late IPC in the 
heart, which works by inducing upregulation of endogenous antioxidant protective mechanisms [16,18]. That protection occurs in lieu of the extreme oxidation of biomolecules and cell damage that usually occurs with I/R-induced oxidative stress and antioxidant depletion.

The current study tested the hypothesis that incorporation of LCn-3 PUFA into myocardial membranes increases their peroxidation potential and basal fatty acid oxidation, which by their constant presence, in turn increases endogenous antioxidant enzymes to confer physiological cardioprotective actions against I/R-stimulated oxidative stress. We propose that this will contrast to the mechanism of early IPC cardioprotection.

\section{Experimental Section}

\subsection{Animals and Diets}

Fifty-four male Wistar rats were randomly assigned to three experimental dietary groups. For six weeks they were fed one of three iso-energetic diets containing either predominantly saturated animal fat, n-6 PUFA or LC n-3 PUFA as the source of fat. The diets were based on the American Institute of Nutrition AIN93 rat diet, containing all essential vitamins and minerals with gelatine as a component of the protein source. The diet was prepared with $10 \%$ (dry wt) fat (23\% metabolisable energy as fat) consisting of: 7\% beef tallow plus 3\% olive oil (SF diet); $5 \%$ sunflower seed oil plus 5\% olive oil ( $n-6$ PUFA diet); or 7\% fish oil (Nu-Mega high DHA tuna fish oil) plus 3\% olive oil (LCn-3 PUFA diet). In addition to delivering diets rich in saturated fat, $n-6$ PUFA or $n-3$ PUFA, the oil blends in the LCn-3 PUFA diet and the n-6 PUFA diet were designed to deliver similar total PUFA, and the oil blends in the LCn-3 PUFA diet and the SF diet were designed to deliver similar total n-6 PUFA, as previously described [5]. All diets contained sufficient PUFA to prevent essential fatty acid deficiency [5].

Animal care and experiments were conducted with the approval of the University of Wollongong, Animal Care and Ethics Committee according to the guidelines of the National Health and Medical Research Council, Australia, Australian Code of Practice for the Care and Use of Animals for Scientific Purposes [19].

\subsection{Heart Preparation}

After six weeks of feeding, rats were anaesthetised (pentobarbital sodium, $60 \mathrm{mg} \cdot \mathrm{kg}^{-1}$ i.p.), the thorax was opened, the heart was rapidly excised, submerged in ice-cold perfusate to arrest beating, and immediately perfused by an aortic cannula in the Langendorff mode at a constant pressure of $75 \mathrm{mmHg}$ delivering warm $\left(37^{\circ} \mathrm{C}\right)$ Krebs-Henseleit bicarbonate buffer gassed with $5 \% \mathrm{CO}_{2}$ in $\mathrm{O}_{2}$ [5]. The left atrium was opened and a thin-walled balloon catheter was introduced into the left ventricle, 
with balloon volume adjusted to maintain end diastolic pressure of 6-8 $\mathrm{mmHg}$. $6-0$ silk suture was passed through the myocardium closely underlying the left anterior descending coronary artery near its origin.

\subsection{Index Ischaemia and Ischaemic Preconditioning}

Each dietary group ( $n=18$ ) was separated into groups of $n=6$ and randomly assigned to one of three perfusion protocols for $180 \mathrm{~min}$ after initial $30 \mathrm{~min}$ equilibration perfusion (Figure 1).

1. Control normoxia protocol ( $n=6$ per diet): Hearts were perfused throughout with oxygenated Krebs-Henseleit solution.

2. Ischaemia protocol $(\mathrm{n}=6$ per diet): Hearts were normoxically perfused for $30 \mathrm{~min}$ followed by $30 \mathrm{~min}$ index-ischaemia and $120 \mathrm{~min}$ normoxic reperfusion. Index-ischaemia was induced by occluding the left anterior descending coronary artery.

3. Ischaemic preconditioning (IPC) protocol ( $n=6$ per diet): Hearts were subjected to three cycles of five minutes global ischaemia (zero perfusion), each followed by five minutes normoxic reperfusion, prior to the $30 \mathrm{~min}$ index-ischaemia then 120 min normoxic reperfusion [5].

On completion of 120 min reperfusion in the ischaemia and IPC protocols, the coronary artery was re-occluded to reveal the ischaemic zone at risk (I-z/r). Hearts were then cut into $2 \mathrm{~mm}$ slices. The central slice was incubated in a buffer containing triphenyl-tetrazolium chloride and sodium phosphate $(\mathrm{pH} 7.4)$, then stored in 10\% formalin until photographed and analysed for infarct size. Infarct size was reported as a percentage of the zone at risk. The remaining slices were separated into non-ischaemic (non-I) and ischaemic (ISCH) segments (Figure 1). Samples of fresh ISCH and non-I tissue were used immediately for lipid hydroperoxide (LPO) analysis, with the remainder rapidly frozen and stored at $-80^{\circ} \mathrm{C}$ for analysis of other markers of oxidation and antioxidant status. Samples of control normoxic heart were always taken from the left ventricle anterior free wall, supplied by the left anterior descending coronary artery, that would have been subject to ischaemia in the other protocols. It represents the basal state of the ischaemic zone at risk.

\subsection{Measurement of Oxidative Stress Biomarkers}

Concentrations of LPO were measured by modification of the ferric thiocyanate assay using a colorimetric assay kit (Lipid Hydroperoxide Assay, Cayman Chemical Company, Ann Arbor, MI, USA) and were expressed per mg of protein. Concentrations of malondialdehyde (MDA) were measured in thawed tissue homogenates by reverse-phase HPLC with fluorescence detection [20]. 


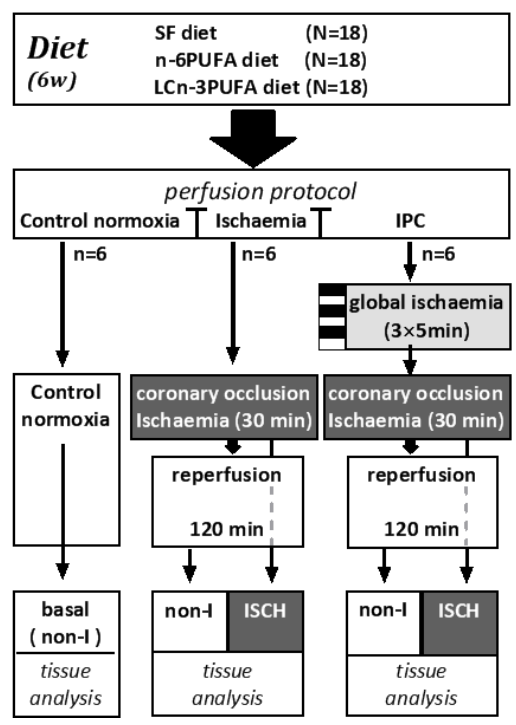

Figure 1. Flow chart illustrating the distribution of dietary groups into: Control normoxic; Ischaemia; and IPC perfusion protocols. In each protocol, isolated hearts were perfused for $180 \mathrm{~min}$. Ischaemia protocol and IPC protocol hearts were dissected into non-ischaemic (non-I) and ischaemic (ISCH) tissue for biochemical analysis. IPC: ischaemic preconditioning.

\subsection{Measurement of Antioxidants}

Endogenous: Total superoxide dismutase (SOD) activity and CuZnSOD activity were measured in ventricle sections of: ISCH tissue; and non-I tissue using a BIOXYTECH $^{\circledR}$-SOD-525 ${ }^{\mathrm{TM}}$ assay kit (Oxis Research ${ }^{\mathrm{TM}}$, Portland, OR, USA). The activity of mitochondrial SOD activated by manganese (MnSOD) was calculated as the difference between total SOD and CuZnSOD. The activity of SOD was expressed per mg of tissue protein. Glutathione peroxidase (GPX) activity was measured in ventricle sections of: ISCH; and non-I ventricle using BIOXYTECH ${ }^{\circledR} \mathrm{GPx}-340 \mathrm{TM}$ assay kit (OxisResearch ${ }^{\mathrm{TM}}$, Portland OR, USA) and was expressed per mg of tissue protein. Exogenous: Myocardial vitamin E (alpha-tocopherol) was measured by HPLC with electrochemical detection, using a modification of the method described by Yang [21].

\subsection{Myocardial Fatty Acid Analyses}

Total lipids were extracted from 100 to $200 \mathrm{mg}$ samples of ventricular myocardium using a modification of the Folch method [22]. Phospholipids were isolated from the total muscle lipid by solid phase extraction using silica Sep-pak ${ }^{\mathrm{TM}}$ cartridges (Waters, Rydalmere, NSW, Australia). Fatty acid methyl esters were 
prepared by direct transesterification of the phospholipid fraction [23] and analysed by gas chromatography using a Shimadzu GC-17A with flame ionization detection using a $30 \mathrm{~m} \times 0.25 \mathrm{~mm}, 0.25 \mu \mathrm{m}$ FAMEWAX column (J and W Scientific, Santa Clara CA, USA) with hydrogen as carrier gas and a step temperature program rising from $150{ }^{\circ} \mathrm{C}$ to $260{ }^{\circ} \mathrm{C}$, over $27 \mathrm{~min}$ and held for $6 \mathrm{~min}$. Individual fatty acids were identified by their retention times with reference to authentic fatty acid methyl ester standards (Sigma-Aldrich, Rydalmere, NSW, Australia) and expressed as a percentage of total phospholipid fatty acids.

\subsection{Statistical Analyses}

Results were expressed as mean \pm SEM. Data were analysed by two-way analysis of variance (ANOVA) for diet and treatment main effects (normoxic perfusion, ischaemia, IPC + ischaemia) and by multi-way ANOVA for diet, treatment and ISCH versus non-I tissue main effects. Tukey's HSD test was used for post-hoc pairwise comparison of individual means and interactions. Within dietary groups, ISCH and non-I sections of the same hearts were compared using repeated measures ANOVA. Statistical analyses were performed using Statistix software, version 10 (Analytical Software, Tallahassee, FL, USA). Linear regression analysis with Pearson's correlation was performed to determine linear associations between lipid oxidation products, antioxidants and infarct size using Prism for Windows, version 6 (GraphPad Software, La Jolla, CA, USA). Statistical significance was accepted at $p<0.05$.

\section{Results}

Neither the starting body weight, the final body weight nor the change in body weight over six weeks differed between dietary groups (Start: SF $348 \pm 6$ g; $n$-6 PUFA $351 \pm 5$ g; LC $n-3$ PUFA $352 \pm 6$ g. Six weeks: SF $460 \pm 11$ g; $n-6$ PUFA $457 \pm 8$ g; LC $n-3$ PUFA $480 \pm 9$ g. Change: SF $112 \pm 7$ g; n-6 PUFA $109 \pm 9$ g; LC $n-3$ PUFA $128 \pm 7$ g. $n=18$ per diet).

\subsection{Myocardial Membrane Phospholipid Fatty Acid Composition}

The relative concentration of DHA (22:6n-3) was greater in phospholipid of LCn-3 PUFA hearts than in either $n-6$ PUFA or SF hearts $(p<0.05)$ (Table 1$)$. The LCn-3 PUFA hearts contained significantly lower concentrations of linoleic (18:2n-6) and arachidonic acids (20:4n-6) compared with $n-6$ PUFA or SF hearts. Total $n-3$ PUFA was greater in LC $n-3$ PUFA hearts compared with either $n-6$ PUFA or SF hearts, and lower in $n-6$ PUFA than SF hearts $(p<0.05)$. The total concentration of $n-6$ PUFA was lower in LC $n$-3 PUFA hearts than in either $n-6$ PUFA or SF hearts and greater in $n-6$ PUFA than SF hearts $(p<0.05)$. 
Table 1. Influence of dietary fat (six weeks) on phospholipid fatty acid composition as percentage of total phospholipid fatty acids of rat heart ventricle.

\begin{tabular}{|c|c|c|c|c|c|c|c|c|c|}
\hline \multirow{3}{*}{$\frac{\text { Fatty Acid }}{16: 0}$} & \multicolumn{9}{|c|}{ DIET } \\
\hline & \multicolumn{3}{|c|}{ SF } & \multicolumn{3}{|c|}{ n-6 PUFA } & \multicolumn{3}{|c|}{ LCn-3 PUFA } \\
\hline & 9.7 & \pm & 0.1 & 10.2 & \pm & 0.2 & 10.8 & \pm & 0.1 \\
\hline $18: 0$ & 23.7 & \pm & 0.2 & 23.8 & \pm & 0.1 & 22.4 & \pm & 0.2 \\
\hline $18: 1 n-9$ & a 9.5 & \pm & 0.1 & b 5.4 & \pm & 0.1 & $\mathrm{~b}_{4.3}$ & \pm & 0.3 \\
\hline $18: 1 n-7$ & 3.6 & \pm & 0.1 & 3.5 & \pm & 0.1 & 3.4 & \pm & 0.1 \\
\hline Total SFA & 33.80 & $\overline{ \pm}$ & 0.13 & 34.70 & \pm & 0.80 & 33.70 & $\overline{ \pm}$ & 0.40 \\
\hline Total MUFA & a 13.50 & \pm & 0.12 & b 8.95 & \pm & 0.30 & b 7.75 & \pm & 1.10 \\
\hline $18: 2 n-6(\mathrm{LA})$ & b 17.50 & $\overline{ \pm}$ & 0.20 & a 18.7 & \pm & 0.40 & c 5.60 & \pm & 0.03 \\
\hline $20: 4 n-6$ (AA) & a 23.30 & \pm & 0.30 & a 23.5 & \pm & 0.20 & b 13.30 & \pm & 0.15 \\
\hline $22: 5 n-6$ & n.d & & & a 1.50 & \pm & 0.12 & a 1.06 & \pm & 0.05 \\
\hline $20: 5 n-3$ (EPA) & n.d & & & n.d & & & 1.30 & \pm & 0.01 \\
\hline $22: 5 n-3$ (DPA) & 1.90 & \pm & 0.04 & 1.02 & \pm & 0.02 & 1.17 & \pm & 0.04 \\
\hline $22: 6 n-3$ (DHA) & b 12.20 & \pm & 0.04 & b 10.02 & \pm & 0.20 & a 28.30 & \pm & 0.04 \\
\hline Total $(n-6)$ PUFA & b 40.80 & \pm & 0.20 & a 43.80 & \pm & 0.60 & c 20.00 & \pm & 0.16 \\
\hline Total $(n-3)$ PUFA & b 14.10 & \pm & 0.06 & c 11.00 & \pm & 0.20 & a 30.70 & \pm & 0.08 \\
\hline Total PUFA & 54.90 & \pm & 4.50 & 54.70 & \pm & 4.50 & 50.70 & \pm & 4.40 \\
\hline UI & b 215.40 & \pm & 1.20 & b 215.10 & \pm & 0.50 & a 260.58 & \pm & 1.20 \\
\hline Peroxidisability Index & b 156.20 & \pm & 1.20 & b 149.50 & \pm & 1.60 & a 201.10 & \pm & 0.70 \\
\hline
\end{tabular}

SF: saturated fat enriched diet; $n$-6 PUFA: $n-6$ PUFA enriched diet; LC $n-3$ PUFA: $n-3$ PUFA enriched diet; SFA: saturated fatty acids; MUFA: monounsaturated fatty acids; PUFA: polyunsaturated fatty acids; LA: linoleic acid; AA: arachidonic acid; EPA: eicosapentaenoic acid; DPA: docosahexaenoic acid; DHA: docosahexaenoic acid. Unsaturation index (UI) was calculated according to the formula: $\mathrm{UI}=1 \times(\%$ monoenoic acids $)+2 \times(\%$ dienoics $)+$ $3 \times(\%$ trienoics $)+4 \times(\%$ tetraenoics $)+5 \times(\%$ pentaenoics $)+6 \times(\%$ hexaenoics $)$ or sum (fatty acid percent) $\times$ (number of double bonds). Peroxidatisability index was calculated from the formula: $(\%$ dienoic acids $\times 1)+(\%$ trienoics $\times 2)+(\%$ tetraenoics $\times 3)+$ $(\%$ pentaenoics $\times 4)+(\%$ hexaenoics $\times 5)$ [17]. n.d: not detected. $n=6$ per dietary group. ${ }^{a, b, c}$ Values not sharing a common letter superscript are significantly different (ANOVA, $p<0.05$ ).

No significant dietary differences were observed in the membrane phospholipid total saturated fatty acids or total PUFA. The SF hearts had greater concentrations of total monounsaturated fatty acids (MUFA). Membrane unsaturation index (UI) and peroxidisability index (Figure 2A) were significantly greater in LCn-3 PUFA than in either $n-6$ PUFA or SF hearts $(p<0.05)$, which were not significantly different from each other. 

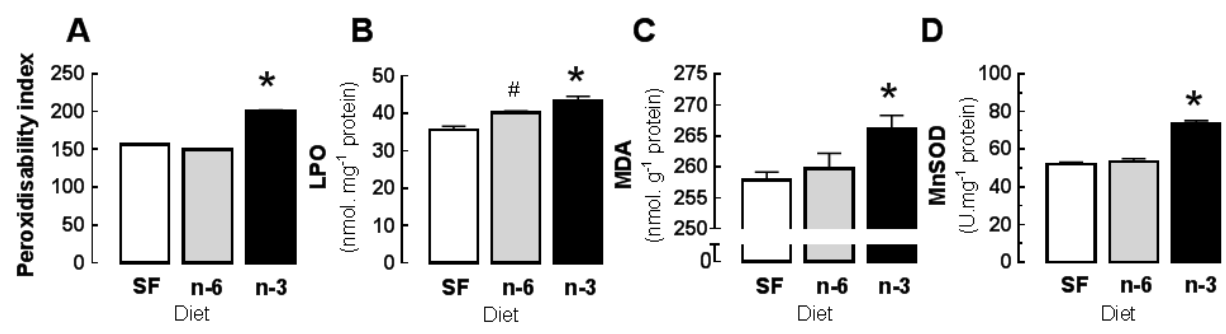

Figure 2. Influence of six weeks dietary fat feeding on basal: (A) membrane fatty acid peroxidisability index; and concentrations of (B) lipid hydroperoxides (LPO); (C) malondialdehyde (MDA); and (D) antioxidant superoxide dismutase (MnSOD) of basal or non-I regions of the heart after $180 \mathrm{~min}$ of isolated perfusion protocol. Open columns (SF): saturated fat diet; shaded columns (n-6): n-6 PUFA rich diet; filled columns ( $n-3)$ : LC $n-3$ PUFA rich fish oil diet. Values are means \pm SEM. $n=18$ per dietary group except peroxidisability index: $n=6$. ${ }^{*}$ different from both other diet groups, $p<0.05$. ${ }^{\#}$ different from SF group, $p<0.05$.

\subsection{Basal Properties: Effects of Diet on Oxidative Stress and Antioxidant Activity}

The basal and non-I tissue derived from the three perfusion protocols exhibited no significant between protocol differences in tissue concentrations of lipid oxidation products LPO or MDA or anti-oxidants within any dietary group (pooled data shown in Figure 2). This establishes the non-I measures as representative of the basal state of the ISCH region.

The concentrations of LPO in basal and non-I tissue were significantly greater in LCn-3 PUFA than in either $n-6$ PUFA or SF hearts and greater in $n-6$ PUFA than SF hearts $(p<0.05)$ (Figure 3A). The concentrations of MDA in basal and non-I tissue were significantly greater in LC $n-3$ PUFA than in either $n-6$ PUFA or SF hearts $(p<0.05)$, which were not different from each other (Figure 3B).

The activity of MnSOD in basal and non-I tissue was significantly greater in LCn-3 PUFA hearts, than in SF or n-6 PUFA hearts (Figure 3C). In basal and non-I tissue there were no significant dietary differences in CuZnSOD activity (basal, non-I (U. $\mathrm{mg}^{-1}$ protein): SF $15.3 \pm 0.9 ; n-6$ PUFA $16.9 \pm 0.7 ;$ LC $n-3$ PUFA $17.4 \pm 0.6 n=18$ ) $(p>0.05)$ or GPX (basal, non-I (mU $\mathrm{mg}^{-1}$ protein): SF $19.2 \pm 1.5 ; n-6$ PUFA $19.7 \pm 1.5$; LCn-3 PUFA $21 \pm 1.2 n=15$ ). The concentration of $\alpha$-tocopherol was significantly greater in $n$-6 PUFA hearts than in either LC $n$-3 PUFA or SF hearts $(p<0.05)$ (basal, non-I $(\mu \mathrm{M})$ : SF $6.1 \pm 0.4 ; n-6$ PUFA $6.9 \pm 0.2$; LC $n-3$ PUFA $5.9 \pm 0.6 n=15)$. 

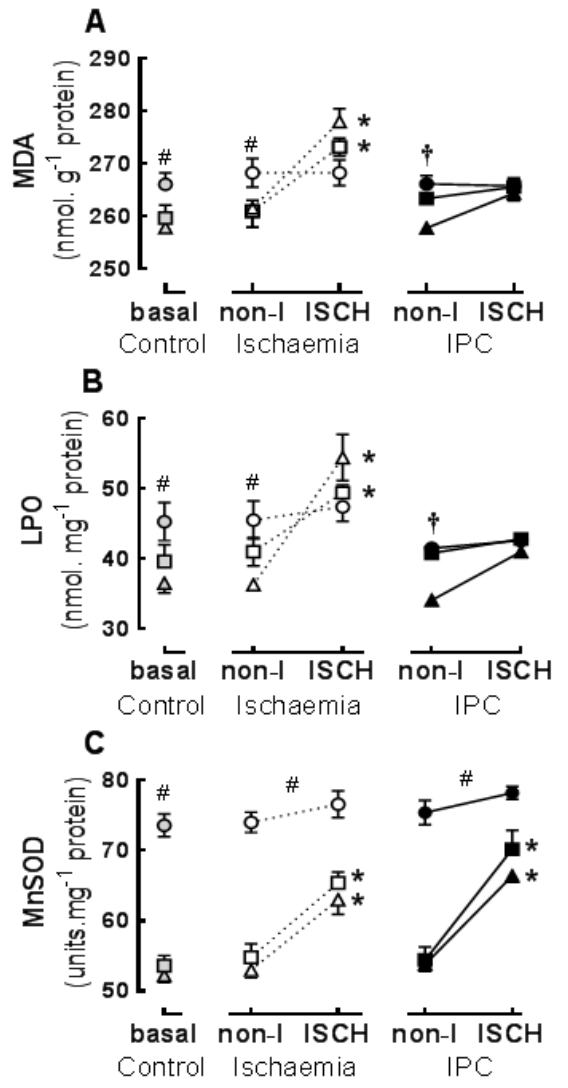

Figure 3. Influence of six weeks dietary fat feeding on cardiac lipid oxidation and antioxidant markers in ischaemic (ISCH) or non-ischaemic (non-I) regions after: Control normoxic perfusion (basal), Ischaemia perfusion, or ischaemic preconditioning (IPC) perfusion protocols: (A) lipid hydroperoxides (LPO); (B) malondialdehyde (MDA); and (C) superoxide dismutase (MnSOD). Data are from hearts that were normoxic throughout (basal), or the non-I and ISCH regions of hearts subjected to $30 \mathrm{~min}$ of regional ischaemia with or without prior IPC. Diet

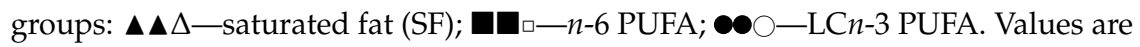
means \pm SEM. $n=18$ per diet, $n=6$ per perfusion protocol. ${ }^{*} \mathrm{ISCH}$ different from non-I region within diet, $p<0.05$; ${ }^{\#} n$-3 PUFA different from SF $p<0.05$; † LCn-3 PUFA and $n-6$ PUFA different from SF.

3.3. Ischaemic Responses: Effects of Diet and Ischaemic Preconditioning on Oxidative Stress and Antioxidant Capacity in Hearts Subjected to Regional I/R

Ischaemia: The concentrations of LPO (Figure 3A) and MDA (Figure 3B) were acutely increased in the ISCH compared to non-I region of $n-6$ PUFA and SF hearts $(p<0.01)$ but not in LC $n-3$ PUFA hearts. The concentrations of LPO and MDA in 
the ISCH region were significantly greater in SF hearts than in LCn-3 PUFA hearts (Figure 3A,B).

IPC: There were no significant acute changes in LPO or MDA in ISCH compared to non-I regions within any dietary group (Figure $3 \mathrm{~A}, \mathrm{~B}$ ), nor were there any significant between-diet differences within the ISCH regions of IPC + ischaemia hearts. Concentrations of LPO and MDA in ISCH regions were significantly lower in IPC + ischaemia hearts than in ischaemia only hearts $(p<0.0001)$. Pairwise comparison indicated that this IPC difference was evident in SF and n-6 PUFA diets only.

Myocardial MnSOD activity was significantly greater in ISCH compared to non-I regions of hearts from SF and $n-6$ PUFA fed rats but not significantly changed within LCn-3 PUFA hearts (Figure 3C). The perfusion protocol incorporating IPC + ischaemia had no different effect on MnSOD activity to ischaemia alone.

\subsection{Infarct}

In hearts subjected to the ischaemia perfusion protocol, infarct size was significantly smaller in LCn-3 PUFA hearts (ischaemia infarct size (\% Iz/r): SF $50 \pm 1 n=6$; $n-6$ PUFA $47 \pm 1 n=6$; LC $n-3$ PUFA $n=611 \pm 1 n=6,(p<0.05))$. In hearts subjected to the IPC + ischaemia protocol, the infarct size was significantly smaller in the SF and n-6 PUFA hearts than in the corresponding ischaemia group $(p<0.05)$. There was no significant difference within the LC $n-3$ PUFA diet. (IPC + ischaemia infarct size (\% Iz/r): SF $13 \pm 1 n=6 ; n-6$ PUFA $12 \pm 1 n=6$; LC $n-3$ PUFA $10 \pm 1 n=6)$.

\subsection{Associations between Oxidation Biomarkers, Antioxidant and Infarct Size}

Ischaemia protocol: Infarct size was positively associated with lipid oxidation biomarker production in the ISCH region, independent of diet (Table 2). The acute increases in LPO and MDA (ISCH compared to the non-I region) correlated better than the absolute ISCH concentrations of LPO and MDA. Lipid oxidation biomarkers LPO and MDA were correlated in the ISCH region. Ischaemic production of LPO and MDA and infarct size were inversely associated with the basal (non-I) MnSOD activity (Table 2). The strongest association was the inverse correlation between basal MnSOD and infarct size (Figure 4).

IPC: In hearts subjected to the IPC + ischaemia perfusion protocol, infarct size was not significantly correlated with LPO, MDA or MnSOD concentrations, and ischaemia-induced increase in MDA but not LPO was correlated with MnSOD activity. Lipid oxidation biomarkers LPO and MDA were correlated in the ISCH region (Table 2 ). 
Pooled analysis of data from both perfusion protocols revealed significant correlations of infarct size with lipid oxidation markers and with MnSOD. Lipid oxidation biomarkers LPO and MDA were correlated in the ISCH region (Table 2).

Table 2. Correlations between lipid oxidation products, antioxidants and infarct size.

\begin{tabular}{|c|c|c|c|c|}
\hline Dependent Variable & Independent Variable & Association & $r^{2}$ & $p$ for Slope \\
\hline \multicolumn{5}{|c|}{ Ischaemia Protocol } \\
\hline Infarct & LPO (ISCH) & positive & $0.337^{*}$ & 0.018 \\
\hline Infarct & LPO increase & positive & $0.478^{* *}$ & 0.004 \\
\hline Infarct & MDA (ISCH) & positive & $0.356 *$ & 0.015 \\
\hline Infarct & MDA increase & positive & $0.517^{* *}$ & 0.004 \\
\hline Infarct & MnSOD (basal) & negative & $0.851^{* *}$ & $<0.0001$ \\
\hline MDA (ISCH) & LPO (ISCH) & positive & $0.481^{* *}$ & 0.006 \\
\hline LPO increase & MnSOD (basal) & negative & $0.397^{* *}$ & 0.009 \\
\hline MDA increase & MnSOD (basal) & negative & $0.617^{* *}$ & 0.001 \\
\hline \multicolumn{5}{|c|}{ IPC + Ischaemia Protocol } \\
\hline Infarct & LPO (ISCH) & positive & 0.039 & 0.483 n.s. \\
\hline Infarct & LPO increase & positive & 0.147 & 0.175 n.s. \\
\hline Infarct & MDA (ISCH) & positive & 0.175 & 0.150 n.s. \\
\hline Infarct & MDA increase & positive & 0.009 & 0.728 n.s. \\
\hline Infarct & MnSOD (basal) & negative & 0.058 & 0.335 n.s. \\
\hline MDA (ISCH) & LPO (ISCH) & positive & $0.764^{* *}$ & $<0.0001$ \\
\hline LPO increase & MnSOD (basal) & negative & 0.128 & 0.174 n.s. \\
\hline MDA increase & MnSOD (basal) & negative & $0.293 *$ & 0.017 \\
\hline \multicolumn{5}{|c|}{ Overall } \\
\hline Infarct & LPO increase & positive & $0.583^{* *}$ & $<0.0001$ \\
\hline Infarct & MDA increase & positive & $0.475^{* *}$ & $<0.0001$ \\
\hline Infarct & MnSOD (basal) & negative & $0.270 *$ & 0.0012 \\
\hline MDA (ISCH) & LPO (ISCH) & positive & $0.760 * *$ & $<0.0001$ \\
\hline
\end{tabular}

LPO: lipid hydroperoxides. MDA: malondialdehyde. MnSOD: manganese superoxide dismutase. ISCH: ischaemic region. Basal: non-ischaemic region of ventricle wall. n.s.: not significant $(p>0.05) ;{ }^{*} p<0.05 ;{ }^{* *} p<0.01$. 


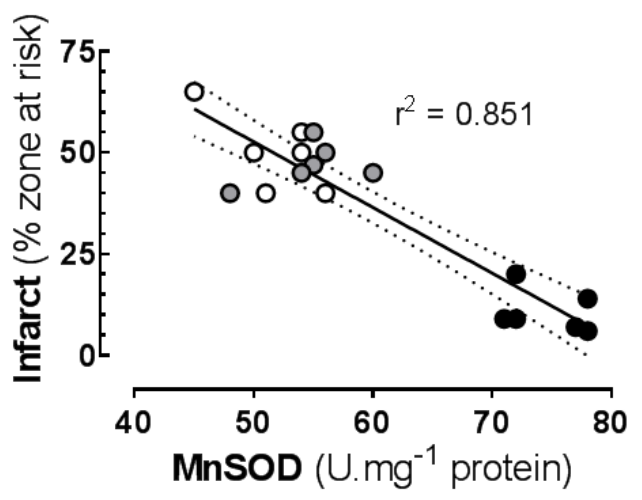

Figure 4. Correlation between: basal (non-I) concentration of superoxide dismutase; and infarct size in isolated rat hearts subjected to $30 \mathrm{~min}$ index ischaemia and 120 min reperfusion. Rats fed supplemented diets for six weeks-open symbols: saturated fat (SF) diet; shaded symbols: $n-6$ PUFA diet; closed symbols: LC $n-3$ PUFA fish oil diet.

\section{Discussion}

A diet rich in LCn-3 PUFA from fish oil modified the fatty acid profile of myocardial membrane phospholipids, increasing the percentage of fat as DHA and the peroxidisability index (predicting an increase in risk of oxidative damage), yet paradoxically reduced the measured oxidative damage following I/R. While the increased myocardial peroxidation potential was associated with an increase in basal fatty acid peroxidation, confirming effects of DHA feeding recorded in plasma and liver [24], it also induced a marked chronic increase in MnSOD (endogenous antioxidant) activity, and inhibited I/R-induced lipid oxidation and infarction. Reactive oxygen species act as both the agents of damage and of conservation in IPC, causing cellular damage yet triggering protective signalling processes [25]. In this respect, LCn-3 PUFA supplementation reflects both the low level generation of ROS through lipid peroxidation [26] and up-regulation of endogenous antioxidants that are implicated as triggers and mediators respectively of late phase IPC [27]. This aligns fish oil nutritional preconditioning [5] not only with this more persistent form of IPC (variously known as late, delayed or second window of IPC), but through LCn-3 PUFA continuous presence as a membrane component, it also provides a persistent tolerance to I/ $R$ injury. This persistent preconditioning is also observed with repeated stresses like exercise and heat exposure [12]. In contrast, early phase IPC did not acutely affect basal lipid oxidation or antioxidant activity during the 150 min post preconditioning time course of this study protocol. Moreover, IPC prevention of lipid oxidation and infarction during the index ischaemia was not 
additive to the effects of fish oil feeding. Admittedly the anti-infarct effects of both fish oil and IPC could be individually regarded as already maximal.

Fish oil-induced chronic increases in basal lipid oxidation directly correlated with basal MnSOD antioxidant activity in myocardium, which in turn was negatively correlated with the I/R-induced increase in lipid oxidation. This interdependence, which reflects the contrasting damaging influence and homeostatic signalling roles of ROS in ischaemia and IPC, can explain some of the lack of consistent correlation between oxidation products and anti-oxidants and sometime failure of oxidation markers to serve as clear criteria for defining oxidative stress [26]. The effects were consistent on LPO (an intermediate common to oxidation of all PUFA) and MDA (a stable end product of a single pathway also not specific for any PUFA family). Ultimately the infarct size was negatively correlated with MnSOD activity and directly correlated with the increase in lipid oxidation products in the ISCH region. Chronic elevation of plasma MnSOD has been previously observed during fish oil feeding [28], consistent with its persistent elevation over several days following multiple exposures to TNF $\alpha$, exercise stress or heat stress [12].

Fish oil induced increases in antioxidant expression and reduced lipid peroxidation products were also reported in hepatic and renal tissue of immune suppressed mice [29,30] and hepatic tissue of hypertensive rats [31], conditions associated with heightened oxidative stress. In those studies, the fish oil diets were effective independently of varied provision of high or low concentrations of natural antioxidants in comparative n-6 PUFA or MUFA enriched diets. In the current study, the n-6 PUFA rich diet with its elevated vitamin E content did not change the membrane fatty acid composition sufficiently to modulate either membrane peroxidisability index or endogenous antioxidant enzyme activity relative to the low PUFA saturated fat enriched diet, and hearts from those diets were equally highly susceptible to oxidative damage. This is consistent with previous findings that both membrane effects and cardioprotective effects of $n-6$ PUFA are readily lost as the PUFA content is diluted by other fat sources [32,33]. This is not the case for LCn-3 PUFA, which sustain membrane composition [34,35], and cardiac [5,10,32,33,36] and other functional effects [35] to very low dietary concentrations. The ability of low (nutritionally relevant) intakes of fish oil to modify membrane composition and cardiac function, including prevention of $\mathrm{I} / \mathrm{R}$ arrhythmias is important, since the provision of extremely high LCn-3 PUFA intakes can be pro-arrhythmic (fish oil concentrate $4 \mathrm{~g} / \mathrm{d} / 20 \mathrm{~kg}$ dog, equivalent to $\geqslant 40 / \mathrm{d}$ standard fish oil capsules in an $80 \mathrm{~kg}$ man) [37], perhaps representing the harmful effects of excessive oxidation. Similarly, in a senescence-prone mouse model, high fish oil feeding in conjunction with high total PUFA enhances oxidative stress and decreases lifespan [38].

The present study suggests that LCn-3 PUFA exert protection from ischaemia by activating signalling pathways that resemble those involved in late IPC or exercise, 
and we describe this as "nutritional preconditioning". The current study used a LCn-3 PUFA intake equivalent to more than $30 \mathrm{~g}$ of fish oil per day in humans [34]. However, even very low doses in the range $0.16 \%-1.25 \%$ FO markedly increase myocardial DHA and peroxidation index (at $0.31 \%$ dietary fish oil equivalent to human 1-2 fish meals per week) DHA is increased from $7.7 \%$ to $14.9 \%$ of phospholipid fatty acids and PI is increased from 149 to 164 (calculated from Slee [34]). This is a dose that modulates skeletal muscle membrane fatty acids and muscle fatigue [35]. In skeletal muscle, reactive oxygen species capable of causing cellular damage when in physiological excess can at lower levels also act to optimise contractile performance and initiate long-term protective adaptations to the intermittent stress imposed by exercise training [39].

The present study deliberately used a high DHA fish oil, which does not reflect the composition of most nutritional supplement fish oils, but rather reflects the main LCn-3 PUFA derived from eating fish [4,40]. As the most abundant $n-3$ PUFA found in myocardium, DHA is also the main fatty acid underpinning the cardiac effects of fish and fish oil [4]. The use of two diets for comparison with fish oil allows specific attribution of the effects of fish oil feeding to its LCn-3 PUFA content, since similar total PUFA content was provided in the $n-6$ and LC $n-3$ PUFA diets; similar $n-6$ PUFA was provided in the SF and LCn-3 PUFA diets; and low saturated fat was provided in the $n-6$ PUFA diet, all without effect.

The increased expression of antioxidants within LCn-3 PUFA hearts was restricted to the mitochondrial form of SOD (MnSOD or SOD2) with CuZnSOD and GSx unchanged. This suggests localisation of the primarily influence of LCn-3 PUFA to the mitochondria. Increased MnSOD activity is similarly selectively implicated in the sustained cardioprotection elicited by heat stress and in late, delayed or second window of IPC [41], whereas over-expression of cardiac MnSOD in mice enhances contractile function, slows heart rate and increases efficiency of myocardial $\mathrm{O}_{2}$ consumption [42], all properties shared by dietary fish oil [5,43,44]. Furthermore, the fish oil-reduced cardiac oxygen consumption and reduced susceptibility to I/R-damage and arrhythmias in rats is linked to mitochondrial $\mathrm{Ca}^{2+}$ handling [44]. In contrast, early IPC inhibited acute lipid oxidation and infarction did not involve upregulation of mitochondrial SOD, confirming its difference from late IPC [41] and highlighting a difference to the more persistent forms of preconditioning including exercise [45-47], late IPC [41] and now fish oil-induced nutritional preconditioning.

This study confirmed that increasing myocardial membrane percentage content of long chain $n-3$ highly polyunsaturated fatty acids by feeding fish oil, increased the basal peroxidation of cellular fatty acids, which in turn increased the activity of endogenous mitochondrial antioxidant superoxide dismutase. When these hearts were acutely subjected to regional I/R, the stimulated lipid oxidation and myocardial damage were reduced. The increase in peroxidation index of myocardial 
membranes through fatty acid compositional change and associated chronic mild elevation in lipid peroxidation products provokes a persistent physiological stress that might better be described as "oxidative shielding" [48], which if confirmed at lower fish oil intakes, could explain much of the cardioprotective effect of regular fish consumption. This readily available and safe nutritional approach appears to represent a natural form of late preconditioning, which, characterised by its persistence over time, would be particularly valuable in the clinical setting, where oxidative insults occur unexpectedly and preclude the use of planned preventative interventions [41]. The observation, however, also raises the possibility that like exercise training [45], effects of fish oil nutritional preconditioning may be blunted by concomitant antioxidant supplementation.

Acknowledgments: This research received no specific grant from any funding agency, commercial or not-for-profit sectors. The research was supported by the donation of high-DHA tuna fish oil from Clover Corporation and Nu-Mega Lipids (Altona North VIC, Australia).

Author Contributions: G.G.A., A.J.O. and P.L.M. conceived and designed the experiments; G.G.A., A.J.O. and T.A.L. performed the experiments and analysed the data; and G.G.A., A.J.O., T.A.L. and P.L.M. interpreted the data and provided important intellectual content for drafting the manuscript. P.L.M. had the primary responsibility for the final content.

Conflicts of Interest: The authors declare no conflict of interest.

\section{References}

1. Trikalinos, T.A.; Lee, J.; Moorthy, D.; Yu, W.W.; Lau, J.; Lichtenstein, A.H.; Chung, M. Effects of eicosapentanoic acid and docosahexanoic acid on mortality across diverse settings: Systematic review and meta-analysis of randomized trials and prospective cohorts. Available online: http://www.ncbi.nlm. nih.gov/books/ NBK91413/pdf/Bookshelf_NBK91413.pdf (accessed on 2 March 2016).

2. Valagussa, F.; Franzosi, M.G.; Geraci, E.; Mininni, N.; Nicolosi, G.L.; Santini, M.; Tavazzi, L.; Vecchio, C.; Marchioli, R.; Bomba, E.; et al. Dietary supplementation with $n-3$ polyunsaturated fatty acids and vitamin E after myocardial infarction: Results of the GISSI-Prevenzione trial. Lancet 1999, 354, 447-455.

3. Mozaffarian, D.; Rimm, E.B. Fish intake, contaminants, and human health-Evaluating the risks and the benefits. JAMA 2006, 296, 1885-1899.

4. McLennan, P.L. Cardiac physiology and clinical efficacy of dietary fish oil clarified through cellular mechanisms of omega-3 polyunsaturated fatty acids. Eur. J. Appl. Physiol. 2014, 114, 1333-1356.

5. Abdukeyum, G.G.; Owen, A.J.; McLennan, P.L. Dietary (n-3) long-chain polyunsaturated fatty acids inhibit ischemia and reperfusion arrhythmias and infarction in rat heart not enhanced by ischemic preconditioning. J. Nutr. 2008, 138, 1902-1909.

6. Zeghichi-Hamri, S.; de Lorgeril, M.; Salen, P.; Chibane, M.; de Leiris, J.; Boucher, F.; Laporte, F. Protective effect of dietary $n-3$ polyunsaturated fatty acids on myocardial resistance to ischemia-reperfusion injury in rats. Nutr. Res. 2010, 30, 849-857. 
7. Cohen, M.V.; Yang, X.M.; Downey, J.M. Conscious rabbits become tolerant to multiple episodes of ischemic preconditioning. Circ. Res. 1994, 74, 998-1004.

8. McLennan, P.L.; Abeywardena, M.Y.; Charnock, J.S. Dietary fish oil prevents ventricular fibrillation following coronary artery occlusion and reperfusion. Am. Heart J. 1988, 116, 709-717.

9. McLennan, P.L. Myocardial membrane fatty acids and the antiarrhythmic actions of dietary fish oil in animal models. Lipids 2001, 36, S111-S114.

10. McLennan, P.; Howe, P.; Abeywardena, M.; Muggli, R.; Raederstorff, D.; Mano, M.; Rayner, T.; Head, R. The cardiovascular protective role of docosahexaenoic acid. Eur. J. Pharmacol. 1996, 300, 83-89.

11. Dana, A.; Baxter, G.F.; Walker, J.M.; Yellon, D.M. Prolonging the delayed phase of myocardial protection: Repetitive adenosine A(1) receptor activation maintains rabbit myocardium in a preconditioned state. J. Am. Coll. Cardiol. 1998, 31, 1142-1149.

12. Hoshida, S.; Yamashita, N.; Otsu, K.; Hori, M. Repeated physiologic stresses provide persistent cardioprotection against ischemia-reperfusion injury in rats. J. Am. Coll. Cardiol. 2002, 40, 826-831.

13. Marber, M.S.; Latchman, D.S.; Walker, J.M.; Yellon, D.M. Cardiac stress protein elevation 24 hours after brief ischemia or heat stress is associated with resistance to myocardial infarction. Circulation 1993, 88, 1264-1272.

14. Bolli, R. The early and late phases of preconditioning against myocardial stunning and the essential role of oxyradicals in the late phase: An overview. Basic Res. Cardiol. 1996, 91, 57-63.

15. Baxter, G.F.; Ferdinandy, P. Delayed preconditioning of myocardium: Current perspectives. Basic Res. Cardiol. 2001, 96, 329-344.

16. Zhou, X.B.; Zhai, X.L.; Ashraf, M. Direct evidence that initial oxidative stress triggered by preconditioning contributes to second window of protection by endogenous antioxidant enzyme in myocytes. Circulation 1996, 93, 1177-1184.

17. Song, J.H.; Fujimoto, K.; Miyazawa, T. Polyunsaturated (n-3) fatty acids susceptible to peroxidation are increased in plasma and tissue lipids of rats fed docosahexaenoic acid-containing oils. J. Nutr. 2000, 130, 3028-3033.

18. Bolli, R.; Becker, L.; Gross, G.; Mentzer, R.; Balshaw, D.; Lathrop, D.A. Myocardial protection at a crossroads-The need for translation into clinical therapy. Circ. Res. 2004, 95, 125-134.

19. National Health and Medical Research Council. Australian Code of Practice for the Care and Use of Animals for Scientific Purposes, 7th ed.; NHMRC: Canberra, Australia, 2004; p. 84.

20. Lepage, G.; Munoz, G.; Champagne, J.; Roy, C.C. Preparative steps necessary for the accurate measurement of malondialdehyde by high-performance liquid chromatography. Anal. Biochem. 1991, 197, 277-283.

21. Yang, C.S.; Jung, L.M. Methodology of plasma retinol, tocopherol and carotenoid assays in cancer prevention studies. J. Nutr. Growth Cancer 1987, 4, 19-27.

22. Folch, J.; Lees, M.; Sloane-Stanley, G.H. A simple method for the isolation and purification of total lipids from animal tissues. J. Biol. Chem. 1957, 226, 497-509. 
23. Lepage, G.; Roy, C. Direct transesterification of all classes of lipids in a one-step reaction. J. Lipid Res. 1986, 27, 114-121.

24. Song, J.H.; Miyazawa, T. Enhanced level of $n-3$ fatty acid in membrane phospholipids induces lipid peroxidation in rats fed dietary docosahexaenoic acid oil. Atherosclerosis 2001, 155, 9-18.

25. Ray, P.D.; Huang, B.W.; Tsuji, Y. Reactive oxygen species (ROS) homeostasis and redox regulation in cellular signaling. Cell. Signal. 2012, 24, 981-990.

26. Dotan, Y.; Lichtenberg, D.; Pinchuk, I. Lipid peroxidation cannot be used as a universal criterion of oxidative stress. Prog. Lipid Res. 2004, 43, 200-227.

27. Stein, A.B.; Tang, X.L.; Guo, Y.; Xuan, Y.T.; Dawn, B.; Bolli, R. Delayed adaptation of the heart to stress-Late preconditioning. Stroke 2004, 35, 2676-2679.

28. Erdogan, H.; Fadillioglu, E.; Ozgocmen, S.; Sogut, S.; Ozyurt, B.; Akyol, O.; Ardicoglu, O. Effect of fish oil supplementation on plasma oxidant/antioxidant status in rats. Prostaglandins Leukot. Essent. Fatty Acids 2004, 71, 149-152.

29. Chandrasekar, B.; Fernandes, G. Decreased pro-inflammatory cytokines and increased antioxidant enzyme gene-expression by omega-3 lipids in murine lupus nephritis. Biochem. Biophys. Res. Commun. 1994, 200, 893-898.

30. Venkatraman, J.T.; Chandrasekar, B.; Kim, J.D.; Fernandes, G. Effects of $n-3$ and $n-6$ fatty-acids on the activities and expression of hepatic antioxidant enzymes in autoimmune-prone NZBxNZW F1-mice. Lipids 1994, 29, 561-568.

31. Ruiz-Gutierrez, V.; Vazquez, C.M.; Santa-Maria, C. Liver lipid composition and antioxidant enzyme activities of spontaneously hypertensive rats after ingestion of dietary fats (fish, olive and high-oleic sunflower oils). Biosci. Rep. 2001, 21, 271-285.

32. McLennan, P.L.; Bridle, T.M.; Abeywardena, M.Y.; Charnock, J.S. Comparative efficacy of $n-3$ and $n-6$ polyunsaturated fatty acids in modulating ventricular fibrillation threshold in marmoset monkeys. Am. J. Clin. Nutr. 1993, 58, 666-669.

33. McLennan, P.L.; Abeywardena, M.Y. Membrane basis for fish oil effects on the heart: Linking natural hibernators to prevention of human sudden cardiac death. J. Membr. Biol. 2005, 206, 85-102.

34. Slee, E.L.; McLennan, P.L.; Owen, A.J.; Theiss, M.L. Low dietary fish oil threshold for myocardial membrane $n$-3 PUFA enrichment independent of $n$ - 6 PUFA intake in rats. J. Lipid Res. 2010, 51, 1841-1848.

35. Henry, R.; Peoples, G.E.; McLennan, P.L. Muscle fatigue resistance in the rat hindlimb in vivo from low dietary intakes of tuna fish oil that selectively increase phospholipid $n-3$ docosahexaenoic acid according to muscle fibre type. Br. J. Nutr. 2015, 114, 873-884.

36. McLennan, P.L.; Owen, A.J.; Slee, E.L.; Theiss, M.L. Myocardial function, ischaemia and n-3 polyunsaturated fatty acids: A membrane basis. J. Cardiovasc. Med. 2007, 8, S15-S18.

37. Billman, G.E.; Carnes, C.A.; Adamson, P.B.; Vanoli, E.; Schwartz, P.J. Dietary omega-3 fatty acids and susceptibility to ventricular fibrillation lack of protection and a proarrhythmic effect. Circ. Arrhythm. Electrophysiol. 2012, 5, 553-560. 
38. Tsuduki, T.; Honma, T.; Nakagawa, K.; Ikeda, I.; Miyazawa, T. Long-term intake of fish oil increases oxidative stress and decreases lifespan in senescence-accelerated mice. Nutrition 2011, 27, 334-337.

39. Powers, S.K.; Jackson, M.J. Exercise-induced oxidative stress: Cellular mechanisms and impact on muscle force production. Physiol. Rev. 2008, 88, 1243-1276.

40. McLennan, P.L.; Pepe, S. Weighing up fish and omega-3 PUFA advice with accurate, balanced scales: Stringent controls and measures required for clinical trials. Heart Lung Circ. 2015, 24, 740-743.

41. Hausenloy, D.J.; Yellon, D.M. The Second Window of Preconditioning (SWOP) Where Are We Now? Cardiovasc. Drugs Ther. 2010, 24, 235-254.

42. Kang, P.T.; Chen, C.-L.; Ohanyan, V.; Luther, D.J.; Meszaros, J.G.; Chilian, W.M.; Chen, Y.-R. Overexpressing superoxide dismutase 2 induces a supernormal cardiac function by enhancing redox-dependent mitochondrial function and metabolic dilation. J. Mol. Cell. Cardiol. 2015, 88, 14-28.

43. Pepe, S.; McLennan, P.L. (n-3) long chain PUFA dose-dependently increase oxygen utilization efficiency and inhibit arrhythmias after saturated fat feeding in rats. J. Nutr. 2007, 137, 2377-2383.

44. Pepe, S.; McLennan, P.L. Cardiac membrane fatty acid composition modulates myocardial oxygen consumption and post-ischemic recovery of contractile function. Circulation 2002, 105, 2303-2308.

45. Gomez-Cabrera, M.C.; Salvador-Pascual, A.; Cabo, H.; Ferrando, B.; Vina, J. Redox modulation of mitochondriogenesis in exercise. Does antioxidant supplementation blunt the benefits of exercise training? Free Radic. Biol. Med. 2015, 86, 37-46.

46. Gomez-Cabrera, M.C.; Domenech, E.; Vina, J. Moderate exercise is an antioxidant: Upregulation of antioxidant genes by training. Free Radic. Biol. Med. 2008, 44, 126-131.

47. Powers, S.K.; Smuder, A.J.; Kavazis, A.N.; Quindry, J.C. Mechanisms of Exercise-Induced Cardioprotection. Physiology 2014, 29, 27-38.

48. Naviaux, R.K. Oxidative Shielding or Oxidative Stress? J. Pharmacol. Exp. Ther. 2012, 342, 608-618. 


\title{
The Effect of Marine Derived $n$-3 Fatty Acids on Adipose Tissue Metabolism and Function
}

\author{
Marijana Todorčević and Leanne Hodson
}

Abstract: Adipose tissue function is key determinant of metabolic health, with specific nutrients being suggested to play a role in tissue metabolism. One such group of nutrients are the $n-3$ fatty acids, specifically eicosapentaenoic acid (EPA; 20:5n-3) and docosahexaenoic acid (DHA; 22:6n-3). Results from studies where human, animal and cellular models have been utilised to investigate the effects of EPA and/or DHA on white adipose tissue/adipocytes suggest anti-obesity and anti-inflammatory effects. We review here evidence for these effects, specifically focusing on studies that provide some insight into metabolic pathways or processes. Of note, limited work has been undertaken investigating the effects of EPA and DHA on white adipose tissue in humans whilst more work has been undertaken using animal and cellular models. Taken together it would appear that EPA and DHA have a positive effect on lowering lipogenesis, increasing lipolysis and decreasing inflammation, all of which would be beneficial for adipose tissue biology. What remains to be elucidated is the duration and dose required to see a favourable effect of EPA and DHA in vivo in humans, across a range of adiposity.

Reprinted from J. Clin. Med. Cite as: Todorčević, M.; Hodson, L. The Effect of Marine Derived n-3 Fatty Acids on Adipose Tissue Metabolism and Function. J. Clin. Med. 2016, 5, 3 .

\section{Introduction}

Adipose tissue, the largest organ in the human body, was historically considered to be metabolically inert. However, white adipose tissue is now considered an endocrine organ as it secretes adipokines (and hormones) which act locally and distally through autocrine, paracrine and endocrine effects [1]. Although adipose tissue is comprised of several cell types, including pre-adipocytes, adipocytes, endothelial cells, fibroblasts, leukocytes and macrophages [2], all of which may impact on tissue function, one of the main functions of adipocytes/adipose tissue is to store fatty acids [3]. Adipose tissue acts to "buffer" the influx of dietary fat into the circulation $[3,4]$, with excess dietary fat being stored in adipose tissue rather than "overflowing" to non-adipose organs. Ectopic fat deposition has been proposed to underlie obesity-associated metabolic diseases [5]. An increase in adipose tissue mass may alter the function of the tissue. For example, when adipose tissue starts to expand (such as with excess nutrition) macrophages infiltrate and orchestrate inflammatory responses via molecules such as tumor necrosis factor $\alpha(\mathrm{TNF} \alpha)$, 
interleukin 6 (IL-6) and monocyte chemoattractant protein-1 (MCP-1), all of which have been implicated in the development of pathological changes in adipose tissue physiology [6-9]. Intriguingly, a proportion of overweight/obese individuals remain metabolically healthy even with further weight gain, whilst others do not; it has recently been suggested this is due to an increased capacity of adipose tissue for lipogenesis [10]. Multiple factors have been suggested to impact on the function of adipose tissue, however as the tissue is the primary site for dietary fat storage and reflects dietary fat intakes [11] it is reasonable to suggest that the composition or type of fat that the tissue is exposed to may also influence the function.

A class of fatty acids that has received a lot of attention over the last 30 years is the $n-3$ (or $\omega-3$ ) fatty acids, specifically those derived from marine sources. $n-3$ fatty acids have been suggested to lower the risk of a number of non-communicable metabolic diseases including cardiovascular disease, obesity and diabetes [12-14]. Here we review the effect of long chain $n-3$ polyunsaturated fatty acids (LCPUFA), specifically eicosapentaenoic acid (EPA, 20:5n-3) and docosahexaenoic acid (DHA, 22:6n-3) on white adipose tissue metabolism and function. Although other $n-3$ fatty acids such as $\alpha$-linolenic acid (ALA, 18:3n-3) and docosapentanoic acid (DPA, 22:5n-3) are of potential interest, data are limited. A number of reviews on the effect of fish oil or $n-3$ fatty acids on adipose tissue have previously been undertaken [15-20], therefore we have chosen to review the evidence from human, animal (rodent and fish) and in vitro cellular studies regarding the specific effects EPA and DHA have on the metabolism and function of white adipose tissue from different depots. Specifically, we will discuss the mechanisms by which EPA and DHA are proposed to reduce adiposity along with discussion regarding how $n-3$ fatty acids may influence markers of adipose tissue inflammation and cytokine production.

\section{Dietary Sources of Eicosapentaenoic Acid (EPA) and Docosahexaenoic Acid (DHA)}

EPA and DHA, commonly referred to as fish oil fatty acids, are not synthesized de novo by fish. Fish accumulate them through consumption of water plants, such as plankton and algae, which are part of the marine food chain [21]. Therefore, if plankton and algae are not a dietary component or if fish oil is replaced by other feed sources, such as in fish farming where a vegetable-oil based diet rich in linoleic acid (18:2n-6) and oleic acid (18:1n-9) may be given, the EPA and DHA content of the fish will decrease $[22,23]$. Marine fish tend to have higher amounts of EPA and DHA than freshwater fish. Fish typically store EPA and DHA mainly as triacylglycerol, at the middle position (sn-2) of the glycerol backbone however, in krill, a shrimp-like crustacean that feed off algae in deep ocean waters, $30 \%-65 \%$ of EPA and DHA is in phospholipids [24]. 
Within the human diet, EPA and DHA can be produced from ALA but the capacity of conversion is low in humans, although higher in women of child-bearing age than men [25]. Thus, it is likely that the majority of EPA and DHA within the body, for most individuals are derived from fish and fish oil intakes. Fish oil is often considered to be the best source of EPA and DHA however, as mentioned above, the amount of EPA and DHA varies amongst species and within a species according to environmental variables such as diet, temperature and salinity of the water.

\section{Fatty Acid Composition of Adipose Tissue}

As the fatty acid composition of adipose tissue has a half-life between 6 months and 2 years, it reflects long-term dietary intake along with endogenous metabolism [11]. The abundance of EPA and DHA in human subcutaneous adipose tissue is low, typically less than 0.2 for EPA and up to $1.0 \mathrm{~mol} \%$ for DHA [11]. The amount of EPA and DHA in adipose tissue has been reported to increase or remain unchanged with increasing age [26-28], which is suggested to be an age-dependent effect independent of dietary intake [28].

Studies measuring the change in adipose tissue fatty acid composition, as a marker of compliance to $n-3$ supplementation are limited and findings inconsistent with some [29-32] but not all [33,34] noting small but significant increases in the abundance of adipose tissue EPA and DHA after varying periods of fish oil supplementation (Table 1). The inconsistency in findings may in part be explained by differences in: duration of supplementation, amount of EPA and DHA consumed, participant age, sex and adiposity, or site where the adipose biopsy was taken. Elegant work by Katan et al. [30] clearly demonstrated that the levels of DHA rose more rapidly in subcutaneous abdominal compared to gluteal adipose tissue depots whilst differences between the depots for EPA were not as obvious (Table 1). The difference in the appearance of DHA in subcutaneous abdominal compared to gluteal adipose tissue, may be explained by the fact that dietary fat extraction (from chylomicron-triacylglycerol) occurs to a greater extent in subcutaneous abdominal than gluteal adipose tissue [35]. Of note, Katan et al. [30] found that the proportion of EPA and DHA in subcutaneous abdominal and gluteal adipose tissue was approximately one-sixth and one-third respectively of dietary intake. It would be of interest to determine the extent to which the fatty acid composition of visceral (intra-abdominal) adipose tissue changed with n-3 fatty acid supplementation. However, as visceral adipose tissue samples are often obtained during elective surgery, it would be challenging to undertake a well-controlled study. Taken together, the data presented in Table 1 clearly demonstrate that even with supplementation the abundance of EPA and DHA in adipose tissue does not increase notably. This suggests that EPA and DHA are not preferentially stored in adipose tissue triacylglycerol long-term, rather they may be partitioned to oxidation pathways or 
to storage in other lipid fractions, such as phospholipids; red blood cell and plasma phospholipids have a notably higher abundance of both EPA and DHA than adipose tissue [11]. However, a change in adipose tissue fat mass and therefore dilution of EPA and DHA abundance cannot be ruled out as the majority of studies do not indicate if there were changes in participants' body weight over the course of the study. Changes in fatty acid composition of adipose tissue have been reported with weight loss, notably there was not change in EPA abundance but an increase in DHA abundance, without a reported change in $n-3$ fatty acid intake, over the weight maintenance period [36]. These changes highlight the importance of weight/fat mass stability in subjects participating in intervention studies where adipose tissue fatty acid composition is being measured as a marker of compliance. 


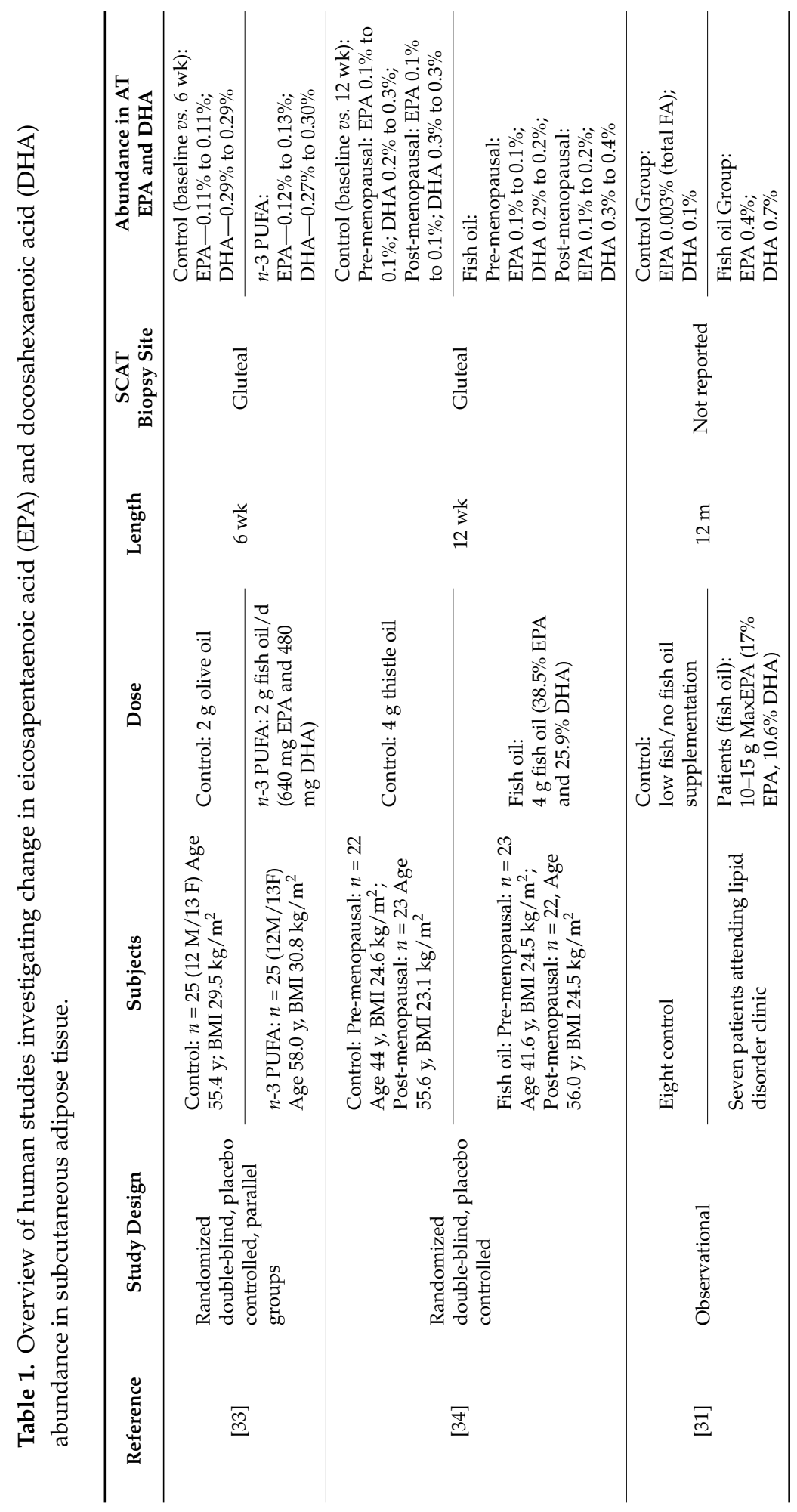




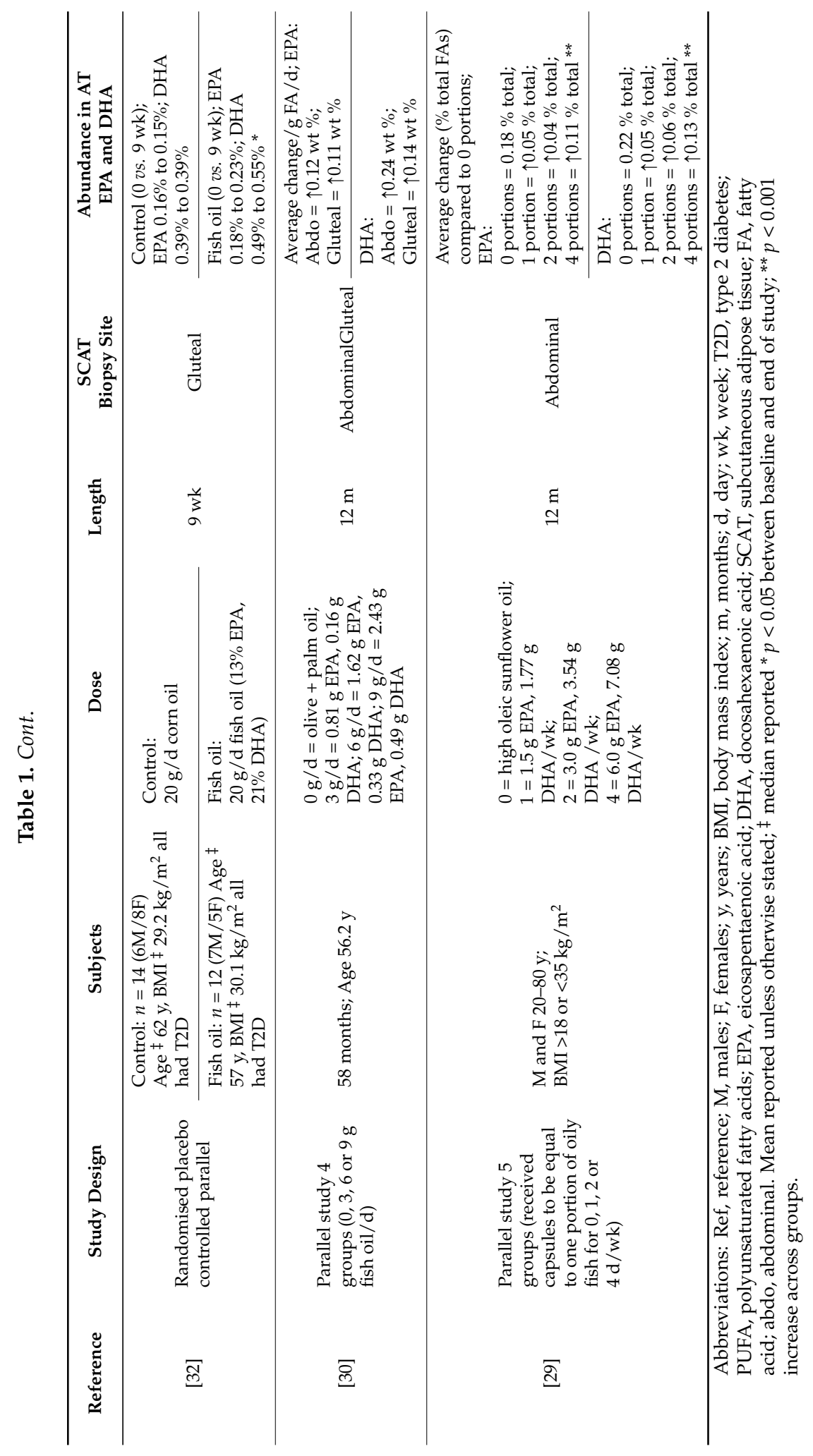




\section{The "Anti-Obesity" Effect of EPA and DHA}

Measuring an anti-obesity effect of increased EPA and DHA consumption in humans is challenging, not least as there are many other factors to control for (e.g., exercise and other dietary components) and methodology sensitive to small changes in adipose tissue mass needs to be used. In 2009, Buckley and Howe [37] reviewed the available evidence for an anti-obesity effect of EPA and DHA. They suggested from the limited human studies, that increased consumption of EPA and DHA may reduce body fat; the majority of these studies were short-term, with a small number of subjects. It remains unclear if similar conclusions can be drawn from longer-term studies. A recent meta-analysis by Du and colleagues [38] identified randomised, placebo controlled trials where adults were assigned to either fish oil/marine group for a period of greater than 4 weeks and had reported at least one anthropometric measure of body composition (i.e., body weight, BMI, waist circumference or waist to hip ratio). From the 21 studies (a total of 1329 individuals) they found no evidence to support an anti-obesity role of $n$-3 LCPUFA [38]. It is plausible that changes were not detected due to the non-specific and insensitive methods used to assess changes in body fat. By using computed tomography Sato et al. [39] noted that 6 months supplementation with EPA only (1800 mg/day) resulted in a significant decrease in epicardial and visceral adipose tissue mass, with no change in subcutaneous abdominal adipose tissue, in individuals with confirmed coronary artery disease. It is possible that subcutaneous abdominal adipose tissue mass did decrease however it was only measured in a single slice at the level of the umbilicus, thus changes in other depots would not have been detected. Results from some, but not all animal studies have suggested EPA and DHA consumption to have an anti-obesity effect with a lack of increase in fat mass even when an obesogenic diet is consumed [40], as well as a reduction in body weight if already obese [41]. Moreover, these studies, along with cellular studies have been used to tease out the mechanisms involved in this process, as discussed below with data from human [42-48], animal [40,41,49-55] and cellular [56-69] studies provided in Tables 2-4.

\subsection{Suppression of Fat Deposition and Adipogenesis}

A decrease in fatty acid deposition within adipose tissue may occur due to a decrease in triacylglycerol synthesis via decreased de novo lipogenesis or re-esterification of fatty acids within the tissue; alternatively it may occur due to a lower flux of fatty acids to the tissue. In the latter situation, fatty acids could be repartitioned to other tissues (such as muscle) for disposal, rather than going to adipose tissue for storage. In humans, the absolute contribution of de novo synthesized fatty acids to adipose tissue triacylglycerol is potentially small [70] and measuring adipose tissue de novo lipogenesis (or fatty acid esterification/re-esterification) in vivo 
in humans is challenging. Therefore, it is not surprising that studies have not been undertaken investigating how EPA and DHA supplementation influence these processes in humans. Although not a direct measure of fatty acid synthesis or esterification/re-esterification within the tissue, the measurement of the expression of genes related to these processes provides some insight to the effect of EPA and DHA on these processes. Camargo et al. [42] reported that consumption of $4 \mathrm{~g} /$ day of fish oil (containing a total of $1.24 \mathrm{~g}$ EPA and DHA) for 12 weeks significantly decreased the expression of genes related to fatty acid uptake and storage in subcutaneous obese adipose tissue (Table 2).

Work in animal models has typically found EPA and DHA to limit lipid accumulation in adipose tissue (Table 3). The majority of studies have reported lower fad pad mass and adipocytes number and size which was suggested to occur via suppression of lipogenic genes and, in some studies, a concomitant activation of lipolytic genes after supplementation with EPA and DHA (Table 3). Despite reporting a significant decrease in inguinal retroperitoneal fat pad mass Hainault et al. [52] did not find any significant change in fatty acid synthase (FAS) activity or mRNA expression in these depots. Of note, one study reported that EPA and DHA consumption resulted in higher total and perigonadal fat mass than control group [55]. This discrepancy in findings maybe in part explained that this study used an LDL receptor deficient $\left(\mathrm{LDLR}^{-/}\right)$mouse model whilst others have typically used C57Bl/ 6 mice or Wistar rats.

Studies investigating the effects of dietary EPA and DHA on adipose tissue function have also been undertaken in fish (Table 3). Todorcevic et al. [40] demonstrated that a diet supplemented with EPA and DHA (20\% of total fatty acids) for 21 weeks repressed the development of adiposity, regulating triacylglycerol accumulation in visceral adipose tissue of Atlantic salmon. A positive influence of dietary EPA and DHA on lipid accumulation in adipose tissue was also reported in grass carp [71]. Diet containing EPA and DHA, (12\% of total fatty acids for 75 days), suppressed lipid accumulation in intraperitoneal adipose tissue and significantly up-regulated the expression lipolytic genes including: lipoprotein lipase (LPL), stearoyl-CoA desaturase 1 (SCD1) and peroxisome proliferator activated receptor $\alpha$ $(P P A R \alpha)$ [71]. Furthermore, similar results were reported by Liu et al. [72] in grass carp treated with dietary EPA and DHA (11\% of total fatty acids) for 95 days. 


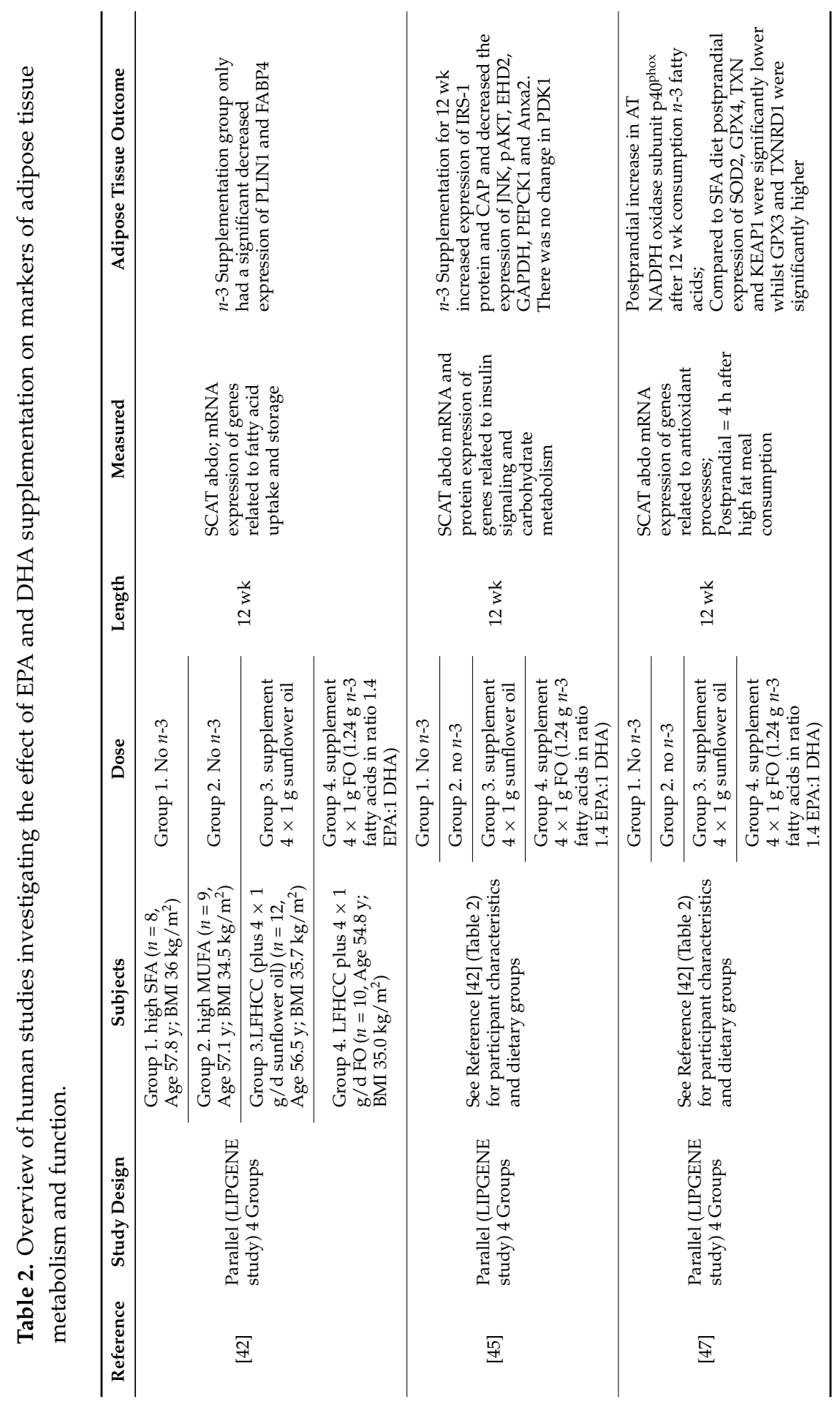




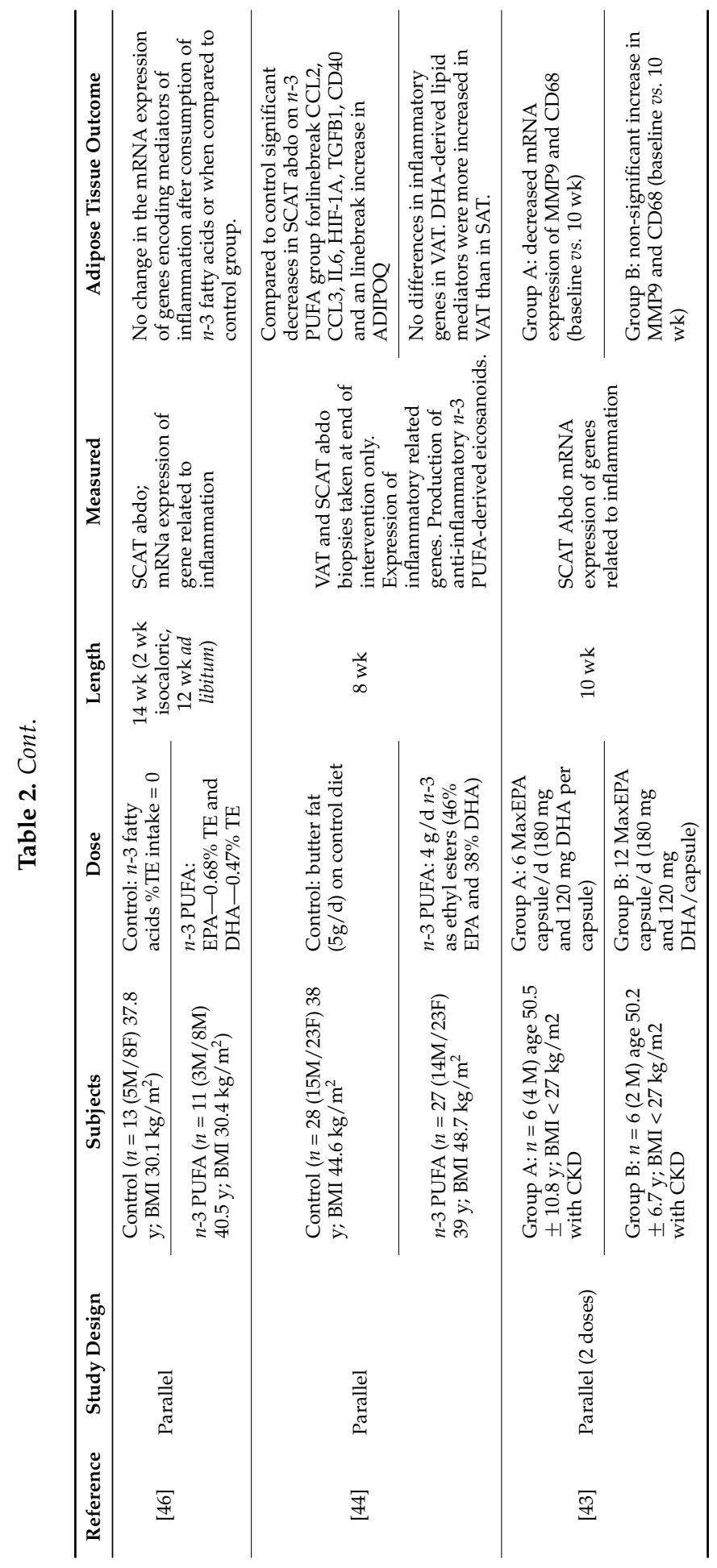




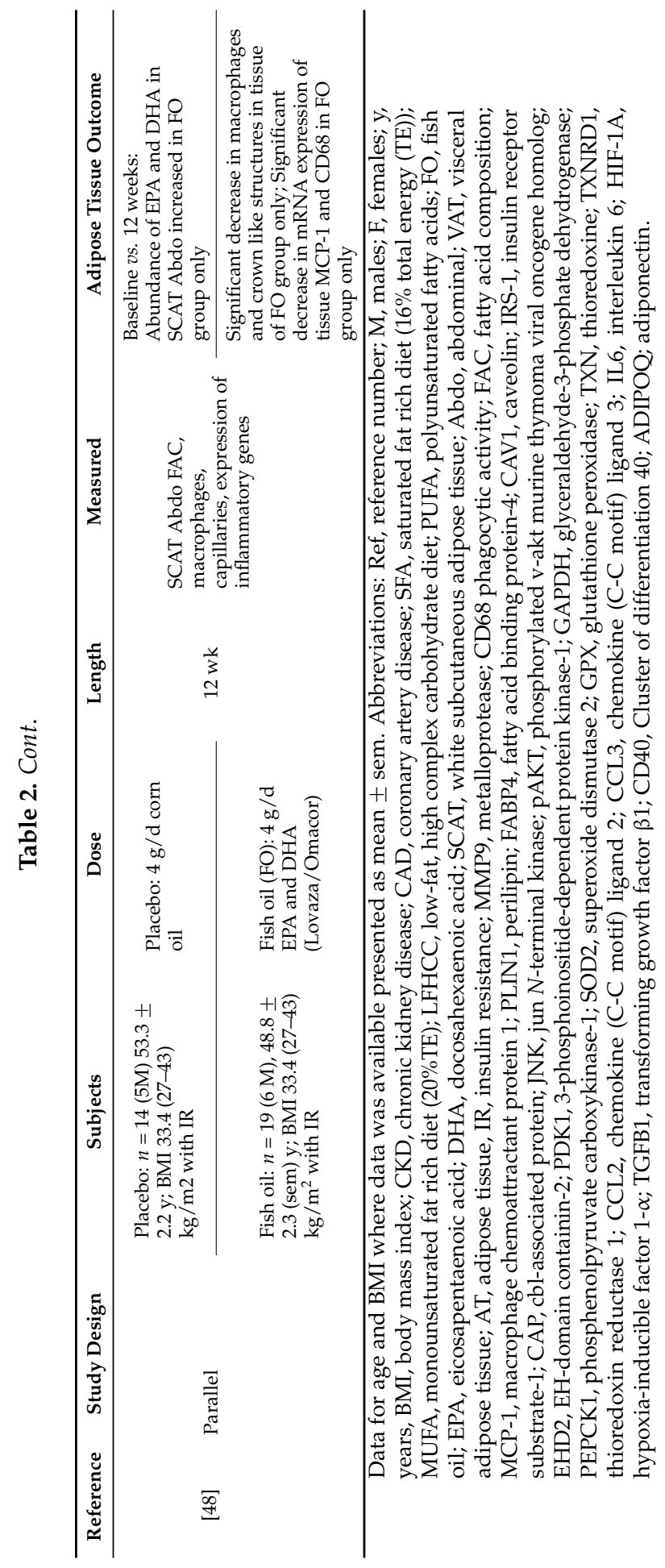




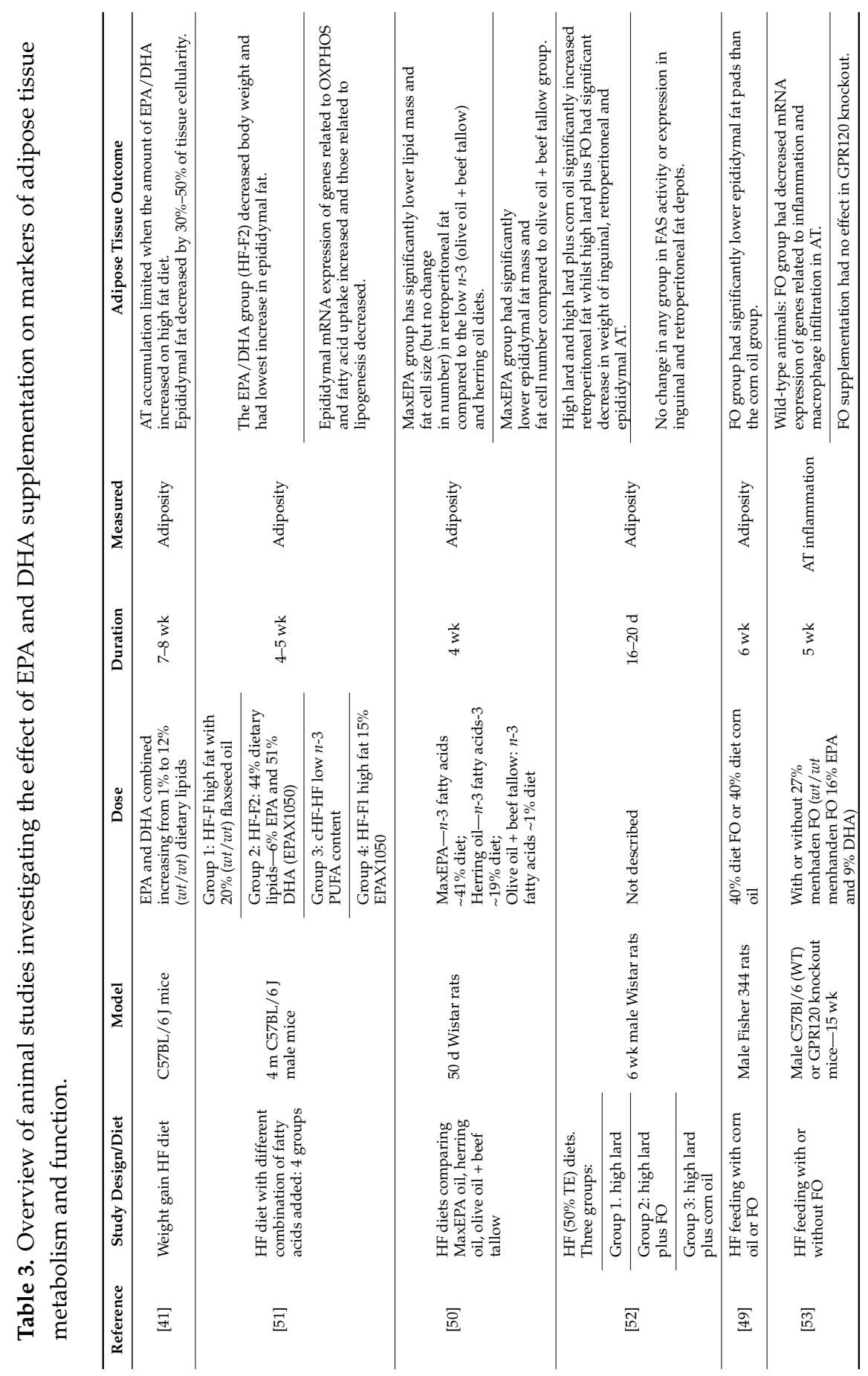




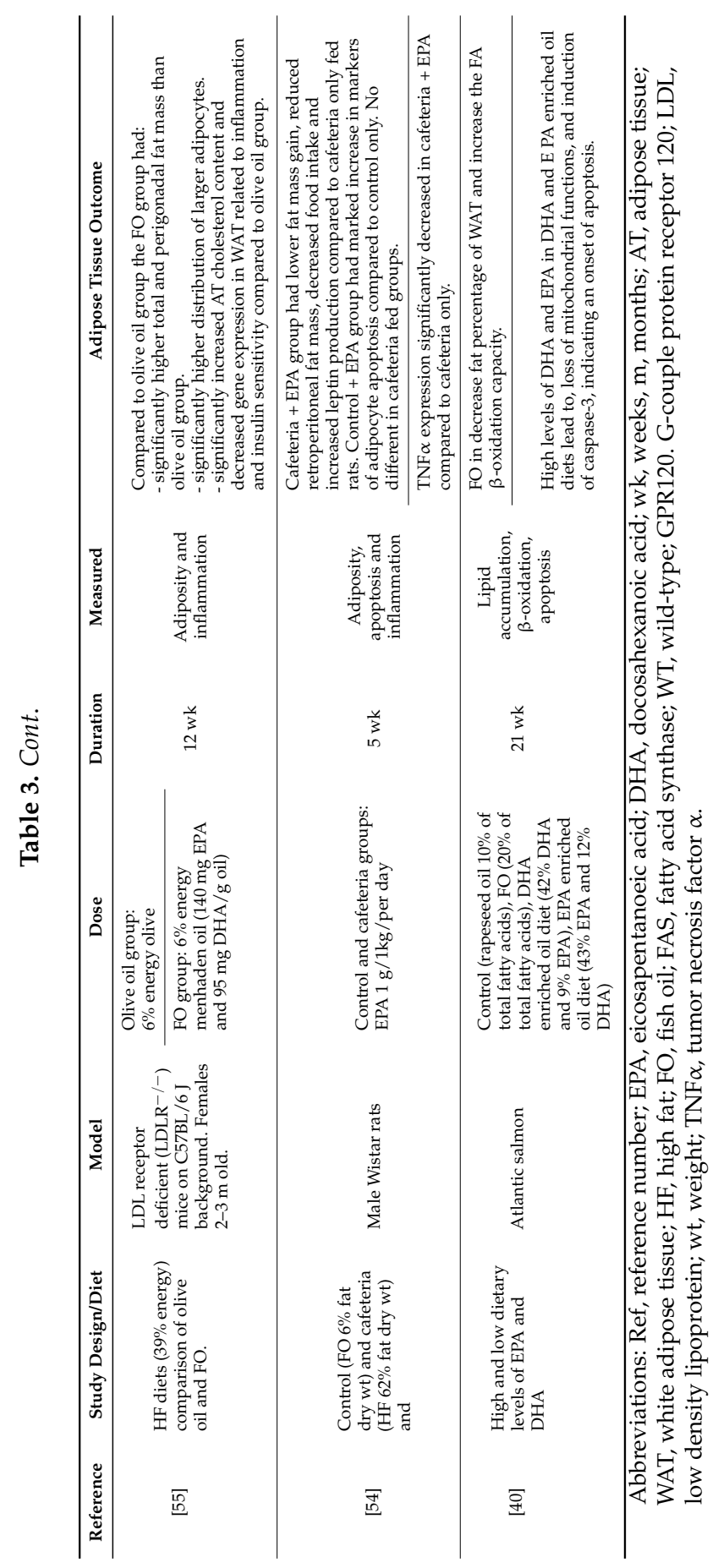


The process of adipogenesis (or an increase in fat mass) involves the differentiation of preadipocytes to mature adipocytes, is a complex and tightly regulated process involving a cascade of transcription factors which are sensitive to the nutritional environment [73]. In a comprehensive review by McMillen and Robinson [74] the role of the nutritional environment an individual is exposed to before birth and in early infancy impacts on risk of obesity and obesity-related diseases later in life was discussed. Evidence from animal studies shows that offspring of mothers fed a diet high in calories or high in fat before birth are heavier and have a higher percentage body fat throughout life [75,76]. Findings from human studies are compelling; children born to mothers who are obese during their pregnancy have an increased incidence of obesity over the life course [75]. Therefore, it has been suggested that targeting maternal nutrition during pregnancy may reduce risk of obesity in subsequent generations [77]; $n-3$ fatty acids may decrease adipogenesis and lipogenesis and thus exposure in utero to these fatty acids may lower the risk of obesity in offspring. In 2011 Muhlhausler and colleagues [77] reviewed animal studies to determine the effects of $n-3$ LCPUFA supplementation during pregnancy and lactation on postnatal body composition of offspring. Although 13 potential studies were identified, only four met the inclusion criteria and the authors found from albeit limited data that there was a suggestion that the offspring from $n-3$ LCPUFA supplemented dams had a lower fat mass [77]. In contrast, supplementation of dams with a high DHA diet (5\% fat of which DHA was $0.95 \%$ total fatty acids) during pregnancy and lactation resulted in offspring that had significantly higher total and subcutaneous fat mass (as percentage of total body weight) at 6 weeks of age, compared to control animals fed a diet containing the same amount of fat but devoid of $n-3$ LCPUFA [78]. Thus it remains unclear if increased exposure in utero to $n-3$ fatty acids decreases adipogenesis and lipogenesis and is an area that warrants further investigation.

To date, the majority of in vitro evidence regarding the mechanistic effects of EPA and DHA on triacylglycerol accumulation/lipid deposition comes from the clonal murine cell line, 3T3-L1 (Table 4). This cell line offers advantages over primary cells, as they are homogenous with regards to cellular population and stage of differentiation; however, their ability to reflect human adipose tissue function and metabolism remains to be clarified. Primary pre-adipocyte cultures have been shown to better reflect in vivo adipose function, than cell lines because they can be isolated from different species and fat depots. The latter is of interest as there are distinct molecular and biochemical hallmarks between different adipose tissue depots and at a cellular level; pre-adipocytes isolated from different adipose tissue depots and cultured in vitro retain depot-specific functional properties [79-81]. Unlike cell lines, the function and metabolism of primary cells will be influenced by the age, sex, 
and genetics of the donor and therefore consideration is needed when comparing across studies.

Results from in vitro cellular studies that have added EPA and DHA to media for periods of $24 \mathrm{~h}$ to 3 weeks are mixed; some suggest EPA and DHA to be anti-adipogenic whilst others find a pro-adipogenic response. EPA and DHA have been found to inhibit, promote or have no effect on the differentiation of pre-adipocytes (Table 4). Typically the markers of adipocytes adipogenesis that have been measured include: the accumulation of triacylglycerol, lipid droplet formation, expression of master adipogenic transcription factors, and lipid genes. Using 3T3-L1 pre-adipocytes, Kim et al. [59] investigated the effects of DHA alone (6 days) on lipogenesis and lipolysis and found mean lipid droplet size, percent lipid area, as well as glycerol-3-phosphate dehydrogenase (GPDH) activity all significantly decreased whilst basal lipolysis increased in fully differentiated adipocytes. The results from this work demonstrate the anti-adipogenic effects of DHA via inhibition of triacylglycerol accumulation and increased lipolysis [59]. When comparing the effects of EPA and DHA on lipid droplet formation in 3T3-L1 cells it was found that although both fatty acids reduced the presence of lipid droplets, DHA was more potent than EPA [56]. In addition to the decreased lipid droplet formation, there were notable reductions in the expression of key protein involved in this process, including perilipin A, caveolin-1 and Cidea [56], however there was no effect of DHA on PPAR $\gamma$ expression [56]. In contrast, Murali et al. [62] reported that incubating 3T3-L1 with EPA and DHA induced adipogenesis; DHA being more potent than EPA in inducing the differentiation process. The authors suggested the differential effects of EPA and DHA on adipognesis could be due to differential accumulation of $n-3$ fatty acids in membrane phospholipids [62]. In line with Murali et al. [62], Wojcik et al. [69] reported increased accumulation of neutral lipids in mature 3T3-L1 adipocytes; however, others have reported no effect on triacylglycerol accumulation at any stage of maturation in 3T3-L1 adipocytes [65]. A reduced expression of both adipogenic and lipogenic genes, including sterol regulatory element-binding protein 1 (SREBP1), FAS, and peroxisome proliferator-activated receptor $\gamma(\operatorname{PPAR} \gamma)$ after EPA and DHA treatment of mature adipocytes has been report by some [69] but not by others [67]. Using human breast adipocytes as a cell model, Wang et al. [68] demonstrated exposure of DHA for $24 \mathrm{~h}$ decreased the expression of lipogenic genes, including FAS, LPL and PPAR $\gamma$, whilst expression of lipolytic genes was increased. Lee et al. [82] found EPA to stimulate glycerol and free fatty acids release which was associated with induction of lipolytic gene expression and suppression of adipogenic gene expression in 3T3-L1 adipocytes. Treatment of fish primary adipocytes with EPA and DHA (for 3 weeks) resulted in decreased triacylglycerol accumulation in mature adipocytes [83]. In an acute study, using mature adipocytes isolated from grass carp Liu et al. [72] found that $6 \mathrm{~h}$ of incubation with EPA and DHA was sufficient 
to notably decreased triacylglycerol accumulation, significantly increased glycerol release and the expression of genes involved in lipolysis (e.g., adipose triglyceride lipase (ATGL), hormone-sensitive lipase (HSL)). The findings from in vitro cellular studies, notably those using primary adipocytes demonstrate that EPA and DHA inhibit triacylglycerol accumulation, which may be the result of effects mediated through genes related to lipogenesis and lipolysis.

Overall, the effects of EPA and DHA as well as EPA versus DHA in modifying adipogensis and lipid accumulation, in particular in humans, and to a lesser extent in murine models, remain unclear. Plausible reasons the discrepancies in findings between in vitro cellular studies are likely to be in part due to the use of different in vitro models, i.e., using primary cells versus immortalized cell lines, studying the cells at different developmental stages, differences in the concentrations of fatty acid(s) the cells were exposed to, along with the duration of exposure.

\subsection{Adipocyte Apoptosis}

To our knowledge, there have been no studies in humans investigating the effect of $n-3$ fatty acids on adipocyte apoptosis and only limited work has been undertaken in animal and in vitro cellular models. Although outside the scope of this review, there have been a large number of studies investigating the effect of $n-3$ fatty acids and cancer in relation to apoptosis, as reviewed by Wendel and Heller [84]. 


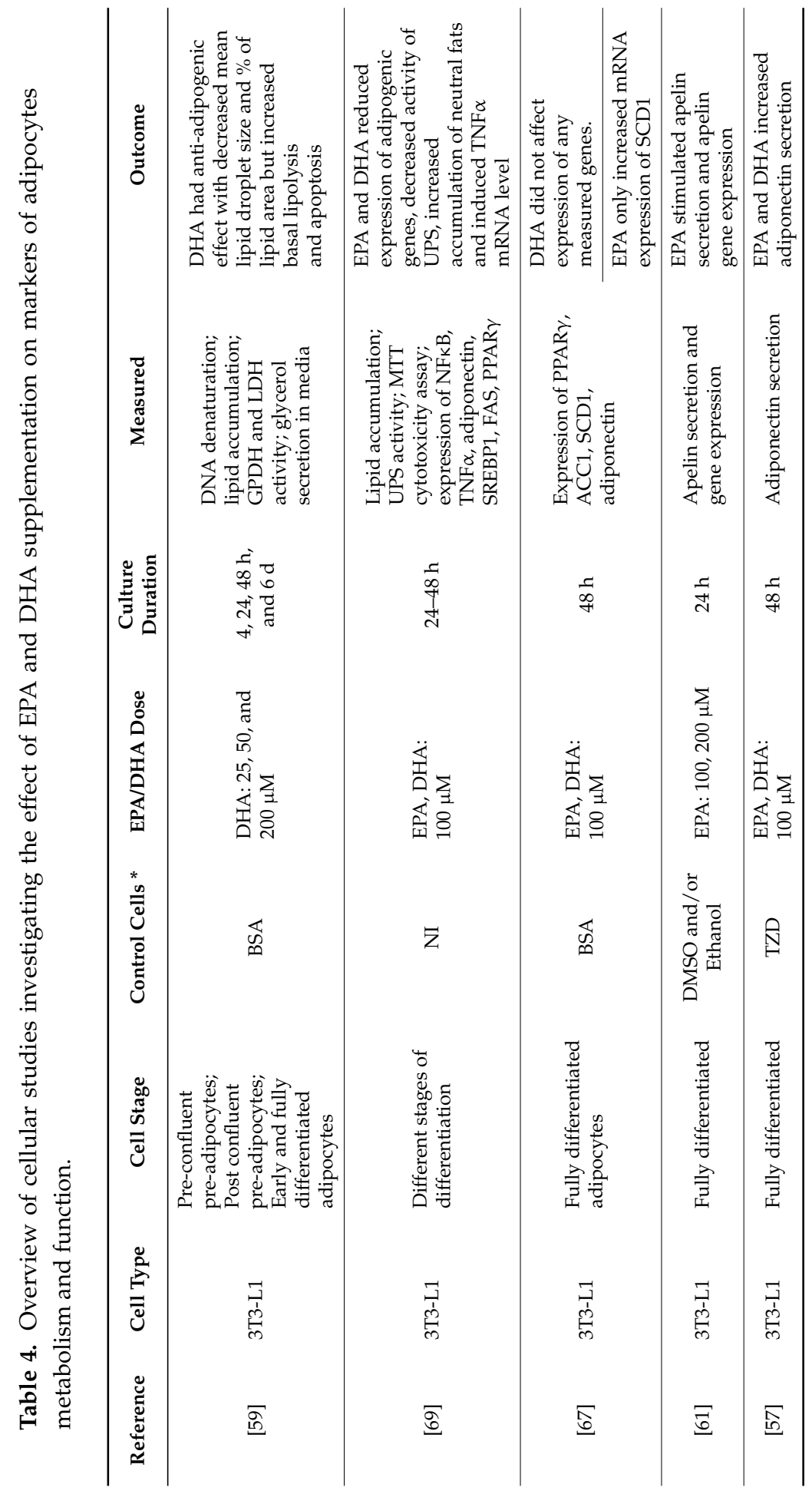




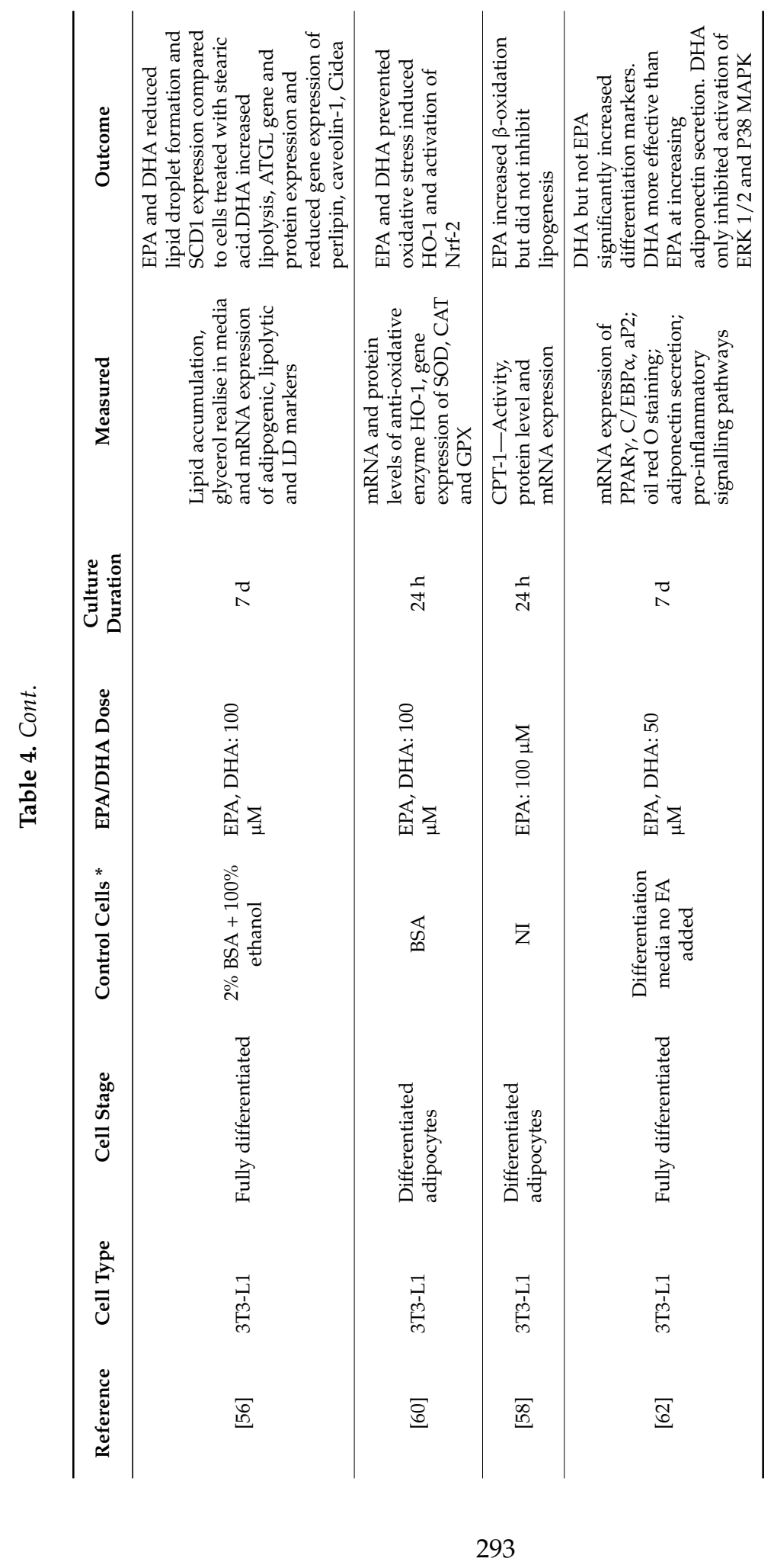




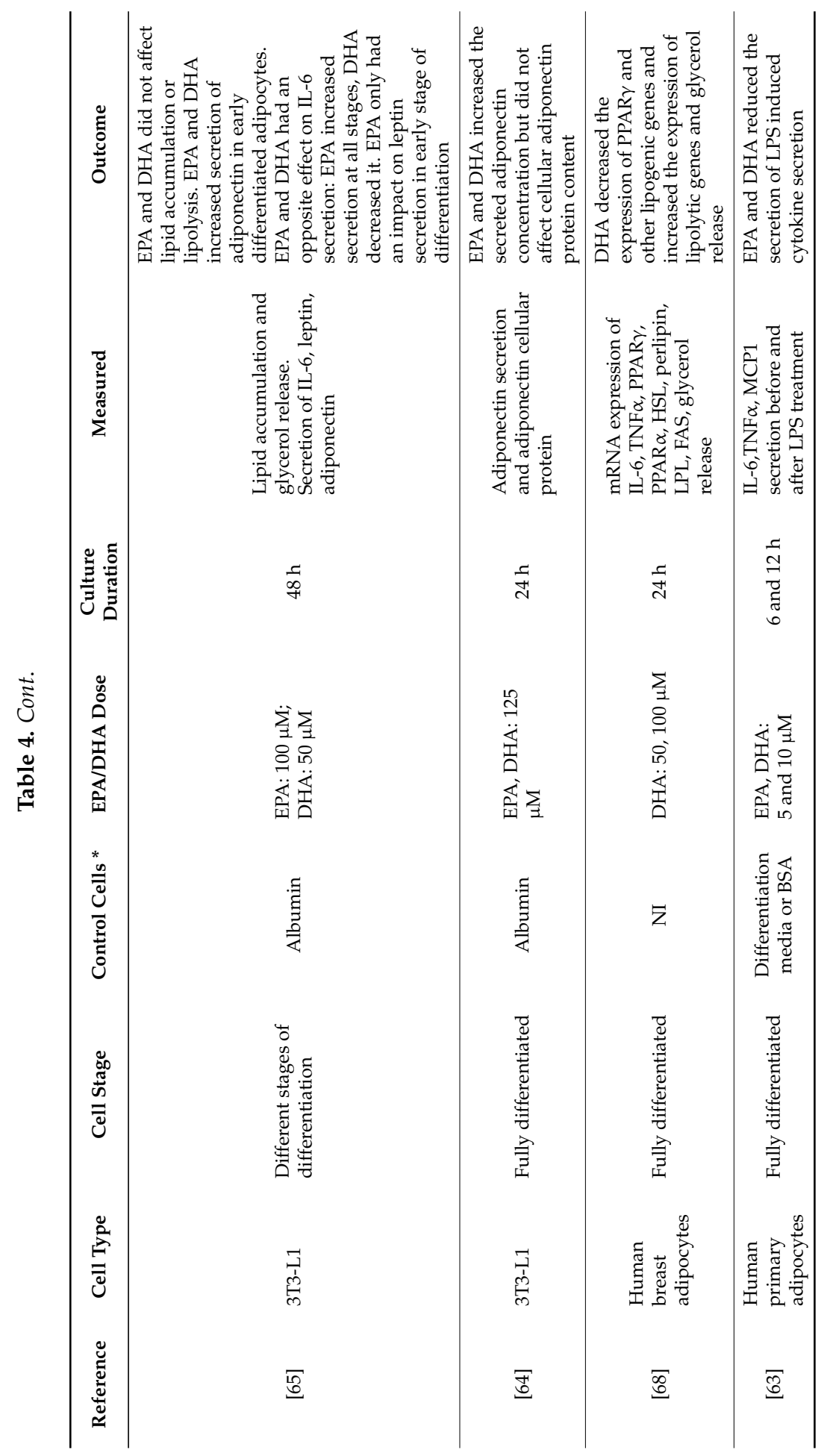




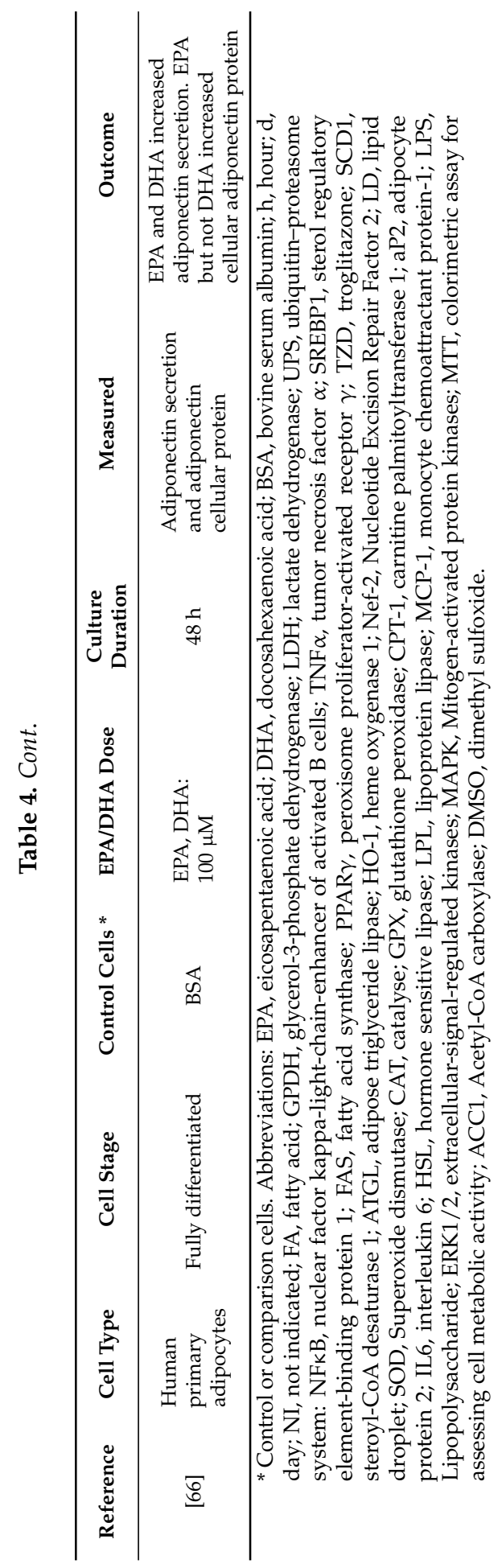


Limiting findings from human in vitro and in vivo studies have reported apoptosis in white adipose tissue along with alternations in adipose tissue mass. Thus consideration is required when looking at adipose tissue mass in relation to cell number as they might be partly regulated by pre-adipocyte/adipocyte apoptosis [85,86]. Nelson-Dooley et al. [87] have suggested targeting apoptotic pathways in adipocytes as a novel way of treating obesity. Apoptosis is often assessed by cytomorphological alterations, DNA fragmentation and condensation, detection of caspases, protein cleavage at specific locations, cell membrane alterations and and increased mitochondrial membrane permeability [88]. In 2004 Ruszickova et al. [41] were the first to suggest the concept of $n-3$ fatty acids and regulation of cellularity in adipose tissue. Using a rodent model the authors suggested that increased intakes of EPA and DHA (Table 3) reduced high-fat diet-induced obesity by decreasing the number of adipocytes in adipose tissue, which could be interpreted as evidence of a pro-apoptotic effect. Perez-Matute et al. [54] demonstrated increased levels of histone-associated DNA oligonucleosomal fragments, classical markers of apoptosis in the white adipose tissue of rats fed a standard diet with additional oral administration EPA ethyl ester $(1 \mathrm{~g} / \mathrm{kg}$ per day) daily for 5 weeks. Moreover, they found a cafeteria diet strongly impaired the apoptotic action induced by EPA and suggested that EPA-induced apoptosis depends on the nutritional and metabolic status of the animals [54]. High dietary- $n$ - 3 fatty acid levels are at increased susceptibility to fatty acid peroxidation which has been reported to occur in different tissues within a fish model [89] including adipose tissue [40]. Fish contain a greater amount of more highly unsaturated fatty acids than mammals which makes them more prone to fatty acid peroxidation leading to apoptosis [90]. Todorcevic et al. [40] were the first to demonstrate that high dietary intakes of EPA and DHA induced oxidative stress and apoptosis in the visceral adipose tissue in Atlantic salmon. Salmon was fed with diets containing 50\% EPA and 55\% DHA of total fatty acid for 21 weeks and found increased activity of caspase-3, indicative of apoptosis occurring in white adipose tissue. The authors concluded that decreased adipocytes cell number due to apoptosis, may be one factor explaining the lower triacylglycerol accumulation occurring in fish white adipose tissue when diets enriched with EPA and DHA are fed [40]. On the basis of these finding, it would be prudent to suggest the measurement of adipose tissue apoptotic markers when EPA and DHA, notably at high dietary doses, are given.

Even though there is a growing literature on the studying the mechanisms for the inhibitory effects of $n-3$ fatty acids on proliferation of various tumor cells (reviewed by [91]) but also on non-cancerous cells [92], there are surprisingly very few in vitro studies that have investigated the effect of EPA and DHA on adipocyte apoptosis. Kim et al. [59] reported significant DHA-induced apoptosis in 3T3-L1 post-confluent pre-adipocytes after $48 \mathrm{~h}$ incubation with $200 \mu \mathrm{M} / \mathrm{L}$ compared 
to $100 \mu \mathrm{M} / \mathrm{L}$ DHA, demonstrating the inhibitory effects of DHA on adipocyte differentiation. Todorcevic et al. [93] treated primary antioxidant glutathione (GSH) depleted salmon adipocytes with high doses of EPA and DHA (600 $\mu \mathrm{M}$ for 6 days) in present or absence of $\alpha$ tocopherol and showed increased expression of genes encoding a set of well-known apoptotic markers in the groups with no added $\alpha$ tocopherol, suggesting that the induction of adipocyte cell death by EPA and DHA likely plays an important part in the adipose tissue homeostasis especially in animals exposed to high dietary $n-3$ fatty acids.

Taken together, the available data from animal and in vitro studies suggests that high doses of EPA and DHA may induce adipocyte apoptosis. How targeting the apoptotic pathway in white adipose tissue would decrease obesity and influence adipose tissue function and overall metabolic health in humans remains to be elucidated.

\subsection{Increased Fatty acid Oxidation (Energy Expenditure)}

Although an increase in fatty acid oxidation, via $\beta$-oxidation has been suggested to play a role in a reduction of triacylglycerol accumulation in adipocytes, evidence for this in white adipose tissue is limited; fatty acid oxidation and mitochondrial function has been studied more often in brown adipose tissue. The number and activity of mitochondria within adipocytes has been suggested to contribute to insulin resistance and type 2 diabetes [94]. Changes in the expression of genes related to insulin-signaling have been reported to increase, whilst the expression of genes related to glycolysis, gluconeogenesis and glyceroneogenesis decreased in subcutaneous abdominal adipose tissue after 12 weeks supplementation with n-3 fatty acids [45] (Table 2). On the basis of these changes, the authors suggested that a low-fat (fat $28 \%$ total energy (TE)) high complex carbohydrate diet supplemented with $1.24 \mathrm{~g} /$ day $n-3$ fatty acids (EPA and DHA) improved adipose tissue insulin sensitivity compared to diets high saturated or monounsaturated fat in individuals with the metabolic syndrome [45]. To lower the risk of obesity-mediated diseases such as the metabolic syndrome, weight loss is often encouraged to decrease fat mass; weight loss by calorie restriction has been suggested to increase subcutaneous abdominal adipose tissue capacity for lipid oxidation [95]. Whether similar changes occur in subcutaneous gluteal or visceral adipose tissue remains to be determined. Moreover, it would be of interest to determine if calorie restriction in combination with EPA and DHA supplementation has an additive effect on up-regulating adipose tissue fatty acid oxidation in different adipose tissue depots. The amount of EPA and DHA has varied between studies, with higher doses tending to be used in animal and in vitro studies, translation to the appropriate dose, along with duration required to see an effect in humans needs to be elucidated. 
In vivo or in vitro cellular studies investigating the effects of EPA and DHA on adipose tissue fatty acid oxidation and/or mitochondrial function are sparse. Specifically measuring markers of adipose tissue fatty acid oxidation in vivo in humans has not, to our knowledge, been undertaken. This is most likely to be due to the challenges associated with assessing adipose tissue fatty acid $\beta$-oxidation directly. Surprisingly no study in humans has yet investigated changes in the expression of relevant genes in adipose tissue before and after supplementation with EPA and DHA. Fasting whole-body fatty acid oxidation (assessed by indirect calorimetry) has been reported to increase in young, healthy men $(n=5)$ after 3 weeks of supplementation with fish oil ( $6 \mathrm{~g} /$ day) when compared to a control diet containing equal amounts of total dietary fat [96]. Only a few animal studies have investigated the effect of EPA and DHA on fatty acid $\beta$-oxidation in white adipose tissue (Table 3). Flachs et al. [51] reported that feeding mice for 4 weeks with diet containing increasing amounts of EPA and DHA, preferentially up-regulated several mitochondrial regulatory genes, increased $\beta$-oxidation and suppressed lipogenesis in white abdominal fat. Using a fish model, Atlantic salmon, Todorcevic et al. [40] reported an increase in adipose tissue fatty acid $\beta$-oxidation after fish consumed fish oil rich in EPA and DHA for 21 weeks.

In vitro cellular studies have found increased $\beta$-oxidation in 3T3-L1 adipocytes after incubation with $100 \mu \mathrm{M}$ of EPA for $24 \mathrm{~h}$ [58]. The increase in $\beta$-oxidation was associated with increased carnitine palmitoyltransferase 1 (CPT-1) activity but mRNA and protein expression did not change [58]. As EPA treatment increased the proportion of EPA in mitochondrial membrane lipids, the authors concluded that the activity of CPT- 1 and $\beta$-oxidation was due to changes in the structure or dynamics of the mitochondrial membranes [58]. EPA and DHA are reported to activate AMP-activated protein kinase (AMPK) in 3T3-L1 adipocytes, which could be a mechanism for their effect on fatty acid oxidation [97]. Todorcevic et al. [83] demonstrated that EPA and DHA increased $\beta$-oxidation in salmon primary adipocytes, which may in part explain the concomitant reduction in adipocyte triacylglycerol. A possible mechanism, by which EPA and DHA may result in increased fatty acid oxidation and therefore less body fat accumulation, is through induction of thermogenesis mediated by mitochondrial uncoupling protein-1 (UCP1); the thermogenic capacity of brown adipose tissue (BAT) is associated with uncoupling whereas white adipose tissue is typically not [98]. In 2013 Flach et al. [98] reviewed the effect of $n-3$ fatty acids on mitochondrial oxidative phosphorylation (OXPHOS) and fatty acid oxidation in white adipose tissue. In this comprehensive review they reported that in a murine model, supplementation with $n-3$ fatty acids in combination with mild calorie restriction induced mitochondrial OXPHOS in epididymal white adipose tissue only, independent of UCP1 induction; other studies in rodents have reported increased levels of UCP1 mRNA and/or protein in BAT 
in response to $n-3$ fatty acid supplementation [98]. Recently, Zhao and Chen [99] using an in vitro cellular model of isolated stromal-vascular (SV) cells from inguinal adipose tissue of suggested that EPA enhanced energy dissipation capacity by recruiting brite adipocytes to stimulate oxidative metabolism. From the limited data available it appears that EPA and DHA increase fatty acid $\beta$-oxidation in adipocytes, however the mechanisms responsible and the effect on mitochondrial OXPHOS and thermogenesis in human adipose tissue remains to be elucidated.

\section{The "Anti-Inflammatory" Effects of EPA and DHA on Adipose Tissue}

An expansion of adipose tissue mass, is often associated with macrophage infiltration which may lead to inflammatory responses, which have been implicated in the development of pathological changes in adipose tissue physiology [6-9]. These changes potentially move the tissue toward a pro-inflammatory phenotype and there is accumulating evidence suggesting pro-inflammatory processes in adipose tissue increase the risk of obesity-related disorders, such as insulin resistance [100-103]. For example, several studies reported positive associations between degree of obesity and the expression of genes related to inflammation in adipose tissue $[7,9]$. A number of studies have investigated the "anti-inflammatory" effect of EPA and DHA in white adipose tissue.

\section{Suppression of Pro-Inflammatory Cytokine Production}

Studies investigating the effect of $n-3$ fatty acid supplementation, for periods between 8 weeks up to 6 months, on the expression of genes related to inflammation in human subcutaneous white adipose tissue have been undertaken. Overall results are variable, with some suggesting consumption of EPA and DHA decreases the expression of genes related to inflammation, whilst other report no change (Table 2). For example, Guebre-Egziabher et al. [43] noted decreased expression of metalloprotease 9 (MMP9) and CD68 in subcutaneous abdominal adipose tissue on a low not high dose of MaxEPA in a small number $(n=12)$ of individuals with chronic kidney disease (CKD) who were randomised to take either a low $(n=6)$ or high $(n=6)$ dose of MaxEPA for 10 weeks. In contrast, Itariu et al. [44] found that high doses of EPA and DHA (total $4 \mathrm{~g} /$ day) for 8 weeks significantly decreased the expression of genes related to inflammation in subcutaneous obese adipose tissue and increased production of anti-inflammatory eicosanoids in visceral adipose tissue (Table 2).

Work in murine models has found consumption of n-3 fatty acids decreased inflammatory gene expression in white adipose tissue depots (Table 3 ). Todoric et al. [104] investigated the effect of an $n-3$ fatty acid diet on macrophage infiltration in white adipose tissue of obese, diabetic mice, as well as on gene expression of several immune genes. They found that consumption of $25.1 \mathrm{mg}$ 
of $n$-3 fatty acids (containing EPA and DHA) per gram of fat for 6 weeks resulted in a reduction in macrophage infiltration in combination with decreased expression of inflammatory genes in white adipose tissue [104]. Sarawathi et al. [55] used LDLR ${ }^{-/-}$ mice and showed similar results to Todoric et al. [104] despite a gain in white adipose tissue mass. They reported a diet supplemented with fish oil containing $140 \mathrm{mg}$ EPA and $95 \mathrm{mg}$ DHA/day for 12 weeks reduced expression of macrophage markers such as MAC-1 and CD68 as well as inflammatory markers such as TNF $\alpha$, metalloprotease 3 (MMP3), and serum amyloid A3 (SAA3) in white adipose tissue [55]. Taken together these data demonstrate that consumption of $n-3$ fatty acids have the potential to modulate immune response in adipose tissue.

In vitro studies, using cell-lines and human primary cells, have been utilised to investigate the potential cellular mechanisms and pathways involved in an $n-3$ fatty acid mediated alteration in immune response (Table 4). Adiponectin, an adipocyte-specific protein, is often suggested to be anti-inflammatory cytokine and it has been postulated that a change in secretion may be associated with visceral obesity [105]. In vitro cellular work has found that incubation of primary human adipocytes isolated from subcutaneous adipose tissue with either with EPA or DHA significantly increased the concentration of secreted adiponectin [106], which is in agreement with several studies performed using primary cultured rat adipocytes [107], 3T3-L1 adipocytes [64] and human adipocyte cell lines [66]. From the work of Oster et al. [64] it appears that EPA and DHA have differential effects on adiponectin secretion, which may be influenced by the cell model used. They found DHA increased adiponectin mRNA expression and secreted adiponectin protein to a greater extent than the same dose of EPA in 3T3-L1 adipocyte after $24 \mathrm{~h}$ incubation [64]. In contrast, Tishinsky et al. [99] found using a commercial line of human adipocytes that EPA significantly increased cellular adiponectin protein content after $48 \mathrm{~h}$ of treatment while DHA did not affect cellular adiponectin protein.

The effects of $n-3$ fatty acids on the adipokine leptin, have been investigated in vitro however results show conflicting effects of $n-3$ fatty acids on leptin mRNA expression and secretion. EPA has been shown to have a stimulatory effect on leptin gene expression and secretion in 3T3-L1 adipocytes [108] and primary cultured rat adipocytes [109]. Reseland et al. [110] reported an opposite effect to the work of Murata et al. [108] and Perez-Matute et al. [109], where exposure to both EPA and DHA reduced leptin mRNA expression in 3T3-L1 adipocytes. Furthermore, the effect of EPA and DHA on leptin expression has been shown to vary depending on the stage of adipocyte maturation [65]. Thus, the discrepancy in reported results could be related to differences in different cell models used (primary cells vs. cell lines) or in measuring the effects of $n-3$ fatty acids on leptin at different stages of adipogenesis. Culturing human primary adipocytes in either EPA or DHA resulted in a down-regulation of IL6 and TNF $\alpha$ secretion [63]. In contrast, differential effects 
of EPA and DHA were found for IL6 secretion in 3T3-L1 cells with EPA increasing and DHA decreasing secretion [65]; the underlying mechanisms for these responses were unable to be clarified by the authors. Another divergent finding is that from Wojcik et al. [69] who noted culturing 3T3-L1 cells in either EPA or DHA increased $\mathrm{TNF} \alpha \mathrm{mRNA}$ expression; it is unclear if this lead to increased secretion as it was not measured. The authors speculated that their finding would not be replicated in adipose tissue in vivo, as the anti-inflammatory effects of EPA and DHA on TNF $\alpha$ expression would be modulated through the direct effect of these fatty acids on macrophages; cells that were not present in their in vitro culture [69]. It remains unclear if EPA and DHA have a differential effect on anti-inflammatory markers in human and animal models as typically these fatty acids have been given together and not directly compared.

\section{Conclusions}

In recent years evidence demonstrating that an increased consumption of EPA and DHA may have a beneficial effect on white adipose tissue function and metabolism is starting to emerge. Although current literature cannot support an exact mechanistic role of EPA and DHA on adipose tissue biology it is apparent that these fatty acids have the potential to be potent modulators of adipose tissue and adipocyte function. More work has been undertaken using animal and cell models therefore consideration is required regarding the dose and duration of EPA and DHA, the animal and cell model used (e.g., primary vs. cell-lines). Moreover, in vitro cellular cells often investigate the effects of EPA and DHA on adipocytes and it is plausible a different response may be found in whole adipose tissue due to the presence of other cell types (e.g., macrophages, endothelial cells) and their interaction with adipocytes. Although the effects of $n-3$ fatty acid supplementation on the fatty acid composition of subcutaneous abdominal and gluteal adipose tissue have been investigated, mechanistic studies (in vivo and in vitro) appears to be limited to primarily subcutaneous abdominal adipose tissue and/or adipocytes. Evidence for an effect of $n-3$ fatty acids in human visceral adipose tissue is sparse and therefore not well understood. Evidence for a reduction in fat accumulation in animal models, along with an anti-inflammatory effect appears to be consistent when intakes of EPA and DHA are high (up to $20 \%$ of total fatty acids); however recommendations for human intakes are between $0.5 \%$ and $2 \%$ of total energy intake [111]. Thus, the duration and amount of dietary EPA or DHA required for beneficial effects in human subcutaneous adipose tissue depots remains to be elucidated.

Acknowledgments: Leanne Hodson is a British Heart Foundation Intermediate Fellow.

Author Contributions: Marijana Todorčević and Leanne Hodson wrote the manuscript.

Conflicts of Interest: The authors declare no conflict of interest. 


\section{References}

1. Mohamed-Ali, V.; Pinkney, J.H.; Coppack, S.W. Adipose tissue as an endocrine and paracrine organ. Int. J. Obes. Relat. Metab. Disord. 1998, 22, 1145-1158.

2. Trayhurn, P. Adipocyte biology. Obes. Rev. 2007, 8, 41-44.

3. Frayn, K.N. Adipose tissue as a buffer for daily lipid flux. Diabetologia 2002, 45, 1201-1210.

4. Lewis, G.F.; Carpentier, A.; Adeli, K.; Giacca, A. Disordered fat storage and mobilization in the pathogenesis of insulin resistance and type 2 diabetes. Endocr. Rev. 2002, 23, 201-229.

5. Unger, R.H.; Orci, L. Lipotoxic diseases of nonadipose tissues in obesity. Int. J. Obes. Relat. Metab. Disord. 2000, 24, S28-S32.

6. Sun, K.; Kusminski, C.M.; Scherer, P.E. Adipose tissue remodeling and obesity. J. Clin. Investig. 2011, 121, 2094-2101.

7. Weisberg, S.P.; McCann, D.; Desai, M.; Rosenbaum, M.; Leibel, R.L.; Ferrante, A.W., Jr. Obesity is associated with macrophage accumulation in adipose tissue. J. Clin. Investig. 2003, 112, 1796-1808.

8. Wellen, K.E.; Hotamisligil, G.S. Obesity-induced inflammatory changes in adipose tissue. J. Clin. Investig. 2003, 112, 1785-1788.

9. Xu, H.; Barnes, G.T.; Yang, Q.; Tan, G.; Yang, D.; Chou, C.J.; Sole, J.; Nichols, A.; Ross, J.S.; Tartaglia, L.A.; et al. Chronic inflammation in fat plays a crucial role in the development of obesity-related insulin resistance. J. Clin Investig. 2003, 112, 1821-1830.

10. Fabbrini, E.; Yoshino, J.; Yoshino, M.; Magkos, F.; Tiemann Luecking, C.; Samovski, D.; Fraterrigo, G.; Okunade, A.L.; Patterson, B.W.; Klein, S. Metabolically normal obese people are protected from adverse effects following weight gain. J. Clin. Investig. 2015, $125,787-795$.

11. Hodson, L.; Skeaff, C.M.; Fielding, B.A. Fatty acid composition of adipose tissue and blood in humans and its use as a biomarker of dietary intake. Prog. Lipid Res. 2008, 47, 348-380.

12. Marik, P.E.; Varon, J. $\omega-3$ Dietary supplements and the risk of cardiovascular events: A systematic review. Clin. Cardiol. 2009, 32, 365-372.

13. Delarue, J.; LeFoll, C.; Corporeau, C.; Lucas, D. n-3 Long chain polyunsaturated fatty acids: A nutritional tool to prevent insulin resistance associated to type 2 diabetes and obesity? Reprod. Nutr. Dev. 2004, 44, 289-299.

14. Buckley, J.D.; Howe, P.R. Long-chain $\omega-3$ polyunsaturated fatty acids may be beneficial for reducing obesity-a review. Nutrients 2010, 2, 1212-1230.

15. Flachs, P.; Rossmeisl, M.; Bryhn, M.; Kopecky, J. Cellular and molecular effects of n-3 polyunsaturated fatty acids on adipose tissue biology and metabolism. Clin. Sci. 2009, 116.

16. Torres-Fuentes, C.; Schellekens, H.; Dinan, T.G.; Cryan, J.F. A natural solution for obesity: Bioactives for the prevention and treatment of weight gain. A review. Nutr. Neurosci. 2015, 18, 49-65. 
17. Puglisi, M.J.; Hasty, A.H.; Saraswathi, V. The role of adipose tissue in mediating the beneficial effects of dietary fish oil. J. Nutr. Biochem. 2011, 22, 101-108.

18. Kalupahana, N.S.; Claycombe, K.J.; Moustaid-Moussa, N. n-3 Fatty acids alleviate adipose tissue inflammation and insulin resistance: Mechanistic insights. Adv. Nutr. 2011, 2, 304-316.

19. Pinel, A.; Morio-Liondore, B.; Capel, F. n-3 Polyunsaturated fatty acids modulate metabolism of insulin-sensitive tissues: Implication for the prevention of type 2 diabetes. J. Physiol. Biochem. 2014, 70, 647-658.

20. Martinez-Fernandez, L.; Laiglesia, L.M.; Huerta, A.E.; Martinez, J.A.; Moreno-Aliaga, M.J. $\omega-3$ Fatty acids and adipose tissue function in obesity and metabolic syndrome. Prostaglandins Other Lipid Mediat. 2015.

21. Berge, J.P.; Barnathan, G. Fatty acids from lipids of marine organisms: Molecular biodiversity, roles as biomarkers, biologically active compounds, and economical aspects. Adv. Biochem. Eng. Biotechnol. 2005, 96, 49-125.

22. Rørå, A.M.B.; Ruyter, B.; Skorve, J.; Berge, R.; Slinning, K.-E. Influence of high content of dietary soybean oil on quality of large fresh, smoked and frozen atlantic salmon (Salmo salar). Aquac. Int. 2005, 13, 217-231.

23. Torstensen, B.E.; Bell, J.G.; Rosenlund, G.; Henderson, R.J.; Graff, I.E.; Tocher, D.R.; Lie, O.; Sargent, J.R. Tailoring of atlantic salmon (Salmo salar L.) flesh lipid composition and sensory quality by replacing fish oil with a vegetable oil blend. J. Agric. Food Chem. 2005, 53, 10166-10178.

24. Schuchardt, J.P.; Hahn, A. Bioavailability of long-chain $\omega-3$ fatty acids. Prostaglandins Leukot. Essent. Fat. Acids 2013, 89.

25. Burdge, G.C.; Finnegan, Y.E.; Minihane, A.M.; Williams, C.M.; Wootton, S.A. Effect of altered dietary $n-3$ fatty acid intake upon plasma lipid fatty acid composition, conversion of $\left[{ }^{13} \mathrm{C}\right] \alpha$-linolenic acid to longer-chain fatty acids and partitioning towards $\beta$-oxidation in older men. Br. J. Nutr. 2003, 90, 311-321.

26. Bolton-Smith, C.; Woodward, M.; Tavendale, R. Evidence for age-related differences in the fatty acid composition of human adipose tissue, independent of diet. Eur. J. Clin. Nutr. 1997, 51, 619-624.

27. Ogura, T.; Takada, H.; Okuno, M.; Kitade, H.; Matsuura, T.; Kwon, M.; Arita, S.; Hamazaki, K.; Itomura, M.; Hamazaki, T. Fatty acid composition of plasma, erythrocytes and adipose: Their correlations and effects of age and sex. Lipids 2010, 45, 137-144.

28. Walker, C.G.; Browning, L.M.; Mander, A.P.; Madden, J.; West, A.L.; Calder, P.C.; Jebb, S.A. Age and sex differences in the incorporation of EPA and DHA into plasma fractions, cells and adipose tissue in humans. Br. J. Nutr. 2014, 111, 679-689.

29. Browning, L.M.; Walker, C.G.; Mander, A.P.; West, A.L.; Madden, J.; Gambell, J.M.; Young, S.; Wang, L.; Jebb, S.A.; Calder, P.C. Incorporation of eicosapentaenoic and docosahexaenoic acids into lipid pools when given as supplements providing doses equivalent to typical intakes of oily fish. Am. J. Clin. Nutr. 2012, 96, 748-758. 
30. Katan, M.B.; Deslypere, J.P.; van Birgelen, A.P.; Penders, M.; Zegwaard, M. Kinetics of the incorporation of dietary fatty acids into serum cholesteryl esters, erythrocyte membranes, and adipose tissue: An 18-month controlled study. J. Lipid Res. 1997, 38, 2012-2022.

31. Leaf, D.A.; Connor, W.E.; Barstad, L.; Sexton, G. Incorporation of dietary $n-3$ fatty acids into the fatty acids of human adipose tissue and plasma lipid classes. Am. J. Clin. Nutr. 1995, 62, 68-73.

32. Mostad, I.L.; Bjerve, K.S.; Bjorgaas, M.R.; Lydersen, S.; Grill, V. Effects of $n-3$ fatty acids in subjects with type 2 diabetes: Reduction of insulin sensitivity and time-dependent alteration from carbohydrate to fat oxidation. Am. J. Clin. Nutr. 2006, 84, 540-550.

33. Gammelmark, A.; Madsen, T.; Varming, K.; Lundbye-Christensen, S.; Schmidt, E.B. Low-dose fish oil supplementation increases serum adiponectin without affecting inflammatory markers in overweight subjects. Nutr. Res. 2012, 32, 15-23.

34. Witt, P.M.; Christensen, J.H.; Ewertz, M.; Aardestrup, I.V.; Schmidt, E.B. The incorporation of marine $n-3$ PUFA into platelets and adipose tissue in pre- and postmenopausal women: A randomised, double-blind, placebo-controlled trial. Br. J. Nutr. 2010, 104, 318-325.

35. McQuaid, S.E.; Humphreys, S.M.; Hodson, L.; Fielding, B.A.; Karpe, F.; Frayn, K.N. Femoral adipose tissue may accumulate the fat that has been recycled as VLDL and nonesterified fatty acids. Diabetes 2010, 59, 2465-2473.

36. Kunesova, M.; Hlavaty, P.; Tvrzicka, E.; Stankova, B.; Kalouskova, P.; Viguerie, N.; Larsen, T.M.; van Baak, M.A.; Jebb, S.A.; Martinez, J.A.; et al. Fatty acid composition of adipose tissue triglycerides after weight loss and weight maintenance: The diogenes study. Physiol. Res. 2012, 61, 597-607.

37. Buckley, J.D.; Howe, P.R. Anti-obesity effects of long-chain $\omega-3$ polyunsaturated fatty acids. Obes. Rev. 2009, 10, 648-659.

38. Du, S.; Jin, J.; Fang, W.; Su, Q. Does fish oil have an anti-obesity effect in overweight/obese adults? A meta-analysis of randomized controlled trials. PLOS ONE 2015, 10, e0142652.

39. Sato, T.; Kameyama, T.; Ohori, T.; Matsuki, A.; Inoue, H. Effects of eicosapentaenoic acid treatment on epicardial and abdominal visceral adipose tissue volumes in patients with coronary artery disease. J. Atheroscler. Thromb. 2014, 21, 1031-1043.

40. Todorcevic, M.; Kjaer, M.A.; Djakovic, N.; Vegusdal, A.; Torstensen, B.E.; Ruyter, B. n-3 HUFAs affect fat deposition, susceptibility to oxidative stress, and apoptosis in Atlantic salmon visceral adipose tissue. Comp. Biochem. Physiol. B Biochem. Mol. Biol. 2009, 152, 135-143.

41. Ruzickova, J.; Rossmeisl, M.; Prazak, T.; Flachs, P.; Sponarova, J.; Veck, M.; Tvrzicka, E.; Bryhn, M.; Kopecky, J. w-3 PUFA of marine origin limit diet-induced obesity in mice by reducing cellularity of adipose tissue. Lipids 2004, 39, 1177-1185.

42. Camargo, A.; Meneses, M.E.; Perez-Martinez, P.; Delgado-Lista, J.; Jimenez-Gomez, Y.; Cruz-Teno, C.; Tinahones, F.J.; Paniagua, J.A.; Perez-Jimenez, F.; Roche, H.M.; et al. Dietary fat differentially influences the lipids storage on the adipose tissue in metabolic syndrome patients. Eur. J. Nutr. 2014, 53, 617-626. 
43. Guebre-Egziabher, F.; Debard, C.; Drai, J.; Denis, L.; Pesenti, S.; Bienvenu, J.; Vidal, H.; Laville, M.; Fouque, D. Differential dose effect of fish oil on inflammation and adipose tissue gene expression in chronic kidney disease patients. Nutrition 2013, 29, 730-736.

44. Itariu, B.K.; Zeyda, M.; Hochbrugger, E.E.; Neuhofer, A.; Prager, G.; Schindler, K.; Bohdjalian, A.; Mascher, D.; Vangala, S.; Schranz, M.; et al. Long-chain n-3 pufas reduce adipose tissue and systemic inflammation in severely obese nondiabetic patients: A randomized controlled trial. Am. J. Clin. Nutr. 2012, 96, 1137-1149.

45. Jimenez-Gomez, Y.; Cruz-Teno, C.; Rangel-Zuniga, O.A.; Peinado, J.R.; Perez-Martinez, P.; Delgado-Lista, J.; Garcia-Rios, A.; Camargo, A.; Vazquez-Martinez, R.; Ortega-Bellido, M.; et al. Effect of dietary fat modification on subcutaneous white adipose tissue insulin sensitivity in patients with metabolic syndrome. Mol. Nutr. Food Res. 2014, 58, 2177-2188.

46. Kratz, M.; Kuzma, J.N.; Hagman, D.K.; van Yserloo, B.; Matthys, C.C.; Callahan, H.S.; Weigle, D.S. N3 PUFAs do not affect adipose tissue inflammation in overweight to moderately obese men and women. J. Nutr. 2013, 143, 1340-1347.

47. Pena-Orihuela, P.; Camargo, A.; Rangel-Zuniga, O.A.; Perez-Martinez, P.; Cruz-Teno, C.; Delgado-Lista, J.; Yubero-Serrano, E.M.; Paniagua, J.A.; Tinahones, F.J.; Malagon, M.M.; et al. Antioxidant system response is modified by dietary fat in adipose tissue of metabolic syndrome patients. J. Nutr. Biochem. 2013, 24, 1717-1723.

48. Spencer, M.; Finlin, B.S.; Unal, R.; Zhu, B.; Morris, A.J.; Shipp, L.R.; Lee, J.; Walton, R.G.; Adu, A.; Erfani, R.; et al. w-3 Fatty acids reduce adipose tissue macrophages in human subjects with insulin resistance. Diabetes 2013, 62, 1709-1717.

49. Baillie, R.A.; Takada, R.; Nakamura, M.; Clarke, S.D. Coordinate induction of peroxisomal acyl-coa oxidase and UCP-3 by dietary fish oil: A mechanism for decreased body fat deposition. Prostaglandins Leukot. Essent. Fat. Acids 1999, 60, 351-356.

50. Belzung, F.; Raclot, T.; Groscolas, R. Fish oil n-3 fatty acids selectively limit the hypertrophy of abdominal fat depots in growing rats fed high-fat diets. Am. J. Physiol. 1993, 264, R1111-R1118.

51. Flachs, P.; Horakova, O.; Brauner, P.; Rossmeisl, M.; Pecina, P.; Franssen-van Hal, N.; Ruzickova, J.; Sponarova, J.; Drahota, Z.; Vlcek, C.; et al. Polyunsaturated fatty acids of marine origin upregulate mitochondrial biogenesis and induce $\beta$-oxidation in white fat. Diabetologia 2005, 48, 2365-2375.

52. Hainault, I.; Carolotti, M.; Hajduch, E.; Guichard, C.; Lavau, M. Fish oil in a high lard diet prevents obesity, hyperlipemia, and adipocyte insulin resistance in rats. Ann. N. Y. Acad. Sci. 1993, 683, 98-101.

53. Oh, D.Y.; Talukdar, S.; Bae, E.J.; Imamura, T.; Morinaga, H.; Fan, W.; Li, P.; Lu, W.J.; Watkins, S.M.; Olefsky, J.M. GPR120 is an $\omega-3$ fatty acid receptor mediating potent anti-inflammatory and insulin-sensitizing effects. Cell 2010, 142, 687-698.

54. Perez-Matute, P.; Perez-Echarri, N.; Martinez, J.A.; Marti, A.; Moreno-Aliaga, M.J. Eicosapentaenoic acid actions on adiposity and insulin resistance in control and high-fat-fed rats: Role of apoptosis, adiponectin and tumour necrosis factor- $\alpha$. Br. J. Nutr. 2007, 97, 389-398. 
55. Saraswathi, V.; Gao, L.; Morrow, J.D.; Chait, A.; Niswender, K.D.; Hasty, A.H. Fish oil increases cholesterol storage in white adipose tissue with concomitant decreases in inflammation, hepatic steatosis, and atherosclerosis in mice. J. Nutr. 2007, 137, 1776-1782.

56. Barber, E.; Sinclair, A.J.; Cameron-Smith, D. Comparative actions of $\omega-3$ fatty acids on in-vitro lipid droplet formation. Prostaglandins Leukot. Essent. Fat. Acids 2013, 89, 359-366.

57. DeClercq, V.; d'Eon, B.; McLeod, R.S. Fatty acids increase adiponectin secretion through both classical and exosome pathways. Biochim. Biophys. Acta 2015, 1851, 1123-1133.

58. Guo, W.; Xie, W.; Lei, T.; Hamilton, J.A. Eicosapentaenoic acid, but not oleic acid, stimulates $\beta$-oxidation in adipocytes. Lipids 2005, 40, 815-821.

59. Kim, H.K.; Della-Fera, M.; Lin, J.; Baile, C.A. Docosahexaenoic acid inhibits adipocyte differentiation and induces apoptosis in 3T3-L1 preadipocytes. J. Nutr. 2006, 136, 2965-2969.

60. Kusunoki, C.; Yang, L.; Yoshizaki, T.; Nakagawa, F.; Ishikado, A.; Kondo, M.; Morino, K.; Sekine, O.; Ugi, S.; Nishio, Y.; et al. $\omega$-3 Polyunsaturated fatty acid has an anti-oxidant effect via the Nrf-2/HO-1 pathway in 3T3-L1 adipocytes. Biochem. Biophys. Res. Commun. 2013, 430, 225-230.

61. Lorente-Cebrian, S.; Bustos, M.; Marti, A.; Martinez, J.A.; Moreno-Aliaga, M.J. Eicosapentaenoic acid up-regulates apelin secretion and gene expression in 3T3-L1 adipocytes. Mol. Nutr. Food Res. 2010, 54, S104-S111.

62. Murali, G.; Desouza, C.V.; Clevenger, M.E.; Ramalingam, R.; Saraswathi, V. Differential effects of eicosapentaenoic acid and docosahexaenoic acid in promoting the differentiation of 3T3-L1 preadipocytes. Prostaglandins Leukot. Essent. Fat. Acids 2014, 90, 13-21.

63. Murumalla, R.K.; Gunasekaran, M.K.; Padhan, J.K.; Bencharif, K.; Gence, L.; Festy, F.; Cesari, M.; Roche, R.; Hoareau, L. Fatty acids do not pay the toll: Effect of SFA and PUFA on human adipose tissue and mature adipocytes inflammation. Lipids Health Dis. 2012, $11,175$.

64. Oster, R.T.; Tishinsky, J.M.; Yuan, Z.; Robinson, L.E. Docosahexaenoic acid increases cellular adiponectin mRNA and secreted adiponectin protein, as well as PPAR $\gamma$ mRNA, in 3T3-L1 adipocytes. Appl. Physiol. Nutr. Metab. 2010, 35, 783-789.

65. Prostek, A.; Gajewska, M.; Kamola, D.; Balasinska, B. The influence of EPA and DHA on markers of inflammation in 3T3-L1 cells at different stages of cellular maturation. Lipids Health Dis. 2014, 13, 3.

66. Tishinsky, J.M.; Ma, D.W.; Robinson, L.E. Eicosapentaenoic acid and rosiglitazone increase adiponectin in an additive and PPAR $\gamma$-dependent manner in human adipocytes. Obesity 2011, 19, 262-268. 
67. Vaidya, H.; Cheema, S.K. Arachidonic acid has a dominant effect to regulate lipogenic genes in 3T3-L1 adipocytes compared to $\omega$-3 fatty acids. Food Nutr. Res. 2015, 59, 25866.

68. Wang, Y.C.; Kuo, W.H.; Chen, C.Y.; Lin, H.Y.; Wu, H.T.; Liu, B.H.; Chen, C.H.; Mersmann, H.J.; Chang, K.J.; Ding, S.T. Docosahexaenoic acid regulates serum amyloid a protein to promote lipolysis through down regulation of perilipin. J. Nutr. Biochem. 2010, 21, 317-324.

69. Wojcik, C.; Lohe, K.; Kuang, C.; Xiao, Y.; Jouni, Z.; Poels, E. Modulation of adipocyte differentiation by $\omega-3$ polyunsaturated fatty acids involves the ubiquitin-proteasome system. J. Cell. Mol. Med. 2014, 18, 590-599.

70. Hodson, L.; Humphreys, S.M.; Karpe, F.; Frayn, K.N. Metabolic signatures of human adipose tissue hypoxia in obesity. Diabetes 2013, 62, 1417-1425.

71. Ji, H.; Li, J.; Liu, P. Regulation of growth performance and lipid metabolism by dietary n-3 highly unsaturated fatty acids in juvenile grass carp, ctenopharyngodon idellus. Comp. Biochem. Physiol. B Biochem. Mol. Biol. 2011, 159, 49-56.

72. Liu, P.; Li, C.; Huang, J.; Ji, H. Regulation of adipocytes lipolysis by $n-3$ HUFA in grass carp (Ctenopharyngodon idellus) in vitro and in vivo. Fish. Physiol. Biochem. 2014, 40, 1447-1460.

73. Desai, M.; Beall, M.; Ross, M.G. Developmental origins of obesity: Programmed adipogenesis. Curr. Diab. Rep. 2013, 13, 27-33.

74. McMillen, I.C.; Robinson, J.S. Developmental origins of the metabolic syndrome: Prediction, plasticity, and programming. Physiol. Rev. 2005, 85, 571-633.

75. Catalano, P.M.; Ehrenberg, H.M. The short- and long-term implications of maternal obesity on the mother and her offspring. BJOG 2006, 113, 1126-1133.

76. Sewell, M.F.; Huston-Presley, L.; Super, D.M.; Catalano, P. Increased neonatal fat mass, not lean body mass, is associated with maternal obesity. Am. J. Obstet. Gynecol. 2006, 195, $1100-1103$.

77. Muhlhausler, B.S.; Gibson, R.A.; Makrides, M. The effect of maternal $\omega-3$ long-chain polyunsaturated fatty acid ( $n$-3 LCPUFA) supplementation during pregnancy and/or lactation on body fat mass in the offspring: A systematic review of animal studies. Prostaglandins Leukot. Essent. Fat. Acids 2011, 85, 83-88.

78. Muhlhausler, B.S.; Miljkovic, D.; Fong, L.; Xian, C.J.; Duthoit, E.; Gibson, R.A. Maternal $\omega-3$ supplementation increases fat mass in male and female rat offspring. Front. Genet. 2011, 2, 48.

79. Hilton, C.; Karpe, F.; Pinnick, K.E. Role of developmental transcription factors in white, brown and beige adipose tissues. Biochim. Biophys. Acta 2015, 1851, 686-696.

80. Pinnick, K.E.; Neville, M.J.; Fielding, B.A.; Frayn, K.N.; Karpe, F.; Hodson, L. Gluteofemoral adipose tissue plays a major role in production of the lipokine palmitoleate in humans. Diabetes 2012, 61, 1399-1403.

81. Pinnick, K.E.; Nicholson, G.; Manolopoulos, K.N.; McQuaid, S.E.; Valet, P.; Frayn, K.N.; Denton, N.; Min, J.L.; Zondervan, K.T.; Fleckner, J.; et al. Distinct developmental profile of lower-body adipose tissue defines resistance against obesity-associated metabolic complications. Diabetes 2014, 63, 3785-3797. 
82. Lee, M.S.; Kwun, I.S.; Kim, Y. Eicosapentaenoic acid increases lipolysis through up-regulation of the lipolytic gene expression and down-regulation of the adipogenic gene expression in 3T3-L1 adipocytes. Genes Nutr. 2008, 2, 327-330.

83. Todorcevic, M.; Vegusdal, A.; Gjoen, T.; Sundvold, H.; Torstensen, B.E.; Kjaer, M.A.; Ruyter, B. Changes in fatty acids metabolism during differentiation of atlantic salmon preadipocytes; effects of $n-3$ and $n-9$ fatty acids. Biochim. Biophys. Acta 2008, 1781, 326-335.

84. Wendel, M.; Heller, A.R. Anticancer actions of $\omega-3$ fatty acids-Current state and future perspectives. Anticancer Agents Med. Chem. 2009, 9, 457-470.

85. Prins, J.B.; Walker, N.I.; Winterford, C.M.; Cameron, D.P. Apoptosis of human adipocytes in vitro. Biochem. Biophys. Res. Commun. 1994, 201, 500-507.

86. Prins, J.B.; O'Rahilly, S. Regulation of adipose cell number in man. Clin. Sci. 1997, 92, 3-11.

87. Nelson-Dooley, C.; Della-Fera, M.A.; Hamrick, M.; Baile, C.A. Novel treatments for obesity and osteoporosis: Targeting apoptotic pathways in adipocytes. Curr. Med. Chem. 2005, 12, 2215-2225.

88. Wyllie, A.H. Apoptosis: An overview. Br. Med. Bull. 1997, 53, 451-465.

89. Tocher, D.R. Metabolism and functions of lipids and fatty acids in teleost fish. Rev. Fish. Sci. 2003, 11, 107-184.

90. Abele, D.; Puntarulo, S. Formation of reactive species and induction of antioxidant defence systems in polar and temperate marine invertebrates and fish. Comp. Biochem. Physiol. A Mol. Integr. Physiol. 2004, 138, 405-415.

91. Biondo, P.D.; Brindley, D.N.; Sawyer, M.B.; Field, C.J. The potential for treatment with dietary long-chain polyunsaturated $n-3$ fatty acids during chemotherapy. J. Nutr. Biochem. 2008, 19, 787-796.

92. Calviello, G.; Palozza, P.; Maggiano, N.; Piccioni, E.; Franceschelli, P.; Frattucci, A.; Di Nicuolo, F.; Bartoli, G.M. Cell proliferation, differentiation, and apoptosis are modified by $n-3$ polyunsaturated fatty acids in normal colonic mucosa. Lipids 1999, 34, 599-604.

93. Todorcevic, M.; Skugor, S.; Ruyter, B. Alterations in oxidative stress status modulate terminal differentiation in atlantic salmon adipocytes cultivated in media rich in $n-3$ fatty acids. Comp. Biochem. Physiol. B Biochem. Mol. Biol. 2010, 156, 309-318.

94. Maassen, J.A.; Romijn, J.A.; Heine, R.J. Fatty acid-induced mitochondrial uncoupling in adipocytes as a key protective factor against insulin resistance and $\beta$ cell dysfunction: Do adipocytes consume sufficient amounts of oxygen to oxidise fatty acids? Diabetologia 2008, 51, 907-908.

95. Bouwman, F.G.; Wang, P.; van Baak, M.; Saris, W.H.; Mariman, E.C. Increased $\beta$-oxidation with improved glucose uptake capacity in adipose tissue from obese after weight loss and maintenance. Obesity 2014, 22, 819-827.

96. Couet, C.; Delarue, J.; Ritz, P.; Antoine, J.M.; Lamisse, F. Effect of dietary fish oil on body fat mass and basal fat oxidation in healthy adults. Int. J. Obes. Relat. Metab. Disord. 1997, $21,637-643$. 
97. Lorente-Cebrian, S.; Bustos, M.; Marti, A.; Martinez, J.A.; Moreno-Aliaga, M.J. Eicosapentaenoic acid stimulates amp-activated protein kinase and increases visfatin secretion in cultured murine adipocytes. Clin. Sci. 2009, 117, 243-249.

98. Flachs, P.; Rossmeisl, M.; Kuda, O.; Kopecky, J. Stimulation of mitochondrial oxidative capacity in white fat independent of UCP1: A key to lean phenotype. Biochim. Biophys. Acta 2013, 1831, 986-1003.

99. Zhao, M.; Chen, X. Eicosapentaenoic acid promotes thermogenic and fatty acid storage capacity in mouse subcutaneous adipocytes. Biochem. Biophys. Res. Commun. 2014, 450, 1446-1451.

100. Hosogai, N.; Fukuhara, A.; Oshima, K.; Miyata, Y.; Tanaka, S.; Segawa, K.; Furukawa, S.; Tochino, Y.; Komuro, R.; Matsuda, M.; et al. Adipose tissue hypoxia in obesity and its impact on adipocytokine dysregulation. Diabetes 2007, 56, 901-911.

101. Trayhurn, P.; Wood, I.S. Adipokines: Inflammation and the pleiotropic role of white adipose tissue. Br. J. Nutr. 2004, 92, 347-355.

102. Wood, I.S.; de Heredia, F.P.; Wang, B.; Trayhurn, P. Cellular hypoxia and adipose tissue dysfunction in obesity. Proc. Nutr. Soc. 2009, 68, 370-377.

103. Ye, J.; Gao, Z.; Yin, J.; He, Q. Hypoxia is a potential risk factor for chronic inflammation and adiponectin reduction in adipose tissue of $o b / o b$ and dietary obese mice. Am. J. Physiol. Endocrinol. Metab. 2007, 293, E1118-E1128.

104. Todoric, J.; Loffler, M.; Huber, J.; Bilban, M.; Reimers, M.; Kadl, A.; Zeyda, M.; Waldhausl, W.; Stulnig, T.M. Adipose tissue inflammation induced by high-fat diet in obese diabetic mice is prevented by $n-3$ polyunsaturated fatty acids. Diabetologia 2006, 49, 2109-2119.

105. Fantuzzi, G. Adiponectin and inflammation: Consensus and controversy. J. Allergy Clin. Immunol. 2008, 121, 326-330.

106. Romacho, T.; Glosse, P.; Richter, I.; Elsen, M.; Schoemaker, M.H.; van Tol, E.A.; Eckel, J. Nutritional ingredients modulate adipokine secretion and inflammation in human primary adipocytes. Nutrients 2015, 7, 865-886.

107. Lorente-Cebrian, S.; Perez-Matute, P.; Martinez, J.A.; Marti, A.; Moreno-Aliaga, M.J. Effects of eicosapentaenoic acid (EPA) on adiponectin gene expression and secretion in primary cultured rat adipocytes. J. Physiol. Biochem. 2006, 62, 61-69.

108. Murata, M.; Kaji, H.; Takahashi, Y.; Iida, K.; Mizuno, I.; Okimura, Y.; Abe, H.; Chihara, K. Stimulation by eicosapentaenoic acids of leptin mrna expression and its secretion in mouse 3T3-L1 adipocytes in vitro. Biochem. Biophys. Res. Commun. 2000, 270, 343-348.

109. Perez-Matute, P.; Marti, A.; Martinez, J.A.; Fernandez-Otero, M.P.; Stanhope, K.L.; Havel, P.J.; Moreno-Aliaga, M.J. Eicosapentaenoic fatty acid increases leptin secretion from primary cultured rat adipocytes: Role of glucose metabolism. Am. J. Physiol. Regul. Integr. Comp. Physiol. 2005, 288, R1682-R1688. 
110. Reseland, J.E.; Haugen, F.; Hollung, K.; Solvoll, K.; Halvorsen, B.; Brude, I.R.; Nenseter, M.S.; Christiansen, E.N.; Drevon, C.A. Reduction of leptin gene expression by dietary polyunsaturated fatty acids. J. Lipid Res. 2001, 42, 743-750.

111. Aranceta, J.; Perez-Rodrigo, C. Recommended dietary reference intakes, nutritional goals and dietary guidelines for fat and fatty acids: A systematic review. Br. J. Nutr. 2012, 107, S8-S22. 
MDPI AG

St. Alban-Anlage 66

4052 Basel, Switzerland

Tel. +41616837734

Fax +41 613028918

http://www.mdpi.com

JCM Editorial Office

E-mail: jcm@mdpi.com

http://www.mdpi.com/journal/jcm

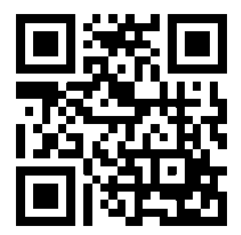



MDPI AG

St. Alban-Anlage 66

4052 Basel

Switzerland

Tel: +41 616837734

Fax: +41 613028918 